\title{
Dogs, working memory and educational achievement: Barking up the wrong tree or an effective mechanism for facilitating cognitive acuity?
}

Janet Oostendorp Godfrey

A thesis submitted to The University of Gloucestershire in accordance with the requirements of the degree of Doctor of Philosophy in the School of Natural and Social Sciences 


\section{Declaration:}

I declare that the work in this thesis was carried out in accordance with the regulations of the University of Gloucestershire and is original except where indicated by specific reference in the text. No part of this thesis has been submitted as part of any other academic award. The thesis has not been presented to any other education institution in the United Kingdom or overseas.

Any views expressed in the thesis are those of the author and in no way represent those of the University.

Signed:

Date: $29^{\text {th }}$ September 2020

doi: $10.46289 / J D I O 5195$ 


\section{Abstract:}

A mixed methods convergent design was used to investigate the involvement and role of a school dog with 13 , seven year olds and 24 university students over three phases: a desk based survey; the identification of children's working memory skills used in the dog's presence when reading; and comparing maths and vocabulary school based tasks in both 'live' and 'Virtual Reality' (VR) settings.

The findings provided insights into how the dogs' presence affected learning within the classroom. Cross-age correlations were found for specific working memory skills between the adults and children. The data from both age groups revealed a significant effect of a live (as opposed to VR) dog's presence for the language-based activities and suggested that there is potential for dogs to increase resilience and confidence in mathematical tasks. Using 'virtual reality dogs', significant effects were found for language abilities, corroborating previous research. Results clearly indicated that it is primarily the individual learner's initial response to the task itself which determines the personal achievement and attainment, with the presence of a dog having a secondary effect. Further research is required into establishing the greatest benefits from working with a dog with respect to the measurement of progress mediated by social, academic and learning needs. The finding in this research that the effect of animal assisted learning is somewhat equivocal and idiosyncratic has implications for the widely used 'read to dogs' schemes in schools, together with the hours, working conditions and the perceived necessity of having full time school dogs. Animal assisted learning, in this study, showed benefits for some participants, but the effect was not universal. Such a finding suggests that a specific, individual, specialist academic teaching approach may be required to gain 
the greatest benefit from animal assisted intervention and animal assisted education in the future. 


\section{Acknowledgements:}

There are many people to thank for helping me through the PhD 'experience.' First and foremost, to husband Frits; for checking my insanity, holding my hand and saying, 'go for it.' To my Mum and Dad; for my early training in how to be 'resilient' and how to stay committed to a long-term project; and to our two Labradors, Maisie and Isla; for their general 'Labrador' enthusiasm, and willingness to take part in all things exciting.

Thanks, must go to: Prof. Graham Edgar (Chief supervisor) Steadfast in advice from start to finish over seven years; Dr Alex Masardo (Supervisor) for his advice and timekeeping; Dave Brookes, for his technical skills, patience, support and time with the VR equipment; and to Sue Turner and the library teams, who pointed me in the right directions for research articles and skills.

Thanks, must also go to all the participants themselves: Jerry (Can't give surname due to anonymity reasons!), handler and ally in all visiting situations; Matt the dog, a whiskery hero and a real gentleman of dogs (currently now in retirement at 15 years old); and to Therapy Dogs Nationwide.

Strong mentions of thanks must go to: Lucy Llewellyn, for acting as a 'sound board;' the A.P.D.T.UK Education team; and to Tigs Smith of "Two Happy Tails," for walking Maisie and Isla to keep me sane while writing.

Finally, a huge thank you to Soumya from Endnote - for rescuing my library twice, despite power cuts and the monsoon, an $11^{\text {th }}$ hour life saver! Top job! 


\section{Table of Contents}

List of Tables

List of Figures

List of Appendices

Chapter 1

Introduction

1.1 Background 30

1.2 Why use dogs? 31

1.3 What is Animal Assisted Intervention? 34

1.4 The 'roots' of Animal Assisted Education, (AAE) 36

1.5 Differences in research styles and stances......... 38

1.6 Research Rationale .... 40

1.7 Aims, definition and scope of the research: 46

1.8 The Structure of the Thesis. 46

Chapter 2 48

Literature Review 48

2.1 Introduction 48

2.2 What is working memory? 49

2.3 How working memory affects school subject areas 52

2.4 Emotions and working memory. 54

2.5 Anxiety and Stress. 55

2.6 Links to school achievement in England 56 
2.8 Identification of "need"

2.9 Introducing Animal Assisted Intervention (AAI).

2.10 Benefits of Human Animal Intervention for those with identified 'needs.'

2.11 Overall Evidence for Animal Interactions in schools .....................................................66

2.12 Issues for Teaching from these reviews

2.13 Truth Effect .70

2.14 Research into Reading using Dogs. .71

2.15 Past research into the presence of the dog for cognitive acuity .76

2.16 Use of Virtual Reality .84

2.17 Summary

Chapter 3

Methodology

3.1 Phase 1 - Online School Website Desk Based Survey: June 2016 - August 2016 .93

3.2 Phase 2-Children's Research (School based): September - December 2016 .94

3.3 Phase 3 Adult based research (University students): May - October 2019 .94

3.4 Thesis Analysis Section . .95

3.5 Total Participant Characteristics. .96

3.6 Recruitment of Participants .96

3.7 The school .97

3.8 Construct Validity 97

3.9 Robustness .98 
Quantitative

3.10.2 Skin Conductance "Pip"

3.10.3 Standardised Tests

3.10.4 Non-Standardised Tests 101

Qualitative Data 102

3.10.5 Video observations 102

3.10.6 Questionnaires 103

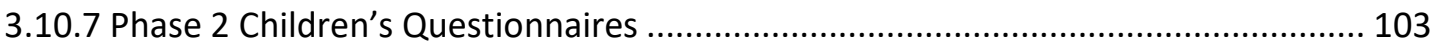

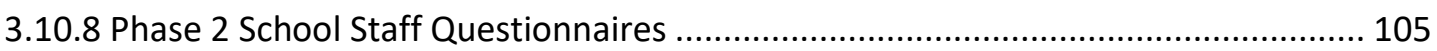

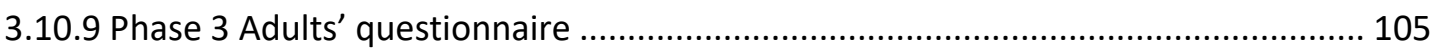

3.11 Other tools, techniques and methods used in the Phase 2 children's research 106

3.11.1 Reading Scheme 106

3.11.2 Selection of suitable rhyme 107

3.11.3 Miscue Analysis 108

3.12 Other tools, techniques and methods used in the Phase 3 Adults project 108

3.12.1 VR Headset. 108

3.12.2 Preparation of Materials... 109

3.12.3 Vocabulary and Mental Maths Questions 109

3.12.4 Creation of the films used for VR and "Live" Research. 110

3.13 The Dogs 111 
3.14.1 Permissions

3.14.2 Anonymity

3.14.3 Respect.

3.14.4 Disclosure and Barring Services check .

3.15.5 Health and Safety

3.14.6 The role of the handler

3.16 Risk Assessment

3.18 Summary.

Background Survey: School dogs in Special Educational Settings in England (2016), revisited 2018.

4.1 Rationale for Background Survey

4.2 Why have dogs been brought into schools?

4.3 Reason 1: Interpretation or "assumption" made of the word "therapy"

4.4 Reason 2: Quality of training 
4.8 Summary

Chapter 5 (Research Phase 2)

School based Investigation: Working Memory Tests

5.0 The Problem

5.1 Tests available for teacher use

5.2 How can teachers recognise working memory difficulties in the classroom?

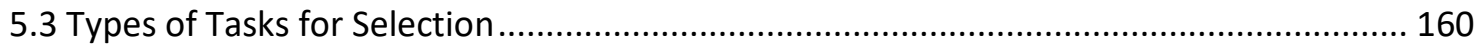

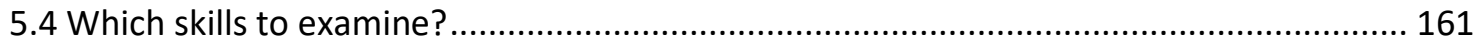

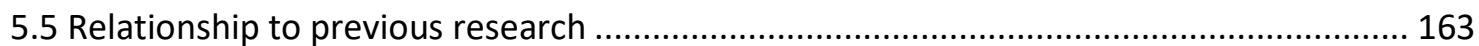

5.6 Preparation of Working Memory Sub Tests for 2016 School-Based Project ...................... 164

5.6.1 Test Details

5.6.2 TOMAL 2 (Test of Memory and Learning, version 2, Reynolds and Voress, 2007)..... 167

5.6.3 WRAT-4 (Wide Range Achievement Test, Wilkinson and Robertson, 2006) 169

5.6.4 TVPS-3 (Test of Visual Perception Skills, Version 3, Martin, 2006) ..... 171

5.6.5 PhAB (Phonological Assessment Battery, Frederickson, Frith and Reason, 1997) ..... 171

5.6.7 Test Comparability 173

5.6.8 Explanation of the Standardised Test Scores 174

5.6.9 Level Descriptors

5.7.0 Design

5.7.1 Ethical Permissions and Health and Safety 
5.8.0 Results of Specific Working Memory Tests

5.8.1 Executive working Memory Group Scores

5.8.2 Working Memory Spans (backwards) 
6.1.3 Reading without dog present session.

6.1.3. Reading Attainment

6.3.5 Which group made most Ratio Gain progress? ....................................................... 217

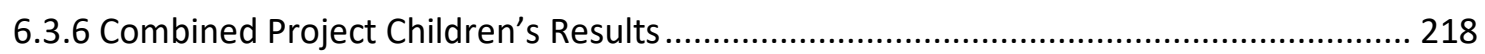

6.4 Comparisons to other School Dog Reading Projects ................................................... 220

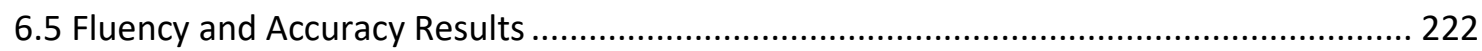

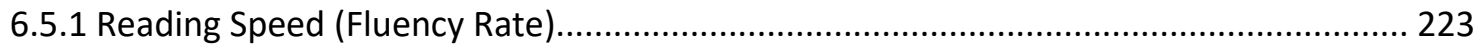

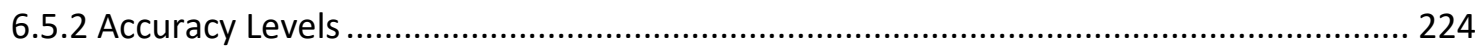

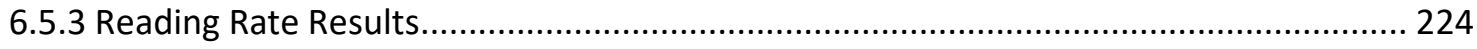


6.7 Comparison of Reading Rhyme Speed with dog present. 
(Age during project: seven years and seven months to seven years and nine months) ......... 246

7.2.1 Introduction and results from questionnaire ......................................................... 246

7.2.2 Results from the non-repeated working memory tests, due to time........................ 247

7.2.3 Working Memory Tests with and without dog present....................................... 247

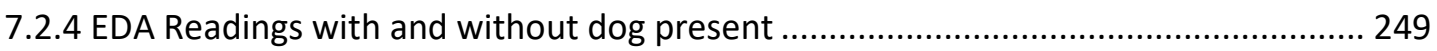

7.2.5 EDA Box and Whisker Charts for all three tasks ................................................... 250

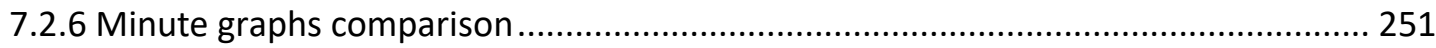

7.2.7 Reading Accuracy and Words Per Minute (WPM) rate............................................ 252

7.2.8 Reading Attainment in both reading age and standardised scores......................... 253

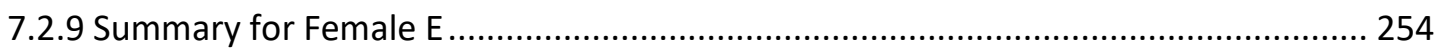

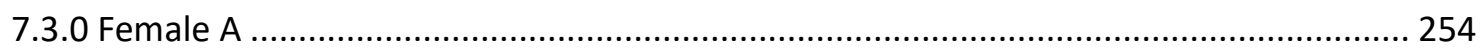

(Age during project: seven years, nine months to eight years) ............................................254

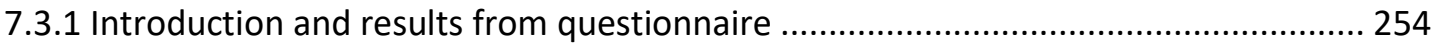

7.3.2 Results from non-repeated working memory tests, due to time .............................. 255

7.3.3 Working Memory with and without dog present ...................................................... 256

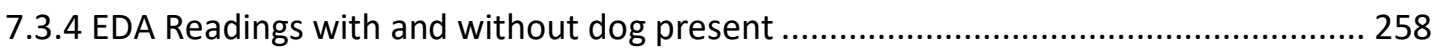

7.3.4 Female A Minute Graphs Comparison ................................................................ 260

7.3.5 Reading Accuracy and Words per Minute (WPM) rate ......................................... 261

7.3.6 Reading Attainment in both reading age and standardised scores........................... 262

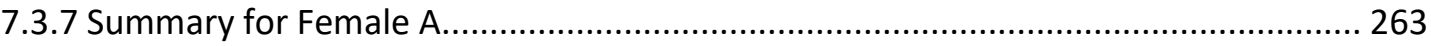

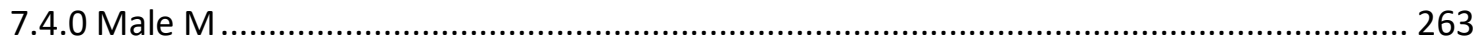


7.4.1 Introduction and results from questionnaire

7.4.2 Results from the non-repeated working memory tests, due to time

7.4.3 Working Memory with and without dog present

7.4.4 Electrodermal Activity Readings with and without dog present.

7.5.0 Male C

(Age during project: seven years, eight months to seven years ten months)

7.5.1 Introduction and results from questionnaire

7.5.2 Results from non-repeated working memory tests, due to time.

7.5.3 Working Memory Tests with and without dog present

7.5.4 Electrodermal Activity Readings with and without dog present.

7.5.5 EDA Box and Whisker charts for all three tasks 
7.6.1 Introduction and results from questionnaire

7.6.2 Results from the non-repeated working memory tests, due to time

7.6.3 Working Memory with dog present and absent

7.6.4 EDA readings with and without the dog present.

7.6.5 EDA Box and Whisker charts for all three tasks.....

7.6.6 Female G Minute Graphs Comparison.

7.6.7 Reading Accuracy and Words per Minute Rate (WPM).

7.6.8 Reading Attainment in both reading age and standardised scores

7.6.9 Summary

7.7.0 Male $\mathrm{H}$

(Age during project: seven years, ten months to eight years, one month).

7.7.1 Introduction and results from questionnaire

7.7.2 Results from the non-repeated working memory tests due to time

7.7.3 Working Memory with dog both present and absent

7.7.4 EDA readings with and without the dog present

7.7.5 EDA Box and Whisker charts for all three tasks

7.7.6 Male H Minute graphs comparison

7.7.7 Reading Accuracy and Words Per Minute (WPM) rate

7.7.8 Reading Attainment in both reading age and standardised scores

7.7.9 Summary for Male $\mathrm{H}$ 
University Investigation: School based tasks with adult students using 'live' and 'virtual reality' dog presence

8.2 Preparation and set up.

8.21 Participants:

8.24 Tasks:

8.27 Virtual Reality Camera / Headset:

8.28 Issues with the preparation stage: 
8.8.2 Questionnaire Preferences - Preferred condition for Relaxation or Concentration...... 319

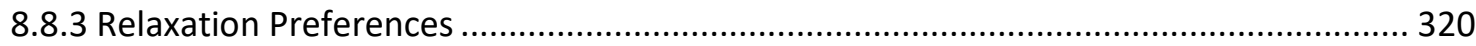

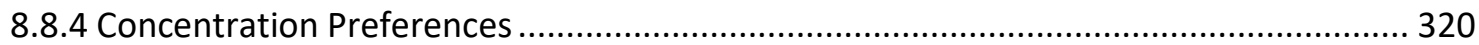

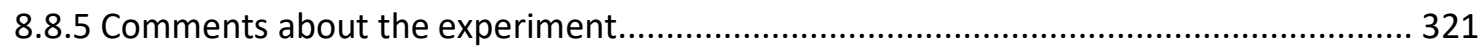

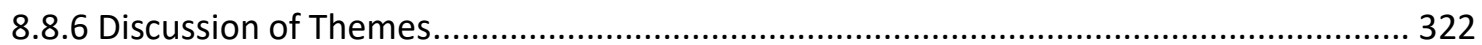

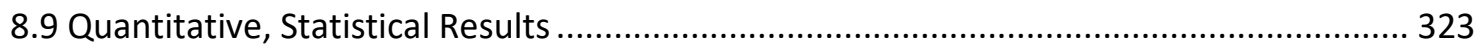

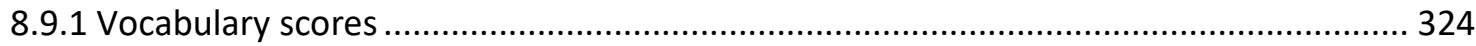

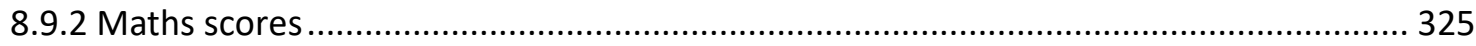

8.93 Electrodermal activity (EDA) scores - entire session.................................................. 327

8.94 Electrodermal activity (EDA) scores - vocabulary sessions only. ................................... 328

8.95 Electrodermal activity (EDA) scores - maths sessions only....................................... 330

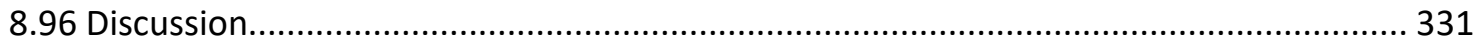

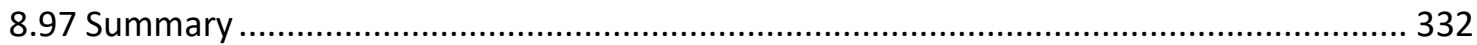

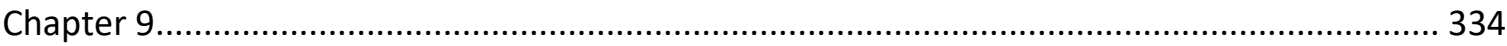

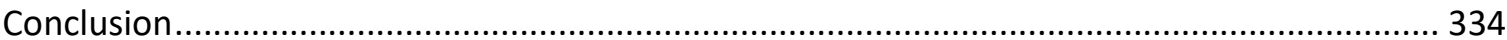

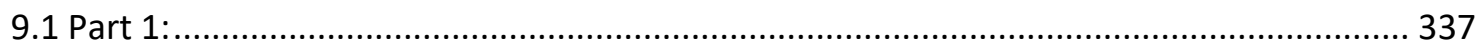

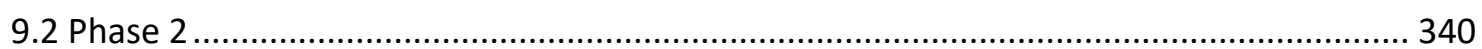

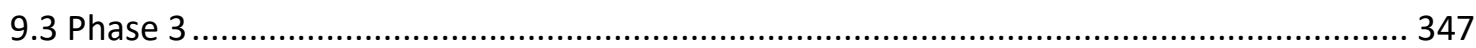

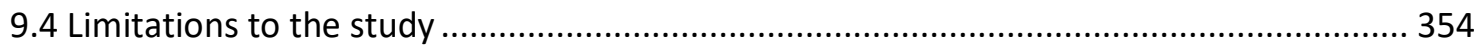

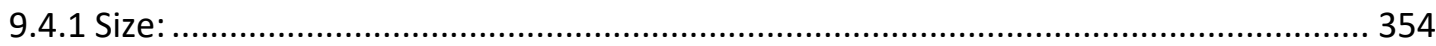

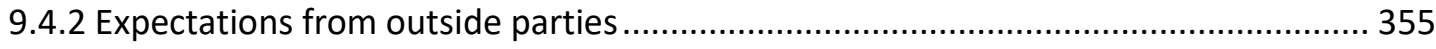


9.4.4 Selection of participants.

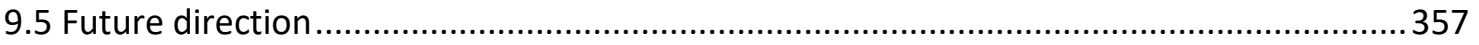

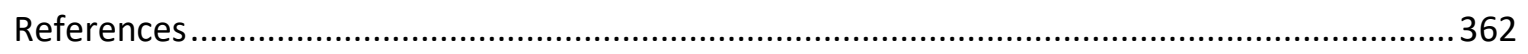

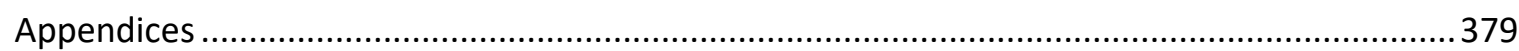




\section{List of Tables}

Table 1: Participant characteristics for the research

Table 2: Equivalent reading ages and reading schemes for the children's books 107

Table 3: School based roles of the dog handlers in the school sample

Table 4: Top five reasons why dogs were brought into this sample of special schools

Table 5: Guidelines for animals used in animal assisted interventions in schools

Table 6: Table of commercial working memory tests available to qualified teachers (2016)..... 165

Table 7: Selected sub-tests (based on memory skills used in reading and mathematics)

Table 8: Standardisation across chosen commercial tests

Table 9: Explanation of level descriptors

Table 10: Working memory skills scores: with and without dog standardised scores

Table 11: Backward spans results

Table 12: Digits and letters forward spans

Table 13: Combined effects of tests on group skills

Table 14: Final Participant Numbers for School-based Project

Table 15: Children's answers to their questionnaire

Table 16: Adult responses to the question about subsequent changes in behaviour after reading to the dog

Table 17: Children in case study group, within SEN group who read to the dog

Table 18: Standardised scores for the children in the case study group and within the SEN group who read to the dog

Table 19: Reading scores for those who were in the SEN group but not in the focused case study group

Table 20: Standardised scores for those children who were in SEN group but not in the focused study group 213 
Table 21: Reading scores for those who did not read to the dog, but were part of the SEN group (Control)

Table 22: Standardised scores for those who did not read to the dog but were part of the SEN group (Control)

Table 23: Total progress in reading ages for "Paws and Read" 215

Table 24: Total progress in standardised scores for "Paws and Read" 215

Table 25: Individual Ratio Gain scores per group

Table 26: Table of focus study case children's reading level choices for reading to the dog over time 219

Table 27: Literacy and numeracy interventions used in schools Table 221

Table 28: Average words per minute rate (wpm) for the reading passages 224

Table 29: Averaged electrodermal activity (EDA) for the focus case study group's reading sessions 225

Table 30: Accuracy scores for the reading passages 226

Table 31: Reading speed rate comparisons between rhyme and prose without dog present....229 Table 32: Average sessional electrodermal activity rates for rhyme, and prose without dog present 230

Table 33: Comparison of accuracy rates between reading rhyme, and reading prose, without dog present 230

Table 34: Reading rate comparisons between unknown rhyme and known prose with the dog231 Table 35: EDA comparisons between reading unknown rhyme to reading known prose with the $\operatorname{dog}$. 232

Table 36: Comparisons in accuracy rate between reading unknown rhyme and reading known prose with the dog 232

Table 37: Male A's standardised scores for the working memory tests unable to be repeated with the dog present 238 
Table 38: Averaged EDA readings for Male A

Table 39: Male A Comparative measure for the box and whisker charts ................................ 242

Table 40: Reading accuracy and words per minute rate for Male A ....................................... 244

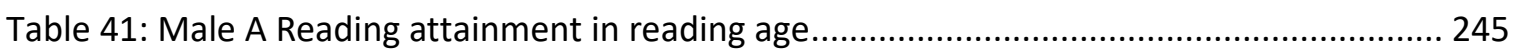

Table 42: Male A Reading attainment in standardised scores ................................................ 245

Table 43: Female E Standardised scores for the working memory tests unable to be repeated with the dog present.

Table 44: Averaged EDA readings for Female E.

Table 45: Female E comparative measures for box and whisker chart. 250

Table 46: Female E Reading speed and accuracy 252

Table 47: Overall reading attainment for Female $\mathrm{E}$ 253

Table 48: Standardised scores attainment for Female E 253

Table 49: Female A Standardised score for working memory tests unable to be repeated with the dog present 255

Table 50: Averaged EDA readings for Female A 258

Table 51: Female E Comparative measures for the box and whisker charts 259

Table 52: Female A Reading accuracy and words per minute rate 261

Table 53: Female A Reading attainment in reading age 262

Table 54: Female A Reading Attainment in Standardised Scores. 262

Table 55: Male M Standardised Scores for working memory tests unable to be completed with dog present 264

Table 56: Male M Averaged EDA readings 267

Table 57: Male M Comparative measures for box and whisker chart 268

Table 58: Male M Reading accuracy and words per minute rate 270

Table 59: Male M Reading attainment in reading age

Table 60: Male M Reading attainment in Standardised Scores 
Table 61: Male C Standardised Scores for the working memory tests unable to be repeated with the dog present

Table 62: Male C Averaged EDA readings 275

Table 63: Male C Comparative measures for box and whisker chart 277

Table 64: Male C Reading accuracy and words per minute rate 279

Table 65: Male $C$ Reading attainment in reading age 280

Table 66: Male C Reading attainment in Standardised Scores. 280

Table 67: Female G Standardised Scores for the working memory tests unable to be repeated with the dog 282

Table 68: Female G Averaged EDA readings 284

Table 69: Female G Comparative measures for the box and whisker charts 285

Table 70: Female G Reading accuracy and words per minute rate. 287

Table 71: Female $\mathrm{G}$ Reading attainment in reading age 288

Table 72: Female G Reading attainment in Standardised Scores 288

Table 73: Male H Standardised Scores for the working memory tests unable to be repeated with the dog present 290

Table 74: Male H Averaged EDA readings 292

Table 75: Male H Comparative measures for the box and whisker charts 293

Table 76: Male H Reading accuracy and words per minute rate 295

Table 77: Male H Reading attainment in reading age..... 296

Table 78: Male H Reading attainment in Standardised Scores 296

Table 79: Pilot - Overall Attainment 313

Table 80: Pilot - Overall EDA for 'relaxed' 313

Table 81: Pilot Maths Attainment 314

Table 82: Pilot Maths Relaxation. 314

Table 83: Pilot Vocabulary Attainment 315 
Table 84: Vocabulary Relaxation.

315

Table 85: Overall Sample of adults' attainment scores in each condition ....

318 


\section{List of Figures}

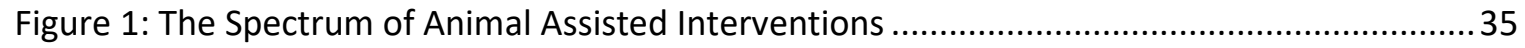

Figure 2: The revised working memory model of Baddeley (2000) ..........................................5

Figure 3: Diagram of a convergent parallel design (Source: Cresswell, 2015, p.38) .....................91

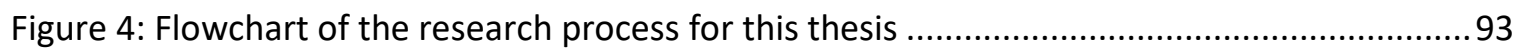

Figure 5: Example of 'emojis' used in the children's questionnaire ............................................105

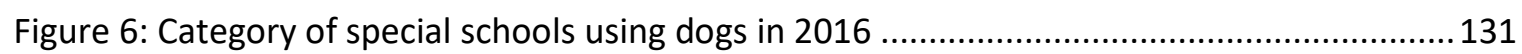

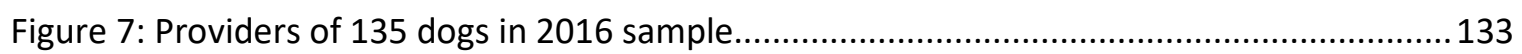

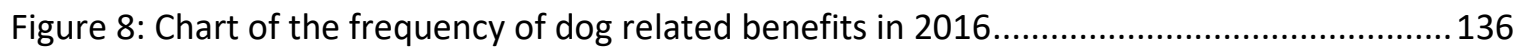

Figure 9: Providers of dogs in special school sample 2018 compared to 2016 .........................151

Figure 10: A standardisation curve showing relationships between standard scores, percentiles

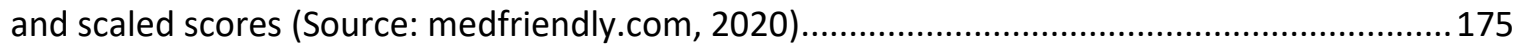

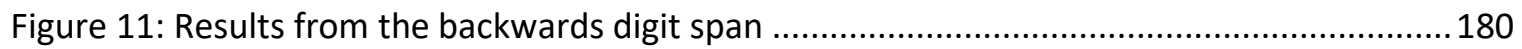

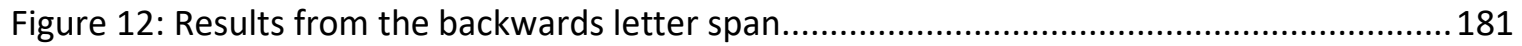

Figure 13: Combined results from both digits and letters backwards scores ............................. 182

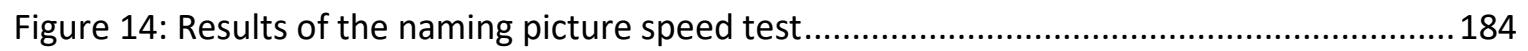

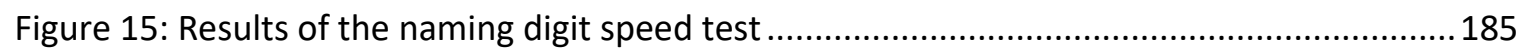

Figure 16: Combined results from both naming picture and digits at speed test ...................... 186

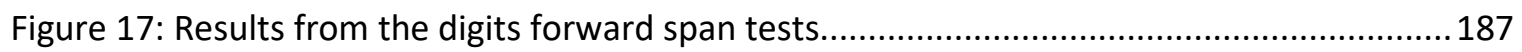

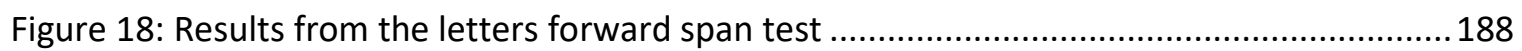

Figure 19: Combined results for digits and letters forwards tests ............................................. 189

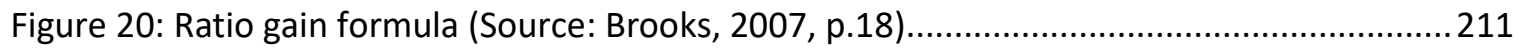

Figure 21: Male A's working memory results, with and without the dog present and key .........240

Figure 22: Male A's EDA comparisons between the three tasks.................................................. 242

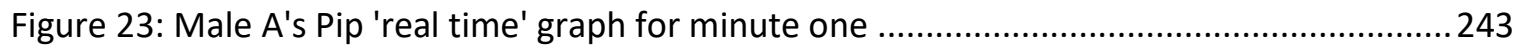

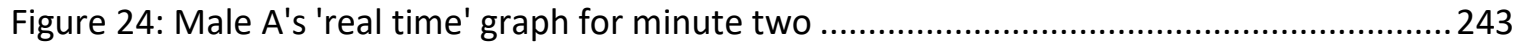


Figure 25: Female E working memory results with and without the dog present and key........

Figure 26: Female E's EDA comparisons between the three tasks

Figure 27: Female E's Pip 'real time' graph for minute one 252

Figure 28: Female E's Pip 'real time' graph for minute two.

Figure 29: Female A's working memory results with and without the dog present and key ...... 257

Figure 30: Female A's EDA comparisons between the three tasks.

Figure 31: Female A's Pip 'real time' graph for minute one 260

Figure 32: Female A's Pip 'real time' graph for minute two 261

Figure 33: Male M's working memory results with and without the dog present and key 266

Figure 34: Male M's EDA comparisons between the three tasks 268

Figure 35: Male M Pip 'real time' graph for minute one 269

Figure 36: Male M Pip 'real time' graph for minute two 269

Figure 37: Male C's Working memory results with and without the dog present and key........ 275

Figure 38: Male C's EDA comparisons between the three tasks 276

Figure 39: Male C's Pip 'real time' graph for minute one 278

Figure 40: Male C's Pip 'real time' graph for minute two 278

Figure 41: Female G's working memory results with and without the dog present and key...... 283

Figure 42: Female G's EDA comparisons for the three tasks 285

Figure 43: Female G's Pip 'real time' graph for minute one 286

Figure 44: Female G's 'real time' graph for minute two

Figure 45: Male H's working memory results with and without dog present and key 292

Figure 46: Male H's EDA comparisons between the three tasks 293

Figure 47: Male H's Pip 'real time' graph for minute one 295

Figure 48: Male H's Pip 'real time' graph for minute two 295

Figure 49: Latin square design 310

Figure 50: Vocabulary scores across all four conditions 325 
Figure 51: Maths scores across all four conditions 326

Figure 52: EDA scores across all four conditions - incorporating both maths and vocabulary tests 327

Figure 53: EDA scores across all four conditions - while participant completed the vocabulary tests 329

Figure 54: EDA scores across all four conditions - while participant completed the maths tests 330 


\section{List of Appendices}

Appendix A Qualification Codes from Pearson as of 05/09/2020 ............................................. 380

Appendix B Therapy Dogs Nationwide Paws and Read Programme ........................................ 382

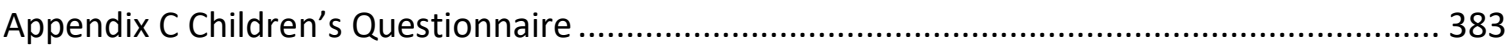

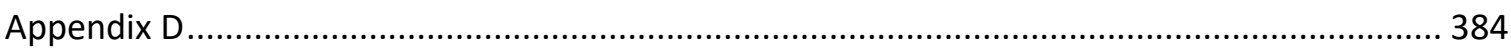

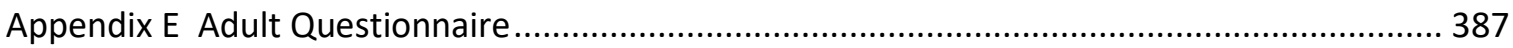

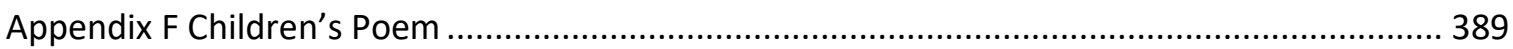

Appendix G Examples of Miscue analysis, Poem and Reading .............................................. 390

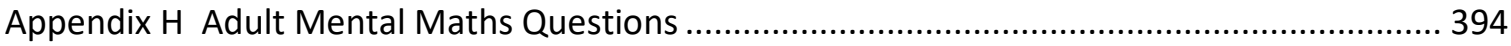

Appendix I Adult Nelson Denny Vocabulary Questions................................................................. 395

Appendix J Example of Powerpoint questions used in VR and 'Live' Conditions ........................ 399

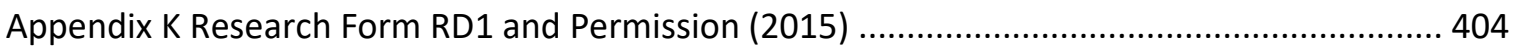

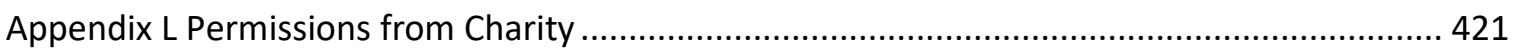

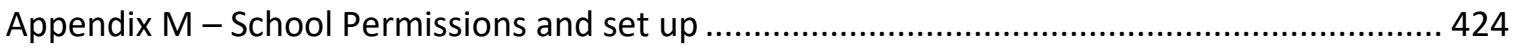

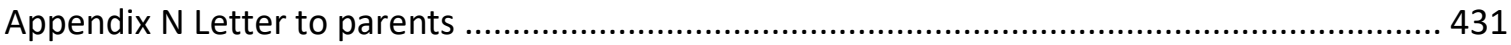

Appendix O Research Permission Adults project 2019.............................................................. 432

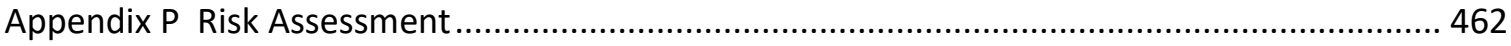

Appendix Q Locations of school dogs in special school sample 2016 ....................................... 464

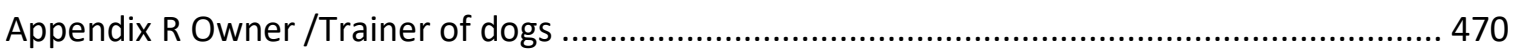

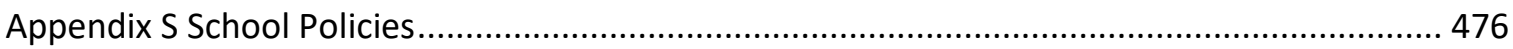

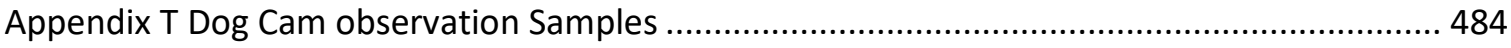

Appendix U Female A - Session 1 Reading Observations .......................................................... 490 
Appendix $V$ Observations between dog and adult participants. 499

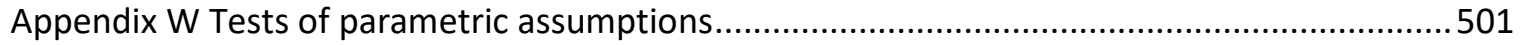

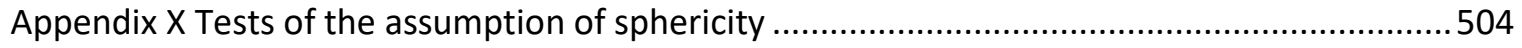

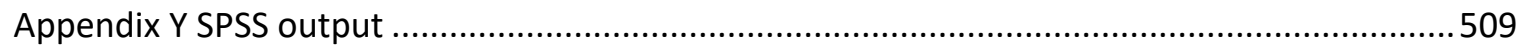




\section{Chapter 1}

\section{Introduction}

\subsection{Background}

Working memory, (Baddeley, 1974, Baddeley, 2007, Cowan, 2005), is involved in a range of cognitive skills used across all school subject areas, such as: visual word recognition, vocabulary retention, recall of facts, comprehension, reasoning, problem solving, space and layout of ideas, number recall and procedural strategies. Working memory, however, is also extremely susceptible to emotion, anxiety, fatigue and stress (Ansari and Nazanin, 2010, Derakshan and Eysenck, 2010). An individual's internal 'state,' (physiological) or 'trait' (psychological) anxieties can lead to inaccurate recall, a forgetting of facts and procedures, an incompletion of work tasks and consequential reduction of self-esteem and motivation at any age.

School aged children are particularly susceptible to information overload, (Elliott et al., 2010, Gathercole et al., 2008b, Pakiam-Alloway and Alloway, 2015, Visu-Petra et al., 2011) which can lead to frustration, worry and anger. Together with immature emotional regulation strategies, children can suffer external emotional outbursts or internal, mental withdrawal leading to longer term issues for health and wellbeing. These are referred to as Social Emotional and Mental Health (SEMH) difficulties within the United Kingdom's schools and academic environments.

According to Cowan (2010), the span and capacity of an individual's working memory, even when mature, is finite and the cumulative effects of the inefficient use of working memory skills and strategies when young will therefore affect later learning. 
It has been suggested that through the reduction of stress and anxiety associated with classroom learning tasks, more facts and knowledge could be retained in the memory linking to greater overall subject attainment (Elliott et al., 2010, Alloway et al., 2009, Jaroslawska et al., 2016b, Gathercole, 2014). However studies and research into motivation and self-efficacy for learning (Deci and Ryan, 2000) has shown that we humans focus on what personally interests us most, or that our emotional states tend to direct our attention towards information that is relevant to our current state of mind (Wilson, 2006). This implies that teaching interventions that positively attract children's attention, interests and emotions would facilitate later adults' performance (Wilson, 2006).

An adapted learning strategy which has become extremely popular in England's classrooms over the past 20 years, has been the involvement of animals, such as dogs within the learning environment, otherwise known as Animal Assisted Activities (AAA), or when instituted on a more formal, regular basis, is known as Animal Assisted Intervention (AAI). While there is much AAI research into the socio-emotional effects on learning, particularly with different "groups" of learners, there is limited work into its efficacy in actual cognitive effects on attention and working memory. The aim of the research described in this thesis is to explore whether AAI can facilitate improvements in cognitive acuity, and whether such improvements can be demonstrated across students of all ages.

\subsection{Why include dogs?}

In 1984, Edward Osbourne Wilson initially proposed biophilia as "the innate tendency to affiliate with natural things." Wilson later specified that when we encounter living things, we experience a wide spectrum of emotions ranging from "attraction to aversion, from 
awe to indifference, [and] from peacefulness to fear driven anxiety" (Wilson, 1993, p. 131, as cited in Kahn and Kellert, 2002).

Wilson's "naturalistic" standpoint denotes that the physiological responses to animals, such as the mutual lowering of blood pressure and relieving of stress of both the human and animal (Odendaal, 2000, Beck and Katcher, 2003, Clark et al., 2018), intensifies the sensitivity towards detail into the surrounding environment and thus not only increases intellectual attention, but also cognition.

Regular association over time to one particular animal or species forms a "bond," both physically and emotionally. Dogs, for instance, have been intimately linked to human life for over 12,000 years according to archaeological evidence (Bradshaw, 2017, Herzog, 2010, Beetz, 2018). This partnership between animals such as dogs and humans is studied as the "Human Animal Bond" (HAB). Research into this area is known as Human Animal Interaction (HAI) or Anthrozoology.

Bowlby proposed that humans have an innate need for supportive relationships with others, from infancy to adulthood (Keefer et al., 2014, Bowlby, 1969). According to Keefer et al, "For Bowlby, the motive for security is an innate psychological tendency" (Keefer et al., 2014 p 525) acquired over the course of evolution which showed that those who could seek and maintain proximity with caregivers for relationships and security, were more likely to survive than individuals lacking this approach.

There are two main forms of security - 'safe haven' - the offering of assistance to an imminent threat to one's safety and 'secure base' - believing that a caregiver would be available if the support was necessary. Bowlby suggested that close interpersonal 
relationships play a unique role in human behaviour and functioning. Stress and trauma in early infancy can be overcome by strong, nurturing relationships with significant others, peers and role models, including teachers in schools, (Blair and McKinnon, 2016).

It is also known that both humans and animals, including dogs communicate through relationships (Bradshaw, 2017, Bekoff and Pierce, 2019, McCardle et al., 2011) whether with positive or negative implications for attachment. The use of an animal in school could be potentially substituted as an emotional caring bond. This might suggest that for most students with positive emotional bonds, the use of animals within the classroom would be secondary to their learning, but for some without a secure attachment, an animal could provide an alternative bond, thus reducing stress and assisting with overall performance, socially, emotionally and cognitively (Amiot and Bastian, 2015, Beetz et al., 2012b, Jalongo, 2015, VanFleet, 2018b, VanFleet and Faa-Thompson, 2017).

However, these "bonds" are initiated by the parent, guardian or adult in allowing a pet to be made available for the child, in the belief that the animal will benefit their child's development and social awareness (McCardle et al., 2011, Blazina, 2013). This belief is usually born from their own childhood experience. Teachers too are using these bonds when bringing dogs into the classroom.

Both Wilson's Biophilia and the Attachment theories developed by Bowlby and others have sustained credibility over time partly due to the advancement of medical science and the now available, measurement of neurochemicals such as oxytocin, cortisol, prolactin, phenylethylamine and dopamine (Beetz, 2013, Beetz et al., 2012b, Schretzmayer et al., 2017, Wohlfarth et al., 2014). These neurochemicals can control and affect the fluidity of working memory, focus and attention. Studies have shown that dogs can activate the 
oxytocin system despite the current limited evidence for other stress-related markers such as epinephrine and norepinephrine (Beetz et al., 2012b). Overall cortisol levels and heart rate $(H R)$ were shown to be attenuated while a 'novel' dog was available during stress tests for anxiety (Polheber and Matchock, 2014). These measurements of neurotransmitters are also often used with cognitive studies of stress and anxiety on the brain when examining specific groups of learners (Bedewy and Gabriel, 2015, Matthews and Campbell, 2010, Carapetian et al., 2008, Mattarella-Micke et al., 2011), although there are ethical, practical and accuracy considerations for attaining such readings from very young children (Dimolareva et al., 2018).

\subsection{What is Animal Assisted Intervention?}

There are many confusing terms used within the area of animal assisted activities, education and associated interventions and it is useful to try to know their definitions and frameworks as a reflection of how the different categories have developed over time. Despite several rewriting of the definitions for clarity (IAHAIO, 2014 updated 2018, Winkle et al., 2019) the boundaries are still open to interpretation. For example the "Read to Dogs scheme" can be considered as an Animal Assisted Intervention, (AAl), as it is a goal orientated and structured intervention, an Animal Assisted Activity, (AAA), where an untrained volunteer hears a child read in school with their dog present, or if a qualified teacher leads the session, Animal Assisted Education, (AAE) (Beetz and McCardle, 2017).

In 2016, Busch et al., classified Animal Assisted Activities (AAA) as providing, “opportunities for motivational, educational, recreational, and/or therapeutic benefits to enhance quality of life. Animal Assisted Activities are delivered in a variety of environments, not just educational, by specially trained professionals, paraprofessionals, 
and/or volunteers in association with animals that meet specific criteria" (Busch et al., 2016). According to Fine (2015, p.415), the International Association of Human-Animal Interaction Organisations white paper (IAHAIO, 2014 updated 2018) gives the definition of both Animal Assisted Activities and Animal Assisted Intervention as, "a goal orientated and structured intervention that intentionally includes or incorporates animals in health, education and human service (e.g. social work) for the purpose of therapeutic gains in humans." The only difference in the categorisation appears to be the qualifications and job role title of whoever is carrying out the intervention and in which type of environment. Animals have been traditionally viewed as having therapeutic benefit through the fields of mental health and education in the following ways: as a social lubricant for therapy; as an extension to a therapist or a method for rapport building; as a catalyst for emotion; an acting adjunct to a clinician, therapist or teacher; or as role model (Fine et al., 2015 p.141148).

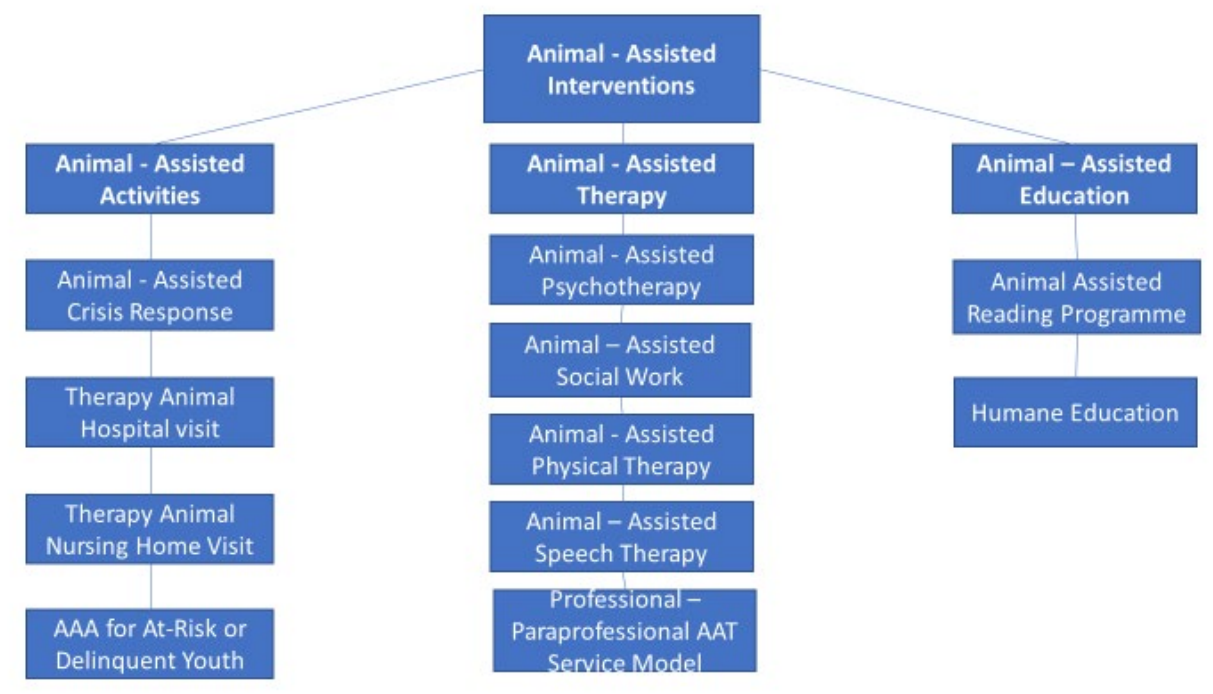

Figure 1: The Spectrum of Animal Assisted Interventions

Source: (Fine et al., 2015 p.21) 
Animal-Assisted Therapy (AAT) is a healing model involving a patient, an animal therapist and handler with a goal of achieving a specified therapeutic outcome (Braun et al., 2009). AAT is directed and/or delivered by a health service professional with specialised expertise, and within the scope of practice of his/ her profession. AAT is designed to promote improvement in human physical, social, emotional, and/or cognitive functioning, is provided in a variety of settings and may be group based or individual in nature (Busch et al., 2016). An intervention with Animal Assisted Therapy can also be regarded as an Animal Assisted Intervention (AAI) depending on the skills, qualifications and training of the handler and / or therapist. If the handler is not a qualified therapist, but is simply present, then the "therapy" is under the direction of the qualified "therapist." The handler has simply provided access to an animal for the length of the intervention.

\subsection{The 'roots' of Animal Assisted Education, (AAE)}

Originally the animal assisted research focus had medical intentions using 'populations' such as those with cardiovascular disease, (Allen, 2003, Wolff and Frishman, 2004), and hospitalised patients, (Jalongo et al., 2004, Braun et al., 2009). This focused on the emotional benefits of the animal assisted 'therapy' (AAT) for the patient through trained practitioners and therapists. Autism, ADHD and special needs, at that time were viewed as part of the medical 'deficit' model (Gill, 1987), a deficit within the person themselves requiring 'curing' or management of an illness and therefore open to and included in clinical trials of therapeutic intervention.

Research from AAl, together with AAT involving younger aged patients, both with and without clinical diagnoses merged over approximately 40 years into what could be described as Animal Assisted Learning (AAL) while beginning to move into school settings. 
The term, Animal Assisted Learning (AAL) was suggested by Friesen (2010) who referred to the work of Nagengast, $(1997$, p. 329) "where trained therapy dogs act as a nonjudgemental supplement to an intervention," i.e. as a classroom companion. These dogs would commonly offer benefits to children who are socially unresponsive, shy, or withdrawn and/or who may experience heightened anxiety in school environments who otherwise might not engage in learning. This can be delivered by a therapist, or staff member." The acronym for Animal Assisted Learning (AAL) is often used in conjunction with the acronym for Animal Assisted Education, (AAE), the subtle change is with the qualifications of the handler, either as a therapist or as a teacher.

According to Smith and Dale (2016), Animal Assisted Education (AAE) is now defined as a goal orientated, planned and structured intervention directed / or delivered by educational and related service professionals such as qualified (with degree) general and special education teachers. Classroom teachers who conduct AAE must have knowledge of the animals involved. An example of general AAE delivered by a classroom teacher is an educational visit that promotes responsible pet ownership. AAE when done by special education teachers, however, can be also considered therapeutic and a goal orientated intervention as often the focus of activities is on academic goals, pro-social skills and cognitive functioning (IAHAIO, 2014 updated 2018). As with all school-based interventions, each student's progress should be measured, with entrance and exit criteria and documented.

Animal Assisted Education (AAE) is now further evolving in that qualified teaching professionals are now developing their own criteria and specific curriculum practices within the classroom for working and learning with dogs. These are qualified educators 
who can now also handle trained dogs in the dual role of both teacher and handler. In the UK, and in particular England, this is still very much a developing area, whereas in Europe, countries such as Germany, Poland, The Netherlands, Austria and Norway, or further afield, in the United States and Australia, training, rules and procedures have already been established. This could mean that another acronym is imminent, Animal Assisted Pedagogy (AAP.) For the sake of clarity in this thesis, however, any suggested pedagogic strategies used with dogs in the classroom will be inclusive of Animal Assisted Education, AAE.

Researchers in AAE continue to investigate 'Educational settings' mainly within the student age range of $4-13$ years as this is a period in which the brain rapidly grows and develops both cognitively and emotionally. Focusing chiefly on the social-emotional aspects of learning, AAE continues to compare 'identified groups' with homogenous student classes. Bias has since been noted however, in that non-significant results are not published which could have major implications for classroom conditions and to the validity and the reliability of having an animal present at all (Reilly et al., 2020).

\subsection{Differences in research styles and stances}

Nezhad and Vahedi remark that, "Psychology gives education the theory of individual differences that every child has different mental ability and learns with a different pace," (Nezhad and Vahedi, 2011, p.327). To date, however, evidenced studies promoting the benefits of animals for learning are often within the 'clinical psychological' field and have taken place in university laboratories or 'pseudo' classrooms. From an educational point of view, this could be regarded as a 'false' situation as actual interaction and learning takes place within busy school environments often undetected. Clinical numbers required for 
'Randomised Control Groups' also do not easily lend themselves to school or classes or the educational 'inclusive' policy. As such, there can be great differences between given policy 'ideals' and actual classroom practice.

From a teaching stance, there are also issues with worldwide differences in identifying and labelling those requiring extra learning and/or emotional support. A universal language needs still to be developed for a greater platform of awareness and aetiological understanding. The term 'learning disabled' that Animal Assisted Intervention experts in the field such as Nancy Gee use in their work (cited in Blazina, 2013) have different emotional and clinical interpretations in other countries of the world (Lissack, 2018). Equality too, is an issue, as teachers are expected to raise standards for all pupils in their care, not just a select few (DfE/DoH, 2015), while another practical issue is the location of suitably assessed, trained and qualified dogs within the schools themselves.

Animal Assisted Intervention researchers have called for further high-quality research investigating the psycho-educational impact of animals to drive evidence-based learning programmes (Gee et al., 2017b, Hediger et al., 2017, Gee et al., 2017a, Fine et al., 2015, Jalongo et al., 2018). It is anticipated that this collaborative research will, in turn inform policy decisions and "pave the way for acceptance of Human Animal Interaction in educational settings" (Gee et al., 2017a, p.213). Gee et.al., continue to state that "Education leaders need to recognise that the catalyst of these efforts is not trivial and that there is a growing body of knowledge indicating that animals can make a significant contribution to education and wellbeing of students. These gatekeepers must be educated and engaged" (Gee et al., 2017a, p.217). 
While some teachers may be impressed or convinced to involve dogs considering Animal Assisted Intervention clinical data findings alone, further investigations are required as to how much of the subsequent findings are transferrable to the classroom and the reality of day to day academic-based learning. After all, whereas the scientists and psychologists may create the theory, it is the teachers who will have to create, and integrate the classroom practice.

\subsection{Research Rationale}

There is a growing body of evidence suggesting that the involvement of therapy dogs in animal assisted interventions have also helped children to achieve greater attainment in their reading (Bassette and Taber-Doughty, 2013, Friesen, 2010, Black, 2009, Fisher and Cozens, 2014, Kirnan et al., 2016, Lenihan et al., 2016, le Roux et al., 2014, Pillow-Price et al., 2014, Scallion, 2010, Schretzmayer et al., 2017, Massengill Shaw, 2013, Stroud, 2012, Wohlfarth et al., 2014). This information has been seized upon by both the general media and social media creating a phenomenon which has become a main reason why visiting charities with dogs enter schools. The introduction of dogs into reading sessions has been sensationalised under such newspaper headlines as "reading with a fluffy friend," or given the equivalent status as a human Teaching Assistant (TA) (Stroud, 2012).

The "read to dogs" scheme is an international programme for enhancing learner's reading skills and although aimed at young learners, can also include adults such as those in prison (Leonardi et al., 2017). Different charities have individual variances in how it is carried out, such as some 'train' the volunteer in simple reading skills, while others do not. Essentially the programme consists of either a single learner or a small group of learners taking it in turns to read aloud to the dog from their own, chosen books. This usually takes place in a 
comfortable area such as a library or a corner of the classroom. The dog is encouraged to lie among the readers for easy access to petting, stroking and 'hugging.' There are differences in opinion about whether the 'handler' should help or assist to correct the learner while reading and many charities use the 'dog as a silent uncritical friend' approach in that there is no correction for the learner while they read (Kennel-Club, 2018, Swift, 2009, Therapy-Dogs-Nationwide, 2020, Pets-As-Therapy, 2016).

The reported effects of this scheme alone are encouraging many schools to investigate the possibilities of introducing school dogs into the curriculum, many without due consideration for the reciprocal effects for the animal. Charities and businesses are now offering 'reading dogs' or even 'fully trained' dogs for schools, but questions still remain as to the accuracy of the results and the extent to which any measurable outcomes can be attributed specifically to the presence of the dog rather than the efforts and motivation of the reader (Connell et al., 2019, Deci and Ryan, 2000). It has also been argued that their presence is purely more of a motivational exercise (Bassette and Taber-Doughty, 2013, Bell, 2013, Friesen and Delisle, 2012, Fisher and Cozens, 2014) which could even be considered as a politically motivated 'gimmick' to raise literacy standards (The-KennelClub, 2019).

Several studies in particular have tried to look at specific, underlying working memory skills for reading such as speed and accuracy, (Schretzmayer et al., 2017, Wohlfarth et al., 2014). Many of these studies have relied on teacher and student self-assessment with generalisations of the success proposed across age ranges and groups or anecdotal evidence. One concern is that teachers with a vested interest in the success of Animal Assisted Intervention, may not be best placed to evaluate the success of such schemes. 
Most recorded research, however, still remains within the 'English' or 'Literacy' based curriculum. There is still limited research as to whether, or how the dog could affect the underlying skills for an individual's attention or working memory which can be cross related to other curriculum subjects. It is theoretically possible that these same skills are used across the entire school-based curriculum (Holmes, 2006, Gathercole et al., 2016, Holmes, 2017). For example, there is no evidence for the effects of dogs on mathematical performance.

There are however, two, recent systematic reviews examining the worldwide efficacy of AAl for children in the classroom but again, specifically with reading (Brelsford et al., 2017, Hall et al., 2016a). These do refer to the beginnings of specific research into working memory skills with young pre-school children through the work of Nancy Gee, a key contributor to the field of Animal Assisted Intervention and working memory (Gee et al., 2015a, Gee et al., 2017a, Gee et al., 2017b).

Gee and her respective research teams have undertaken six separate projects about working memory tasks with pre-school children aged between three and five and a half years in the United States (Gee et al., 2010b, Gee et al., 2010a, Gee et al., 2007, Gee et al., 2012b, Gee et al., 2009, Gee et al., 2012a) and two examining sequencing tasks, requiring the same use of the children's working memory skills with adult university students (Gee et al., 2014, Gee et al., 2015b). The results were mixed, with a lack of corroborating evidence and depended on the use of classification tasks, following instructions and sequencing tasks. While they may be relevant for nursery aged students in a United States pre-school laboratory pseudo-classroom setting, they have not been further developed for an actual school classroom involving students above six years old. 
In England, the official age of school entry is earlier at age four, than the United States, usually at age six, therefore the equivalent aged children to Gee's studies in England are already being taught to recognise sounds and phonemes to read, count and write by the age of five years and have already had their school entry baseline assessments by their Early Years Foundation teachers. Despite their subjects' young ages, Gee and her teams encourage the involvement of dogs in all schools to improve subject attainment and working memory skills (Gee et al., 2017b, Gee et al., 2015a).

Research has also suggested that while a dog can be present within the vicinity of the room, the learner does not need to be physically touching the animal to benefit (Gee et al., 2010a, Hediger et al., 2017, Hediger and Turner, 2014, Prothmann et al., 2009). The effect of the mere presence of a dog is particularly apparent when comparing robotic dogs or simply stuffed toy dogs to 'live' dogs with young students. Students reportedly (Gee et al., 2014, Kerepesi et al., 2006) prefer the 'live' dog situations achieving higher attainment in set tasks, yet there is still a debate as to whether the 'live' dog versus the 'traditional' adult teacher enhances learning overall for all when following instructions, even with adult students (Gee et al., 2009, Gee et al., 2012a, Gee et al., 2015b, Trammell, 2019). While children are aware of the characteristics of a robot or stuffed dog, animal assisted research has not yet considered the use of a 'virtual' dog for learning purposes.

If only the 'presence' of the dog is of significance, then introducing virtual reality environments, in which 3D films of dogs are presented in combination with the learning tasks while in a headset could be investigated and compared. Previous studies in virtual reality simply using photographs of dogs have had significant, effects reducing the anxieties with those suffering from 'cynophobia,' the fear of dogs (Rohani and 
Puthusserypady, 2015). While there would be ethical and safety considerations for the use of virtual reality headsets with young children, adult students would be capable of undertaking such an investigation. These adult based studies could not only highlight whether memory and attention skills are congruent across age groups but give the possibility to develop further considerations for the virtual classroom environment, subject areas and remote learning.

While current educators across the board do recognise the value of animals to meet emotional, developmental, general social goals and needs of some students, there still appears to be many unresolved issues in linking Animal Assisted Intervention to its actual efficacy for classroom curricula attainment and performance. There is also a debate as to whether involving a dog can help every single student, rather than a select few. Both Beetz and Gee have noted that not all children prefer to work with dogs (Beetz, 2013, Gee et al., 2015b) or indeed have definitely benefitted because a dog was present. Currently, there is no, consistent, regulated, standardised system of tracking animal assisted practice, or ultimate progress - a point raised in four systematic reviews covering not just Animal Assisted Interventions in schools, but those also focusing on curriculum subjects such as reading, or with specific group populations such as those with autism (Brelsford et al., 2017, Hall et al., 2016a, Davis et al., 2015, O'Haire, 2013).

Quantifying the measurements of the success of animal assisted schemes appears to be an overriding issue in education. Some trained teachers, however, with post-graduate qualifications in specific learning difficulties, do have access to commercial working memory diagnostic tests used by both psychologists and specialists for identifying students with suspected dyslexia and dyscalculia (TOMAL2, WRAML). These tests and 
subtests could be adapted in such a way that perhaps all teachers could then have a screener which could not only highlight if the dog's presence is making a difference to the child's abilities, but depending on the type of test used, where the dog is affecting the thinking process, such as processing speed. Tests and screeners such as these may then begin to widen classroom and individual learner links to research and the efficacy of using Animal Assisted Intervention.

However, Animal Assisted Intervention, as it currently stands, is still generally perceived within the educational environment as inconsistent in robustness both in approach and results (Brelsford et al., 2017, Herzog, 2011, Herzog, 2010). What may work for one group / cohort in one school year, may not work as well for subsequent years.

According to the Animal Assisted Intervention researchers themselves this unpredictability is due to "too many variables" (Beck and Katcher, 2003, Fine et al., 2015, Gee et al., 2017a, Crossman and Kazdin, 2016, Hediger et al., 2017) to make credible claims in general attainment learning, let alone of the efficacy of including animals in classrooms. Sroufe (2017) in his introduction to the book, "How Animals Help Students Learn," written for educators, neatly organises the variables into four main groups human variables, dog variables, interaction variables and time (Gee et al., 2017b Introduction p. x). This would suggest that investigating the effects of the involvement of animals to enhance students' learning is a complex subject, which will require further 'unpicking' to highlight if any of these 'variables' can be reduced in a practical way for the classroom.

Schools, however, are expected to cope with student variables on a day to day basis and as previously argued, be able to justify and account for the purpose of using of an animal 
as an intervention to outside regulatory bodies such as Ofsted (Office for Standards in Education).

\subsection{Aims, definition and scope of the research:}

The aims of this thesis research therefore are as follows: i) To explore whether animal assisted intervention can facilitate performance in cognitive acuity, particularly working memory and attention, for school-based tasks; ii) If so, could the subsequent effects of the presence of the dog be re-enacted in virtual reality; and finally iii) would either of the above two aims apply across students of all ages?

The research objectives are: To design and carry out a mixed methods-based research enquiry into the effects of dogs on attention, memory and stress for both school and university aged students, using classroom-based tasks such as reading, vocabulary recalls and mental mathematics. Within the second study with adults, investigate whether a dog

is as effective in a virtual reality form. Drawing from any combined data, to then determine the emotional and learning (attention and memory) consequences for all students of having an animal (dog) "working" within the classroom environment.

\subsection{The Structure of the Thesis}

The structure of the thesis will take the form of nine chapters. The first three are an Introduction, a Literature Review and a Methodology discussing the background and subsequent convergent parallel designed approaches and methods used. These are followed by 'Phase one' of the research - a desk-based study as given in chapter four to gather background information into how and why dogs are used in some schools in England. Phase two of the research comprises chapters five, six and seven, working in a primary school with seven-year olds. Chapter five gives the selection of tests for working 
memory tasks and the subsequent group results with a case study group of seven children with and without a dog present. Chapter six includes a small-scale investigation with 13 children, inclusive of the original seven case study children, examining the effects and measurement of a 'read to dogs' scheme. Chapter seven, the final chapter of that section, gives the individual overall results for the efficacy of the dog for cognitive acuity in reading and working memory for each of the original seven case study children. Phase 3 , presented in chapter eight, was an experimental paradigm using both 'live' and virtual reality settings with a dog for 24 adult students completing vocabulary recall and mental mathematical tasks. The overall results from both the children and the adults' studies, together with future recommendations are given in chapter nine. 


\section{Chapter 2}

\section{Literature Review}

This chapter intends to discuss the background theory of working memory, through attention, focus and emotional stability when applied to effective academic attainment for schools and educational establishments. The chapter also examines the theories, concepts and evidence behind Animal Assisted Intervention (AAI) for those with, and without learning differences, and discusses the current reasoning, support and practicalities for including a dog in a school curriculum. The chapter also scrutinises both past and current AAI research into how cognitive acuity skills have been identified, and investigates the concepts and techniques required to begin a methodological study in chapter three.

\subsection{Introduction}

Cognition, according to Groome $(2014$, p.3), is a "rather broad umbrella term, which has many component processes," including perception, attention, memory, thinking and language; while 'acuity' is the ability to improve clarity of thought, a 'sharpness' or 'keenness' of thought, vision or hearing through concentration. 'Cognitive acuity,' for the duration of this thesis will simply imply the heightening of cognitive processes leading to clarity and improvement in attainment within the classroom-based setting. The last 20 years have seen an increased pedagogic interest for strategies relying on memory and attention in order to enhance the learning of all students (Gathercole, 2013, Elliott et al., 2010, Alloway et al., 2004, Gathercole and Alloway, 2008). Specialist teachers have been 
questioning how intrinsic or extrinsic motivation, can increase memory retention and individual autonomy for attainment. Working memory for example, has become a significant area for diagnostic discussion within the official assessment process for those labelled with 'special educational needs' (Jones, 2013, Gathercole, 2013). If there is a possibility of increasing cognitive acuity by simply bringing a dog into a learning environment, it is worthy of an investigation.

\subsection{What is working memory?}

Working memory as a concept is important for individual learning both within the school environment and outside the classroom (Alloway and Gathercole, 2006, Baddeley, 2002, Cowan, 2014, Posner and Rothbart, 2017). Working memory has been termed as the brain's post it note' (Pakiam-Alloway and Alloway, 2015) or 'mental workspace' (Gathercole, 2013) where the memory holds, stores and manipulates current, cognitive information, while simultaneously processing or combining, previous knowledge (Dehn, 2015, Dehn, 2008). It is the 'thinking' part of the memory, influencing language, vocabulary, actions and ultimately overall cognition. Without the fluent use of a 'working memory,' processing instructions, adapting current facts and data, deducing comprehension and retention for later retrieval is impossible and subsequently affects overall attainment and performance (Cowan, 2014, Elliott et al., 2010, Gathercole et al., 2016, Gathercole et al., 2008a, Gathercole and Pickering, 2001, Pickering and Gathercole, 2004, St Clair-Thompson et al., 2010). Working memory is implicit in all school curriculumbased subjects including English language, mathematics and science. 
The definitive conceptualisation of working memory is that of Baddeley (2000), which is a revised version of the original Baddeley and Hitch (1974) model. Working memory, as proposed by Baddeley (2000) is shown in Figure 2.

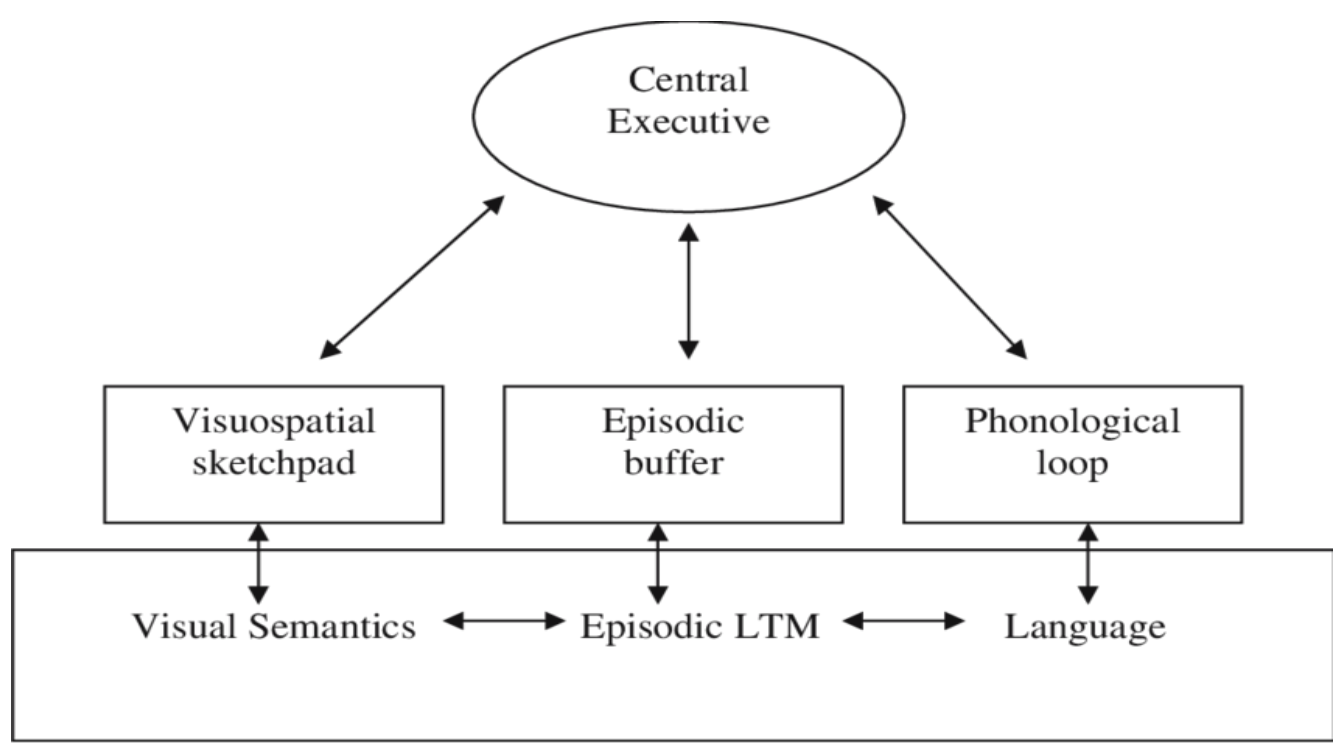

Figure 2: The revised working memory model of Baddeley (2000)

Working Memory has many elements. Executive control is a key component of Working memory since the original conception of Baddeley (Baddeley, 2007, Baddeley, 2002). One of the vital roles of this "central executive" is to control what is brought into the working memory - a process closely linked to attention (Dehn, 2015, Rueda et al., 2004, Cowan, 2014).

The Central Executive functions are: the capacity to focus the attention, the capacity to divide attention, to switch attention between tasks and to provide the link between working memory and long term memory (Baddeley, 2007 p.118).These skills assist the focus and concentration necessary for cognitive functioning, placing the attention on the activity and teacher, rather than the distractions from the surrounding environment or "mind wandering" (McVay and Kane, 2012). Together with "capacity," and attention 
"span," (Cowan, 2005, Kane et al., 2006) these skills dictate accurate subject content recall underlying classroom academic performance (Dehn, 2015, Dehn, 2008, Kaufman, 2010, Brock et al., 2009, Schweppe and Rummer, 2014, Baddeley, 2007, Gathercole, 2013, Pickering and Gathercole, 2004, Rueda et al., 2004, Posner, 2012). According to Miyake et al., (2000) this concentration, impulse and control of the memory also give rise to cognitive flexibility, which is, in turn, related to self-motivation, self-reflection and personal achievement.

Executive functioning has links to the hypothalamus, hippocampus and amygdala (the emotion centre) of the brain and is "mature" at birth (Gabard-Durnam et al., 2018). Learning is therefore affected by maturity, self-regulation and genetic individuality (Posner and Rothbart, 2009, Posner and Rothbart, 2007). Executive functioning skills can also be regarded as either "hot," involving the use of emotional regulation - whether subconscious or not, and "cool or cold" linking with cognitive development and specific skills for problem solving (Brock et al., 2009, Poon, 2018). Emotional attachments and subjectivity to learning can also be "reprogrammed" due to life events such as trauma and previous experiences (Blanchette and Caparos, 2016, Dodaj et al., 2017, Lilley et al., 2009).

The phonological loop is capable of temporarily holding and storing new audio and verbal information, both direct to the central executive and to the episodic buffer; a temporary storage space which links both visual and verbal information with the central executive and long-term memory. This verbal information is liable to decay over a few seconds and thus relies on both the acoustic memory trace of the letters phonemes, words or phrases seen or heard, and rehearsal, whether sub vocally overt or covert (Baddeley, 2007). The visuo-spatial sketchpad performs as similar function to the phonological loop, but with 
visual and spatial information, including three-dimensional location and rotation (Baddeley, 2007).

The essence of the working memory model is that it provides a plausible integrating structure for systems that manipulate different types of information, particularly visual and auditory (smell, touch, etc, were not addressed in detail by Baddeley). Later theories, for example, Postle (2006) have, however, challenged the essentially reductionist 'boxand-arrow' model of working memory, and have suggested that the functions ascribed to working memory are emergent properties of integrated brain systems. That is, there are no discrete 'boxes', only processes arising from distributed brain activity that could be labelled as, for example, the visuo-spatial sketchpad. Although there is some debate as to how physiologically discrete the functions of working memory are, the basic concept of separable processes for handling different types of information is common across the different models. In this thesis, therefore, the term 'working memory' and the subcomponents (e.g. visuo-spatial sketchpad, phonological loop, episodic buffer), as defined by Baddeley (2000) will be used as descriptors of processes. It is acknowledged, however, that the different functions may not be localised in a discrete 'box' or brain region as implied by the box-and-arrow model but may represent processes arising from activity across multiple brain regions. Particular tasks may also recruit more than one of the components of working memory and utilise links to other systems such as long-term memory (LTM).

\subsection{How working memory affects school subject areas}

Mathematics, for example, involves manipulating facts and sequences, layout and processing speed. Calculations and arithmetic involve selecting, implementing, and 
evaluating alternative solution based strategies (Holmes, 2017, Dowker, 2019). Students with superior working memories tend to have a 'head start' in mathematical achievement and attainment (Bull et al., 2008, Passolunghi and Cornoldi, 2008, Wu et al., 2017) showing fluent verbal or visuo-spatial recall, access to phonological loop and a complex memory span (Holmes et al., 2010). The development of counting skills and subsidisation is dependent upon facilitating numbers through supporting known number facts from the long-term memory, speed of processing using Rapid Automised Naming (RAN) and sequential recall of instructional processes (Brandenburg et al., 2018, Koponen et al., 2016). Formula recall, number bonds, place value, interpretation of written problems, and arithmetic explanations all use visuo-spatial skills. Slower counters, and the continued use of finger counting strategies beyond the early years, reflects weaker number networks and often incomplete, or incorrect schoolwork tasks. Older children who show a reduction in mathematical competencies often revert back to visuo-spatial aspects of the working memory system during complex problems - such as referring to fingers or the use of practical equipment and resources (Brandenburg et al., 2018, LeFevre et al., 2013, Mattarella-Micke et al., 2011). Studies have linked numerical competence and mathematical achievement with working memory deficiencies (Adams, 2007, Astle et al., 2019, Gathercole et al., 2016, Colomer et al., 2013, Ganley and Vasilyeva, 2014, Henry and Winfield, 2010, Brandenburg et al., 2018, Koponen et al., 2016, LeFevre et al., 2013, Passolunghi and Cornoldi, 2008, Wilson et al., 2015, Wu et al., 2017).

'Literacy' skills include reading, writing, spelling, articulate speech, vocabulary and comprehension. Academic success and attainment in literacy relies on rhythm, rhyme and speech amplitude (Goswami, 2004). Skills with verbatim recall, following instructions, 
lexical semantics, and inference assist with the creation of alternative solutions to problems (Gathercole et al., 2006, Hayes et al., 2013, Király et al., 2017, Swanson et al., 2010, Wilson et al., 2015, Acheson et al., 2010, Jaroslawska et al., 2016a). These enhance the ability to process fluently, spot, reduce and correct errors (Chiarenza et al., 2014, Astle et al., 2019, Koponen et al., 2016). Reading, spelling and writing also involve good phoneme / grapheme correspondence, phonological awareness, lexical access, decoding skills, visual word recognition, prosody, syllabic awareness, comprehension and graphical motor skills linked to motor memory for writing (Shiran and Breznitz, 2011, Henry et al., 2015, Goswami, 2004, Muter and Snowling, 1998, Oakhill et al., 2011, Shankweiler et al., 1979). Thus, the importance of a good working memory, together with a stable emotional response can dictate the individual's learning potential for overall school-based achievement.

\subsection{Emotions and working memory}

Neuroimaging has shown that there are two distinct areas of the brain used in cognitive problem solving. The 'cool' skills involve the dorsolateral pre-frontal cortex and the 'hot' skills the processing area of the orbitofrontal cortex (Posner and Rothbart, 2009, Posner and Rothbart, 2007, Rueda et al., 2004). Research with kindergarten students showed that the 'cool' executive function deficits even at age three could identify students who would need additional support academically, including those who may develop behaviour problems over time (Brock et al., 2009). This concurs with other age-related research (Bull et al., 2008, Conejero and Rueda, 2017, Wiebe et al., 2011). Investigations into adolescents' academic performance and scholastic achievement between 12 and 18 years (Poon, 2018) showed a 'bell curve' effect where students showed greater behavioural 
responses to risk taking, reward magnitude, choice and impulsivity, with vulnerability to emotional challenges peaking around the ages of 14-15 years (Gruska, 2010, Kauffman, 2005, Nelson, 2009), reflecting implications in achievement in major exams taken at age 16 in England.

Student performance is therefore modulated by emotion, processing efficiency through these emotions, stress, workload and arousal (De Lissnyder et al., 2010, Derakshan and Eysenck, 2010, Eysenck and Calvo, 1992, Matthews and Campbell, 2010). Selfacknowledgement and self-recognition of these perceived mental states may be further assisted in personal response to favourable environmental factors within the school and home, such as acknowledging hobbies, interest, sports or including a pet.

\subsection{Anxiety and Stress}

Increased anxiety affects three major areas of executive function: inhibition, shifting and updating, while clinical depression can affect the processing speed and efficiency (Gruska, 2010). Ansari's research into cognitive overload suggests that anxious individuals deplete more working memory resources when compensating for deficits, in order to achieve comparable performance outcomes with their peers (Ansari and Nazanin, 2010). Research into arousal through skin conductance variability and orthostatic, systolic blood pressure regulation (OBPR) with children suggests that students with a low OBPR may lead to reduced levels of classroom effort, and be a marker for depression, fatigue, anxiety, reduced attention, impulsive behaviour and reduced volition (Carapetian et al., 2008, Chen et al., 2015, Hindes et al., 2009). A lowering in skin conductance levels is not only positively linked to the visuo-spatial aspects of learning, rather than verbal, but also 
aggression, under-arousal and depression (El-Sheikh et al., 2010, Brenning et al., 2012, Smithson and Nicoladis, 2016).

State (psychological reaction of the moment) and trait (psychological reactions over a length of time) emotional attachments to experiences of learning can affect not only selfesteem and confidence, leading to long term mental health issues, (Ansari and Nazanin, 2010, Yair et al., 2010, Gruska, 2010, Brenning et al., 2012) but also a lack of overall progress and therefore an eroding of confidence in particular subject areas (Chinn, 2004, Bedewy and Gabriel, 2015, Ganley and Vasilyeva, 2014, Owens et al., 2008, Visu-Petra et al., 2011, Waters and Kershaw, 2015).

Gago et al (Almeida and Martins deGago, 2013) when investigating pleasant and neutral stimuli on anxiety found that, "emotion was not the only factor that influences memory, and other factors appear to be important, such as prior knowledge and cognitive, social and physiological factors including personal history, the environment and culture." (Almeida and Martins deGago, 2013 p 351). Self-esteem, motivation and intrinsic qualities should be more stable in adults, despite influences from childhood (Charron et al., 2008, Çiftçi and Yıldız, 2019, Sarkova et al., 2013, Ayotola and Adedeji, 2009).

\subsection{Links to school achievement in England}

The National School Curriculum for England relies on a hierarchical skills learning basis through the four Key Stages for students aged 4-18 years, using regular summative and formative assessment (DfE, 2014). The knowledge of mental maths and the requirement to be able to recall simple numerical facts such as times tables, number bonds and algebraic equations, together with verbal and written comprehension, spelling and grammar are required in order to pass peer comparison 'National Standard' tests and 
exams throughout a student's school career (Dehn, 2015). This clearly affects future life skills, qualifications and long -term job opportunities. Astle et al. (2018) record that 'struggling learners' - those with poor communication, emotional regulation, phonological processing and reading are already making up $30 \%$ of the current school population who fail to attain "expected" targets in reading and maths at age 11(DHE, 2017).

While attention and working memory spans can be said to be limited and 'reflect the moment' (Cowan, 2014, Cowan, 2005, Ricker and Cowan, 2014, Schweppe and Rummer, 2014) it is difficult to measure and link these psychological effects into the long-term accuracy and subsequent accumulated effects in day to day thinking and learning within the classroom. Specific scholastic and academic performance, however, can be recorded using formal and informal assessment for tasks which have included the use of working memory skills, such as reading, speech rate, reading rate, accuracy, fluency and selected questions from comprehension can reveal verbal flexibility, whereas written answers, diagrams, layout of work, types of errors made can also highlight visuo-spatial understanding and thinking. Consequently, individual academic performance can be 'tracked.'

The English National Curriculum's emphasis on academic attainment relies upon 'set' year and key stage 'goals' for every child. Attainment is linked to the subsequent tests given at school entry (4 years old), Year 1 phonics check (5-6 years old), Year 2 Statutory Attainment Tests (SATs) Reading and Mathematics (6-7 years old), Year 4 multiplication test (8-9 years old) and Year 6 SATs in reading, spelling, punctuation, grammar and mathematics (10-11 years old) of the primary curriculum. In the Secondary curriculum, 
despite Key Stage 3 SATS being dropped in 2010, many schools still use them as a form of assessment in Year 9 (13-14 years) giving options for the formal General Certificate of Secondary Education (GCSE) choices taken in Year 11 (15-16 years). In 2007, the National school leaving age changed from 16 years to 18 years old which meant that young people were now required to stay in school, training or a workplace. This gave subsequent implications for most students to take on either Business and Technology Education Council (BTEC) 'Diplomas' or 'Extended Diplomas,' the International Baccalaureate (IB) or 'Advanced' levels (A Levels) at Year 13 (17-18 years) required for college and University entrance levels (DfE, 2014).

As students are in school from the age of four in the UK, it is the school who initially observes and assesses each student's academic progress. The British Dyslexia Association suggests that within a mainstream class between $10-30 \%$ of pupils will have a learning difference of some description (BDA, 2018) while according to the British Institute of Learning Disabilities (BILD, 2018), a further $20-30 \%$ will also have subsequent mental health issues affecting their academic performance. These differences in learning are currently coming under the banner of 'Special Educational Needs and Disability' (SEND) within the English school system under the latest "Code of Practice" (DfE/DoH, 2015).

\subsection{Working Memory and links to learning difficulties / differences}

Special needs diagnoses and their consequential teaching strategies still refer to the term 'difficulties' when describing differences in learning and thinking, denoting to a medical, deficit model of referral. More recently the more socially aware term 'neuro-diverse' has been substituted to explain the difference, but not necessarily the difficulty, between individuals' leaning approaches and styles, (Kirby, 2020). Whether 'difficulties' or 
'differences' in working memory, its fluency and capacity, can reflect intermittent learning which in combination with genetical dispositions can lead to diagnoses of dyslexia, and dyscalculia (Alloway et al., 2009, Alloway, 2007, Alloway and Gathercole, 2006, Gathercole et al., 2008a, Holmes, 2006, Lissack, 2018, Posner et al., 2014, Posner and Rothbart, 2007).

Dyslexia and dyscalculia can co-occur with many neuro-diverse differences such as Attentional Deficit Hyperactivity Disorder (ADHD), Attentional Deficit Disorder (ADD) or Autism, yet can also occur singularly in individuals. Dyslexia affects not just reading skills but also written and comprehension difficulties. Dyscalculia affects sequencing skills, overall mathematical knowledge, and reduces mental capacity for number facts and spatial awareness in presenting findings. Other differences such as dysgraphia and dyspraxia can affect both thinking and muscle control through the control of neural pathways (Alloway, 2007, Jeffries and Everatt, 2004, Terrell, 2011). Evidence also suggests that students with speech and language difficulties also suffer from differences in working memory and fluent, verbal communication (Hasselhorn and Grube, 2003, Henry et al., 2015, Moll et al., 2015, Muter and Snowling, 1998, Shankweiler et al., 1979, Veenendaal et al., 2014). Individual approaches or adapted 'personalised' learning strategies are often the most successful way to maintain accuracy in memory retention, confidence, selfesteem and autonomy for these learners.

\subsection{Identification of "need"}

Special Educational Needs (SEN) was originally a title given in the 1996 Education Act, to denote those students who had a 'learning difficulty' in that they had, "significantly greater difficulty in learning than the majority of the children of the same age," or "had a 
disability which prevented or hindered them from making use of educational facilities of a kind generally provided for children of the same age in schools within the area of the Local Education Authority" (DFES, 2001p. 6, Section 1.3). Previous to this, these children were labelled either physically or mentally handicapped or 'maladjusted' (Warnock, 1978).

Students' individual needs require adaption to different styles of teaching and learning. Schools now use a tiered process called 'Quality First' teaching, set up in 2015 whereby 'high quality teaching' is differentiated for those pupils who have, or may have Special Educational Needs (SEN) (DfE/DoH, 2015 Section 6.37 p. 99). This 'quality' of teaching, however is reliant on, "improving teachers' understanding of strategies to identify and support vulnerable pupils and their knowledge of the SEN most frequently encountered," (DfE/DOH, 2015 p. 99) while documenting student social and academic progress in consultation with national data, desired outcomes and expectations of progress including those at risk of underachievement (DfE/DoH, 2015 Section 6, 6.36-6.41, p.99).

There have been huge efforts to improve pedagogic strategies (EEF, 2017) and subsequent quests for robust interventions for small group or 1:1 teaching for the academic success of all students, regardless of ability, whether with learning differences or not. Those under achieving can be given extra funding, for example 'pupil premium' (EEF, 2019, Gov.UK, 2019, NASEN, 2014) if they fit the 'disadvantaged' criteria. Access to 'interventions' are given on an attainment basis in relation to low scores for reading, spelling, writing or mathematical schemes. Schools are now carefully studying which interventions will work best for the required achievement expected from their students 
The introduction of the latest OFSTED framework (2019) further emphasises new changes in the school inspection criteria for 'curriculum breadth' and the pupils' 'effort and success' in completing their work, both in and outside lessons. This is in combination with the Mental Health Act (DHE, 2017). School inspectors themselves are still required to examine the intent, implementation and impact of teaching of reading and mathematics, particularly in the 'Early Years' through their 'deep dive' approach to the curriculum (Ofsted, 2019, Ofsted, 2018).

Continuous assessment, whether for young students learning to read and calculate or for subject knowledge learning and exam stress are still emotive issues with many students, teachers and parents. As maintained, attainment is regulated by previous knowledge, working memory and attention, together with emotional motivation and regulation of anxiety, fear and stress. It is therefore plausible that an intervention can be implemented which not only can manipulate these levels of stress but also improve the levels of working memory performance and educational attainment.

\subsection{Introducing Animal Assisted Intervention (AAI)}

Beetz (2013) uses the reasoning that that dogs should be incorporated in to schools due to the socio-emotional correlates to learning, in that a 'positive attitude' increases motivation and potential to support learning (2013, p.1). Beetz further explains that dogs are useful when children read due to the following factors; dogs can affect motivation and self-efficacy, attract motivation through the 'biophilia' hypothesis (Wilson, 1984), assist with attachment and stress reduction via social support, promote learning pre-requisites and activate the oxytocin system (Beetz and McCardle, 2017). However, it should be noted that the 'biophilia' hypothesis involves all living organisms including plants or people and 
not just animals. It could therefore be argued that people are equally responsive to other human beings and as such these human to human interactions would also be of equal benefit (Busch et al., 2016).

Nevertheless, according to Gee (Gee et al., 2017b), the 'success' of including animals within educational settings follows the principles of major sociocultural educational philosophers, such as Piaget, Vygotsky and Montessori via a relationship built with the environment and exploring the use of experimental holistic approaches through integration and interaction. Children are naturally drawn to explore and play in novel environments, including those with animals. Simply by having the dog present, it is suggested that students can relax, increase their attention and therefore their performance (Hediger and Turner, 2014). While teachers do not disagree with this principle, they are responsible for measuring and evaluating such responses in relation to each student's subsequent attainment.

\subsection{Benefits of Human Animal Intervention for those with identified 'needs.'}

In the past 10 years, overall claims for the benefits of AAI and AAT with Autism or ADHD have included an increase in social skills, either with the animal, or with initiating conversation with others, (Becker et al., 2017, Stevenson et al., 2015, Grandgeorge et al., 2015, Solomon, 2010, Keino et al., 2010). An increase in vocabulary (Grandgeorge and Hausberger, 2011, Gabriels et al., 2012), a creation of an awareness of 'Theory of Mind' and empathy with others, (Becker et al., 2017, Grandgeorge et al., 2015, Grandgeorge and Hausberger, 2011, Marine et al., 2012). Greater pro-social skills have been promoted by the students such as 'assertion,' reduction in 'irritability,' 'adaptability,' and 'conflict management,' (Carlisle, 2015, Hall et al., 2016b, Hall et al., 2017, Gabriels et al., 2012). 
These social skills have led to transference of communication skills to adults, (Prothmann et al., 2006, Prothmann et al., 2009, Marine et al., 2012, Martin and Farnum, 2002, Kršková et al., 2010), reduction in solitary or repetitive behaviours (Becker et al., 2017, Stevenson et al., 2015, Redefer and Goodman, 1989) and an increase laughter and playfulness (Martin and Farnum, 2002). The dogs have acted as 'social buffers' (O'Haire et al., 2015) with claims that they 'prime' children ready for therapeutic interactions, (Silva et al., 2011), become a motivational tool for school activities (Stevenson et al., 2015) and decrease feelings of isolation and depression (Becker et al., 2017).

Several claims have also been made for the particular perceived relationship between those with autism and dogs, such as those with dog ownership have greater social skills the stronger the bond the greater the social skills (Carlisle, 2015), reduction of stress in families (Hall et al., 2017, Hall et al., 2016b, Hall et al., 2016c) dogs body language being more easily "read" by those with autism (Grandgeorge et al., 2009, Marine et al., 2012, Prothmann et al., 2009) with several references to Temple Grandin's work with many species (Grandin et al., 2009, Grandin, 2007). Reading has also been an area of success for researchers with many international links to charities supporting and promoting the use of 'read to dogs' schemes; simply reading to the dog will improve overall reading skills, confidence and self-esteem for the learners.

Most AAl studies refer to the American Psychological Association Diagnostic Manual of Mental Disorders version five, (DSM-5) as baseline criteria for diagnoses of neurodevelopmental differences. In 2013, the latest version however, changed the criteria for autism and now the diagnosis of "autism spectrum disorder" encompasses the previous diagnostic criteria for Autistic Disorder, Asperger Disorder, Childhood 
Degenerative Disorder and Pervasive Developmental Disorder (autism.org.uk., 2019). Currently the UK also refers to the latest International Classification of Diseases, version 10, (IDC-10) from the World Health Organisation. This means that there is now a wide spectrum not just in the diagnoses but also in the different interpretations of the criterion involved and that much of the AAI research for 'autism' may not now truly reflect the scope of the condition.

Research has assumed that all those with autism have social and communication difficulties, a lack of understanding social and interpersonal skills, non-verbal communication, repetitive behaviours and need to develop the skills of 'theory of mind.' However, as is present in many schools, not every student with autism displays all or some of these difficulties all the time.

Information from statements can therefore be misconstrued. For example, Bystrom \& Lundqvist Persson (2015) reason that, “As children with ASD [Autism Spectrum Disorder] are a very heterogeneous group, with different personalities and different kinds of problems within the autism spectrum which hamper their development, various kinds of treatments are needed,"(Byström et al., 2015 p 264). Animal Assisted Therapy or Animal Assisted Education would therefore help as it is, "well documented that animals can provide social support to people"(Byström et al., 2015 p 264). Their evidence, however, is related to animal support in general, rather than to the specifics of ASD. This can be misleading, as those reading the research may well assume that all animals help all children or adults rather than it can potentially help with different skills for some individuals. 
There have been six systematic reviews for AAl and autism over the last ten years, covering the research period between 1980 - 2017 (O'Haire, 2013, Davis et al., 2015, Hoagwood et al., 2017, McDaniel Peters and Wood, 2017, Shen et al., 2018). Most of the studies reviewed have been with horses (McDaniel Peters and Wood, 2017, Shen et al., 2018).

Critiques of this body of work have focused on study numbers, with many single case studies, parental or teacher based questionnaires showing bias and non - blind judgements, insufficient control groups, difficulties in separating just one aspect of therapy as many had simultaneous designs, non-standardised measurements used, including 'rating' based assessments, differing assessment of ASD, anecdotal reports and overall design. Time and length of the 'interventions' were highlighted as problematic, as many were only for the duration of the intervention study only, and so long-term effects were not measured, included or discussed. 'Theory of Mind,' according to Davis (2015), was still yet to be considered as solid 'proof' of concept.

At best the evidence for improvement in ASD behaviour and cognition overall was considered 'weak' and a stark warning was given by Davis et al, "Caregivers and practitioners are cautioned in selecting AAl as it is unclear the impact this may have in treating impairments in social communication and restricted, repetitive behaviours in children with ASD"'(Davis et al., 2015 p 326), although O'Haire is more positive, suggesting that at best, the reviewed studies show, "that AAI is in the first phase of research on a new psychosocial interventions for ASD - proof of concept"(O'Haire, 2013 p 1619).

The research for ADHD and AAI gave a similar picture to that of the ASD researchers. The body of work has incorporated emotional and behavioural difficulties, together with 
'attachment' issues under the banner of 'Attention Deficit (Hyperactivity) Disorder' behaviours, even though under the DSM 5 description, attachment is not mentioned. Different experimental designs and case studies have still shown a lack of significant, unbiased differences between control groups not receiving treatment and those who have had animal intervention (Kogan et al., 1999, Ewing et al., 2007, Somervill et al., 2009, Beetz et al., 2012a, Bassette and Taber-Doughty, 2013, Schuck et al., 2015, Rabbitt et al., 2014, Busch et al., 2016). Difficulties again occurred with sample size, experimental set up, with some groups being held as 'self-controls' so that all students could take part in AAl, different interpretations of behavioural diagnoses, individual differences and although there was much anecdotal evidence of improvement in behaviours with students on encountering the animals, the conditions would be difficult, if not impossible to recreate, for standardisation and replication.

The most common measured outcomes of research into AAl for both the ASD and ADHD groups were the behavioural consequences, interpersonal interactions and subsequent sensory and movement processing. Classroom behaviour outcomes were expressed as having 'easier times at school,' 'interacting with peers' or to 'paying attention to the consequences of behaviour,' but these same outcomes did not further relate these skills into cognitive benefits for class subject or curriculum based learning (McDaniel Peters and Wood, 2017).

\subsection{Overall Evidence for Animal Interactions in schools}

There have been two, recent, systematic reviews trying to examine the overall evidence for the efficacy of animals in school-based curriculums. Brelsford et al., (2017) and Hall et al., (2016). Hall et al (2016), conducted their systematic review of Human Animal 
Interaction (HAI) articles with the intention to highlight those reviews which not only added to overall literacy and frequency of reading, but also overall enjoyment of school. Using the Preferred Reporting Items for Systematic Reviews and Meta-Analyses (PRISMA) technique; an evidence based minimum set of items aimed at helping authors to report a wide array of systematic reviews and meta-analyses that asses the benefits and harms of a health care intervention, they gathered 48 documents written in English using multiple data bases between January to March 2015.

The second systematic review, Brelsford et al., (2017) examined "Animal Assisted Interventions in the Classroom." Their aims were to, "scrutinise the empirical research literature relating to animal assisted interventions conducted in educational settings" reviewing 25 papers, 21 from peer reviewed journals and four from 'grey' literature databases" (Brelsford et al., 2017 p 1). 'Grey literature' describes a wide range of information produced from different sources often outside of the distribution and publishing channels, which is not well represented in indexing databases. Both reviews, Hall et al., (2016) and Brelsford et al., (2017), had the same contributor, Dr Nancy Gee, who had also included her own research within the Oxford Centre for Evidence-based Medicine (OCEBM) criteria which questions the partiality of the reviews.

Both reviews had similar results in that most of the AAI evidence was on an "ad hoc" basis and conclusions drawn from small sample sizes (these can be as small as three students.) The AAI were usually studies of typically developing children in comparison to those with a special educational need or reading difficulties. Studies did not use 'blind scoring' nor appropriately randomly allocate children to intervention or control groups. There was not a priority to use standardised tests which would show reliability and validity to their 
results, and that standardised scales would need to be required to assess the relationship between reading performance and behavioural processes. Further documentation of the intervention sessions should take place such as the number and duration of sessions, details of the dog/s and demographic variables of the children - including their experience with dogs. Animal welfare and safety precautions need to be considered and included as part of the research design, including risk assessments not just for the humans but also the animals themselves. Testing should be known to all involved and study results should be reported with all necessary detail and background information for replication.

Despite these points, it was concluded there was enough evidence from both reviews to suggest that there was an argument for animals to increase socio-emotional influences in learning including the effects of animals on mood, emotional regulation and social behaviours. There is a positive effect of animals for those with insecure / disorganised attachment behaviours and despite there being no random control trials and rigorous controls in many investigational designs, in general, the reviewers felt that there was enough evidence to suggest the validity in using dogs with reading for Human Animal Interaction (HAI).

Overall, the reviewers' conclusion raised the issues that, "It is important that studies use appropriate sample sizes to enable confidence in the detection of meaningful effects. Additionally, there is a need for practitioners and researchers to work together to evaluate specific reading to dog's curricula in order to ensure any benefits are maximised and studies comparable." (Hall et al., 2016a, p. 8). 


\subsection{Issues for Teaching from these reviews}

The main concerns that teachers could question from these reviews would be those of the selection of the groups of children and the necessity of standardised control tests. A random standardised control test assumes a scientific and medical stance towards a choice of the participant for the conditions, one the intervention, and the other a control. To be 'randomised' entails simply being given a number and placed accordingly into a group. Alternatively, it means being labelled by a diagnosis, and on the grounds of the diagnosis alone, being chosen for that intervention. It would also imply that there is one set of criteria universally used for that diagnosis and that these remain static throughout the experiment.

This is a major area of contention as each student, regardless of age, is an individual and has strengths and weaknesses in their learning profile which may fluctuate daily depending on mood and cognitive maturity, requiring differing learning styles. Under the movement of "inclusion," the emphasis is on all students regardless of need, integrating to support one another. Too often the selected children used in these AAl interventions appeared to be in specialist classes or gained from 'specialist groups' from across several schools - which is impractical to reproduce unless a set of 'cluster' schools (i.e. those neighbouring schools working collaboratively together) or a Multi Academy Trust (MAT) could get funding within their own schools. Arguably, a failing of these studies is the lack of samples drawing on typically developing children across a single school.

This is also problematic when working in schools with diverse populations / catchment areas and it is difficult to justify why one child receives one intervention over another, without 'labelling' the child. Children who are receiving 'pupil premium' or are 'looked after' by the authority may also potentially benefit from working with the dog, yet they 
may not be showing any learning differences or needs in their schoolwork. Schools would argue that working with AAI should involve all students across the school, or due to the large numbers of students within some schools, within certain year groups.

Standardised tests are used within schools and are a useful measure to link with other results from other subject areas for attainment. These can add to the overall learning profiles of the children concerned, yet depending on some of the tests used, teachers require postgraduate qualifications and training for tests measuring more than simple reading, comprehension, spelling or mathematical ages. Much of the AAl research presented has used tests and assessments which can only be accessed by qualified psychologists. This would suggest that reading ages, for example, given by teachers, may be simply too crude to measure the difference the presence of the dog would make to a student.

\subsection{Truth Effect}

Dechêne et al., (2010) in investigating the 'truth effect' suggests that the fluency of truth is often complicated by the repetition of previously heard information, in that people's trust in statements' is increased if it had been encountered before - regardless whether it was a true or false statement (De keersmaecker et al., 2019, Hasher et al., 1977, Nadarevic and Aßfalg, 2017, Kahneman, 2011). This can lead to a semi-conscious bias when interpreting the results and anecdotal stories from animal assisted interventions, for example the influence on the teaching community from the heavy bias from the social and National media when reporting the efficacy of AAI programmes such as reading (Anstice, 2016, Black, 2009, Lane and Zavada, 2013, Pillow-Price et al., 2014, Powell, 2018, Massengill Shaw, 2013, Stroud, 2012). It is often for this reason that dogs are invited into 
schools under the guise of assisting with the pupils' academic skills. On the basis of this 'evidence,' schools are now taking the concept of the 'visiting' dog further, in that they are now introducing their own full time school dog as part of the 'staff team' regardless of the suitability or necessity of the role. This implies that the students in these schools will have ready access to the animal, throughout school hours, and the dog can be thus employed in several roles, such as reading dog, comfort dog, and companion as required. This is an onerous task for any dog, let alone a very young dog or even a puppy.

The repetition of how dogs can affect working memory and attention too, could be considered illusory when just one major author is quoted in many different sources yet has limited research facts and figures to substantiate the claim. It is important to also mention that sponsorship from large foundations and trusts is also given to smaller school-based charities on the understanding that dogs can help students with their learning. Likewise, certain journals promote certain views, and have employed 'lead' researchers through parent companies, such as the case of Waltham Mars, (a producer of animal feed) which sponsors the journal 'Anthrozoos,' a major source for Human Animal Interaction research. These articles are then subsequently re-written by education-based researchers for teaching communities, thus reiterating the implied results. Often what is not considered is the amount of research which also exists that may negate some findings but remains either unpublished or has not made certain journals' requirements or standards.

\subsection{Research into Reading using Dogs}

Research into reading using dogs has suggested that reading skills improvements have been found (Pillow-Price et al., 2014, Schretzmayer et al., 2017, Lenihan et al., 2016, 
Smith, 2010, Kirnan et al., 2016, Beetz and McCardle, 2017, Swift, 2009, Jalongo, 2005). However, on further examination, these studies have very limited numbers, refer to reading "attitudes" or behaviours, rather than phonological or processing assessment and there are still difficulties in replication, with many studies in languages other than English. The English language for reading and spelling can be considered more complicated than others, due to its aetiology (Snowling, 2013, Thompson et al., 2015). Bearing in mind that reading is an area covering many executive functioning skills, it is useful to also check the methodologies against the claims.

Bassette and Taber Doughty's USA study (2013) drawing on qualitative methodology, indicated that indicated that on task, reading aloud behaviours, such as accurate decoding or speed improved with three 'elementary' (UK Key Stage 2 Primary - 7-11 years) students with diagnosed emotional and behavioural difficulties, two were male and one female. The students worked through the 'upper instructional/ lower frustration' levels of reading and actively chose to read to the dog. These behaviours were apparently maintained after the intervention had finished. The individual case studies of each reader also showed more general positive reading behaviours centred around the dog while it was present. This study reiterated that dogs can help with reading motivation.

Wohlfarth (2010 as cited in Wohlfath et al, 2014) reported on Smith's thesis research in that the standardised reading baseline assessments both pre and post intervention using the Grey Oral Reading Test (GORT), a test of oral fluency and comprehension used in the United States, showed significantly improved scores than a control group when 26 homeschooled children read for 30 minutes to a dog for six weeks. 
Wohlfarth then used a quantitative approach with a group of 24 , German speaking 6-7year olds to test reading both with and without a dog present using a cross-over design, where each of the participants experience all the conditions during the experimental timeframe period. Statistical analysis was used with the results which examined all reading errors, content comprehension, reading time, and text comprehension (which was later removed as it was shown to have too many simple questions which could be easily answered.) There was a slight increase in the mean percentile of $2 \%$ in the accuracy of reading with the dog, a $12 \%$ increase in the mean percentiles for the punctuation and $1 \%$ increase in the time spent reading (although these were 'set' reading pieces rather than the pupil's own choice of book.) Wohlfarth et al then suggested that the t-test combination of the correct word recognition, the punctuation and correct line breaks created a "concentration" index, showing raised results from the dog condition in comparison to the control. Another area that did arise from their study was that the longer the actual physical, intensive contact with the dog, the slower the reading performance, which could reflect low stress levels or alternatively cognitive or sensory overload.

Le Roux et al., (2014) in South Africa, used an experimental, mixed methods design using pre and post-test standardised reading tests; the ESSI Reading Test, a South African reading and spelling test for both Afrikaans and English, and the Neale Reading Analysis, a test of oral reading, also available in the United Kingdom. The purpose was to study the effects for reading rate, accuracy and comprehension with 102 poor readers aged between 7 and 13 years of age, randomly assigned to three groups, reading to the dog, reading to an adult and reading to a teddy bear, over a 10-week programme. Each group 
met with their 'team' for 20 minutes each week for 10 weeks. There was a difference in this study in that none of the children had attended pre-school before the age of seven and had no access to outside literacy resources such as a community library. During her study four children then left school and it is questionable as to whether 12- and 13-year olds were wanting to read to a stuffed teddy. SPSS statistical analysis was used with the results. The results showed a difference between the genders in the overall reading levels with females scoring higher for both rate and accuracy at both pre and post baselines. The study showed that the length of reading time would naturally have an effect for overall comprehension and that the students from the dog group read at a significantly higher rate than all the other groups - even eight weeks later after the intervention - although the motivation of being given a book for every completed reading book may also have been an incentive.

Lenihan et al., (2016) used the Reading Education Assistance Dogs (R.E.A.D., Pet Partners charity) with 18 children ( 9 test and 9 control) aged between 7-8 year olds over two half hour sessions using a 'Curriculum Based Measurement' (CBM) through reading timed passages together with an Elementary Reading Attitude Survey (ERAS) using statistical analysis. The results showed there was no statistical difference in the CBM between the control or dog groups, although there were reductions in the ERAS $(p=0.04)$ attainment and attitude for the control, showing that those who had not read to the dog, had reduced attitudes towards their reading.

Schretzmayer et al., (2017) in her Masters dissertation, investigated the short-term effects of dogs on reading performance on 36 , nine to ten year olds in three Austrian schools. These included physiological data gathering such as heart rate and heart rate variability, 
salivary cortisol and behavioural variables from the children. Schretzmayer et al., used a quantitative cross over design, including observational data using Solomon Coding, a technique using error correcting codes, together with standardised pre and post-test reading tests, using the sub test of sentence comprehension and text comprehension. She also used a non-standardised reading test, for 'Repeated Reading' which allowed the assessment of short term, spontaneous improvement in reading performance. This was taken from two, selected short texts which had to be read out aloud as quickly as possible, i.e. within two minutes, on two occasions and their speed and accuracy was compared. The results of the study confirmed that the dog had no effect on the reading performance scores but did have an improved effect from the first to second runs of the 'Repeated Reading' - but only when the dog was present. They found no significant impact on Heart Rate or Heart Rate variability but there was more excitement in the expectation of the dog with the children being more aroused when the dog was present. They concluded that the dog had a positive effect only on the minor short-term motivation and reading performance of the children.

Consequently, there have been similar results from more recent research. Connell et al., (2019) worked with 63 children aged 6-8 years, grouped and assigned to three conditions. The clearly defined parameters were; 15-20 mins per week over 4 weeks to train a dog to complete an obstacle course; $15-20$ mins to read to a stationary dog; and the third group to continue with normal classroom activities with a dog present. Although there were overall improvements in reading ability across the time period for all three groups, there were no significant changes between the group or interaction effects, showing the dog had made no impact of significance at all. The weakest readers did seem to make the most 
progress. Measurements used included the York Assessment of Reading Comprehension (YARC) and the Single Word Reading Test (SWRT) which were considered for this dissertation. Despite there not being a "non-dog participating" group to compare results, the Australian researchers concluded that, "Our study contributes to available research on the benefits of dog-reading programs by suggesting that reading gains may occur regardless of the type of activity engaged in. This is potentially important because, if how children interact with the dog is not a critical factor in improving reading, with the mere presence of a dog providing the support children require to optimally function, there may be little need for dog-assisted programs to be overcomplicated by focused activities; simply having a dog in the classroom may be equally as beneficial as specific interventions," (Connell et al., 2019 p. 356). The original suggestion that simply the presence of the dog assisted with classroom tasks came from research set by Nancy Gee in 2009.

\subsection{Past research into the presence of the dog for cognitive acuity}

Professor Nancy Gee was part of both panels for the Brelsford and Hall reviews and included her articles in the overall criteria (Brelsford et al., 2017, Hall et al., 2016a). As a result of her presence, several articles from these reviews were also suggested for highlighting the research of using dogs for cognitive skills, in particular the improvement of working memory and attention. Gee's own work implied that increased arousal from the dog's presence can heighten cognitive performance for some tasks - although the cited sources were examining the effects of exercise on children and emotional effects of sport rather than the use of dogs. Dogs, according to Gee, can assist with specific skills such as: reading rate, accuracy, comprehension, adherence to instructions and 
instructional prompts, categorisation, object recognition, motor tasks and general classroom behaviour (Gee et al., 2009, Gee et al., 2012a). A dog could, "improve the motivation of children to engage and accurately complete set tasks," (Hall et al., 2016a p 7) and that there is enough evidence to show that children prefer to interact with, and pay greater attention to, real animals including dogs, with reference to the studies suggesting that children prefer to work with animals rather than stuffed toy dogs (Gee et al., 2010a, Gee et al., 2012b, Gee et al., 2010b). This claim has been further supported by two further studies which report that children, particularly with ADHD diagnoses, also respond with more attention and engagement to animals (LoBue et al., 2013, Schuck et al., 2015).

Gee's research has been based on seven studies with children and two with adults. Gee and her teams recruited their children from a nursery pre-school attached to their campus laboratory and used her own two therapy trained dogs that had regularly visited the children, sometimes up to twice a week (Gee et al., 2010a). As the children were very young, aged between three and five years, Gee and her teams concentrated on the prerequisites for formal, academic learning with this age group, such as fine and gross motor skills, following instructions or categorisation tasks. In every experiment, story scenarios were used to explain the activity to the young children and although the children would have realised that 'Louie' and 'Nicki' or 'Duncan' were dogs, the canines were often included in the stories as if they were human characters (Gee et al., 2009, Gee et al., 2010b, Gee et al., 2010a). The suggestion of 'assistance' may have simply motivated and encouraged the young children to complete the tasks in itself. 
In project one (2007), 14 children aged between four and six years, nine 'identified' with speech and language difficulties and five 'typical' were asked to complete ten gross motor skills (such as long jump, high jump etc.) and an object categorisation task with and without the presence of a dog. The dog interacted by performing the task immediately prior to, or at the same time as the child, giving an overall project significance effect. However, despite all the children completing the tasks faster when the dog was present, this did not overall affect the ratings data.

The project concluded that the dog was a motivator and did not affect motor task accuracy yet the authors recommended a "role for therapy dogs in speech and language development programmes for school age children"(Gee et al., 2007 p.375).

Despite these findings for gross motor skills, a second project was initiated. In project 2 (2009) 11 children, six of whom were 'identified' with either speech and language or fine and gross motor movement difficulties were asked to complete several motor skill tasks such as 'long jump,' 'crawl' or use a balance beam under three conditions; modelling where the children were asked to emulate the behaviour of the 'model,' whether adult, stuffed toy dog or real dog; competitive, where the children were asked to do the tasks faster than the competitor; and tandem where the children did the tasks at the same time as a co-performer. Both dogs were interactive in that they could take part in all the motor movement-based exercises (based on their agility training skills.) While the adult proved most effective in the tandem conditions using verbal instructions, the dog presence was strongest during the modelling condition. There was no significant difference in the competition tasks. 
The project concluded that the dog was beneficial for tasks requiring modelling or copying behaviour within the classroom and the dog was a highly 'salient' stimulus, restricting the children's focus to the task in hand (Gee et al., 2009 p.273). This was despite the statistics portraying the adult and dog as equal importance or the noted fact that the children enjoyed playing with the dogs.

In 2010, Gee's teams published two more articles about children's memory and attention in the presence of dogs (Gee et al., 2010b, Gee et al., 2010a). In the first, Gee used a group of 12, 3-4-year olds, five of whom were considered 'cognitively delayed' for an 'object categorisation task' involving matching a given object to another from a given selection of 'taxonomic,' 'thematic' and 'different' features, for example matching a brown rabbit with either a grey rabbit, a carrot or a book. Again, these tasks were compared between the presence of a real dog, a stuffed toy dog and an adult. The results concluded that "the presence of the dog resulted in significantly fewer irrelevant choices in either the stuffed toy dog or the human condition.... the real dog does not appear to alter the typical performance patterns seen in object categorisation, but the fact that fewer errors were made in the presence of the dog does have a positive impact on performance of this cognitive task" (Gee et al., 2010a, p. 223). The task was then repeated with one variable per week, for 10 minutes with each child, who was asked to 'help' to create a picture book for either the real dog, stuffed toy or human. Depending on when the dog session was held, it is possible that the children could have remembered the novelty of the task, plus the actual initial stimulus pictures were given to the child, rather than 'free choice' to pair any card as the child saw fit. There was also an unexpected issue raised when the younger children (aged three to four years) made more taxonomic choices than thematic, 
reflecting their stage of developmental awareness. The team's experiment substantiation also included the statement, "The presence of the dog may have a calming effect (based on Friedmann et al, 1983), or it may serve to increase focus on task demands," quoting Gee's previous findings in 2009.

The second article that year involved two experiments (Gee et al., 2010b). The initial task was with 12, three to five-year-old children, seven of whom were "identified" (no further information given) while five were considered "typical." Gee et al., used two "instructional prompt tasks" while the children were in the presence of a real dog, a stuffed toy dog and an adult. Prompts included general instructions like, "Face this way," and task specific prompts included "Pick one of these objects." The first experiment involved the use of three-dimensional objects placed on a toy 'train.' There were three parts to the experiment. Initially the child was introduced to the 10 target items and was then given a 'distractor' game to complete. After this, the child had to pick the same 10 target objects out from a group of 20 objects (10 as distractors) from taxonomic themes, e.g. the child needed to pick a stuffed bear from a choice between a stuffed bear and a stuffed lamb. In all conditions the object chosen was confirmed and touched by either the dog's nose, the stuffed dog's nose or had a "good job" said from the adult.

Initial results showed that the dog's presence was of significance in both the general and specific prompt instructions with the least instructions required. This was accredited to either the children perceiving the dog as requiring more help than the adult or the stuffed dog, or that the dog, being well behaved and 'nosing' the object had both kept the children focused and gave a model for the copied behaviour. Novelty was not seen as a factor as the children were 'used' to the dog. It was noted however, that the children 
viewed the stuffed dog in a similar way to the real dog, but as the stuffed dog was not real, the toy was simply not motivating enough when next to a real dog.

The second half of the experiment, several months later, used photographs of the objects and was repeated in the same way as the first. The results were that the dog situation required the least number of instructional prompts during the tasks, followed by the stuffed toy, and then the adult. Gee explains this as the dog provided a motivator for the children and refutes any 'novelty effect' for the task as the dog was a regular visitor to the class, -"Many of the children would rush to hug him when he entered the room," (Gee et al., 2010b $\mathrm{p}$ 182). This questions whether the results are the motivational effect of that particular dog's bond with the children, or if the novelty of a play task in a 'pseudoclassroom environment' with the dog gives greater enjoyment to the children in general. Gee suggests that it is, "possible that the dog provided that highly salient stimulus needed to help the children restrict their attention to the demands of the task," in requiring fewer instructional prompts. Another suggestion was that the children were possibly modelling "the calm and obedient behaviour of the well behaved dog" and that perhaps children do not feel the need to model that behaviour of an adult human or stuffed dog" (Gee et al., 2010b p 183).

From an educational view, it was noted that in this study the 'identified' children did not require more prompts than the 'typical' children. Gee suggests this is due to the possibility of the dog helping them with their improved comprehension. Neither experiment was examining the extent of the impact of the dog on memory performance itself, which would be useful to compare with both dog present and without. 
In 2012, Gee and her team developed the 2010 object recognition task further, removing the need for instructional prompts but now included, 'one versus four distractors' conditions. This was to enhance the use of visual strategies. This implied that the child was to select a 'target' picture, e.g. a bike from either one other choice or from four, very similar choices, differing perhaps in slight shape or colour. The 20 , three and a half to fiveyear-old children, 12 considered 'typical' and eight considered 'identified' with language delay were prepared in advance of the experiment to recognise the objects on the cards and were marked according to an 'identity score' depending if any verbal prompts had to be required. The children were then told a story about how the dog or adult had seen certain objects on their way to the 'store'(shop) and then asked to complete a simple matching distractor activity before being asked to identify the objects seen. The children could touch the dog throughout, but due to social barriers this may not have then applied in the same way to the adult. According to Gee, the children performed the object recognition task significantly faster and more accurately in the presence of the therapy dog relative to an adult, suggesting that the presence of the dog, "serves to turn down the volume on other sources of distracting stimulation," (Gee et al., 2012a p.298) and despite not all the children in the sample being drawn to the dog, they suggested that they could still benefit from its presence for learning.

In 2012, Gee's team also worked with a further 17 pre-schoolers, 11 'typical' and six identified with at least one language delay. The children were asked to 'categorise' 24 animate and inanimate pictures of objects into two environments, the ocean and the farm. This was again in three conditions, with a dog present, a stuffed toy dog and an adult. The results again showed that, "The animation variable interacted with the 
collaborator, such that in the presence of the real dog the impact of the animation was significant" (Gee et al., 2012b p.187). However, during the tests, the children were verbally given the labels to the pictures and the dog interacted with his nose, whereas the adult just took the card.

In 2014, Gee continued her work with 58 university students (aged 18-41) recording heart rate variation while competing a geometrical sequence working memory task in the presence of a real dog, stuffed dog or human. In this experiment the stress rate of the working memory task did not vary in either of the three conditions, nor did the dog reduce arousal in the students as was hypothesised. Gee concluded that, "A dog may not be effective at reducing stress responses to this type of stressor." She proposed the possibility that, "the presence of the dog is more effective than touching the dog at reducing stress responses," but would require further, future investigation (Gee et al., 2014 p.513). By working with adult students in this manner, however, Gee et al., have identified that there are also effects for the presence of the dog across age ranges, which would also indicate either a learned behaviour or response from childhood, or that the presence of the dog is, in some way is affecting cognitive skills which can be manipulated by more mature executive skills.

In 2015 , Gee and her then team returned to the study of college students (31, aged 18-23 years) who while wearing a heart rate variability (HRV) monitor were asked to complete a working memory sequential task involving coloured lights under five counterbalanced conditions; dog-touch, dog-no-touch, person-touch, person-no-touch and alone. The best memory scores were when either the dog or human were present, but the worst scores were when the participant was touching the dog. Gee and her team concluded that, "If 
there is a beneficial effect on cognition from a dog, physical contact may not be a necessary component" (Gee et al., 2015b p.483). They also proposed that the relationship with the dog and its breed can also affect cognitive performance.

These experimental investigations would suggest that there is huge scope for further examination into the effectiveness of a dog's presence on working memory skills and attention, both in young children and in adults, particularly within classroom situations. Different styles of classroom-based tasks aimed at older students, i.e. those over six years old, have not been investigated, such as mathematics. The area of reading still has sufficient breadth to further examine the effects of the dog's presence, in both the identification of underlying working memory skills and individual responses for phonological awareness and overall reading ability. There is also room to examine the adult responses with the dog and if the physiological effects of higher or lower rates of skin conductance can be re-created with tasks other than sequencing. Ultimately, if as Gee's overall work suggests, that children and adults respond to the presence of the dog, rather than the task itself, would this then open up opportunities for investigating any learning task outcomes while having dogs present in virtual reality? There has been recent research into teaching at a Higher Education (University) levels using virtual reality (VR) with bi-lingual language learning which has suggested that students with SEN and their preferred learning styles can be enhanced in this environment (Tsun-Ju and Yu-Ju, 2015, Huang et al., 2020).

\subsection{Use of Virtual Reality}

Technology has also previously been used in classroom contexts and with academic subjects such as Geography, Mathematics and English to examine attention and 
concentration rates. Bester and Brand (2013) showed that 45 Grade 8 students (11-12 year olds) showed greater correlation rates for motivation, attention and concentration over sustained periods, linked to higher overall subject attainment when using simple audio visual technology such as interactive PowerPoints and videos in lessons. Investigations into action video gaming and virtual reality have also been argued to increase reading fluency, visual attention span and verbal working memory (Jaroslawska et al., 2016b, Pedroli et al., 2017, Antzaka et al., 2017) particularly with those with dyslexia or ADHD.

Indeed, it has been demonstrated that robotic dogs also interest children, such as Sony's AIBO, a robotic dog from a series of robotic pet. The AIBO was given 'physical essences mental states, sociality and moral standing' as if it were a real dog by 72 children aged from 7 to 15 years when compared to a 'real' Australian Shepherd dog (Melson et al., 2009). This suggest that there could be strong links through the Human Animal Bond (HAB) and technology to help improve future educational outcomes. For example, the use of mixed reality technology has also encouraged interaction with virtual pets to improve children's health through 'exercising' virtual puppies during a summer camp. By gaining tokens for physical exercise or eating more fruit and vegetables in 'real life' the children were motivated to play allowing their virtual avatar 'puppies' to become slimmer, be quicker at tricks and win prizes (Johnsen et al., 2014, Ahn et al., 2016).

Rohani and Puthusserypady (2015) also used animal pictures inside VR headsets with six adult students aged between $24-32$ years also increasing attention, showing the same benefits for adults as with children, while Suied et al., also showed that the VR headsets 
had a significant effect for auditory and visual processing revealing successful treatment of 10 adults with cynophobia, the fear of dogs (Suied et al., 2013).

However, during a review of over 20 studies involving the use of VR with school aged students for possible treatments for ADHD, Bashiri et al., heavily emphasised the benefits, but also illustrated the negative points, in that for some individuals, wearing a VR headset can cause side effects. These included cybersickness, headaches, seizures, nausea, fatigue, drowsiness, disorientation and apathy which for safety, perhaps should preclude those under 18 years old (Bashiri et al., 2017). Oculus, the creators of the VR headset used in this thesis, suggest this age should be even lower, at age 13 years (Oculus, 2019).

This implies that that due to ethical concerns and parental and student permissions, any research using dogs and attainment in this PhD study would have to be limited to adult students only and each participant would have a series of safety questions asked before commencement.

\subsection{Summary}

The use of dogs for an intervention for working memory and attention is clearly feasible in the context of research investigations for several different areas. Differences in working memory and attention with young children have been recorded, but there are still gaps in the research for school aged children, whether classed as having 'special needs' or not. School based curriculum subject intervention research has appeared to be restricted to reading only, which has not been truly examined in terms of how the working memory could be affected with or without the dog's presence. If any specific working memory skills in such subjects as reading can be identified, then despite the maturity in cognition, they too should also be apparent in adults, showing similar results for fluency, accuracy 
and attainment. This means that similar classroom tasks can be given to both groups and compared. Further investigation into the use of certain working memory tests accessible by teachers (albeit with post graduate qualifications, see appendix A) can also occur to give educators the autonomy to initiate their own research into any areas associated with working memory, such as visuo-spatial learning. This could point us in the direction of new areas for specific interventions, both with and without dogs for students.

A further under-researched area is the role of how the dog is employed within the classroom. The studies examined have been limited as to how the dog physically interacts and is incorporated into the classroom. Despite Gee's work, there is still minimal direct observation of the cognitive aspects of interaction with the dog and the subsequent effects this has on the learner for the actual task in hand. The experience from the view of the dog, such as enjoyment, play or boredom would surely also affect the emotional feedback to the learner and thus both the future abilities, attainment and enthusiasm for future partnerships as a learning team. This would question the role, knowledge and training of the handler, whether as a volunteer, a staff member or even a teacher in the preparation of the dog for interaction, and thus if the handler's role too, influences the learning process.

The use of virtual reality in comparison to 'live' situations can help to explore future implications for remote learning. The presence of the dog could have strong, emotional and cognitive influences in VR, which could lead to increased possibilities of alternative schooling for individuals, in some instances. The investigation may also reveal whether some subject areas are easier than others to attain while in the presence of the dog in VR. 
Again, any findings too could have implications for specific interventions with certain learners.

With some of the methodologies suggested through the reading research, there is also the scope to examine the promotion around AAI in terms of reading to dogs, particularly with the presentation for teachers, media and the social media. There is opportunity to examine the measurement of 'perceived' progress against 'actual' progress of these schemes and if the subsequent personal choice of the school 'gate-keepers' themselves influences the popularity of the scheme by perpetuation. Further investigation is required into the suggestion of the dog greater benefitting those with lower reading skills, or whether it is simply due to outside environmental influences, personal determination and maturity of reading skills.

Finally, negotiation may need to be explored as to how heart rate and physiological measurements can be measured in a 'normal' classroom with young children and with adults without breaking any ethical or school protocols. The practicality of everyday classroom situations may prove to be huge factor for consideration in future research investigations. There is plenty of scope for further research from a teacher or educator's point of view. 


\section{Chapter 3}

\section{Methodology}

As discussed in chapter one, this thesis had three linked aims:

I. To explore whether animal assisted intervention can facilitate performance in cognitive acuity, particularly with working memory and attention, for schoolbased tasks.

II. If so, could the subsequent effects of the presence of the dog still be re-enacted in virtual reality?

III. Would any effects of the above two apply across students of all ages?

These aims were based on the theory that if dogs could reduce the stress and anxiety associated with classroom-based learning tasks, cognitive acuity would increase and subsequently reflect higher achievement levels (Gee et al., 2010b, Gee et al., 2014, Gee et al., 2015b, Beetz and McCardle, 2017, Schretzmayer et al., 2017). However, an alternative theory could be formed that it is the self-determination of the student, together with the cognitive reaction to the given task that creates the 'achievement,' rather than the presence of the dog (Ryan and Deci, 2006, Posner and Rothbart, 2017, Bergen et al., 2018). The dog is present for motivational purposes only.

The main objective of the thesis was to design and carry out a mixed methods research inquiry into the effects of dogs on stress, attention and memory in both live and virtual reality (VR) situations for two groups of learners using academic based tasks such as 
reading, vocabulary recalls and mental mathematics. The secondary objective was to simultaneously use research methods used within the context of 'educational' research, to provide a practical, pragmatic view for classroom teachers themselves.

Previous research into Animal Assisted Intervention with school-based study tasks has tended to favour empirical techniques. The findings have been rather equivocal. Wohlfarth (Wohlfarth et al., 2014) used a crossover design (in which two groups of students took part in two separate conditions and swapped these over, so they experienced both) including quantitative, statistical analysis when examining differences reading to a dog, and to an adult with 12 , seven to eight year olds. This design recorded that the presence of the dog assisted with correct punctuation, word recognition and correct line breaks, yet there was no significant correlation with either eye or body contact or in length or reading time between the dog or adult. However, the generalisation was created that reading to therapy dogs was of benefit to older readers. Lenihan (Lenihan et al., 2016) used a random controlled trial and standardised tests with 15 , seven to eight year olds. The results showed no attainment difference between the control group or the dog group but perhaps near some significance for reduction in attitude from the group without the dog. Schretzmayer (Schretzmayer et al., 2017) used a crossover approach with standardised pre and post-tests, plus physiological measurement of heart rate, heart rate variability and cortisol readings with 36 , nine to ten year old Austrian students for a single session of reading with and without a dog present. The results were compared through using a statistics package showed that the dog had no effect on reading scores, and no impact on physiological measurements, although there was less 'nervous' behaviour seen while reading to the dog and the first group to read to the dog showed a 
higher average heart rate overall. From this, conclusions were drawn that the dog has "minor, short term effects on children's motivation and performance" (Schretzmayer et al., 2017 p.9). These examples show that under clinical statistical analysis, there is no benefit for attainment, no physiological differences and only minor short-term benefits when to reading to a dog. This would seriously undermine the purpose of having any reading to dogs' programmes in schools. Therefore, perhaps a less clinical approach is required to interpret the results and try to understand why dogs have been brought into the classroom. Such an approach could involve both quantitative and qualitative data gathering.

Cresswell (2015) recommends a convergent design when considering both qualitative and quantitative data together for an investigation into a problem. Although the data is gathered simultaneously during the research period, it is considered as two different areas for analysis which are then combined into one to contemplate the problem from multiple angles.

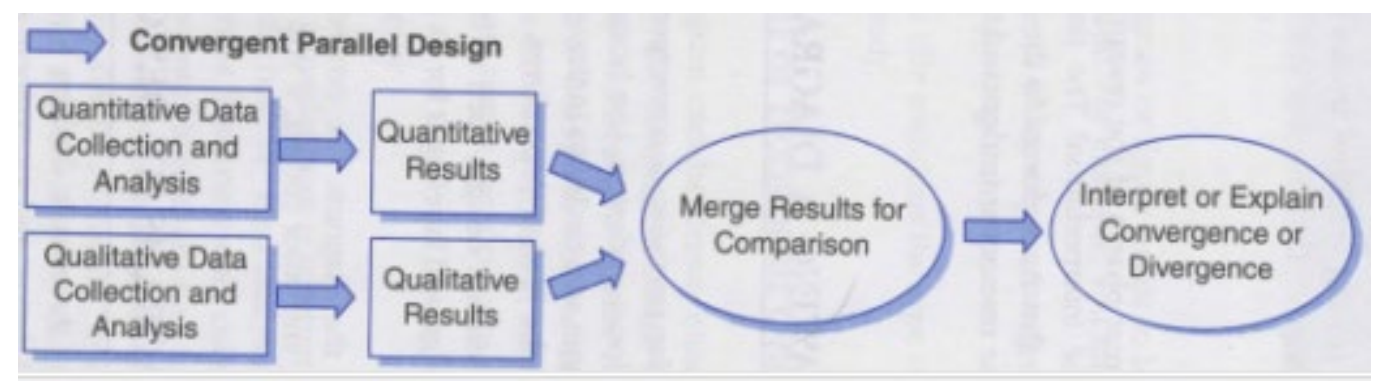

Figure 3: Diagram of a convergent parallel design (Source: Cresswell, 2015, p.38)

According to Hart, (2005 p.194) Interpretivism is the role used to interpret, to understand as well as to explain, "the truth or falsity" of a situation which are relative concepts to one another; "And that the subjective nature of human behaviour with its complex, different social values being the defined properties of the social life that create the different 
realities." The problems we recognise are therefore often the outcome of our own personal biography and do not come value free. To paraphrase Weber, "We live in webs of signification which we, our contemporaries and forerunners have created" (Geertz 1973, p.3, as cited in Hart, 2005, p.221). Despite this inheritance, Robson, (2002, p.18 as cited in Hart, 2005, p.197) suggests that scientific attitude should still be "carried out systematically sceptically and ethically" and that research should have practical value (Hart, p.197). Therefore, for this thesis several internal designs were woven together and delivered through three phases as shown in figure four below:
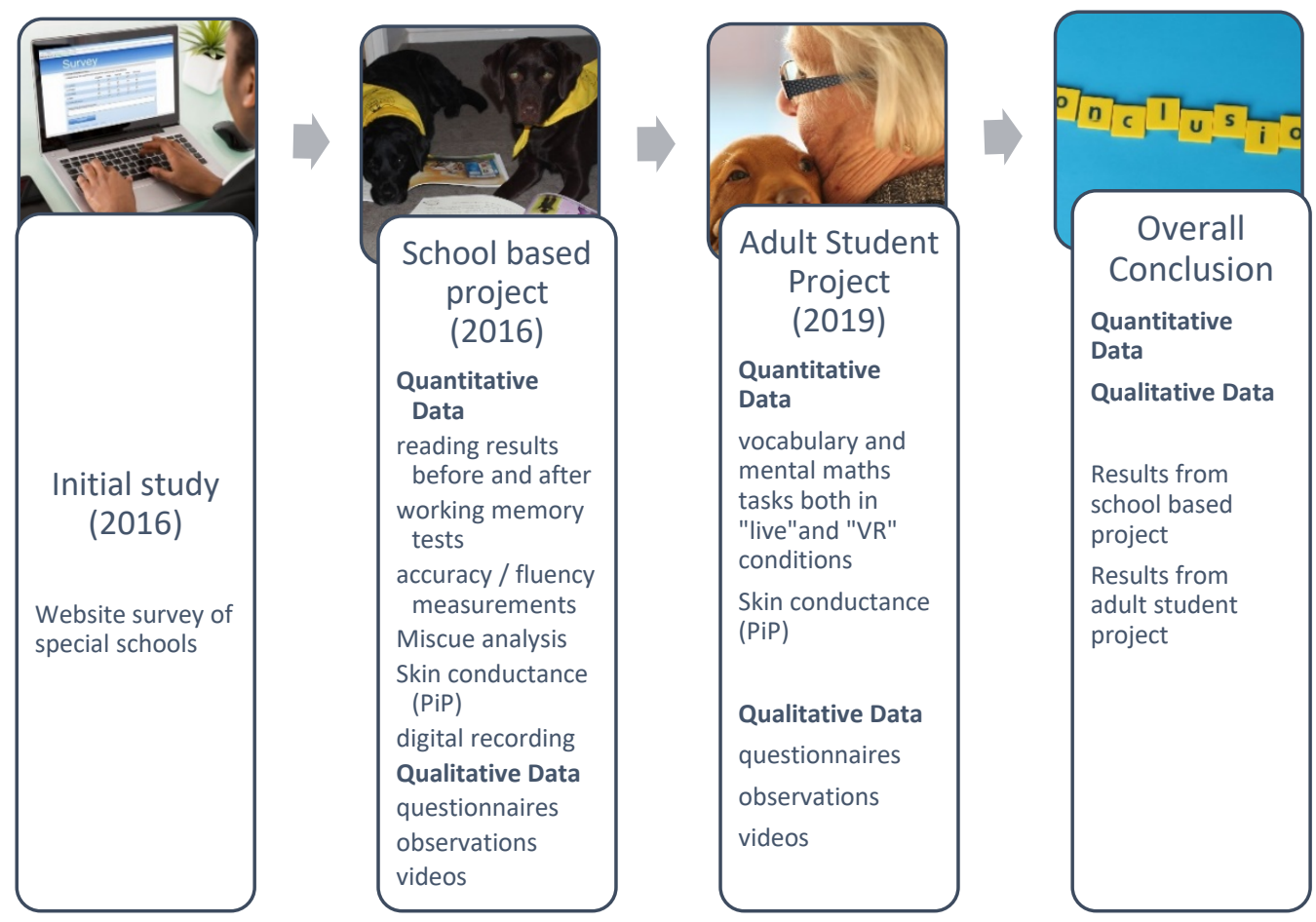


\subsection{Phase 1 - Online School Website Desk Based Survey: June 2016 - August 2016}

An initial quantitative desk-based survey was created to provide a baseline for knowledge since there was no apparent data for how many dogs were available in England's schools in 2016.

According to Cohen, Manion and Morrison (2018), the British Psychological Association had internet guidelines in 2013, defining internet research as "any research involving the remote acquisition of data from or about human participants using the internet and its associated technologies" (BPS, 2013, p.3). Although Denzin (1999, as cited in Cohen et al., 2018 p.361) suggested that any "postings on the bulletin boards are automatically public and do not need informed consent," the BPS society's "Ethics guidelines for internet mediated research" (BPS, 2013) give four key principles for consideration: respect for autonomy and dignity of persons, scientific value, social responsibility and maximising benefits and minimising harm. As information was gathered from school websites and from open pages to the public, the survey about dogs, conducted by one person simply for numerical purposes, was set to "do no harm" (Cohen et al., 2018 p.152).

A list of all schools within England was selected (Schoolswebdirectory, 2016), but with the number so large for a solo researcher $(32,770$ schools), a smaller Special Needs School subset just for England was used. The list was worked through county by county and all references to dogs found were added to an MSExcel table, according to school type of need allocation, name of dog, owner / handler and their role. This table was simply totalled into columns and subsequently gathered into groups for enquiring into the source, assessment and training of the dog. When dogs were found, the websites were 
then further searched for any policies or risk assessments mentioning its presence and welfare. Where there were broken links to school websites, these were followed up by direct 'google' searches of the school, and in some cases, an email to the school itself to confirm the presence. Two years later, the website survey was repeated for only those dogs found in the previous schools. The findings are recorded in chapter five.

\subsection{Phase 2 - Children's Research (School based): September - December 2016}

Phase two included a small-scale investigation of 13, Year 3 (7-8-year-old) school children, including a small control of three children, for the before and after testing of overall reading attainment while using the read to dog programme. From the group of thirteen children selected, there was also a further focused study of seven children to highlight the individuality of the findings. For those seven, a quantitative approach was also applied to examine electro-dermal activity (EDA), speed, fluency and accuracy using a technique called miscue analysis, and a quasi-experimental approach taken in using six sub-tests from commercial, standardised working memory tests with and without a dog present. Questionnaires were given to both the seven children and the school-based adults involved with read to dogs to ask for opinions for the scheme or the benefits of reading to the dog. Video recordings were made of the reading sessions to reflect upon body language and interaction while the dog was and was not present. The findings are recorded in chapters five, six and seven.

\subsection{Phase 3 Adult based research (University students): May - October 2019}

The University adult students project used an 'experimental paradigm' in collecting the quantitative data portion. This followed an experimental approach using dependent and independent variables and protocols, based on the theoretical ideas raised from the 
school-based students' findings. The experimental construction should allow other researchers to re-create the experiments using the same standards, procedures, set ups and layouts if starting from the same basepoints allowing for individuality, personality and backgrounds.

A repeated measures design, using a 'Latin Square' was chosen so all participants could take part in all four conditions and to control for order effects such as fatigue and practice. Complete counterbalancing of the conditions required a sample of at least 24 participants. A prospective-power analysis using G-Power (Faul, Erdfelder, Buchner, \& Lang, (2009) suggested that 21 students are required for each condition ( 84 students in total) but this was reduced due to concerns for the welfare, stress and fatigue of the dogs. There were four conditions; i) live with handler and dog present; ii) live with just handler present; iii) VR with dog and handler previously recorded; iv) and VR with just handler previously recorded. As with the younger school-based students, questionnaires were given to all participants and the sessions recorded on video. Electrodermal activity was measured using the same devices that the children had previously used (Pip). The findings are recorded in chapter eight.

\subsection{Thesis Analysis Section}

The results from both projects were then combined, both quantitatively and qualitatively to form the overall thesis conclusion. This also allows the triangulation of the data and increases the validity, robustness and reliability of the results by cross relating the themes and evidence from both sections. The University project was able to support and strengthen themes and issues raised from the school-based project, which due to its small numbers could have been argued as "weak evidence." This analysis assists the overall 
thesis in its internal validity and robustness. The overall findings are included in chapter nine.

\subsection{Total Participant Characteristics}

The total participant characteristics are shown in table 1 below:

Table 1: Participant characteristics for the research

\begin{tabular}{|l|l|l|l|l|}
\hline Source & Number & Male / Female & Age & Pet Owner? \\
\hline Junior School & 13 & $9 \mathrm{~m} \mathrm{4f}$ & $\begin{array}{l}7: 02-7: 11 \\
\text { years }\end{array}$ & All \\
\hline University & 24 & $6 \mathrm{~m} \mathrm{18f}$ & $18-58$ years & All but one \\
\hline Total & $\mathbf{3 7}$ & $\mathbf{1 5 m ~ 2 2 f}$ & & \\
\hline
\end{tabular}

\subsection{Recruitment of Participants}

The original children's school based project was planned for two parallel Year 3 mainstream classes (60 children) within the same school over an academic year, but was reduced in size by the school and Special Education Needs Co-ordinator (SENCO) to a selected group of 13, Special Educational Needs (SEN) students chosen by the SENCO and limited to 12 weeks only due to curriculum pressures. Nevertheless, the research parameters were kept in place with the smaller group available.

The adult student volunteers were recruited through emailing lecturers, high visibility on charity run, "wellbeing" visiting days to the campuses with the actual dogs and through campus wide, media events screen advertising boards. Although many more than 24 students signed up for the project, not all of them turned up for their available slotted appointments. Most volunteers were from the psychology department. 


\subsection{The school}

The School was a Victorian built mainstream Juniors (pupils aged between 7-11 years old) situated on the outskirts of a large town, with 240 pupils on role in double entry classes. The school was Church of England Foundation based and 'Voluntary Aided.' There were less than five pupils with English as an Additional Language (EAL) with Pupil Premium (PP) at less than $1 \%$. At its last OFSTED the rating was 'good.' A further OFSTED inspection took place during the project itself (October 2016.) While a small control group was established for the pilot study, the school however, allowed only limited access to the classroom for 12 weeks and declined to take part in any mathematical projects with the dog due to 'curriculum pressures.'

\subsection{Construct Validity}

Validity, according to Robson (2002 p.170) also requires the studies or projects contained within the overall thesis to be "accurate, correct or true." Studies can be threatened by inadvertent or direct reactivity, respondent biases and researcher biases (Lincoln and Guba as cited in Robson, 2002, p,172). An example shown in this thesis is that all the students (child and adult) except one, had either currently owned pets, had pets as a child or had owned pets in the past. Thus, each of the studies can be argued as biased in the favour of having a dog present in learning situations. Despite recruitment for all volunteer students not specifying for pet owners to take part, the thesis reflects that, when animals are concerned, pet orientated owners were more interested in taking part than non-pet owners. As the researcher's role requires to be an 'observer' or 'clarifier' rather than to intervene, conclusions and recommendations drawn from this research can only be made 
according to the evidence found. Therefore, due to the mixed methods approach, this thesis is still valid for the evidence, thoughts and opinions of these pet loving participants. Internal validity can also be affected in quantitative, experimental research through the history and maturation of the research periods (Cohen et al., 2011). Events other than the research intervention during the period of time between pre and post-test can be mistakenly attributed to the overall effect and the maturity of the students can "produce differences that are independent of the research" (Cohen et al., 2011 p.183). The research into the children's reading intervention (Chapter 6 ) therefore not only included pre and post tests and a small control, but also took care to both cross relate the findings with other similar projects, and to include outside measurements such as 'Ratio Gain,' a calculation of achievement over time (Brooks, 2016) and other recommended reading interventions commonly used in schools.

\subsection{Robustness}

An important aspect of the methodology and methods used throughout is that they are familiar to and can be used by teachers for replication. A difficulty raised from the background reading and literature review was the inadequate time for teachers to undertake such research, and the limited studies examining how Animal Assisted Interactions can link to attainment in the classroom, therefore any techniques and processes used need to be highlighted for educational research practice replication. Consequently, wherever possible, commercial tests, commonly used in diagnostic testing and available to qualified teachers were used. The whole thesis aims to be accurate, given the participant numbers taking part, thorough, in using many different approaches to cross-examine thoughts and ideas, and honest in its interpretation of the results. 


\subsection{Methods of Data Collection and Selection}

\subsubsection{Tools, Methods and Techniques Common to both Phase 2 and Phase 3}

Several tools, methods and techniques were used in both phases two and three of the data gathering so comparisons could be made between both groups of participants.

\section{Quantitative}

\subsubsection{Skin Conductance "Pip"}

Phases two and three both used the pip. The Pip is a portable wireless biofeedback device which detects stress through electrodermal activity (EDA). It consists of a small, ergonomic, plastic, white apple 'pipped' shape with electrodes on both sides which are held between the finger and thumb. It is small enough for a child's hand but needs to be held at all times as the individual completes the task. The wireless feedback works with both android (Windows on a laptop) and with Apple products (i-pad). The feedback is in real time and can be recorded as an overall file. Each file for an individual, however, requires the 'Pip' to be re-loaded. These files were converted to MSExcel for analysis. Since using the Pip for the children's project (2016), the Pip according to their website, has now been used in many schools to help reduces stress through 'mindfulness' and 'wellbeing' programmes and is used as an aid to relaxation.

Using an in house EdExcel calculation programme, an average Electrodermal Positive Change (EPC) was calculated giving the overall average Electrodermal Activity (EDA) for the session's inputted data. Alternatively, the data was also represented in a graph form as seen in chapter seven. Both graphs and session averages were used with the case study group in the school children's project due to the small numbers (seven children.) 
In the adults' phase, the averaged EPC data was analysed using SPSS (version 25). Average EDA recordings were collected for general overall sessions in the four conditions and then sub-divided into the maths and vocabulary activities for each one. Performance between each condition and the curriculum-based tasks were analysed using a one-way analysis of variance ANOVA.

\subsubsection{Standardised Tests}

As suggested in the Brelsford et al., review (2017) there was a requirement for a baseline and post intervention assessment of reading for both the intervention groups and the control. Controversially in 'education' this usually refers to a measured 'reading age' or use of standardised scores / percentile ranks. The scores can then be compared, both within themselves and with other assessments using standardised scores and therefore reflect a profile of the student's current ability and achievement. As has been previously argued in Chapter 1 and 2, the qualifications for these tests required further training (Pearson, 2020).

The school SENCO chose the Salford Sentence Reading Test (SSRT, Vincent \& Crumpler, 2002, updated 2012), classed as able to be used by any teacher, as the school was already familiar with the test using it for whole school assessment. The test involves a student reading a series of graded, decodable sentences until six mistakes are recorded, whereupon a 'reading age' or standardised score is calculated.

As discussed in chapter five, several subtests from commercially produced diagnostic test batteries were considered and used within the children's phase. These were the Test of Memory and Learning (TOMAL, version 2, Reynolds and Voress, 2007), The Phonological Assessment Battery (PhAB 2, Frederickson, Frith and Reason, 1997) the Test of Visual 
Perception Skills (TVPS, Version 3, Martin, 2006) and the Wide Range Achievement Test (WRAT - 4, Wilkinson and Robertson, 2006.)

To keep all the tasks within an educational, academic skills theme, the adult tasks selected were a timed vocabulary multiple choice task and timed mental arithmetic tasks from English Key Stage 4 Foundation maths papers. These tasks were kept to short right / wrong answers, partly for ease of measurement and to create a sustainable level of stress as if in a sustained concentration period such as an exam. The fluency and processing speed under this stress would hopefully try to recreate the same pressure the children were under when sight reading their books.

A suitable vocabulary test for adults was found in the American standardised Nelson Denny Vocabulary test, part of the Nelson Denny Reading tests, using forms $\mathrm{G}$ and $\mathrm{H}$ (Brown et al., 1993). The whole test is designed for students between 14 and 25 years old for reading assessment purposes and is standardised for age and grade equivalents although these were not used in this instance.

\subsubsection{Non-Standardised Tests}

In 2019 there were no suitable commercially produced mental mathematical attainment tests for the adults' wide age ranges. The mental maths questions, therefore, were taken from a variety of England's Key Stage 4 foundation level curriculum mathematics papers and study books. These questions are for students working towards the General Certificate of Secondary Education exam, levels 4 and 5 including the EdExcel and AQA examining boards (the main two boards used in England). These exam levels are now the entrance levels for most UK university courses under the 'new' level system (DfE, 2018 - 
equivalent Grade C, UCAS, 2020). The versions of the tests were then trialled by two professional teachers and found challenging, but not impossible.

\section{Qualitative Data}

\subsubsection{Video observations}

To observe behaviours in both phases two and three, both with and without the dog present, a video of the sessions was made which were then preserved for analysis at a later date. Permission for this was given by the parents of the children. In the children's phase the camera was with a 'front on' view to the situation so that both the dog body language and that of the reader was observed. A small hand-held camera was more discreet than a full camera on a tripod and in the children's phase was attached to a 'music trolley.' The emphasis was to keep the reading sessions as valid, and as close to a normal protocol as possible rather than to have an obtrusive camera within the room, which could potentially have disturbed both the dog and the reader. A Panasonic HDC-SD600, 35mm handheld was chosen with 14.2 megapixels, 1920x1080 High Definition Video. The recordings were invaluable for the running records of the children reading using 'Miscue Analysis' as the video recorded the individual's timings, hesitancies and mis pronounced words in a permanent way. The children were also familiar with these handhelds as they were usually used in most schools for project work. In the adults' phase, due to lack of any available discreet spaces the same camera was placed on a tripod and placed carefully so it could record all conditions.

The data were analysed from all the videos at a later point using a simple behavioural observation technique. Using the video meant that the whole session was being recorded in general and would increase the likelihood of natural behaviours of the reading and 
interaction with the dog within the school environment. In phase two, the actual period of specific observation was started when the dog and child were settled ready to start reading and finished after the child had given the dog a treat for 'good listening.' The videos were time stamped so timed differences in body movement could be recorded, rather than at every minute intervals. The movement of the child was recorded first, with the dog's body movements recorded second and subsequently woven into the overall timings. After all body movements were recorded, then conclusions could be made about the quality of interaction. The timed length of these observations did vary between each reader, due to the level and fluency of what was being read.

Due to the larger number of adults $(n=24)$, running field notes from the video data were also created for the 'live' dog condition to watch the amount and type of interaction between the individual adults and the dog. The request for videoing was incorporated into the informed consent forms.

From both sets of observations, it was then possible to compare the interaction and movement not only with and without the dog in both the children's and adults' videos, but also the differences between the children and adults' behaviours compared when concentrating on a task.

\subsubsection{Questionnaires}

Both phases two and three used questionnaires.

\subsubsection{Phase 2 Children's Questionnaires}

A questionnaire was created to gain some background knowledge about the children and their attitudes to reading, mathematics and their hopes for reading to the dog. 
Consideration was given to the length and format of the questions, the understanding behind the language and 'readability' for those with limited skills, together with the amount of effort required to fill in the answers, depending on whether the children were already familiar with the concept of a questionnaire and giving their own opinions. The questions were planned so that either the child themselves could read and understand them, or an adult could guide them through a mix of open and closed questions and assist with the scribing if necessary. Robson (2002) suggests that a good questionnaire, "Provides a valid measure of the research questions, but also gets the co-operation of the respondents, and elicits accurate information (Robson, 2002 p 242).

For the questions asking for the children's own opinions, a 3-point Likert Scale was used using well known visual face expression "Emojis" which the children could circle, while a space was created for them to elaborate on their feelings if they so wished. As the children were young and relatively inexperienced at filling in questionnaires for themselves, the choices were kept simple, hence only three items in the Likert scale. Two of the questions were created to see if there was any anthropomorphising with the dog to see if this influenced their thinking (Hawkins and Williams, 2016). The questionnaire was then typed in a "dyslexic friendly" typeface using "Dyslexie-font" (DeBoer, 2019) in blue and then pilot tested on three students of the same age at a local tuition centre to check for clarity in understanding. (For the children's questionnaire see Appendix C.)

The example of emojis used is seen in Figure 5:

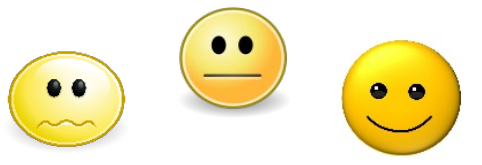




\subsubsection{Phase 2 School Staff Questionnaires}

The teaching staff in this school were not directly involved with the children as they read to the dog, therefore it was still important to have their opinions about the reading scheme and how they felt about its practice, because it had previously applied to the children within their classes. A 12-question multiple-choice format was chosen which could be quickly filled in the staff member's free time yet could then be analysed. Apart from the first question, the questions were kept "neutral" as any member of staff could answer them, rather than having specific biases for the senior management team, teaching staff and ancillary staff. Spaces were given for any further comment expansion. The completion of the questionnaire was voluntary. (For the Staff questionnaire see Appendix D.)

\subsubsection{Phase 3 Adults' questionnaire}

After the completion of the four conditions, the adult participants were asked to complete a questionnaire to gain their views and opinions of the experience (see chapter eight.) Again the design was aimed at being quickly self-administered and was semi-structured, allowing for some initial, dichotomous data to be rapidly gathered and for more in depth answers to open questions (Cohen et al., 2011, Bell, 1992). It was to be completed out of choice. Double questions were avoided (Bell, 1992). Two, separate focus questions were asked. These were; in which condition did the participant feel most relaxed; and in which condition did they feel that they had concentrated the most? As was warned by Youngman (1984 as cited in Cohen, 2011, and again in Bell, 1992) there could have been 
a natural human tendency from the participants to not want to pursue with statements given, however a definite choice was implied.

The questionnaire had been organised into four sections: the first section contained the personal data, the second, contained the previous experience with animals, third section contained the day's experimental data and the fourth section was for any other information which may have impacted on the scores or opinions. (For the adult questionnaire see Appendix E.)

\subsection{Other tools, techniques and methods used in the Phase 2 children's research}

\subsubsection{Reading Scheme}

The school used the 'Accelerated Reader' programme which incorporates popular children's reading schemes such as the 'Oxford Reading Tree.' 'Accelerated Reader' (Renaissance-Learning, 2019) is an American online based reading scheme, accessible to the teacher, parent and child, designed to increase the progress of readers through to fluency through reading levelled texts, independent choice of books within certain levels and comprehension tests based on these books. Within this scheme, any children's book can be formulated into sentence length, structure and difficulty and given a 'level' to help guide a child. This structure is particularly useful for research purposes as these levels have already been calculated. All the children's books read in Phase 2 had been levelled using this scheme and were between 0.7 and 2.7 or between US school grades Kindergarten to Grade 2 (equivalent of the UK Year 3, 7-8 years old.)

Table two below shows the equivalent reading ages between the different reading schemes and books used by the children in the school: 
Table 2: Equivalent reading ages and reading schemes for the children's books

\begin{tabular}{|l|l|l|l|}
\hline $\begin{array}{l}\text { Accelerated } \\
\text { Reader Book Level }\end{array}$ & $\begin{array}{l}\text { Oxford Reading Tree } \\
\text { Level }\end{array}$ & $\begin{array}{l}\text { Colour Book } \\
\text { Band }\end{array}$ & Approximate Reading Age \\
\hline 0.7 & Level 3 & Yellow & Year 1 (5-6 Years old) \\
\hline $1.4-2.0$ & $\begin{array}{l}\text { Level 5 } \\
\text { Level 6 }\end{array}$ & $\begin{array}{l}\text { Green } \\
\text { Orange }\end{array}$ & Year 2 (6-7 Years old) \\
\hline 2.1-2.7 & $\begin{array}{l}\text { Level 7 } \\
\text { Level 8 } \\
\text { Level 9 }\end{array}$ & Purquoise & Year 3 (7-8 Years old) \\
& & Gold & \\
\hline
\end{tabular}

\subsubsection{Selection of suitable rhyme}

A simple reading text was located to compare whether reading to a dog or reading an unknown piece could be possibly as stressful as each other. Knowing that rhyme is a difficult concept to gain through the stress beats on the syllables while maintaining the rhythm and meter of the reading, a simple rhythmical poem was chosen. "Sitting by a Swamp" by Kristyn Crow had been researched from a teaching website (TimesEducational-Supplement, 2019). This poem had a simple phonetical structure and included all the phonemes covered within the "Letters and Sounds" structure of the UK's English Phonics Curriculum which is usually covered by the end of Key Stage 1 (Years 1 and 2, 5-7 years old) in all primary schools. This phonetical structure would suggest that all children from Key Stage 2 should be able to access and read it, although those with underlying literacy difficulties, such as dyslexia, may have problems with recognising the rhyme. The children in the project were in Year 3 (7-8 years old) and at the beginning of Key Stage 2 (Years 3-6). The poem was presented without illustrations, in a dyslexic friendly font to help ease any visual difficulties. (See Appendix F). 


\subsubsection{Miscue Analysis}

The reading speed and accuracy was analysed using a technique called 'miscue analysis.' Miscue analysis, developed by Kenneth Goodman in the 1980s, is a means of using a "running record" for diagnosis to identify students' specific reading difficulties. By using a systematic code for types of errors made while the student is reading, the examiner or teacher creates a running record which not only records reading rate and reading accuracy, but also can assess reading behaviours which require support. It is an integral part of many reading scheme approaches, a requirement for training in diagnostic testing and is also used in reading tests themselves such as the York Assessment of Reading Comprehension (Snowling et al. 2011). Examples of miscue analysis for the children's reading, are in Appendix G.

\subsection{Other tools, techniques and methods used in the Phase 3 Adults project}

\subsubsection{VR Headset}

In this study, all the participants were adults over the age of 18 and were therefore eligible to able to wear VR headsets without risk of harming the brain's development. All participants were made aware that the VR headset was crucial to the experiment from the outset, so that anyone with known difficulties associated with virtual reality headset wearing, such as suffering from seizures, epilepsy, had a fitted pace maker or hearing aids, or suffered from emotional stress or anxiety, could decide whether to participate in this research or not. An Oculus CV1 headset was used, powered by an Asus G752VS notebook with an Intel core i-7 2820HK CPU @ 2.90Ghz, 32 GB RAM and a GeForce GTX 1070 graphics card. 


\subsubsection{Preparation of Materials}

Two test environments were created, one 'live' and one in Virtual Reality (VR) to test and compare whether the physical presence of the 'real' dog had an effect on learning and attainment, or whether a VR dog had an equivalent effect. The participant was to work through all four conditions completing the maths and vocabulary tasks in each:

I. Real or 'live' world and dog

II. Real or 'live' world, no dog just handler

III. Virtual Reality (VR) world with dog and handler

IV. VR world, no dog, just handler

The same Pip recording device was maintained to show the fluctuance and variance of the individuals while in the four conditions. This allows for cross-comparisons to be made with the children's earlier experiences.

\subsubsection{Vocabulary and Mental Maths Questions}

For this project, 4 different versions of the same vocabulary test, together with 4 similar versions of mathematical mental problems were created at an adult level. (See Appendices, $\mathrm{H}$ and I.)

The Nelson Denny vocabulary test was carefully split into sections of 20 questions each. There was no gradience to the test, so the questions were equally selected throughout the original 80 question test. Each word had multiple choice answers as to its definition. Each question was placed into a MS PowerPoint slide, using Calibri typeface, 28 point size and set to change every 10 seconds. 
Each of the four mental maths versions contained the same number of questions on the same themes: linking long term memory mathematical vocabulary for mean and range, rearranging lists of numbers, percentages of numbers, simplification of algebraic term, equivalent fractions, negative numbers, addition and subtraction of decimals and simple orders of operation. Many of these operations required mental 'visualisation' of processes linking to the theme of using visuo-spatial memory. There were two questions for each 'topic' area. These questions were then placed into MS PowerPoint slides, using Calibri typeface, point size 48 and then set to automatically change every 10 seconds. The slides were added to the vocabulary questions so that four joint maths and vocabulary sets of questions were produced, containing 10 slides of maths and 20 slides of vocabulary each. (See Appendix J.)

\subsubsection{Creation of the films used for VR and "Live" Research}

These four sets of slides were then filmed twice each, once within the VR studio headset with dog and handler interacting, and once with just the handler in the VR headset to produce eight VR films. Each sequence of questions in VR was then 'superimposed' onto the whiteboard in the VR studio headset as a focus point, although the filming clearly allowed for viewing around the whole room. For each film the order of the mathematics or vocabulary questions was changed, therefore four started with the mental maths section first and four with the vocabulary.

A second set of just the PowerPoint slides was produced for the whiteboard within the same physical VR studio for the 'real situations.' In each condition there were now eight slide sets or films which could also be rotated, ensuring that the participant did not receive the same questions, or indeed the same film twice, regardless of 'real' or 'VR' situation. 
Although the questions were initially difficult to read while in the VR headset, adjustments were made to the screen within the headset to compensate. Several typeface versions, including 'Dyslexie-font' (DeBoer, 2019) were trialled for initial clarity, with Calibri body finally chosen for clarity.

The eight different VR films were recorded using 360 degrees camera with the two different therapy dogs, both Labradors, playing and trying to interact with the camera as much as possible. Each film had just one dog present at a time.

\subsection{The Dogs}

Under the conditions set by the British Psychological Society and University of Gloucestershire Ethical committee, for health and safety reasons, the dog had to be trained and assessed as either a therapy dog or assistance dog who was familiar with a school situation and happy working in a classroom with young children. A year passed while contacting many charities including 'Dogs for Good' and 'Pets as Therapy' to visit schools, the project almost stalled due to subsequently finding that the dog was either not assessed, ill, handler unavailable or that an internal exam period during the dates was planned. The research proposal then went to the newly established visiting volunteer charity 'Therapy Dogs Nationwide' and was passed by their committee in May 2016. 'Therapy Dogs Nationwide' was created by ex-members of 'Pets as Therapy.' Due to the difficulties in finding suitably assessed, local dogs, in 2016, the researcher began to also train and work with her own two dogs towards the charity's assessment criteria.

All three dogs used in the thesis came from the same volunteer, visiting charity, had been charity assessed as having calm temperaments required in therapy dogs and were very used to interacting with people. All dogs were over nine months old, had been with their 
owners for at least 6 months and had been acting as regular therapy dogs for over three years. The dogs were all insured under the charity's insurance and had been regularly reassessed for continuing suitability to work with children and vulnerable people. They were groomed, clean, vaccinated, regularly treated for parasites and in good health. While the charity itself does promote raw feeding, all three dogs had been kibble fed for at least 90 days to reduce any fears of zoonotic transference between the dogs and the humans (S.C.A.S., 2019, section 2.5).

\subsubsection{Phase 2 - Matt the Border Terrier}

Matt was a male, neutered, pedigree ten-year-old Border Terrier who had already had many years of 'therapy visits' behind him with two volunteer charities, 'Pets as Therapy' and 'Therapy Dogs Nationwide.' He was one of the first dogs to join his current charity, 'Therapy Dogs Nationwide' at their creation in April 2016. Matt was already known to the school. He usually visited the children on a weekly basis throughout the academic year, regularly visited a local hospice, and the local 'Riding for the Disabled Association.' He went on to represent the charity at 'Crufts' in 2017. After this project, Matt regularly also visited the university, taking part in wellbeing and mental health visits. Matt worked for up to one hour at a time when visiting the school.

\subsubsection{Phase 3 - Maisie and Isla, both Labrador Retrievers}

In Phase three, due to the larger participant numbers and possible fatigue for the dogs, it was decided that two dogs would take part. Maisie (aged 7) and Isla (aged 5) were female, neutered, pedigree Labrador Retrievers, both certified and charity insured as visiting therapy dogs registered with the same national charity as Matt. Maisie and Isla were also insured for unpaid visiting therapy work by their medical insurer (Petplan) and had also 
been independently assessed by a member of the local vet's behavioural team. The dogs had regular experience in 'one off' school visits, including 'read to dogs,' a mental health charity, a local hospice, numerous open and outdoor events and with general university students on campus.

\subsection{Ethical Considerations}

\subsubsection{Permissions}

In phase two, permissions were sought and granted from the school, the Head teacher, SENCO, parents, children, charity, handler and the dog in accordance with the then British Psychological Society and University of Gloucestershire ethical committee guidelines available in 2016 (See Appendices, K,L, M and N.) The parents were consulted by the school Headteacher and the SENCO to gain permission for the research and signed on behalf of their children. As this was seen as a school-based research project, the actual permission slips were kept in the school by the SENCO as part of school protocol and included three 'waves' of permission; i) To take part in read to the dog scheme throughout the year without becoming part of the research project; ii) To take part in the research project for the reading to the dog only; and iii) To fully take part in the reading to dog project, including the working memory tests, questionnaires and electrodermal activity measurement. The SENCO then gave a list of children's names to the researcher after the different levels of permission was granted. At the beginning of every session the children themselves were also asked for their verbal permission. Only once was it refused, in favour of playing football for that session Male C.)

As the adult phase was introduced after the Data Protection Act (2018), the General Data Protection Regulations (GDPR, 2018) were in place. The participants were provided with 
an information sheet and consent form (see appendices) which stated their role within the purpose of the study and their ethical rights as a participant. The researcher was on hand throughout the study to answer any questions and to ensure that the participant had fully understood the information provided to them. At any point they could stop their participation without having to provide a reason. Informed consent was available throughout the period of the study until the closing date. Debriefing sheets were prepared and given to the participants after completion of the research (See Appendix O).

\subsubsection{Anonymity}

As with all the schools found in phase one for the research, all school and children's data were anonymised and while videos were used, these were for research purposes only and will not be published. After the completion of the thesis they will be destroyed.

All adult participants were assigned a unique participant number which was initially linked to their data. Data from each participant was then combined with that of other participants, and it is this data, rather than individual data, that was then analysed. Therefore, no information that allowed the participant to be uniquely identified was stored with the data.

In line with GDPR (2018) rules, all information gathered was stored securely, on password protected laptops within locked premises, with only the researcher and her supervisors having access to this. Personal information, including videos will then be destroyed in line with British Psychological Society and data protection guidelines when the thesis completed. 


\subsubsection{Respect}

At all times the children's interests and needs were paramount. Their questions such as "What does this [pip] do?" were answered immediately and in a child friendly way, keeping language simple, yet informative. They were curious, often coming to look at their immediate data graphs in the sessions and noting the differences. The ethos was taken that they were being "practical hands-on scientists" creating their own data for the dogbased project. Their results were simply, verbally explained as, "You worked better with the dog in... and you were better on your own with..." when the results were finalised. The diagnostic nature of the tests themselves were chosen carefully in that they would not have any long-lasting negative effects but would highlight individual strengths and weaknesses (B.D.A., 2019, P.A.T.O.S.S., 2016). All sessions were with individual students, so there was no comparison with others and breaks were included as and when necessary. The adult participants were similar in their curiosity but would also reveal personal anecdotal stories about their own experiences with animals and their pets. There were two childhood incidents revealed. Both adults were happy just to state the facts but were more interested in relating to how important their pets were to them during those times. Phone numbers for helpful organisations had been included on the debriefing sheet.

Respect was also given to all the dogs. For instance, their body language was checked, the children and adults were asked not to crowd the dog or stand over them and 'escape' ways were assured so that the dogs could move away if necessary. A selected area was created behind a barrier, so that the dogs could choose to retreat from the adults if desired. The adults were taught to 'ask permission' before giving the dogs full scratches, i.e. to scratch for a few seconds, to wait and see the response from the dog, before 
continuing if permission was granted (VanFleet and Faa-Thompson, 2017). Any treats given were done so in a calm, ordered way and both dogs and handlers wore the charity's uniform to be easily identifiable.

\subsubsection{Disclosure and Barring Services check}

As with all adults working in schools, basic background checks into the researcher were undertaken including using the Disclosure and Barring Services. These are also recommended by all animal assisted associations, including the direct volunteer charities, such as 'Therapy Dogs Nationwide' (IAHAIO, 2014 updated 2018, Winkle et al., 2019, S.C.A.S., 2019). In phase two, the safeguarding policies were read, and the child protection / safeguarding member of staff made known to the researcher. The school SENCO was to act as the liaison between the school, parents and the researcher. In phase two, whenever the researcher was on her own with a child, simple protocols were kept, such as leaving the door open so anyone passing could check and view what was happening, and the seating positions were angled so that the researcher could keep a respectful distance from the child, while still following any test manual guidance (B.D.A., 2019, P.A.T.O.S.S., 2016). The school relied on the trusted conduct of the researcher as a qualified teacher and SENCO, although for most sessions the school SENCO did keep a quick, visual check (DfE, 2013, p.14).

\subsubsection{Health and Safety}

The small Victorian built 'music room,' used weekly in phase two, was always checked for accessibility before the dog handling team arrived. Instruments were tidied away so that there was a clear floor space with no hazards, for either the dog, children or adults. Electrical wires, such as those used for the laptop were carefully place out of reach. A 
window was opened to allow for movement of air during the sessions. Hot drinks were consumed, and mugs returned before the arrival of the children.

In phase three, the Virtual Reality laboratory, although full of computing equipment, was wide and large enough to have plenty of space for movement of dogs, adults and equipment. All major wires were either suitably covered or taped down. The working area for the study and the participant's chair was clearly marked out on the floor with safety tape. The room had good ventilation and light, a secured door requiring a pass for access, and the technician was always present when unlocked. The dogs were not left unattended when working in the room. There was a small atrium, hallway space adjacent to the laboratory which the dogs used as their 'safe space' while waiting to take part.

All participants were asked if they had any known allergies to dogs, dander or dog hair. All replied in the negative except one adult who chose to continue the experiment with her preventative medication (S.C.A.S., 2019, section 2.5).

In phase two to ensure that the young children were 'dog aware' i.e. aware of suitable behaviours around a dog and knowledgeable of basic dog body language, links were made with a child friendly dog awareness course, 'Stop the 77 Workshop' with a registered licensee from a local, registered Dog Training Centre to visit the children in school (Stopthe-77, 2019). This visit was later declined by the school SENCO due to time and perceived as unnecessary as the 'dog would be on a lead.'

The school also provided a 'volunteer' to be available, should anything untoward happen under the rules of the visiting dog charity for the "health and Safety" of both the children and also the dog (Therapy-Dogs-Nationwide, 2020). The volunteer was a part time 
member of the staff who was a teaching assistant. On occasion, she was unavailable, so the researcher became the volunteer for those sessions.

The adult participants were only in the presence of a live therapy dog for up to five minutes in one of the four conditions, yet all the participants were given the option to withdraw from the study without ever meeting the live dog if they wished. At all times the dog was under the control of the handlers. The VR filmed sections within the VR headset also only took five minute each.

The Oculus health and safety procedures preclude anyone under the age of 13 years to wear their headset due to harming the developing nature of the visual development at that age. Side effects, even for adults are shown in Figure 6 below:

- Seizures;

- Loss of awareness;

- Eye strain;

- Eye or muscle twitching;

- Involuntary movements;

- Altered, blurred, or double vision or other visual abnormalities;

- Dizziness;

- Disorientation;

- Impaired balance;

- Impaired hand-eye coordination;

- Excessive sweating;

- Increased salivation;

- Nausea;

- Light-headedness;

- Discomfort or pain in the head or eyes; 
- Drowsiness;

- Fatigue;

- Any symptoms similar to motion sickness.

Figure 6: Side effects of wearing a virtual reality head set (Source: Oculus, 2019)

A protocol of questions was followed with each participant before the use of the equipment and participants were regularly asked how they were feeling throughout the VR sections.

\subsubsection{The role of the handler}

For phase two the charity, Therapy Dogs Nationwide, had located the experienced volunteer handler and dog team in a school local to the University. The handler / owner was also familiar with primary aged children and trained both in safeguarding and schools' literacy policies as an ex-governor of that particular school. The handler / owner, who was also one of the charity dog assessors, was required to be control of the dog and to always advocate and oversee the animal's welfare, health and interests (Therapy-DogsNationwide, 2020). This handler had also received some extra 'handler training' from the University of Lincoln, having previously been involved as a dog team in one of their 2016 human animal interaction research projects.

The main handler for the dogs in phase three was the researcher herself. This was for several reasons; i) lack of other suitably qualified dogs available on a regular basis; ii) convenience in the accessibility for the timing and dates of the filming sessions for the videos to be made; iii) flexibility for the dates for the participants to take part; iv) congruence for the experiment in that they, and the handler, were the same dog teams as the ones in the VR videos for comparison to the live situations; v) the researcher's knowledge of the dogs themselves; and vi) the fact that the dogs were already visitors to 
the university and knew the rooms in question. (The dogs were not familiar to the students in the project as they had come from a different cohort to those previously visited.) As the researcher was videoing the observed animal and human body language in one of the 'live' situations, she could sit with her dogs as she would in any visiting situation. The interaction could be simply videoed and analysed separately at another date.

By 2019, the researcher / handler had three years' experience of being a visiting therapy dog handler and was also an assessor for the therapy dog charity itself. She knew her own dogs' behaviour and body language well, having had her two dogs from 8-week puppies. In line with advice from animal interaction guidelines available (Winkle et al., 2019, IAHAIO, 2014 updated 2018) the researcher / handler had also undertaken further training in canine behaviour including completing a theory and practical based eight day course as a 'Foundation Dog Training Instructor' with the Association of Pet Dog Trainers (APDT- UK) and two courses of theoretical and hands on training (16 days) through the International Institute For Animal Assisted Play Therapy ${ }^{\circledR}$ (VanFleet, 2018a) all requiring knowledge of classical and operant conditioning and using positive reinforcement techniques only. All courses had included written assessment and heavy emphasis on reading canine (and equine) body language. This is in line with suggested standards of training for dog handlers as given by the Society for Companion Animal Studies (S.C.A.S., 2019) and the Animal Behaviour and Training Council (A.B.T.C., 2018).

In order to work with two dogs, a second charity assessed owner / handler was required as per the rules of the charity (Therapy-Dogs-Nationwide, 2020). The second owner / handler was also a qualified teacher, who also had three years' volunteer handler's 
experience. He was well acquainted with both dogs, having been included in the dogs' training and welfare since they were puppies. Both handlers had been trained in Mental Health First Aid for both Adults and Youths had there been any mental health issues raised about the dogs from the participants, although suitable websites and phone numbers had been included in the permission sheets.

\subsection{Dog Ethics}

In Phase 2, Matt, who lived within five minutes walking distance of the school, was expected to attend the sessions as was the routine and either 'settle' within the environment while being petted or encouraged to actively take part in the lesson following cues from the handler - whichever was the normal procedure. As per charity rules, treats, water and a dog bed were always provided by the handler / owner and easily accessible to the dog. Regular breaks were given between pupil visits and treats given between visits and when the sessions ended. Therapy Dogs Nationwide followed the guidance as set in the Bark and Read guidelines (Kennel-Club, 2018).

In Phase 3, upon arriving at the venue, (a 45-minute drive in an air-conditioned car) both dogs' body language was observed to see if there was permission and enthusiasm for taking part that day (Winkle et al., 2019). Both dogs had regularly been visiting the Psychology Department and taking part in Mental Health and Stress Weeks with many students over the past three years and were familiar with being in the Virtual Reality laboratory room. They had been introduced to it on several occasions. Both staff and handlers had encouraged play and interaction in that environment, before and during the setting up of the experiment. Both dogs showed complete trust in the personnel involved and were very happy to work with them. They were also accustomed with the automatic 
doors, lifts and stairs in the building after some initial focused, positive reward training (Winkle et al., 2019).

The dogs had the rights to be withdrawn from the study at any time under the direction of either handler, had a quiet space or bed to 'retreat' to if necessary, including the 'safe space' of the car, always had provision of fresh water and regular breaks for rest, snacks, play or toileting under the guidance of both the handlers. At all times they had the choice of whether to work or not with the handlers very observant of the dogs' body language and any vocal signs.

Each dog took it in turn to be in the experiment, leaving the other to rest. There were breaks between each participant and the length of the availability for the sessions was dependent on the dogs' energy levels. The dogs worked for up to a total of two 'solid' hours only in a day as per charity rules but had the advantages of many breaks in between participants, thereby lengthening the times they could be physically present on campus.

\subsection{Risk Assessment}

As part of the ethical considerations a risk assessment for the dogs was undertaken. The risk assessment based on the charity's guidelines is available in Appendix P.

\subsection{Challenges}

The immediate challenges to the school-based research from the outset were the number of children the original research project requested (60 children across one-year group,) the length of time for the project (an academic year), the inclusion of mathematics in comparison to the reading with the dog and the use of blood pressure monitors. Exploratory planning meetings with the school took place in June 2016, before the project's commencement date of the following September. Over the Summer, the 
research request for the measurement of the children's blood pressure became a concern to the parents who did not wish their children to wear such monitors as they, and the information they contained would be too obtrusive and could highlight potential health problems. These research issues required compromise with the SENCO, who had established by July 2016, that the school was unable to give assurances for pupil numbers and time due to curriculum pressures and the school's focus and planning for the following academic year. An important decision was also made to swap the blood pressure monitors to the hand-held Pip electrodermal monitors. The size, timing and choice of students, therefore, were at the school's discretion. Once these decisions were made and accepted, the research project was able to start.

The school-based situation also presented itself with several day to day challenges, although none were any different from any other working primary school. The researcher role (as a qualified teacher) collaborated with all partners including teachers, SENCOs, pupils, parents and dog handlers, their ideas and opinions, which also provided internal validation of the selected resources used for data collection. This collaboration also required tact and diplomacy as although the researcher was expected to carry out the research and often substitute for the school based volunteer required for the 'read to dogs' intervention to take place, she was not included in any of the school based meetings regarding disseminating the ideas behind the research and obtaining parental and child permissions. Efforts were made to inform the school of any changes to the regular routine of visiting on Tuesday afternoons. Upon arrival, the researcher would 'check in' with the Head Teacher, School Administrative Officer and SENCO for the weeks' news and availability of the children and classes. These quick meetings heightened the awareness 
of flexible routines required in schools, such as the inclusion of the visit from OFSTED, staff absences, the collection of children from the sports field which was adjacent to the school property but within a few minutes' walk, individual children's absences for the dentist or the hospital, working with the time table of the visiting speech and language therapist, school trips and the play and Christmas concert rehearsals. This unpredictability meant that the researcher often had to 'think in the moment' and adapt the plans for the 'read to dog' sessions accordingly. The researcher also had to contend with an assembly group who regularly required access to the room to store equipment. Skills, when undertaking research in schools, require open, honest and good communication with many different stakeholders. This can take many forms including emails, letters, texts and verbal phone calls, or simply visiting people's offices. Issues which are important to the research process may not be as apparent to the host establishment.

One teacher, however, was not happy with the necessity of the research and felt negatively towards the ethos of the 'read to dogs' scheme in general, believing it to be of no benefit to the readers. He argued that as the children would be regularly out of class, this would negatively affect overall routines and classroom planning. The SENCO and Head teacher intervened on the researcher's behalf so that access to his class' children could still be established.

A further challenge occurred when one child showed a profile of underlying learning difficulties after completing the initial set of working memory tests without the dog's presence. For the benefit of having appropriate identification and intervention in place to assist with any further diagnosis, the group's working memory results was shared with the SENCO. 
In Phase 3 challenges also occurred when trying to recruit the students. Although named slots were booked, many students did not turn up. This situation was due to many reasons such as forgetting the slot times, dissertations and reports taking precedence, the arrival of the summer holidays, students returning home, equipment not working thus slowing down the time slots, and participants wanting to talk and share about their experiences with dogs, both general and therapeutic, which over ran their sessions. Some of these conversations were extremely personal in nature although not at a stage of requiring reference to other professionals.

For one adult participant, it was discovered that she was unable to read anything within the VR headset. This was due to having had previous laser, lens eye surgery. This may have been unique to this individual or this possibility of occurrence could be a consideration for the health and safety features for future studies.

\subsection{Summary}

The research methodology, methods, tools and welfare have been carefully considered in terms of purpose, appropriateness, practicality and within ethical contexts, standards, guidance and regulations for this thesis. Equal consideration has been given to the welfare, wellbeing and safety needs of the participants both child and adult, and of the dogs themselves in accordance to British Psychological Guidelines (B.P.S., 2018, B.P.S., 2012), the British Educational Research Association (BERA, 2011), the University of Gloucestershire research ethics guidelines (Gloucestershire, 2018) the Animal Assisted Intervention International Standards of Practice (Winkle et al., 2019), The International Association of Human Animal Interaction Organisation Definitions and Guidelines (IAHAIO, 2014 updated 2018), and the Society for Companion Animal Studies Code of 
Practice (S.C.A.S., 2019). For the standardised assessments suggested in the Brelsford et al., (2017) the conduct and ethics for carrying out assessments under the British Dyslexia Association and the Professional Association of Teachers and Students with Specific Learning Difficulties were adhered (B.D.A., 2019, P.A.T.O.S.S., 2016). This should ensure that while random controls were not used, and the number of participants was small, the results from the research should have relevance for the integrity of the study itself and for future dissemination. 


\section{Chapter 4 (Research Phase 1)}

\section{Background Survey: School dogs in Special Educational Settings in England (2016), revisited 2018.}

\subsection{Introduction}

This chapter, originally written in 2016, but since updated, was the basis for the Phase 2 research areas including the investigation into the 'Read to Dogs' within a working primary school and the motivation for the working memory tasks. The training and welfare issues also raised within this chapter subsequently led to the preparation and training of the dogs for Phase 3.

\subsection{Rationale for Background Survey}

In 2016, the United Kingdom did not have a central or government informed specific definition of what the title of a 'school dog' implied and how this role then compared to other countries for comparison for the expectations of both the dog and handler when in school environments. This chapter therefore relies upon an Austrian definition as given by a lead researcher in the field of Animal Assisted Education and Learning, Dr Andrea Beetz and the Animal Assisted Intervention International Guidelines for Animal Assisted Education (Winkle et al., 2012) available in 2016 (since updated in 2019, now referred to in this thesis as the "AAIl Guidelines.") Beetz (2018) explains that the Austrian Ministry of Education defines a school dog as one that: spends class time in (one) classroom on a regular basis; is handled by a teacher who had had specific training in dog-assisted education; is assessed for its suitability, health and behaviour; is trained and socialised for 
the clients / students it will meet; and is assessed in the class / school on a regular basis. A school visiting dog, by contrast, is defined as one that: visits classes (one at a time) once or a few times for 1-3 hours; is handled by a person with training in dog assisted education and is usually not a teacher at the school; is assessed for its suitability, health and behaviour; is trained and socialised for the clients/students it will meet; and is assessed in school on a regular basis (BMUKK, 2013, as cited in Beetz, 2018).

In the UK, the visiting dog model is interpreted as a dog brought into school usually by a charity volunteer; a member of the public, who may or may not have had specific training in dog assisted education, other than by the experience of bringing the dog into school itself. The dog is usually trained for simple obedience commands, is temperament assessed only and is referred to as a 'therapy' dog, despite the term 'therapy' itself having a different clinical definition. These underlying differences between the interpretation of the international standards of practice are why research into the role of the school dog requires further identification, clarification and understanding in the United Kingdom.

In 2016, there appeared to be no actual statistical information about how many dogs were being used within schools in the United Kingdom; where they came from, how they were trained and what their purpose was. In order to try to gather not just an approximate number of dogs, but also interest into their general roles, training and welfare, the researcher initiated an online desk-based survey of school websites. Due to the overall numbers of schools within the UK $(24,281$, GOV.UK, 2016) however, the research task was deemed unmanageable for a single researcher to complete over the short time period of three months. In 2016, as most Animal Assisted Intervention research published tended to involve those considered to have "special needs," this school sector therefore seemed 
a logical, and manageable place to start. To reduce group numbers further, only England's special school internet websites, including Pupil Referral Units, were checked for an acknowledgement of a school and / or therapy dog. England was selected due to the researcher's own experience and knowledge of the English school system as a qualified primary (ages 4 to 11 years) trained schoolteacher.

According to the UK Government Statistics in 2016, there were 1,037 state funded and non-maintained special schools in England. These could be broken down into group classification of: 662 with Autism Spectrum Differences, 556 Severe Learning Differences and 537 schools for those with Moderate Learning Differences (GOV.UK, 2017b). There were also 380 Independent Special Schools and Colleges (GOV.UK, 2017a). The total of all the special schools was 1,417. A list of all the schools and colleges was established through a search engine (Schools-Web-Directory, 2016) and systematically worked through county by county including academy chains and Local Education Authorities (LEAs) between June and August 2016.

Only information for public knowledge is placed upon these school websites such as general news, policies and information for Office for Standards in Education (OFSTED) therefore all the information about the dogs was in the 'public domain.' By using the search engine features on the individual school sites for, 'dog,' 'dogs,' 'dog policy' and 'therapy' where possible and looking at 'news,' 'gallery,' 'events' and 'staff' or 'our team' for those websites which did not have a search feature, 1368 schools (96.5\%) were checked, revealing 135 dogs in 104 schools. Only five schools also included an accessible 'dog policy' on their web sites. These included 'broken links' where a 'follow up' was included by using a direct google search of the schools (For the tables of school dogs and 
handlers, please see Appendices, Q and R.) Two years later, a second survey of only those dogs found in the 2016 sample was initiated to check the longevity of the school dog remaining in the school.

The survey of web-based information in the public domain revealed that $9.87 \%$ of Special Schools in England in 2016, did have dogs in the schools surveyed, but that the dogs were also working in a variety of different roles. Six schools had more than one dog recorded on their websites, with five being the highest number in total in one school. Schools using dogs often had the dog with its own page, blog, or photo as a 'staff' member. In one school for those with Autism, however, there was a therapy cat noted amongst the staff. The other $90.13 \%$ of Special Schools, although they may not have directly used a dog, showed strong links to 'Forest Schools,' 'Community Farms,' 'Outdoor Learning Centres' and 'Riding for the Disabled' which reflects the current, positive national consensus for the use of alternative educational methods involving animals and nature, with those considered to have 'special or additional needs.'

Special schools in England, similarly to the other UK nations, are allocated different categories, dependent on the needs of their pupils. Difficulties arose when several schools covered all categories of need within their websites. As each school is allocated a specific category according to the government website (GOV.UK, 2017b), the 'Head Teacher's Welcome,' 'School SEND policy' and latest 'OFSTED' reports were then checked for these schools to try to group the different types of schools for the survey. The chart below in Figure 7 shows the percentage of dogs available for each category of school. 


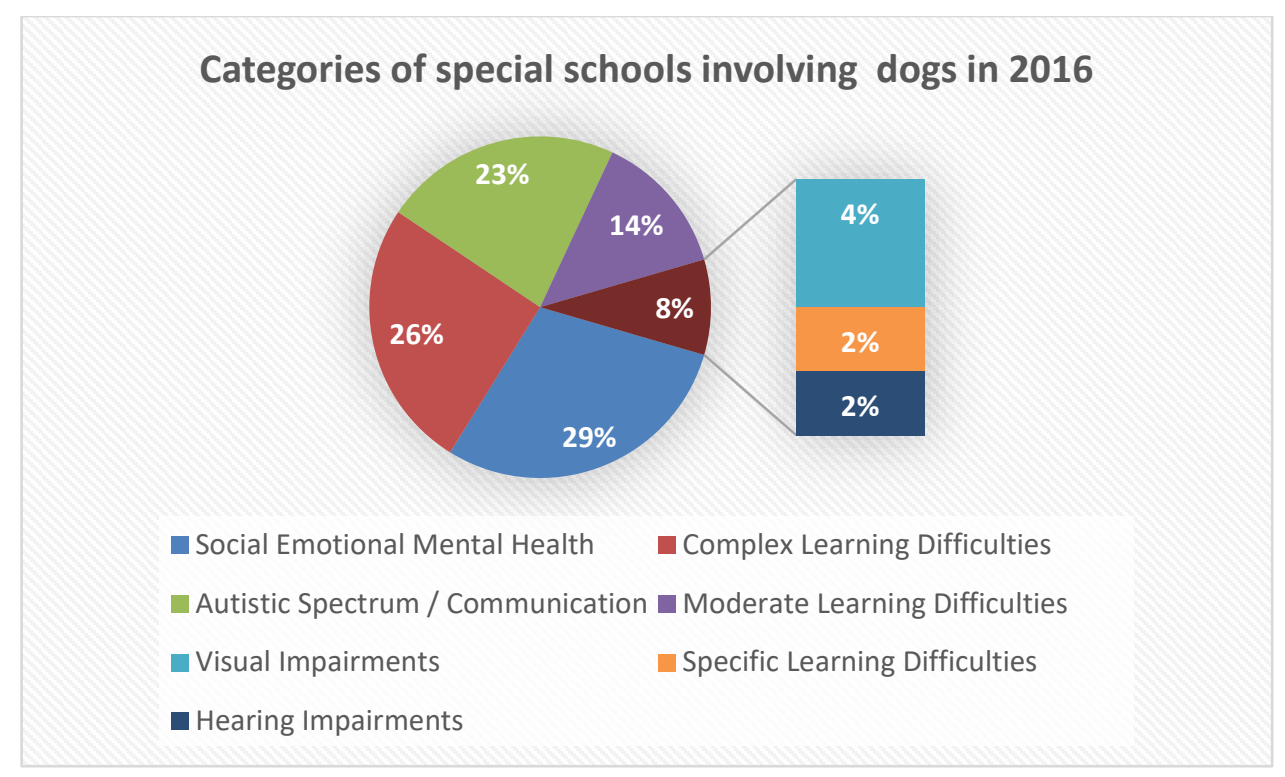

\section{Figure 6: Category of special schools involving dogs in 2016}

The main portion of dog users were the Social Emotional Mental Health group (29\%), the Complex Learning Difficulties Group (26\%) and the Autistic Spectrum / Communications groups (23\%). These findings reflected the then current areas and trends of worldwide research into the efficiency of dogs for certain diagnosable groups such as Autism, ADHD and socio-emotional difficulties, highlighting the associated links and strengths towards dogs assisting mental health and wellbeing in previous research (Fine et al., 2015, Gee et al., 2017a, Hawkins, 2012, Hergovich et al., 2002, Hoffmann et al., 2009, Jenkins et al., 2014, McCardle et al., 2011, Watts and Everly, 2009, Wolff and Frishman, 2004). This chart suggests that the Animal Assisted Intervention research was being read and acted upon by the special needs' schools at this time.

As previously explained, specialist assistance dogs were still included in the survey numbers, such as Guide dogs, Hearing dogs and Autism Support dogs. These dogs tended to work with an individual on a 1:1 basis for a specific role, rather than being a general 'class' dog. Out of 135 dogs found, there were 13 dogs who were pure assistance dogs for 
individuals and were therefore discounted in the school dog research set. The remaining 122 dogs therefore were classed as 'School Dogs' whether provided by the school itself or a charity / organisation. According to the websites, 48 of these were in school 'full time,' while 70 dogs were in school 'part time' i.e. not for the full working school week, and for four dogs their hours were unclear.

While 70 dogs were trained, assessed or supplied by named charities, there was a major number ( $n=52$ ) of 'Independent' or 'unknown' dogs (i.e. source not given, or information about any training or preparation shown on the website.) These dogs could have received training from independent dog training schools, following such schemes as the UK Kennel Club's Good Citizen Dog Training Scheme, or as was also hinted on the websites, no screening, assessment or training had been given to either the dog or handler, other than it belonged to one of the teachers or staff members. The sources of the named providers are shown in Figure 7 below:

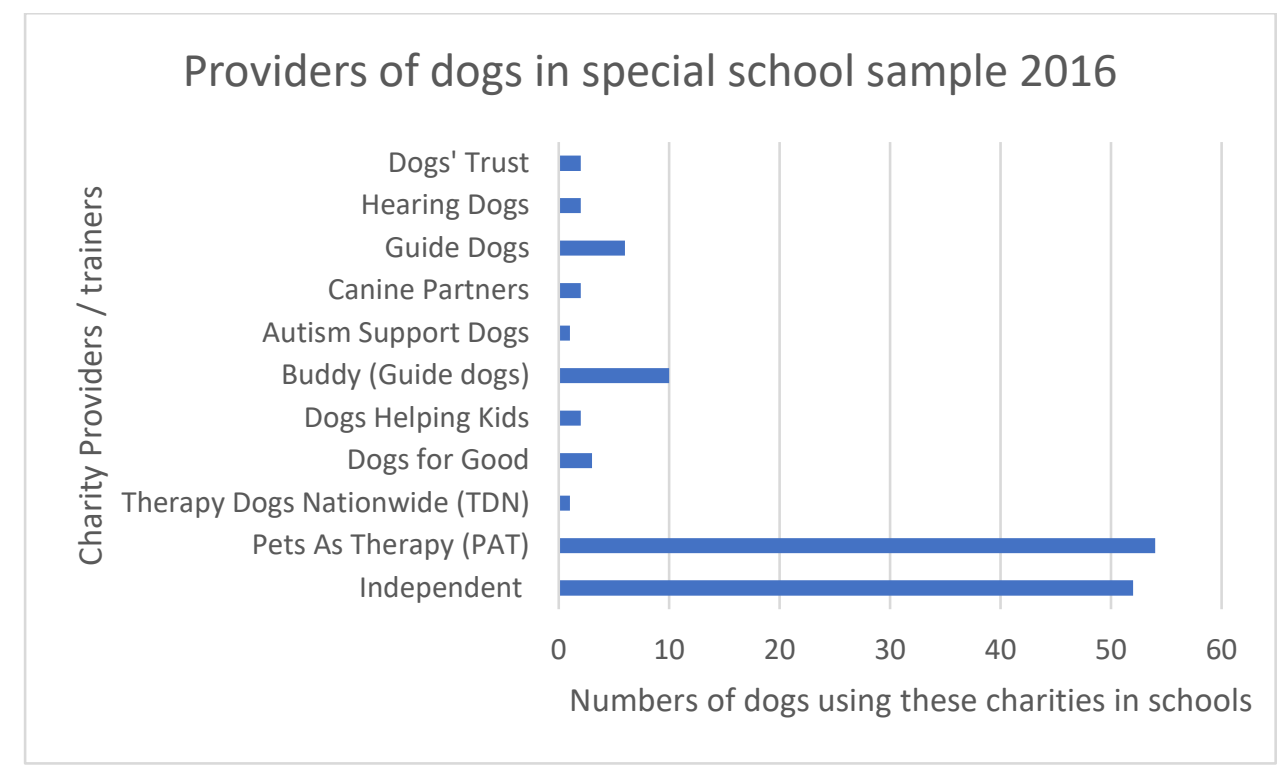




\section{Figure 7: Providers of 135 dogs in 2016 sample}

The main provider of the 'school based' dogs in $2016(n=54)$ was the national 'visiting' dog charity Pets as Therapy (P.A.T., 2016). At this point, this charity was allowing teachers with their own P.A.T. 'temperament assessed only' dogs to a remain in school for an indefinite period as recognised therapy dog.

In 2016, these temperaments tested dogs would have only been tested once, on entry, to this charity. The temperament test consisted of a 'pet' or 'family' dog of over nine months old, being assessed for calmness, quickness in recovering from sudden noise, not minding being petted all over, could take treats gently, be brushed by strangers and was amiable to meeting lots of different people. The charity assessed dogs were intended to stay in schools for short periods of time (1-3 hours) and then return home with their owners / handlers once the task was finished.

It was also clear from the websites however, that these 'P.A.T.' dogs remained in school all day and often on a daily basis - even as small puppies (between 8-12 weeks old, is a crucial period of socialisation and learning for the puppy, regardless of having to cope with the school environment.) The schools' websites promoted that these young puppies would become 'therapy dogs' through this charity when old enough and that by remaining in school from such a young age, the puppies were gaining 'experience.'

The chart also shows that there were small numbers, usually single figures, working from other established charity based organisations such as Dogs for Good and 'Buddy' dogs trained by the Guide Dog Society following the 'assistance training model,' in that the dog had been selected and trained from birth from brood bitches, using puppy handlers and training programmes designed for the eventual roles, individual handlers and for the 
environments in which they would be working. Dogs Helping Kids (DHK) used a mixed approach in that they chose selected puppies but then raised them with their intended school families while incorporating regular 'in house' assessments set up by the charity itself. The distinction between providing Animal Assisted Interaction or Animal Assisted Education, was dependent on the handler's official role in school.

Out of the 122 'school' dogs found, 55 were clearly the responsibility of school staff members. Not all of these staff were trained, qualified teachers, thus pertaining to Animal Assisted Education per se, but because they were staff members with roles in the classroom, they would be providing Animal Assisted Intervention for learning purposes (Beetz, 2018). This purpose was chiefly through socio-emotional intervention and literacy development through "Read to Dogs" programmes. Table 3 below shows the school roles of the "handlers" of the 55 dogs concerned.

\section{Table 3: School based roles of the dog handlers in the school sample}

\begin{tabular}{|l|l|}
\hline Role & Frequency \\
\hline Head Teachers & 6 \\
\hline Deputy Head Teachers & 5 \\
\hline Teacher & 7 \\
\hline Teaching Assistant specifically mentioned & 4 \\
\hline Clinical staff such as therapists & 5 \\
\hline General staff in school & 28 \\
\hline Total: & 55 \\
\hline
\end{tabular}

Another 67 dogs were also visiting schools regularly without their handlers having an educational background at all, other than to be there to assist the school pupils for some 
educational activity. These activities were presumably planned by the staff members themselves. The 13 assistance dogs were in school to assist their owners and have not been included in this number.

\subsection{Why have dogs been brought into schools?}

The five available dog policies from the 104 schools, together with their website information about the dogs, revealed that while schools appeared to be taking past, published research into Animal Assisted Intervention and Animal Assisted Therapy quite seriously when generally arguing for the role of the school dog, the depth of this research may not have specifically applied to their own individual school situation or animal ( See policies in Appendix S). The evidence showed that dogs had mostly been brought in to assist with reading, self-esteem, confidence, social and behaviour issues, but to a lesser extent to promote animal awareness and safety from a child's perspective, as an educational resource (such as an ASDAN Animal Awareness Therapy qualification for Secondary students) or to reflect individual schools' ethos, e.g. in one school for the deaf, a deaf dog was chosen to be their 'mascot.' Only two policies specifically mentioned the dogs' welfare when both in and away from the school environment.

From each of the five policies available and in combination with information on the individual school websites' pages, the reasoning given for benefits of having a dog in school were grouped according to the number of times mentioned. The top ten benefits are to the left of the red line of Figure 9 below. Table 4 then clarifies the top five reasons the dogs had been brought into these schools. 


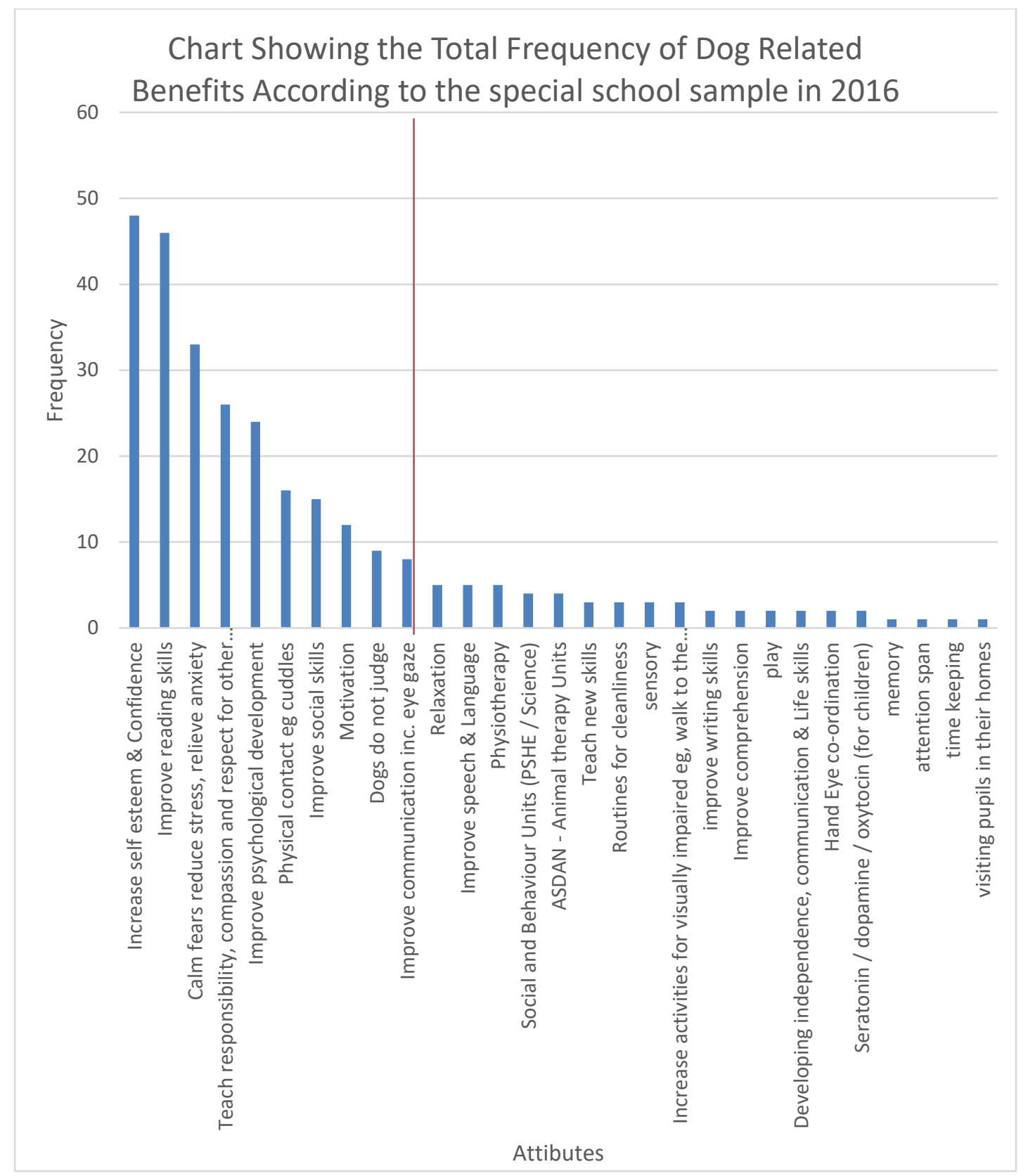

Figure 8: Chart of the frequency of dog related benefits in 2016

Table 4: Top five reasons why dogs were brought into this sample of special schools

\begin{tabular}{|l|l|}
\hline Reason & Frequency \\
\hline Increase self-esteem & 48 \\
\hline Improve reading skills & 46 \\
\hline Calm fears, reduce stress, relieve anxiety & 33 \\
\hline Teach responsibility, compassion and respect for other animals & 26 \\
\hline Improve psychological development & 24 \\
\hline
\end{tabular}


The top five priorities, minus reading, were about the 'socio-emotional' presence of the dog, rather than the ability to improve academic skills, yet there was limited information on how the socio-emotional effects were recorded. This lack of recorded accountability suggests that the dog was present simply as a vague 'companion' in those schools, without a defined role other than as a 'reading dog.' Overall, the emphasis points towards how the dog could 'assist' the student more as a behavioural modification tool for learning. Executive functioning skills such as memory and attention, leading to comprehension, communication and subsequent written and verbal skills did not appear as key benefits to these schools as much as the emotion-based regulatory functions of improving selfconfidence, self-esteem and motivation of their students. The schools' response reflects a behaviourist, rather than a cognitive approach to the inclusion of dogs within classroom learning, which also may replicate these school's aspirations and educational expectations for their pupils.

This evidence would imply that the specifics of purposely planning or integrating a dog in the classroom was on an 'ad hoc,' spontaneous basis and thus differed between schools with little standardisation. There appeared little interpretation as to any other educational possibilities the dog could offer, other than simply by being 'present.' In addition, this research further revealed that any subsequent, spontaneous activity was not even led by the same member of staff who was responsible for the dog. For example, in two of the policies it was recorded that, in the first, that the pupils could at any time, take the dog for a walk within the school grounds or sit with him for a few minutes in a 
quiet space, accompanied by an adult, but with the second, the dog had "responsibilities" for cheering up students by letting them stroke and hug them whenever they wanted.

In contrast, according to the original Animal Assisted Intervention International (AAII) guidelines available in 2016 (which have since been updated in 2019) the specifics for AAE in educational environments were quite clear:

Animal Assisted Education (AAE)- "AAE teams offer goal directed interventions designed to promote improvement in cognitive functioning of the person(s) involved and in which a specially trained dog handler team is an integral part of the education process. AAE is directed and/or delivered by an education professional with education, licensure, and specialized expertise and within the scope of education. AAE may be provided in a variety of settings, may be group or individual in nature and may be implemented for persons of any age. There are specific educational goals for each student involved and the process is evaluated and documented." (Winkle et al., 2012 updated 2019).

These guidelines would suggest that there was very little Animal Assisted Education apparent in the sample schools shown.

The incorporation of the teachers and /or handlers in the integral planning of the dog's specific activities, would not only improve the session's learning goals but would allow a more adaptive, focused and creative outcome, where the dog can initiate exercises, join in activities and supply supportive games to enhance the student's learning depending on the interests and character strengths of the individual dog. Dogs are very capable of learning "tricks" to assist those with or without needs e.g. they can utilise their retrieving skills to fetch and carry items, place paws on cards, use noses to interact and push items such as dice, learn to use head rests for "snuggles" and due to visual shape, learn to read simple words (Beetz, 2013, Childs, 2019, Childs, 2018). Dogs in Austria, Netherlands and Norway are far more interactive with the students than just lying on a cushion or waiting in an office. For example, Sigrid, in Norway, asks her dogs to assist with spelling, by holding, carrying and fetching "plastic ducks" with phonemes written on them to be 
placed in the right order by the child (Sigrid, 2018), Marlies, in the Netherlands uses an alphabet blanket with pockets for treats and reading activities and dog-sized number mats for basic mathematical concepts such as counting (Marlies, 2020). These dogs can work with individuals, pairs and small groups of students. There is a huge difference between a dog joining in for enjoyment and fun rather than just for tolerance and perhaps boredom (VanFleet, 2018b, VanFleet and Faa-Thompson, 2017).Yet, using a dog in this way requires skills in training, knowledge of the dog's interests together with the time for practice. The five school dog policies in combination with the school dog websites showed possible reasons why this potential was not being realised in these sample schools, namely: the interpretations or assumptions made of the word 'therapy'; the quality of training; the personification and generalising of the effects of the dog; and the consequent health and safety issues for all parties concerned.

\subsection{Reason 1: Interpretation or "assumption" made of the word "therapy"}

The websites and polices revealed differences in the interpretation of the term 'therapy.' Therapy can be used in the popularist, general sense as wellbeing, happiness and a sense of self care, but the term 'therapy' is also defined in 'The Concise Oxford Dictionary' as a "medical treatment of disease" requiring professional, qualified personnel working within clinical, medical provisions (Fowler and Fowler, 1989). Only one school had interpreted it in its clinical sense as the owner / handler of the dog was an occupational therapist (School in Cheshire.)

American school teachers and dog handlers such as Mary Renck Jalongo and Lori Friesen, (Friesen and Delisle, 2012, Friesen, 2010, Jenkins et al., 2014, Jalongo et al., 2004) suggests that, "The term "therapy" dog [within schools] may imply that these animals have the 
ability to treat children's emotional, behavioural or physical difficulties," (Friesen, $2010 \mathrm{p}$ 264). Indeed, under a trained clinician, such as a licensed therapist, this can be possible particularly in special schools, such as those previously in the sample.

In the UK, however, two of the largest volunteer nationwide charities who are involved in schools, Pets as Therapy (PAT, established in 1983) and Therapy Dogs Nationwide (TDN, established in 2016) use the term 'therapy' in their charity titles. For PAT and TDN this 'therapy' is not defined as a specific clinical background, but of a general, wellbeing nature, in that none of the volunteers are clinical qualified therapists. Both charities' volunteers do visit a variety of places, such as schools, hospitals, prisons, mental health locations, sheltered housing, old people's homes and end of life care establishments with their dogs for petting activities. Those volunteers who visit schools, often take part in the 'Read to Dogs' scheme whereby the volunteer and dog hear students reading aloud and assist with the reading process, in a similar manner to trained school staff (See Read to Dogs leaflet in Appendix B). Misunderstandings can take place where the tasks these volunteers are given, can cross the professional boundaries of qualified staff, with the assumptions that what the volunteers are providing are of the same standard and quality. Another pertinent issue is that these volunteer dogs are heavily promoted as reading and 'therapy' dogs through the charities' websites, nationwide television campaigns and on social media, including huge, international dog-based events such as 'Crufts,' which is why many of the volunteers choose to join these charities in the first instance. Over time, therefore, the public has become indifferent of the professional differences. This has caused difficulties with other, usually very small, charities who are specifically working to the clinical guidelines and ethos of animal assisted therapy (Winkle et al., 2019). 


\subsection{Reason 2: Quality of training}

The five policies plus the websites themselves revealed a wide variety and difference in the selection and training of the dogs and their handlers / owners. The websites also revealed that many of the teachers or staff involved were also 'first time' dog owners with altruistic notions about the capabilities of their chosen puppies / dogs. Breeds selected were usually the teacher's personal choice after reading breeders' recommendations. The sample revealed a mix of gun dog breeds such as Labradors, Golden Retrievers or Labrador crosses with poodles; spaniels, both springer and cocker; and spaniel crosses such as cockerpoos, springadors, etc. but there were also terrier breeds: border terriers, Jack Russells; 'schnoodles' plus a dalmatian and a chihuahua. All these breeds are highly driven, requiring a lot of exercise, training, patience and work especially in the younger months and years. None of the policies or websites mentioned any learning or training techniques to be encouraged in schools such as positive reinforcement, clicker training or classical and operant conditioning (Winkle et al., 2012).

Apart from the dogs trained in the 'assistance dog' methods, i.e. careful selection and exposure to environments, puppy handler for the first year, intense training for specific tasks, requiring up to two years to become fully trained before entering the classroom, puppies were appearing in schools at eight weeks old. Puppies have always required long periods of sleep to consolidate their learning, so it is questionable as to how much quality interaction and exposure is required at that age. In addition, it is also a known dog training fact that different breeds of both pedigree dogs and 'cross breed' dogs all mature at different ages (Dennison, 2015, Parry, 2012, Fisher and Miller, 2018). Larger dogs, such as labradors for example, can take a longer time to settle and lose their 'bounciness' 
(15months to 2 years) - yet some school dog charities still used these breeds while very small puppies (8-12 weeks) for their training. There are also general acceptance periods for 'fear' in young dogs, usually between $6-14$ months, which if not handled correctly can have long term training consequences, while different breed groups, e.g. 'working' versus 'gun dogs,' require different training schedules and activities (Fisher and Miller, 2018, Dennison, 2015, Parry, 2012). There was evidence of very inexperienced teachers taking on some quite challenging dog behaviours. The crucial task would be whether the individual dog chosen did portray the required characteristics as they grew into a teenage and older dog.

In fact, one charity within the survey did claim on their website that there was an attrition rate of up to $25 \%$ of the dogs leaving the scheme. This school dog charity had therefore put in place, many interpretations of the levels of dog 'roles' created so the dog could maintain a social role within the school (Dogs Helping Kids.).

In 2016 all school dogs required basic obedience skills and behaviour for the safety and understanding of all. Promises of training for these 'school' dogs was normally selected with 'good intentions' and 'commitment' by their owners, usually from 'local' dog clubs or trainers. Often Kennel Club $(\mathrm{KC})$ awards or Good Citizen awards such as those from the KC itself or the Association of Pet Dog Trainers (APDT- UK) were followed. These local dog trainers however, whether accredited to a dog organisation or not (such as the UK Association of Pet Dog Trainers, APDT-UK, Institute of Modern Dog Trainers, IMDT or Association of Pet Behaviour Counsellors, APBC), and despite heavily emphasising positive reinforcement and force free techniques, were also without school teaching qualifications or experience with Animal Assisted Intervention, Animal Assisted Therapy or Animal 
Assisted Education background training themselves - thus being totally unaware of the preparation and complexities required for working within school environments. From the survey, two schools had selected independent dog training businesses who claimed not only to supply the puppy, but to train the dog to a level ready to simply interact with the school. The whole area of dog training in the UK in 2016 was unlicensed, unregulated and training often relied on the personality, style and beliefs of the individual trainer involved.

In 2016, despite the A.A.I.I. guidelines (Winkle et al., 2012), volunteers or teachers were also unlikely to have had the recommended specific training in spotting canine stress behaviours in body language unless personally pursued through short, online courses where even in 2020, it is a case of 'caveat emptor.' Training courses, whether 'hands on,' 'practical' or 'online' can be set up by anyone, and despite many fantastic claims on websites, do not always rise to the equivalent educational standards required for a college or university level certificate, diploma or degree. Since 2018, however, the Animal and Behaviour Council have issued a new set of guidelines for the beginnings of standardisation across dog trainers and associations (A.B.T.C., 2018). Another feature raised from the 2016 survey, however, was that there were at that time no national requirements by law for therapy dogs or school dogs to be formally assessed. There are still no national requirements as of 2020 .

A dog within the school environment can be expected to be amenable and adaptable to a wide variety of situations. These can include curriculum awareness, time constraints, changing staff members, child or adult absence, safeguarding issues, permissions to see certain children, school inspections, events, assemblies and assessment. Dogs need to be 
prepared for any sudden, loud, strange or confusing behaviour from children or adults, be happy to work in any environment in a school, indoors or out, cope with strange sounds, different surfaces and smells, leave equipment alone unless asked and be constantly attentive. They are expected to know in which areas it is allowed to be present, and the areas where it is not, cope with changing timetables and routines - including overriding the dog's own inbuilt timing and routine such as toileting, while still being able to remain calm in emergency drills, including 'lock down' procedures in cases where schools may face threats posed by an intruder or an emergency situation outside the school that prevents the evacuation of students from the building.

In 2016, there was and still is (as of 2020) no specific 'central' training given to PAT or TDN charity handler volunteers. This suggests that unless the schools gave specific requirements as to how they wanted 'read to dogs' to run, volunteers were reliant on the often one-page downloads provided to them from the charities' websites. These downloads should have also included task specific dog training, observation of dog body language and communication, reading tuition, or basic counselling skills.

In contrast, in the United States, Canada, Australia and mainland Europe, for the dog to be a true 'therapy' or 'school' dog it must undergo more thorough environmental skills based training and experience than just a 'temperament test,' together with regular, specific training of the owner/handler in Animal Assisted Intervention or Education (DeltaSociety, 2019, Intermountain-Therapy-Animals, 2019, Pet-Partners, 2019, R.E.A.D, 2019). In 2012, the A.A.I.I. guidelines were advocating for mentorship within charities and up to 10 hours of self-appointed education for each member (Winkle et al., 2012). For instance, Reading Education Assistance Dogs (R.E.A.D®) provides eight hour basic workshops, or an 
eight to ten week course of sessions, plus mentoring from more experienced members for simple reading 1:1 reading with the dog and child for their volunteers (Klotz, 2019) .

In 2016, there was also the underlying assumption that teachers and school staff would be able to cope with the extra training a dog would require, despite their usual school contracts. The staff member of the dog team was still expected to continue to carry out their own school role and responsibilities as per school teaching contract. This assumption negates the required hours out of school with the dog at training classes, usually during the evenings and weekends. There were and currently still are no Animal Assisted practitioners teaching contracts advertised, which either acknowledge the complexities of the role in the job description, reduces the direct classroom teaching hours with the students or specifies the percentage of time the dog and handler are to work together during the school day in the UK.

In Norway, specific university undergraduate courses in anthrozoology are provided towards the overall classroom teaching qualification for those intending to use animal assisted education (Sigrid, 2018). In the Netherlands, the Open Universiteit has a course, written in the English language, which covers Animal Assisted Education available (Enders-Slegers, 2018). There are also annual conferences throughout Europe specifically for those engaged in Animal Assisted Interventions in schools to share common practice (Ludziom, 2019). In contrast, in 2016, there were limited conferences discussing the use of dogs for educational purposes in the UK. These were not advertised in the main teaching websites or journals at that time.

The special school sample charity dog providers also revealed that often corporate sponsorship was expected from outside 'business' partners, such as local firms or 
companies to pay for training, travelling costs and upkeep of the dogs as schools could not afford this on their own. A suggested conservative estimation of training costs alone is upwards of $£ 8000$ per dog (Dogs-for-Good, 2019) with one school apparently spending over $£ 12,000$ on its dogs (Carr, 2019). One specific school dog charity, Dogs Helping Kids also suggested costs should be paid for mandatory training at the charity's Head Quarters for both the handler and dog up to four times a year, and that the handler was to also annually raise funds within the school for the charity. This factor alone may well have contributed to the small numbers of dogs for this charity in the school sample in 2016. Cash strapped schools were, and still are, reliant on the owners and teachers themselves to totally fund the dogs, including feeding, paying for routine health visits from the vet, insurance, training, toys and equipment.

\subsection{Reason 3: Personification and generalising of the effect of the dog}

The personification of the dog was becoming an issue in 2016 with school dogs or puppies being given very human like qualities and responsibilities. One school referred to the dog as a pastoral staff team member, thus implying the dog had special training (Liverpool school.) The personification of the dog in many schools may have influenced the overall enhancement that simply the presence of the dog would suffice for educational activities in schools, and therefore no further explanatory teaching was necessary. For example, one website claimed that, "an adorable four-legged visitor improved self-esteem, acceptance from others and lifted moods often provoking laughter. The companionship with the dog would simulate memory problem solving and game playing," (School in Doncaster.) Another school suggested that, “The dog won't judge the child's skills, enabling them to relax, pat the dog and focus on reading. The dog has an important role 
to play in school day from 'his' scheduled walks, meeting the children, listening to children read and being shown special pieces of work," (School in Southampton.) Some schools did apply a more scientific approach, but these were in the minority, "Dogs can elevate levels of serotonin and dopamine which are known to calm and relax. Dogs can encourage communication and provide a motivation for learning," (school in Solihull.) There were several schools which used the direct personal approach, for example, "[Dog's name] will provide a non-stressful, non-judgmental learning environment for our children. By reading to [dog's name], it will help the children relax and focus on the dog and build their self-confidence with reading skills," (School in the Wirral). "[Dog's name] will listen to children without interrupting which helps to produce competent readers," (school in Blackpool.) While this humanistic approach may emphasis the ethos of the whole school community appearing as a 'family,' the underlying implication ensures that those with non-teaching or educational backgrounds could then simply oversee the reading projects as the dog's presence as the 'uncritical friend' was all that was required. Thus, the act of reading then became a stress free, unaccountable process. Any measurement of progress, if desired, was still up to the individual school's choice of reading test, which simply took place back in class on a separate occasion. This approach may also explain why there is limited research into other academic curriculum subjects with dogs, such as mathematics as there has been no time, will or training available to investigate the area.

\subsection{Reason 4: Health and Safety for all parties concerned}

All five schools with online policies available in the 2016 survey mentioned health and safety. These policies revealed the emphasis on the human interpretation of safety. Only two of these schools showed some acknowledgement of the animals' own needs and 
'natural behaviours' and only one policy mentioned specific dog body language for stressed and unstressed behaviours (Oxfordshire School.) All five found policies contained short statements about allergies; usually that those suffering would be 'kept away' from the dog. These statements did not differentiate between the pupils or teachers having allergies, as a teacher suffering with these could have difficulties completing their role in school (Clayton, 2019).

The five policies emphasised 'controlled' interaction, with the adults or teachers in charge, even though the roles these animals had were to actively initiate interaction with students, both off and on lead. Understandably, if a mistake was made, the dog would be removed from the situation and the school, regardless or not of whether if it was the animal's fault. There were also no specific acknowledgements in the five policies of how children / dog interactions would be taught in a positive manner, despite many schemes available at the time (Brelsford et al., 2017, Dogs-Helping-Kids, 2017, Dogs-for-Good, 2019, Meints et al., 2017).

Undoubtably, if the dog's body language can be quickly read by all present then many perceived "dangers" can be reduced or even avoided. Jalongo (2004) emphasises the importance of the health and safety aspect of being able to read the dog's body language to prevent any dog bites or stress from the animal, stressing that both adults and children themselves also require 'training in how and when to interact appropriately with the dog (Jalongo, 2006, Jalongo et al., 2004, Friesen, 2010, Friesen and Delisle, 2012). A dog, like a person, also needs to feel safe when in school, or any environment (Bekoff and Pierce, 2019) and that its needs are read, listened and responded to. Any animal used in AAI or $A A E$, needs to show active and natural behaviours, rather than coerced, human 
orientated, directed behaviours (excluding those for health and safety of all.) Animals should show the awareness of choice - whether to participate or not in an activity and have their wishes respected (Bekoff and Pierce, 2019, Bradshaw, 2017, Rugaas, 2006, VanFleet, 2018b, VanFleet and Faa-Thompson, 2017). 'Choice' does not mean just for that occasion or during that lesson, it means every time a dog enters a room or situation. Many dogs do decide that they no longer wish to take part in activities and handlers need to be alert to their signals. Only one policy referred to the consideration of the 1979 UK Animal Farm Welfare Committee's 'Five Freedoms' for any animal, since adapted and updated in 2019 by Marc Berkoff into the "Ten Freedoms":

Freedom from hunger and thirst

Freedom from pain

Freedom from discomfort

Freedom from fear and distress

Freedom from avoidable or treatable illness and disability

Freedom to be themselves

Freedom to express normal behaviour

Freedom to exercise choice and control

Freedom to frolic and have fun

Freedom to have privacy and "safe zones"

(Bekoff and Pierce, 2019 p. 11)

In 2016, there appeared to be very little mention of 'risk assessments' or insurance on either the websites or in the dog policies. The visiting dogs were presumably covered by their charities' insurance and general policies, yet the fact that there was still a required bare minimum of a risk assessment when any dog was regularly on the premises was not highlighted. In 2016, the advice to Senior Management Teams in schools was to rely on 
the handler to provide the insurance for the dog (The-Key, 2019, The-School-Bus, 2019, NEELB, 2016). General household insurance or pet insurance ( $3^{\text {rd }}$ party) did not cover in enough depth for visits to educational establishments, however it did appear that many schools were relying on this. Consequently, since 2016, some insurers have now introduced specific public and liability products for animals into schools, but the emphasis still lies with the handler for overall responsibility (Winkle et al., 2019, Dogs-Helping-Kids, 2017, The-Dogs-Trust, 2017). Paradoxically even in 2020, in order to insure a dog as a therapy dog, it needs to have passed at least a temperament assessment or have the charity's requirements for assessment listed by the insurance company. Not all the insurance companies are aware of the different charities' requirements and standards for their dogs (Petplan email 2019). There are also different rules depending on whether the handler is 'paid' while in role, and still no policies exist for injury to the dog or therapy animal itself while in an establishment. It was then, and still is (as of 2020) assumed that any injury will be covered under the dog's own personal medical insurance supplied by the handler / owner.

\subsection{How long do 'trained / assessed' dogs remain in schools?}

In 2016, 135 specific special school dogs were accounted for, but when the same sample was rechecked in 2018 only 73 of the dogs remained (see Figure 10). 


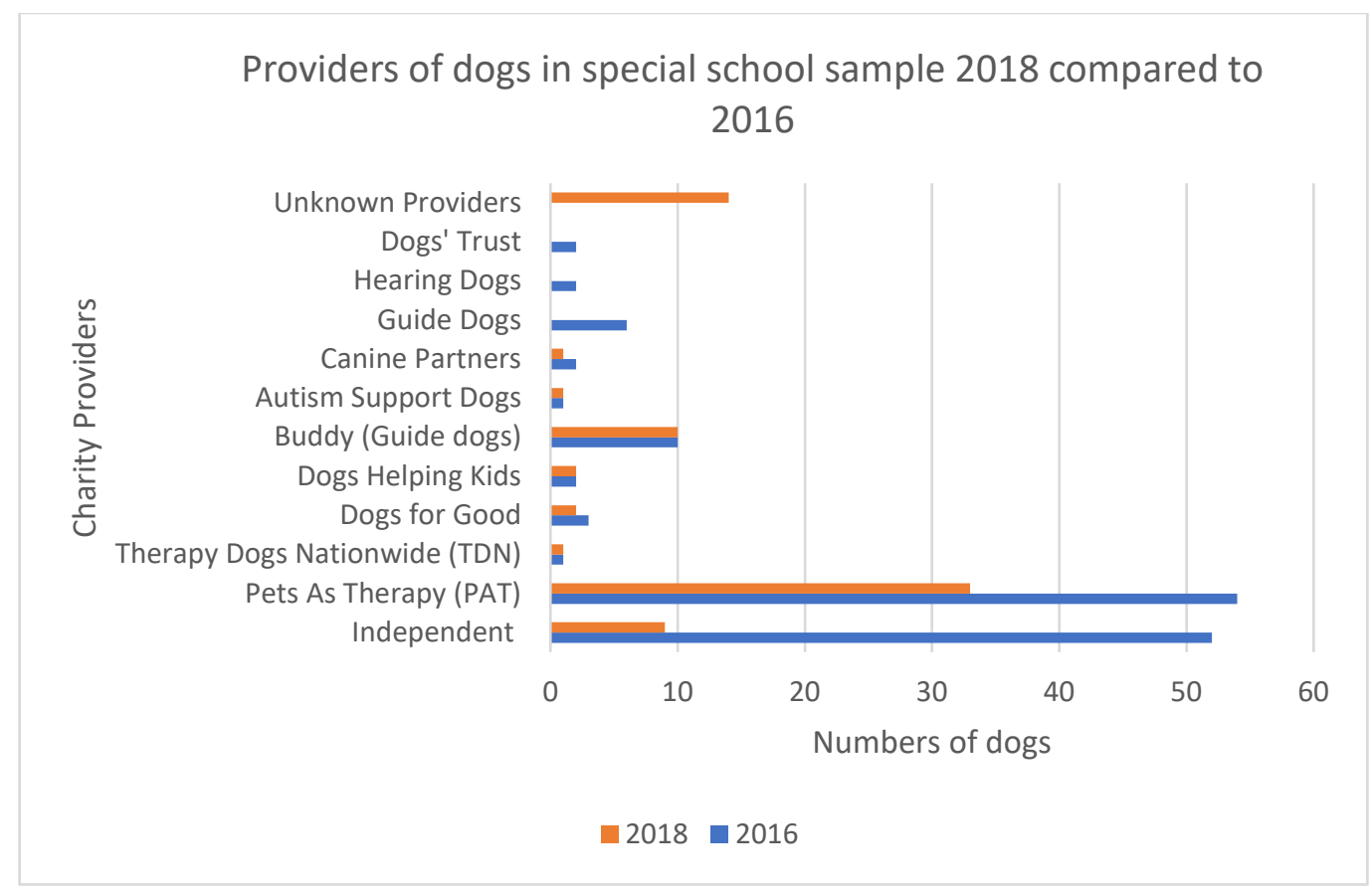

Figure 9: Providers of dogs in special school sample 2018 compared to 2016

Figure 10 shows that out of the sample of 135 school-based dogs in 2016, only 73 remained in their roles in 2018, a reduction of 62 dogs. Several sectors have shown significant change. For example, there has been movement in the volunteer sector of this sample. Originally in 2016, 55 dogs were with volunteer visiting dog charities. By 2018 only 34 remained. Twenty-one of these dogs no longer visited these schools, with the previous school websites revealing that they no longer had any dogs at all. There could be many reasons for this change in numbers: natural retirement or illness of the dog, lack of time on behalf of the volunteer, or has been the case, a change of senior management staff or a change to an academy status. A cut back in funding could result in a reshuffle of staff positions leaving no member of staff to 'monitor or oversee' the sessions as per charity requirements, or that the relationship between the dog and school did not positively progress over the longer time period.

Again the 'visiting volunteer' dog charity group, was more flexible than other charity groups, as most were in schools on a part time basis. The volunteers and their dogs 
therefore were transferrable between both mainstream and special schools, often visiting more than one school during the week. Although the dogs here, are no longer present in the original sample of schools, they may be visiting in different schools outside of the sample. It is often the practice that once one school has a visiting dog, others within the same school cluster or academy chain also ask for visits (T.D.N., 2019).

The numbers of dogs receiving 'Independent' training have also decreased significantly by 43 dogs, yet there is a new, 'unknown' provider category. This was due to the websites no longer giving clear information as to the provider or training for the dogs. This could be related to 'outside influences' such as ongoing discussions between the charities or show that many dogs were still being brought into schools privately, with no specific training planned.

At this time new guidelines were being discussed between the British UK Kennel Club and all the charities involved with dogs in school environments. These discussions went on to become the basis for the 'Bark and Read Standards of Practice for providers of animal assisted interventions in schools' (Kennel-Club, 2018). These guidelines, based upon the Animal Assisted Intervention International Guidelines (Winkle et al., 2012) suggested that: the dogs should not be 'owned' by the school itself but should have registered owners responsible for their daily health care and when not in school, must live as a family pet; be trained at least to the level of the Kennel club's 'silver' award for canine 'Good Citizen' in behaviour and obedience; be re-assessed every two years as a minimum when working with young children or vulnerable people or above; should only work for limited amounts of time before having a complete rest, away from distractions; be insured; and that further training for both the dog and handler would be required if the dog was to remain 
in school as a specific 'school dog' rather than as a visitor (Kennel-Club, 2018, Sections 1.5, 1.10, 3.1, 5.1f, 5.1n.). This meant that the simple, once only 'temperament' PAT dog test which many schools had previously relied upon was no longer a viable option for the school dog owned by a teacher or member of staff.

In contrast to the volunteer visitor dog, in 2018 however, the graph results also suggest that, despite the small numbers, the assistance trained dogs, or those charities using those longer-term training approaches, were still in their original placements after two years. These retained placements could reflect the implications of the cost in time, dedication, training and awareness of international guidelines and long waiting lists for these dogs.

Concurrently to this period, however, updates to Animal Assisted Intervention guidelines both international and national were beginning to highlight the concerns and welfare for both the dogs and handlers in schools. These updated standards and guidelines were also being openly discussed in and between the members of the charities and organisations' social media groups online (ISAZ, 2014, VanFleet, 2018a, S.C.A.S., 2019). Several documents arrived in short succession which required further dissemination in the raising of schools' general awareness of issues. (See table 5 below.) In July 2019, Dogs for Good also held an "Animal Assisted Intervention Insight Day," for the general public and interested charities examining the width and breadth of animals assisted activities in the UK. It was well attended (Dogs-for-Good, 2019) by schools and charities alike, encouraging more charities which involved dogs in school environments to question and re-align their practices. To further highlight safety aspects steps have since been taken to produce awareness documents of possible scenarios for incidences in the UK (Westgarth et al., 
2018, Kennel-club, 2020, Kennel-Club, 2015) which have also been taken up by some schools.

Further joint conferences for dogs working with children have now taken place (Paws for play - PAWS for progress, Scotland, October 2019; S.C.A.S, September 2019.) The Dog's Trust has now developed several pages on its website dedicated to the use of dogs in school environments (Dogs' Trust, 2020.) One of the advantages of using social media during the Covid Crisis (2020) has been that many conferences have been placed online, allowing greater access to wider audiences for a smaller price. These audiences are from many different animal-interested backgrounds including teaching (ISAZ International Conference 2020, SCAS Conference, 2020.) The 'snowball' of information and knowledge is beginning to grow.

However the choice to adapt, develop or even follow these guidelines is up to the charities' board members - and if a charity visits more than schools, say for instance hospitals as well as care homes, then priorities can be challenged, especially when other environments have new guidelines and codes of practice of their own.

Table 5: Guidelines for animals used in animal assisted interventions in schools

\begin{tabular}{|l|l|l|l|}
\hline Date & Charity / Society & Origin & Document \\
\hline 2017 & Dogs' Trust - Dog in schools' guidelines & U.K. & $\begin{array}{l}\text { Dogs' Trust Statement and advice } \\
\text { on using dogs in school's fact } \\
\text { sheet }\end{array}$ \\
\hline 2017 & $\begin{array}{l}\text { Dogs Helping Kids / Dogs for Good } \\
\text { Guidelines }\end{array}$ & U.K. & $\begin{array}{l}\text { Guidance for schools - Key factors } \\
\text { when considering the } \\
\text { introduction of a dog into your } \\
\text { school for }\end{array}$ \\
\hline 2018 & Bark and Read Guidelines & U.K. & $\begin{array}{l}\text { Standards of Practice for } \\
\text { Providers of Animal Assisted } \\
\text { Interventions in schools }\end{array}$ \\
\hline
\end{tabular}




\begin{tabular}{|l|l|l|l|}
\hline 2018 & $\begin{array}{l}\text { International Association of Human } \\
\text { Animal Interaction Organisations } \\
\text { IAHAIO update }\end{array}$ & U.S. & $\begin{array}{l}\text { The IAHAIO Definitions for Animal } \\
\text { Assisted Intervention and } \\
\text { Guidelines for Wellness of } \\
\text { Wellness of Animals Involved in } \\
\text { AAI }\end{array}$ \\
\hline 2018 & $\begin{array}{l}\text { Animal Assisted Intervention } \\
\text { International (A.A.I.I.) }\end{array}$ & U.S. & $\begin{array}{l}\text { A.A.I.I. Standards of Practice } \\
\text { updated }\end{array}$ \\
\hline 2019 & $\begin{array}{l}\text { Society for Companion Animal Studies } \\
\text { (S.C.A.S) }\end{array}$ & U.K. & $\begin{array}{l}\text { Animal Assisted Interventions: } \\
\text { SCAS Code of Practice for the UK }\end{array}$ \\
\hline
\end{tabular}

\subsection{Summary}

This chapter has raised several challenges for the current inclusion of dogs in England's schools. While just under $10 \%$ of schools had dogs in 2016 , their roles were for socioemotional 'therapeutic' interests, unless examining reading. In much of the suggested activities, however, the dog was simply present as a companion rather than as active partner. The potential to create and develop a role for Animal Assisted Education by which the dog would assist with specific learning goals in all subject areas had not been recognised. Significant issues remained in how and where the dog could be incorporated for academic learning; dog welfare; and what experience and training both the dog and the handler receive for the classroom environment.

Whereas both the European 'visitor' and 'in school' models have been applied, the survey highlighted insufficient consistency between schools, combined with a lack of general or central guidance for the practical aspects of developing these roles further. Developments such as examining teaching contracts and the possibility of upskilling willing volunteers were not explored. Since 2016, however, there have been signs of an 'overhaul' in approach, attitudes and standards required for including dogs within the classroom. From 2018 , these standards have appeared to have been further discussed, refined and begun 
to be adapted by English based charities. Yet while these guidelines are available, they remain "advised" rather than mandatory - and still very young dogs even now, can be taken into schools with no assessment whatsoever.

Other possible benefits to teaching and learning for subject areas are being missed. Books produced to help teachers plan and consider the use of AAE in the classrooms are still few in number, (Gee et al., 2017b, Jalongo et al., 2018). These do not always reveal a clear way to introduce activities, say for social programmes, mathematics or spelling target or lesson objectives, step by step approach, that teacher manuals and interventions often give. Therefore, further, in depth research is required into how the skills of learning can be affected when a dog is available.

What ultimately underlies all these activities, however, is the relationship and knowledge of the dog which comes from nurture, training, trust, and ultimately time - a commodity which is inherently poor in schools and classrooms. Perhaps starting with reading, the one area recognised by all the schools and charities as involving cognitive, academic skills would be of benefit. An investigation into how dogs affect the underlying skills for reading and how these could be measured will be required. 


\section{Chapter 5 (Research Phase 2)}

\section{School based Investigation: Working Memory Tests}

\subsection{The Problem}

If a dog can affect the executive functioning and working memory skills associated with classroom learning, then ideally there should be a test, or perhaps a battery of tests that could be used by teachers to assess any dog-contingent changes in academic performance. Currently, such tests do not exist.

Tests for working memory are used in schools when assessing for specific learning difficulties by qualified teachers and educational psychologists. As they are already commercially produced, they should also show validity and reliability. Such tests may not, however, be available to all staff. Teachers have to undertake postgraduate training at Level 7 (Master's Level - see Appendix A) to be able to access the tests and report back on the subsequent findings or alternatively undertake a Certificate of Competence in Educational Testing (CCET). Currently these qualifications are part of the Specific Learning Difficulties Assessment Standards Committee (SASC) requirements for an Assessing Practicing Certificate (APC) in the diagnostic assessment of learning difficulties such as Dyslexia. Training can take up to two years to complete and is also part of the Associate Membership of the British Dyslexia Association (AMBDA) award. This is considered the "gold" standard for teachers of specific learning difficulties.

\subsection{Tests available for teacher use}

Education as an area, is therefore still limited to certain 'teacher version' tests for working memory. In 2016, examples of these tests were: 
- $\quad$ The Automated Working Memory Assessment test (AWMA, Alloway, 2007)

- $\quad$ The working memory rating scale (WMRS, Alloway et al. 2008)

- The Working memory test battery for children (WMTBfC, Pickering and Gathercole (2008),

- The Test of Memory and Learning (TOMAL 2, Reynolds and Voress, 2007) or

- The Wide Range Assessment of Memory and Learning (WRAML 2, Shezlow and Adams, 2003).

Although much educational and psychological research into aspects of measuring working memory with children has previously used the AWMA, mostly through the work of Gathercole and Alloway, it was taken out of print in December 2016.

Within each of these test batteries, there are short, individual activities examining different skills such as speed of processing. The activities are designed to be child-friendly in that stories, pictures and symbols are used, while the interpretation of the results is via training and manuals. Often two similar products such as the TOMAL 2 and the WRAML 2 cover the same areas of working memory such as an 'Attention Concentration Index' and can be used by an assessor / examiner to cross check skills and results for an individual. The cost of each of these tests, however, can be off-putting for the self-employed assessor or school, and so only one test is referred within a diagnosis.

There are several tests available to teachers linked to visuo-spatial awareness such as the Test of Visual Perceptual Skills (TVPS, Martin, 2006) or parts of the Test of Memory and Learning (TOMAL, Reynolds, 2007). Phonological processing, speed and rapid automatic naming are also available through the Phonological Assessment Battery (PhAB, 
Frederickson et al., 1997) To check whether the dog's presence affected these skills, individual tests could be simply adapted.

As these tests are already standardised to population means and every attempt has been made to remove cultural and ethical biases. Although they have not been used within a dog's presence before it is proposed to use these tests as they are widely used and validated within education. This fits with Brelsford et al.,(2017) and Hall, Gee and Mill's (2016a) requests for standardised measures, that can be used with students with no known developmental or learning differences to give a more objective view on how the dog affects cognition in general (Brelsford et al., 2017, Hall et al., 2016a).

Test baselines would have to be established, and any improvement upon these tasks would have to clearly indicate that the presence of the dog had made a significant change. Different measures using the provided individual skill tests from these tests would have the potential to show the dog's effects related to overall skills. General statements such as "the dog's presence affected the child" (Gee et al., 2012a) could then be analysed to determine how the dog had affected the child, ideally linking back to any influence the dog may have on such things as working memory performance.

\subsection{How can teachers recognise working memory difficulties in the classroom?}

Most teachers are now aware of Gathercole and Alloway's lists of classroom behaviour, (Gathercole and Alloway, 2008, Packiam Alloway and Alloway, 2015, Gathercole, 2008) yet having the time and knowledge to 'spot' these difficulties in individuals still takes time. A student with working memory difficulties in the classroom can show many different behaviours such as: rarely volunteering information in class discussions, giving simple answers to questions and are usually not achieving to academic expectations in either or 
both, literacy and numeracy. They can be slow to map sounds for phonics and spellings, struggle to interpret and retain comprehension when reading for themselves, have difficulties retaining basic number facts such as times tables and subsequent poor progress in mathematical concepts requiring overlearning of areas such as time or fractions. Other complications can include difficulties in following lengthy instructions, problems with storage and processing procedural information, e.g. when asked to organise and write a paragraph or story or to carry out 'steps' in mathematical problems, while retaining place-keeping throughout those sequences.

\subsection{Types of Tasks for Selection}

The commercial tests discussed can be used both with and without the dog present for a comparison of performance. This may highlight not just the areas of weakness in the child, but also where the dog could facilitate differences in cognition and attainment, such as with visuo-spatial tasks. The tests selected, such as the TOMAL 2, should reflect those associated with working memory skills used in the classroom such as phonological or numerical recognition, accuracy of automisation, speed of processing, verbal, or visuospatial short-term memory biases, which would also be relevant when working with a dog. The tests do what they are designed to do, and that is to give an indication of performance linked to working memory. What they do not do is to give any indication of the 'state' of the child in terms of, for example any stress or anxiety that may be induced by the task and possibly ameliorated by the presence of the dog. To assess such general measures of state, it is proposed to use a simple and non-invasive physiological measure electrodermal activity (EDA). These measures will be discussed in more detail in the next chapter (Chapter 6). 


\subsection{Which skills to examine?}

Working memory capacity, is usually measured in traditional ways such as the backward digit span, where a participant is asked to verbally repeat a series of given numbers (or letters) in reverse order. This tests the capacity to retain and re-sequence the numbers given. The commercial tests, however, also examine other skills such as verbal short-term memory and visuo-spatial short-term memory and processing speed which can incorporate executive functioning skills. An alternative to the digit or verbal spans is the 'spatial span' where a participant can be asked to touch a set of cubes or shapes in a given sequence which increases in length. This shows the visual span and can be used to show the difference between the visual and verbal, which can also highlight receptive language difficulties.

Visuo-spatial skills would be essential with young children learning to read. The 'visuo' element is linked to the static visual features of an item, e.g. the objects and their colour. The 'spatial' element relates to the position, motion and direction of the item (Baddeley, 2007). This is useful for reading as the grapheme, for example, is visually encoded to create the letters and allows for tracking across the page in reading (Alloway, 2011). Similarly, in mathematics, the visuo-spatial sketchpad uses a more holistic approach and is more dependent on the central executive than the phonological loop (Gathercole and Pickering, 2006 as cited in Dehn, 2008). Young children however, are more reliant on the visuo-spatial sketchpad for the solutions to maths problems up to around seven years old, when they become more reliant on the phonological loop (Gathercole et al., 2008a, Holmes, 2017, Holmes, 2006, Passolunghi and Cornoldi, 2008). Holmes and Adams, (2006) suggest that the visual spatial short-term memory (VSSTM) acts as a mental blackboard 
or number line. Bourke and Adams (2010) discovered that it was also the VSSTM scores which predicted writing skills at age four to five years.

Similarly, verbal short-term memory, together with the phonological short-term memory are associated with both mathematical and language difficulties. Under the Baddeley and Hitch working memory model, the phonological loop, (also known as the articulatory loop) briefly stores verbal words in the working memory in the order they are perceived. Storage is for about 2 seconds before decay occurs, unless items are rehearsed (Hulme and Mackenzie,1992, as cited in Dehn, 2008). Rehearsal relies on subvocal repetition, articulation and speech rate (Baddeley, 2007; Henry 2012). Short, monosyllabic words are retained better which has implications for the word length effect for reading and attainment (Gathercole and Martin 1996 as cited in Dehn 2008). Impairment in the phonological loop and central executive underpins Snowling's research into phonological deficits for Dyslexia (Snowling, 2000; Goswami, 2004) and the double deficit hypothesis, citing speed of processing and phonological awareness (Wolf \& Bowers, 1999 as cited in Snowling 2000). Other difficulties experienced by young learners and those with learning differences include difficulties with mental arithmetic in terms of mental strategies for calculations and the sub vocal rehearsal in counting (Passolinghi and Conoldi; 2008, Maehler and Schuchardt, 2009; Holmes and Adams, 2006; Andersson, 2008). Henry and Winfield (2010) suggest that the ability to self -rehearse is also linked to success in reading with Gathercole et al (1992 as cited in Boudreau and Constanza-Smith, 2011) showing that the Phonological Short-Term Memory (PSTM) scores at age four, are a significant predictor of reading at age eight. 


\subsection{Relationship to previous research}

Under the 'Biophilial theory' (Kellert and Wilson, 1993) many AAT researchers, e.g. Beetz

et al. (2012b), have used physiological evidence such as electrodermal activity (EDA), blood pressure and cortisol measurements to check the cognitive / emotional response of the human to the dog, whereas many of the working memory researchers use activities and traditional cognitive mind exercises using equipment, puzzles and activities.

Gee and her research teams, however, have used physical tasks (Gee et al., 2007) such as object categorisation tasks, by using picture cards or soft toys linking subjects thematically, taxonomically and a 'different' category, or instructions and 'distraction' activities (Gee et al., 2010b, Gee et al., 2010a). Adult students used a geometrical sequence activity, similar to an electronic lights version of the game of 'Simon' on an ipad with and without the presence of the dog for up to five conditions (Gee et al., 2015b, Gee et al., 2014). For each experiment she provided 'distractor activities' such as cartoon characters on cards and listening to an audio recording of a book. While these can be shown as classroom-based tasks, it could also be argued these tasks are not only complicated within themselves but almost impossible to indicate where the dog is affecting the person's thought or memory process. For instance, it would be difficult to show if the dog affects the speed of processing, the verbal responses or the assimilation of the tasks. Her results were analysed using ANOVAs, which are usually not covered as a part of an educationalist's training curriculum and so are difficult to 'translate' for classroom practitioners who are more familiar with standardised scores. Perhaps a combination therefore of Gee's tasks plus 'teacher-friendly' versions of working memory 
tests could be used to investigate where, if any, the dog's presence could influence learning and attainment.

\subsection{Preparation of Working Memory Sub Tests for 2016 School-Based Project}

If Working Memory is to be investigated quantitatively, knowledge of suitable normative criterion referenced standardised tests for specific underlying skills are required. These tests can highlight areas of strength and weakness in comparison to other peer groups, or for individual differences with or without the dog present.

For recording any improvement after a period of intervention It would also be necessary for a baseline and post-intervention assessment. Many of these tests do require a certain amount of time between retesting so parallel 'test papers' will be required. As time is a huge factor in schools, these tests need to be simple, child friendly and quick and accurate to complete.

A range of tests have been identified (See Table 6) that assess specific areas of skill (in reading and mathematics ) relating to working memory were carefully selected based on the research of Dehn (Dehn, 2008, Dehn, 2015), and from a wide range of reading from educational psychology articles on working memory for children. Qualifications, experience and the testing knowledge of the teacher (which is often not at the level 7 required), together with costs and availability, were all taken into consideration. 
Table 6: Table of commercial working memory tests available to qualified teachers (2016)

\begin{tabular}{|c|c|c|c|c|}
\hline Name & Author & Description & Specific Tests for WM? & Teacher Accessible? \\
\hline $\begin{array}{l}\text { TOMAL (Test of } \\
\text { Memory and } \\
\text { Learning) } \\
\text { Age: 5:00-59:11 }\end{array}$ & Reynolds (2007) & $\begin{array}{l}\text { TOMAL- } 2 \text { provides of memory assessment currently available } \\
\text { in a standardised battery. This assessment permits a direct } \\
\text { comparison across a variety of aspects of memory in a single } \\
\text { battery. This allows the assessment of strengths and } \\
\text { weaknesses, as well as potentially pathologic indicators of } \\
\text { memory disturbances. }\end{array}$ & $\begin{array}{l}\text { Verbal Memory Index } \\
\text { Non-Verbal Memory Index } \\
\text { Composite Memory Index } \\
\text { Verbal Delayed Recall } \\
\text { Learning Index } \\
\text { Attention and Concentration } \\
\text { Sequential Memory } \\
\text { Free Recall } \\
\text { Associative Recall }\end{array}$ & $\begin{array}{l}\text { Yes but Need higher } \\
\text { training e.g. AMBDA, } \\
\text { Level } 7 \text { or APC }\end{array}$ \\
\hline $\begin{array}{l}\text { WRAML (Wide Range } \\
\text { Assessment of } \\
\text { Memory and } \\
\text { Learning) } \\
\text { Age: } 5: 00-90: 00\end{array}$ & $\begin{array}{l}\text { Sheslow \& Adams } \\
\text { (2003) }\end{array}$ & $\begin{array}{l}\text { The WRAML2 is a standardised instrument that allows the } \\
\text { user to evaluate an individual's memory functioning. It } \\
\text { provides evaluation of both immediate and delayed memory } \\
\text { ability, as well as the acquisition of new learning. }\end{array}$ & $\begin{array}{l}\text { Verbal Memory Index, } \\
\text { Visual Memory Index, } \\
\text { Attention/Concentration Index } \\
\text { General Memory Index. } \\
\text { Working Memory Index, Design } \\
\text { Recognition, Picture Recognition, } \\
\text { Verbal Recognition, and Story Memory } \\
\text { Recognition. }\end{array}$ & $\begin{array}{l}\text { Yes but Need higher } \\
\text { training e.g. AMBDA, } \\
\text { Level } 7 \text { or APC }\end{array}$ \\
\hline
\end{tabular}




\begin{tabular}{|c|c|c|c|c|}
\hline $\begin{array}{l}\text { WRAT } 4 \text { (Wide Range } \\
\text { Ability Test) } \\
\text { Age: 5:00 - 94:00 } \\
\text { Has been superseded } \\
\text { by WRAT } 5 \text { (2017) }\end{array}$ & $\begin{array}{ll}\text { Wilkinson } & \& \\
\text { Robertson (2006) }\end{array}$ & $\begin{array}{l}\text { The WRAT- } 4 \text { accurately measures the basic academic skills of } \\
\text { word reading, sentence comprehension, spelling, and maths } \\
\text { computation. } \\
\text { Now superseded by WRAT } 5 \text { (2017) }\end{array}$ & $\begin{array}{l}\text { Sentence Comprehension } \\
\text { Oral Maths } \\
\text { Maths Computation }\end{array}$ & $\begin{array}{l}\text { Yes but need higher } \\
\text { training e.g. AMBDA, or } \\
\text { Level } 7 \text { or APC }\end{array}$ \\
\hline $\begin{array}{l}\text { PhAB (Phonological } \\
\text { Assessment Battery } \\
\text { Age: 6:00-14:00 } \\
\text { (updated 2014 but } \\
\text { was used within } 2 \\
\text { years of update) }\end{array}$ & $\begin{array}{l}\text { Fredrickson, Frith \& } \\
\text { Reason (1997) }\end{array}$ & $\begin{array}{l}\text { Phonological Assessment Battery }(P h A B) \text { is designed to assess } \\
\text { phonological processing in individual children. It identifies } \\
\text { children who have significant phonological difficulties and } \\
\text { need special help in processing sounds in spoken language. }\end{array}$ & $\begin{array}{l}\text { Naming Speed tests } \\
\text { Spoonerisms Test } \\
\text { Fluency Tests } \\
\text { Non-Word Reading Test }\end{array}$ & Yes \\
\hline $\begin{array}{l}\text { TVPS }-3 \text { (Test of Visual } \\
\text { Perception Skills) } \\
\text { Age: } 4: 00-18: 11\end{array}$ & Martin (2006) & $\begin{array}{l}\text { The TVPS-3 is intended for a reliable and valid measure of a } \\
\text { child's perceptual abilities. Since visual perceptual abilities } \\
\text { are used in a number of academic pursuits, including learning } \\
\text { to read, it is important to know which processes the child may } \\
\text { be having difficulty with. }\end{array}$ & $\begin{array}{l}\text { Visual Memory } \\
\text { Spatial Relationships } \\
\text { Form Constancy } \\
\text { Sequential Memory } \\
\text { Visual Closure }\end{array}$ & Yes \\
\hline $\begin{array}{l}\text { TAPS-3 (Test of } \\
\text { Auditory Processing) } \\
\text { Age: } 4: 00-18: 11\end{array}$ & $\begin{array}{l}\text { Martin \& Brownwell } \\
(2005)\end{array}$ & $\begin{array}{l}\text { The TAPS- } 3 \text { represents a test of Auditory Perceptual Skills: } \\
\text { Phonological Segmentation, Phonological Blending, Auditory } \\
\text { Comprehension, and Auditory Reasoning specifically designed } \\
\text { to tap auditory cohesion, a higher-order process. }\end{array}$ & $\begin{array}{l}\text { Number Memory Forward } \\
\text { Number Memory Backward } \\
\text { Word Memory } \\
\text { Sentence Memory }\end{array}$ & Yes \\
\hline
\end{tabular}


The subsequent 'skills' tests selected are as follows (Table 7). Care has been taken to try to use as many teacher-accessible sub tests as possible. The Test of Memory and Learning (TOMAL) 2 Letters and numbers forward and backwards is the standard working memory span, which is used in many tests of memory, some of which do not require advanced training skills.

Table 7: Selected sub-tests (based on memory skills used in reading and mathematics)

\begin{tabular}{|c|c|}
\hline Subtest & Skills \\
\hline Memory for Stories (TOMAL) & Verbal Working Memory / Auditory Memory \\
\hline Memory for Stories Delayed (TOMAL) & Verbal Working Memory \& Long-Term Memory \\
\hline Letters Forward (TOMAL) & Auditory Phonological Short-Term memory \\
\hline Letters Backwards (TOMAL) & Executive Working Memory \\
\hline Digits Forward (TOMAL) & Auditory Phonological Shirt Term Memory \\
\hline Digits Backwards (TOMAL) & Executive Working Memory \\
\hline Naming Picture Speed (PhAB) & $\begin{array}{l}\text { Visuo-spatial Short-Term Memory, Verbal Short- } \\
\text { Term Memory, Processing Speed }\end{array}$ \\
\hline Naming Digit Speed (PhAB) & $\begin{array}{l}\text { Visuo-spatial Short-Term Memory, Verbal Short- } \\
\text { Term Memory, Processing Speed }\end{array}$ \\
\hline Visual Memory (TVPS) & $\begin{array}{l}\text { Visuo-Spatial Short-Term Memory and Working } \\
\text { Memory (application) }\end{array}$ \\
\hline Sentence Comprehension (WRAT) & Verbal Working Memory and Processing \\
\hline Oral Maths (WRAT) & $\begin{array}{l}\text { Visuo-Spatial Long-term Memory and Working } \\
\text { Memory }\end{array}$ \\
\hline Maths Computation (WRAT) & $\begin{array}{l}\text { Visuo-Spatial Long-term Memory and Working } \\
\text { Memory }\end{array}$ \\
\hline
\end{tabular}

\subsubsection{Test Details}

\subsubsection{TOMAL 2 (Test of Memory and Learning, version 2, Reynolds and Voress, 2007)}

The Test of Memory and Learning was established in the mid-1990s and was designed to give reliable information for a variety of memory functions for children and adolescents. It was subsequently updated to include adults up to 59 years of age with the TOMAL 2 (2007). It allows interested parties such as specialist teachers or psychologists to assess the strengths and weaknesses of participants' memory 
functions and strategies used for learning tasks and is often utilised to assess those with learning difficulties. TOMAL2 was standardised across a United States population, incorporating gender, social background and ethnicity. The test is designed to be administered individually. A booklet is provided for the examiner with scripts to follow in each test for consistency. Each test is designed to be quick so that if necessary, the whole battery can be used at one sitting, which should take up to 30 minutes with a 'practised' examiner. Sections been designed, however, to only have four or five tests so that a 'composite index' can be formed examining certain skills only e.g. attention / concentration.

Tests chosen for the children in the 2016 school-based project were 'Memory for Stories' and 'Memory for Stories Delayed.' In 'Memory for Stories,' the participant, after hearing a short story from the examiner, is required to use their attention skills, short-term memory and auditory processing to create meaningful recall, including the use of semantics, sequencing and direct details to immediately retell the given story back to the examiner. These features were written down by the examiner. This is then repeated with a second story also recorded in the same way.

In 'Memory for Stories Delayed,' fifteen minutes later after completing several other tests, they are asked to recap both stories again with as much detail as they can remember. This compares immediate auditory recall and longer-term memory recall, with the comparison and recollection of the details between the two. This uses not only short-term memory but also working memory in using techniques in remembering details, associations with words and sequential re-telling. 
Other sub-tests chosen from the TOMAL 2 were the 'Digits Backward' and 'Letters Backward' recall, which are traditional tests of working memory and capacity. The 'Digits Forward,' and 'Letters Forward' tests can give an indication as to rote memory or immediate short-term recall ability. The letters will give a comparison for language stimuli versus digits. Within all the tests there are 'ceilings' provided where an individual can access the test at an appropriate stage for themselves and a 'cut off' point where after several consecutive mistakes have been made, the test is stopped. The resulting spans are then converted from raw scores to standardised scores, scaled scores and percentile ranks.

\subsubsection{WRAT-4 (Wide Range Achievement Test, Wilkinson and Robertson, 2006)}

The Wide Range Achievement Test version 4, is a classroom-based tasks for assessing, e.g. reading, writing and spelling, and was also standardised in the United States using a cross-section of individuals aged between 5 to 94 years and across gender, ethnicity, educational attainment and geographic region. It has also been tested for internal and external reliability and validity. The WRAT 4, now superseded by the updated WRAT 5 (2017), is still used in the diagnosis of specific literacy difficulties in the United Kingdom, as there is currently no alternative using UK populated norms.

According to the WRAT instructions, "The sentence comprehension measures an individual's ability to gain meaning from words and to comprehend ideas and information contained in sentences through the use of modified cloze technique" (WRAT, p.2). This relies on working memory, long term lexical knowledge, and visual memory. 
The sentence comprehension test, available in two parallel forms contain 50 items on a 'cloze' procedure format, (a sentence with one vital word missing), in which all the answers can be answered correctly with a one-word response. Prompting and questioning from the examiner can be used asking for one to three-word responses, such as, "Can you give me a different word?" if the participant has just repeated the same word already in the sentence. The sentences have demonstration answers that are available to the examiner, but the rest of the section is to be read internally by the participant. The scores are converted into raw scores which are then transformed into standardised scores.

The oral maths test includes finger counting through to simple four operations completed mentally. It is to be read by the examiner with the participant answering verbally.

The Maths Computation Test measures an individual's ability to perform basic mathematical computations through counting, identifying numbers, solving simple oral problems, and calculating written mathematics problems. Maths computation is a timed test where the participant needs to read and understand mathematical questions and, after being given specific instructions by the examiner as to how to fill it in, completes it by themselves. Several starting points are given dependent on age. The questions are graded in difficulty and the participant Is given 15 minutes to complete as many as they can in a written format, working internally. The raw score can then be calculated.

The WRAT test already has 2 parallel forms, which can be used while the dog is, and is not, present. 


\subsubsection{TVPS-3 (Test of Visual Perception Skills, Version 3, Martin, 2006)}

The Test of Visual Perception Skills version 3 (TVPS-3) is designed to determine the visual perceptual strengths and weaknesses of students between the ages of 4 years through to 18 years, using a series of black and white line drawings. The items are in a multiple-choice format, and answers can be given either verbally or by pointing, depending on the cognitive needs and requirements of the student. There are 16 plates in each area of Visual discrimination, visual memory, spatial relationships, form constancy, sequential memory, figure-ground and visual closure which all involve aspects of the working memory in holding and manipulating visual mental information. Ceilings are given so as not to tax younger students. It is timed and estimated to take about 30 minutes to complete. The difficulty with this test is that the plates are graded in difficulty which can be awkward to re-create for before and after comparisons. This may be a test that needs to be given annually, or bi-annually, rather than for shorter time periods such as a 12-14-week school term.

\subsubsection{PhAB (Phonological Assessment Battery, Frederickson, Frith and Reason, 1997)}

The phonological assessment battery and the newer phonological assessment battery, primary update, $2^{\text {nd }}$ edition (2014) are aimed at school aged children. The original version can still be used for students up to 18 years of age, whereas the PhAb2 Primary was updated in 2014 for children between the ages of 5:00 to 11:11 years. For the 2016 children's school-based study, the original PhAB was used as it may still be used up to two years after the new version had arrived. The tests have been standardised with a UK-based population and has been internally and externally 
validated with the Neale Assessment of Reading Ability (NARA) and the British Ability Scales II (BAS II) also used in diagnostic assessments.

The test was originally designed to assess the awareness of phonology or the ability to process sounds within the English language, and helps to seek and identify any underlying difficulties in reading or spelling words which can hinder reading and comprehension attainment, thus the three main areas of assessment are phonological awareness, phonological production and speed and language fluency. The tests chosen were of the same presentation and order in both versions $\mathrm{PhAB}$ and PhAB 2 and have a script for the examiner to follow.

Each test is quickly administrated and includes word and non-word tests. This test is regularly used in schools. Certain tests can be used with immediacy, rather than waiting for a retest period, such as the fluency tests and naming picture speed tests which can be easily adapted within the test's framework. This can give comparison for when the dog is and is not present.

The sub tests chosen were: Fluency (Part 1) The student is asked to say as many words which rhyme with a given target word within a minute. (Part 2) The student is asked to give as many words which alliterate with a given word within a minute. (Part 3) The student is asked to give as many words which link semantically to a given topic within one minute.

These tests happen frequently within the Key stage 1 (5-7 years old) and Key Stage 2(7-11 years) classrooms so their reproduction should pose no difficulty when working with and without the dog. 
PhAB sub test Naming Speed: - These tests assess the fluency and automaticity with which the student can recall verbal information. The student is given a series of either pictures or digits and told their names. They are then required to name a random sequence of 50 stimuli (either pictures or digits) as quickly as possible, with the time to do so recorded (seconds). This can get quite competitive and exciting. For both the pictures and the digits, there are two sheets available to get an average of the scores. By simply swapping the sheets the naming speed test can easily be replicated with the dog present or not and the scores compared.

\subsubsection{Test Comparability}

If the results are to be comparable in terms of standardised scores, each test used needs to be standardised for the target population. The selected commercial assessments all standardise to a median of 100 to allow comparability (see table 8).

\section{Table 8: Standardisation across chosen commercial tests}

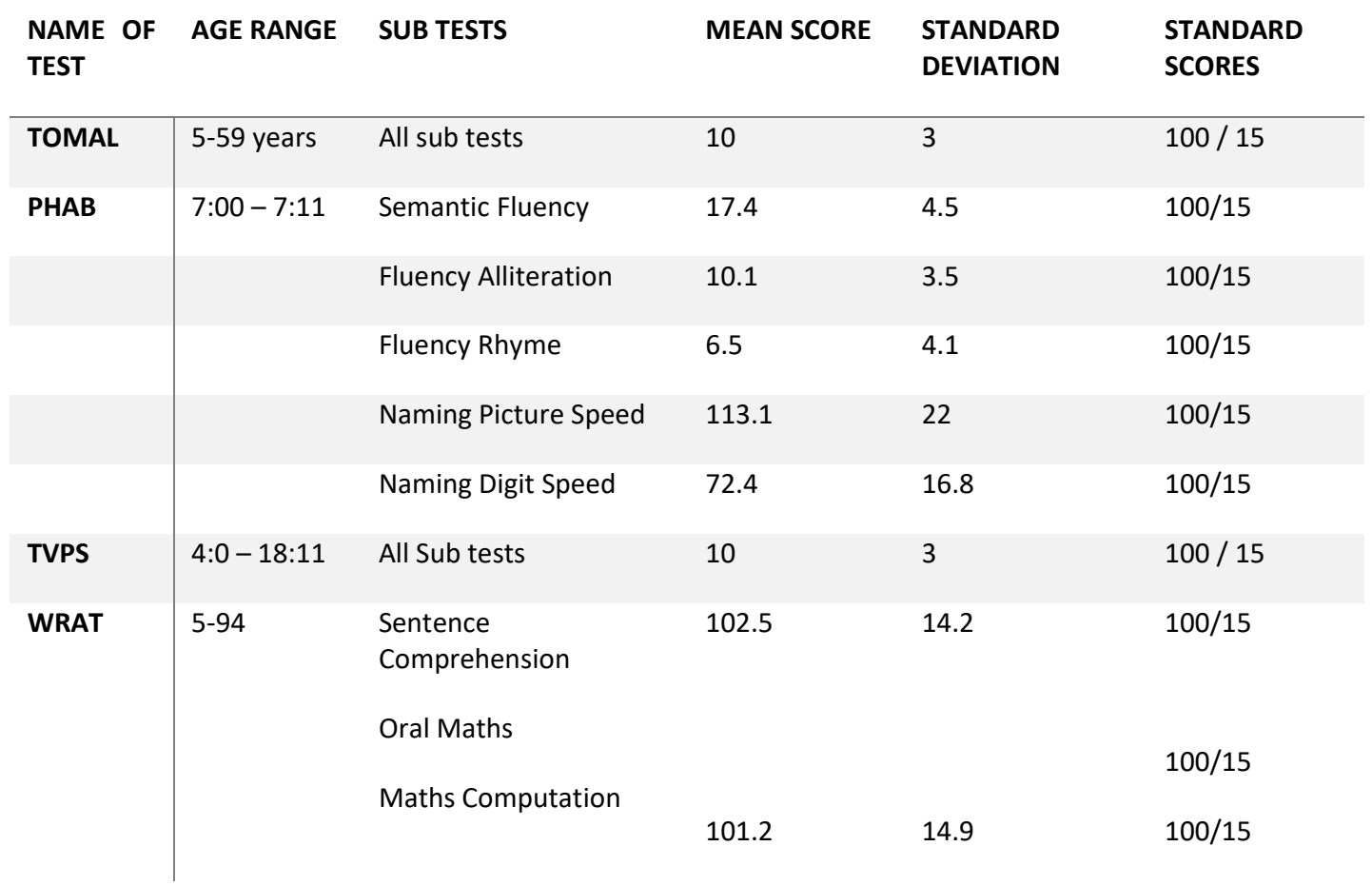




\subsubsection{Explanation of the Standardised Test Scores}

Standardised Scores: These scores enable us to compare the performance of one person to the performance of others who are the same age. They also enable us to compare an individual's performance on one test with performance on another. A standard score of 100 is, by definition, average. For other scores please see the level descriptor table below.

\subsubsection{Level Descriptors}

Although some manuals use different terms, for the purpose of this study and to aid accessibility for the non-specialist, the labels and level descriptions below are used to describe performance. They are the same as those set by the UK authorities in relation to examination access arrangements (see table 9 below.)

\section{Table 9: Explanation of level descriptors}

\begin{tabular}{|c|c|}
\hline Standard Score Range & Suggested Descriptors \\
\hline 131 or more & Well above average \\
\hline $116-130$ & Above Average \\
\hline $111-115$ & High Average \\
\hline $90-110$ & Average \\
\hline $85-89$ & Low Average \\
\hline $70-84$ & Below Average \\
\hline 69 and below & Well below average \\
\hline
\end{tabular}


For an understanding of how a standardised (standard) score compares to other measurements used in other tests, such as scaled scores, please see Figure 10, below.

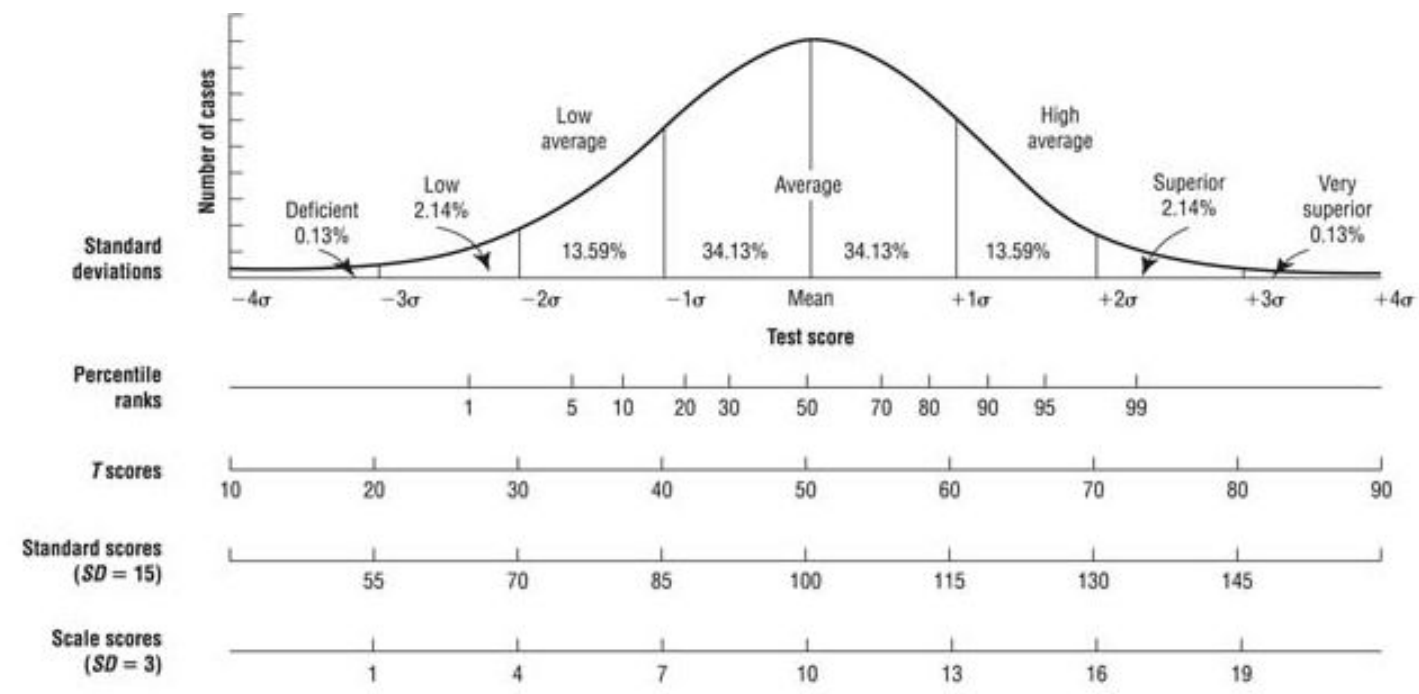

Figure 10: A standardisation curve showing relationships between standard scores, percentiles

and scaled scores (Source: medfriendly.com, 2020)

This thesis refers mainly to the standardised scores, percentile ranks, and scaled scores used in these sub tests. An average standardised score is 100 and this has the equivalent percentile rank of 50 and a scale score of 10 . Thus, while each test may be scored separately using different scoring scales, there can be conversion and cohesion for an overall understanding. The 'Bell' curve shows the natural distribution of scores across the sampled population.

\subsubsection{Design}

The tests were used to examine whether there was a difference between the dog present or absent. There were two testing points: one without the dog present and 
a second phase when the dog was present. Sufficient time was needed between the two points of data collection.

This was a working school environment with only a 12-week window for the entire school-based study to be completed. Ideally the two points would be at weeks 1 or 2 of the school term and again at weeks 11 and 12 . In reality, it was closer to eight weeks apart.

\subsubsection{Ethical Permissions and Health and Safety}

Consideration was given as to how the dog could possibly interact during the second session. The dog was alongside for stroking before and during the activity. There were also safety concerns depending on the excitability of the child completing the tests and whether this would affect the dog in any way. For safety and consistency, it was decided that the child would complete the set tasks while sitting at the table with the examiner in both situations. The dog and handler were available but sat slightly away from the table setting and closer to the door, so that should the dog have been affected or not responded to any verbal commands, there was an available 'emergency' exit into a hallway and to an outside playground for calming and distraction.

The school had agreed to the testing as part of the main reading project. All permissions were gained from parents, the SENCO, and from the charity dog handler as were insurances and safety checks for child protection etc. Verbal permission was asked of the children before testing on both occasions. No child refused and they were very interested in the experiment itself, asking questions about what was to happen. They all thought they would be 'better' in the dog situation. The whole study 
was 'open' to the children in that it was verbally explained what was happening, why it was happening and that they were all, "Scientists conducting research." The children also were happy to be out of class.

\subsubsection{Participants}

The participant group was smaller than preferred. It consisted of only the seven children who had permission to be recorded as case studies for the 'read to dog' sessions. All were between seven and eight years old with the average age of 7 years 6 months. This group had been selected by the school SENCO as part of her special needs group. The SENCO did not reveal why these children had been labelled as such as she wanted to see if anything would be revealed as part of the research. Ideally, including the whole group of 15 children for the reading project would have given a better indication of any effect of the presence of a dog on performance.

\subsubsection{Results of Specific Working Memory Tests}

Individual tests from specific Working Memory Assessments available in schools had been planned for fluency in semantics, alliteration and rhyme, visual memory and medium to long term verbal memory both with and without the dog's presence. Unfortunately, due to class and school timetabling, many of the chosen tests were too time consuming for every child to complete in both conditions. On average, the initial testing phase took between $30-40$ minutes per child for the 12 tasks, but this was considered too long a period for being out of class by one teacher. Therefore, a compromise had to be made in that only those tests for processing speed, short term and working memory executive skill changes could be repeated in both conditions. Had there been more time, then long term, verbal and visuo-spatial memory could 
have been compared and possibly links could have been made for the effects of the dog in other curriculum areas.

Tests which had to be removed were the Visual Memory test from the TVPS3, The Maths oral and computations from the WRAT, The Memory for Stories and The Memory for Stories Delayed, tests from the TOMAL 2, Maths computation and counting back in fours from 100, and the Semantic, Alliterative and Rhyme Fluency Tests from the PhAB.

The tests that remained, were chosen to be good indicators of changes in the backwards digit and letters spans, revealing longer attentional periods, attentional capacity, switching of attention, inhibition, error reduction and links between long term memory and working memory. The children's results for these tests are shown in table 10. 
Table 10: Working memory skills scores: with and without dog standardised scores

\begin{tabular}{|c|c|c|c|c|c|c|c|}
\hline Name & Chronological Age & $\begin{array}{l}\text { Naming Speed } \\
\text { Pictures }\end{array}$ & Naming Speed Digits & Digits forward & Letters Forward & Digits Backwards & Letters Backwards \\
\hline Male A & 7:03 & SS 111 & SS 88 & SS 80 & SS 85 & SS 90 & SS 90 \\
\hline Dog & 7:05 & SS 131 & SS 124 & SS 75 & SS 85 & SS 95 & SS 100 \\
\hline Female E & 7:08 & SS 69 & SS 93 & SS 100 & SS 90 & SS 105 & SS 95 \\
\hline Dog & 7:09 & SS 93 & SS 89 & SS 85 & SS120 & SS120 & SS 100 \\
\hline Female $\mathrm{A}$ & 7:09 & SS 80 & SS 75 & SS 76 & SS 80 & SS 90 & SS 90 \\
\hline Dog & $7: 11$ & SS $<69$ & SS $<69$ & SS 80 & SS 80 & SS 95 & SS100 \\
\hline Male M & 7:04 & SS 114 & SS 118 & SS 75 & SS 75 & SS 95 & SS 100 \\
\hline Dog & 7:05 & SS 107 & SS 122 & SS80 & SS 80 & SS 100 & SS 100 \\
\hline Male C & 7:09 & SS 87 & SS 107 & SS 95 & SS 80 & SS 85 & SS 85 \\
\hline Dog & 7:10 & SS 85 & SS 112 & SS 80 & SS 80 & SS 90 & SS 85 \\
\hline Female G & 7:06 & SS 99 & SS 92 & SS 100 & SS 75 & SS 100 & SS 95 \\
\hline Dog & 7:06 & SS 104 & SS 91 & SS 95 & SS 95 & SS 100 & SS 95 \\
\hline Male H & $8: 00$ & SS 77 & SS 104 & SS 85 & SS 85 & SS 105 & SS 95 \\
\hline Dog & 8:00 & SS 101 & SS 91 & SS 75 & SS 90 & SS 85 & SS 95 \\
\hline $\begin{array}{l}\text { Average Group No Dog } \\
\text { Score }\end{array}$ & & ss 91.0 & ss 96.7 & ss 87.3 & ss 81.4 & ss 95.7 & ss 92.9 \\
\hline $\begin{array}{l}\text { Average Group Dog } \\
\text { Score }\end{array}$ & & Ss 98.6 & Ss 99.7 & Ss 81.4 & Ss 90.0 & Ss 97.9 & SS 96.4 \\
\hline
\end{tabular}




\subsubsection{Executive working Memory Group Scores}

The classic measurement of Working memory span is usually the backwards digit test. To compare with a reading -related analogue, the letters backward span was also included. This compared the differences in auditory memory for both digits and letters (See Figure11 below.)

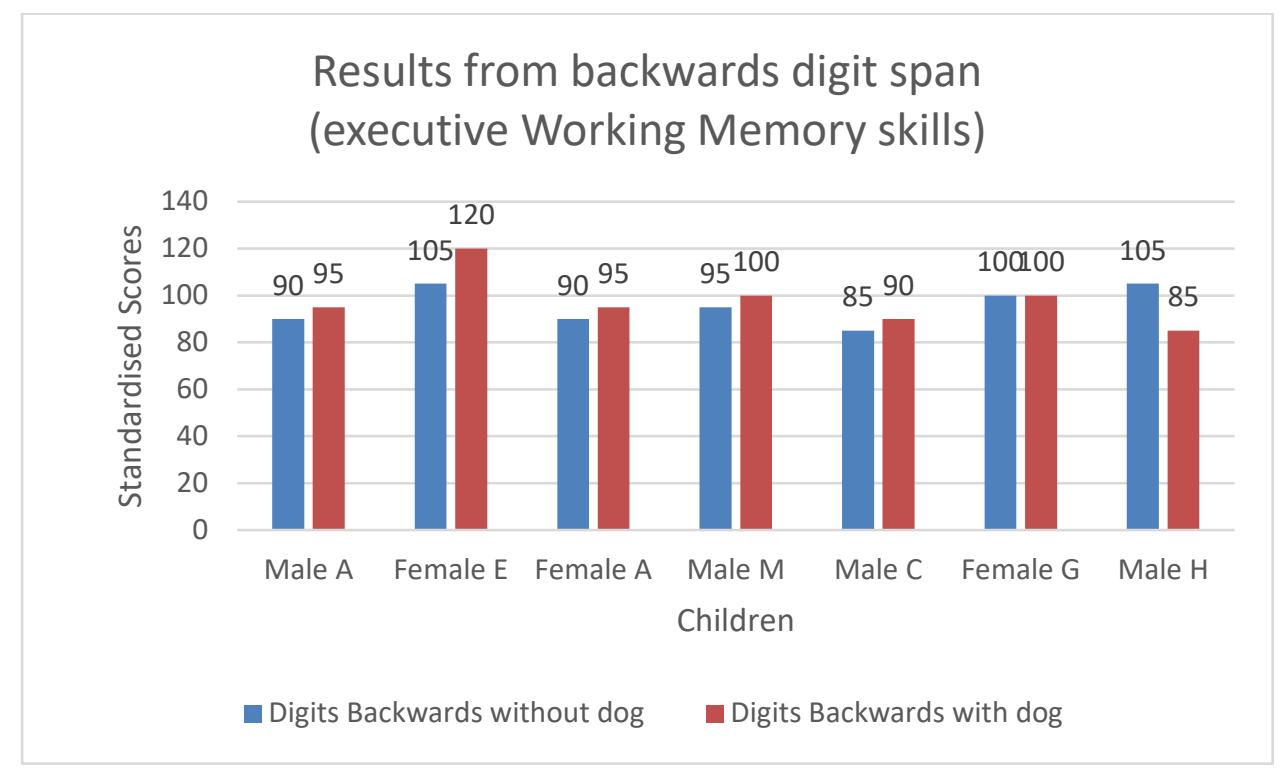

Figure 11: Results from the backwards digit span

The average group standardised score without dog was 95.7. The average group standardised score with dog was 97.9.

For the digits backwards span, overall, there was a group average increase of 2.2 in standardised scores or $2 \%$ increase generally in scores for the group, when the dog was present. Due to the small sample size, no further statistical tests were conducted.

The results from the backwards letter span are shown in Figure 12, below. 


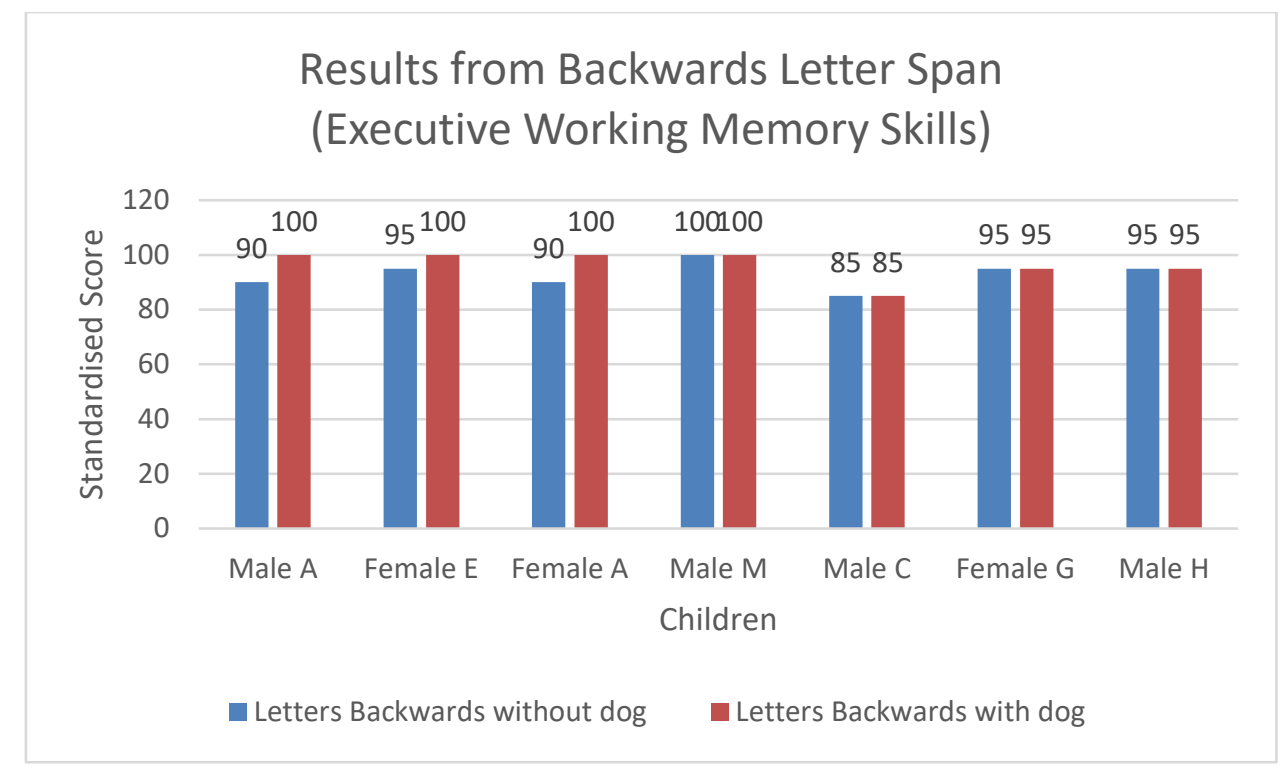

Figure 12: Results from the backwards letter span

The average group standardised score without dog was 92.9. The average group standardised score with dog was 96.4 .

For the backwards letter span (see Figure 13) there was a group average increase of 3.5 in standardised scores, or $4 \%$ generally, for the group when the dog was present.

The combined results from both the letters and digits tests for all children are shown in Figure 13, below.

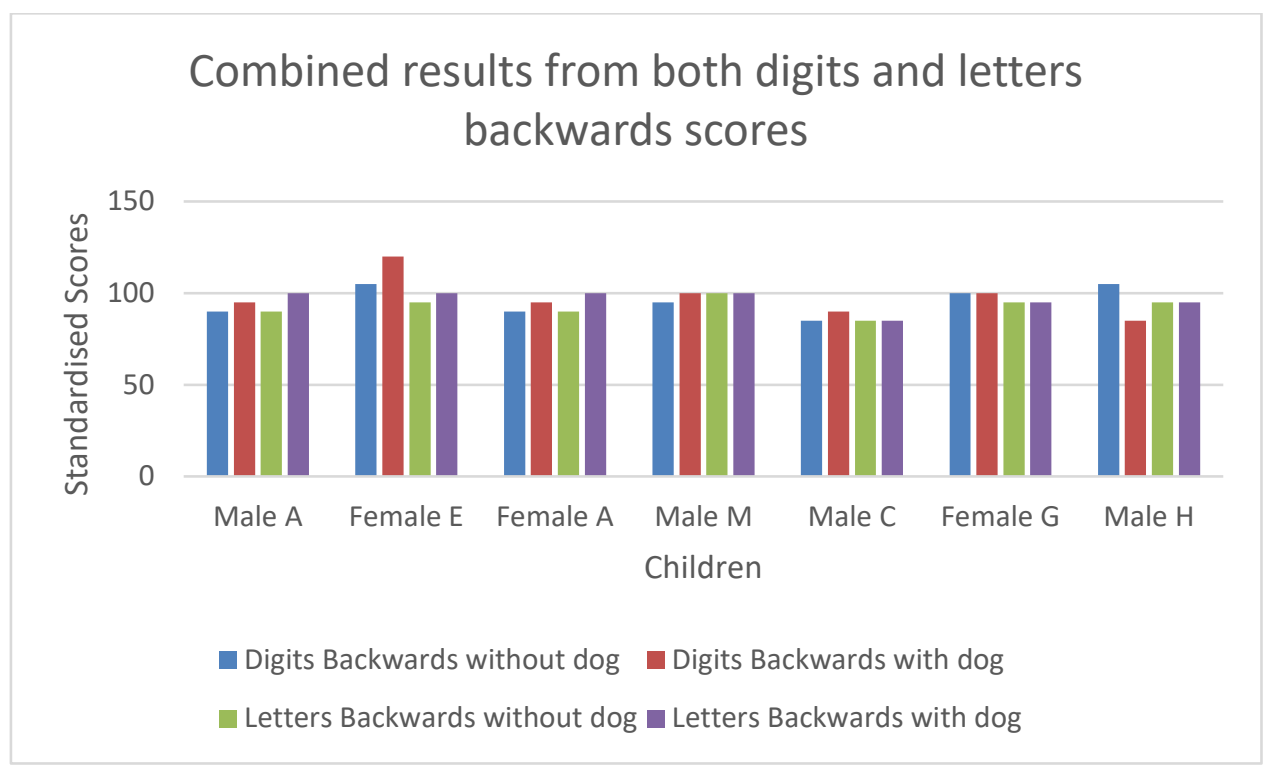


Reynolds (1997)suggest that the backwards digit span in particular, tests the visuospatial elements of memory and retention.

The combined tests with both the digits and the letters backward shows that for five children, their working memory span increased with the dog present. For two however, it did not. This could be down to individual profiles. The original, baseline individual standardised scores for the group showed that none of these children were below average for this measure of their working memory (i.e. scores lower than a standardised score of 85 ). Also indicated are the differences in the individual children's preferences for digits or letters, as the presence of the dog may affect individual scores on one or the other.

\subsubsection{Working Memory Spans (backwards)}

To examine if length of spans, or capacity was affected, raw scores with and without the dog were compared with the longest digit span recorded for each child. Alloway suggests that for children aged 5-6 years the span would be two items, and children aged 7-9 it is three (Alloway \& Alloway 2015). The table below shows the longest spans that these children reached during these tests. There was a difference between the digit results and the letter results. Digits backwards with the dog increased the span for three children, stayed the same for two, but reduced it for one. In letters backward with the dog, only two children had their spans increased while the other five remained the same. Only two female children had improvements in both the digits and letters backwards spans when the dog was present. This would suggest that the dog has a greater effect on the visuo-spatial component, rather than the language element of the task. 
The modal backwards digit span for this group of 7-8-year-old children without the dog is a span of 2.5 , yet with the dog, this increased to 3 itself. With letters, the backwards modal span was 2 , but with the dog, it increased to 2.5 . This could be signs of evidence that the dog is affecting their attentional executive skills for some individuals when it is present. The length of the backwards span is recorded for each child in Table 11 below.

Table 11: Backward spans results

\begin{tabular}{|c|c|c|c|c|c|c|}
\hline Child & Test & Raw Score & $\begin{array}{l}\text { Best } \\
\text { Span }\end{array}$ & Test & Raw Score & $\begin{array}{l}\text { Best } \\
\text { Span }\end{array}$ \\
\hline \multirow[t]{2}{*}{ Male A } & $\begin{array}{l}\text { Digits Back no } \\
\text { dog }\end{array}$ & 7 & 2 & $\begin{array}{l}\text { Letters Back -no } \\
\text { dog }\end{array}$ & 6 & 2 \\
\hline & $\begin{array}{l}\text { Digits back with } \\
\text { dog }\end{array}$ & 9 & 2 & $\begin{array}{l}\text { Letters back with } \\
\text { dog }\end{array}$ & 10 & 2 \\
\hline \multirow[t]{2}{*}{ Female $\mathrm{E}$} & $\begin{array}{l}\text { Digits back no } \\
\text { dog }\end{array}$ & 12 & 3 & $\begin{array}{l}\text { Letter back - no } \\
\text { dog }\end{array}$ & 8 & 2 \\
\hline & $\begin{array}{l}\text { Digits back with } \\
\text { dog }\end{array}$ & 22 & 4 & $\begin{array}{l}\text { Letters back - } \\
\text { with dog }\end{array}$ & 10 & 3 \\
\hline \multirow[t]{2}{*}{$\begin{array}{l}\text { Female } \\
\text { A }\end{array}$} & $\begin{array}{l}\text { Digits back no } \\
\text { dog }\end{array}$ & 7 & 2 & $\begin{array}{l}\text { Letters back no } \\
\text { dog }\end{array}$ & 7 & 2 \\
\hline & $\begin{array}{l}\text { Digits back with } \\
\text { dog }\end{array}$ & 9 & 3 & $\begin{array}{l}\text { Letters back with } \\
\text { dog }\end{array}$ & 11 & 3 \\
\hline \multirow[t]{2}{*}{ Male M } & $\begin{array}{l}\text { Digits back no } \\
\text { dog }\end{array}$ & 9 & 3 & $\begin{array}{l}\text { Letters back no } \\
\text { dog }\end{array}$ & 10 & 3 \\
\hline & $\begin{array}{l}\text { Digits back with } \\
\text { dog }\end{array}$ & 10 & 3 & $\begin{array}{l}\text { Letters back with } \\
\text { dog }\end{array}$ & 10 & 3 \\
\hline \multirow[t]{2}{*}{ Male C } & $\begin{array}{l}\text { Digits back no } \\
\text { dog }\end{array}$ & 4 & 2 & $\begin{array}{l}\text { Letters back no } \\
\text { dog }\end{array}$ & 2 & 2 \\
\hline & $\begin{array}{l}\text { Digits back with } \\
\text { dog }\end{array}$ & 9 & 3 & $\begin{array}{l}\text { Letters back with } \\
\text { dog }\end{array}$ & 5 & 2 \\
\hline \multirow[t]{2}{*}{$\begin{array}{l}\text { Female } \\
\text { G }\end{array}$} & $\begin{array}{l}\text { Digits back no } \\
\text { dog }\end{array}$ & 13 & 3 & $\begin{array}{l}\text { Letters back no } \\
\text { dog }\end{array}$ & 9 & 2 \\
\hline & $\begin{array}{l}\text { Digits back with } \\
\text { dog }\end{array}$ & 11 & 3 & $\begin{array}{l}\text { Letters back with } \\
\text { dog }\end{array}$ & 8 & 2 \\
\hline
\end{tabular}




\begin{tabular}{|l|l|l|l|l|l|l|}
\hline Male H & $\begin{array}{l}\text { Digits back no } \\
\text { dog }\end{array}$ & 14 & 4 & $\begin{array}{l}\text { Letters back no } \\
\text { dog }\end{array}$ & 12 & 3 \\
\hline & $\begin{array}{l}\text { Digits back with } \\
\text { dog }\end{array}$ & 9 & 3 & $\begin{array}{l}\text { Letters back with } \\
\text { dog }\end{array}$ & 11 & 3 \\
\hline
\end{tabular}

\subsubsection{Group Processing Speed Results}

To investigate whether the presence of the dog affected the processing rate and the rapid recall of visual memory and verbal labelling, two tests were undertaken, one with pictorial symbols and the other with a list of digits. A graph of the naming picture speeds is seen in Figure 14, below.

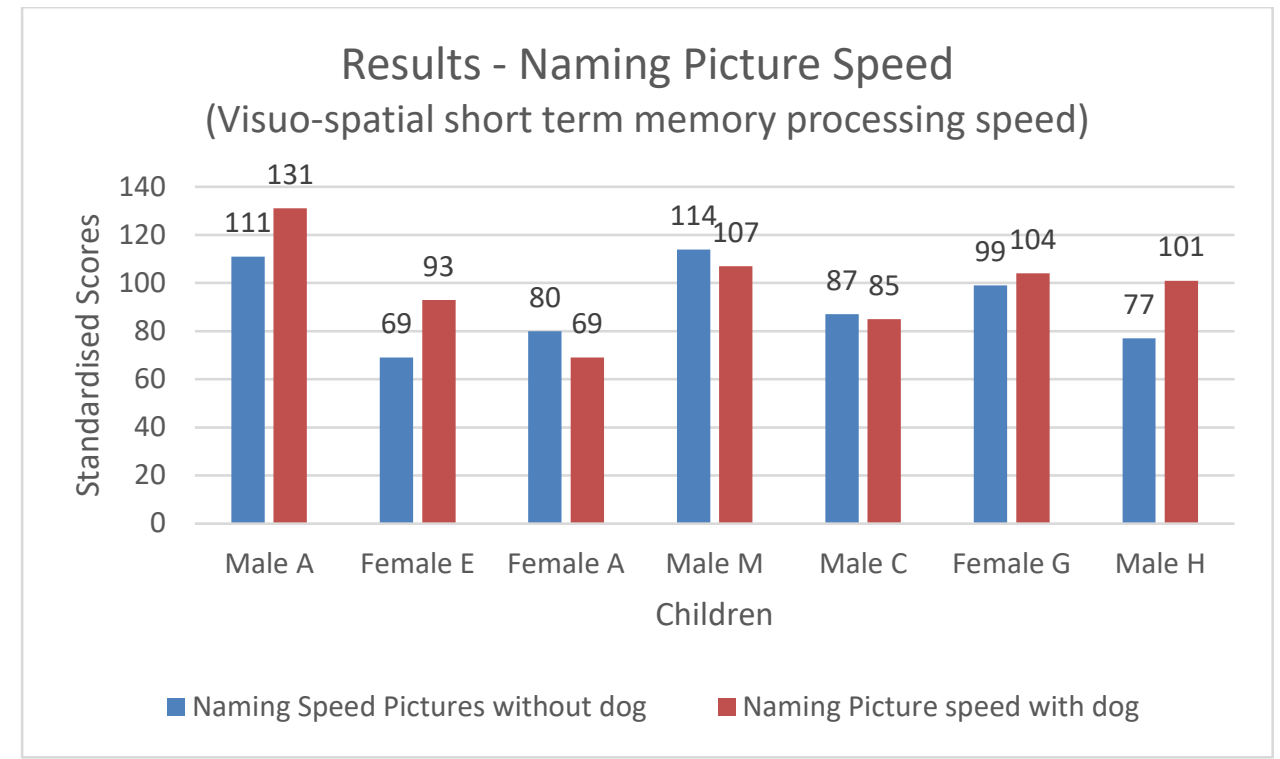

Figure 14: Results of the naming picture speed test

The average group standardised score without dog was 91.0. The average group standardised score with dog was 98.6.

The presence of the dog appeared to improve the average naming picture speed by

7.5 standardised scores for the group, or $8 \%$ overall.

A similar graph was produced to portray the difference in digit naming speed (see

Figure 15.) 


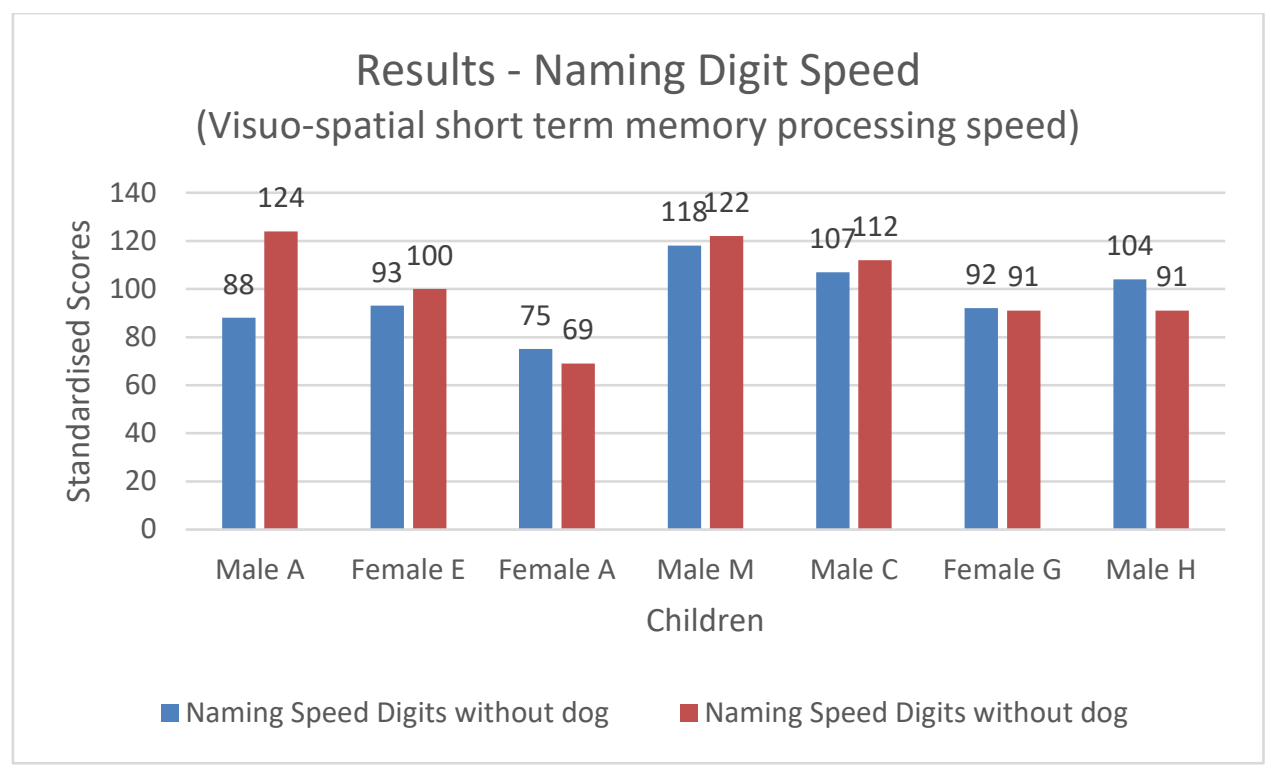

Figure 15: Results of the naming digit speed test

The average group standardised score without dog was 96.7. The average group standardised score with dog was 99.7.

Overall, the presence of the dog appeared to improve the average Naming digit speed by 3.0 standardised score points for the group, or by $3 \%$.

The graph below shows the combined differences of both the pictures and digits speed tests for each child (see Figure 16.)

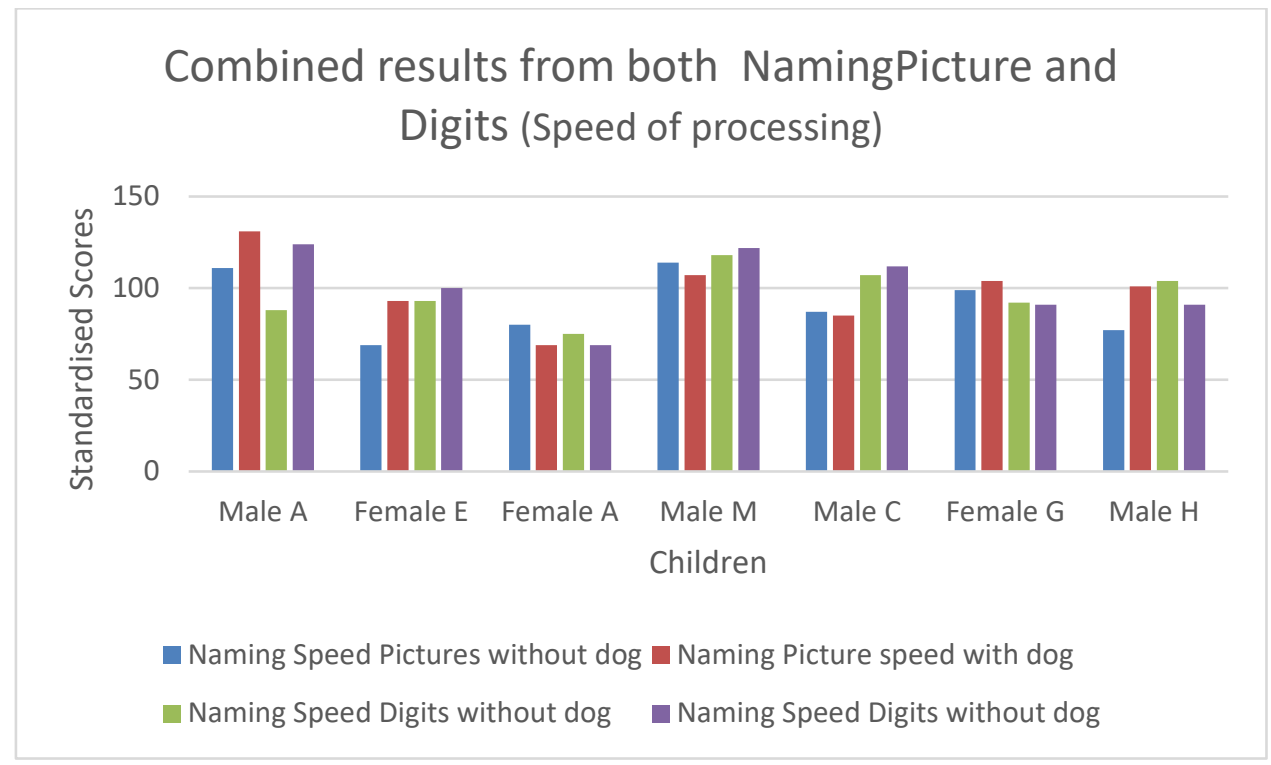


When examining the naming speed pictures and digits tests three of the children reduced their scores with the dog present. This measurement did seem to be reliant on the individual's processing skills, as for four children, the presence of the dog increased their "picture" processing speeds, using visuo-spatial short-term memory, yet this was not the case for the digits. For digits test three different children (compared to naming speed tests) showed decreased scores, with only one child showing a decrease on both scores with the dog present.

\subsubsection{Section Discussion}

The findings from the digits and pictures at speed tests may indicate that from a very early age (pre-toddler), children are 'hard-wired' to look for visual images, whereas digits are limited to just 10, learned symbols Frith's, 'Model of Reading Acquisition' theory, suggests that between the ages of 6-8 years old, children pass from the logographic, through the alphabetic, to the orthographic stages to the individual letter, phoneme chunk and written image for reading and spelling, so possibly the children are reflecting that stage in their development (Uta Frith 1985). For these children, the 'pictures' were quicker to name as symbols.

Another consideration is that the demands on short-term memory of the recall of the speech and language items, together with the speech muscle memory itself, can also affect the automaticity and fluency with some children - especially those with developmental or speech disorders, which can affect both pictorial and digit fluidity of recall. These demands can lead to 'cognitive overload' where too much information being processed at once has led to verbal breakdown. Three of the 
children indicated these tendencies with scores lower than one standard deviation from the norm (below Standard Scores 85-115) in their baseline, (no dog scores) for the processing speed with pictures. Two improved their scores into the average range with the dog's presence. The one child with low scores in both pictures and digits (under SS 85) unfortunately slipped further with her standardised scores in the presence of the dog. This could hint at either a negative reaction to the dog's presence, capacity limitations or indicate an overall developmental condition involving processing at speed, such as dyslexia.

\subsubsection{Group Auditory Short-Term Memory Results}

The auditory short-term memory tests used sequences of both letters and digits forwards spans. The children had to listen to both the given letters or digits before repeating them back in the same order as they were given. The results from the digits forwards spans are shown in Figure 17 below.

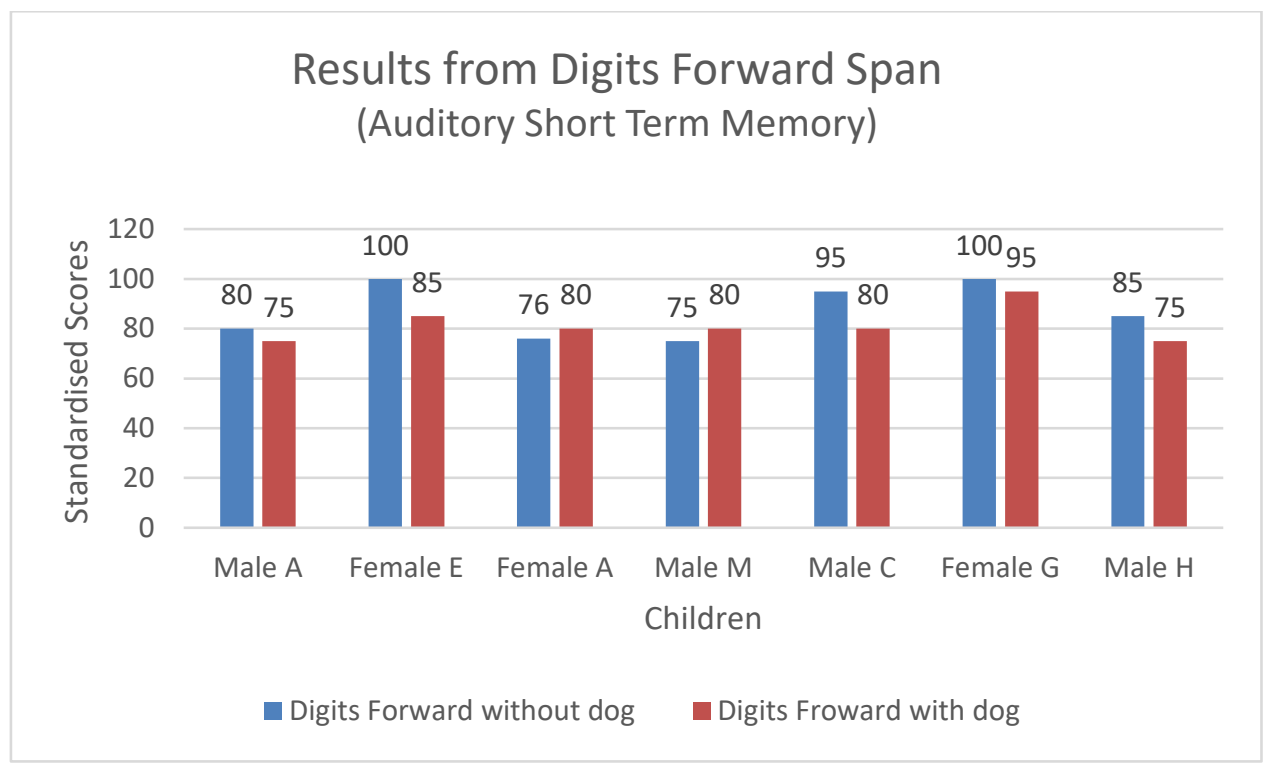

Figure 17: Results from the digits forward span tests

The average group standardised score without dog was 87.3. The average group standardised score with dog was 81.4. 
The presence of the dog decreased the performance of the digits forward span by an average of 5.9 standardised score points for the group or by $7 \%$. This would show a significant difference between digits and letters for some individuals.

The presence of the dog only increased scores for two children, with some significant decreases for the other five - up to 15 standardised points with two children. This could indicate that either there were some auditory issues for the retention of numbers or that the dog could have been a distraction in this task.

The results from the letters forwards spans are seen in Figure 18 below.

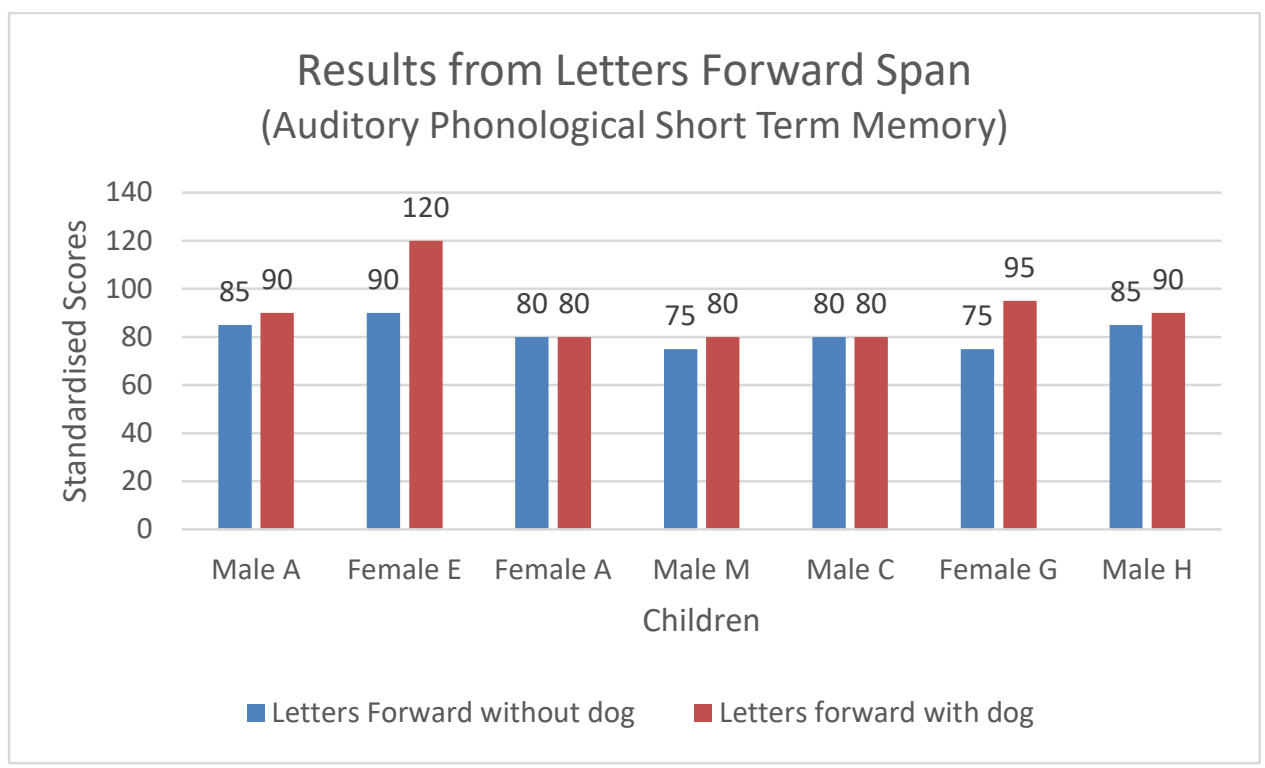

Figure 18: Results from the letters forward span test

The average standardised score without dog was 81.4. The average standardised score with dog was 90.0 .

Overall, the presence of the dog increased the performance of the Letters Forward span on average by 8.6 standardised scores for the group or by $9 \%$. Five of the children improved their scores when the dog was present, with only two remaining the same. For two children the positive differences were significant, up to 30 standardised points for one and 20 for another. Only one child showed a distinct 
profile of dropping 15 points for the digits while gaining 30 points for the letters.

This could suggest that for her, due to the huge differences, that this was task related, rather than the presence of the dog.

Again, these two scores, letters and digits forward showed pronounced individual differences in the children's results. The combined graph for both letters and digits forwards is seen in Figure 19.

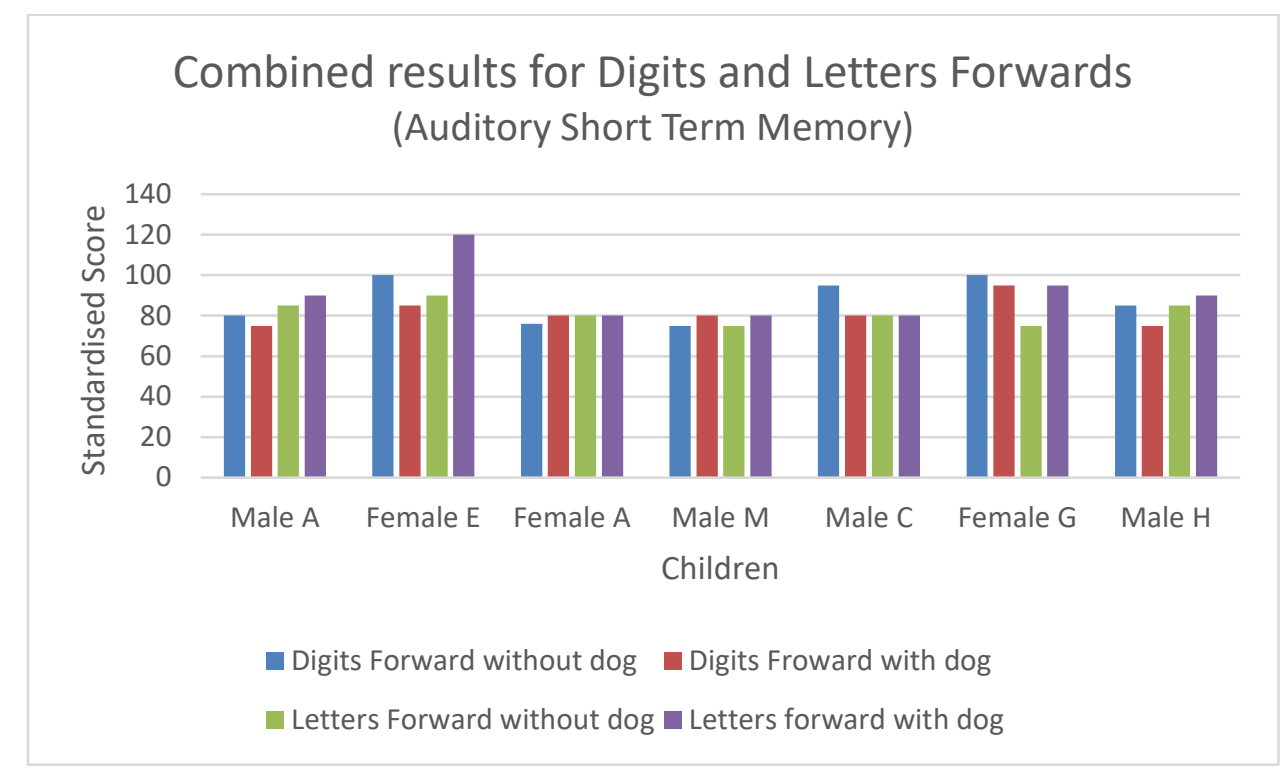

Figure 19: Combined results for digits and letters forwards tests

Looking at the Digits and letters forward tests (traditionally auditory and phonological short-term memory) only one child performed at a higher level with the dog present for both letters and digits in their standardised scores. This indicates that despite the differences in scores for some, the overall group results for the presence of the dog in this small sample does not affect auditory short-term memory.

\subsubsection{Working Memory Spans Forwards}

As with the length of the backwards spans, the forward spans were compared for the group. The forward span relates to auditory short-term memory used when carrying 
out instructions in the classroom. A poor auditory memory span would suggest that attention and focus would be affected negatively during learning tasks. The digits and letters forward spans are recorded in Table 12 below.

Table 12: Digits and letters forward spans

\begin{tabular}{|c|c|c|c|c|c|c|}
\hline Child & Test & Raw Score & $\begin{array}{l}\text { Best } \\
\text { Span }\end{array}$ & Test & $\begin{array}{l}\text { Raw } \\
\text { Score }\end{array}$ & $\begin{array}{l}\text { Best } \\
\text { Span }\end{array}$ \\
\hline \multirow[t]{2}{*}{ Male A } & $\begin{array}{l}\text { Digits forward } \\
\text { no dog }\end{array}$ & 15 & 4 & $\begin{array}{l}\text { Letters Forward - } \\
\text { no dog }\end{array}$ & 14 & 4 \\
\hline & $\begin{array}{l}\text { Digits forward } \\
\text { with dog }\end{array}$ & 13 & 4 & $\begin{array}{l}\text { Letters forwards } \\
\text { with dog }\end{array}$ & 15 & 4 \\
\hline \multirow[t]{2}{*}{ Female E } & $\begin{array}{l}\text { Digits forward } \\
\text { no dog }\end{array}$ & 30 & 4 & $\begin{array}{l}\text { Letters Forward - } \\
\text { no dog }\end{array}$ & 17 & 3 \\
\hline & $\begin{array}{l}\text { Digits forwards } \\
\text { with dog }\end{array}$ & 21 & 4 & $\begin{array}{l}\text { Letters forwards } \\
\text { with dog }\end{array}$ & 31 & 5 \\
\hline \multirow[t]{2}{*}{$\begin{array}{l}\text { Female } \\
\text { A }\end{array}$} & $\begin{array}{l}\text { Digits forward } \\
\text { no dog }\end{array}$ & 14 & 3 & $\begin{array}{l}\text { Letters Forward - } \\
\text { no dog }\end{array}$ & 9 & 3 \\
\hline & $\begin{array}{l}\text { Digits forward } \\
\text { with dog }\end{array}$ & 17 & 4 & $\begin{array}{l}\text { Letters forwards } \\
\text { with dog }\end{array}$ & 12 & 3 \\
\hline \multirow[t]{2}{*}{ Male M } & $\begin{array}{l}\text { Digits forward } \\
\text { no dog }\end{array}$ & 12 & 3 & $\begin{array}{l}\text { Letters Forward - } \\
\text { no dog }\end{array}$ & 7 & 3 \\
\hline & $\begin{array}{l}\text { Digits forwards } \\
\text { with dog }\end{array}$ & 15 & 3 & $\begin{array}{l}\text { Letters forwards } \\
\text { with dog }\end{array}$ & 11 & 3 \\
\hline \multirow[t]{2}{*}{ Male C } & $\begin{array}{l}\text { Digits forward } \\
\text { no dog }\end{array}$ & 27 & 5 & $\begin{array}{l}\text { Letters Forward - } \\
\text { no dog }\end{array}$ & 12 & 3 \\
\hline & $\begin{array}{l}\text { Digits forwards } \\
\text { with dog } *\end{array}$ & 16 & 4 & $\begin{array}{l}\text { Letters forwards } \\
\text { with dog* }\end{array}$ & 11 & 3 \\
\hline \multirow[t]{2}{*}{$\begin{array}{l}\text { Female } \\
\text { G }\end{array}$} & $\begin{array}{l}\text { Digits forward } \\
\text { no dog }\end{array}$ & 27 & 5 & $\begin{array}{l}\text { Letters Forward - } \\
\text { no dog }\end{array}$ & 21 & 4 \\
\hline & $\begin{array}{l}\text { Digits forwards } \\
\text { with dog }\end{array}$ & 30 & 5 & $\begin{array}{l}\text { Letters forwards } \\
\text { with dog }\end{array}$ & 14 & 4 \\
\hline \multirow[t]{2}{*}{ Male $\mathrm{H}$} & $\begin{array}{l}\text { Digits forward } \\
\text { no dog }\end{array}$ & 22 & 4 & $\begin{array}{l}\text { Letters Forward - } \\
\text { no dog }\end{array}$ & 15 & 4 \\
\hline & $\begin{array}{l}\text { Digits forwards } \\
\text { with dog }\end{array}$ & 16 & 3 & $\begin{array}{l}\text { Letters forwards } \\
\text { with dog }\end{array}$ & 20 & 4 \\
\hline
\end{tabular}

*Child said they felt tired, but wanted to continue 
The modal length of the span decreased from 4 items for the forward digits to 3 items for the letters without the dog present, whereas when the dog was present, the modal span for digits remained the same at 4 items, while the letters lowered to evenly spread between 3 and 4 items. These scores would suggest that with or without the dog present, auditory short-term memory span is further affected when letters are used, in comparison to digits.

\subsubsection{Overall Group Working Memory Skills}

A summary of the effect of the dog's presence for Working Memory Skills using the above tests is in Table 13, below.

Table 13: Combined effects of tests on group skills

\begin{tabular}{|l|l|l|l|l|l|l|}
\hline & \multicolumn{2}{|l|}{$\begin{array}{l}\text { Executive } \\
\text { (Traditional } \\
\text { Memory) }\end{array}$} & \multicolumn{1}{|l|}{$\begin{array}{r}\text { Skills } \\
\text { Working }\end{array}$} & \multicolumn{2}{l|}{$\begin{array}{l}\text { Rapid Automatic Naming } \\
\text { (Processing Skills) }\end{array}$} & $\begin{array}{l}\text { Short Term Memory } \\
\text { (Auditory \& Phonological } \\
\text { Skills) }\end{array}$ \\
\hline Test & $\begin{array}{l}\text { Digits } \\
\text { Backwards }\end{array}$ & $\begin{array}{l}\text { Letters } \\
\text { Backwards }\end{array}$ & $\begin{array}{l}\text { Picture } \\
\text { Speed }\end{array}$ & $\begin{array}{l}\text { Digits } \\
\text { Speed }\end{array}$ & $\begin{array}{l}\text { Digits } \\
\text { Forward }\end{array}$ & $\begin{array}{l}\text { Letters } \\
\text { Forward }\end{array}$ \\
\hline With Dog & $+2 \%$ & $+4 \%$ & $+8 \%$ & $+3 \%$ & $-7 \%$ & $+9 \%$ \\
\hline & Overall 6\% & \multicolumn{2}{|l|}{ Overall 11\% } & \multicolumn{2}{|l|}{ Overall 2\% } & \\
\hline
\end{tabular}

In this sample of seven children, the dog's presence has helped with executive and processing skills, whereas the dog has had less effect on auditory and phonological performance, particularly where digits are concerned. The implications of this pilot investigation are that the executive functioning skills, including span are to some extent, affected by the dog - but it should be borne in mind these findings are based on tests designed for assessing cognitive functions, rather than performance within actual classroom learning situations. 
It is the speed of processing which shows the greatest effect of the presence of the dog. This finding relates back to work by Poon (2018) where language itself did not seem to be affected, although executive control and processing was used. This effect was also seen in the accuracy and processing scores in the earlier group results, as despite these attentional skills and areas being used, the actual word per minute rates, fluency and accuracy was not affected by the presence of the dog but, apparently, by the activity of reading itself.

The Letters forward short-term memory result may also reflect classroom practice in spelling and reading skills and the difficulties in phonological awareness and retention of information for some of these individuals. This would explain why the SENCO has considered these children for this project. There is a difference between the language-based skills than the digits. Both backwards and forwards group scores with the dog present have shown more positive results than the digits when compared with each other.

The executive attention skills results do echo the results of the work of Hediger using PIR-HEG technology whose research suggested that the presence of the dog heightened executive skills, visual search and inhibition while the dog was interacting with the students (Hediger et al., 2017, Hediger and Turner, 2014) although in this small sample it did not enhance the performance.

\subsection{Summary}

This investigation, using commercially available tests, has shown the potential for further research into the effect of the presence of a dog on working memory skills. Executive functioning, phonological and auditory short-term memory, visuo-spatial 
and processing speed can be affected, at least in some children. These tests are available and can be used by teachers to provide a 'battery' of areas for investigation.

The use of selected commercial tests can be applied to examine speed of processing, visual and verbal abilities and to compare results with, or without a dog present. The data indicate that the classroom attainment for phonological and language awareness is a function of the individual, but that the efficiency and fluidity of processing skills, used in the thinking behind these skills, may be affected and improved by the presence of the dog.

If this study was to be repeated with a larger sample, showing similar results, this would be further evidence to suggest that a dog has a positive effect on executive functioning and processing and increase the 'field' of knowledge further for research. This sample group is, however, very small and therefore more research is needed to allow for replication across a variety of settings and school populations.

The use of visual spatial memory through the backwards span tests would help maths subjects and layout with maths as already noted. The data strengthen the case for a dog being of use in other subject areas like mathematics. Unfortunately, due to time constraints, which is the case in many schools, we were unable to do any of the mathematical tasks in the original selection of tests. The fact that the investigation took place in the Autumn term created conflicts with the time used for Christmas plays and rehearsals which is the same in any school in the United Kingdom. Any future work with the dog looking at working memory would require more than one terms worth of evidence and should certainly be on a longer scale. 
If these data are replicated this would suggest that individuals with Special Educational Needs (SEN) who have working memory difficulties could potentially have a chance to catch up if they were in the presence of a dog while working. This suggestion needs to be treated with caution, however, as the presence of a dog may not work with every child or for long periods of time. The effects of the presence of a dog have not been investigated in a longitudinal study, nor has this study incorporated those without perceived SEN difficulties. It could be that, under different circumstances (such over longer time periods) the presence of a dog can affect all children's memory skills, not just a few.

In this study, individual children also had different reactions to the dog depending on the tasks, suggesting that there is no area showing consistent results for all children. Therefore, it is possible that not every child would benefit from this approach. As has been shown, even with a very small sample, the dog does not always have a positive effect for every 'skill' with every child. There are strong individual differences in the effect of having the dog present. For example, one child (Male $\mathrm{H}$ ) showed that when concentrating on a test using digits that, for him, there was clearly a reaction to the test rather than to the dog. It is not known whether this translates to anxiety with numbers, or perhaps for that time, he was distracted by the dog. Therefore, blanket policies of having a dog in the classroom are not necessarily uniformly beneficial, and it may be worth working on a one to one trial basis only.

Executive functioning helps with emotional regulation so therefore having a dog available to be used within a classroom, although not necessarily on a full-time basis, 
will help some of those children with social emotional and mental health difficulties. This socio-emotional approach to using the dog has been found beneficial elsewhere (Jalongo et al., 2018, Beetz, 2013, Jenkins et al., 2014).

There are, potentially, other benefits to having a dog present when assessing aspects of children's' performance. The difference between the reaction to the sessions' tasks with, or without a dog present may reveal, to an experienced observer whether a child has specific difficulties independently of any issues (such as anxiety) that may be ameliorated by the presence of a dog. Such an approach would require a significant amount of training for qualified teachers. At present, the available research, including that described here does not suggest that dogs can be used to facilitate the diagnosis of learning difficulties. 


\section{Chapter 6 (Research Phase 2)}

\section{School-Based Reading Investigation: 'Paws to Read' Project}

This study took place in a working primary school environment to investigate how 'Read to Dogs' or 'Paws to Read,' takes place on a practical level and to consider the benefits and disadvantages of having a dog present while reading. The titles 'Read to Dogs' (Pets-As-Therapy, 2016) or 'Paws to Read' (Therapy Dogs Nationwide, 2016) depends on how each charity labels the same scheme.

The aims of the 'Paws to Read' research project were as follows:

1. A whole-group measurement comparison for pre-dog and post-dog reading attainment including a small control group.

2. Using a case study focus group, of seven children, record any differences in reading accuracy, fluency and speed both with and without a dog present, for children as a group and as individuals.

3. Using the case study focus group to compare reading speed and accuracy in both reading texts and poetry to investigate if what is read has a difference, rather than, or as well as, the presence of the dog.

4. Use physiological measures such as electrodermal activity (EDA) to check for over-or-under arousal with and without the dog present for both the focus group as a whole and as individuals in order to examine whether this has any influence on any of the above skills. 


\subsection{Method}

\subsubsection{Participants}

Initially during the Summer Term 60 children from two parallel form entry classes were suggested for the project by the school. This selection included randomly selected children from the classes, with or without needs, but by the Autumn Term this was then reduced to 14 children with considered 'Special Educational Needs' by the school's Special Education Needs Co-ordinator (SENCO.) After parental consent for individual working memory investigation, videoing and recording was gained, this group was then further reduced to just eight students; although permissions for the baseline and post reading assessments for the whole group of 14 were given and could be used in comparison. Unfortunately, one of the eight students with full permission then went into hospital during the project and his partial results had to be removed from the data.

The children were from two parallel classes within Year 3 (aged between 7 and 8 years old - average age 90 months, 7:06) and had been selected as having Special Educational Needs or Disability (SEND) by the SENCO from their previous, linked infant school. There were nine males and four females. The nature of their needs was not entirely obvious in every case, but they were generally under the four areas of need as suggested by the 2014 Special Needs and Disability code of Practice (DfE/DoH, 2015) of: communication and interaction, cognition and learning, social emotional and mental health and sensory and/or physical needs. Of those 13 children, the seven who were to be assessed in detail comprised four males and three females. Although the question was asked but not answered, it was assumed that 
none of the children had a current Educational statement or Educational and Health Care Plan (EHCP.) The final participant numbers are given in table 14, below.

Table 14: Final Participant Numbers for School-based Project

\begin{tabular}{|l|l|l|}
\hline Group & Number & Male / Female \\
\hline $\begin{array}{l}\text { Read to dog and } \\
\text { investigation }\end{array}$ & 7 & $4 \mathrm{~m} \mathrm{3f}$ \\
\hline Read to Dog only & 3 & $2 \mathrm{~m} \mathrm{1f}$ \\
\hline $\begin{array}{l}\text { Did not read to Dog or was } \\
\text { investigated (Control) }\end{array}$ & 3 & $3 \mathrm{~m}$ \\
\hline Total & 13 & $9 \mathrm{~m} \mathrm{4f}$ \\
\hline
\end{tabular}

\subsubsection{Materials, procedure and measures}

Two initial visits had been made by the researcher to the school, to meet and observe the dog with the Paws to Read scheme during the previous summer term to organise and prepare for the children reading in the Autumn term. This allowed for planning of the project with the handler, and for determining the scope of the children reading and their timings. It also allowed for trialling equipment such as the 'dogcam.'

\subsubsection{Electrodermal Activity (EDA-Pip)}

There had also been a discussion held with the SENCO for permission to use and record a wrist blood pressure monitors with the children. This was, however later rejected at the beginning of the project as the SENCO felt that it could highlight blood pressure (BP) problems with the children, as it had with herself. Instead, a 'Pip' wireless electro-dermal activity monitor was found, which, with hindsight, proved to be far more interesting than the original BP monitor as it could also record in 'real time' using a laptop, the reactions of the child throughout the session. 


\subsubsection{Environment}

The small music room was used for the sessions with the dog and all but three of those without the dog. The three without the dog took place in the 'Interventions' room. Room changes were kept to a minimum. Some children were already familiar with the music room as this was where they had their individual music lessons. It was also a room familiar to the dog and handler as this is where the sessions had taken place in the past. The sessions ran within the school timetable.

\subsubsection{Dog}

The registered, experienced therapy dog, Matt, a 10-year-old border terrier, attended with his handler for 10 sessions over the Autumn term. This helped to ensure that all those children both being investigated and as 'read to dog control' were able to read to Matt at least twice and those in the case study group had enough time to complete the working memory tasks in his presence. Each session with Matt lasted about an hour, with up to five visits from individual children and the timing allowed for short rest breaks in between. This was also the usual routine for Matt and his handler, and the handler and dog also very helpfully did an extra session on a different day to help to finish all the recording before the end of term.

\subsubsection{Time}

The researcher made an extra five visits, totalling $7 \frac{1}{2}$ hours at the school, working with the children on the working memory activities, rhyme and reading without the dog present. 


\subsubsection{Charity Volunteer Protocol}

For each session, besides the dog handler, a volunteer also needed to be present as per the rules of health and safety from the charity. Often this was the researcher herself. On three occasions, the school did provide its own volunteer who oversaw to the running of the scheme and the collection and returning of the children to their classes. This did mean that at times there were three adults present in the tiny music room, which could have presented as quite daunting to the children and the dog.

\subsubsection{Recording Equipment}

During the sessions with the dog present, the children were digitally recorded, videoed and used the Pip while they read. Matt too, also wore a Go Pro 'dogcam' (a small camera attached on a comfy harness) to gather his 'eye-view' of the situation. The hand-held video recorder (Panasonic HDC-SD600 35mm) was strapped and 'hidden' within the music trolley so that it was not too obtrusive but was aimed at the scene allowing for the dog and child's movements to be captured. The Olympus digital recorder was placed horizontally upon an empty music stand, close to the child's head.

\subsubsection{Procedures}

The children were collected from their class individually and brought to the room with their reading books of their own choice, following the school's 'Accelerated Reading' scheme. This scheme was a finely graded scheme where the children could have several books at a similar level to practice their reading skills. Often the children chose a book that they had already 'pre-read' making it easier to re-read, or it was a book that they thought that the dog would like. On arrival, following the charity's 
paws and read protocol, they would greet the dog and handler, chatting for a minute or two. They then read to the dog for approximately five minutes each and afterwards could pet and fuss the dog and give him a biscuit for his 'good listening' skills. They were then encouraged to 'wash their hands' using an alcohol-based hand cleanser and then received a stamp in their reading books to say that they had read to Matt. After this they went back to class, often to alert the next child to come and visit. All the sessions of reading to the dog took place during the afternoons.

\subsubsection{Questionnaire procedure for the case study children}

Initially, while the dog was not present, the seven children were individually asked to complete a small, simple questionnaire using emojis to allow for their age and unfamiliarity with the research process. It quickly became clear that the children did not have the corresponding writing skills for the answers that they had expressed, so the researcher acted as a scribe. This allowed for a far more 'in depth' conversation, even if the children were unfamiliar with expressing their own opinions in this way. It also allowed the children to get to know the researcher better and to establish the relationship with each other's roles. The researcher is aware that while she made every effort to remain neutral in recording the answers, there is always a possibility of unconscious bias.

\subsubsection{Working memory tests procedure without dog present}

Each case study child then completed the selection of working memory tests without the dog present. This activity took about 30 mins per child to go through, but with one child who scored consistently high scores, the time ran out due to the school timetable. Due to these time constraints, when the tests were repeated with the dog 
present, fewer tests were used. The tests which were re-selected were those which had shown lower average scores for the children across the group. The whole test battery did give clear indications as to the nature of some of the individual needs of the children.

\subsubsection{Reading without dog present session}

A second opportunity to read without the dog present was arranged for each of the children. This session was to gather information to compare with the dog present. Each case study child was asked to read a short, rhythmical poem, partly to assess their stress levels when reading something from 'cold,' or unrehearsed - and partly to see whether the children could identify rhyme and rhythm. After the children had read their poem, they were then asked to read their reading books as normal. For five children, this reading was also recorded on video. Due to a room change, for the other two, however, the video recording was not available as there was not an easy set-up. The Pip and digital recorder were still able to be used with all seven.

\subsubsection{Reading Attainment}

The reading tests were administered by the SENCO using the Salford Sentence Reading Test. (McCarty, 2012) in week 1 and week 12 of the term, using the latest Salford Sentence Reading Test version 4, which was routinely used throughout the school. Only the reading progress was recorded, despite there being an option for comprehension scores within the test. 


\subsubsection{Qualitative Results}

\subsubsection{Children's questionnaires - research group}

The questionnaires were the first meeting of the researcher with the children on their own. Through completing them, it became apparent that for some, there was still a reliance on an adult to write the responses, even though they had been designed to require minimal written information. Time was of the essence, as the children were not allowed out of the classroom for long periods. Each questionnaire took approximately 20 minutes to complete, including listening to the children's responses and the time to arrive and return to the classroom. The children appreciated the emoji format for indicating their likes and self-ability levels for reading and maths.

In this sample, it appeared that all seven of the children had pets at home: five dogs, two cats and two rabbits. All the cats and dogs were in the singular. Three children, two of whom were cat owners, said that they read to their pet regularly or 'sometimes.' All were unanimous that they would like to read to Matt.

Out of the seven children, four were confident and happy with their reading in general. Two marked themselves as 'OK' and one said that he didn't like it. When it came to maths, three children thought they were confident and happy, three thought they were 'OK,' and one didn't like maths. No children thought they were good at both reading and maths, and only one child said he didn't like either. This shows that at age seven, the children were very aware of their perceived strengths and weaknesses in subject areas. 


\subsubsection{Questionnaire Group Answers - Children}

The answers the children gave to their questionnaire are grouped in table 15 below:

Table 15: Children's answers to their questionnaire

\begin{tabular}{|c|c|}
\hline Question & Answers \\
\hline $\begin{array}{l}\text { How does reading to Matt make you } \\
\text { feel? }\end{array}$ & $\begin{array}{l}\text { Happy } \\
\text { Not sure } \\
\text { Excited and happy } \\
\text { Really calm and gentle } \\
\text { Pretty good because he is kind } \\
\text { A bit nervous } \\
\text { Happy - he cheers me up } \\
\text { Exciting because I'm making him relax }\end{array}$ \\
\hline How do you think it makes Matt feel? & $\begin{array}{l}\text { Good because he lies next to me } \\
\text { Happy } \\
\text { Excited } \\
\text { Happy and settled - he snuggles up } \\
\text { He likes it because I can read really well } \\
\text { I think he likes it } \\
\text { Happy he likes jumping on me and stuff } \\
\text { He likes to relax }\end{array}$ \\
\hline $\begin{array}{l}\text { How can you help Matt with his } \\
\text { reading? }\end{array}$ & $\begin{array}{l}\text { He enjoys it } \\
\text { Yes, but I don't know how } \\
\text { Read to him lots of times to make him understand } \\
\text { books } \\
\text { Yes (no further explanation) } \\
\text { Yes, I can say the word and then he can read the } \\
\text { word } \\
\text { Help spell out the words for him } \\
\text { Yes, if he gets stuck on a word, I can help him }\end{array}$ \\
\hline
\end{tabular}




\begin{tabular}{|l|l|}
\hline & I don't really know dog language! \\
\hline $\begin{array}{l}\text { How can Matt help you with your } \\
\text { reading? }\end{array}$ & $\begin{array}{l}\text { I don't know - I read it myself } \\
\text { He can listen to me } \\
\text { By listening carefully } \\
\text { Don't know } \\
\text { By giving me perseverance } \\
\text { He can put his paw out to point to the words } \\
\text { I don't know } \\
\text { Helps me to relax and read all the book }\end{array}$ \\
\hline
\end{tabular}

The children at 7-8 years old anthropomorphised Matt. All of them could suggest ways of helping Matt, based on their own experiences of being helped by the adults around them. Matt, according to the children, was apparently able to say words and to have difficulties reading, allowing the children to phonetically help him with his sounds. All the children actively wanted to help Matt and could imagine themselves doing so.

There was a difference in the perceived help from Matt. All children acknowledged that Matt could either listen and help by helping with 'perseverance' (school assembly word that week) or helping them to relax. Three children answered that they didn't know how he could help, as they had consciously realised that he was a dog and that his verbal communication would be limited - only one suggested that he could use his paw to point to the words. Further questioning suggested that at that point, she thought Matt could actually read.

This 'belief' could suggest that the children can easily imagine and portray their thoughts and experiences towards another person, in this case a dog, but are still 
developing their awareness of viewing from another's point of view or perspective. What was clear was the powerful motivation to want to read to the dog and the belief that it was helping both Matt and them in some way.

\subsubsection{Questionnaire Group Answers - Staff}

Out of the eight questionnaires given to the staff (including both teachers, the Head and SENCO), four were returned. These were from three teaching assistants and one teacher. (Return rate 50\%)

The original forms had 12 questions. Out of the four returned, two staff members had pets of their own. The three Teaching Assistants (TAs) all thought that dogs had a role in education, but the teacher was 'not sure.' All the members of staff had been involved in the 'Paws and Read' scheme for over 3 years. It also became clear that the SENCO had only asked for the SEN children to be heard or read to that term. A comment from the teacher read, "This term we selected for the SEN project. Sometimes we choose pupils with behaviour issues but normally we allow all children in Year 3 access to Matt because they want to spend time with him."

Results from the open question as to "What did they believe the Paws to Read scheme was about?" brought about the following answers:

"Giving children who have no home support the opportunity to read in a nonthreatening environment: encouraging reluctant readers and building confidence"

"Making children feel at ease and give them confidence in reading"

"Enabling children to be able to relax and have more confidence in reading and so enjoy reading more" 
"Giving children the opportunity to meet and interact with Matt. Read and share the enjoyment of a book with a non-judgemental listener."

The overall result from "Who selects the readers for reading to the dog?" came back with a clear indication that the TAs did not select the readers, but the teacher did comment that the SEN readers had been specifically chosen for the project. One TA did mention that they thought it was always the SEN and pupil premium children who were chosen.

There was a unanimous, "Yes," to the question whether they thought that it made a difference for the individual child.

When asked if the staff had "noticed any changes for a child who had used the scheme" the multiple answers were as follows (see Table 16).

Table 16: Adult responses to the question about subsequent changes in behaviour after reading to the dog

\begin{tabular}{|l|l|l|}
\hline $\begin{array}{l}\text { Have you noticed any changes in the following for any child who } \\
\text { has used the scheme? }\end{array}$ & Tally & Count \\
\hline Improved attitude to reading when back in class & 1 & 1 \\
\hline Increase in motivation to read when back in class & 11 & 2 \\
\hline Increase in fluency and accuracy when back in class & 1 & 1 \\
\hline Overall confidence and self-esteem when back in class & 111 & 3 \\
\hline The child has regressed in their reading & & 0 \\
\hline Other (please specify) & & \\
\hline
\end{tabular}

There was one TA who chose not to answer this question as she didn't work in that class after they had read to the dog on a weekly basis. Clearly confidence, motivation 
and self-esteem were the indicators that the staff viewed. Regression was not considered a change by any.

All the staff members felt, that in term of progress, those who had read to the dog made about, "the same progress as those who had not read to the dog."

The evaluation of the scheme was done termly as indicated by the teacher - again the TAs indicated that they were not involved. What was not clear from the teacher's answers, was whether the teachers monitored the progress of 'Paws to Read' within their classes or whether someone else in the school had 'overall responsibility' for the scheme or 'intervention' and monitored it as a group.

A question about 'anecdotal evidence' gave more evidence about the staff perception of the children's attitudes to the dog:

"Just that children really enjoy reading to the dog and talk about what they have read and how the dog really was listening because his ears would perk up!"

"When I was classroom based, I did see children grow in confidence when they had been reading to Matt."

"One year, Y5 SEN had a project to write a school newspaper. They interviewed the Handler and Matt. Matt likes chasing toads! The children like to choose books about cats or bones to keep Matt interested!"

All the staff wanted to continue the scheme. 


\subsubsection{Observations of the dog}

When examining the videos of the dog it became clear that while all the children were reading, the dog remained still, usually in a down position, with no interaction with the child. The dog was listening to the tone of the voice as he could pre-empt the, "well done" to the child from the handler, indicating for the child to stop. Matt enjoyed interacting with the children, accepting ear tickles and belly rubs, but was also carefully watching the handler's hands for treats. If he did not think a treat was forthcoming, he would whine. The 'dog cam,' at his head level, clearly backed this up. On reflection, however, the 'dog cam' was preventing Matt from rolling onto his back, either to get comfy, or to have a full belly scratch. This was unintentional by the researcher. Therefore, in any future planning of projects involving dogs, the 'dog cam' will not be used. (See Appendix T for the Dog Cam observation samples.)

\subsubsection{Qualitative Summary}

It was clear from the evidence that the children at seven years old had anthropomorphised the dog and similarly to the work by Gee and her colleagues, also wanted to 'help' or assist the dog with his reading. There was some difference of opinion, however, about how this would help would occur, as four of the children had realised that their efforts would be limited. This finding may reflect their developing cognitive and emotional maturity for reality over fantasy. All the children were motivated to read to Matt, except for one boy for one session, where he asked to play football instead.

The teacher / teaching assistants also believed that the dog's presence was good for self confidence and self-esteem, particularly with those who had SEN or behavioural 
needs, although they were not convinced that the dog assisted with attainment. They were happy to continue to allow the children to read to Matt, yet the responsibility for the overall scheme was not clear. This gave an "ad hoc" feel to the approach and despite being assessed every term, it is not clear how they measured assessment other than the reading ages. Perhaps it is the fact that all the children and teachers taking part had pets of their own and so their opinions could be biased in favour of the dog's attendance. There was one teacher who declined to fill in the questionnaire and was not happy with the project, despite having to allow his children to attend their sessions. He clearly stated that he did not believe in dogs helping and that it was a complete myth. This response indicates that, despite school leaders planning for Animal Assisted Interaction, not everyone wants to take part. Rather, it is often a case of having to engage as part of the expected teaching role.

\subsection{Quantitative Results}

\subsubsection{Investigation 1 - Overall Reading Attainment}

Ten children had at least two sessions of reading to the dog including those not involved in the case study recorded research over the 12-week (Autumn Term) period. Three children were the control read as normal, without the dog, in school.

\subsubsection{Reading Attainment (as measured by schools in 2016)}

The results show the children's chronological ages against their Salford scored 'reading ages.' This comparison gives a baseline to indicate how much progress has been made over the 12-week period. These scores have then been converted into Ratio Gain scores. Ratio Gain (RG) defined by Topping and Lindsay (1992, p 210) as cited in Brookes \& NFER, (2007 p 129), is "the gain in reading age made by a subject 
on a reading test during a chronological time span, expressed as a ratio of that time span; that is, ratio gain equals reading age gain in months divided by chronological time in months." (See figure 22 below).

(average reading age in months at post-test) - (average reading age in months at pre-test)

Time elapsed in months

Figure 20: Ratio gain formula (Source: Brooks, 2007, p.18)

A ratio Gain of 1.0 means that the child is developing at a normal pace, but they will not be catching up with their peers. Brooks (Brooks, 2016, Brooks, 2007) suggests that:

- Ratio Gains of less than 1.4 are of "doubtful educational significance"

- Between 1.4 and 2.0 of "modest impact"

- Between 2.0 and 3.0 of "useful impact"

- Between 3.0 and 4.0 of "substantial impact" and

- Above 4.0 of "remarkable impact" (Brooks, 2007 p. 289)

There are also 'Standardised Scores' which many schools are now also using as reference. These scores can be grouped, and progress tracked, through progress matrices and by children showing attainment gains putting them into the 'higher groups.' Expected progress is within the standardised score bracket of $85-115$, those between $116-130$ is 'above progress' and $130+$ is 'well above progress' (Pembroke, 2019). These scores are also comparable to those used in the Working Memory tests.

\subsubsection{Group Reading Results}

Tables 17 and 18, present the reading progress across the case study group, both as reading ages and as standardised scores. Within Table 17 are the converted ratio gains for each child. Under Table 18 is the group mean standardised score. 
Table 17: Children in case study group, within SEN group who read to the dog

\begin{tabular}{|c|c|c|c|c|c|c|}
\hline Name & $\begin{array}{l}\text { Chron } \\
\text { Age } \\
\text { Begin }\end{array}$ & $\begin{array}{l}\text { Salford } \\
\text { Reading } \\
\text { Age }\end{array}$ & $\begin{array}{l}\text { Chron } \\
\text { Age End }\end{array}$ & $\begin{array}{l}\text { Salford } \\
\text { Reading Age }\end{array}$ & $\begin{array}{l}\text { Total } \\
\text { Progress }\end{array}$ & $\begin{array}{l}\text { Ratio } \\
\text { Gain }\end{array}$ \\
\hline Male A & $7: 02$ & $7: 05$ & $7: 05$ & $8: 11$ & $+18 m$ & +6 \\
\hline $\begin{array}{l}\text { Female } \\
\text { E }\end{array}$ & $7: 07$ & $8: 11$ & $7: 09$ & $9: 02$ & $+3 m$ & +1 \\
\hline $\begin{array}{l}\text { Female } \\
\text { A }\end{array}$ & $7: 09$ & $6: 04$ & $8: 00$ & $7: 06$ & $+14 m$ & +4.6 \\
\hline Male M & $7: 02$ & $10: 10$ & 7:05 & $10: 09$ & $-1 m$ & -0.3 \\
\hline Male C & $7: 08$ & $8: 00$ & $7: 10$ & $8: 00$ & $0 \mathrm{~m}$ & 0 \\
\hline $\begin{array}{l}\text { Female } \\
\text { G }\end{array}$ & $7: 04$ & 9:00 & 7:07 & 9:03 & $+3 m$ & 1 \\
\hline \multirow[t]{2}{*}{ Male H } & $7: 10$ & 7:08 & $8: 01$ & $8: 05$ & $+9 m$ & 3 \\
\hline & & & & & Total $46 \mathrm{~m}$ & $\begin{array}{c}\text { Total } \\
15.3\end{array}$ \\
\hline
\end{tabular}

The total average progress, for the children in the focus group in reading ages is 6.6 months, - the total average ratio gain per child is 2.9

Table 18: Standardised scores for the children in the case study group and within the SEN group who read to the dog

\begin{tabular}{|l|l|l|l|}
\hline Standardised Scores & SS Beginning & SS End & Difference \\
\hline Male A & 103 & 118 & +15 \\
\hline Female E & 117 & 119 & +2 \\
\hline Female A & 87 & 94 & +7 \\
\hline Male M & $130+$ & $130+$ & 0 \\
\hline Male C & 104 & 102 & -2 \\
\hline Female G & 120 & 122 & +2 \\
\hline Male H & 101 & 104 & +3 \\
\hline & Mean SS 108.9 & Mean SS 112.7 & Difference 3.8 SS \\
\hline
\end{tabular}


The average Standardised Score points have been raised by a difference of 3.8 (103 \%)

Tables 19 and 20, present the reading progress for the children who read to the dog, but were not part of the focused case study group, both as reading ages and as standardised scores. Within Table 19 are the converted ratio gains for each child. Under Table 20 is the group mean standardised score.

Table 19: Reading scores for those who were in the SEN group but not in the focused case study group

\begin{tabular}{|l|l|l|l|l|l|l|}
\hline Name & $\begin{array}{l}\text { Chron } \\
\text { Age } \\
\text { Begin }\end{array}$ & $\begin{array}{l}\text { Salford } \\
\text { Reading } \\
\text { Age }\end{array}$ & $\begin{array}{l}\text { Chron } \\
\text { Age } \\
\text { End }\end{array}$ & $\begin{array}{l}\text { Salford } \\
\text { Reading Age }\end{array}$ & $\begin{array}{l}\text { Total } \\
\text { progress }\end{array}$ & $\begin{array}{l}\text { Ratio } \\
\text { Gain }\end{array}$ \\
\hline Henri & $7: 09$ & $7: 05$ & $8: 00$ & $8: 00$ & $+7 \mathrm{~m}$ & 2.3 \\
\hline Greg & $7: 11$ & $8: 00$ & $8: 01$ & $9: 07$ & $+19 \mathrm{~m}$ & 6.3 \\
\hline Kerry & $7: 04$ & $8: 11$ & $7: 06$ & $10: 02$ & $+15 \mathrm{~m}$ & 5 \\
\hline & & & & & Total & Total \\
& & & & & $+41 \mathrm{~m}$ & 13.6 \\
\hline
\end{tabular}

The average Reading Age Progress while reading to the dog per child is 13.6 months, average Ratio Gain per child is 4.5

Table 20: Standardised scores for those children who were in SEN group but not in the focused study group

\begin{tabular}{|l|l|l|l|}
\hline $\begin{array}{l}\text { Standardised } \\
\text { Scores }\end{array}$ & SS Beginning & SS End & Difference \\
\hline Henri & 102 & 99 & -3 \\
\hline Greg & 101 & 116 & +15 \\
\hline Kerry & 99 & 102 & +3 \\
\hline & Mean SS 100.6 & Mean SS 105.7 & Difference 1.1 SS \\
\hline
\end{tabular}

The average standardised scores for this group have been raised by 1.1 or $105 \%$ 
Tables 21 and 22, present the reading progress across the control, who did not read to the dog, but were still within the SEN group both as reading ages and as standardised scores. Within Table 21 are the converted ratio gains for each child. Under Table 22 is the group mean standardised score.

Table 21: Reading scores for those who did not read to the dog, but were part of the SEN group (Control)

\begin{tabular}{|l|l|l|l|l|l|l|}
\hline Name & $\begin{array}{l}\text { Chron } \\
\text { Age } \\
\text { Begin }\end{array}$ & $\begin{array}{l}\text { Salford } \\
\text { Reading } \\
\text { Age }\end{array}$ & $\begin{array}{l}\text { Chron } \\
\text { Age End }\end{array}$ & $\begin{array}{l}\text { Salford } \\
\text { Reading } \\
\text { Age }\end{array}$ & $\begin{array}{l}\text { Total } \\
\text { Progress }\end{array}$ & Ratio Gain \\
\hline Ben & $7: 02$ & $8: 02$ & $7: 06$ & $8: 07$ & $+5 \mathrm{~m}$ & 1.6 \\
\hline Louie & $7: 11$ & $8: 00$ & $8: 01$ & $8: 02$ & $+2 \mathrm{~m}$ & 0.6 \\
\hline Simon & $7: 05$ & $5: 05$ & $7: 08$ & $6: 02$ & $+9 \mathrm{~m}$ & 3 \\
\hline & & & & & Total & Total \\
& & & & & $+16 \mathrm{~m}$ & 5.2 \\
\hline
\end{tabular}

The average Reading Age Progress per child while project was taking part is 5.3 months and Ratio Gain per child, while not reading to the dog is 1.7

Table 22: Standardised scores for those who did not read to the dog but were part of the SEN group (Control)

\begin{tabular}{|l|l|l|l|}
\hline $\begin{array}{l}\text { Standardised } \\
\text { Scores }\end{array}$ & SS Beginning & SS End & Difference \\
\hline Ben & 112 & 113 & +1 \\
\hline Louie & 101 & 99 & -3 \\
\hline Simon & 82 & 85 & +3 \\
\hline & Mean SS 98.3 & Mean SS 99 & Difference 0.7 SS \\
\hline
\end{tabular}

The average standardised scores for this group have been raised by 0.7 or $100.7 \%$ 
Therefore, total average amount of progress in reading to the dog (whether in case study group or not) versus the control is presented in Table 23 and Table 24 below

Table 23: Total progress in reading ages for "Paws and Read"

\begin{tabular}{|l|l|l|}
\hline & $\begin{array}{l}\text { Average Reading Age } \\
\text { Progress per child }\end{array}$ & $\begin{array}{l}\text { Average Ratio Gain } \\
\text { Progress per child }\end{array}$ \\
\hline Total Read to Dog Group & 8.7 months & 2.9 \\
\hline $\begin{array}{l}\text { Non Read to Dogs } \\
\text { (3 children) }\end{array}$ & 5.3 months & 1.7 \\
\hline
\end{tabular}

Table 24: Total progress in standardised scores for "Paws and Read"

\begin{tabular}{|l|l|l|l|l|}
\hline & $\begin{array}{l}\text { Average } \\
\text { Standard Score } \\
\text { Beginning }\end{array}$ & $\begin{array}{l}\text { Average } \\
\text { Standard } \\
\text { Score End }\end{array}$ & $\begin{array}{l}\text { Difference in } \\
\text { Standard } \\
\text { Scores }\end{array}$ & $\begin{array}{l}\text { Percentage } \\
\text { Improvement }\end{array}$ \\
\hline $\begin{array}{l}\text { Total Read to } \\
\text { Dog Group } \\
\text { (10 children) }\end{array}$ & 106.4 & 110.6 & 4.2 & 103.9 \\
\hline $\begin{array}{l}\text { Non Read to } \\
\text { Dogs } \\
\text { (3 children) }\end{array}$ & 98.3 & 99 & 0.7 & 100.7 \\
\hline
\end{tabular}

The scores revealed that reading to the dog had improved the group scores in reading age, ratio gain and standardised scores in comparison to the control.

\subsubsection{Summary of Reading Assessment}

Reading to Dogs can improve reading levels, showing 'useful' impact (ratio gain between 2.0 and 3.0,) in comparison to 'modest' (ratio gain between 1.0 and 2.0) 
impact, if overall scores are only based on group reading age averages. The individual scores require a more in-depth analysis.

Despite the study being projected for 12 weeks, it took 10 weeks allowing for term to start, permissions to be finalised and Christmas Play rehearsals to be taken, as is the Autumn Term reality in UK primary schools. Each hour-long session was divided into four 15-minute sections for up to four different children, giving forty slots. Time was required to collect and return the children to their classes and certain children 'swapped' sessions depending on their attendance or absence on that day. This meant that from the original study numbers $(n=13)$ each child could be heard twice with the dog, with some three times.

The students are only actually reading to the dog for periods between 5-10 minutes, equalling 20-30 minutes maximum for the whole school term where the school was also promoting reading - with great efforts being made to contact all families to hear their children read at home. This 'useful impact' score is therefore not reflective of the entire school, the non-SEN groups, and does not record the efforts made from other outside influences such as family reading, family cultures, libraries, summer reading schemes and outside tuition etc. These additional influences would have inevitably also impacted the children's reading.

If, however, just the overall average group 'reading age' or Ratio Gain results were used, then it perhaps explains why so many schools believe in dogs helping children to read. In class, time constraints do not allow for complete, individual analysis. If the 'dog-reading' sessions, despite smaller groups have higher averages of reading scores, they are seen in a positive light. Thus, the repeated evidence of the 
advantages of reading to dogs in peer reviewed research, together with the media and social media bias can create a 'positive picture.' In this study, individual reading age gains ranged from -1 month to +19 months with the 'read to dog' groups whereas the control ranged from +2 to +9 months. The range of 'improved' reading scores with the dog present, would link to the positive research both in the USA and Australia with similar charities using a visiting therapy approach (Massengill Shaw, 2013, Pillow-Price et al., 2014, Stroud, 2012).

\subsubsection{Which group made most Ratio Gain progress?}

The children's reading results were further analysed for where individual ratio gains had been obtained. The results are shown in Table 25 below:

Table 25: Individual Ratio Gain scores per group

\begin{tabular}{|l|l|l|l|}
\hline Brooks R.G Score & $\begin{array}{l}\text { Read to Dog Focus } \\
\text { Group }\end{array}$ & Read to Dog & Control \\
\hline Less than 1.4 & 4 & & 1 \\
\hline $1.4-2.0$ & & 1 & 1 \\
\hline $2.0-3.0$ & 1 & & 1 \\
\hline $3.0-4.0$ & 2 & 2 & \\
\hline Above 4.0 & & & \\
\hline
\end{tabular}

Analysis of the 'ratio gains' reveals a different picture. Out of the thirteen children, five children did not make any significant 'ratio gain' and under Brook's definition (2016) the gains are therefore of 'doubtful educational significance.' The children that did not show gains included four in the case study group and one control. Of the four children in the case study group, all bar one, were already reading at least a year 
above their chronological ages and in one case up to three years above. This finding would raise questions about the possibility of the 'Hawthorne Effect' or could suggest that there is a plateau or 'ceiling effect' where reading to the dog is not as effective with fluent, competent readers.

Researchers have previously suggested that those children with the lowest starting points often have the most success when reading to the dog (Bassette and TaberDoughty, 2013, Hall et al., 2016a, Jalongo, 2015, Lane and Zavada, 2013). The four children making "significant or remarkable" impact i.e. ratio gain of above 4 in Table 25 were originally reading within 3-4 months of their chronological age, or in one case, reading a year below. Although these findings do lend some support to the claim that the scheme is more successful if the children are stating from a lower basepoint, the small cohort limits the conclusions that can be drawn.

To conclude, dogs may be a motivational aid, but it is doubtful that their presence is the entire cause of changes in reading achievement. Perhaps the system of using 'reading ages' for measurement is not as accurate as scientific research would require, yet this has been the culture established in many of England's schools over previous years. It would also be interesting to compare these scores for a larger school in a less affluent area, with a higher percentage of free school meals and greater pupil diversity.

\subsubsection{Combined Project Children's Results}

Not every child's accelerated reading level went up - in fact, some children's levels regressed. This is interesting as under the Accelerated Reader scheme the children had been given 'book bands' to read to, although the books themselves were 'free 
choice' within those bands. Many of these books, according to the reading records had also been pre-read at home. The scheme would assume an incremental increase in the 'levels' overtime. The lower the number awarded to the book, the 'easier' the reading level. In England there is a huge emphasis on using progressive phonics so the 'lower books' would also have less words to read and would be simpler to decode. This may be a motivational factor for the reader's choice of book. There were difficulties with individuals "forgetting" book bags, however and having to read a 'stand in' book. This is typical behaviour in a primary school classroom. The different reading levels of the books read by the focus case study group as shown in Table 26 over time, reveal the differences in reading progress.

Table 26: Table of focus study case children's reading level choices for reading to the dog over time

\begin{tabular}{|l|l|l|l|}
\hline $\begin{array}{l}\text { Child in } \\
\text { Project }\end{array}$ & $\begin{array}{l}\text { Level Reading 1 } \\
\text { Time 1: Sept 16 }\end{array}$ & Level Reading 2 & $\begin{array}{l}\text { Level Reading 3 } \\
\text { Time 3: Dec 16 }\end{array}$ \\
\hline Male A & Fizz kid Liz 1.7 & The Outing 2.0 & Look Out 1.6 \\
\hline Female E & $\begin{array}{l}\text { Camping } \\
\text { Adventure 1.4 }\end{array}$ & $\begin{array}{l}\text { Noah's Adventure 1.7 } \\
\text { Adv }\end{array}$ & Rainbow Machine 2.6 \\
\hline Female A & $\begin{array}{l}\text { The Motorway } \\
2.2\end{array}$ & $\begin{array}{l}\text { The } \\
\text { Elephant 2.5 }\end{array}$ & By the Stream 0.7 \\
\hline Male M & Pocket Money 2.7 & In the Net 2.2 & Don't look Down 2.2 \\
\hline Male C & My Weekend 1.1 & The Outing 2.0 & $\begin{array}{l}\text { The Outing 2.0 (forgot } \\
\text { own book) }\end{array}$ \\
\hline Female G & $\begin{array}{l}\text { Christmas } \\
\text { Adventure 2.1 }\end{array}$ & $\begin{array}{l}\text { Lions and Buffaloes } \\
2.5\end{array}$ & $\begin{array}{l}\text { I took the moon for a walk } \\
2.1\end{array}$ \\
\hline Male H & $\begin{array}{l}\text { Birds that can think } \\
2.1\end{array}$ & The Quest 2.7 & What was it like? 2.6 \\
\hline
\end{tabular}




\subsection{Comparisons to other School Dog Reading Projects}

To provide a comparison to the pilot project, a request was made to the visiting dog charity for any data from similar reading projects. One volunteer replied, with permission from her school.

In a 12-week project at a large secondary, urban academy school in Staffordshire, a group of 18 students with SEN needs also read to a volunteer visiting dog on a weekly basis. All the students were in Year 7 (aged $11-12$ years). The group was split into four students reading to the dog, four students on a reading intervention for comparison and two groups of five students for controls, during the Autumn Term 2018. This school also used reading age measurement using the Salford Sentence Reading Test.

The results were that the students who read to the dog on average improved their reading scores by 9 months (Ratio Gain 3 months - 'useful impact,') in comparison to the control group who made no extra progress (Ratio Gain 0.33 months - 'doubtful impact.') Those students on the reading intervention, 'Switch on Reading' improved their scores on average by 8 months (Ratio Gain 2.6 months - 'useful impact') compared to the control group that made only 2 months progress (Ratio Gain 0.6 months - 'doubtful impact'). At this school both the read to dog group and the taught intervention had similar impact (Private email March 2019).

In comparison to the study described in this chapter, the Staffordshire group of Year 7 students (older by four years) with both the read to the dog groups, and the control group made similar progress to the primary school study. The Secondary school study, however, suggests that an older child may also be more motivated to read 
with a dog, yet as the baselines for the individual secondary aged students are unknown, the progress could reflect that it is simply the maturity in cognitive functioning, which has raised the reading skills to the levels of their peers.

This could further add to the argument that there could be a plateau or 'cut off point' for progression with the dog. Perhaps the cognitive skills of reading then supersede the motivational aspects. Further research is required to investigate whether the reading skills are affected by the dog's presence according to age or ability.

Attainment in reading with the dog can also be compared with other literacy-based intervention schemes used in schools. The Education Endowment Fund (2018) in the UK provides an account of the expected progress of Literacy and Maths interventions which are commonly used in England and Wales' schools (see Table 27 below). These also show ratio gains for progress. Reading to dogs in both the studies above appear to surpass these progress attainment levels.

\section{Table 27: Literacy and numeracy interventions used in schools Table}

\begin{tabular}{|c|c|c|c|c|c|}
\hline Age & Name & Length & Group & Session & Ratio Gain \\
\hline $\begin{array}{l}\text { KS1 } \\
\text { (4-7 years) }\end{array}$ & $\begin{array}{l}\text { Reading } \\
\text { Recovery }\end{array}$ & $\begin{array}{l}12-20 \\
\text { weeks }\end{array}$ & $1: 1$ & $\begin{array}{l}\text { Daily } \quad 30 \\
\text { mins }\end{array}$ & +2 months \\
\hline $\begin{array}{l}\text { KS1 \& } 2 \\
\text { (4-11 } \\
\text { years) }\end{array}$ & $\begin{array}{ll}\text { Catch Up } \\
\text { Literacy }\end{array}$ & 30 weeks & $\begin{array}{l}\text { 1:1 or small } \\
\text { group }\end{array}$ & $\begin{array}{l}2 \times 15 \text { mins } \\
\text { per week }\end{array}$ & $+2 m$ \\
\hline $\begin{array}{ll}\text { KS1 \& } 2 & \\
\text { (4- } & 11 \\
\text { years) } & \end{array}$ & $\begin{array}{l}\text { Catch Up } \\
\text { Numeracy }\end{array}$ & 30 weeks & $\begin{array}{l}\text { 1:1 or small } \\
\text { group }\end{array}$ & $\begin{array}{l}2 \times 15 \text { mins } \\
\text { per week }\end{array}$ & $+3 \mathrm{~m}$ \\
\hline KS3 & $\begin{array}{l}\text { Switch On } \\
\text { Reading }\end{array}$ & $\begin{array}{ll}10- & 12 \\
\text { weeks } & \end{array}$ & $1: 1$ & $\begin{array}{l}15 \text { mins } \\
\text { Weekly }\end{array}$ & $+3 \mathrm{~m}$ \\
\hline
\end{tabular}




\begin{tabular}{|l|l|l|l|l|l|}
\hline $\begin{array}{l}(11-14 \\
\text { years })\end{array}$ & & & & & \\
\hline
\end{tabular}

\subsection{Fluency and Accuracy Results}

The data presented so far used a formative assessment of reading, with and without the presence of the dog. Another factor that should be considered are the skills within reading, particularly those which are affected by executive functioning skills.

According to Beetz and McCardle (2017) the presence of dogs can positively affect children's fluency levels when reading. Hediger (Hediger et al., 2017, Hediger and Turner, 2014) has also investigated the presence of a dog increasing the attention and concentration performance of $24,10-14$ year olds using passive infrared hemoencephalography (PIR-HEG) technology -finding that working memory skills (digit backwards) visual search and heightened inhibition skills are enhanced while the dog is available and interacting.

Francisco et al., (2017) following research by Melby -Lervag and Hulme(2013), Snowling (2001) and Stanovich (1994), suggest, however, that in adults it is the audio visual sensitivity that contributed uniquely to variance in reading errors and thus fluency. Horowitz-Kraus' (2014) research also suggests that reading-training improved contextual reading speed, speed of processing, memory and visual screening. Bearing in mind that for many generations, children have learned to read without a dog available, it would be wise to investigate these skills further. This finding would also imply that as some reading difficulties are neuro-developmental 
in origin, such as dyslexia, it would be difficult to establish how the dog would affect these skills, if indeed it does.

Situational evidence therefore is required to establish any effects arising from the dog and child interaction, such as the fluency levels of speech and reading rate, skin conductance for emotional variance and general working memory levels.

\subsubsection{Reading Speed (Fluency Rate)}

According to the Key Stage 1 National Curriculum Tests for 2016, the expected standard for a Year 2 child's reading speed should be over 90 words per minute (wpm). As this sample of children are in Year 3, it would be expected that they can read to this speed and above. As the next section shows, this is not the case. Fluency rate is linked to the flow and comprehension of the piece, involving a combination of skills: processing speed, expression, articulation, awareness of punctuation, and accuracy, affecting working and short-term memory. The two skills which are easiest to measure for reading are speed and accuracy. Reading speeds and reading rates were compared while the children read both with and without the dog present. A poem was selected which was to be read twice in comparison. The rate was calculated by using the child's verbal recordings, in conjunction with the written words / copies of the texts read and timed using a stopwatch. Depending on the length of the pieces these rates were then averaged for each child. These baseline scores, without the dog, may indicate why these children have been placed within the special needs group. 


\subsubsection{Accuracy Levels}

The accuracy rates were calculated using the 'Running Record and Miscue Analysis' technique which is taught in learning difficulties courses for teachers and is used in such reading assessments as the YARC (York Assessment of Reading and Comprehension (Snowling et al., 2009). This involves a 'running record' where the teacher notes all the words uttered by the child when reading a text, paying careful attention to mispronunciations, omitted words and substitutions. If a child selfcorrects then the mistake is not counted. The number of correct words is then calculated from the total number of words for the extract and converted into a percentage. These running records were analysed using the digital and video recordings of the sessions.

The following data was taken from the 2016 results for the 'focused case study' group of 7 students.

\subsubsection{Reading Rate Results}

The results for the reading rate for the focused case study children are in Table 27 below. To compare if the reading speed was associated with raised or lowered electoral dermal activity (EDA), the EDA averages for the session are given in Table 28.

Table 28: Average words per minute rate (wpm) for the reading passages

\begin{tabular}{|l|l|l|l|}
\hline Name & Without Dog & With Dog & $\begin{array}{l}\text { Reading Speed } \\
\text { with dog }\end{array}$ \\
\hline Male A & $94 \mathrm{wpm}$ & $111 \mathrm{wpm}$ & $17 \mathrm{wpm}$ increase \\
\hline Female E & $74 \mathrm{wpm}$ & $87 \mathrm{wpm}$ & $13 \mathrm{wpm}$ increase \\
\hline
\end{tabular}




\begin{tabular}{|l|l|l|l|}
\hline Female A & $47 \mathrm{wpm}$ & $33 \mathrm{wpm}$ & $14 \mathrm{wpm}$ decrease \\
\hline Male M & $91 \mathrm{wpm}$ & $88 \mathrm{wpm}$ & $3 \mathrm{wpm}$ decrease \\
\hline Male C & $88 \mathrm{wpm}$ & $98 \mathrm{wpm}$ & $10 \mathrm{wpm}$ increase \\
\hline Female G & $75 \mathrm{wpm}$ & $71 \mathrm{wpm}$ & $4 \mathrm{wpm}$ decrease \\
\hline Male H & $50 \mathrm{wpm}$ & $38 \mathrm{wpm}$ & $12 \mathrm{wpm}$ decrease \\
\hline Mean & $\mathbf{7 4 . 1 4} \mathbf{~ w p m}$ & $\mathbf{7 5 . 1 4} \mathbf{~ w p m}$ & Ave Diff 1 wpm \\
\hline
\end{tabular}

Green is higher rate of reading speed

Table 29: Averaged electrodermal activity (EDA) for the focus case study group's reading sessions

\begin{tabular}{|l|l|l|}
\hline Name & $\begin{array}{l}\text { Reading without } \\
\text { dog }\end{array}$ & Reading with dog \\
\hline Male A & 0.41653858 & 0.075774591 \\
\hline Female E & 0.16699076 & 0.332322034 \\
\hline Female A & 0.159303749 & 0.036849274 \\
\hline Male M & 0.231725186 & 0.163453497 \\
\hline Male C & 0.252235266 & 0.138107919 \\
\hline Female G & 0.117970004 & 0.398895233 \\
\hline Male H & 0.300260455 & 0.079886496 \\
\hline
\end{tabular}

Blue is higher average of $\mathrm{SCL}$ arousal measurements

\subsubsection{Accuracy of Reading Results}

The results for the accuracy rate for the focus case study children are in Table 30 below. To compare if the reading speed was associated with raised or lowered electoral dermal activity (EDA), the EDA averages for the session are given in Table 29 above. 


\begin{tabular}{|l|l|l|l|}
\hline Name & Without Dog & With Dog & Greater Accuracy \\
\hline Male A & $97.4 \%$ & $97 \%$ & $\begin{array}{l}\text { Reading in } \\
\text { general }\end{array}$ \\
\hline Female E & $96.4 \%$ & $96.7 \%$ & $\begin{array}{l}\text { Reading in } \\
\text { general }\end{array}$ \\
\hline Female A & $100 \%$ & $86 \%$ & Without dog 14\% \\
\hline Male M & $91 \%$ & $97 \%$ & With dog 6\% \\
\hline Male C & $90.4 \%$ & $98 \%$ & With dog 8.4\% \\
\hline Female G & $92.8 \%$ & $96.4 \%$ & With dog 3.7\% \\
\hline Male H & $95 \%$ & $92 \%$ & Without dog 3\% \\
\hline Mean & $\mathbf{9 4 . 7 1 \%}$ & $\mathbf{9 4 . 7 2 \%}$ & \\
\hline
\end{tabular}

Salmon denotes greater accuracy rate

The group mean results for reading to the dog, and without, show only one-word difference and no statistical change in the average group accuracy. This suggests that, on average, the presence of the dog does not make any difference in reading rate, accuracy and, therefore, fluency. On an individual basis, there is a clear indication, however, that for three of the individuals, reading with the dog helped to improve their wpm rate although the other four in that group do not show a similar improvement. Two children slowed by $12-14$ words per minute while the dog was present. This finding links back to the research by Wohlfarth et al., (2014) who reported that, with some individuals the longer the dog petting time, the longer the reading time - possibly linked to the child's distractibility (Wohlfarth et al., $2014 \mathrm{p}$. 69). 


\subsubsection{Electrodermal activity (EDA)}

In general, the electrodermal activity (EDA) is decreased when the dog is present showing lower arousal from the individuals. It became clear after working through the results that arousal for some does not mean that they are either more or less stressed, but that for some the arousal appeared to help them concentrate and focus on the task in hand, whereas for others, the arousal readings may have helped with calm and focus. There are large individual differences in arousal levels, and other factors such as reading performance and accuracy can give a clearer suggestion as to how arousal can affect the individual concerned.

The electrodermal activity levels show a higher arousal level in the "without dog" situation, which when compared to reading accuracy, show that for the majority of students $(n=5)$, reading with the dog either led to no difference to their reading accuracy or improved their accuracy $(n=3)$. As fluency and accuracy involve visual processing, syntax, semantics and grapheme/phoneme rapid recall, this could suggest that the dog is affecting the speed of processing, the verbal short-term memory and / or the visual spatial memory, with these students.

These data would link to the findings of Odendaal (Odendaal, 2000, Beck and Katcher, 2003) which found that the presence of a dog lowered stress rates. Yet this may not necessarily agree with the theory that simply the presence of the dog, through the lowering of the stress rates, has improved accuracy - as the individual results are split between little to no difference $(n=2)$, increase in accuracy $(n=3)$ and decrease in accuracy $(n=1)$. 
There could be a debate as to whether the slower pace of the reading also leads to greater accuracy, but other factors need to be considered, such as are the children more familiar with reading poetry, or with general texts, as this may affect the stress or EDA levels. The effects, or familiarity of the type of reading task may also have given the apparent fluency (wpm) increase, rather than the dog's presence.

By comparison the AAE / AAI research uses reading of selected prose passages, single word reading, or a precise comprehensive reading test, rather than any other forms of text commonly found in classroom reading settings (Schretzmayer et al., 2017). These approaches can give a false reading as, with single word reading, other strategies can be used, - such as the visual shape of the word to "guess" the accuracy, or during "cloze procedure" tests, there is usually a multiple choice of answers. When children are aware that they are reading texts within a testing situation, i.e. graded texts with comprehension questions such as used in the YARC, this awareness can affect physiological readings due to anxiety and stress. Future studies should consider 'real' literacy classroom settings and baselining of EDA levels in 'standard' reading for all children, before considering the presence of a dog.

To investigate whether the nature of the task affected EDA, accuracy and fluency, a second task was created for comparison using words read from 'sight' or unrehearsed, with no preparation.

Reading of rhyme was used for this second task, as the length of the pieces are shorter than prose. Each line has limited numbers of words, and while all phoneme / graphemes should have been known, the underlying meter together with the auditory onset and rhyme should assist the reader with decoding and thus accuracy. 
Poetry reading also is an area of difficulty for those with neurodevelopmental differences such as dyslexia as it is the constant maintaining and renewal of the rhythm through the executive functioning skills and the working memory which sustains the overall fluency of reading. Simply reading a poem in comparison to reading a piece of prose, or text, may show differences in speed and accuracy. If these effects are measurable without the dog present, then an argument for what is read, may well be affecting the perceived 'success' of reading to the dog.

\subsection{Comparison of Reading Rhyme with Reading without dog}

The results for the accuracy rate for the focus case study children for rhyme are in Table 31 below. To compare if the speed of reading rhyme was associated with raised or lowered electoral dermal activity (EDA), the EDA averages for the session are given in Table 32 below.

Table 31: Reading speed rate comparisons between rhyme and prose without dog present

\begin{tabular}{|l|l|l|l|}
\hline Name & Unknown Rhyme & $\begin{array}{l}\text { Reading Without } \\
\text { Dog }\end{array}$ & $\begin{array}{l}\text { Reading } \\
\text { with dog }\end{array}$ \\
\hline Male A & $50 \mathrm{wpm}$ & $94 \mathrm{wpm}$ & 44 wpm increase \\
\hline Female E & $94 \mathrm{wpm}$ & $74 \mathrm{wpm}$ & 20 wpm decrease \\
\hline Female A & $27 \mathrm{wpm}$ & $47 \mathrm{wpm}$ & 20 wpm increase \\
\hline Male M & $90 \mathrm{wpm}$ & $91 \mathrm{wpm}$ & 1 wpm increase \\
\hline Male C & $53 \mathrm{wpm}$ & $88 \mathrm{wpm}$ & 35 wpm increase \\
\hline Female G & $91 \mathrm{wpm}$ & $75 \mathrm{wpm}$ & 16 wpm decrease \\
\hline Male H & $34 \mathrm{wpm}$ & $50 \mathrm{wpm}$ & 16 wpm increase \\
\hline Mean & $\mathbf{6 2 . 7}$ wpm & $\mathbf{7 4 . 1 4} \mathbf{~ w p m}$ & Ave Diff 11 wpm \\
\hline
\end{tabular}

Green is higher rate of reading speed 


\begin{tabular}{|l|l|l|l|}
\hline Name & Unknown Rhyme & $\begin{array}{l}\text { Reading without } \\
\text { dog }\end{array}$ & $\begin{array}{l}\text { Hear rhythm to the } \\
\text { rhyme? }\end{array}$ \\
\hline Male A & 0.399867176 & 0.41653858 & Yes \\
\hline Female E & 0.070337059 & 0.16699076 & Yes \\
\hline Female A & 0.054034099 & 0.159303749 & No \\
\hline Male M & 0.177902914 & 0.231725186 & Yes \\
\hline Male C & 0.151724912 & 0.252235266 & No \\
\hline Female G & 0.144936593 & 0.117970004 & Yes \\
\hline Male H & 0.086053362 & 0.300260455 & Yes \\
\hline
\end{tabular}

Blue shows higher average arousal EDA rates

Table 33: Comparison of accuracy rates between reading rhyme, and reading prose, without dog present

\begin{tabular}{|c|c|c|c|}
\hline Name & $\begin{array}{l}\text { Unknown Rhyme } \\
\text { Accuracy }\end{array}$ & $\begin{array}{l}\text { Reading Without } \\
\text { Dog }\end{array}$ & Preferred Reading \\
\hline Male A & $76 \%$ & $97.4 \%$ & Text \\
\hline Female E & $92.8 \%$ & $96.4 \%$ & Text \\
\hline Female A & $65.17 \%$ & $100 \%$ & Text \\
\hline Male M & $95.6 \%$ & $91 \%$ & Poem \\
\hline Male C & $71.4 \%$ & $90.4 \%$ & Text \\
\hline Female G & $92.8 \%$ & $92.8 \%$ & Either \\
\hline Male H & $78.5 \%$ & $95 \%$ & Text \\
\hline Mean & $81.75 \%$ & $94.71 \%$ & \\
\hline
\end{tabular}

Salmon denotes greater accuracy rate

The reading of the rhyme showed lower average reading speeds than those for reading in general. Out of the two tasks, the higher EDA levels were clearly associated with the reading task rather than the poetry. Only one student found the poem of 
higher arousal than the reading, but this did not affect her accuracy. As predicted, there were differences in maintaining speed and accuracy between the two pieces, base on familiarity - with two children unable to recognise the meter of the rhyme.

This finding supports the theory that it is 'what is read' (the specific task) which assists fluency rather than the presence of the dog. To check this hypothesis, the poetry results were compared to the reading with the dog scores, which should show higher EDA rates when reading the poem.

\subsection{Comparison of Reading Rhyme Speed with dog present}

The results for the comparison in accuracy and speed between the rhyme and reading to the dog for the focus case study children, are in Table 34 and Table 36 below. In comparison, the EDA averages for the session are given in Table 35 below.

Table 34: Reading rate comparisons between unknown rhyme and known prose with the dog

\begin{tabular}{|l|l|l|l|}
\hline Name & Unknown Rhyme & $\begin{array}{l}\text { Read with Dog } \\
\text { (known prose) }\end{array}$ & $\begin{array}{l}\text { Accuracy with } \\
\text { Dog }\end{array}$ \\
\hline Male A & $50 \mathrm{wpm}$ & $111 \mathrm{wpm}$ & $\begin{array}{l}61 \text { wpm } \\
\text { increase }\end{array}$ \\
\hline Female E & $94 \mathrm{wpm}$ & $87 \mathrm{wpm}$ & $7 \mathrm{wpm}$ decrease \\
\hline Female A & $27 \mathrm{wpm}$ & $33 \mathrm{wpm}$ & $6 \mathrm{wpm}$ increase \\
\hline Male M & $90 \mathrm{wpm}$ & $88 \mathrm{wpm}$ & 2 wpm decrease \\
\hline Male C & $53 \mathrm{wpm}$ & $98 \mathrm{wpm}$ & $\begin{array}{l}45 \\
\text { increase }\end{array}$ \\
\hline Female G & $92 \mathrm{wpm}$ & $71 \mathrm{wpm}$ & $\begin{array}{l}21 \\
\text { decrease }\end{array}$ \\
\hline Male H & $34 \mathrm{wpm}$ & $38 \mathrm{wpm}$ & 4 wpm increase \\
\hline Mean & $62.85 \mathrm{wpm}$ & $\mathbf{7 5 . 1 4} \mathbf{w p m}$ & Ave diff 21 wpm \\
\hline
\end{tabular}

Green is higher rate of reading speed 


\begin{tabular}{|l|l|l|}
\hline Name & Unknown Rhyme & $\begin{array}{l}\text { Reading with dog } \\
\text { (known prose) }\end{array}$ \\
\hline Male A & 0.399867176 & 0.075774591 \\
\hline Female E & 0.070337059 & 0.332322034 \\
\hline Female A & 0.054034099 & 0.036849274 \\
\hline Male M & 0.177902914 & 0.163453497 \\
\hline Male C & 0.151724912 & 0.138107919 \\
\hline Female G & 0.144936593 & 0.398895233 \\
\hline Male H & 0.086053362 & 0.079886496 \\
\hline
\end{tabular}

Blue is higher average of SCL arousal measurements

Table 36: Comparisons in accuracy rate between reading unknown rhyme and reading known prose with the dog

\begin{tabular}{|l|l|l|l|}
\hline Name & $\begin{array}{l}\text { Unknown Rhyme } \\
\text { Accuracy }\end{array}$ & Reading with Dog & Greater Accuracy \\
\hline Male A & $76 \%$ & $97 \%$ & With dog \\
\hline Female E & $92.8 \%$ & $96.7 \%$ & With dog \\
\hline Female A & $65.17 \%$ & $86 \%$ & With dog \\
\hline Male M & $95.6 \%$ & $97 \%$ & With dog \\
\hline Male C & $71.4 \%$ & $98 \%$ & With dog \\
\hline Female G & $92.8 \%$ & $96.4 \%$ & With dog \\
\hline Male H & $78.5 \%$ & $92 \%$ & With dog \\
\hline Mean & $\mathbf{8 1 . 7 5 \%}$ & $\mathbf{9 4 . 7 2 \%}$ & \\
\hline
\end{tabular}

Salmon denotes greater accuracy rate

As predicted the EDA readings were greater for the unfamiliar poem. The higher EDA levels overall indicated that there were higher stress levels associated with the 
different style of reading presentation. The group average reduction in both speed and accuracy for rhyme, reflects that the children were affected by the poem's layout, length, decodability and choice of words. The presence of the dog in this final comparison has now appeared to increase the accuracy of all the students when reading prose over the poem. The prose was more familiar to the children, and they had read these books before.

\subsection{Overall Summary of Paws to Read Project}

The examination of the group results in the first sections of this chapter, showed that overall reading to the dog appeared to be of more benefit to students than those who had not. The groups involving the dog reflected higher attainment in ratio gain, standardised scores and reading ages than the control. These were of similar finding to the secondary school comparison and were also the equivalent, or slightly higher to the school-based literacy and numeracy interventions used in classrooms, which did not require a dog. When examined in depth, however, the distribution of individual's ratio gains within the different group scores revealed a more complex picture. Some students within the overall 'higher' attaining dog reading group were not making any 'impact' or progression in their reading at all, in fact several students had negative results.

Comments from the focus group children, at the age of seven years, however, revealed that the children believed in the dog 'helping' them to read, some using more anthropomorphic language than others, but overall they enjoyed the experience of reading with the dog present. The teachers and adults revealed that they enjoyed the scheme as it increased their own perception of the children's' self- 
esteem, motivation and confidence when back in class after the sessions. The staff members were unsure of the actual effects on individual's reading attainment, however, with all four members suggesting that the reading progress was the 'about the same' as those who had not taken part. These staff members were not involved in the evaluation or monitoring of the Paws to Read project in this school as that was delegated to a senior member of staff.

In this small study sample, individual differences in children's reading skills predicted the success of the reading overall, showing that for some there appeared to be a 'plateau' after which the effects of a dog may have been motivational only. The ratio gains showed that the dog did not affect the accuracy or fluency rates as suggested by other research, rather it was the individual's familiarity with the level and type of text which dictated their speed and accuracy.

This would suggest that while the dog is present in this school for perceived academic attainment, the evidence within this chapter does not support this. The dog in this school, did not seem to be bothered whether he was read to or not, but did enjoy the interaction for the treats. Overall, these findings will question the whole validity of the Read to Dogs programme. 


\section{Chapter 7 (Research Phase 2)}

\section{School based Investigation: Individual Children's Case Studies}

\subsection{Introduction}

The data presented to date suggests that reading to a dog on an individual basis rather than a group basis is more likely to yield positive results. In order to test this proposition further, the results of the dog and child interactions were analysed on an individual basis to determine the nature, if any, of how the presence of the dog may have affected each individual. To consider this personal approach, previously surmised qualitative and quantitative data, such as the working memory tests, reading age comparison scores, reading speed, accuracy rates and comments for each child, was gathered together from chapters five and six to examine each child's personal profile.

These results were placed together with the results from the original range of working memory tests, which, due to the school timetable restrictions and lack of time, could not be repeated while the dog was present. These baseline results did highlight the nature of some of the specific needs in the children' profiles and why they may have been considered for the Special Educational Needs group by the school's Special Educational Needs Co-ordinator (SENCO.) The single use of these working memory tests, as opposed to the repetition with the dog present, is the usual, common practice when assessing individuals for specific learning differences. As such, they remain a valid record in themselves for each child. These tests use 
standardised scores (SS) for clarity. The level descriptors for the classification of the scores are represented in Table 9, Chapter 5.

Each profile also contains the observations recorded while the reading sessions took place, both with and without the dog, including the reading of the rhyme, together with the individual fluency and accuracy rates gathered from chapters five and six.

\subsubsection{Working out the individual stress rates for reading using the Pip}

The Pip data revealed the individual's physiological differences between the dog being present or not during the tasks by recording the amount of positive or negative changes in the interaction as integers. The Pip recordings for each session yielded streams of data points individual to the reader and recorded these tiny changes at approximately eight times a second. The data from these recorded changes could then be collated into minutes to create graphs which could be linked back to the timed video recordings for when the child was reading.

\subsubsection{Box and Whisker Charts}

The overall session data collated from the Pip could also be converted into box and whisker charts. These charts gave a pictorial representation of the difference between each child's reaction to the dog or task. The calculated range between the highest and lowest scores revealed the overall variance in that session. While the range gives the extremes, the quartiles give the more common, general range measurements through the time period. The quartile locations also show the overall 'lean' or 'bias' towards the positive or negative integer scores. The medians of these quartiles can be compared between the tasks themselves: reading to the dog, reading without the dog and reading the rhyme on their own. Each recording thus 
showed the personalisation and individuality of each child's overall readings. For some, the dog's presence raised the session's average of electrodermal activity (EDA) variability, both in variance and rate, while for others it was lowered.

\subsection{Individual Profiles}

\subsubsection{Male A}

\section{(Age during project: seven years, three months to seven years six months)}

\subsubsection{Introduction and results from questionnaire}

Male A's family owned a small dog, but he said that he did not usually read to it while at home. He considered himself 'good' at reading and liked the subject, but while he also liked maths as a subject, he circled the 'ok' emoji, suggesting that he may have felt less confident in this subject area. Male A said he was "happy" to read to Matt the dog, and that the dog would be happy too, "because he lies next to me." When asked if he could help the dog with his reading, Male A may have misheard the question and answered, "he enjoys it." Male A was not sure how the dog could help him with his own reading, saying, "I don't know. I read it myself." Male A appeared to be very aware of the dog's limitations and did not anthropomorphise the dog at all. Male A appeared to have attentional issues with verbal instructions. Twice he was collected from the Head's office at the end of lunchtime for not using his "listening" skills.

Throughout all the testing Male A was constantly talking and telling the researcher details about what he had been doing, what was happening in the room and asking why certain tests were being done. While this is commendable from a curious, 
inquisitive point of view, this distraction will mean that Male $A$ is missing out on relevant and instructional information in the classroom in order to achieve his set tasks, and that when explained, he did not always listen to the answers given.

\subsubsection{Results from non-repeated working memory tests due to time}

The working memory results for Male A are shown in Table 37 below. These were the tests which were included in the original selection for the screener, (See chapter five) but were unable to be repeated when the dog was present, due to time. These tests do give an indication to aspects of his abilities. Standardised Scores (SS) are also given in table 37 below, which are in comparison to a nationwide group of children of the same age. Average standardised scores are usually between 85 and 115 .

Table 37: Male A's standardised scores for the working memory tests unable to be repeated with the dog present

\begin{tabular}{|l|l|l|l|l|l|l|}
\hline Name & $\begin{array}{l}\text { Memory } \\
\text { for Story }\end{array}$ & $\begin{array}{l}\text { Memory } \\
\text { for Story } \\
\text { delayed }\end{array}$ & $\begin{array}{l}\text { Fluency - } \\
\text { Semantic }\end{array}$ & $\begin{array}{l}\text { Fluency - } \\
\text { Alliteration }\end{array}$ & $\begin{array}{l}\text { Fluency } \\
\text { - Rhyme }\end{array}$ & $\begin{array}{l}\text { Visual } \\
\text { Memory }\end{array}$ \\
\hline Male A & SS 95 & SS 85 & SS 106 & SS70 & SS 88 & SS125 \\
\hline
\end{tabular}

(Tests taken at seven years, two months)

Male $\mathrm{A}$ at the chronological age of seven years and three months was able to memorise and maintain certain details for the immediate recall of 'memory for stories' from the 'Test of Memory And Learning, (TOMAL 2, see Chapter five), yet he had a low average score (SS 85) for their retention and verbal recall, 30 minutes later. He was able to fluently access his long-term memory to quickly label and name items for 'things to eat' and 'animals' for his semantic fluency tests from the Phonological Assessment Battery (PhAB), but had below average difficulties limiting the lists for 
the initial sound only (SS 70). He was able to suggest only one word to rhyme with more (muse) which was incorrect and only two others, a non-word 'swip' and tip to rhyme with 'whip,' despite having 30 seconds. These findings would suggest that he has issues with cognitive overload, when he must maintain, manipulate and adapt auditory and verbal information, particularly involving initial sounds. While Male $A$ can recognise rhyme, he is unable to initiate it at this point in time.

Male A's strength, however, is as a visual learner with an above average standardised score. Male A relies on visual strategies and visual knowledge to help him with his learning at this stage of development.

\subsubsection{Working Memory with and without dog present}

Male A's working memory test results with and without the dog present are given in Figure 21 below

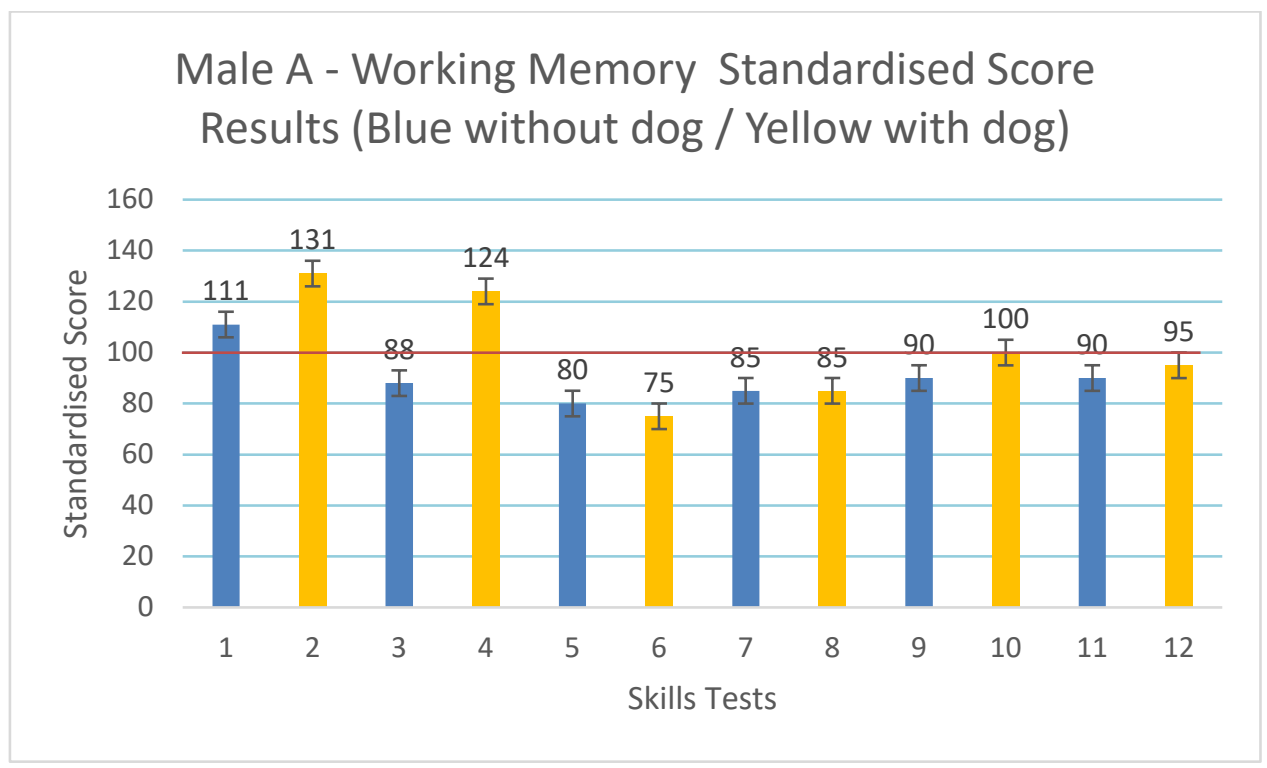

Key to skills tests:

\begin{tabular}{|l|l|l|l|c|}
\hline Skill Tests & & Test & S.S & Improvement? \\
\hline $\begin{array}{l}\text { Processing } \\
\text { Speed }\end{array}$ & 1 & Naming Picture Speed no dog & 111 & \\
\cline { 2 - 5 } & 2 & Naming Picture speed with dog & 131 & Yes \\
\hline
\end{tabular}




\begin{tabular}{|c|c|c|c|c|}
\hline & 3 & Naming speed digits no dog & 88 & \\
\hline & 4 & Naming speed digits with dog & 124 & Yes \\
\hline \multirow{4}{*}{$\begin{array}{l}\text { Auditory } \\
\text { Short-Term } \\
\text { Memory }\end{array}$} & 5 & Digits forward no dog & 80 & \\
\hline & 6 & Digits forward with dog & 75 & No \\
\hline & 7 & Letters forward no dog & 85 & \\
\hline & 8 & Letters forward with dog & 85 & Same \\
\hline \multirow{4}{*}{$\begin{array}{l}\text { Executive } \\
\text { Skills \& } \\
\text { Working } \\
\text { Memory } \\
\text { Span }\end{array}$} & 9 & Digits backward no dog & 90 & \\
\hline & 10 & Digits backward with dog & 100 & Yes \\
\hline & 11 & Letters backward with dog & 90 & \\
\hline & 12 & Letters backward with dog & 95 & Yes \\
\hline
\end{tabular}

Figure 21: Male A's working memory results, with and without the dog present and key

Figure 21 has grouped the tests according to their underlying components: processing speed; auditory short term memory; and underlying executive skills and working memory span (Dehn, 2015, Dehn, 2008). The right-hand column is for comparison whether the dog's presence has made a difference.

For Male A, when visual sources were available, the dog's presence improved his processing skills for object and digit naming, giving above average or well above average standardised scores. The dog appeared to have little to no effect on his auditory short-term memory skills when letters or numbers were given verbally. Male A's digits forwards scores reiterated the low average short term auditory memory, which was not improved by the dog's presence. This finding would suggest that the dog may have been a distraction for this task. The digits and letters backwards span tasks, where Male A had to manipulate the given, verbal information, did show improvement, which suggests that the presence of the dog helped Male A with executive skills, visuo-spatial reasoning and memory span. 


\subsubsection{Electrodermal Activity Readings with and without dog present}

The averaged electrodermal activity readings for both reading sessions with the dog present and without for Male A are below in Table 38.

\section{Table 38: Averaged EDA readings for Male A}

\begin{tabular}{|l|l|l|l|l|}
\hline Name & & $\begin{array}{l}\text { Sum of SC } \\
\text { increments } \\
\text { (micro- } \\
\text { siemens) }\end{array}$ & $\begin{array}{l}\text { Sum of time } \\
\text { differences } \\
\text { (seconds) }\end{array}$ & $\begin{array}{l}\text { EDA Positive } \\
\text { Changes (micro- } \\
\text { siemens / second) }\end{array}$ \\
\hline Male A & No Dog & 131.4878587 & 325.583 & 0.406349712 \\
\hline Male A & Dog & 20.01390779 & 286.463 & 0.069865594 \\
\hline
\end{tabular}

(Green shows the higher variance of EDA changes)

Male A's overall averages for EDA positive changes during the session showed greater variance when the dog was not present.

\subsubsection{EDA Box and Whisker charts for all three tasks}

Male A's Comparisons in electrodermal activity for the three tasks of reading a rhyme, reading to an adult without a dog present and reading with a dog present are shown in Figure 22 below:

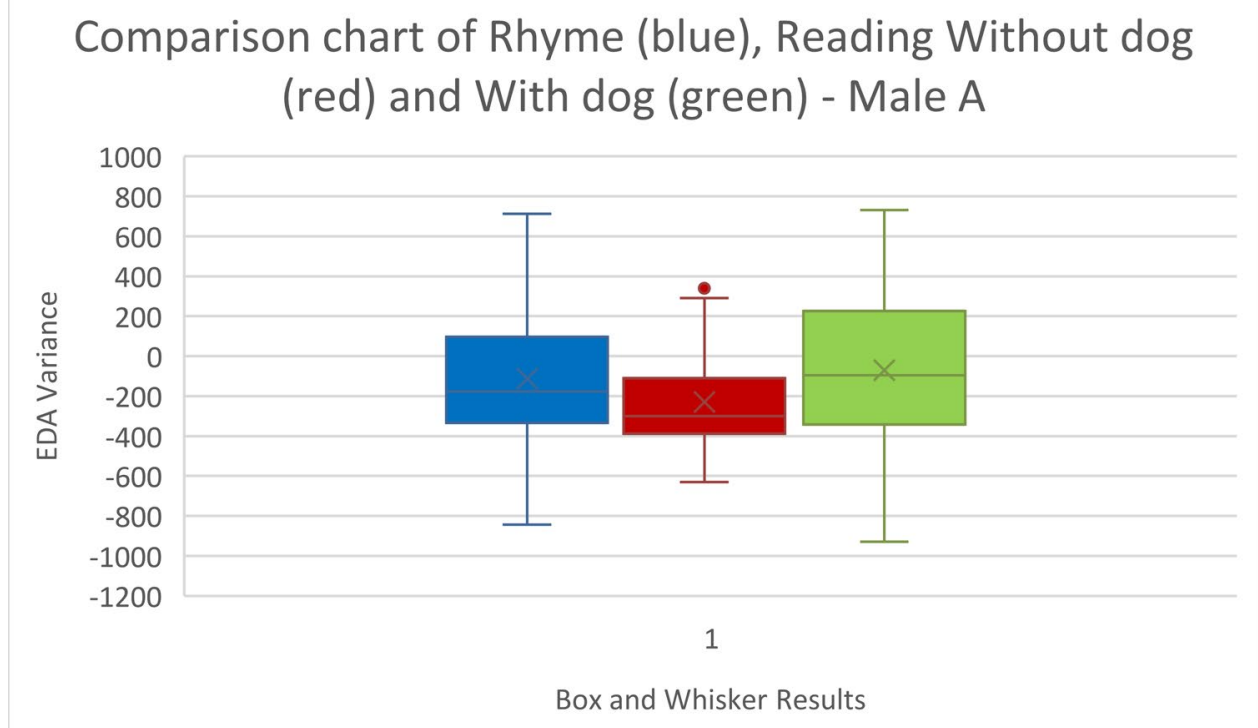


Figure 22: Male A's EDA comparisons between the three tasks

The comparative measures from the box and whisker charts for Male A are in Table 39 below.

Table 39: Male A Comparative measure for the box and whisker charts

\begin{tabular}{|l|l|l|l|}
\hline & Overall Range & Quartile Range & Median \\
\hline Rhyme without dog & 1557 & 423 & -176 \\
\hline Read without dog & 1445 & 380.75 & -208 \\
\hline Read with dog & 1667 & 594.25 & -65 \\
\hline
\end{tabular}

The range of each task shown in Table 39 has revealed that the greatest overall variance for Male A was in the read to the dog situation, despite the averaged session scores in Table 38, above. The quartile range for read to the dog is also the widest out of the three tasks, showing a wider, continual variance during this task in comparison to reading the rhyme and reading to the adult. The difference of median levels shows a slightly higher level of averaged arousal when the dog was present over the rhyme and reading on his own. The outliers in the reading without dog result, show that there were moments of distraction. This data confirms that for Male A, out of the three tasks, reading to the dog was the most physiologically arousing activity, reading a rhyme also gave a higher arousal response, while the least variance and electrodermal activity occurred was when he was reading on his own. This final measurement may reflect his usual EDA response to this task.

\subsubsection{Male A Minute graphs comparison}

The 'real time' effects can be viewed in the Pip graphs for the first two minutes of reading as seen in Figures 23 and 24 below. The dog clearly shows greater variance 
of measurement, while the blue line, read to an adult show, more regular and smaller overall variance.

(Blue is reading without dog. Orange is reading with dog)

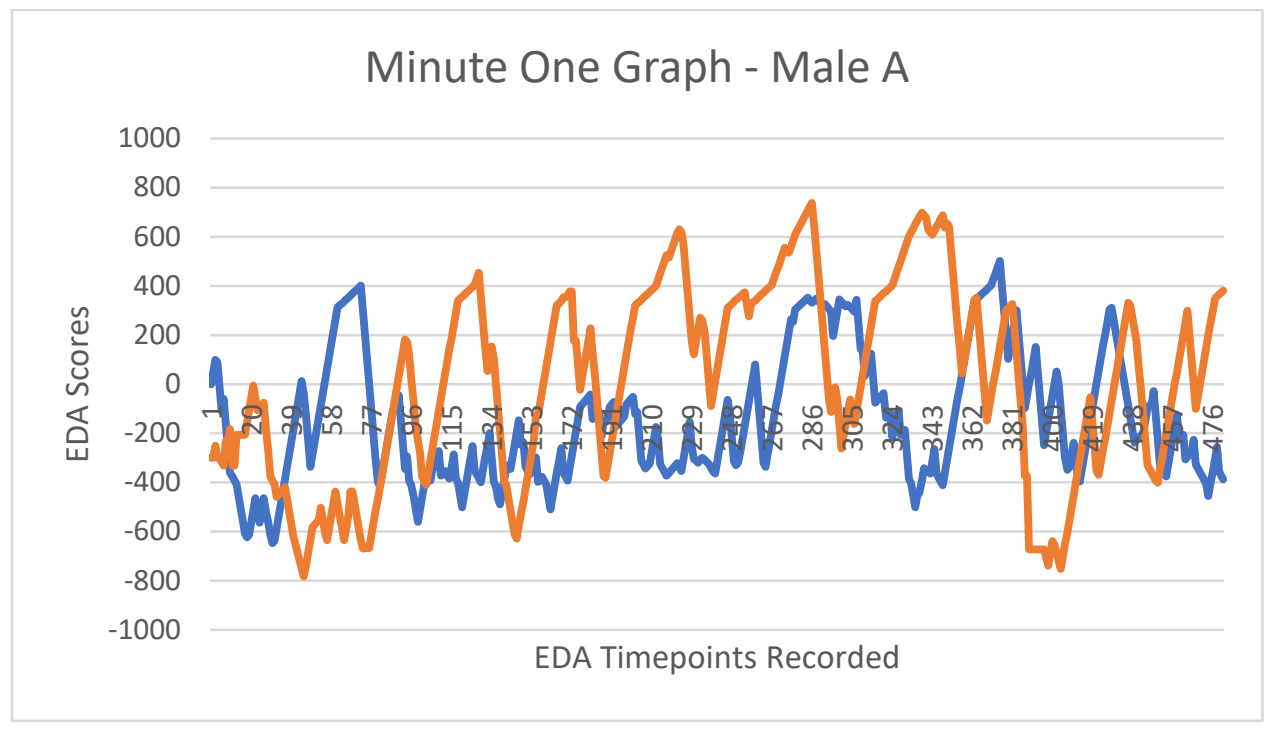

Figure 23: Male A's Pip 'real time' graph for minute one

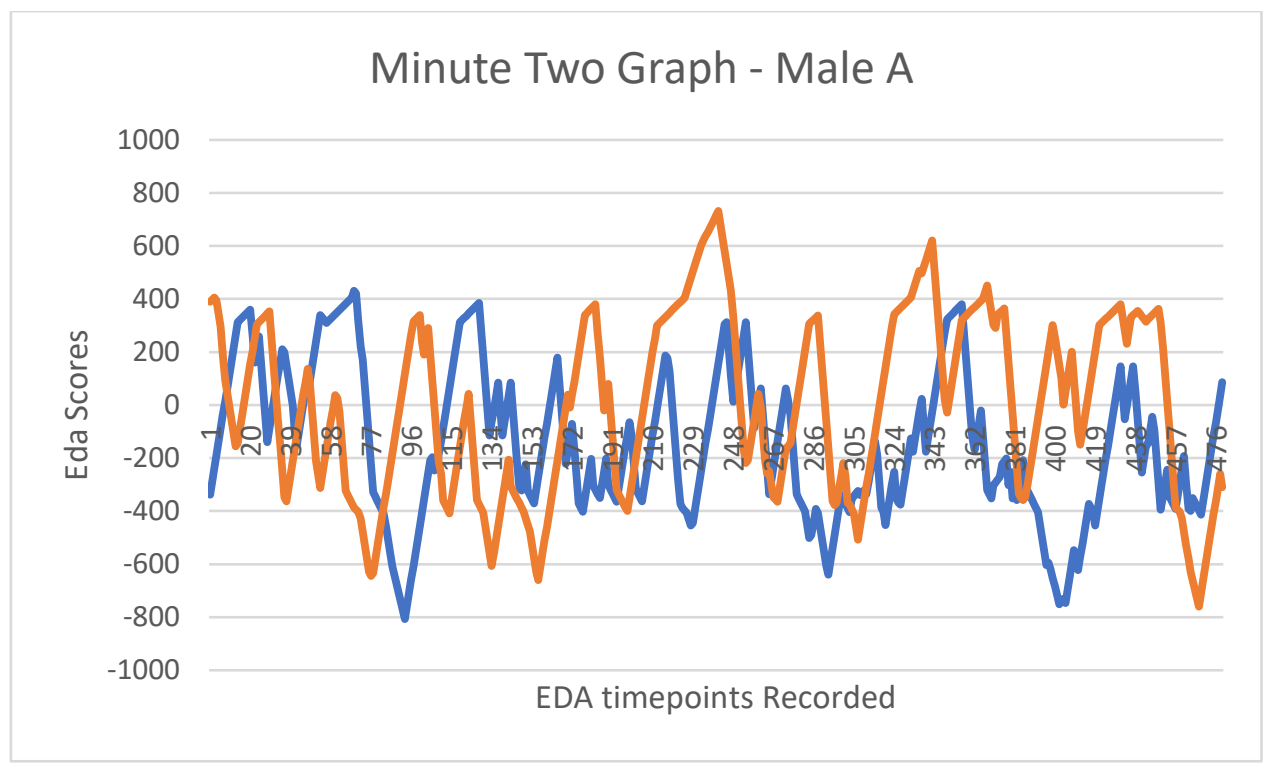

Figure 24: Male A's 'real time' graph for minute two

In order to see the effects of the different levels of arousal on his reading, the accuracy and words per minute rates need to be examined. 


\subsubsection{Reading Accuracy and Words Per Minute (WPM) rate}

The accuracy and speed effects of Male A's EDA arousal response are shown in Table 40 below.

Table 40: Reading accuracy and words per minute rate for Male A

\begin{tabular}{|l|l|l|}
\hline Reading Rhyme (No dog) & $\begin{array}{l}\text { Reading Book (No dog) } \\
\text { 1.9 Reading Level }\end{array}$ & $\begin{array}{l}\text { Reading Book (with dog) } \\
\mathbf{2 . 0} \text { Reading Level }\end{array}$ \\
\hline 76\% Accuracy & $97.4 \%$ Accuracy & $97.0 \%$ \\
50 wpm & $93.6 \mathrm{wpm}$ & $111 \mathrm{wpm}$ \\
\hline
\end{tabular}

If Male A's reading is examined in the context of the reading rate words per minute (wpm) and the overall reading attainment and achievement, it can be interpreted that, for Male A, having a dog present while reading, has improved his reading speed, while his overall accuracy is negligible in these instances.

When observing the videos of Male A reading, he shows a good connection to what he is reading, in that he is alert, can answer questions and will tackle unknown words carefully. While Male A reads, however, he makes no acknowledgement of the dog when it is present - clearly the reading task is of greater attentive focus. Throughout all the recordings he reads clearly. There is slightly better use of intonation and punctuation only when the dog is absent. Male A's total focus on the task showed that he found turning the page difficult with the Pip in his hand, yet he still managed to also follow the words with his finger.

In all recordings his body position for reading was the same, sat on the beanbag, closed body language with legs together and head down, reading into his lap. During 
the dog session, when he had finished reading, however, his full attention turned to the dog. Male A showed open body language, smiling, laughing, eye contact, stroking and giving ear and tummy tickles. He made a lot of comments in comparison to his own dog and would happily chat to the adults although not directly to the dog itself.

\subsubsection{Reading Attainment in both reading age and standardised scores}

Male A's overall reading progress is recoded in tables 41 and 42 below

\section{Table 41: Male A Reading attainment in reading age}

\begin{tabular}{|l|l|l|l|l|l|l|l|}
\hline Name & Chron Age & $\begin{array}{l}\text { Salford } \\
\text { Age } \\
\text { Start }\end{array}$ & $\begin{array}{l}\text { Difference } \\
\text { between }\end{array}$ & $\begin{array}{l}\text { Chron } \\
\text { Age End }\end{array}$ & $\begin{array}{l}\text { Salford } \\
\text { Age End }\end{array}$ & $\begin{array}{l}\text { Difference } \\
\text { between }\end{array}$ & Ratio Gain \\
\hline Male A & $7: 02$ & $7: 05$ & $+3 m$ & $7: 05$ & $8: 11$ & $+18 m$ & +6 \\
\hline
\end{tabular}

Table 42: Male A Reading attainment in standardised scores

\begin{tabular}{|l|l|l|l|l|}
\hline & $\begin{array}{l}\text { Standardised Score } \\
\text { Start }\end{array}$ & Percentile Start & $\begin{array}{l}\text { Standardised } \\
\text { Score End }\end{array}$ & Percentile End \\
\hline Male A & 103 & 58 & 118 & 89 \\
\hline
\end{tabular}

Overall, Male A did manage to make 12 months reading age progress during the project's 12-week duration. As argued in chapter six, this improvement was due to a lot of effort from his parents and teachers as reading was a specific target for him at that point in time. Male A only managed to read to the dog three times during this period. He ended the 12-week study reading 18 months above his chronological age. This gave him a ratio gain of 6 or "Remarkable impact" (Brooks, 2007) and an increased standardised score of 15 points. 


\subsubsection{Summary for Male A}

Male A did comment that he "enjoyed reading to the dog." Despite this opinion, it could be argued that the presence of the dog for Male A's academic attainment during this study was negligible, other than for motivation and reading speed.

\subsubsection{Female $\mathrm{E}$}

(Age during project: seven years and seven months to seven years and nine months)

\subsubsection{Introduction and results from questionnaire}

Female E 's family owned a dog and two rabbits and when asked if she read to them at all, she replied, "sometimes." Female E considered herself to be good at PE (Physical Education) and maths in school. She liked reading, but she was only, "ok" in maths. Reading to the dog made Female E feel, "excited and happy" and in return, the dog was also excited when she read. Female E also give the dog human qualities - when asked how she could help him read, she suggested that she could read to him lots of times to make him understand books and that he could help her by listening carefully.

When reading, Female E showed no interaction with the dog. Her full focus was on the task. After finishing, although Female E gently scratched the dog's head, she was looking around the room and making more eye contact with the adults. Female E was keen to give the dog a biscuit, paying him full attention while he took it, but afterwards she preferred to converse with the adults, rather than the dog. 


\subsubsection{Results from the non-repeated working memory tests, due to time}

Table 43: Female E Standardised scores for the working memory tests unable to be repeated with the dog present

\begin{tabular}{|l|l|l|l|l|l|l|}
\hline Name & $\begin{array}{l}\text { Memory } \\
\text { for Story }\end{array}$ & $\begin{array}{l}\text { Memory } \\
\text { for Story } \\
\text { delayed }\end{array}$ & $\begin{array}{l}\text { Fluency - } \\
\text { Semantic }\end{array}$ & $\begin{array}{l}\text { Fluency - } \\
\text { Alliteration }\end{array}$ & $\begin{array}{l}\text { Fluency } \\
\text { - Rhyme }\end{array}$ & $\begin{array}{l}\text { Visual } \\
\text { Memory }\end{array}$ \\
\hline $\begin{array}{l}\text { Female } \\
\text { E }\end{array}$ & SS 91 & SS 90 & SS 101 & SS 91 & SS 72 & SS 95 \\
\hline
\end{tabular}

Tests taken at Seven years and seven months

The data from Table 43 above reveals that Female $\mathrm{E}$ has an average working memory for her age and could retain certain facts for up to 30 minutes through her scores on "Memory for Stories" and "Memory for Stories Delayed" (TOMAL2.) In the phonological assessment battery tests, (PhAB) Female E could easily recall and label different foods and animals from her semantic long-term memory and could retain and use the rules for alliteration. However, Female E had significant difficulties with her rhyme, changing the given end sound from "ip" to "ick" which is auditorily similar, despite being reminded. This led to a below average standardised score in this task (SS 72.) Female $E$ has a slight speech and language difficulty in the pronunciation of certain phonemes. During her visual memory test from the TVS-P, she worked slowly but accurately and gained an average standardised score (SS 95). Although that test was not timed, Female E took longer to complete it than other children, revealing that she required a slightly longer processing time for accuracy in visual images.

\subsubsection{Working Memory Tests with and without dog present}

Female E's working memory test results with and without the dog present are given in Figure 25 below 


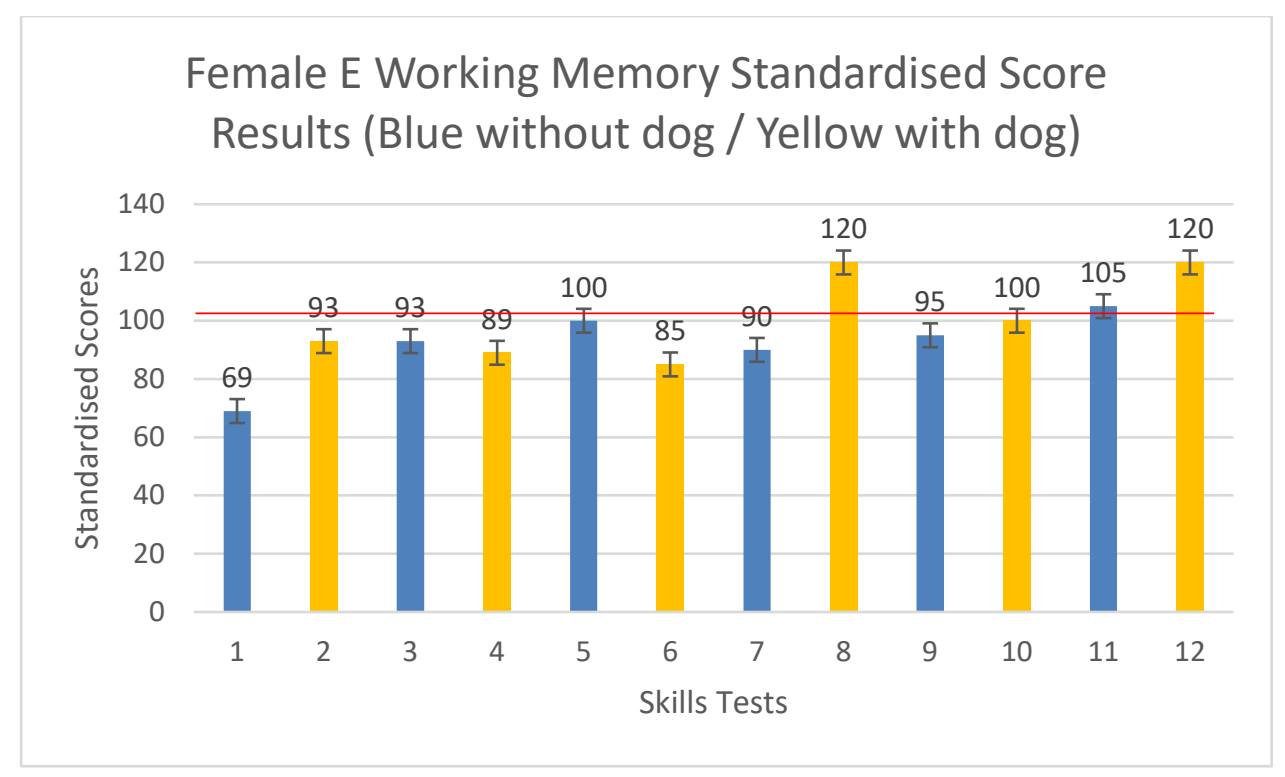

Key to skills tests:

\begin{tabular}{|c|c|c|c|c|}
\hline Skill & & Test & S.S & Improvement? \\
\hline \multirow{4}{*}{$\begin{array}{l}\text { Processing } \\
\text { Speed }\end{array}$} & 1 & Naming Picture Speed no dog & 69 & \\
\hline & 2 & Naming Picture speed with dog & 93 & Yes \\
\hline & 3 & Naming speed digits no dog & 93 & \\
\hline & 4 & Naming speed digits with dog & 89 & No \\
\hline \multirow{4}{*}{$\begin{array}{l}\text { Auditory } \\
\text { Short-Term } \\
\text { Memory }\end{array}$} & 5 & Digits forward no dog & 100 & \\
\hline & 6 & Digits forward with dog & 85 & No \\
\hline & 7 & Letters forward no dog & 90 & \\
\hline & 8 & Letters forward with dog & 120 & Yes \\
\hline \multirow{4}{*}{$\begin{array}{l}\text { Executive } \\
\text { Skills \& } \\
\text { Working } \\
\text { Memory } \\
\text { Span }\end{array}$} & 9 & Digits backward no dog & 95 & \\
\hline & 10 & Digits backward with dog & 100 & Yes \\
\hline & 11 & Letters backward with dog & 105 & \\
\hline & 12 & Letters backward with dog & 120 & Yes \\
\hline
\end{tabular}

Figure 25: Female E working memory results with and without the dog present and key

Without the dog present, Female E's picture naming at speed dramatically slowed for the second picture card giving her a well below average score for her age (SS 69), yet her digit naming gave an average score (SS 93). When the dog was present, Female E showed great improvement between the score for her picture naming 
speed (SS 93), but not with the digits (SS 89). Rapid processing and labelling, particularly with pictures (automisation) appears difficult for Female E, possibly through cognitive overload and difficulties in accurate speech production. The shortterm auditory recall with the dog, improved the forwards letters span into the above average range (SS 120), yet reduced the original digits forward span into the low average range (SS 89). Female A's executive skills, visuo-spatial reasoning and span were improved with the presence of the dog especially with the letters (SS120).

\subsubsection{EDA Readings with and without dog present}

The averaged electrodermal activity readings for both reading sessions with the dog present and without for Female E are below in Table 44.

\section{Table 44: Averaged EDA readings for Female $E$}

\begin{tabular}{|l|l|l|l|l|}
\hline Name & & $\begin{array}{l}\text { Sum of SC } \\
\text { increments } \\
\text { (micro- } \\
\text { siemens) }\end{array}$ & $\begin{array}{l}\text { Sum of time } \\
\text { differences } \\
\text { (seconds) }\end{array}$ & $\begin{array}{l}\text { EDA Positive } \\
\text { Changes (micro- } \\
\text { siemens / } \\
\text { second) }\end{array}$ \\
\hline Female E & Dog & 97.73529092 & 325.045 & 0.300682339 \\
\hline & No Dog & 173.6567263 & 1621.269 & 0.107111606 \\
\hline
\end{tabular}

(Green shows the higher variance of EDA changes)

The higher EDA positive changes score for Female E showed that the presence of the dog was affecting her through arousal, but as shown in the graph below, she could control this reaction, possibly providing greater focus. 


\subsubsection{EDA Box and Whisker Charts for all three tasks}

Female E's Comparisons in electrodermal activity for the three tasks of reading a rhyme, reading to an adult without a dog present and reading with a dog present are shown in Figure 26 below:

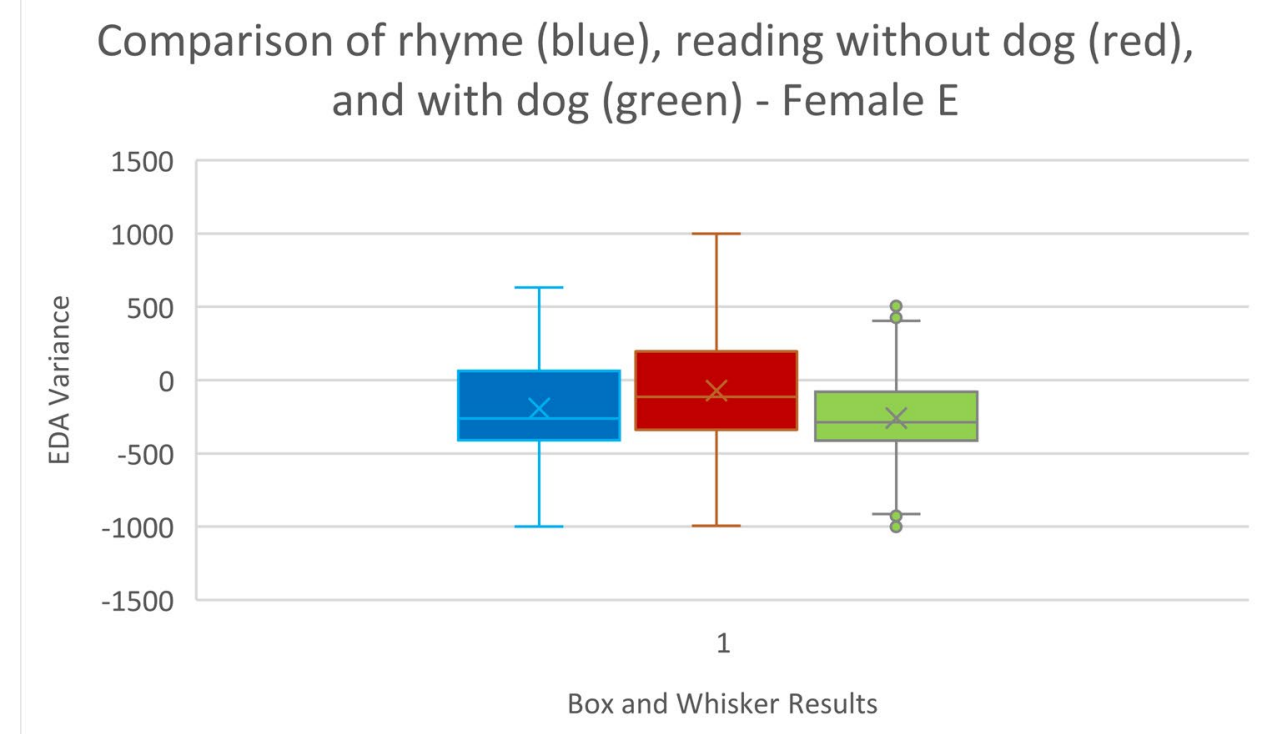

Figure 26: Female E's EDA comparisons between the three tasks

The comparative measures from the box and whisker charts for Female $E$ are in Table 45 below.

Table 45: Female E comparative measures for box and whisker chart

\begin{tabular}{|l|l|l|l|}
\hline & Overall Range & Quartile Range & Median \\
\hline Rhyme without dog & 1633 & 473 & -261 \\
\hline Read without dog & 1933 & 537 & -144 \\
\hline Read with dog & 1320 & 334 & -288 \\
\hline
\end{tabular}

For Female $\mathrm{E}$, the dog's presence reduced the both the overall range of variance, and quartile range in comparison to reading the poem and reading to the adult only. This intensity may suggest Female $\mathrm{E}$ had greater focus and concentration, with the dog, 
although there were momentary outliers and distractions. The difference of only 100 between the rhyme and read with dog medians does suggest that for Female $\mathrm{E}$ reading the poem, while producing less arousal than reading the book, was on a similar stress level to reading to the dog. This would imply that for Female $E$ the greatest physiological reactions were to the reading to the adult and that she may have found that task more difficult to complete. This could indicate that for Female $\mathrm{E}$, while she may be a fluent reader, there may be some differences between the styles of texts and her own awareness of her response, possibly through visual and speech differences.

The "real time effects" can be further revealed in the graphs for the first two minutes of reading:

\subsubsection{Minute graphs comparison}

Female E's Pip graphs of the first two minutes of reading are shown in Figures 27 and 28 below.

(Blue - without Dog, Orange - with dog)

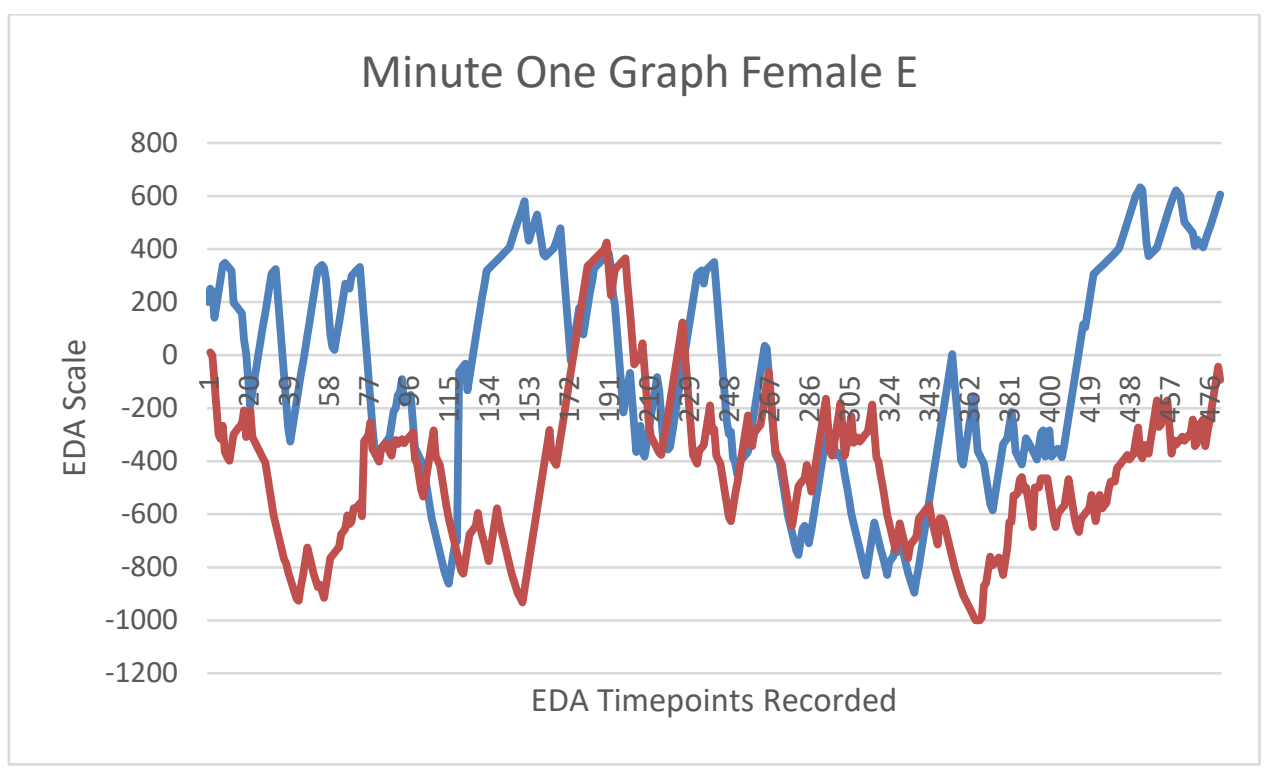




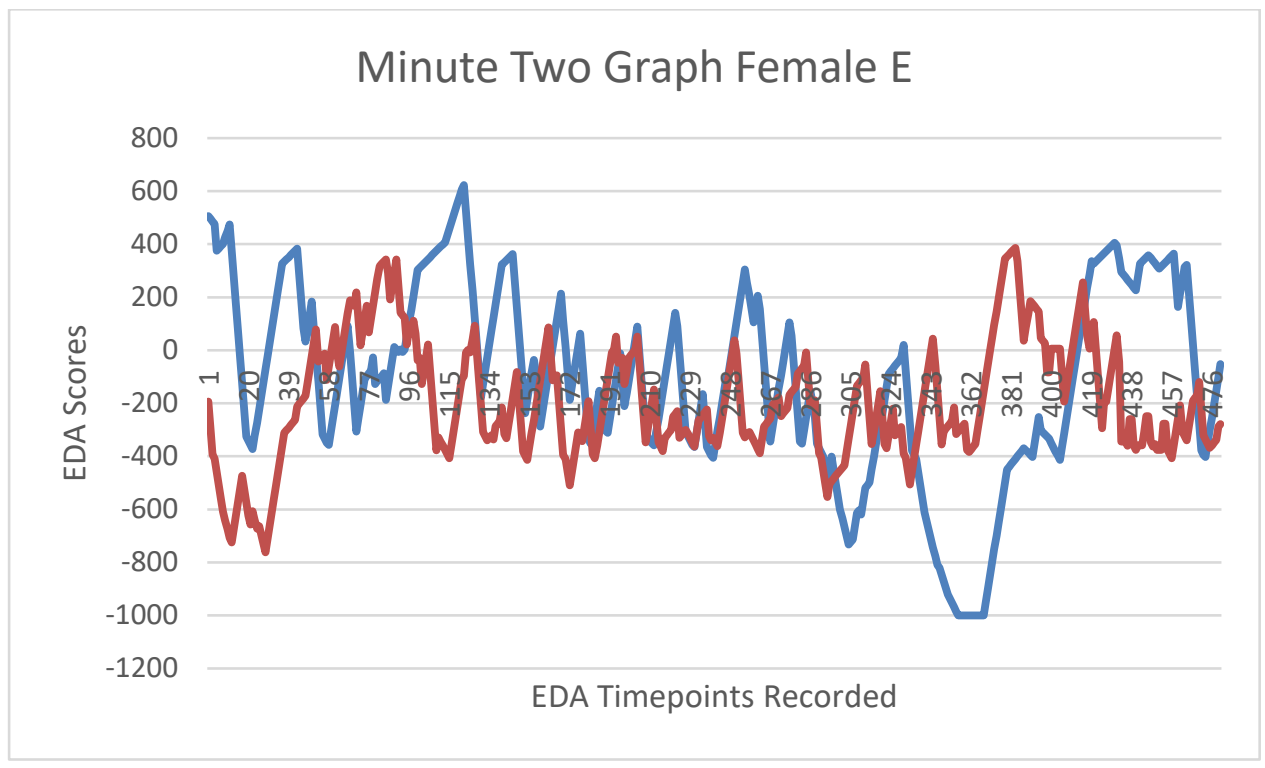

Figure 28: Female E's Pip 'real time' graph for minute two

Female E shows that during the first minute of reading there was a greater variance in EDA changes while the dog was not present. This could show that she was anxious about reading in general, but while the dog was present, she was able to regulate this. Looking at the minute graphs, the intensity of the variance is more acute in the dog present setting.

Overall, the dog suggests a calming influence, which in turn, would imply an improvement in concentration and memory for comprehension. This should be shown in Female E's overall accuracy and speed rates.

\subsubsection{Reading Accuracy and Words Per Minute (WPM) rate}

Female E's reading accuracy and speed are shown in table 46 below

Table 46: Female E Reading speed and accuracy

\begin{tabular}{|l|l|l|}
\hline Reading Rhyme (No dog) & $\begin{array}{l}\text { Reading Book (No dog) } \\
\text { 1.9 Reading Level }\end{array}$ & $\begin{array}{l}\text { Reading Book (with dog) } \\
\mathbf{2 . 0} \text { Reading Level }\end{array}$ \\
\hline $92.8 \%$ Accuracy & $96.4 \%$ Accuracy & $96.7 \%$ \\
\hline
\end{tabular}


The words per minute rate in Table 46 shows that overall, the poem had the most speed and fluency, yet a lower rate for accuracy for Female E. The dog's presence when reading gave the most accuracy although Female E slowed down her reading to the dog, in comparison to the poem. For Female E, however, the dog's presence has hardly made a difference to her accuracy rate in reading her book to an adult, but the recordings showed that while reading to the dog, Female E's speech was clearer in general. Female $\mathrm{E}$ also slowed down her speech when reading to the adult, but as a fluent reader, she may have been asked to do this when reading back in class.

\subsubsection{Reading Attainment in both reading age and standardised scores}

Female E's overall reading attainment, both in reading age and standardised scores are shown in tables 47 and 48 below:

Table 47: Overall reading attainment for Female $E$

\begin{tabular}{|l|l|l|l|l|l|l|l|}
\hline Name & Chron Age & $\begin{array}{l}\text { Salford } \\
\text { Age } \\
\text { Start }\end{array}$ & $\begin{array}{l}\text { Difference } \\
\text { between }\end{array}$ & $\begin{array}{l}\text { Chron } \\
\text { Age End }\end{array}$ & $\begin{array}{l}\text { Salford } \\
\text { Age End }\end{array}$ & $\begin{array}{l}\text { Difference } \\
\text { between }\end{array}$ & Ratio Gain \\
\hline Female E & $7: 07$ & $8: 11$ & $+16 \mathrm{~m}$ & $7: 09$ & $9: 02$ & $+17 \mathrm{~m}$ & $+1 \mathrm{~m}$ \\
\hline
\end{tabular}

Table 48: Standardised scores attainment for Female E

\begin{tabular}{|l|l|l|l|l|}
\hline & $\begin{array}{l}\text { Standardised Score } \\
\text { Start }\end{array}$ & Percentile Start & $\begin{array}{l}\text { Standardised } \\
\text { Score End }\end{array}$ & Percentile End \\
\hline Female E & 117 & 87 & 119 & 90 \\
\hline
\end{tabular}

Overall, Female E's final reading age was still almost 18 months above her chronological age throughout the 12-week project, despite only attaining a ratio gain 
of 1 or having 'no significant impact' (Brookes, 2007). This was not recognised by her teachers. Female E showed the skills of an advanced reader, clearly comprehending the story and was a fluent reader. It is possible that she was not being adequately challenged with these levels of texts.

\subsubsection{Summary for Female E}

For Female $E$, reading to the dog appeared to allow her to focus, concentrate and reduce distractions. When asked, "Which do you prefer, reading to the teacher or reading to the dog?" she answered immediately, "Reading to the dog - it's easier!" clearly showing she was clearly motivated by the opportunity of working with him, but it was questionable as to the necessity of adding the dog for academic attainment and progress other than for enjoyment.

\subsubsection{Female A}

\section{(Age during project: seven years, nine months to eight years)}

\subsubsection{Introduction and results from questionnaire}

Female A's family owned a dog which in subsequent conversation, did not appear too friendly at home. Therefore, Female A did not read to him. Female A considered herself good at art and reading, although she admitted that she found the latter "hard." She gave herself the 'ok' emoji for reading, but a 'happy' emoji for maths. Female A said she would like to read to the dog, even though she felt, "a bit nervous," and she thought that he would enjoy being read to. Female $A$ suggested that she could help the dog by spelling out the words for him and him, in turn could help her by pointing out the words with his paw - thus Female A was associating the dog with human qualities. 
When reviewing her reading videos, however, although Female A said she was very happy to be with the dog, as soon as she started reading, her body language changed to closed postures with nervous tweaking of hair, and her voice becoming quieter and more hesitant. When finished, Female A immediately began to stroke and pat the dog, her voice became a normal level and speed. While reading she had showed no interaction or acknowledgement of the dog at all. This would suggest that it is the task of reading aloud, regardless of whether a dog or adult is present, which causes Female A stress.

\subsubsection{Results from non-repeated working memory tests, due to time}

The working memory tests which could not be completed with the dog due to time for Female A are shown below in table 49.

Table 49: Female A Standardised score for working memory tests unable to be repeated with the dog present

\begin{tabular}{|l|l|l|l|l|l|l|}
\hline Name & $\begin{array}{l}\text { Memory } \\
\text { for Story }\end{array}$ & $\begin{array}{l}\text { Memory } \\
\text { for Story } \\
\text { delayed }\end{array}$ & $\begin{array}{l}\text { Fluency - } \\
\text { Semantic }\end{array}$ & $\begin{array}{l}\text { Fluency - } \\
\text { Alliteration }\end{array}$ & $\begin{array}{l}\text { Fluency } \\
\text { - Rhyme }\end{array}$ & $\begin{array}{l}\text { Visual } \\
\text { Memory }\end{array}$ \\
\hline $\begin{array}{l}\text { Female } \\
\text { A }\end{array}$ & SS110 & SS80 & SS106 & SS87 & SS81 & SS75 \\
\hline
\end{tabular}

(Tests taken at age seven years, nine months)

Female $A$ shows what is known in the specific learning difficulty training world as a 'spiky' profile, in that her strengths and weaknesses from these working memory tests showed no overall consistency. While Female A had an average score for the 'Memory for Stories' test (SS 110) involving immediate auditory recall, she scored a low average score (SS 80 ) when she tried to retell the facts and details 30 minutes later. Female A could easily list things to eat and animals for the semantics fluency 
test with an average score (SS 106) but then had some difficulties recalling any items beginning with the letter ' $b$ ' or ' $m$ ' only, just giving three examples in the 30 second time periods. During the rhyme section, Female A gave phonetically different endings to the word 'whip,' such as 'pick.' Female A also appeared to have some issues with accurate long-term memory recall for visual memory designs, particularly with shapes, rotation and orientation. Female A's visual memory test gave a below average standardised score (SS75). The nature of these findings would suggest that Female A would also be struggling in class with accurate letter and phoneme recall, reading and processing texts, overall fluency, spelling and writing tasks.

\subsubsection{Working Memory with and without dog present}

Female A's working memory tests with and without the dog present are given in Figure 29 below

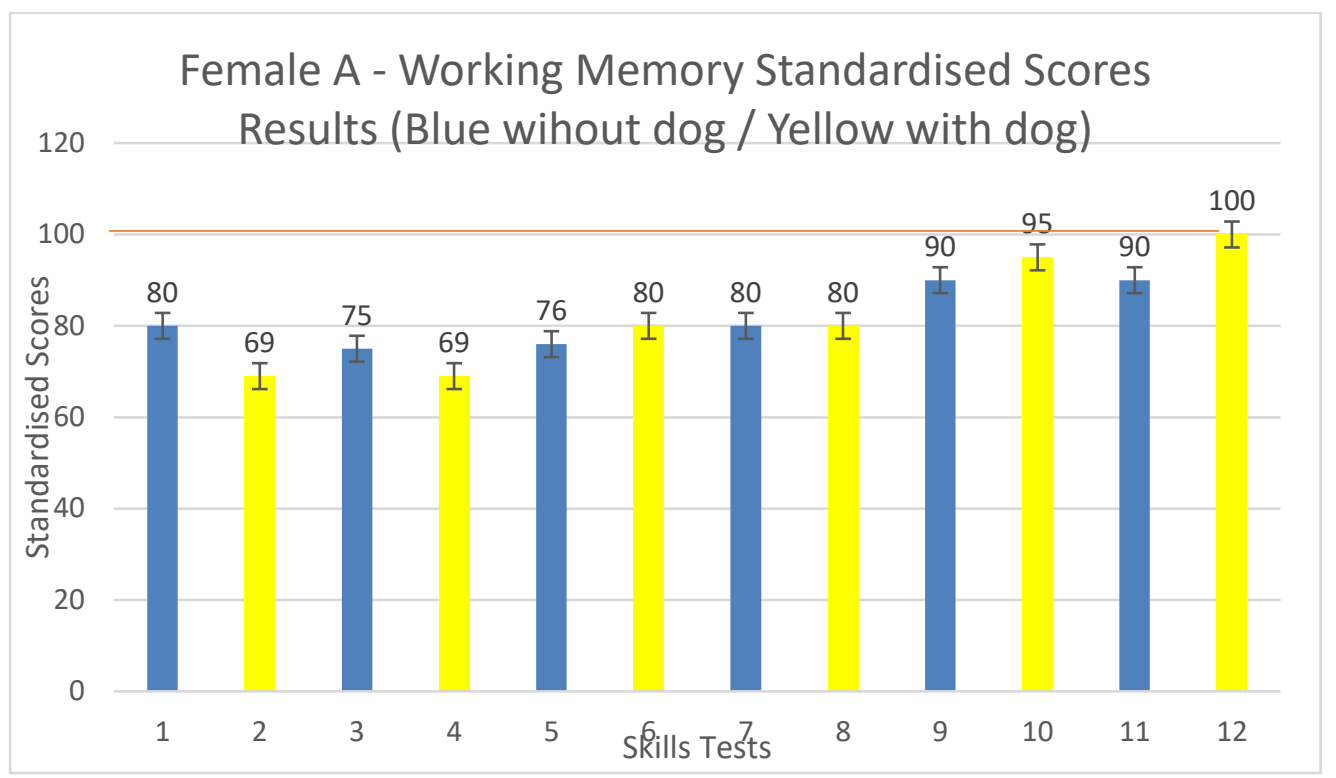

Key to skills tests:

\begin{tabular}{|l|l|l|l|l|l|}
\hline Skill & & 1 & Naming Picture Speed no dog & 80 & \\
\hline & & 2 & Naming Picture speed with dog & 69 & \\
\hline
\end{tabular}




\begin{tabular}{|l|l|l|l|l|l|}
\hline $\begin{array}{l}\text { Processing } \\
\text { Speed }\end{array}$ & & 3 & Naming speed digits no dog & 75 & \\
\cline { 2 - 6 } & & 4 & Naming speed digits with dog & 69 & \\
\hline $\begin{array}{l}\text { Auditory } \\
\text { Short-Term } \\
\text { Memory }\end{array}$ & & 5 & Digits forward no dog & 76 & \\
\cline { 2 - 6 } & & 6 & Digits forward with dog & 80 & \\
\cline { 2 - 6 } & 7 & Letters forward no dog & 80 & \\
\cline { 2 - 6 } & & 8 & Letters forward with dog & 80 & Same \\
\hline $\begin{array}{l}\text { Executive } \\
\text { Skills } \\
\text { Working } \\
\begin{array}{l}\text { Memory } \\
\text { Span }\end{array}\end{array}$ & 9 & Digits backward no dog & 90 & \\
\cline { 2 - 6 } & 10 & Digits backward with dog & 95 & \\
\cline { 2 - 6 } & 11 & Letters backward with dog & 90 & \\
\cline { 2 - 6 } & 12 & Letters backward with dog & 100 & \\
\hline
\end{tabular}

\section{Figure 29: Female A's working memory results with and without the dog present and key}

Female A's baseline scores for naming pictures and digits at speed were already considered below average for her age (SS 80 and SS75). Female A scored lower for the naming speed pictures and digits with the dog present. This could reflect her anxiety and nervousness with the dog, but the results could also be due to possible visual recall difficulties at speed or automisation. Her standardised scores for the skills of auditory short-term memory were also below average in comparison to others her age (SS 76 and SS 80). The presence of the dog did make a slight, positive, difference for the phonological single letters task, but all her scores still remained within the low average band (SS 70-84). The scores for Digits and letters backwards (traditionally a standard test for working memory) has improved with the dog's presence and shows average standardised scores (SS 90-110). This shows that her executive functioning skills are improved with the presence of the dog. 


\subsubsection{EDA Readings with and without dog present}

The averaged electrodermal activity readings for the reading sessions with the dog present and without for Female A are below in Table 50.

Table 50: Averaged EDA readings for Female A

\begin{tabular}{|l|l|l|l|l|}
\hline Name & & $\begin{array}{l}\text { Sum of SC } \\
\text { increments } \\
\text { (micro- } \\
\text { siemens) }\end{array}$ & $\begin{array}{l}\text { Sum of time } \\
\text { differences } \\
\text { (seconds) }\end{array}$ & $\begin{array}{l}\text { EDA Positive } \\
\text { Changes (micro- } \\
\text { siemens / } \\
\text { second) }\end{array}$ \\
\hline Female A & Dog & 19.87325008 & 281.549 & 0.070585405 \\
\hline & No Dog & 34.23405458 & 487.245 & 0.070260453 \\
\hline
\end{tabular}

Overall Female $A$ has shown that the averaged positive EDA changes with and without the dog present are very similar. This similarity in physiological responses suggests difficulties with the task rather than being affected by the dog's presence.

\subsubsection{EDA Box and whisker charts for all three tasks}

Female A's comparisons electrodermal activity for the three tasks of reading a rhyme, reading to an adult without a dog present and reading with a dog present are shown in Figure 30 below 


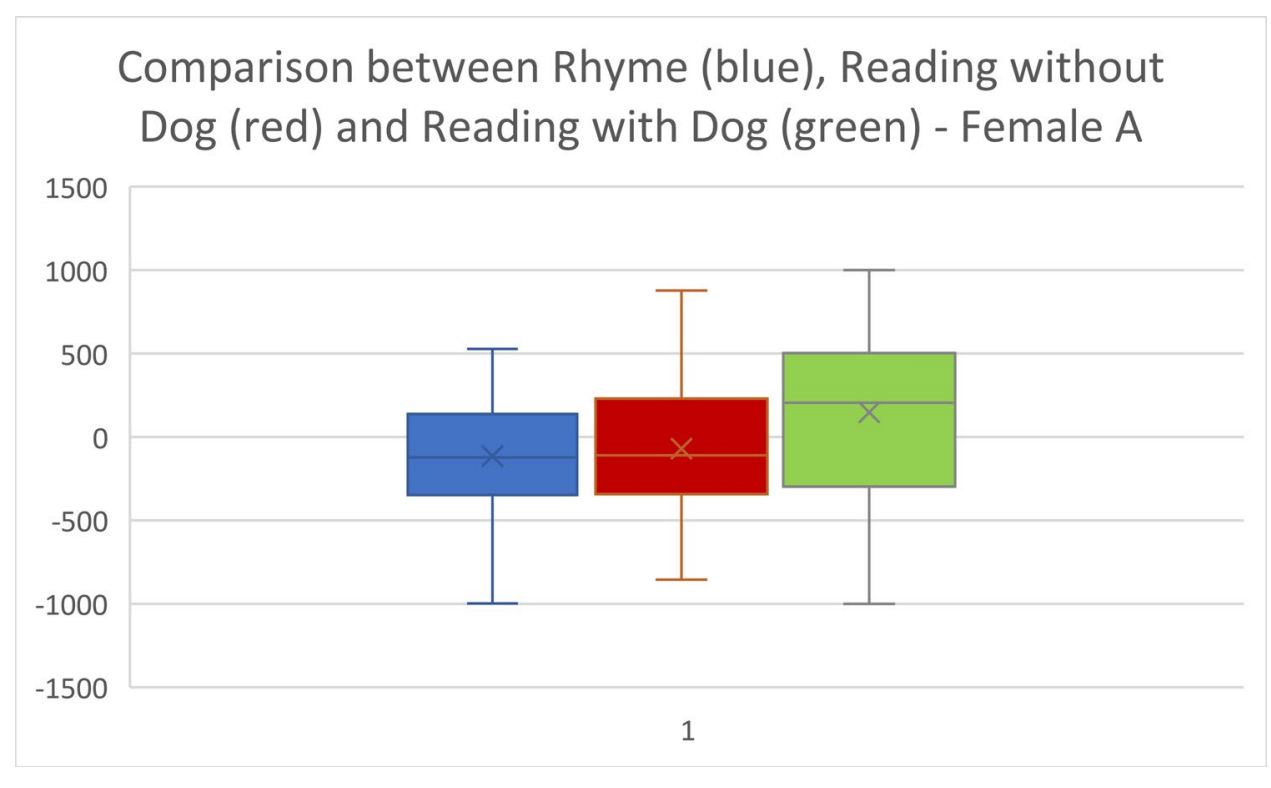

Figure 30: Female A's EDA comparisons between the three tasks

The comparative measure from the box and whisker charts for Female $A$ are in Table 51 below.

Table 51: Female E Comparative measures for the box and whisker charts

\begin{tabular}{|l|l|l|l|}
\hline & Overall Range & Quartile Range & Median \\
\hline Rhyme without dog & 1525 & 485 & -121 \\
\hline Read without dog & 1732 & 573.5 & -109 \\
\hline Read with dog & 2000 & 800 & +205 \\
\hline
\end{tabular}

The range of each task shown in Figure 30 shows that the greatest variance for Female $A$ is in the read with dog situation. The smaller differences of both the quartile ranges and medians for the poetry and the reading would possibly show that she was focusing on the tasks and found them stressful, although familiar to her. The range and median for the dog shows that she was possibly highly aroused, either anxious or excited to read to the dog which may have affected her attainment. This can be clearly observed in the minute graphs below. 


\subsubsection{Female A Minute Graphs Comparison}

The 'real time' effects can be viewed in the Pip graphs for the first two minutes of reading are seen in Figures 31 and 32 below.

(Blue - without Dog, Orange - with dog)

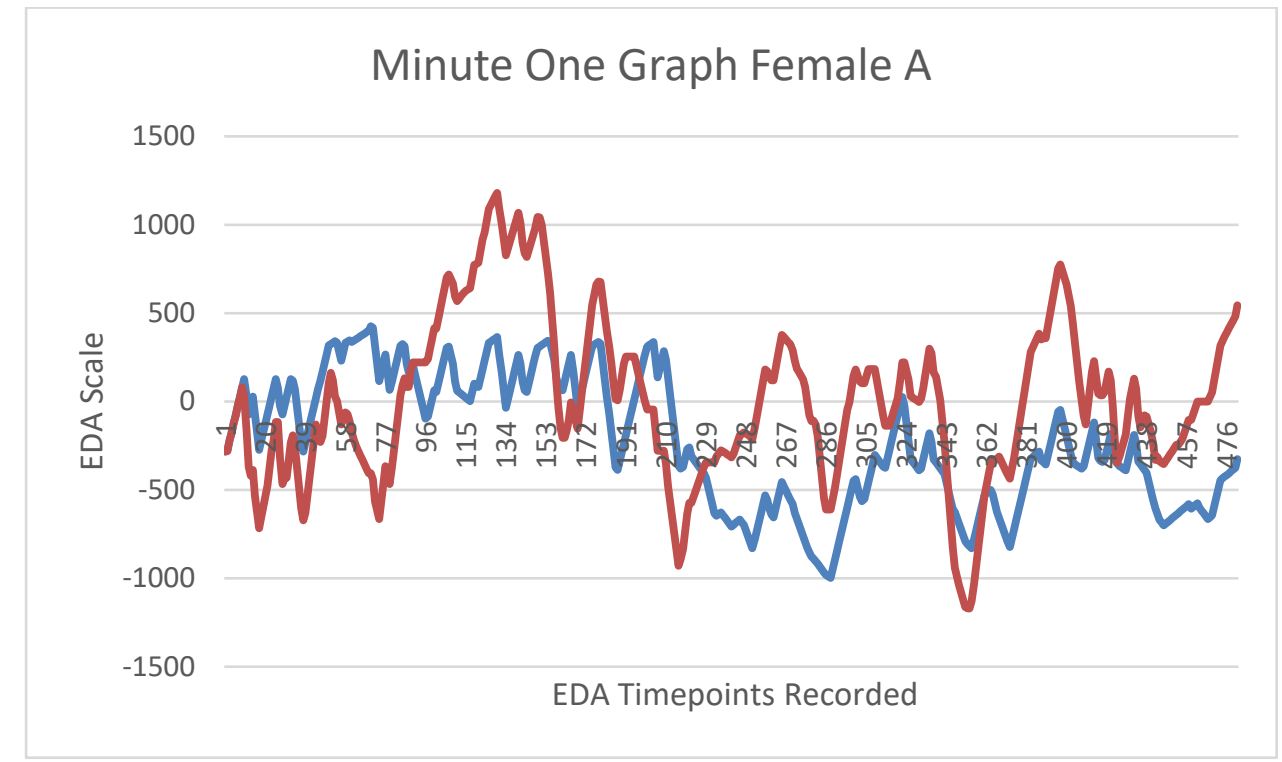

Figure 31: Female A's Pip 'real time' graph for minute one

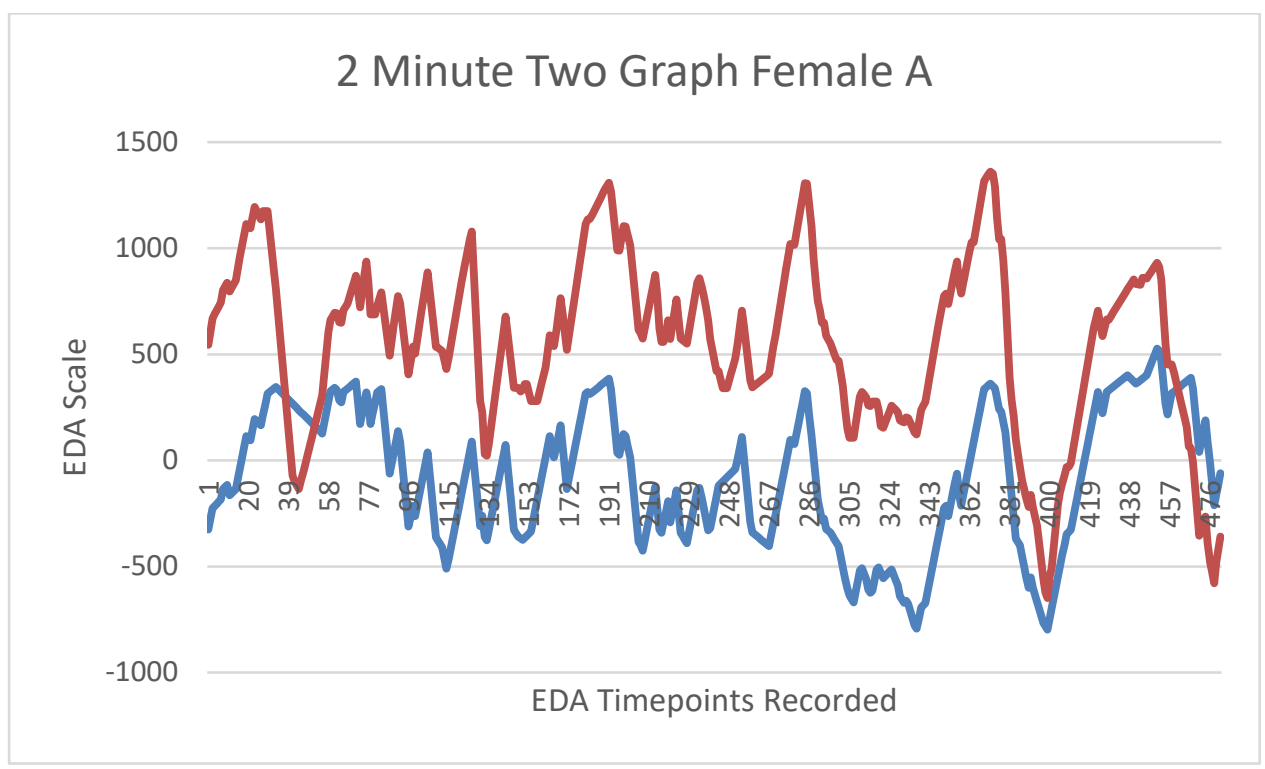


Figure 32: Female A's Pip 'real time' graph for minute two

Female A's minute graphs reflect that the dog's presence was affecting her physiologically, but by the second minute the variance had reduced. Overall, the rate for the dog's presence remained higher than that with the adult only.

\subsubsection{Reading Accuracy and Words per Minute (WPM) rate}

The accuracy and speed effects of Female A's arousal response are shown in Table 52 below

Table 52: Female A Reading accuracy and words per minute rate

\begin{tabular}{|l|l|l|}
\hline Reading Rhyme (No dog) & $\begin{array}{l}\text { Reading Book (No dog) } \\
\text { Reading Level 0.7 }\end{array}$ & $\begin{array}{l}\text { Reading Book (with dog) } \\
\text { Reading Level 2.2 }\end{array}$ \\
\hline $\begin{array}{l}\text { 65.17\% Accuracy } \\
26.5 \mathrm{wpm}\end{array}$ & $\begin{array}{l}100 \% \text { Accuracy } \\
47 \mathrm{wpm}\end{array}$ & $\begin{array}{l}86 \% \text { Accuracy } \\
33 \mathrm{wpm}\end{array}$ \\
\hline
\end{tabular}

Female $A^{\prime}$ s words per minute rate and accuracy scores reflected that she is not confident in her own performance and has difficulties in recognising and blending phonemes together, particularly in longer syllabic words. The poem, reflecting all the phonemes which should have been learned in Year 2, did give her some challenges; she misread certain sounds leading to difficulties in overall word comprehension. The time taken to distinguish the correct phoneme recall also meant that she had forgotten the whole word or the meaning of the entire sentence. She required a lot of teacher prompting and encouragement, and when given the word, would not recognise it when it was repeated. She also did not distinguish the meter or rhyme. 
It is very likely that she did not pass her phonics check at the end of Year 1 or Year 2 and that this child may have a developmental difference such as dyslexia. Due to the levels of difficulty she was having, her immediate results were discussed with the school SENCO.

By the end of the 12-week project, her teachers had placed her back on the lower book levels to increase her fluency, thus her reading without the dog book was at 0.7 , rather than at 2.2 with the dog. This change was meant with great intentions, in that through the success of reading all the words fluently she would increase her selfesteem and confidence in her reading skills. Female A, however, did not seem happy with this, despite acknowledging that she finds reading difficult. She had previously read all the books at that level and considered them, "babyish." Perhaps an alternative 'catch up' reading scheme would have been a more suitable alternative to motivate Female A.

\subsubsection{Reading Attainment in both reading age and standardised scores}

Female A's overall reading progress is recorded in Tables 53 and 54 below

Table 53: Female A Reading attainment in reading age

\begin{tabular}{|l|l|l|l|l|l|l|l|}
\hline Name & Chron Age & $\begin{array}{l}\text { Salford } \\
\text { Age Start }\end{array}$ & $\begin{array}{l}\text { Difference } \\
\text { between }\end{array}$ & $\begin{array}{l}\text { Chron } \\
\text { Age End }\end{array}$ & $\begin{array}{l}\text { Salford } \\
\text { Age End }\end{array}$ & $\begin{array}{l}\text { Difference } \\
\text { between }\end{array}$ & Ratio Gain \\
\hline Female A & $7: 09$ & $6: 04$ & $-15 \mathrm{~m}$ & $8: 00$ & $7: 06$ & $-6 \mathrm{~m}$ & +4.6 \\
\hline
\end{tabular}

Table 54: Female A Reading Attainment in Standardised Scores

\begin{tabular}{|l|l|l|l|l|}
\hline & $\begin{array}{l}\text { Standardised Score } \\
\text { Start }\end{array}$ & Percentile Start & $\begin{array}{l}\text { Standardised } \\
\text { Score End }\end{array}$ & Percentile End \\
\hline Female A & 87 & 20 & 94 & 34 \\
\hline
\end{tabular}


Overall, according to the Salford Reading Tests, Female A did make progress throughout the project's time, achieving 'good' progress (Brooks, 2007). Again, this is not entirely down the presence of the dog. She was also assessed by the Educational Psychologist during this period and then received 1:1 extra tuition for reading and literacy skills from a trained teacher in specific learning difficulties including increased practice from home. Female A's end of the 12-week project showed that she was now within the standardised scores for her age. It is to be noted, however, that the Salford Reading Test is not a timed assessment and Female A would have completed this in her own time, thus her 'average reading score' does not consider the fact that she would not truly be able to keep up with her peers within the classroom setting.

\subsubsection{Summary for Female A}

For Female $A$, the presence of the dog was a comforter which may have helped with her own self confidence for the session. Evidence from her body language and performance in all three tasks showed that it was the activity of reading itself which caused her great anxiety. Had just the Salford Reading Test results just been used to track her progress across the school, this child may not have been identified as having a specific learning difference or need. This project did help the school to identify her.

\subsubsection{Male $\mathrm{M}$}

(Age during the project: seven years, three months to seven years, six months)

\subsubsection{Introduction and results from questionnaire}

Male $\mathrm{M}$ had a cat at home, to which he said he sometimes read. In school he said he was good at quizzes on the computer and Maths, Science and French. He liked 
reading and maths, giving himself both smiling emojis for his own competence. He thought that reading to the dog made him feel "pretty good" as the dog was kind. The dog apparently 'liked' his reading as Male M could read very well. He could help the dog to read by saying the word as he read the word (splitting into phonemes) and that the dog could help him by giving him, "perseverance" (which was the assembly word for that day.) At this point Male $M$ was still equating the dog with human like qualities.

While reading, however, Male $\mathrm{M}$ showed no interaction with the dog, with full focus on the task. Only when he had been asked to stop did he then look at the dog, giving him full eye contact and a stroke to the head. He happily gave the dog the biscuit and another stroke, but only at the handler's request. Male M showed no spontaneous interaction with the dog throughout the session. The dog's own behaviour showed more interest in the handler and biscuits than the child.

\subsubsection{Results from the non-repeated working memory tests, due to time}

The working memory tests which were unable to be repeated due to time are shown in Table 55 below.

Table 55: Male M Standardised Scores for working memory tests unable to be completed with dog present

\begin{tabular}{|l|l|l|l|l|l|l|l|}
\hline Name & $\begin{array}{l}\text { Chron. } \\
\text { age }\end{array}$ & $\begin{array}{l}\text { Memory } \\
\text { for Story }\end{array}$ & $\begin{array}{l}\text { Memory } \\
\text { for Story } \\
\text { delayed }\end{array}$ & $\begin{array}{l}\text { Fluency - } \\
\text { Semantic }\end{array}$ & $\begin{array}{l}\text { Fluency - } \\
\text { Alliteration }\end{array}$ & $\begin{array}{l}\text { Fluency } \\
\text { - Rhyme }\end{array}$ & $\begin{array}{l}\text { Visual } \\
\text { Memory }\end{array}$ \\
\hline Male M & $7: 02$ & SS100 & SS100 & SS119 & SS115 & SS73 & SS95 \\
\hline
\end{tabular}


Male $\mathrm{M}$ had arrived in the UK just before he started school at 4 years old and was raised and encouraged bilingually in English and a West Slavic language. He could easily read, spell and write in English, but still had some difficulties in initiating rhyme, despite recognising it when he heard it. This was shown in his standardised score of 73 which is classed as below average for his peers. Male $M$ showed average skills in the retention of auditory information given as a story and could accurately repeat features of the given information after 30 minutes. His fluency scores for semantic knowledge and alliteration were also of high average when compared to his peers and he also had a good visual memory for patterns and shapes (SS 95).

\subsubsection{Working Memory with and without dog present}

Male M's working memory test results with and without the dog present are given in

Figure 33 below

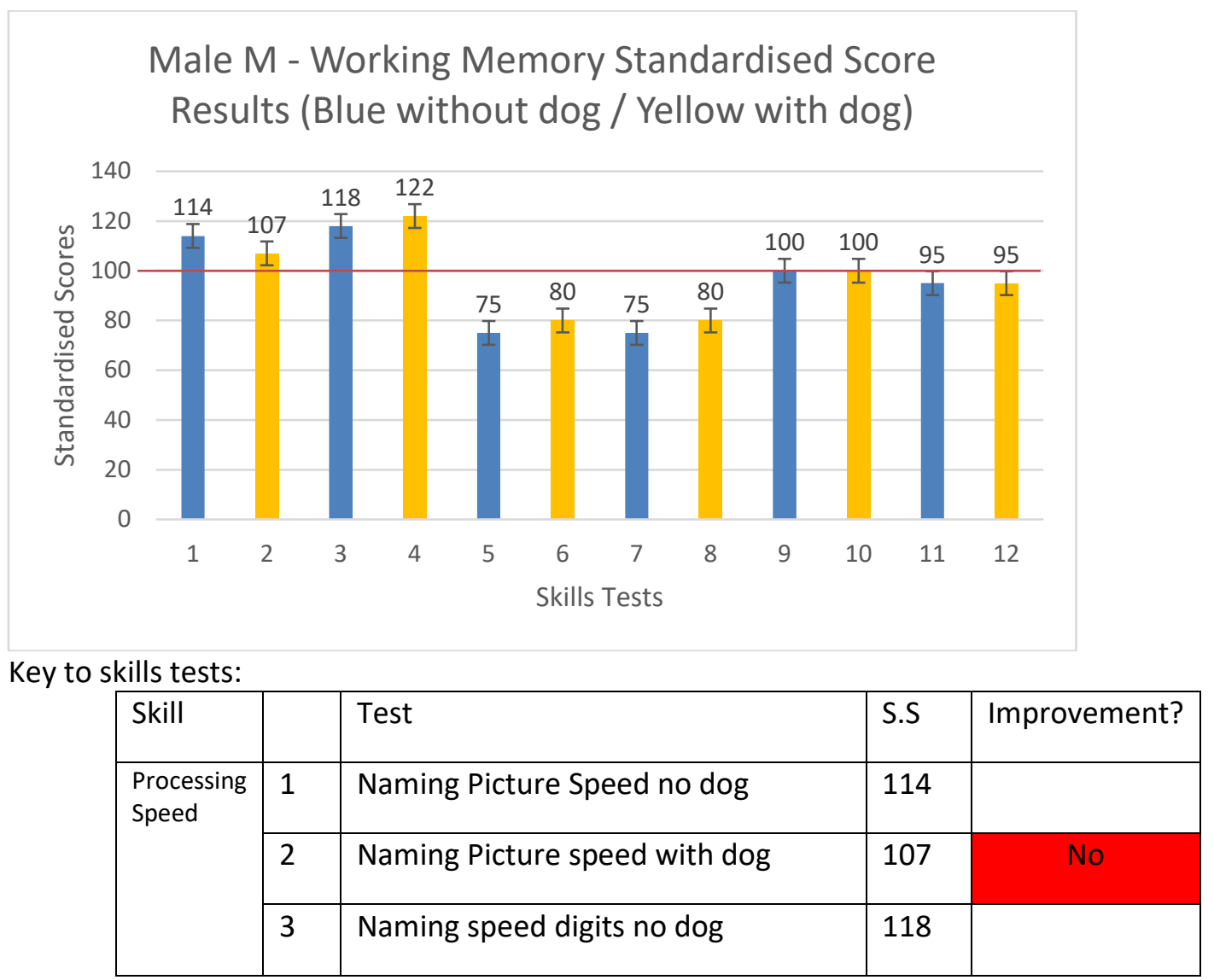




\begin{tabular}{|c|c|c|c|c|}
\hline & 4 & Naming speed digits with dog & 122 & Yes \\
\hline \multirow{4}{*}{$\begin{array}{l}\text { Auditory } \\
\text { Short } \\
\text { Term } \\
\text { Memory }\end{array}$} & 5 & Digits forward no dog & 75 & \\
\hline & 6 & Digits forward with dog & 80 & Yes \\
\hline & 7 & Letters forward no dog & 75 & \\
\hline & 8 & Letters forward with dog & 80 & Yes \\
\hline \multirow{4}{*}{$\begin{array}{l}\text { Executive } \\
\text { skills and } \\
\text { Working } \\
\text { Memory } \\
\text { Span }\end{array}$} & 9 & Digits backward no dog & 100 & \\
\hline & 10 & Digits backward with dog & 100 & Same \\
\hline & 11 & Letters backward with dog & 95 & \\
\hline & 12 & Letters backward with dog & 95 & Same \\
\hline
\end{tabular}

Figure 33: Male M's working memory results with and without the dog present and key

Male M showed that when naming picture icons at speed, the dog's presence did not help on this occasion, yet the dog did assist for processing digits at speed, with an above average standardised score attained (SS 122). His standardised scores for processing were all well above average (SS 100) perhaps reflecting that he uses these skills simultaneously during conversations as a bi-lingual speaker. In these tests, Male M's scores for both digits and letters forwards showed low average results for auditory short-term memory skills, yet with the dog's presence he was more accurate (SS 80). These scores however were still below average standard in comparison to his peers. This may reflect that he prefers to see words, letters and phonemes 'visually' rather than auditorily. The Memory for stories tests above, which Male M completed to an average standard score (SS100), used visual imagery. The direct auditory repetition of letters, common to a digit forward span, may affect his general understanding of classroom instructions. The dog's presence made no difference with the tests for classical working memory and executive skill span showing average scores for Male M. 


\subsubsection{Electrodermal Activity Readings with and without dog present}

The averaged electrodermal activity readings for the reading sessions with and without the dog present for Male M are shown in below in Table 56.

Table 56: Male M Averaged EDA readings

\begin{tabular}{|l|l|l|l|l|}
\hline Name & & $\begin{array}{l}\text { Sum of SC } \\
\text { increments } \\
\text { (micro- } \\
\text { siemens) }\end{array}$ & $\begin{array}{l}\text { Sum of time } \\
\text { differences } \\
\text { (seconds) }\end{array}$ & $\begin{array}{l}\text { EDA Positive } \\
\text { Changes (micro- } \\
\text { siemens / } \\
\text { second) }\end{array}$ \\
\hline Male M & Dog & 74.91521025 & 350.515 & 0.231728971 \\
\hline & No Dog & 53.81541017 & 276.480 & 0.194644857 \\
\hline
\end{tabular}

(Green shows the higher variance of EDA changes)

Overall Male $\mathrm{M}$ showed that the dog's presence did affect him through slightly

higher arousal when reading.

\subsubsection{EDA Box and Whisker charts for all three tasks}

Male M's comparisons in electrodermal activity for the three tasks of reading rhyme, reading to an adult without a dog present and reading with a dog present are shown in Figure 34 below: 


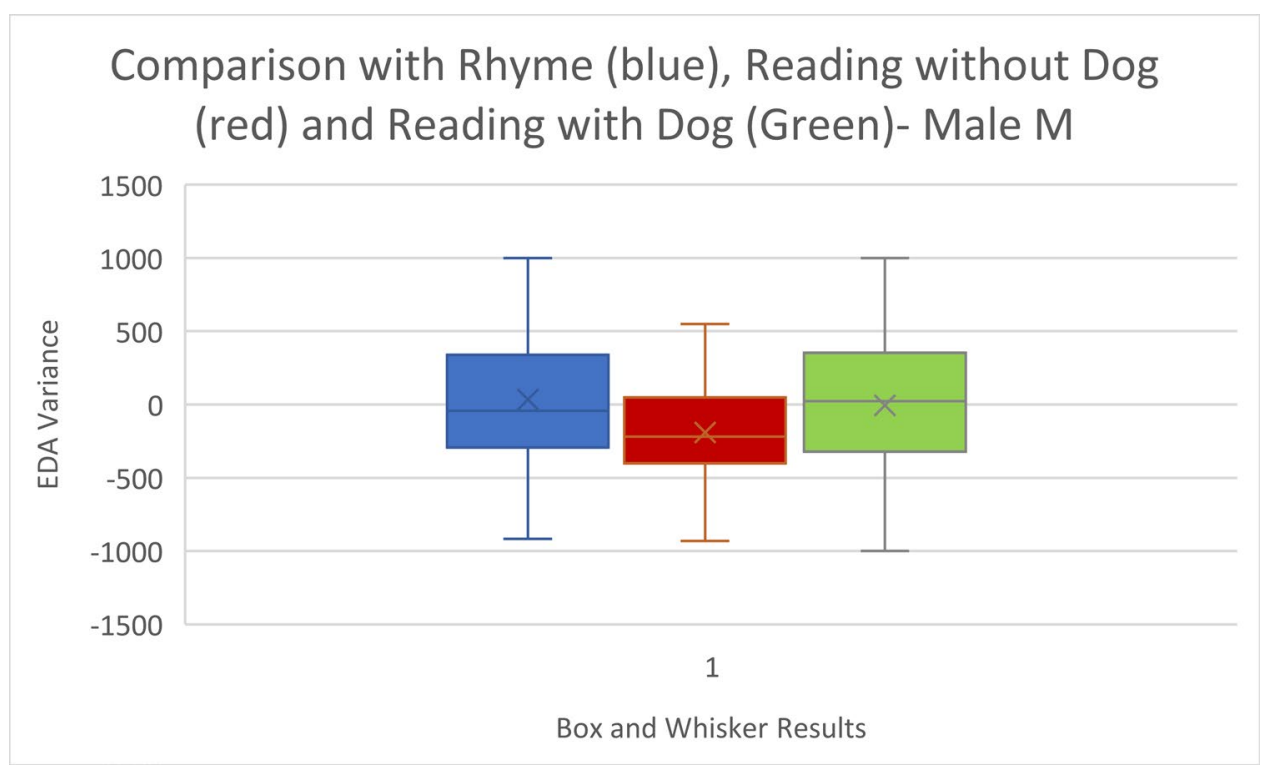

Figure 34: Male M's EDA comparisons between the three tasks

The comparative measures from the box and whisker charts for Male $\mathrm{M}$ are in Table 57 below:

Table 57: Male M Comparative measures for box and whisker chart

\begin{tabular}{|l|l|l|l|}
\hline & Overall Range & Quartile Range & Median \\
\hline Rhyme without dog & 1916 & 631.5 & -43.5 \\
\hline Read without dog & 1482 & 452 & -219 \\
\hline Read with dog & 2000 & 621 & +24 \\
\hline
\end{tabular}

The results from Figure 34 and Table 57 suggest that Male $M$ found both the poem and the presence of the dog more arousing than the reading on his own. The similarities in the overall ranges, quartile ranges and medians indicate that the arousal was comparable for both the poem and the dog's presence. In contrast, reading to the adult without a dog showed a reduced range and quartiles, which may represent greater focus and concentration, as the situation of reading to an adult was more familiar to Male $\mathrm{M}$. 


\subsubsection{Male M Minute graph comparisons}

The 'real' time effects can be viewed in the Pip graphs for the first two minutes of reading as seen in Figures 35 and 36 below.

(Blue - without dog, orange with dog)

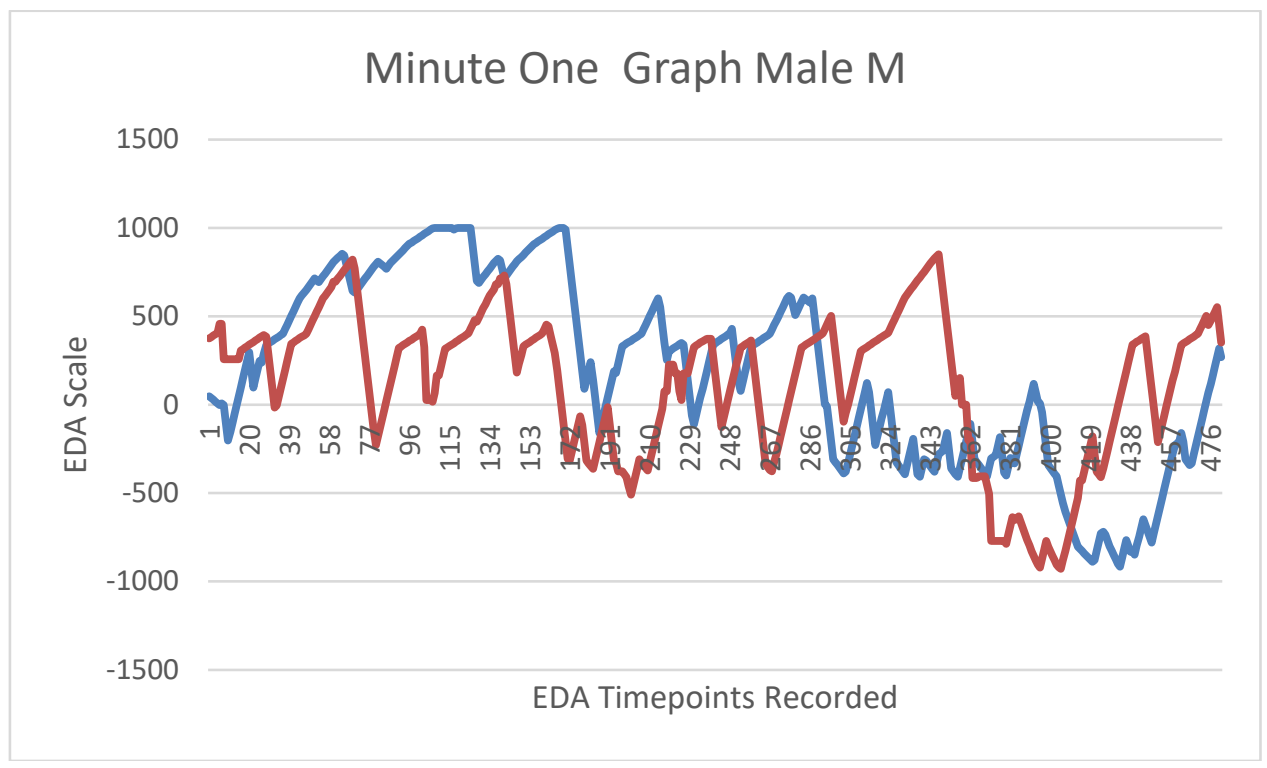

Figure 35: Male M Pip 'real time' graph for minute one

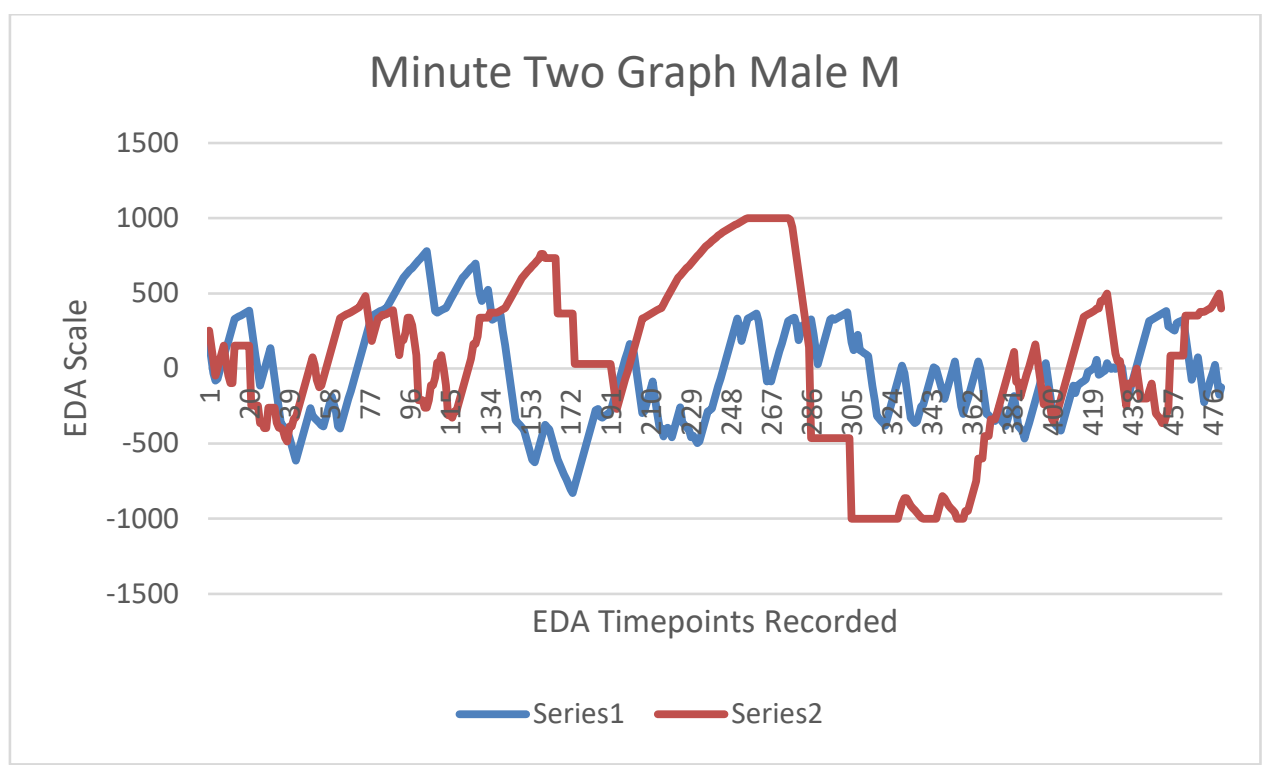

Figure 36: Male M Pip 'real time' graph for minute two 
The minute graphs indicate that in the presence of the dog, Male M's variance between the highest and lowest points was greater, yet he was able to settle and focus quickly to the reading task thus averaging out his overall score. At two points during the second recorded minute in the line graphs, he loosened his grip on the Pip as he was concentrating on the words.

\subsubsection{Reading Accuracy and Words Per Minute rate (WPM)}

The accuracy and speed effects of Male M's arousal response are shown in Table 58 below.

Table 58: Male M Reading accuracy and words per minute rate

\begin{tabular}{|l|l|l|}
\hline Reading Rhyme (No dog) & $\begin{array}{l}\text { Reading Book (No dog) } \\
\text { Reading Level 2.2 }\end{array}$ & $\begin{array}{l}\text { Reading Book (with dog) } \\
\text { Reading Level } 2.2\end{array}$ \\
\hline $95.6 \%$ Accuracy & $99.14 \%$ Accuracy & $97 \%$ Accuracy \\
$90.3 \mathrm{wpm}$ & $91 \mathrm{wpm}$ & $88 \mathrm{wpm}$ \\
\hline
\end{tabular}

The accuracy in Table 58 shows that there was little difference between the dog present and dog absent conditions, although while reading to the dog, his reading speed slowed, and he was far more aware of his sentences and punctuation. While reading without the dog although every word was accurate and fluent, there were extremely brief pauses for ends of sentences or paragraphs and only a little variance in expression and tone. Male M's reading speed both with the adult and the poem, showed that he had tried to slow down his words for the dog's benefit.

\subsubsection{Reading Attainment in both reading age and standardised scores}

Male M's overall reading progress for the study period is recorded in Tables 59 and 60 below. 
Table 59: Male $M$ Reading attainment in reading age

\begin{tabular}{|l|l|l|l|l|l|l|l|}
\hline Name & Chron Age & $\begin{array}{l}\text { Salford } \\
\text { Age Start }\end{array}$ & $\begin{array}{l}\text { Difference } \\
\text { between }\end{array}$ & $\begin{array}{l}\text { Chron } \\
\text { Age End }\end{array}$ & $\begin{array}{l}\text { Salford } \\
\text { Age End }\end{array}$ & $\begin{array}{l}\text { Difference } \\
\text { between }\end{array}$ & Ratio Gain \\
\hline Male M & $7: 02$ & $10: 10$ & $+44 \mathrm{~m}$ & $7: 05$ & $10: 09$ & $+40 \mathrm{~m}$ & $-0.3 \mathrm{~m}$ \\
\hline
\end{tabular}

Table 60: Male M Reading attainment in Standardised Scores

\begin{tabular}{|l|l|l|l|l|}
\hline & $\begin{array}{l}\text { Standardised Score } \\
\text { Start }\end{array}$ & Percentile Start & $\begin{array}{l}\text { Standardised } \\
\text { Score End }\end{array}$ & Percentile End \\
\hline Male M & $130+$ & $98+$ & $130+$ & $98+$ \\
\hline
\end{tabular}

Male $\mathrm{M}$ is another example of a child being able to fluently read ahead of his peers (by over $3 \frac{1}{2}$ years) and with extremely high standardised scores; 'well above average' in the level descriptors. As he has such high scores, despite Brooks' ratio gain showing no significant progress, the school will need to consider an alternative test, as the Salford does not have high enough ceilings for this learner. Consideration also must be given as to whether this high level of reading is actually "sight reading" of the words and if his comprehension from these texts would be to the same standard.

\subsubsection{Summary for Male $M$}

Male $M$ enjoyed reading to the dog and showed that there was a physiological reaction to when the dog was present, yet this effect seems minimal in his overall reading, attention, focus and accuracy results. Apart from providing and audience for Male $M$, the dog's presence, may simply have been a motivational, yet a novelty exercise. It is still questionable in that, had a teacher suggested to Male $M$ to slow his reading and look at his punctuation and expression, there would have been even greater similarity of the results across all three tasks. 


\subsubsection{Male C}

\section{(Age during project: seven years, eight months to seven years ten months)}

\subsubsection{Introduction and results from questionnaire}

Male C's family owned a cat and he said that he read to his pet. Male C placed himself "in the middle" in his class in terms of his opinion of his own skills and he enjoyed football and tennis. Male $\mathrm{C}$ also gave two wobbly emoji faces for his self-perceived attainment for reading and maths and said he didn't like either. He wasn't sure how the dog made him feel when he read to him, but the thought that the dog was happy. Male C didn't know how he could help the dog with his reading, but the dog could listen to him. Male $\mathrm{C}$ was very aware that Matt was a dog and in no way would be able to directly or verbally help him to read.

Male C was often seen working 1:1 outside the classroom and had a Speech and language therapy session on the same afternoons as the 'Read to Dog' sessions. His teacher was not keen on him being out of lessons for so long and complained to the project. Therefore, in negotiation with the Speech and Language teacher, he was collected directly from his therapy session and was kept to the minimum number of sessions for the project to limit disruption. Male $C$ did, on one occasion, preserve his right not to attend a reading session in favour of a PE lesson on the school field. Male $\mathrm{C}$ also had organisational issues and left his required book bag at home on several occasions, which was apparently the normal behaviour for him, according to his class teaching assistant.

When reading to the dog, Male $\mathrm{C}$ appeared to be 'rushing' his reading. His body language showed no indication that the dog was there, turning away from him. When 
he finished, he quickly closed his book, handed back the pip and gave the dog a biscuit with hardly any eye contact. Although he was encouraged to stroke the dog, he only did a quick stroke of the neck, but Male C's body posture clearly indicated that he did not want any more interaction.

\subsubsection{Results from non-repeated working memory tests, due to time}

The working memory tests which were unable to be repeated due to time are shown in Table 61 below.

Table 61: Male C Standardised Scores for the working memory tests unable to be repeated with the dog present

\begin{tabular}{|l|l|l|l|l|l|l|l|}
\hline Name & $\begin{array}{l}\text { Chron. } \\
\text { age }\end{array}$ & $\begin{array}{l}\text { Memory } \\
\text { for Story }\end{array}$ & $\begin{array}{l}\text { Memory } \\
\text { for Story } \\
\text { delayed }\end{array}$ & $\begin{array}{l}\text { Fluency - } \\
\text { Semantic }\end{array}$ & $\begin{array}{l}\text { Fluency - } \\
\text { Alliteration }\end{array}$ & $\begin{array}{l}\text { Fluency } \\
\text { - Rhyme }\end{array}$ & $\begin{array}{l}\text { Visual } \\
\text { Memory }\end{array}$ \\
\hline Male C & $7: 09$ & SS95 & SS85 & SS121 & SS115 & SS72 & SS80 \\
\hline
\end{tabular}

Male C's overall working memory scores showed a very high average score for fluency in semantics and alliteration, but this was after a speech and language session where these skills had just been practised. His fluency for rhyme and visual memory skills gave below average standardised scores for his age. The visual memory results showed that Male $\mathrm{C}$ was not spending enough time looking at the designs, and simply was working as fast as he can. The Memory for stories and stories delayed showed that Male $\mathrm{C}$ could transfer auditory information into long term storage, but he was slower, time wise and in articulating his responses than his peers when repeating the details (these tests are not timed.) Male $\mathrm{C}$ also showed a very short span for focusing on tasks before becoming distracted, or in his words, 'bored.' 


\subsubsection{Working Memory Tests with and without dog present}

Male C's working memory test results with and without the dog present are given in

Figure 37 below

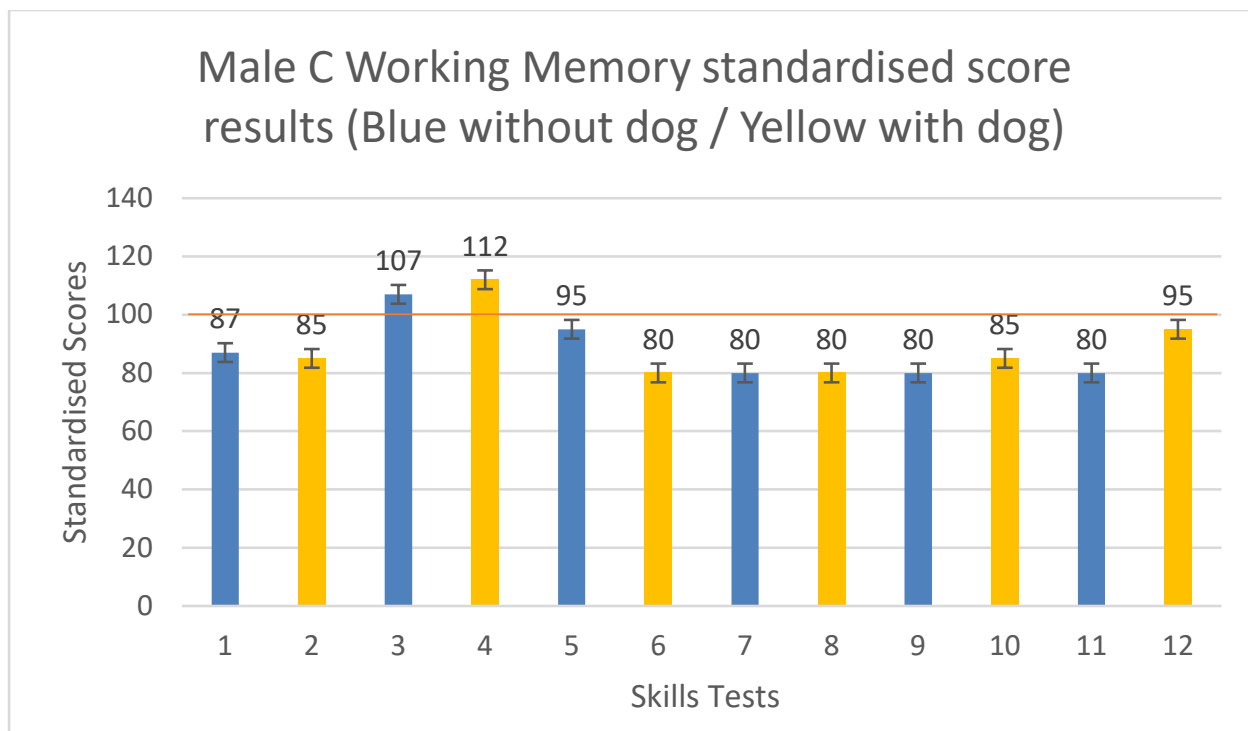

Key to skills tests:

\begin{tabular}{|l|l|l|l|c|}
\hline Skill & & Test & S.S & Improvement? \\
\hline \multirow{4}{*}{$\begin{array}{l}\text { Processing } \\
\text { Speed }\end{array}$} & 1 & Naming Picture Speed no dog & 87 & \\
\cline { 2 - 5 } & 2 & Naming Picture speed with dog & 85 & No \\
\cline { 2 - 5 } & 3 & Naming speed digits no dog & 107 & \\
\cline { 2 - 5 } & 4 & Naming speed digits with dog & 112 & Yes \\
\hline $\begin{array}{l}\text { Auditory } \\
\text { Short-Term } \\
\text { Memory } \\
\text { Skills }\end{array}$ & 5 & Digits forward no dog & 95 & \\
\cline { 2 - 5 } & 6 & Digits forward with dog & 80 & No \\
\cline { 2 - 5 } & 7 & Letters forward no dog & 80 & \\
\cline { 2 - 5 } & 8 & Letters forward with dog & 80 & Same \\
\hline $\begin{array}{l}\text { Executive } \\
\text { Skills and } \\
\text { Working } \\
\text { Memory } \\
\text { Span }\end{array}$ & 9 & Digits backward no dog & 80 & \\
\cline { 2 - 6 } & 10 & Digits backward with dog & 85 & Yes \\
\cline { 2 - 5 } & 11 & Letters backward with dog & 80 & \\
\cline { 2 - 6 } & 12 & Letters backward with dog & 95 & Yes \\
\hline
\end{tabular}


Male C's results showed very little difference in the picture processing speeds with the dog present and without as there was a one-point difference in his raw scores. Male C's processing was of a low average speed for pictures, possibly due to the repeated recall of the images through automaticity or through the articulation of the names of the objects. Male $\mathrm{C}$ did much better with his digits, achieving a high average score (SS 112) with the dog present. Male C's auditory short-term memory scores showed little to no difference whether the dog was present or not, scoring a below average score and a flat profile, in all but one test. The auditory processing and shortterm memory clearly were issues for him and had been a skill that Male C had practiced through his extra speech and language lessons and out of class work. His overall reverse spans were improved with the dog present for executive memory spans, with his standardised scores moving from 'below average' (SS 80) into the' low average' category (SS 95.)

\subsubsection{Electrodermal Activity Readings with and without dog present}

The averaged electrodermal activity readings for the reading sessions with the dog present and without for Male C are below in Table 62.

\section{Table 62: Male CAveraged EDA readings}

\begin{tabular}{|l|l|l|l|l|}
\hline Name & & $\begin{array}{l}\text { Sum of SC } \\
\text { increments } \\
\text { (micro- } \\
\text { siemens) }\end{array}$ & $\begin{array}{l}\text { Sum of time } \\
\text { differences } \\
\text { (seconds) }\end{array}$ & $\begin{array}{l}\text { EDA Positive } \\
\text { Changes (micro- } \\
\text { siemens / } \\
\text { second) }\end{array}$ \\
\hline Male C & Dog & 127.7953162 & 990.922 & 0.128966070 \\
\hline & No Dog & 172.2810264 & 1018.179 & 0.169205048 \\
\hline
\end{tabular}

(Green shows the higher variance of EDA changes) 
On average, the EDA differences between the dog being present or absent when reading for Male $\mathrm{C}$ show a slight increase when the dog is absent and overall show that there is greater variance and rate when Male $\mathrm{C}$ reads on his own, than when he is with the dog.

\subsubsection{EDA Box and Whisker charts for all three tasks}

Male C's comparisons in electrodermal activity for the three tasks of reading rhyme, reading to an adult without a dog present and reading with a dog present are shown in Figure 38 below.

Male C's reading graphs are interesting in that due to forgetting his book bag, he was reading the same book on both occasions.

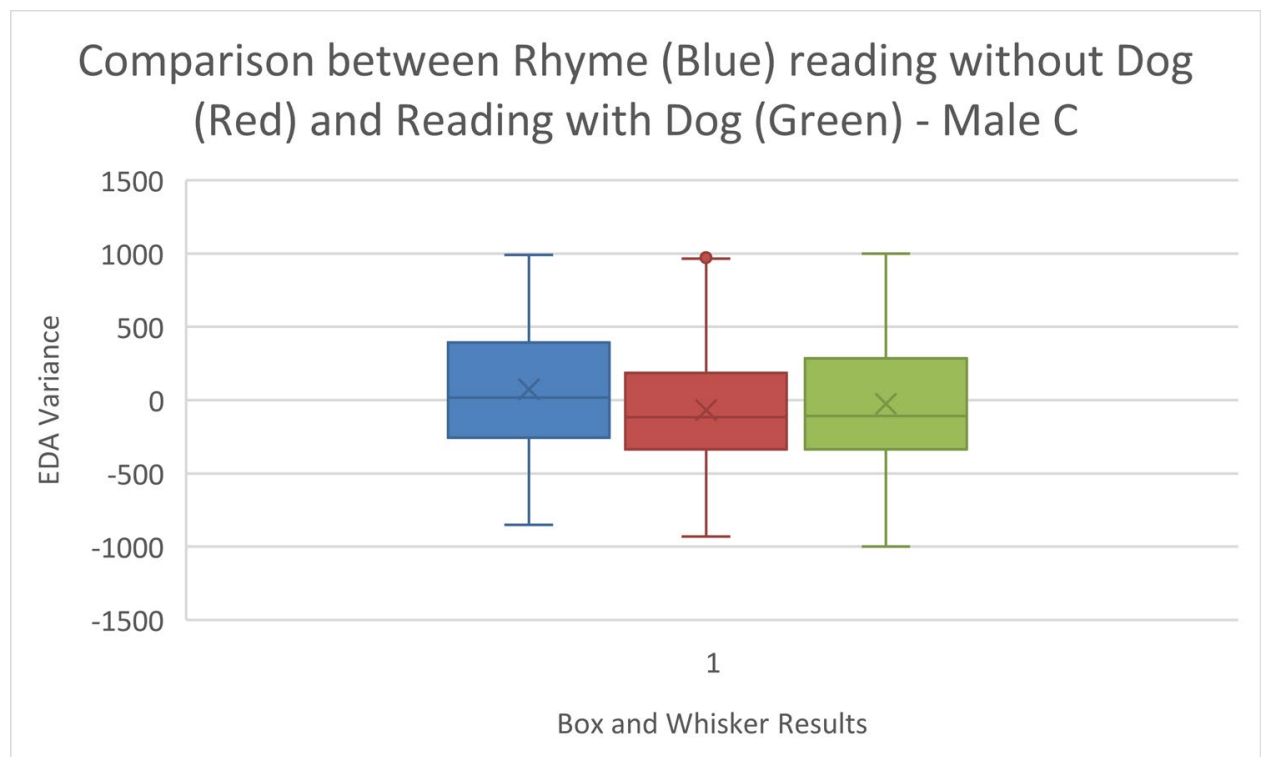

Figure 38: Male C's EDA comparisons between the three tasks

The comparative measures from the box and whisker charts for Male $\mathrm{C}$ are in Table 63 below. 
Table 63: Male C Comparative measures for box and whisker chart

\begin{tabular}{|l|l|l|l|}
\hline & Overall Range & Quartile Range & Median \\
\hline Rhyme without dog & 1844 & 649.5 & +17 \\
\hline Read without dog & 1903 & 521 & -118 \\
\hline Read with dog & 2000 & 621 & -108 \\
\hline
\end{tabular}

These results would suggest that all three activities showed a similar overall range with each other. The reading to the adult showed a more compact quartile range than the rhyme or reading to the dog. This may show that the concentration and focus for this activity was more familiar to him, and he was used to the routine. As the charts, both with the dog and without were similar in both median and interquartile ranges, any differences in speed or accuracy of reading should signify that it is the reading task affecting the variance.

\subsubsection{Male C Minute graphs comparison}

The 'real' time effects can be viewed in the Pip graphs for the first two minutes of reading as seen in Figures 39 and 40 below.

Blue - without dog, orange with dog 


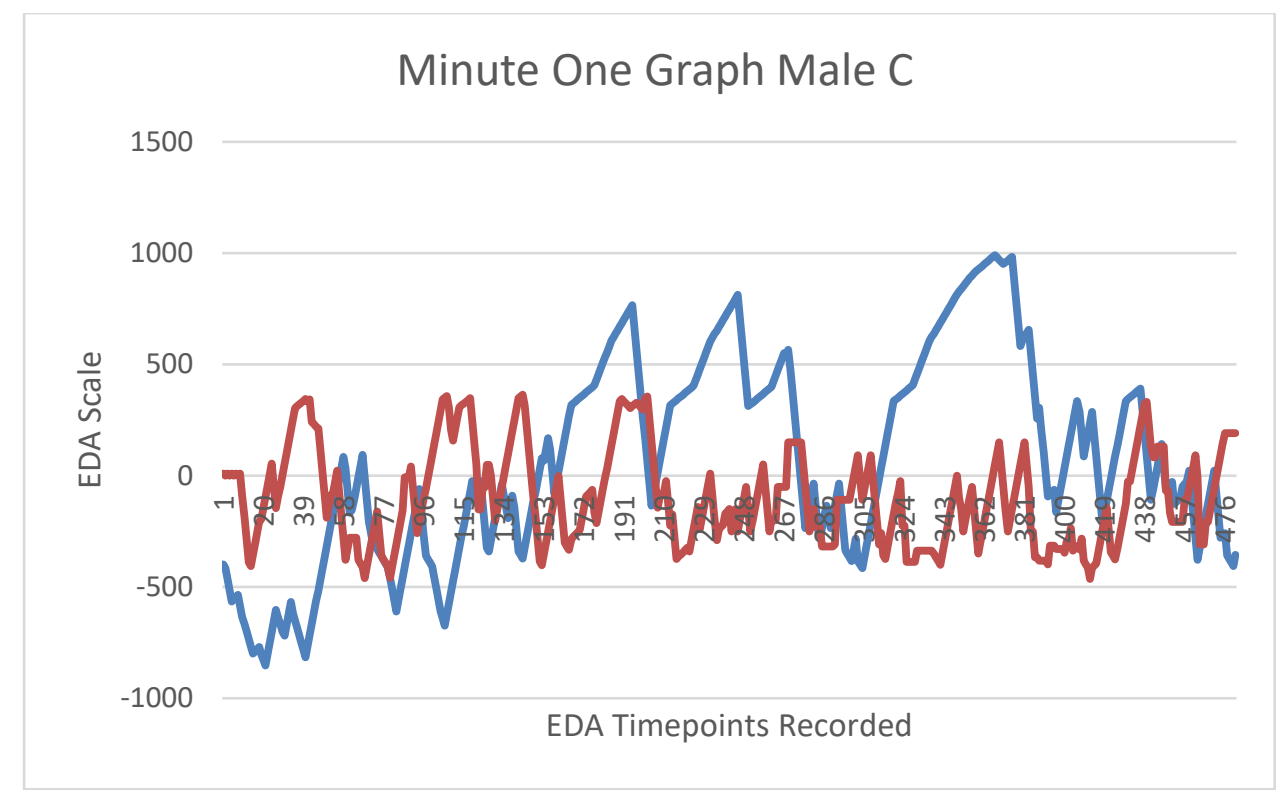

Figure 39: Male C's Pip 'real time' graph for minute one

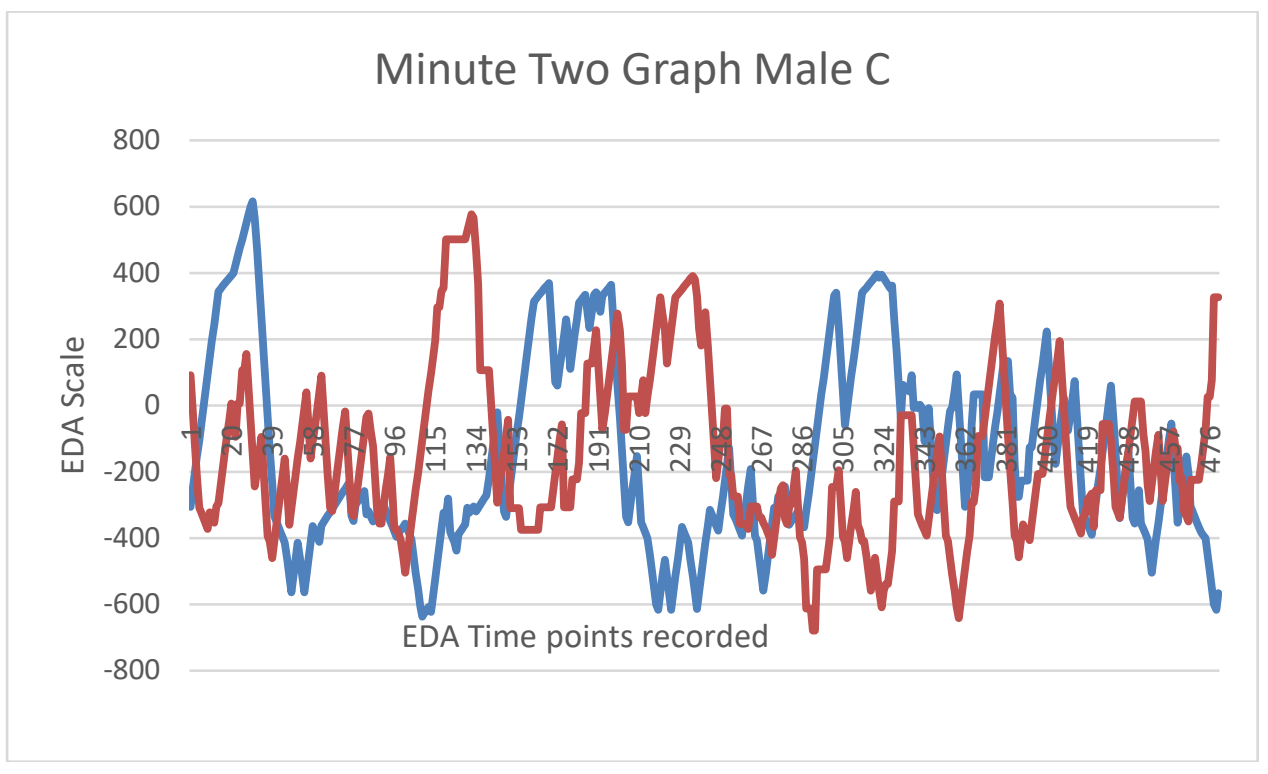

Figure 40: Male C's Pip 'real time' graph for minute two

The first minute graph does suggest that the dog may have affected his focus and concentration at the start, possibly priming him for the reading to come. The second graph shows the similarities in range and intensity of reading the same book 
for the two occasions. The similarity may show that it is the reading task which is affecting his EDA, rather than the presence of the dog.

\subsubsection{Reading Accuracy and Words Per Minute (WPM) Rate}

The accuracy and speed effects of Male C's EDA arousal response are shown in Table 64 below

Table 64: Male C Reading accuracy and words per minute rate

\begin{tabular}{|l|l|l|}
\hline Reading Rhyme (No dog) & $\begin{array}{l}\text { Reading Book (No dog) } \\
\text { Reading Level 2.0 }\end{array}$ & $\begin{array}{l}\text { Reading Book (with dog) } \\
\text { Reading Level 2.0 }\end{array}$ \\
\hline $\begin{array}{l}\text { 71.4\% Accuracy } \\
53 \mathrm{wpm}\end{array}$ & $\begin{array}{l}90.4 \% \text { Accuracy } \\
88 \mathrm{wpm}\end{array}$ & $\begin{array}{l}89.6 \% \text { Accuracy } \\
98 \mathrm{wpm}\end{array}$ \\
\hline
\end{tabular}

Male C's accuracy and reading rate shows that with the dog present, he sped up his reading rate although the differences in his accuracy scores was negligible between the dog being present or not. As he was reading the same book for both occasions it would be expected that his accuracy would be similar. The rates for the rhyme, however, show that Male $C$ was unfamiliar with many of the words and phonemes used and was struggling. With the dog present, there was a slight increase in expression, whereas there was overall clearer diction when reading to the adult. When reading to the dog, it was hard to determine some of the words, as he did not articulate so well.

\subsubsection{Reading Attainment in both reading age and standardised scores}

Male C's overall reading progress is recorded in tables 65 and 66 below. 
Table 65: Male C Reading attainment in reading age

\begin{tabular}{|l|l|l|l|l|l|l|l|}
\hline Name & Chron Age & $\begin{array}{l}\text { Salford } \\
\text { Age } \\
\text { Start }\end{array}$ & $\begin{array}{l}\text { Difference } \\
\text { between }\end{array}$ & $\begin{array}{l}\text { Chron } \\
\text { Age End }\end{array}$ & $\begin{array}{l}\text { Salford } \\
\text { Age End }\end{array}$ & $\begin{array}{l}\text { Difference } \\
\text { between }\end{array}$ & Ratio Gain \\
\hline Male C & $7: 08$ & $8: 00$ & $+4 \mathrm{~m}$ & $7: 10$ & $8: 00$ & $+2 \mathrm{~m}$ & $0 \mathrm{~m}$ \\
\hline
\end{tabular}

Table 66: Male C Reading attainment in Standardised Scores

\begin{tabular}{|l|l|l|l|l|}
\hline & $\begin{array}{l}\text { Standardised Score } \\
\text { Start }\end{array}$ & Percentile Start & $\begin{array}{l}\text { Standardised } \\
\text { Score End }\end{array}$ & Percentile End \\
\hline Male C & 104 & 60 & 102 & 55 \\
\hline
\end{tabular}

Overall, Male C appears to have regressed in his reading scores by 2 months for the project, with zero ratio gain (Brookes, 2007) score. This is despite extra reading practice at home and in school.

\subsubsection{Summary}

Male $\mathrm{C}$ is a difficult example to judge as there were so many influences in his life for the 12-week period. Although he was agreeable about reading to the dog, he was not as enthusiastic like his peers. The dog may have helped him with his focus and fluency but did not motivate his effort to read.

\subsubsection{Female $\mathrm{G}$}

(Age during project: seven years, four months to seven years, seven months)

\subsubsection{Introduction and results from questionnaire}

Female $\mathrm{G}$ had her own dog at home, but she said that she didn't read to it at all. Female $\mathrm{G}$ enjoyed art and music in school, and she wanted to play the guitar. While she liked reading, she wasn't so keen on maths as a school subject, giving it the "ok" emoji. She thought that reading to the dog made her feel calm and gentle and 
Female $\mathrm{G}$ liked the fact that he snuggled up to her and was settled. Female $\mathrm{G}$ said she could help the dog with his reading but did not explain this any further. Female $G$ did not know how the dog could help her with her reading. Female $G$ was aware that the dog did not have such human qualities. Female $\mathrm{G}$ wore glasses, was very active and appeared to be easily distracted. She would happily chat and play with any equipment or musical instruments around the room while waiting. Female $G$ also was a good mimic of her teachers and showed a good sense of humour.

During the reading sessions, Female G showed quick, sudden movements around the dog and a lot of "hands on touching" while the reading was being set up. The dog expressed anxiety through lip licking although he was very tolerant of her actions. While reading, her focus was on the task, showing that she had a wide range of strategies to decode unknown words. Female $\mathrm{G}$ stroked the dog twice during the reading, without taking her eyes from the book and by the end of the session her whole body was leaning towards the dog. Each time he got a stroke, the dog looked to the handler but did not change position. At the end, Female $G$ asked the dog for his opinion and gave him a sudden, quick, full body hug. The dog ignored this as he was concentrating on the forthcoming treat. Female G quickly gave him the biscuit, after which the dog put himself at a slight distance to her.

\subsubsection{Results from the non-repeated working memory tests, due to time}

The working memory tests which were unable to be repeated due to time are shown in Table 67 below 


\begin{tabular}{|l|l|l|l|l|l|l|l|}
\hline Name & $\begin{array}{l}\text { Chron. } \\
\text { age }\end{array}$ & $\begin{array}{l}\text { Memory } \\
\text { for Story }\end{array}$ & $\begin{array}{l}\text { Memory } \\
\text { for Story } \\
\text { delayed }\end{array}$ & $\begin{array}{l}\text { Fluency - } \\
\text { Semantic }\end{array}$ & $\begin{array}{l}\text { Fluency - } \\
\text { Alliteration }\end{array}$ & $\begin{array}{l}\text { Fluency - } \\
\text { Rhyme }\end{array}$ & $\begin{array}{l}\text { Visual } \\
\text { Memory }\end{array}$ \\
\hline Female G & $7: 06$ & SS100 & SS115 & SS98 & SS100 & SS91 & SS75 \\
\hline
\end{tabular}

Female $G$ showed that she had a high average memory (SS 115) for story detail and could retain the information easily for longer term recall. Female $G$ easily gave examples of food and animals the for semantical test and could follow the rules for the alliterative and rhyme tasks. In her visual memory test, Female $\mathrm{G}$ very quickly looked at the images and gave her answers, rather than taking her time. Female $\mathrm{G}$ admitted that for the semantic and alliterative sub tests she looked around the room for ideas. None of the other children had appeared to try this technique.

\subsubsection{Working Memory with dog present and absent}

Female G's working memory test results with and without the dog present are given in Figure 41 below. 
Female G - Working Memory Standardised Score

Results (Blue without dog / Yellow with dog)

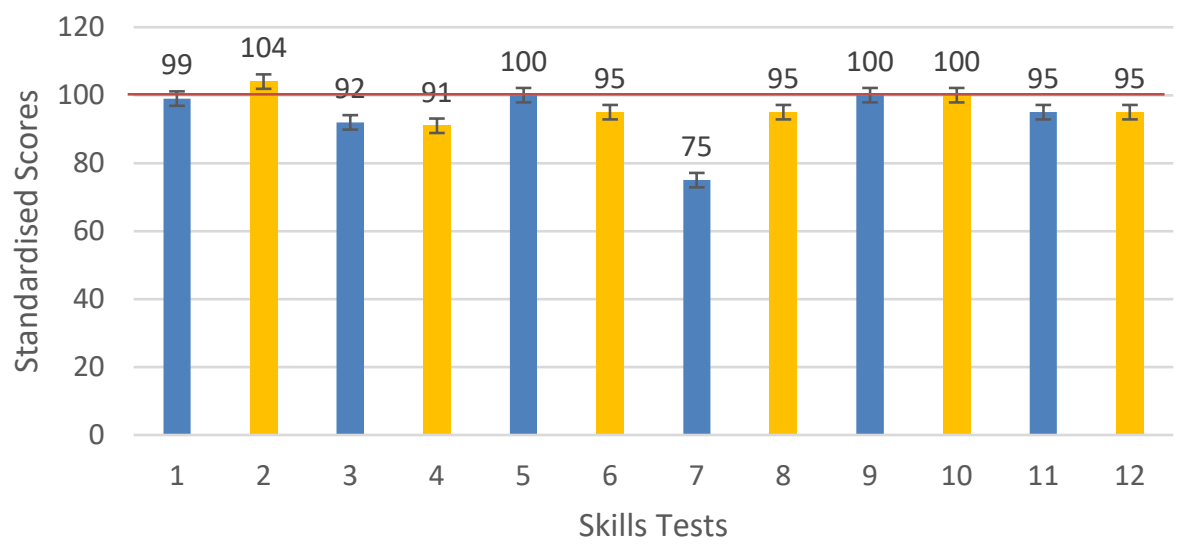

Key to skills tests:

\begin{tabular}{|c|c|c|c|c|}
\hline \multirow{4}{*}{$\begin{array}{l}\text { Processing } \\
\text { Speed }\end{array}$} & 1 & Naming Picture Speed no dog & 99 & \\
\hline & 2 & Naming Picture speed with dog & 104 & \\
\hline & 3 & Naming speed digits no dog & 92 & \\
\hline & 4 & Naming speed digits with dog & 91 & \\
\hline \multirow{4}{*}{$\begin{array}{l}\text { Auditory } \\
\text { Short- } \\
\text { Term } \\
\text { Memory }\end{array}$} & 5 & Digits forward no dog & 100 & \\
\hline & 6 & Digits forward with dog & 95 & \\
\hline & 7 & Letters forward no dog & 75 & \\
\hline & 8 & Letters forward with dog & 95 & \\
\hline \multirow{4}{*}{$\begin{array}{l}\text { Executive } \\
\text { Skills \& } \\
\text { Working } \\
\text { Memory } \\
\text { Span }\end{array}$} & 9 & Digits backward no dog & 100 & \\
\hline & 10 & Digits backward with dog & 100 & Same \\
\hline & 11 & Letters backward with dog & 95 & \\
\hline & 12 & Letters backward with dog & 95 & Same \\
\hline
\end{tabular}

Figure 41: Female G's working memory results with and without the dog present and key

Female $\mathrm{G}$ could easily name the images at speed and appeared to enjoy the challenge when the dog was present, improving her score. Her enthusiasm for numbers however was clearly different, and despite achieving an average score for her age, the presence of the dog hardly made any difference to her processing speed. Female G's letters forwards task showed possible difficulties in retaining direct auditory 
information as she appeared distracted during the task. Female G's initial 'letters forward' score was below average (SS 75) yet this improved with the dog presence into the average range (SS 95). The digits forward, however, was not improved by the dog's presence yet remained within the average standardised scores. The dog's presence did not affect Female G's executive skills or memory span in these tests.

\subsubsection{EDA readings with and without the dog present}

The averaged electrodermal activity readings for Female $\mathrm{G}$ for the reading sessions with the dog present and without, are below in Table 68.

Table 68: Female G Averaged EDA readings

\begin{tabular}{|l|l|l|l|l|}
\hline Name & & $\begin{array}{l}\text { Sum of SC } \\
\text { increments } \\
\text { (micro- } \\
\text { siemens) }\end{array}$ & $\begin{array}{l}\text { Sum of time } \\
\text { differences } \\
\text { (seconds) }\end{array}$ & $\begin{array}{l}\text { EDA Positive } \\
\text { Changes } \\
\text { siemens } \\
\text { second) }\end{array}$ \\
\hline Female G & Dog & 116.5678719 & 283.751 & 0.410810436 \\
\hline & No Dog & 207.3729884 & 1634.122 & 0.126901779 \\
\hline
\end{tabular}

(Green shows the higher variance of EDA changes)

The overall average readings for Female $G$ from the sessions of reading with and reading without the dog showed that the dog was affecting her physiologically with increased arousal. Female $\mathrm{G}$ said she was excited that he was there.

\subsubsection{EDA Box and Whisker charts for all three tasks}

Female G's comparisons in electrodermal activity for the three tasks of reading rhyme, reading to an adult without a dog present and reading with a dog present are shown in Figure 42 below: 


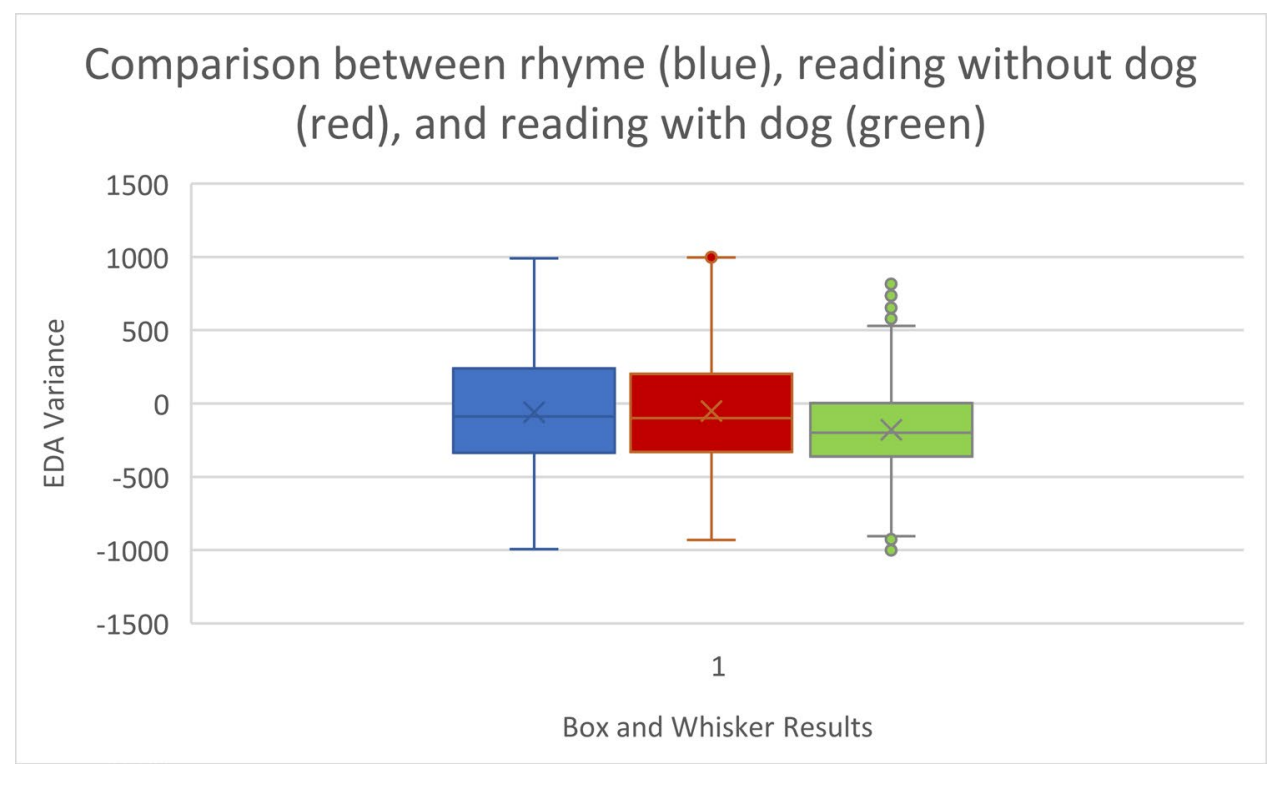

Figure 42: Female G's EDA comparisons for the three tasks

The comparative measures from the box and whisker charts for Female $G$ are in Table 69 below.

Table 69: Female G Comparative measures for the box and whisker charts

\begin{tabular}{|l|l|l|l|}
\hline & Overall Range & Quartile Range & Median \\
\hline Rhyme without dog & 1984 & 576 & -87 \\
\hline Read without dog & 1929 & 531 & -100 \\
\hline Read with dog & 1506 & 363.25 & -200 \\
\hline
\end{tabular}

The comparisons with the rhyme and reading without the dog show there was minimal difference between the tasks, but there was more focus and concentration when the dog was present, despite many outlier moments of distraction. Female $G$ was able to refocus back to the task quickly afterwards. 


\subsubsection{Female G Minute Graphs Comparison}

The 'real time' effects can be viewed in the Pip graphs for the first two minutes of reading as seen in Figures 43 and 44 below.

Blue - without dog, orange with dog

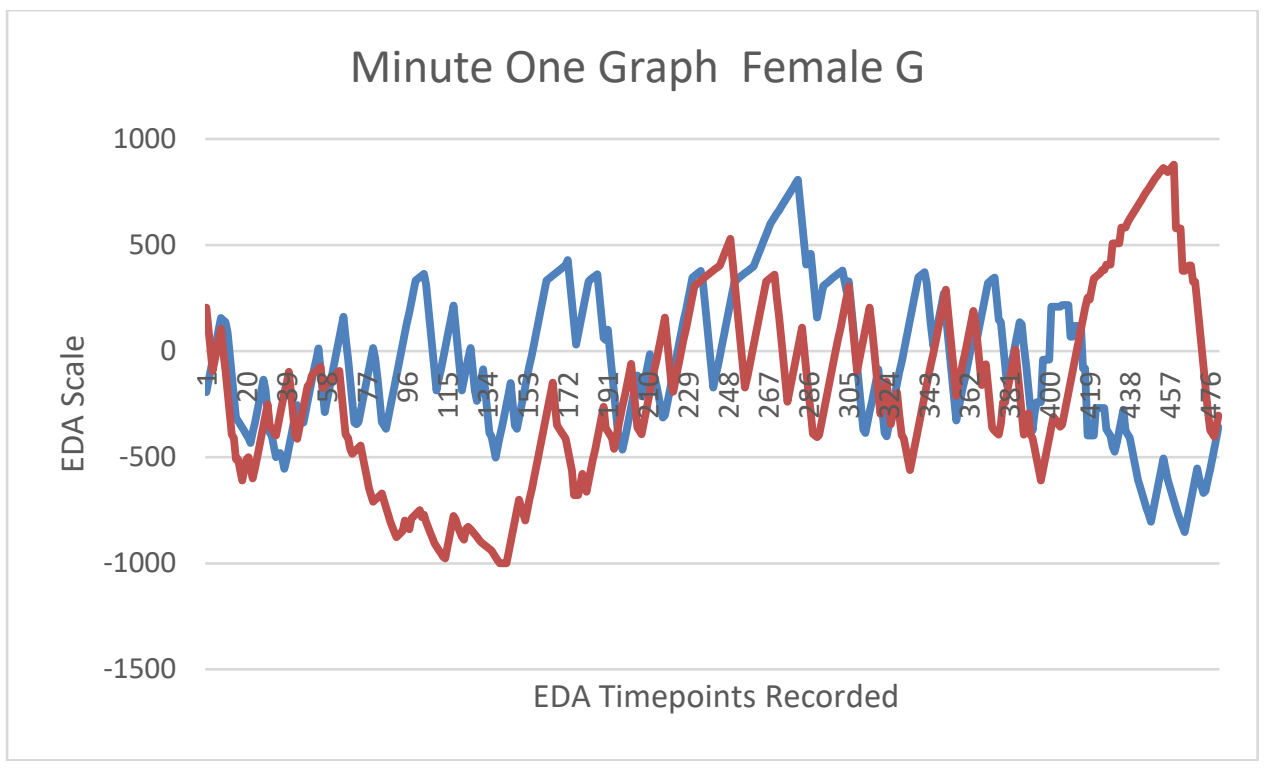

Figure 43: Female G's Pip 'real time' graph for minute one

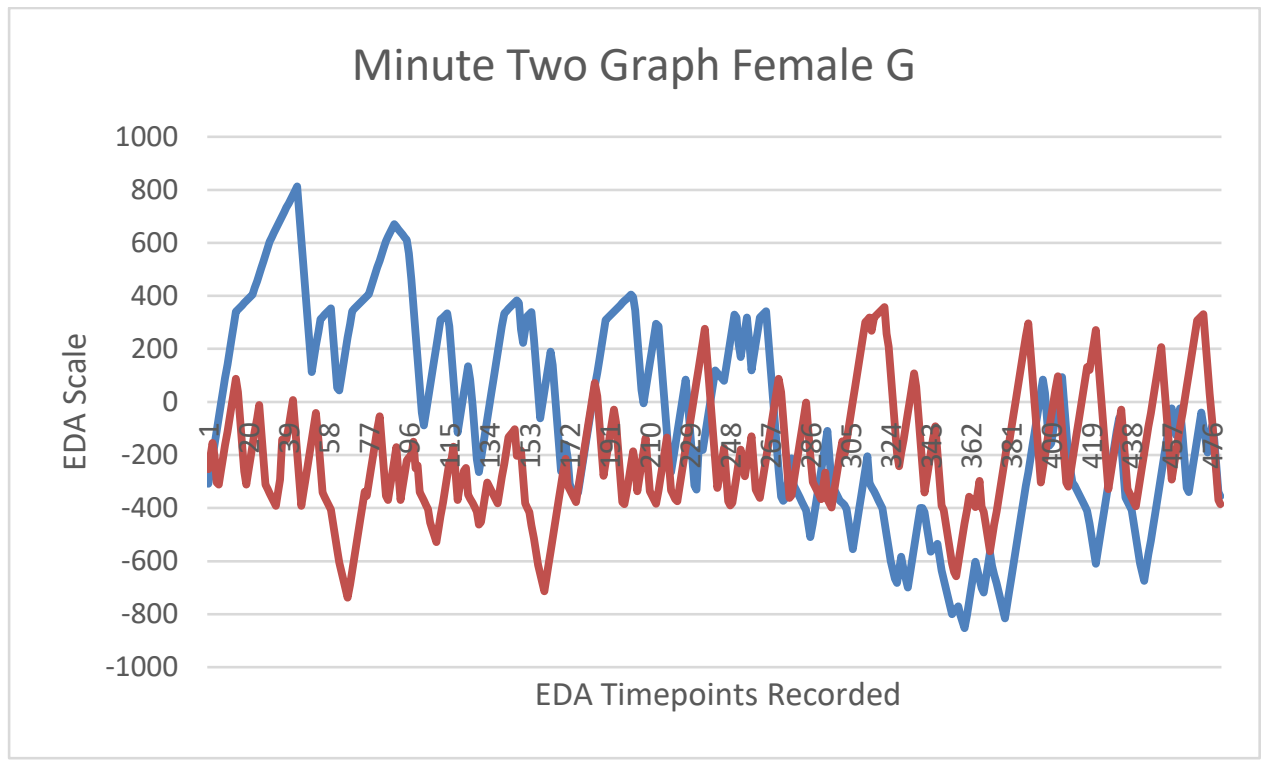


Figure 44: Female G's 'real time' graph for minute two

Female $\mathrm{G}$ commented while she was working the dog was making her concentrate harder and that she enjoyed reading to him. Female G's graphs show that the rate of variance has increased while the dog was present, and that the level of variance is of a much narrower band than when reading alone.

\subsubsection{Reading Accuracy and Words per Minute Rate (WPM)}

The accuracy and speed effects of Female G's arousal response are shown in table 70 below

Table 70: Female G Reading accuracy and words per minute rate

\begin{tabular}{|l|l|l|}
\hline Reading Rhyme (No dog) & $\begin{array}{l}\text { Reading Book (No dog) } \\
\text { Reading Level 2.5 }\end{array}$ & $\begin{array}{l}\text { Reading Book (with dog) } \\
\text { Reading Level 2.1 }\end{array}$ \\
\hline $\begin{array}{l}\text { 92.8\% Accuracy } \\
91 \mathrm{wpm}\end{array}$ & $\begin{array}{l}92.8 \% \text { Accuracy } \\
75 \mathrm{wpm}\end{array}$ & $\begin{array}{l}96.4 \% \text { accuracy } \\
71 \mathrm{wpm}\end{array}$ \\
\hline
\end{tabular}

Female G's wpm rate shows a slower rate of reading while reading to the dog, yet the video confirms that more care was taken with punctuation, expression and clarity of her words. Female G's high accuracy rates for reading generally show she was taking care and attention to her task, even though when reading text passages aloud, her overall rate was slower than the expected rate for Key Stage 1 readers. Her rhyme was accurate and speedy, and Female $\mathrm{G}$ said she enjoyed the task.

\subsubsection{Reading Attainment in both reading age and standardised scores}

Female G's overall reading progress is recorded in Tables 71 and 72 below. 
Table 71: Female $\mathrm{G}$ Reading attainment in reading age

\begin{tabular}{|l|l|l|l|l|l|l|l|}
\hline Name & Chron Age & $\begin{array}{l}\text { Salford } \\
\text { Age } \\
\text { Start }\end{array}$ & $\begin{array}{l}\text { Difference } \\
\text { between }\end{array}$ & $\begin{array}{l}\text { Chron } \\
\text { Age End }\end{array}$ & $\begin{array}{l}\text { Salford } \\
\text { Age End }\end{array}$ & $\begin{array}{l}\text { Difference } \\
\text { between }\end{array}$ & Ratio Gain \\
\hline $\begin{array}{l}\text { Female } \\
\text { G }\end{array}$ & $7: 04$ & $9: 00$ & $+20 \mathrm{~m}$ & $7: 07$ & $9: 03$ & +20 & $1 \mathrm{~m}$ \\
\hline
\end{tabular}

Table 72: Female G Reading attainment in Standardised Scores

\begin{tabular}{|l|l|l|l|l|}
\hline & $\begin{array}{l}\text { Standardised Score } \\
\text { Start }\end{array}$ & Percentile Start & $\begin{array}{l}\text { Standardised } \\
\text { Score End }\end{array}$ & Percentile End \\
\hline Female G & 120 & 91 & 122 & 93 \\
\hline
\end{tabular}

Throughout the project, Female $\mathrm{G}$ had already above high averages of standardised scores for reading, but had not increased her reading ages, despite only one month's ratio gain ("no gain," Brookes, 2007). Again, as Female G already had high levels of reading fluency her 'lack of progress' may not have been noticed. It is possible that Female $\mathrm{G}$ needed to be further challenged in her reading with more complex texts requiring greater comprehension skills.

\subsubsection{Summary}

Female $\mathrm{G}$ enjoyed the experience of reading to the dog, which personally motivated her. In terms of whether the dog helped to increase her attention and memory skills it appears that he helped her to concentrate and focus. As she had such high scores for reading anyway this may also be another case of the plateau of achievement in reading and the dog was there as an "audience" only. 


\subsubsection{Male $\mathrm{H}$}

\section{(Age during project: seven years, ten months to eight years, one month)}

\subsubsection{Introduction and results from questionnaire}

Male $\mathrm{H}$ was a cat owner and enjoyed running, art and guitar lessons at school. Male $\mathrm{H}$ considered himself an 'ok' reader, with a straight-faced emoji, but a good mathematician with a happy emoji. Male $\mathrm{H}$ had previously felt that the dog had cheered him up after an incident on the playground where he had been upset. Male H said that the dog was happy to be with him as he, "jumped on me and stuff." Male $\mathrm{H}$ felt that he could help the dog with his reading if he got stuck on a word, but he wasn't sure how the dog could help him. Male H wasn't too sure whether dogs had the same abilities as humans.

While Male $\mathrm{H}$ read, he showed very closed body language, with legs and body turned away from the animal and head bowed. He was neutral towards the dog. As Male $\mathrm{H}$ read, he would hesitate at words, attempt them and then wait for adult assistance. When the reading was finished, Male $\mathrm{H}$ was encouraged to tentatively stroke the dog and Male $\mathrm{H}$ did give the dog biscuits, Male H's his body position was still very closed, with no interaction or talking to the dog or the adults in the room. This was very different from the behaviour of the boy who walked to and from the sessions with the researcher and the one who read without the dog, where Male $\mathrm{H}$ was chatty, relaxed and happy to respond to questions about his book. Clearly, Male $\mathrm{H}$ found reading while the dog was present an anxious experience. 


\subsubsection{Results from the non-repeated working memory tests due to time}

The working memory tests which were unable to be repeated due to time are shown in Table 73 below.

Table 73: Male H Standardised Scores for the working memory tests unable to be repeated with the dog present

\begin{tabular}{|l|l|l|l|l|l|l|l|}
\hline Name & $\begin{array}{l}\text { Chron. } \\
\text { age }\end{array}$ & $\begin{array}{l}\text { Memory } \\
\text { for Story }\end{array}$ & $\begin{array}{l}\text { Memory } \\
\text { for Story } \\
\text { delayed }\end{array}$ & $\begin{array}{l}\text { Fluency - } \\
\text { Semantic }\end{array}$ & $\begin{array}{l}\text { Fluency - } \\
\text { Alliteration }\end{array}$ & $\begin{array}{l}\text { Fluency } \\
- \text { Rhyme }\end{array}$ & $\begin{array}{l}\text { Visual } \\
\text { Memory }\end{array}$ \\
\hline Male H & $8: 00$ & SS80 & SS95 & SS74 & SS97 & SS89 & SS100 \\
\hline
\end{tabular}

Male $\mathrm{H}$ also had what is known from the specific learning difficulty training as a 'spiky' profile, in that his strengths and weaknesses from these working memory tests showed no overall consistency. Male $\mathrm{H}$ showed a below average score for Memory for Story task (SS 80) but was able to achieve an average score (SS 95) when asked to recall the details 30 minutes later. Male $\mathrm{G}$ had significant difficulties initiating and generating words on the theme of animals and food, with long pauses at the beginning of the task, although he could then manipulate the rules of alliteration and rhyme. Male H's strengths lay in his visual skills and he enjoyed spotting the visual patterns and rotations, scoring his highest 'average' score (SS100). The long pauses and delay in answering questions, together with the average score for retention of the story details may suggest that Male $\mathrm{H}$ has a verbal processing difficulty or that he has difficulty initiating speech. Male $\mathrm{H}$ has particular difficulties when tasks are timed which may mean that he does not finish all his work in class, and therefore his abilities could be underestimated or masked. 


\subsubsection{Working Memory with dog both present and absent}

Male H's working memory test results with and without the dog present are given in

Figure 45 below

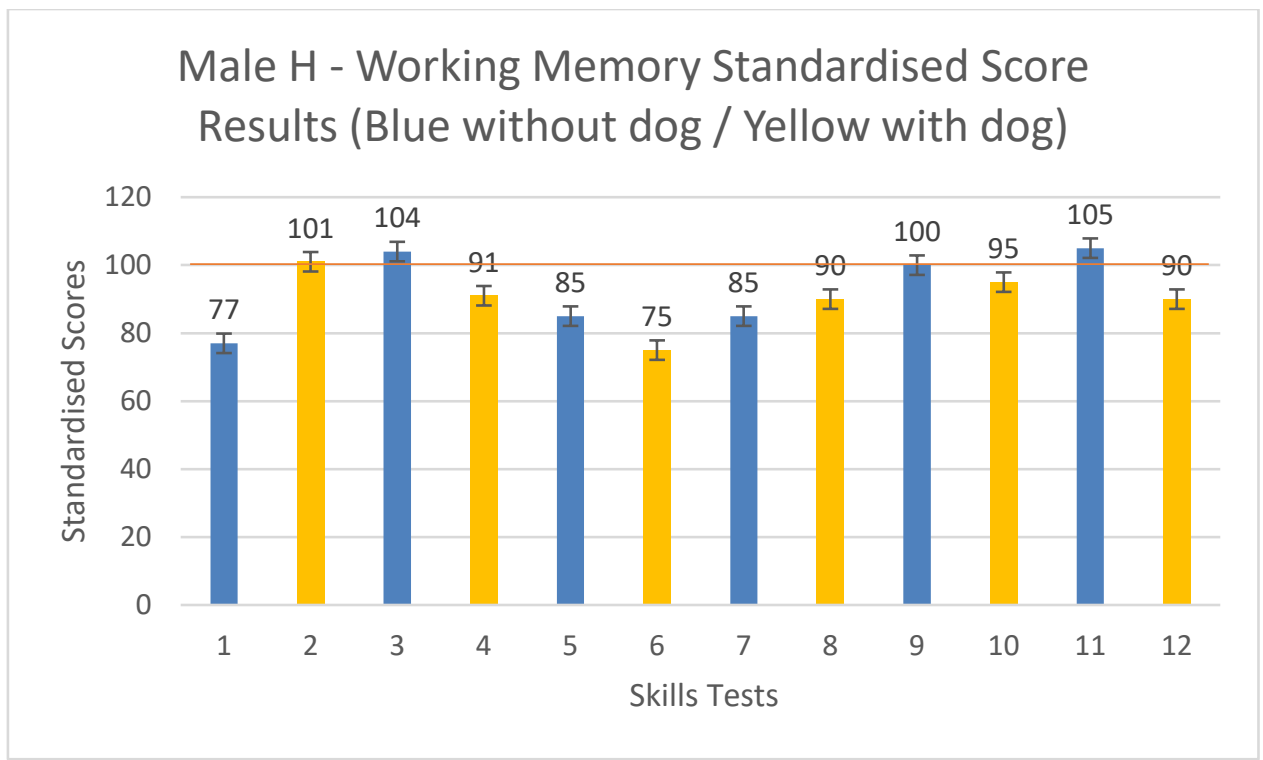

Key to skills tests:

\begin{tabular}{|c|c|c|c|c|}
\hline Skill & & Test & S.S & Improvement? \\
\hline \multirow{4}{*}{$\begin{array}{l}\text { Processing } \\
\text { Speed }\end{array}$} & 1 & Naming Picture Speed no dog & 77 & \\
\hline & 2 & Naming Picture speed with dog & 101 & Yes \\
\hline & 3 & Naming speed digits no dog & 104 & \\
\hline & 4 & Naming speed digits with dog & 91 & No \\
\hline \multirow{4}{*}{$\begin{array}{l}\text { Auditory } \\
\text { Short- } \\
\text { Term } \\
\text { Memory }\end{array}$} & 5 & Digits forward no dog & 85 & \\
\hline & 6 & Digits forward with dog & 75 & No \\
\hline & 7 & Letters forward no dog & 85 & \\
\hline & 8 & Letters forward with dog & 90 & Yes \\
\hline \multirow{3}{*}{$\begin{array}{l}\text { Executive } \\
\text { Skills \& } \\
\text { Working } \\
\text { Memory } \\
\text { Span }\end{array}$} & 9 & Digits backward no dog & 100 & \\
\hline & 10 & Digits backward with dog & 95 & No \\
\hline & 11 & Letters backward with dog & 105 & \\
\hline
\end{tabular}




\begin{tabular}{|l|l|l|l|l|}
\hline & 12 & Letters backward with dog & 90 & No \\
\hline
\end{tabular}

Figure 45: Male H's working memory results with and without dog present and key

Speed of processing appeared to be an issue for Male $\mathrm{H}$, as he would start the icons test well but by the second row was struggling for speed and accuracy. This was much improved when the dog was present. Male H's processing and naming of digits was more fluent, even though the dog's presence lowered his score. Results for Male H's auditory short-term memory were also mixed, with a lower score with the dog present for digits forward, but higher with the letters forward task. In Male H's executive skills, the presence of the dog did not assist him, yet his skills were still within the average range for his age. Throughout these tests, Male $\mathrm{H}$ did show some signs of anxiety both with and without the dog present through his body language pacing and fiddling with his clothes.

\subsubsection{EDA readings with and without the dog present}

The averaged electrodermal activity readings for the reading sessions with the dog present and without for Male $\mathrm{H}$ are below in Table 74.

Table 74: Male H Averaged EDA readings

\begin{tabular}{|l|l|l|l|l|}
\hline Name & & $\begin{array}{l}\text { Sum of SC } \\
\text { increments } \\
\text { (micro- } \\
\text { siemens) }\end{array}$ & $\begin{array}{l}\text { Sum of time } \\
\text { differences } \\
\text { (seconds) }\end{array}$ & $\begin{array}{l}\text { EDA Positive } \\
\text { Changes } \\
\text { siemens } \\
\text { second) }\end{array}$ \\
\hline Male H & Dog & 31.59498042 & 200.258 & 0.157771377 \\
\hline & No Dog & 69.8911869 & 460.235 & 0.151859782 \\
\hline
\end{tabular}

Male H's average EDA positive changes with and without the dog showed hardly any changes at all, although the no dog situation showed a slight variance and rate. This 
could suggest, that again, the task of reading itself, is also producing some anxiety arousal for this child.

\subsubsection{EDA Box and Whisker charts for all three tasks}

Male H's comparisons in electrodermal activity for the three tasks of reading rhyme, reading to an adult without a dog present and reading with a dog present are shown in Figure 46 below:

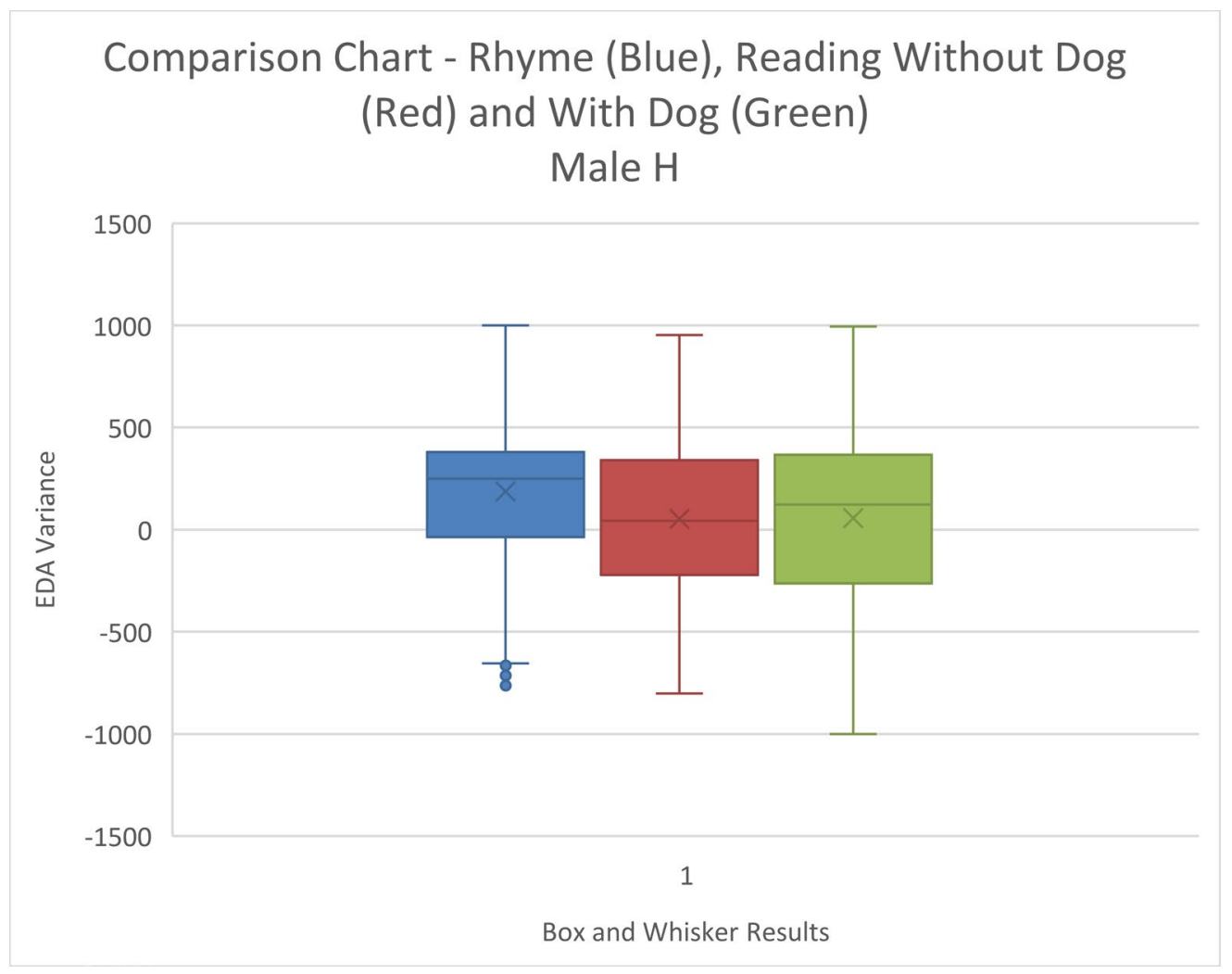

Figure 46: Male H's EDA comparisons between the three tasks

The comparative measures from the box and whisker charts for Male $\mathrm{H}$ are in Table 75 below.

Table 75: Male H Comparative measures for the box and whisker charts

\begin{tabular}{|l|l|l|l|}
\hline & Overall Range & Quartile Range & Median \\
\hline Rhyme without dog & 1654 & 414.5 & +249 \\
\hline
\end{tabular}




\begin{tabular}{|l|l|l|l|}
\hline Read without dog & 1757 & 559 & +43.5 \\
\hline Read with dog & 1995 & 629.5 & +124.5 \\
\hline
\end{tabular}

The results from this table would suggest that general reading is stressful for Male $\mathrm{H}$. Male $\mathrm{H}$ had significant problems reading the rhyme, requiring lots of verbal prompting by the adult, although the narrower box suggests he concentrated harder as a consequence. This may be due to the familiarity of the situation of reading to an adult back in class. Both reading the rhyme and reading to the adult showed similar box and whisker profiles. Reading with the dog present did have the greatest overall physiological variance for Male $\mathrm{H}$.

\subsubsection{Male H Minute graphs comparison}

The 'real time' effects can be viewed in the Pip graphs for the first two minutes of reading as seen in Figures 47 and 48 below.

Blue - without dog, orange with dog

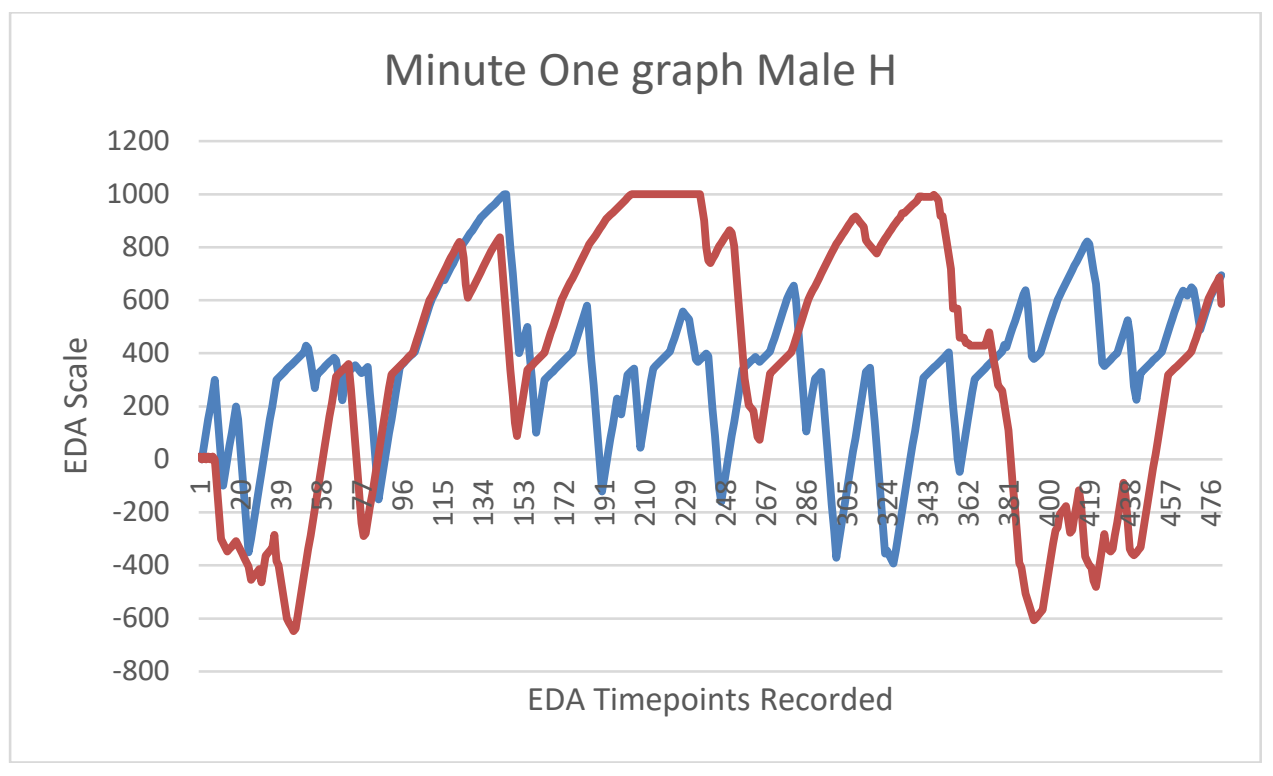




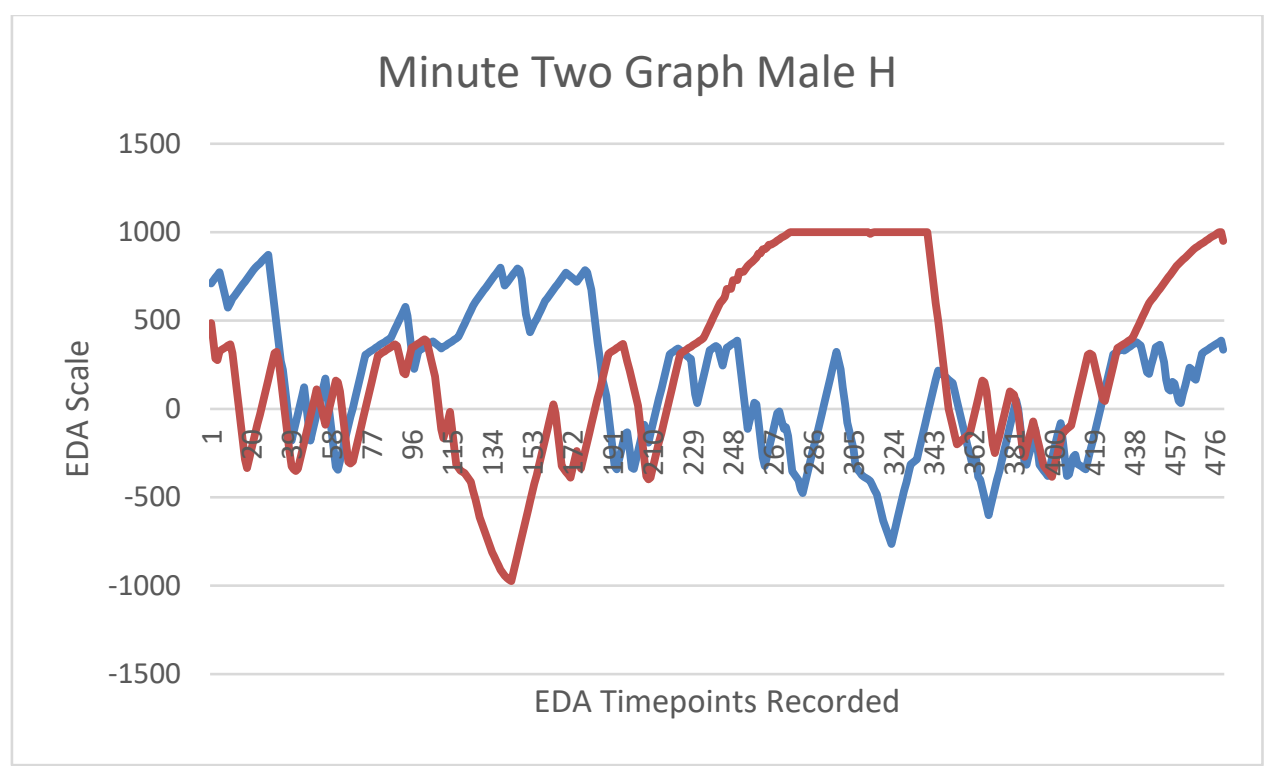

Figure 48: Male H's Pip 'real time' graph for minute two

Male H's graphs show that the presence of the dog showed anxious / high arousal scores as he dropped the Pip at least twice in his dog assisted recordings.

\subsubsection{Reading Accuracy and Words Per Minute (WPM) rate}

The accuracy and speed effects of Male H's EDA arousal response are shown in Table 76 below.

Table 76: Male H Reading accuracy and words per minute rate

\begin{tabular}{|c|c|c|}
\hline Reading Rhyme (No dog) & $\begin{array}{l}\text { Reading Book (No dog) } \\
\text { Reading Level } 1.6\end{array}$ & $\begin{array}{l}\text { Reading Book (with dog) } \\
\text { Reading Level } 2.1\end{array}$ \\
\hline $\begin{array}{l}78.5 \% \text { Accuracy } \\
34 \mathrm{wpm}\end{array}$ & $\begin{array}{l}95 \% \text { Accuracy } \\
50 \mathrm{wpm}\end{array}$ & $\begin{array}{l}92 \% \text { Accuracy } \\
38 \mathrm{wpm}\end{array}$ \\
\hline
\end{tabular}

Male $\mathrm{H}$ found reading the rhyme tricky, requiring support, but was happy to try to sound out the words on his own. Male $\mathrm{H}$ had difficulties with blending and paying attention to the ends of words. He could identify the rhythm, particularly when it was 
read back to him. When Male $\mathrm{H}$ read to the adult, he was happy to read his book about a dog called Max and was speedy and accurate, commenting on the story and questions as he went. When Male $\mathrm{H}$ was with the dog, however, he slowed his rate of speech and hesitantly attempted the words. The word per minute rate with the dog present was similar to Male $\mathrm{H}$ having read the poem from 'cold.' This difference in attitude and reading attainment would suggest that Male $\mathrm{H}$ may be reacting to the dog from anxiety.

\subsubsection{Reading Attainment in both reading age and standardised scores}

Male H's overall reading progress is recorded in Tables 77 and 78 below

Table 77: Male H Reading attainment in reading age

\begin{tabular}{|l|l|l|l|l|l|l|l|}
\hline Name & Chron Age & $\begin{array}{l}\text { Salford } \\
\text { Age } \\
\text { Start }\end{array}$ & $\begin{array}{l}\text { Difference } \\
\text { between }\end{array}$ & $\begin{array}{l}\text { Chron } \\
\text { Age End }\end{array}$ & $\begin{array}{l}\text { Salford } \\
\text { Age End }\end{array}$ & $\begin{array}{l}\text { Difference } \\
\text { between }\end{array}$ & Ratio Gain \\
\hline Male H & $7: 10$ & $7: 08$ & $-2 \mathrm{~m}$ & $8: 01$ & $8: 05$ & $+6 \mathrm{~m}$ & $1.3 \mathrm{~m}$ \\
\hline
\end{tabular}

Table 78: Male H Reading attainment in Standardised Scores

\begin{tabular}{|l|l|l|l|l|}
\hline & $\begin{array}{l}\text { Standardised Score } \\
\text { Start }\end{array}$ & Percentile Start & $\begin{array}{l}\text { Standardised } \\
\text { Score End }\end{array}$ & Percentile End \\
\hline Male H & 101 & 52 & 104 & 60 \\
\hline
\end{tabular}

Despite this, Male $\mathrm{H}$ had worked hard in his reading over that term and project period, increasing his reading age by 6 months, upping his standardised score and with a ratio gain of 1.3 months, despite this being considered of 'little impact' (Brookes, 2007). This was due to practice at home and in school rather than the two reading to dog sessions that he had. 


\subsubsection{Summary for Male $\mathrm{H}$}

For Male $\mathrm{H}$, the dog created an anxious situation and while the accuracy of the words may have increased, clearly the experience, as shown through his observations had caused him stress. The dog's presence may have assisted with Male H's executive functioning skills, but the speed of processing and accurate use of phonemes from the long-term memory were affected, possibly by cortisol flooding.

\subsubsection{Overall Summary of the seven focused case studies}

The seven individual case studies have shown that the presence of the dog has not truly assisted with the individual attainment for learning in their reading scores. Each child responded personally and differently to both to the dog's presence and their then current level of reading skills and attainment.

The overall averaged EDA increased in the three cases of fluent, confident readers when the dog was present (Female E, Male $\mathrm{M}$ and Female G), remained similar for two who showed distinct reading difficulties (Female $A$, Male $\mathrm{H}$ ) and lowered with the two remaining cases, one who was receiving extra speech and language help (Male C) and the other receiving extra help for reading (Male A). The difference in variance and the median showed that for all but two cases (Female $A$ and Female G) the presence of the dog caused greater EDA extremes when present. This extreme width of variance in EDA was not linked to improving accuracy or speed when reading, but reduced scores. For Female $G$ and Female $E$, the narrower variance only marginally improved the accuracy and speed. The argument that the dog would lower stress allowing for greater accuracy did not come to fruition in these cases and 
suggests that the presence of the dog was causing a sub-conscious distraction while they were reading.

Under the Brooks impact scores only two children made 'remarkable progress' during the reading project - five cases 'made no significant impact' despite mainly improving their reading scores. Those individuals making progress, Male A and Female A both showed a significant amount of extra intervention and teaching both at home and in school over the 12-week period. This also incorporated the fact that Female A had been highlighted through the project's memory tests as requiring immediate attention from the Educational Psychologist for a specific learning difficulty. Under a 'duty of care' the school was obliged to intervene and adjust their teaching strategies with this pupil accordingly. The question is raised as to whether had the child not been part of the project she would have been highlighted as struggling with her learning at this stage. This study does reflect common practice in most schools currently working with dogs for their curriculum, in that if there is progress in the reading, the dogs' presence is perceived as being of benefit. This study shows that perhaps many 'struggling' students are being 'overlooked' due to this attitude.

There was enthusiasm for the dog's presence. All the participants stated that they liked having the dog with them, despite the one case where the dog had clearly caused greater stress revealed in the individual's overall raised EDA levels, hesitancy and body language (Male H). Two examples showed that the dog made very little difference to the averaged session overall physiological awareness at all (Female $A$, Male C.) Despite comments of, "helping me concentrate," (Female G), or "reading to the dog is easier," (Female E), showing the children were motivated to read to the 
dog, the benefits of having the dog did not significantly impact on the overall attainment, other than to make the children feel more confident. Unfortunately, the confidence and possible rise self-esteem also did not seem to translate back to the attainment scores.

These findings would suggest that the emphasis on using dogs in schools is literally only from a motivational and 'comfort' perspective and thus it could be argued that it is the gatekeepers and adults within the school who believe in this positivity, despite little accurate evidence for links to academic attainment. This assumption would imply that, for actual scholastic success, the students still require adequate tuition in the skills of reading even when the dog is present. Perhaps this could be somewhat remedied if the visiting dogs and handlers were equipped with some basic skills for the teaching of reading in schools such as the guidelines given to parents for reading at home. This would ensure that the child is supported when selecting decoding strategies and maximise the benefits for attainment during the time of the dog's presence, not just for the self-esteem and self-confidence of the child, but also for capitalising on any strengths that the dog has brought in terms of executive functioning or processing skills.

This chapter's focus on the individuals has shown that each case study child has been at different but critical stages with their skill sets for reading. The results here suggest that there may be a 'plateau' effect for fluent readers, as seen by Female $E$, Male $M$ and Female $\mathrm{G}$ where working with a dog becomes more a comforting issue. Having a dog in class. therefore, may only be truly beneficial as a motivator while teaching for those who are younger or are further behind with these skills. This would raise issues 
for dogs generally visiting younger readers, or those, possibly older readers who are 'at risk of' or have been 'diagnosed' with specific learning difficulties. Yet, as this study has also shown, it is the individual reactions to the dog which show positive benefit, therefore the proposition of working with the dog for these particular reading categories may not be realised with all learners.

The limitations of this small sample, however, may yet reveal truly where the dog can affect individual memory and attainment. There appears to be very small positive signs in linking the dog to speed and fluency with some readers. This finding will have to be further researched and explored to identify the specific traits of these learners and how this links to the dog. If working memory and attention spans change significantly between childhood through to adult hood, perhaps the incorporation of the effects of the dog's presence in an adult study using school-based activities may be able to reveal further information. 


\section{Chapter 8 (Research Phase 3)}

\section{University Investigation: School based tasks with adult students}

\section{using 'live' and 'virtual reality' dog presence}

\subsection{Rationale}

The purpose of this experimental investigation was to extend the previous work with children and to discover whether the presence of the dog could; i) affect stress levels and performance on academic tasks relying on the working memory skills of adult students; ii) explore if the dog's presence would affect skills for achievement in different subject areas, such as maths and language differently and iii) compare whether a Virtual Reality (VR) dog also had equivalent effects for stress and subject academic performance, as compared to a real dog. These factors would have implications not just for overall learning when dogs are available for the classroom, but also for future possible teaching strategies involving the direct role of the dog.

The children's Beliefs About Animal Minds (BAM) questionnaire (Hawkins and Williams, 2016) has shown that children's intrinsic motivation through their beliefs in animals would positively affect the way they perceive their learning. This was, to an extent, also evident in the case studies (See Chapter 7) where the children commented on their thoughts about the dog's role in assisting in their reading. With adults who are less susceptible to conscious anthropomorphic tendencies, any differences in stress or performance in academic tasks should be due to the consequence of the dog itself. Adults are also assumed to have greater control with 
inhibition skills and should also remain on task, rather than being distracted by the animal. If it could be demonstrated with a group of adults that higher achievement and lower physiological response results from the presence of the dog, this may then strengthen the case for the presence of the dog improving memory and attention skills across age ranges. Such a finding would demonstrate therefore, that the dog's presence affects the learning and it is not simply a case of age-related maturity in executive functioning creating higher achievement in tasks. The effects of the dog on task achievement could also highlight any proposed visual spatial effects on learning (if any) in the mathematical tasks, as was tentatively suggested by the individual working memory tests with the children.

Four separate conditions were used to measure the effects of the dog's presence on physiological markers and attainment with an adult while completing academic tasks:

i) "Live" or "real" dog and handler

ii) "Live" or "real" handler only

iii) Virtual Reality dog while wearing a headset

iv) Virtual Reality handler only (no dog) within a headset.

\subsection{Preparation and set up}

\subsection{Participants:}

With four conditions for this study, a prospective-power analysis using G-Power (Faul et al., 2009) would suggest that 21 students are required for each condition (84 students in total). Given that welfare, stress and fatigue of the dogs to be used in the investigation was an issue, it was decided that it was not possible to test 84 
participants. A sample of 24 participants was used as this allowed counterbalancing for order-effects. As all the participants were adults over the age of 18 , they were able to wear VR headsets in line with health and safety guidance without harming the young brain's development. The volunteer adult students were recruited mainly from the psychology department and were aged between 18- 56 years.

\subsection{The Dogs and Handlers}

Two dogs were used, both Labrador retrievers, alternately present for the "real" dog situations. Please see Chapter 3 (Methodology) for their backgrounds and training. A risk assessment was also undertaken (See Appendix P) and two experienced handlers were available who were also the owners of the dogs, who monitored the dogs' welfare at all times. Please see Chapter 3 (Methodology) for the backgrounds of the handlers. Charity policies and insurance documents are to be found in Appendix 0 .

\subsection{Electro Dermal Activity (EDA):}

The same Pip recording device was used (as described in Chapter 3) to monitor participant EDA in the four conditions. Monitoring of EDA allows for crosscomparisons to be made with the children's data. As with the children's studies, the recordings were analysed using an "in house" MS Excel programme which gave average EDA for the whole sessions in all four conditions and could then be further sub-divided into the results for the maths and vocabulary tasks in each one. These data could then be analysed statistically.

\subsection{Tasks:}

Scores from a timed vocabulary multiple choice task selected from the Nelson Denny Reading Test (Brown et al., 1993) and from timed mental arithmetic tasks from 
English Key Stage 4 Foundation maths papers were also recorded as dependent variables.

Four variants of 20 questions based on the same mental maths topic areas were created. These included: linking long term memory mathematical vocabulary for mean and range; rearranging lists of numbers; percentages of numbers; simplification of algebraic terms; equivalent fractions; negative numbers; addition and subtraction of decimals; and simple orders of operation. Many of these operations required mental "visualisation" of processes linking to the use of visuospatial memory. There were two questions for each 'topic' area. For the verbal recall, selection and matching element, the Nelson Denny vocabulary test of 80 preprepared multiple-choice questions was also split into four sections of 20 questions. Each question was presented on a single Powerpoint slide.

Thus, there were four joint maths and vocabulary sets of questions produced, containing 10 slides of maths and 20 slides of vocabulary each. To have any set of questions in any condition, real or in VR, all sets of slides were filmed twice, once within the VR studio headset with dog and handler interacting, and once with just the handler in the VR headset to produce eight VR films. When in the 'real' condition, the sets of slides were projected on the whiteboard within the room. For each set of slides, whether in VR or not, the order of the mathematics or vocabulary questions was changed, therefore four started with the mental maths section first and four with the vocabulary section first. Examples of the questions used are in the appendices. The timings of the recordings of the questions took just under 3 minutes each, therefore in theory if the four conditions were back to back, then the whole process 
would take 12 minutes per individual. In practice it did take longer. For animal welfare, the dogs would only be needed for one session of 3 mins per individual, so there would be time to rest.

\subsection{Questionnaire}

As with the children's phase, a questionnaire was created to gain the views and opinions of the participants towards the tasks and dog's effectiveness. The questionnaire was organised into four sections: the first section collected personal data of the participant; the second collected details of previous experience with animals; the third section contains the day's experimental data; and the fourth section was for any other information which may have impacted on the scores or opinions.

\section{Section 1 - Personal Data}

The personal data gave information about the participant's age, whether they were male or female and for which level of degree they were studying. Data on gender was collected as previous research suggests that more females prefer to work with animals than men.

\section{Section 2 - Previous experience with animals}

This section covered the participant's previous experience with animals, asking: i) whether they were currently an animal or pet owner; ii) had they owned animals or family pets in the past; and iii) did they have animals or family pets as children? This was done in order to determine whether there were any natural tendencies or biases towards completing this project, which may influence overall results. 
Section 3 - Personal Preference of the Conditions

Section 3 used two numerical scales between 1 to 4, (1 representing most and 4 representing least), for levels of concentration and relaxation. This scale was to determine whether the Pip and attainment data were congruent with the subjective ratings. Studies have shown that human opinion of biofeedback data is often not accurate but is a register of a perceived emotional status. Also, in section 3 was a dichotic question asking whether Maths or Vocabulary was preferred overall, which may have influenced the electrodermal activity feedback for certain sections of the tasks.

Section 4 - Extended Feedback

In section 4 there was an option for any other information such as any voluntarily acknowledged specific learning differences which may have affected the data. There was also a section asking for any thoughts, comments or opinions about the day's experiment which the participant wished to make. Here it was stressed that they could write about anything they considered important about the whole study experience.

\subsection{Camera:}

In line with the children's investigation, a second camera was set up within the room, to record the direct answers, interaction and body language of the participants with the real dog and the handler.

\subsection{Virtual Reality Camera / Headset:}

An Oculus CV1 headset was used, powered by an Asus G752VS notebook with an Intel core i-7 2820HK CPU @ 2.90Ghz, 32 GB RAM and a GeForce GTX 1070 graphics 
card. Please see the Methodology Chapter (Chapter 3) for the health and safety conditions.

\subsection{Issues with the preparation stage:}

Two previous attempts at filming the dogs in VR proved unsuccessful in that, if the dogs were to act in the same way as the non-VR, real dog version, they needed to interact with a human. This was difficult when filming in VR with just an inanimate object (the camera on a tripod) within the room. The dogs found this a challenging exercise to complete. In the original films the dogs simply lay on their mats, or wandered about, which provided no emotional feedback either to the camera or therefore the person wearing the head set at all.

A way around the issue of providing stimulation for the dogs was to incorporate the dog's toys rolled across the floor, and involved the handler trying to interact with the dogs within the immediate vicinity of the camera, encouraging the dogs to walk up to the tripod as if they were walking up to the human. This gave the desired effect, yet the handler was having to be over exertive than would have been usual in that condition in the real situation. The handler was careful not to walk in front of the whiteboard screen, and so tried to keep all her action away, behind the direct, frontal view of the headset. The dogs themselves ran and interacted where they liked.

\section{Issues with filming Virtual Reality handler only}

In the VR version with the handler only, the handler then had to re-create the motion of the movement of the toys by herself. Again, she tried to keep the movement to a minimum within the direct view of the whiteboard, although the occasional toy or ball did come into view. 
The real dog situation involved the same two dogs that were filmed in VR working alternately, with the same handler present and were able to interact with the participants. Both dogs were already very familiar with this practice. As the charity insurance insisted on leads, the longest leads available were used, so the dogs had a choice whether to interact in close quarters, stand back or not to interact with the participants. The participants were still able to read the vocabulary and maths tests from the actual whiteboard, and could choose to invite the dog towards them for ear scratches, belly rubs etc. The dogs were given regular breaks between each participant and were with their owner for the remainder of the time.

Issues with filming Handler only "Live" situation

The handler was sat in the vicinity of the participant but was unable to interact, otherwise a new variable would have been introduced. The participant was able to read the vocabulary and the maths from the whiteboard. The dog toys were left scattered around the room to recreate the previous conditions. It was planned that they would be occasionally rolled in a similar style to the VR version by the handler. During piloting, it was found that this became very distracting for the participant, so the handler just sat next to the participant.

\subsection{Proposed data analysis}

Quantitative analysis was undertaken for the recorded average EDA rates for the four main conditions. These were broken down into the maths and vocabulary components within each condition. The results were analysed using SPSS (version 
26). Between-task comparisons were analysed using a one-way ANOVA. Qualitative data were taken from the questionnaires and the dog observations.

\subsection{Organisation and set up}

To control for order effects (e.g. fatigue and practice), the order of presentation of the conditions was counterbalanced using a Latin Square design. Designating the four conditions A, B, C, and D gives an order as follows (Figure 50 ): 


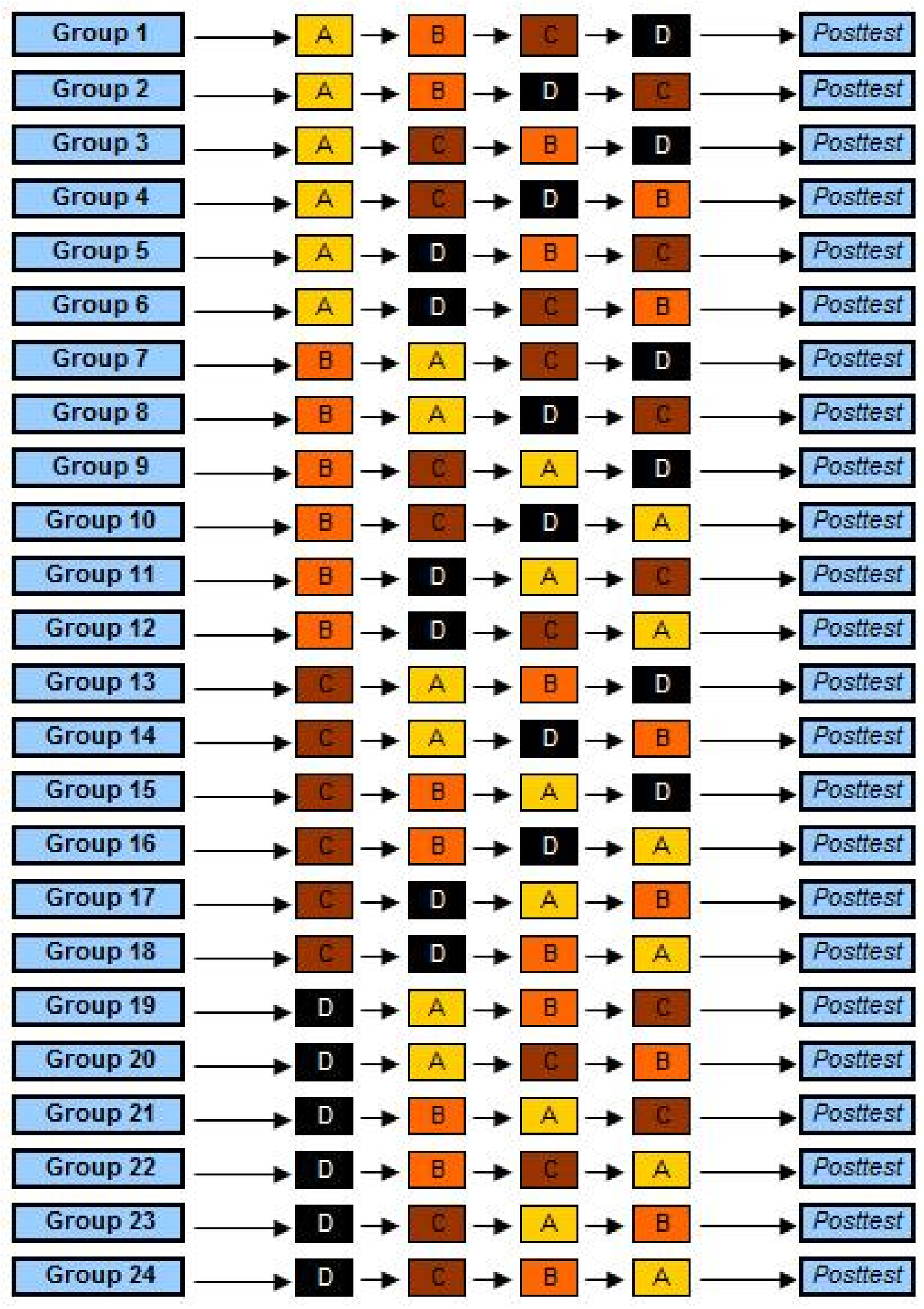

Figure 49: Latin square design

Key to the Latin Square design as seen in Figure 50:

A - real world dog and handler, 
B - real world, no dog just handler,

C - VR dog and handler

D - VR no dog, just handler

\subsubsection{Procedure including the Technician's role}

The testing took place in the virtual reality laboratory at University of Gloucestershire, in which the participant participated in all four conditions. Markers were placed on the floor for the participant's chair, the cameras and the wheelable laptop table which ran the VR. This meant that the participant could remain in one seat while the VR and real situations were adjusted around them. Next to the participant's chair was a dog blanket or area and the researcher /handler's chair set parallel to this. In the corner of the room sat a technician who also operated the VR headset system, the laptop, the video camera and the Pip recorder. During the VR sessions the researcher was able to sit in the handler's chair and record the answers manually as back up to the video camera. Before starting, a protocol of asking several health and safety questions for the VR was given to every participant, and the reassurance that the experiment could be stopped at any point if necessary. After the four conditions were completed, the participant was asked to fill in the questionnaire while the equipment was re-set.

\subsection{Pilot testing}

Initial difficulties arose with the legibility of the filmed power point presentations within the VR environment, but these were solved by adjusting the focus of the VR headset. In future, however, further regard still needs to be taken with the font size and typeface when reading within VR, perhaps using more bold lettering. The instructions for how to 
answer the vocabulary sections were therefore given verbally to the participant before putting the headset on.

When filming with the dogs, the researcher tried to keep her interaction away from the whiteboard screen set in the room, yet the participant could still hear the dog moving around. The test participant for the pilot said she liked this as she felt that she could relax better when in VR, knowing that the dog was there.

The Pip during the pilot, was not as reliable as it had been in in the children's study with a long 10 minutes where it could not find a signal - however once found, the Pip was able to record just as it had done during the children's project. Therefore, the test run took much longer than the predicted 30-40 minutes per person (closer to an hour).

The power-point 'real' environment presentations were clearer than those in VR, simply because of the direct link to the whiteboard in the room. The test participant also suggested that the slides within the VR films seemed to change much quicker than those in the 'real' environment. This is likely to be an artefact of time perception in VR.

While concentrating on the VR films and marking the verbal responses, the importance and value of the video camera being available as feedback for enquiries became clear although a conscious effort had to be made to ensure it was turned on, as it often became an after-thought.

The questionnaire seemed to be appropriate and highlighted the subjective experience of the participant, including the scaled questions. The fact it was a paper version also helped to reduce the time, rather than having a full interview and the questionnaire could be completed outside of the testing area. The participant was able to ask questions 
throughout the experiment and the main comment was that, although the experiment was formal, it was enjoyable.

\subsection{Pilot Test Results}

The pilot test participant showed that although she thought she had concentrated better with the real dog and researcher her results showed a different pattern. Her results are shown below in Tables 79 and 80 (below).

Table 79: Pilot - Overall Attainment

\begin{tabular}{|l|l|}
\hline Condition & Percentage of questions correct \\
\hline Real - No dog (Handler Only) & $67.5 \%$ \\
\hline Real - With dog and handler & $52.5 \%$ \\
\hline VR - with dog and handler & $50 \%$ \\
\hline VR - No dog just handler & $47.5 \%$ \\
\hline
\end{tabular}

Table 80: Pilot - Overall EDA for 'relaxed'

\begin{tabular}{|l|l|}
\hline Condition & Average EDA arousal \\
\hline Real No Dog (Handler Only) & 0.010060335 \\
\hline VR Handler and Dog & 0.039536461 \\
\hline VR No Dog (Handler Only) & 0.047993434 \\
\hline Real Dog and handler & 0.0545639693 \\
\hline
\end{tabular}

These tables would suggest that while the real dog is present, despite 'feeling' relaxed, the session averaged EDA scores are showing higher stress / arousal levels in recording. The participant's perceived feeling of this 'relaxation' has also not actually led to the presence of the dog assisting with the overall attainment scores. Clearly it is the least stress / arousal situation, which also gave the highest attainment was with the 'real' 
handler only. This could reflect previous learning patterns from school where there was only one adult in the classroom. However, the 'real dog' situation has given the second highest attainment score, despite showing the highest stress / arousal rates and supersedes the VR dog condition. In this case, there is a difference between the dog being present in 'reality' and in 'VR.'

The differences between the maths and vocabulary tasks was further examined to see if there was any difference between the conditions. The participant's Maths and vocabulary attainment and relaxation results are set out in in Tables 81 and 82 (below):

Table 81: Pilot Maths Attainment

\begin{tabular}{|l|l|l|}
\hline Condition & Raw Score & Percentage \\
\hline Real Handler Only & $10 / 20$ & $50 \%$ \\
\hline Real dog and handler & $6 / 20$ & $30 \%$ \\
\hline VR no dog & $3 / 20$ & $15 \%$ \\
\hline VR dog & $0 / 20$ & $0 \%$ \\
\hline
\end{tabular}

Table 82: Pilot Maths Relaxation

\begin{tabular}{|l|l|}
\hline Condition & EPC/EDA \\
\hline Real Handler Only & 0.027941594 \\
\hline Real Dog and handler & 0.031322386 \\
\hline VR Dog & 0.048530832 \\
\hline VR No Dog & 0.054220282 \\
\hline
\end{tabular}

Again, for maths, the least stressful situation in combination with the highest attainment was the 'real' handler only. The presence of the 'real' dog during the mathematical tasks 
recorded a lower stress / arousal rate, but now gives the second highest attainment scores. Once more there is a significant difference between the 'real' situations and the 'VR' presenting the VR situation as more stressful / arousal yet with less attainment. The participant acknowledged that she preferred the vocabulary to the maths sections. Her vocabulary and relaxation scores are set out in Tables 83 and 84 (below):

Table 83: Pilot Vocabulary Attainment

\begin{tabular}{|l|l|l|}
\hline Situation & Raw Score & Percentage \\
\hline VR Dog & $19 / 20$ & $95 \%$ \\
\hline VR no dog & $17 / 20$ & $85 \%$ \\
\hline Handler Only & $17 / 20$ & $85 \%$ \\
\hline Real Dog and handler & $15 / 20$ & $75 \%$ \\
\hline
\end{tabular}

Table 84: Vocabulary Relaxation

\begin{tabular}{|l|l|}
\hline Situation & EPC/EDA \\
\hline VR Dog & 0.005727876 \\
\hline VR No Dog & 0.012081316 \\
\hline Handler Only & 0.023978028 \\
\hline Real Dog and handler & 0.042590351 \\
\hline
\end{tabular}

In the vocabulary tasks the 'real dog' situation gave the least attainment and the highest stress / arousal rate. The VR dog situation however gave the highest attainment for the lowest stress arousal. The handler only situations, whether in VR or 'real' also gave better attainment scores than the 'real' dog situation. This could suggest that the participant was 
distracted by the presence of the 'real' dog or felt comfortable enough with the vocabulary tasks to allow her concentration to wander.

The test run participant showed that the experimental set up worked and that useable results could be obtained. As this was a pilot run, no statistical analysis was conducted.

\section{Video Recording}

The video was critical not just in recording answers but also showed the body language of the test participant both in 'real' and VR situations. It also was able to easily record the body language of the dog. In the VR with just the handler the participant's body movement was fairly static, but in the VR with dog - there was more body movement and looking from side to side. The 'real' environment with the dog showed the most interaction, both from the dog towards the subject and vice versa. The dog was looking for attention and ear scratches. After a settling down period the subject relaxed and then concentrated on the activities rather than the dog, leaving the dog redundant and stretched out in a relaxed position. The participant also showed reduced body movement with the researcher only 'real' condition.

\section{Marking Sheets}

It did become essential to have blank "marking sheets available" which could not only record the question answers as they were given but could be prepared in advance. Also, two copies of the participants' rota record were required for both the researcher and technician, referring to which condition needed to be prepared for the participant and to help to run things smoothly. 


\subsection{The Data Collection Periods}

There were two "data collection" periods where volunteer participants were recruited, and data collected. This was due to many students initially volunteering to sign up, but then did not turn up to their allocated slot in May 2019, which subsequently affected the timings of the slots for other participants This then ran into the exam season and then into the academic summer holidays. Therefore, the two collection periods were May / June 2019 and the following academic year, October / November 2019.

\subsection{General Results}

Twenty-four students took part in the project, six males and 18 females. This could reflect the current trend of previous research where females appeared to be more interested in working with the dogs than males, or the fact that there is a higher percentage of females studying at the University rather than males for psychology subjects. In total there were 9 undergraduate students and 15 postgraduate students. The ages of all the student participants ages between 18 and 58 years.

Out of these students, 17 were current pet owners, while seven students currently had no pets in their lives. There were however 21 students who owned pets in the past and had grown up with pets as children. There was only one student who had no current pets, had not owned pets in the past and did not have a family pet as a child. Each student reacted differently to the dogs. (For observation notes see Appendix V)

\subsection{Overall Attainment Scores}

The attainment scores for each participant in each of the conditions are outlined in Table 85 below: 


\begin{tabular}{|c|c|c|c|}
\hline Total VR with dog & Total VR No Dog & $\begin{array}{l}\text { Total Real } \\
\text { with Dog }\end{array}$ & $\begin{array}{l}\text { Total Real } \\
\text { Handler only }\end{array}$ \\
\hline 23 & 20 & 28 & 37 \\
\hline 10 & 10 & 24 & 20 \\
\hline 21 & 26 & 36 & 37 \\
\hline 30 & 25 & 29 & 30 \\
\hline 16 & 9 & 16 & 18 \\
\hline 24 & 27 & 32 & 29 \\
\hline 20 & 20 & 26 & 23 \\
\hline 19 & 18 & 18 & 22 \\
\hline 31 & 21 & 28 & 24 \\
\hline 19 & 20 & 21 & 27 \\
\hline 8 & 10 & 19 & 18 \\
\hline 11 & 13 & 16 & 15 \\
\hline 17 & 19 & 20 & 18 \\
\hline 26 & 20 & 31 & 30 \\
\hline 32 & 27 & 33 & 29 \\
\hline 7 & 10 & 27 & 29 \\
\hline 15 & 12 & 22 & 19 \\
\hline 11 & 11 & 25 & 27 \\
\hline 17 & 19 & 23 & 23 \\
\hline 18 & 12 & 21 & 23 \\
\hline 7 & 17 & 27 & 28 \\
\hline 13 & 7 & 19 & 20 \\
\hline 24 & 21 & 33 & 32 \\
\hline 26 & 21 & 30 & 29 \\
\hline 445 & 415 & 604 & 607 \\
\hline
\end{tabular}

The total achievement scores attained by all participants per condition are in bold.

As seen in Table 85, the higher achievement scores were for the 'real' situations, rather than those within VR.

Actual attainment scores, together with average EDA scores were gathered for each of the participants in the four conditions for comparison and placed in an MSExcel table. The highest and lowest achievement scores for each individual participant were then 
highlighted and compared with the questionnaire's perceived attainment and relaxation responses from the participants. (see table $\mathrm{x}$ in the appendix)

\subsubsection{Questionnaire Preferences - Vocabulary or Mathematics?}

The vocabulary activities were overwhelmingly preferred to the mental maths by the participants. Only three students chose the mental maths over the vocabulary. Four students (three female and one male) suggested that they felt they had undiagnosed dyscalculia and were very nervous during the mental maths sections, shown both in body language and in the verbal answer feedback. This nervousness was not always reflected in their attainment and skin conductance results. It did portray the self-esteem and low sense of confidence that people have within this sample, for mathematical achievements. Issues occurred with cognitive overload or 'flooding' of the memory particularly with the maths sections. Nine participants found that after the first two answers of a maths section, they could no longer think clearly enough to answer the remaining questions. This was described by the pilot participant as her brain 'freezing.'

\subsubsection{Questionnaire Preferences - Preferred condition for Relaxation or}

\section{Concentration}

The pattern of the favoured conditions for relaxation and concentration was, however, very different from the pilot. There was a core of 12 respondents who chose the same condition for both concentration and relaxation. When questioned, the participants stated that they could not distinguish between relaxation and concentration when working on the tasks themselves, so therefore they had retrospectively chosen the situation which they believed was the best for their coping with the tasks at that time. 
This may reflect the intensity of the focus on the completion of the tasks rather than the emotional state of mind.

\subsubsection{Relaxation Preferences}

There were just five participants who felt they were more relaxed in the activities with the real handler only, while 18 felt they were more relaxed with the presence of the real dog. Only one student thought that they were more relaxed with the VR dog.

When checked against the average EDA recordings for the sessions preferred, out of the 18 who chose the real dog, six had the highest readings across the conditions, suggesting high arousal, whereas the other 12 just had mid-range averages. There were no lowest averages recorded. The group of five who felt most relaxed in the handler only condition, only had one person with their lowest EDA scores. The single student that indicated they were more relaxed with the VR dog also had a mid-range score.

These findings could suggest that perceived relaxation may not be linked to physiological (e.g. EDA) changes. Those who thought they were most relaxed in the presence of the dog had chosen or preferred that situation.

\subsubsection{Concentration Preferences}

Fifteen students believed they concentrated better in the "real" handler only situation. Nine of these students had their highest attainment in that condition.

Nine students, however, believed they concentrated better in the real dog with handler condition and 5 of these had their highest attainment scores.

These findings suggest that even though participants preferred to have a dog in the room for relaxation they were aware that it could be a distraction and that they would 
concentrate better if they were in either a one-to-one, or without dog, condition. The overall score of $14 / 24(58.3 \%)$ that were correct in their assumptions of better concentration and attainment in the handler only condition, could also reflect common previous experience from school situations where they were expected to learn from one teacher, or indeed attainment itself as a concept, is easier to personally recognise and remember than stress / arousal.

\subsubsection{Comments about the experiment}

Fifteen participants chose to write comments about the dogs and the experience in general. Several themes were generated.

Theme 1 -The dog as a distraction

- "The dog was a sweetheart who was difficult to say no to. I had the feeling though that while her company made me happier and perhaps more confident, I did get more distracted."

- "The dogs running around in VR was a bit distracting at times. If it was running around less, it might be less distracting."

- "The dog approaching while in VR was very distracting."

- "I found the activity of the dog and handler very distracting in the VR conditions."

Theme 2 - Novelty

- "As a task went on, I felt more comfortable in general as I was more prepared for what was to come and able to better mentally prepare for the later sessions."

- "It is the first time I have experienced a virtual reality with the presence of dogs."

Theme 3 - Relaxation 
- "Definite feeling of relaxation with a real dog."

- "For me, pets always take the stress out of the situation, so I am biased in that regard."

- "It was very interesting, and I would like to see the results - having the dog by my side was so calming."

Theme 4 - Technical Difficulties

- "Time seemed quicker in VR."

- "The slides are very hard to read and changed very fast."

- "It wasn't really easy to see the dogs in the VR experience (possibly due to the focus on reading) but lovely dogs."

Theme 5 - Enjoyment

- "It was fun."

- "I'm allergic to dogs but they are lovely."

- "Very enjoyable exercise and very friendly dogs."

- "Enjoyable."

- "The experience was interesting in terms of the approach the researcher used to carry out the study. Personally, I have really enjoyed taking part."

- "Really enjoyed the experiment."

\subsubsection{Discussion of Themes}

Theme 1 suggests that when focusing on the task in hand, (i.e. the completion of mental maths and vocabulary problems) adults do have greater control over self-inhibition. These adults clearly wanted to succeed and concentrate to do well and for some of these 
individuals, comments suggest that having a moving dog was an irritating distraction. However, they were able to maintain the focus and continue to complete the task, despite the novelty factor (Theme 2). The implications of this adult determination can be compared with the younger children's learning. There clearly is a case for youngsters to be easily distracted and tasks to be left unfinished by the presence of the dog. The motivation to complete a task when in the presence of the dog therefore is up to the individuals inner drive and executive functioning, inhibition skills.

Theme 3 and Theme 5 showed that the adults perceived a general feeling of relaxation, calmness and enjoyment when the dogs were present. Due to these feelings, participants commented that they felt they had performed better. This attitude has been reflected throughout the research by most of the adults involved. However, as both stress and arousal recording plus attainment levels have shown, the subjective impression does not match the results. The argument for adults' opinions and biases to be considered when choosing to have an animal present in the classroom is still strong. Thus the 'gatekeepers' of classroom decisions still need to be involved in discussions as to the academic validity of having an animal in the classroom.

Theme 4 suggested that under stress, time appears to 'speed up' such as in exam situations where there is intense focus. This was also noticed by the pilot participant.

\subsection{Quantitative, Statistical Results}

Vocabulary scores, maths scores, and electrodermal activity (EDA) were recorded across four conditions:

- VR dog. Virtual reality (VR) with dog in VR.

- VR no dog. Virtual reality with no dog. 
- Real dog. 'Real' (non-VR) with real dog.

- Real no-dog. 'Real' (non-VR) with no dog.

The parametric assumptions were assessed across the data for vocabulary scores, maths scores and EDA (see Appendices W and X). As there were significant violations of the parametric assumptions across all data, non-parametric statistics were used for the analysis. All SPSS output is given in Appendix Y.

\subsubsection{Vocabulary scores}

The mean vocabulary scores across the four conditions are shown in Figure 51 (below).

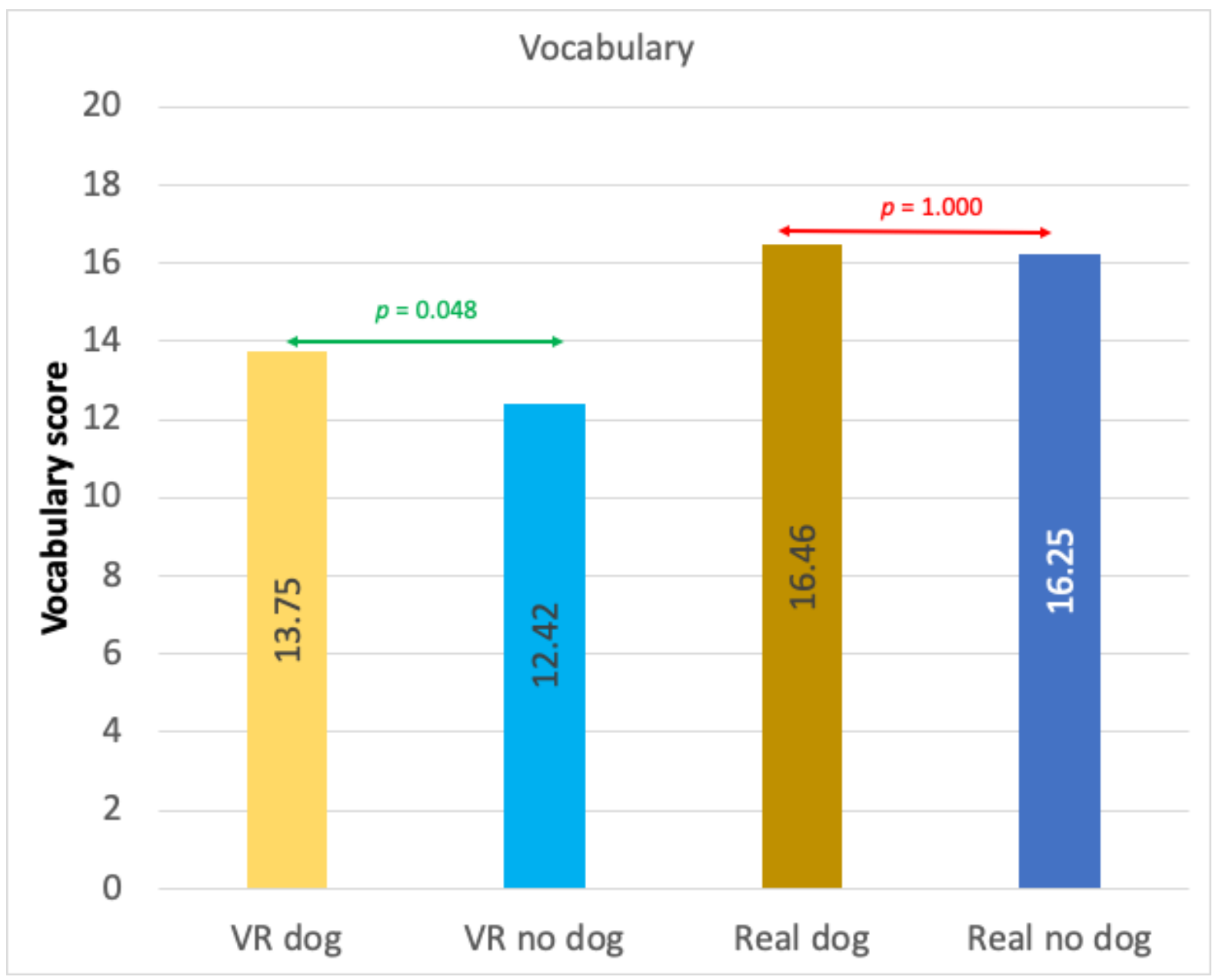




\section{Figure 50: Vocabulary scores across all four conditions}

Figures within the bars are mean scores. Figures on arrows between conditions are $p$ values from Wilcoxon signed-ranks tests. A green arrow indicates statistical significance at $p<0.05$. A red arrow indicates no statistical significance at $p<0.05$.

A Friedman test revealed that vocabulary scores varied significantly across the four conditions: $\left.\chi^{2}(3, N=24)=22.123, p<0.0005\right)$.

Pairwise comparisons using Wilcoxon matched-pairs signed-ranks tests (Bonferroni corrections applied for multiple comparisons) were conducted.

There was a significant difference in the vocabulary scores for the VR dog and VR no dog conditions $(z=-2.259, N-$ Ties $=7, p=0.048)$.

There was no significant difference in the vocabulary scores for the Real dog and Real no dog conditions $(z=-0.265, N-$ Ties $=6, p=1.000)$.

\subsubsection{Maths scores}

The mean maths scores across the four conditions are shown in Figure 52. 


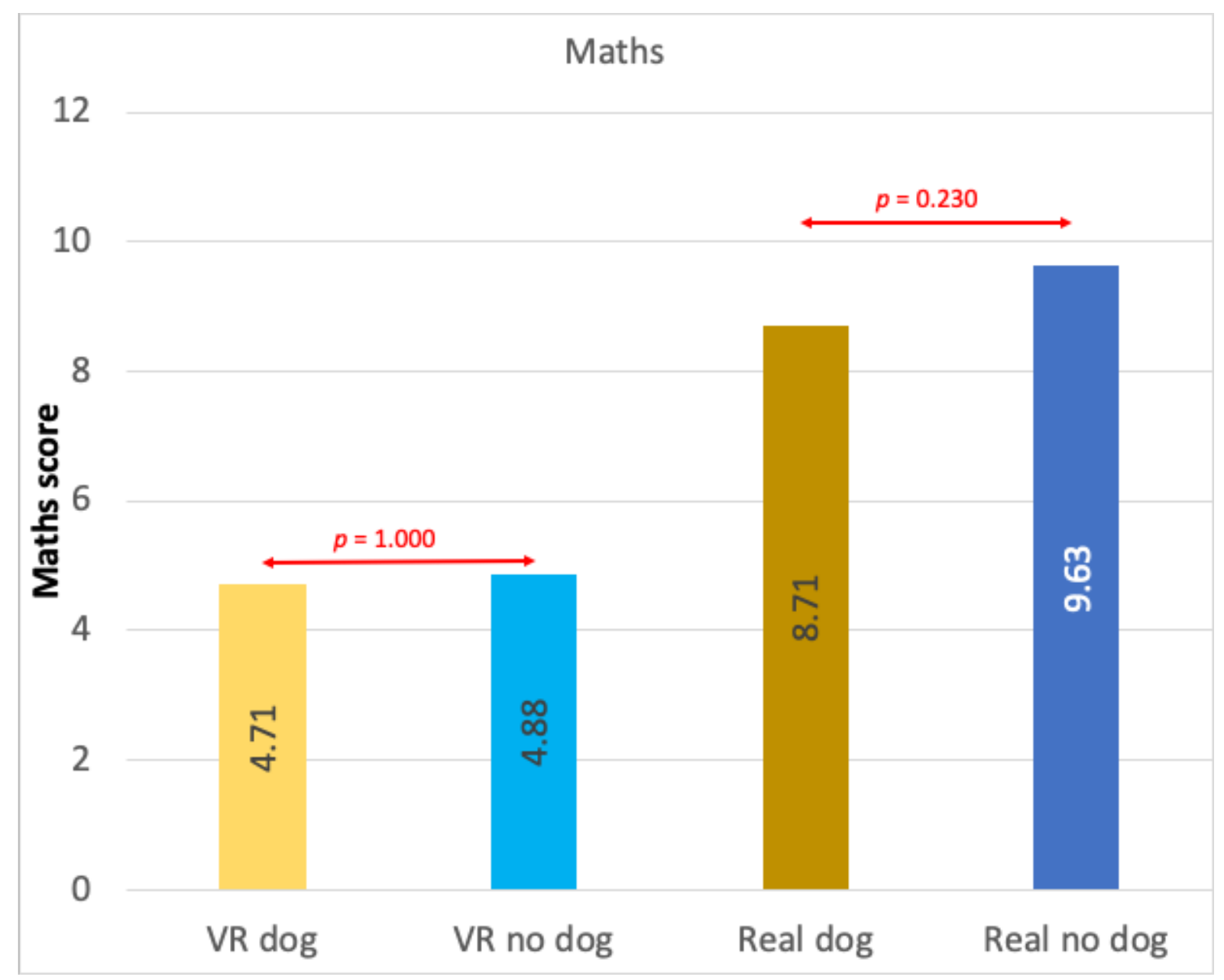

Figure 51: Maths scores across all four conditions

Figures within the bars are mean scores. Figures on arrows between conditions are $p$ values from Wilcoxon signed-ranks tests. A red arrow indicates no statistical significance at $p<0.05$.

A Friedman test revealed that maths scores varied significantly across the four conditions: $\left.\chi^{2}(3, N=24)=43.060, p<0.0005\right)$.

Pairwise comparisons using Wilcoxon matched-pairs signed-ranks tests (Bonferroni corrections applied for multiple comparisons) were conducted.

There was no significant difference in the maths scores for the VR dog and VR no dog conditions $(z=-0.138, N-$ Ties $=1, p=1.00)$. 
There was no significant difference in the maths scores for the Real dog and Real no dog conditions $(z=-1.576, N-$ Ties $=3, p=0.230)$.

\subsection{Electrodermal activity (EDA) scores - entire session.}

The electrodermal activity scores recorded across an entire session incorporating both vocabulary and maths tests are shown in Figure 53.

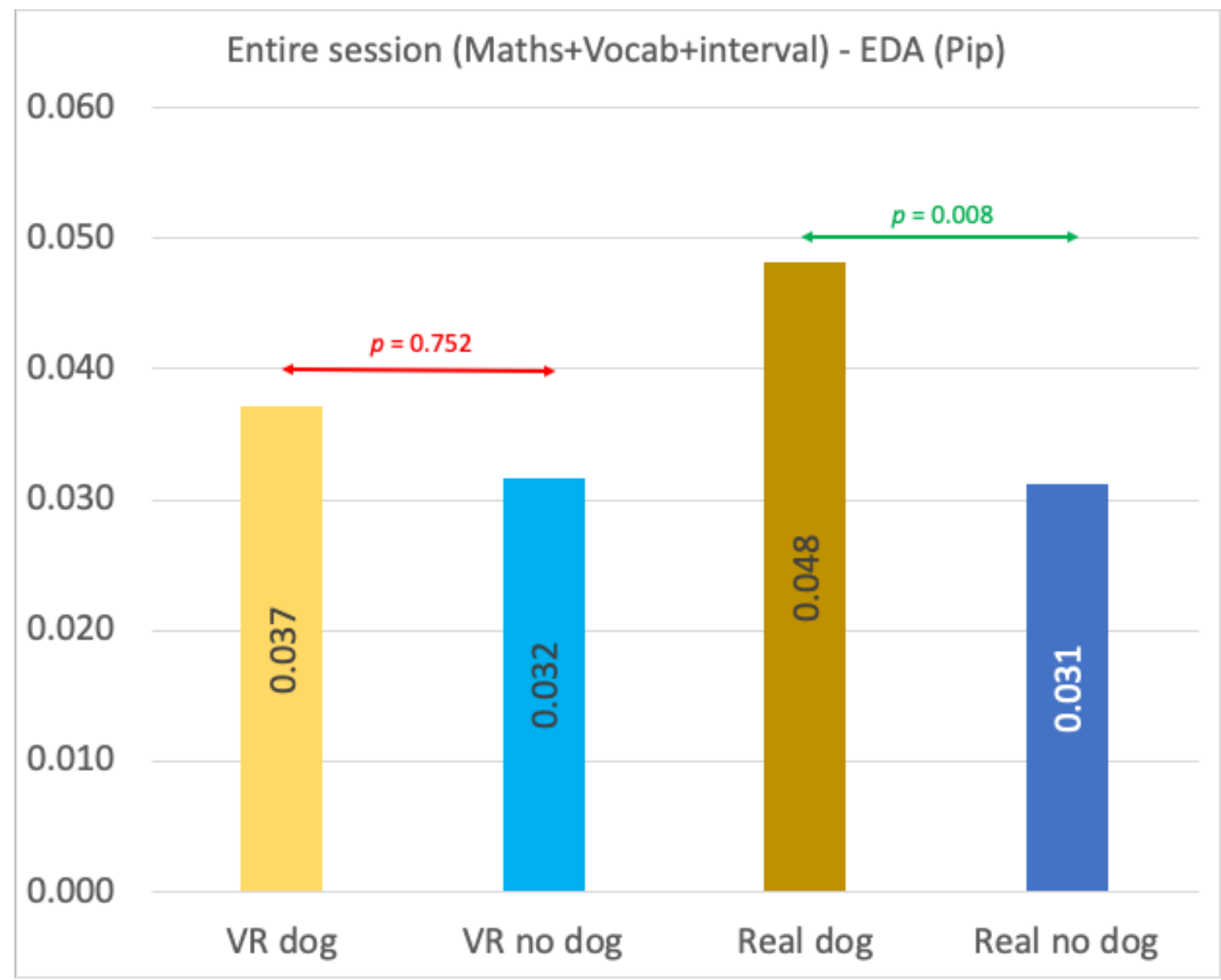

Figure 52: EDA scores across all four conditions - incorporating both maths and vocabulary tests

Figures within the bars are mean scores. Figures on arrows between conditions are $p$ values from Wilcoxon signed-ranks tests. A green arrow indicates statistical significance at $p<0.05$. A red arrow indicates no statistical significance at $p<0.05$. 
A Friedman test revealed that EDA scores varied significantly across the four conditions: $\left.\chi^{2}(3, N=24)=7.950, p=0.047\right)$.

Pairwise comparisons using Wilcoxon matched-pairs signed-ranks tests (Bonferroni corrections applied for multiple comparisons) were conducted.

There was no significant difference in the EDA scores for the VR dog and VR no dog conditions $(z=-0.886, N-$ Ties $=0, p=0.752)$.

There was a significant difference in the maths scores for the Real dog and Real no dog conditions $(z=-2.886, N-$ Ties $=0, p=0.008)$.

\subsection{Electrodermal activity (EDA) scores - vocabulary sessions only.}

The electrodermal activity scores recorded across the period in which the participant was doing the vocabulary tests are shown in Figure 54. 


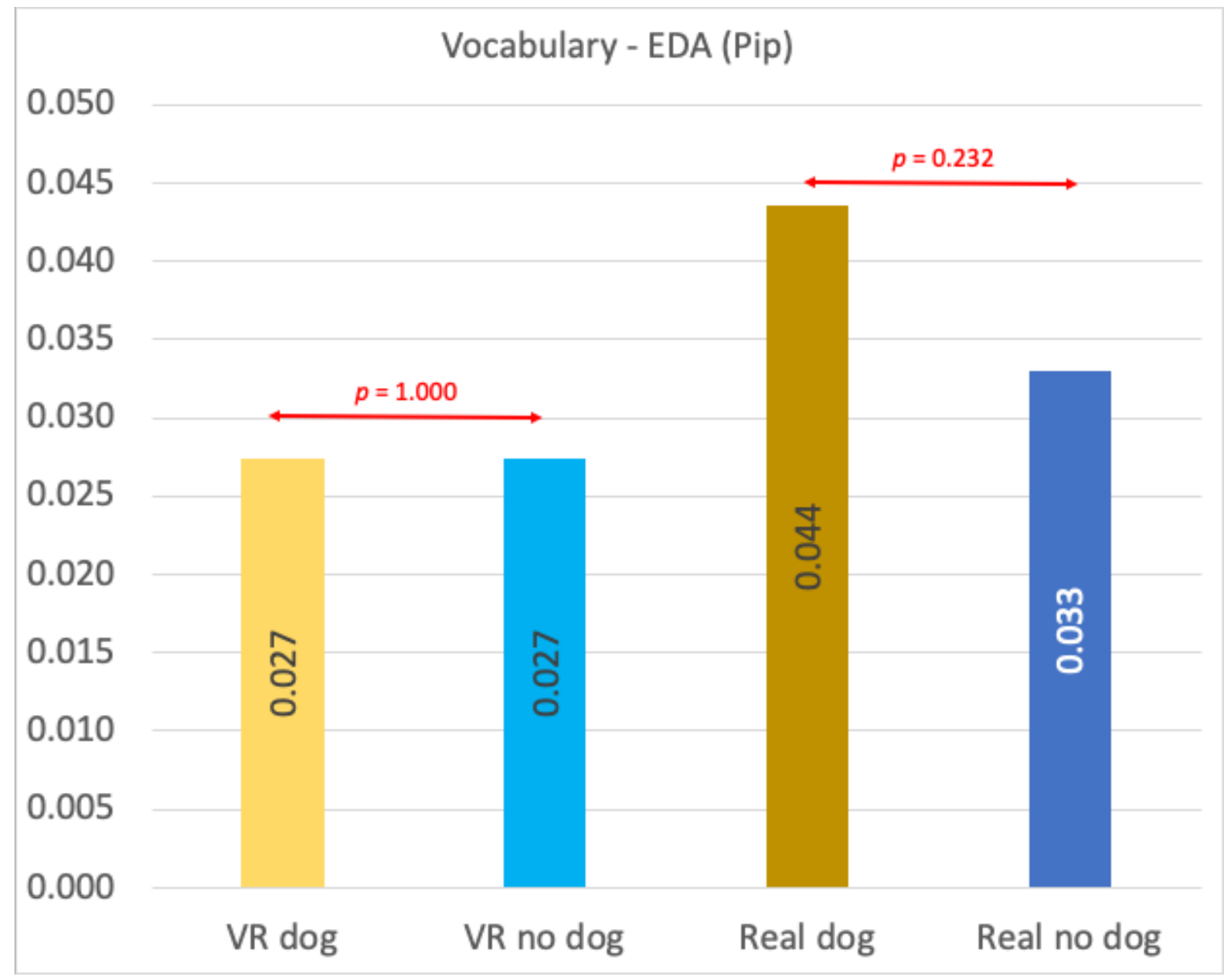

Figure 53: EDA scores across all four conditions - while participant completed the vocabulary tests

Figures within the bars are mean scores. Figures on arrows between conditions are $p$ values from Wilcoxon signed-ranks tests. A red arrow indicates no statistical significance at $p<0.05$.

A Friedman test revealed that EDA scores varied significantly across the four conditions: $\left.\chi^{2}(3, N=24)=8.096, p=0.044\right)$.

Pairwise comparisons using Wilcoxon matched-pairs signed-ranks tests (Bonferroni corrections applied for multiple comparisons) were conducted.

There was no significant difference in the EDA scores for the VR dog and VR no dog conditions $(z=-0.457, N-$ Ties $=0, p=1.000)$. 
There was a significant difference in the maths scores for the Real dog and Real no dog conditions $(z=-1.571, N-$ Ties $=0, p=0.232)$.

8.95 Electrodermal activity (EDA) scores - maths sessions only.

The electrodermal activity scores recorded across the period in which the participant was doing the maths tests are shown in Figure 55.

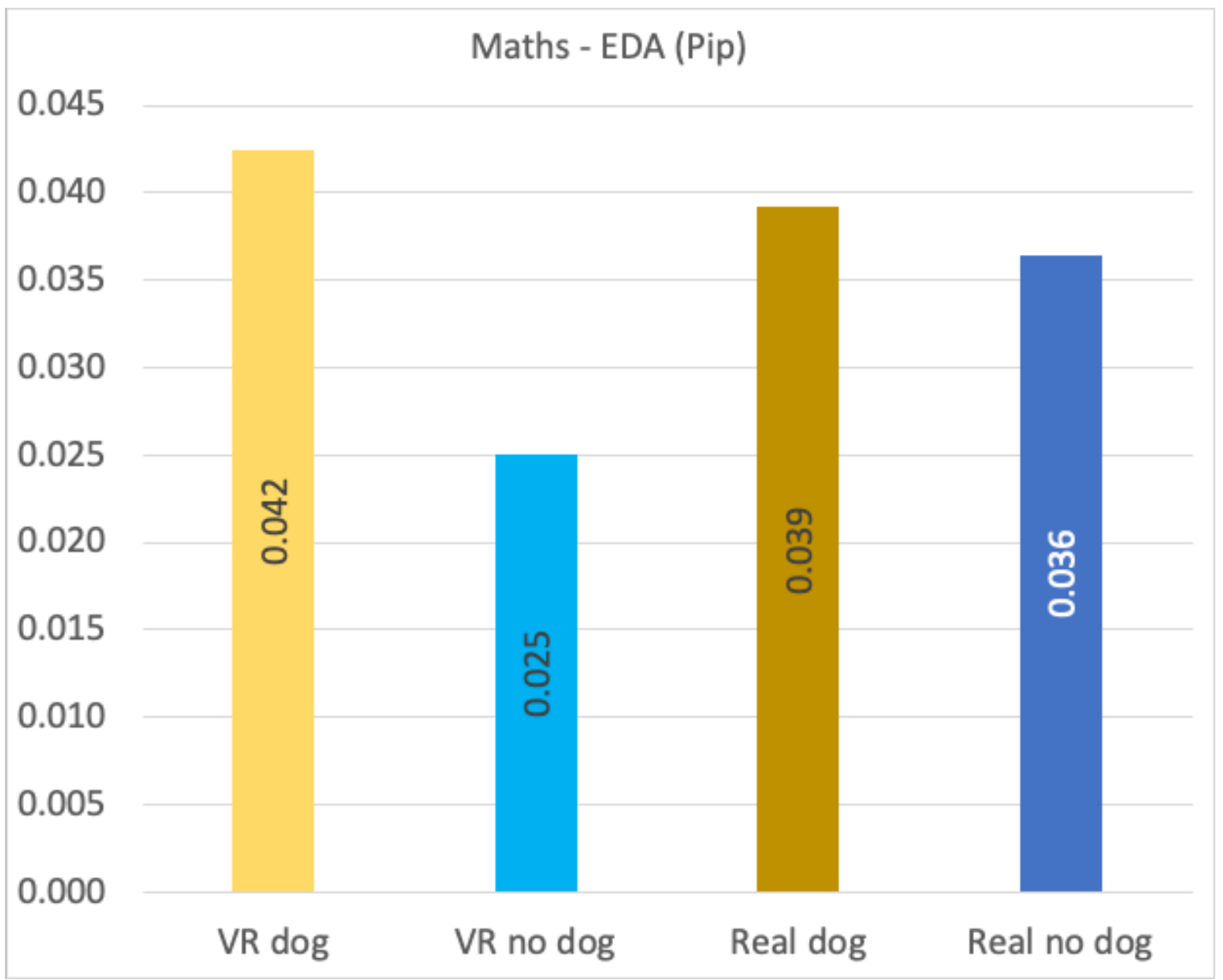

Figure 54: EDA scores across all four conditions - while participant completed the maths tests

Figures within the bars are mean scores.

A Friedman test revealed that EDA scores did not vary significantly across the four conditions: $\left.\chi^{2}(3, N=24)=6.967, p=0.073\right)$. 
As the overall Friedman test was non-significant, no further pairwise comparisons were conducted.

\subsection{Discussion}

There was a significant difference in vocabulary scores across the four conditions with pairwise comparisons revealing that the difference was between the VR dog/VR no dog conditions. Pairwise comparisons were only conducted on the VR and real conditions (not between them) in an effort to reduce the number of comparisons tested.

There was a significant difference in maths scores across the four conditions (the means suggesting that this difference was between the VR and real situations) but there were no significant differences in performance between the dog/no dog conditions. That is, whether the maths task was conducted in VR or not, made a difference to the scores, but the presence of the dog (real or virtual) did not.

Analysis of the EDA scores over the whole session (maths and vocabulary) revealed an overall significant difference across the conditions with the pairwise comparisons suggesting that the significant difference between EDA scores was between the real dog/no dog conditions.

Considering the EDA scores for the vocabulary trials, there was an overall significant difference across conditions, but no significant difference between VR dog/VR no dog, or Real dog/real no dog.

Considering the EDA scores for the maths trials, there was no overall significance across the four conditions and so no pairwise comparisons were conducted. 


\subsection{Summary}

The significant differences are for the overall EDA scores for the "real" situations, particularly with the "live" dog and handler presence, and the vocabulary tasks when in VR. The "live" situations appear not to significantly affect the overall achievement scores for either the maths or the vocabulary tasks. The achievement results only show a significant difference for vocabulary tasks when in VR. This result would suggest that there is greater focus and attention on the vocabulary task when in the VR environment.

As the vocabulary tasks were planned with a heavy emphasis on verbal recall, incorporating both long term memory and working memory strategies in order to answer these sections, it could suggest that when in VR, the dog's presence has helped to focus verbal attention through mediating mental forms of visual representation, articulatory and auditory rehearsal. This would suggest that the dog is affecting the executive skills and phonological loop verbal skills of the working memory with this group of adults. This is considered a "top down" skill, subconsciously made, rather than conscious effort.

The overall EDA results for the live conditions also showed significant differences. These differences suggest that this sample of adults preferred the normality of the real situations, presumably because they were more congruent with their previous experiences of learning or that using the VR headset was still too novel an experience. The achievement scores however, for both maths and vocabulary tasks, were still overall higher in these conditions than those in VR.

Considering the EDA graph (Figure 55), despite the pairwise comparisons not reaching significance, both situations involving the dog show higher scores. This finding could suggest that despite the "live" situations not showing a significant effect of the presence 
of the dog, there could still be some effects when the dog is present. It is possible that the participant preferred the dog's presence in the live situations, thus reducing anxiety, or that due to the maths tasks being very similar in all four conditions, the dog was having some subconscious effect on the mental processes and self-regulation, possibly assisting with the dual coding of not only reading the question mathematically but the visualisation of the visuo-spatial processes and skills used to solve it. This may have improved some of the individual scores of the participants, although overall it should be stressed that there was very little difference in the group achievement total scores between the handler only present (607) and the Live dog and handler (605).

This phase of the study has again shown that while 'group' effects can be achieved, there appear to be strong individual differences. If each participant was examined in the same way as the children's case studies for their responses to the tasks in the conditions and their personal EDA, there would be 24 different conclusions drawn.

This study has confirmed earlier findings that subjective impressions may not match measured performance. The data indicate that care should be taken when using personal impressions as to the utility of a dog, as a factor in determining the utility of a dog in assisting academic learning. 


\section{Chapter 9}

\section{Conclusion}

This thesis addressed the research question of whether the presence of a dog could assist with educational achievement by enhancing cognitive acuity through facilitating executive functioning, working memory and attention. This research question was based on a body of research by several groups of researchers that suggested the presence of a dog, through reducing stress, and with concomitant changes in neurotransmitters, blood pressure and electrodermal activity (EDA), would facilitate improvements in learning. The research evidence for these improvements was based on classification tasks, following instructions and sequencing activities with very young children and adults and reading tasks (Gee et al., 2007, Gee et al., 2010a, Gee et al., 2012a, Hediger et al., 2017, Gee et al., 2015b, Beetz et al., 2012b, Beetz, 2013, Beetz and McCardle, 2017, Schretzmayer et al., 2017, Wohlfarth et al., 2014). Findings were related back to the 'biophilial hypothesis' proposed by E.O Wilson (Kellert and Wilson, 1993) where the human has a genetically determined affinity with the natural world, so that through the visual perception of the animal and subsequent heightened arousal, this would result in improved general awareness and relaxation. Results from the research with young children, however, remained inconclusive and very much depended upon small numbers of mixed age groups, selected 'diagnosed' groups, i.e. those considered with the same particular need and a range of different methodologies, with little evidence found to link results to actual 'real life' classroom learning tasks. The only activity that had been suitably developed for 
use in classroom with a dog was the 'Read to Dogs' schemes, also known as 'Paws and Read,' where children are asked to read aloud in the presence of a dog. The dog, as an 'uncritical listener,' was claimed to enhance fluency, accuracy, self-esteem, confidence and ultimately reading attainment for the children concerned (Pets-As-Therapy, 2016, Bassette and Taber-Doughty, 2013, Lenihan et al., 2016, le Roux et al., 2014, Connell et al., 2019).

This thesis aimed to expand the research by investigating the effects of the presence of a dog in both 'virtual' and 'live' situations in a practical, classroom situation within a primary school and also a university in England, while investigating specific curriculum-based tasks such as reading, vocabulary and mathematics. It was hoped that there would be clear physiological differences in the responses to the dog in the immediate, 'within the moment' situation, which over a longer period would raise subsequent academic achievement with all the participants. The effect of the presence of the dog would also hopefully be similar across age groups of both primary-aged school children and adults included in the studies, suggesting that any improvements in performance are underpinned by changes in specific cognitive skills, rather than simple maturity in overall thinking. The cognitive skills examined were working memory and attention, both key skills for classroom-based learning, affecting all school curriculum subject areas.

Electrodermal activity (EDA) was used as a measure of physiological stress during the tasks. Due to the age of the children, a small, plastic, handheld 'Pip' was used for this EDA measurement, which was also repeated successfully with the adult participants for comparison, while the adults also used a 'virtual reality' headset. 
Consideration was also given to the role of the teacher or handler within the classroom, in that measurements and methods used within the thesis research would be congruent to those currently used in classroom research, and that any subsequent testing could be verified by a trained practitioner in order to replicate any results. This approach was designed to facilitate the access for schools programmes to become more involved in creating a teacher led research base for the use of dogs in classroom learning - as promoted by two, recent, British, systematic reviews (Brelsford et al., 2017, Hall et al., 2016a).

Teaching, incorporating dogs within school environments as a qualified, trained teacher/practitioner is referred to as Animal Assisted Education, or if by an untrained staff member or educator, Animal Assisted Activities. If the intervention is on a regular basis, then this becomes Animal Assisted Intervention (IAHAIO, 2014 updated 2018, Winkle et al., 2012).

Previous research has tended to be limited in scope when adequately promoting, designing and evaluating methods for linked classroom practice for teachers (Brelsford et al., 2017, Smith and Dale, 2016). The mixed methods approach to this thesis was as a response, split into three phases; Part one, a background desk based survey to establish what was already existing in England's schools and how the role of the dog was, as then in (2016), understood and interpreted (Chapter 4); Part two, an in depth study including working memory tests, available to some teachers in schools, following a 'Paws and Read' charity led scheme with 13 , seven year olds, seven of whom were then taken on as individual case studies (Chapters 5, 6 and 7); and Part three, an investigation with 24 adult participants, using both virtual reality and live conditions to examine the effects on 
attainment and electrodermal activity when answering to classroom-based vocabulary and mental maths tasks with and without the presence of a dog (Chapter 8.)

\subsection{Part 1:}

In 2016 there was limited published evidence into how many dogs were in the United Kingdom's schools and their purpose. The 2016 background research into how many dogs, their roles and expectations for educational achievement, presented in Chapter 4, revealed that just under $10 \%$ of special schools within England were using dogs. These were in different roles, but all were referred to as the 'school dog.' These dogs attended schools both in part time and full-time roles and a small number (totalling 55) were often 'owned' or 'handled' by general members of staff or with those with specific teaching roles. The vast majority of 'school dogs' however (totalling 67), were brough in on a regular basis by volunteers from the public, often not educators themselves.

In 2016, issues became apparent as to the standards, welfare, training and the costs of the dogs involved, many of which were in school for improving the social and emotional benefits of the classroom, rather than academic purposes. Teachers appeared to be relating to the then, current research into the therapeutic benefits of including a dog, such as raising self-esteem, self-confidence, reducing fears, worries and anxieties, increasing classroom attitudes to learning and improving social skills, but not considering the academic, teaching strengths, as was also suggested by the likes of Beetz (Beetz, 2018, Enders-Slegers, 2018, Gee et al., 2015a, Hediger et al., 2017). The desk-based study findings identified that many dogs, brought in by staff members, simply were in school to be 'present'" usually occupying an office space until 'required.' The potential of the dog as an interactive partner for specific learning or teaching tasks was usually underplayed. 
There was little evidence of organised interaction or planning for activities, other than reading to the dog.

Despite a small core of individual organisations, businesses and charities adapting a purposeful training approach based on the 'assistance' dog model, the largest group of 'handlers' within these sample schools were 'visiting volunteers.' These members of the public, together with many school staff members, were entirely relying an individual charity's, one time only, 'temperament assessment test,' to 'qualify' their dogs as 'therapy' animals, which could then be brought into schools on a permanent, full time basis. This revealed an 'ad hoc' approach of the scope for the dog's role to develop over a period of years. Consideration towards the planned safety of the interaction with the children was underemphasised, despite planned, organised child /dog programmes being available (Meints et al., 2017). Insurance and risk assessments also appeared limited within the sample. Out of 104 schools, the five school dog policies found, were sporadic in their explanations of how the dog was incorporated into lessons, its welfare and procedures for working. In fact, it appeared that, overall, the standards for Animal Assisted Education or Animal Assisted Intervention (Winkle et al., 2012, Beetz, 2018) had hardly been developed within this group of special schools within the UK at that time. When it did occur, it was referenced as 'reading to dogs' only. England appeared to be behind Europe and the rest of the World when it came to animal assisted education in the classroom.

This lack of cohesion was due to issues of the lack of transparency, regularity and consistency in the awareness and training of both the dogs and handler/teachers/owners' teams, within the sample schools, together with the tendency of the personification of 
the dogs' roles as if human 'staff' members and differing interpretations of the word 'therapy.' When the number of dogs from these sample schools was re-checked in 2018, however, the numbers had fallen, particularly in the 'volunteer' sector. This may have been due to several sets of guidelines being issued or updated within a short period of time, changing the qualification of the school dog, no longer allowing simple, once only charity 'temperament assessments' but considered thought as to the animal's welfare, training and experience (Kennel-Club, 2018, IAHAIO, 2014 updated 2018, Winkle et al., 2019). Dogs trained using assistance dog techniques, however, were still in place after the two-year period, but this was likely due to the associated costs and long-term commitment required from the sponsors and schools.

The desk-based search also reflected that teachers were still unaware of the potential of animal assisted learning, due to the lack of time given within their teaching roles, to develop different schemes and planning which could be used for pets across many schools. (Teacher job vacancies, even as of 2020, have never addressed the dual role of teacher and dog handler.) Addressing this issue would require some central guidance, created National standards or leadership. Since 2016, the schools have become competitive in nature with no practical, standardised guidance or vision as to how to promote dogs in an ethical and academical playful way, therefore both 'positive' and 'negative' classroom-based practices are still being promoted between them.

The desk-based study also revealed that often only basic attainment measures were being deployed to show the efficacy of the dog for academic activities, such as reading; usually reading tests or self-esteem or behavioural questionnaires. The background collated from this study, was given to the representatives from the 'Therapy Dogs Nationwide' 
volunteer charity team when attending the initial meetings for the creation of the 'Bark and Read' guidelines (Kennel-Club, 2018). The lack of rigorous assessment of the efficacy of dogs in classrooms also created the groundwork for the following chapters and studies.

\subsection{Phase 2}

Phase two is outlined in Chapters 5, 6 and 7, of this thesis. These findings presented are from research data collected in 2016, in response to the identification and nature of working memory differences when a dog was present; the following of a 'read to dogs' scheme set within a 'real' school environment, revealing the practicalities of the project and how attainment was, and could be, measured; the subsequent effects of the presence of the dog within the groups for the reading project; and individual case study profiles into the efficacy of the dog for immediate and longer term academic targets.

Chapter 5 explored whether if dogs could affect working memory skills used within all academic learning, how they could potentially be measured, and if so, in which areas those effects could benefit academic achievement. Working memory skills and sub tests were available and used in schools at the time (2016) and could have assisted certain, qualified members of staff to investigate if the dog was influencing cognitive skills. These would, however, have been limited to specific "specialist" teachers who were level 7 qualified (i.e. Master's Level and above.) A future adaptation, therefore, will be necessary to increase access to these tests for less qualified classroom teachers, such as a specific screening test being created.

Cognitive tests proposed were from working memory test batteries, phonological assessments, cognitive profile achievement tests and visuo-spatial reasoning and memory tests (Reynolds and Voress, 2007, Frederickson et al., 1997, Gibbs and Bodman, 2014, 
Wilkinson and Robertson, 2006). Sub tests selected were trialled using the seven focused case study children while in a school environment. It quickly became clear that the length of time required to fully explore the width of the proposed assessments, with and without the dog present, did not coincide with the school timetable, thus only six could be used within 30 minutes: Naming picture speed, naming digits speed, letters and digits forward spans and letters and digits backwards spans.

The group results showed that the dog's presence appeared to be affecting executive functioning, phonological and auditory short-term memory, visuo-spatial processing speed and increased memory span, although each child's profile was unique both with respect to baseline and consequential profiles. While this was a single measurement taken at a specific point in time, and thus may not be truly reflective of the individual's cognitive responses to the dog, the concept of creating a screener for use while a dog is present could be considered in the future. The tests chosen, however, did indicate for two individuals, why they may have been chosen for the 'read to dog / paws and read' project within school by the school SENCO due to the baseline specific strengths and weaknesses in their working memory profiles.

Chapter 6 followed 13 children (three of whom were the control) through 12 weeks, (Autumn Terms 1 and 2) attending the "Paws and Read" project back in school. Several measurements were taken: Pre and post dog reading attainment with the entire group using reading ages and standardised scores; timed reading speed and recorded accuracy to examine any effects on fluency and processing with the focus case study group of seven children; and also with this case study group, electrodermal activity measurements to check for over and under arousal through stress, which may have influenced the skills. 
Measurements for the case study group were compared from recordings taken both with and without the dog present. Questionnaires were given to the children and adults involved with the project to gather their views and opinions.

The evidence showed that the seven case study children, when reading to the dog at the age of seven, still believed that they were able to assist the dog with its reading but were mixed in their responses as to how the dog could help them. There was a growing awareness that the dog was non-human when it came to communication, despite two children still anthropomorphising completely with the dog's abilities (Airenti, 2018). This highlighted the fact that as the children read to the dog, they were unaware of any potential reading measures being used by the adults in schools as the reading tests were usually taken back in class away from the dog. The children read to the dog purely for enjoyment and motivation. At this age, however, children do tend to follow adult led directives, therefore some answers may have been fabricated by the children to please the adult (in this case the researcher.) The school based staff members however revealed that they assumed the scheme was successful as they felt the children had gained in selfconfidence when back in class, but they were sceptical of the scheme raising academic achievement, as were not involved in the selection, monitoring or assessment of the project in school to know if it made any difference. One teacher however did reveal that the group of current students had been selected by the SENCO, which was not usual, and that not all staff members were enthusiastic about the scheme, however as a member of staff, they had to allow their pupils to. These general finding of teachers' personal opinions affecting AAI research is currently under investigation at the University of Swansea. 
The school SENCO used the Salford Sentence Reading Test (McCarty and Lallaway, 2012) with all the Year 3 intake before and after the 'Paws and Read' project for comparison. The reading results suggested that, on a group basis, the ten children reading to the dog, including the case study group members, appeared highly successful. The apparent progress within the group, both in reading age and standardised scores, in comparison to the three children used as the control, superseded even the 'impact' of other non-dog, literacy-based interventions available in schools (EEF, 2018). This 'impact' was due to measuring 'ratio gain' which gave this study's dog reading group result of 2.9 or 'useful impact' in comparison to the control of 1.7 or 'modest impact' of the non-dog readers (Brooks, 2007, Brooks, 2016, EEF, 2018).

Closer inspection showed that within the read to dog case study group, some individuals' high reading 'age' and 'standardised scores' were raising the overall average for the group, whereas other individuals had made little to no progress, or had regressed. Less reading progress was shown for those who had greater fluency in their reading, particularly those who started with reading ages 'higher' than their chronological ages - one child by up to three years (Male M). The four children who were struggling with the skills of reading made more 'apparent' progress than those who had already achieved fluency over the 12-week time period. This finding corroborated recent research by Conell et al., (2019) and Kirnan et al., (Kirnan et al., 2016) who also reported that the Special Educational Needs and the younger readers had the greatest gains when reading to a dog. The appearance of 'improved' reading skills, with these 'struggling reader' case study children could have been simply due to the level of reading books that these children had selected 
at the time, which contained shorter sentences and easier decoded words than their more fluent peers, or that extra practice had been encouraged from home.

Electrodermal activity also reflected highly individual and varied responses. Overall, the group EDA was lower in the 'with dog' condition. This would also suggest that group wise, the visuo-spatial aspects of learning were being employed (Brenning et al., 2012, Smithson and Nicoladis, 2016, Boulet-Craig et al., 2017, O'Haire et al., 2015). This would echo the working memory findings in Chapter 5. The individual responses however, appeared not to have markedly influenced reading between the two conditions; dog or no dog, at all. This suggested that the individual reading response was to the reading task itself, not to the presence of the dog. This echoes the recent work of Janet Trammell who posited that stress effects recorded by EDA are often linked to the attributed emotions to the task, rather than the dog (Trammell, 2019).

To check this hypothesis, a reading of a selected piece of poetry was also recorded, by the seven case study children without the dog's presence. These results were compared with the reading 'without a dog present' sample. The poetry returned very different responses for each child in terms of accuracy, speed and electrodermal activity. This showed that it was very likely to be the individuals own self agency and determination for the successful completion of reading tasks, rather than the dog clearly assisting with processing. It became clear it was 'what was read' which reflected in the stress levels, accuracy and speed, which depended on the individual motivation, personal likes and dislikes of reading material, skills and strategies relied upon for decoding used by each child. These strategies, although mostly taught in school, also depended on genetics, consistency of 
practice and regularity of print exposure (Bergen et al., 2018). The dog, therefore, was possibly only a minor part of this motivation.

To further check if or how a dog's presence had or had not influenced skills for the case study individuals, Chapter 7 examined all the data available for each child as case studies.

Chapter 7 revealed that the children had very different profiles and diverse areas of enhancement in accuracy, reading speed and electrodermal activity between each other when the dog was present. No two profiles were alike and thus it was difficult to accurately predict the effects of the dog on the results. Simply measuring 'reading age,' even with standardised scores and 'ratio gain' used by schools, may be too crude a method when investigating the subtle effects of the dog's presence.

Cross-comparison of the findings from the working memory tests with the four, lower reading skilled individuals, (Female $A$, Male $A$, Male $C$, Male $H$ ) had showed that, with the exception of Male $\mathrm{H}$, the 'dog present' results showed clearer, positive results for processing speed, executive functioning and visuo-spatial recall - skills used within the reading process. As all the children had read only up to three times, maximum, to the dog, the read to dog sessions could not be held responsible for the entire reading achievement.

The video evidence revealed that, despite the children's various anthropomorphic beliefs of the ability of the dog and motivation to read to him, the children were so focused on the task of reading, that they ignored the dog during these periods. This would question the purpose of including the dog for reading sessions at all. One boy, Male C, showed such indifference towards the dog that for one session, he chose not to attend. 
The dog was not always a positive experience. At least two children recorded an anxious state when reading to the dog, which negatively affected their speed and accuracy (Male $\mathrm{H}$ and Female A). Moreover, these skills were not improved with the non-dog reading or the poem, suggesting that there were, in depth, neurological differences affecting their reading skills. The struggling readers' overall success in reading 'gain' through improving their reading ages was probably due to other, outside influences of reading, encouraged by parents, the reading culture, increase to access for reading and the school promotion to hear every reader.

The dog appeared as a distraction for two others, shown clearly in their electrodermal activity variance box and whisker charts. The more fluent readers, however managed to maintain their focus and energy, returning their attention to the task if distracted by the dog's presence (Female G.) The comparative evidence revealed only small differences for fluency and speed with three fluently reading children, who marginally slowed their speech when the dog was present. Again, this suggests a plateau of benefit when reading to the dog, after which the dog appears to be superfluous. These findings would contrast with suggestions by Beetz that the dog is of benefit to all readers (Beetz et al., 2012b, Beetz and McCardle, 2017).

The read to dog scheme was based on this perceived motivation to assist the reading process, which most children appreciated, but may not have required. Despite the evidence from Chapter 5 and the working memory tests, the benefits from the predicted differences in processing speed, visuo-spatial, and phonological recall were not being sufficiently reproduced in the actual children's reading, either due to the fact that they did not exist, or they were too subtle to be measured by simply calculating speed, 
accuracy and fluency and electrodermal activity. The prediction of who would most likely benefit from reading to a dog is too complicated and results cannot be generalised enough to suggest 'groups' of children who would benefit from specific intervention with a dog for academic benefit. This would imply that some form or internal calibrated measurement is required, such as scanning, or measuring electrical activity within the brain while these tasks are taking place (Hediger and Turner, 2014). Obviously, there would be huge ethical considerations for these types of studies with young children.

Perhaps the multi-faceted task of reading involves too many simultaneous skills, making it unclear if the dog has an effect or not. At this stage, in trying to find clear evidence for cognitive acuity improvements with dogs in schools, we may well be literally 'barking up the wrong tree.'

\subsection{Phase 3}

Chapter 8 saw the study switch to adults. While Gee (2017) used adults with a working memory test, suggesting the mere presence of the dog was more favourable for adult attainment, Trammell (2017), used time intervals before an actual exam to improve attainment, this study used classroom based activities such as vocabulary knowledge and maths tasks, not just with a 'live dog' but also with a 'virtual' dog to compare and contrast the effects. This approach was for several reasons, including the practical use of virtual reality headsets without so many health and safety implications associated with young children's cognitive development. A larger, experimental set up with 24 adults was investigated, which included both vocabulary and mathematical activities. Evidence suggested that while adults were more aware of the anthropomorphic tendencies when involving animals such as dogs in activities, they should have been less susceptible to their 
influence, remaining 'on task,' thus any differences in achievement or subsequent averaged electrodermal activity should be because of the dog's presence. Whereas the children were still following given strategies and structures for their reading, which limited and influenced some individuals' overall results, the adults were free to use a combination of pre-learned mental strategies from their long term and working memories to answer either vocabulary or maths questions verbally. The adults also had the comparison between four, separate conditions: Dog and handler in VR; Handler only in VR; 'Live' dog and handler; and 'Live' handler only thus extending the research into whether presence of the dog influences mental acuity in both virtual and live form.

Again, the Pip (electrodermal activity monitor) was used to measure individuals' stress levels while the dog was present in both 'virtual' (VR) and 'live' form. Unfortunately, due to the health and safety considerations in wearing headsets for under the age of 18 , the children had been unable partake in a virtual reality setting to complete a similar, comparative study, nevertheless the adults, with minimal safety implications were all able to do so

This study encountered some unexpected issues which arose when trying to film the dogs for the virtual reality headsets. The thesis relies on the interaction between the human and the dog. The dogs quickly showed that they were unable to interact with a simple $360^{\circ}$ camera on a tripod as they would have done with a human participant available. They totally ignored the camera and tripod and wandered into the far corners of the room where they waited for the filming to finish. Other attempts for asking the dogs to lie on their blankets next the handler sitting still, resulted in them falling asleep giving no interactive feedback to the camera and thus the participant. Despite the children 
appearing to 'ignore the dog' in the previous studies, they were able to interact if they wished. Clearly there was a stronger requirement for the relationship between the dog and the 'virtual participant.' A solution was to include the handler, rolling their toys towards the tripod camera 'participant,' while trying to refrain from being in obvious view. The dogs would follow the toys to the 'participant.' The use and filming of animals in virtual reality for targeted, specific tasks, requires further development and practical consideration in the future, including careful ethical planning, a little explicit training and watchful implementation.

Unintentional issues appeared with the recruitment of the adult participants. The study had been open to all students who wished to take part on a volunteer basis. From those who had signed up, it quickly became clear via the questionnaire, that all but one adult participant had either had pets as a child, currently lived with one or had owned a pet in the past. This biased the findings in the favour of those who were pro-animal interaction. This was similar in the fact that all the children and all but one adult in the school-based study also had the same, unintentional, backgrounds. It could be argued that in future, perhaps non animal or pet 'guardians' (Bekoff and Pierce, 2019) could be recruited for a sample. Yet as this study is examining the presence of the dog 'in the moment' with tasks and differing conditions, the measurements are focusing on the individual's reaction and results, rather than their opinions. Nevertheless, the adults for this study did comment that they had expected to feel more 'relaxed' in the presence of the 'live' dog, with 18 out of the 24 participants reflecting that they had done so, after all four conditions were completed. 
The data showed a clear difference between the group 'live' and 'virtual' achievement scores for the overall tasks, with the 'live' conditions showing the higher attainment overall. These scores were taken from the simple right / wrong answers verbally given to the questions in all four conditions. The virtual reality (VR) condition results were on average, 175 points lower than the live dog conditions. This may have reflected the 'novelty' of wearing the headset and for many, the first time they had experienced VR. An alternative argument could be that subconsciously, the participants preferred the less claustrophobic 'normality' of interacting with the open environment around them.

The 'VR dog' condition had higher achievement scores than the 'VR handler only' condition, whereas the 'live dog' and 'live handler only' conditions scored within three points of each other. This will suggest that both the dog conditions whether in VR or live were affecting the overall achievement scores.

The adults found it difficult to distinguish between the condition they felt the most relaxed or concentrated the hardest in, with 12 choosing the same condition for both. These were not always the dog conditions, although 18 of the students did choose this. When comparing the actual electrodermal activity average readings with the personal choices for the sessions, the individual predicted readings did not match the participants' thoughts, with neither the expected highest nor lowest average readings recorded for those sessions. This shows that what we generally believe is happening consciously, stress wise, is not always the body's subconscious reality. This was also shown with the individual Pip scores for the case study children, where clearly two children had anxiety issues when reading with the dog present, despite being motivated to read to him. 
In terms of preference, the vocabulary tasks were favoured over the maths by all but three participants. This revealed a significant amount of lack of self-confidence and self-esteem for mathematical ability with this group of adult participants. This portrays negative, emotional responses to learning this subject in schools. As with the children, the adults portrayed this reluctance through their body language and hesitancy in their answers during their sections, with closed body posture, hunched shoulders and static positions. One participant started inadvertently rocking while thinking her way through these sections. Even with the live dog present, participants stopped all interaction and maintained full visual focus on the mathematical questions on the board. In contrast, during the vocabulary, participants showed more open body postures, more interaction with the live dog or live handler through eye contact, head turning, and physical contact with the dog during or between the questions. Similarly, to the fluently reading children, during the vocabulary sections, the participants quickly selected the answer, gave it and then had time to return the focus to the dog and back again for the next question. The positive, more confident interactions were like those shown from the children after they had finished reading to the dog and could relax. These observations and findings also show that it is the perception of the activity which may be affecting the results, rather than the presence of the dog, and the strengths and the reliance on the familiar verbal automised language skills at an adult age is far stronger than the often repeated, under the breath, confirmation required when remembering particular steps to complete a maths problem.

The differences between the live and virtual reality maths and vocabulary scores did reach statistical significance. As the selection of adult students was not indicative of a normal distribution of the population (parametric assumption), non-parametric assumptions 
were used in the SPSS analysis. The Friedman test did reveal that the scores varied significantly across all four conditions, but the Wilcoxon pairwise comparisons only showed significance between the Virtual Reality conditions and live conditions for vocabulary.

For maths, again the Freeman test revealed that the scores varied significantly across the four conditions but with the Wilcoxon pairs, no significant difference was found in the VR maths $(p=1.000)$ either with the VR dog or VR handler condition, or in the 'live' maths with 'live' dog or 'live handler.' This finding could be due to the participants finding that they could not think or remember the answers or correct strategies to answer the questions, possibly indicating memory overload. The maths questions contained several different elements to the test: i) mean and range of numbers; ii) percentages; iii) simplification of algebraic terms; iv) equivalent fractions; v) negative numbers; vi) addition and subtraction of decimals; and vii) simple order of operation. This may have been too many concepts to remember at once.

This response was also seen with the children's working memory task overload (Alloway, 2009, Alloway 2007, Gathercole et al., 2008). A 'freeze, flight or fight response' ensued, but whereas the adults tended to use a technique of moving on to the next question to be answered, the children would stop and take far longer to recover before attempting the next question or task. This difference reflects the maturity of the self-regulation strategies used, which have either been developed through self-experience or direct teaching.

The electrodermal activity scores did show significant differences between the 'live' dog sessions and the virtual dog sessions. This was unsurprising, given that the preference 
from the adult participants was for the live dog condition, but this gave the statistical support to the claim. With vocabulary EDA, the Friedman test revealed scores varied significantly across the four conditions. but when using pair-wise Wilcoxon tests there were no significant differences between the four conditions, either in VR or live. This finding could support the comments, backed by the recordings of the body language from the participants, in that they enjoyed and preferred the vocabulary tasks as they were more familiar with their presentation from past school experience and could see and hear where the dog was in its positioning in the room. As the group average electrodermal activity was not significantly different across any of the conditions, this could imply that when a familiar case of knowledge is called upon, such as the long term recall of vocabulary, the extenuating circumstances do not affect the group electrodermal activity in terms of stress. This may be different, however, for each individual within the group.

The EDA scores for maths sections across the four conditions also showed no significance as the Freeman test showed that scores were not significantly different across the four conditions. This implies that the overall group response during the maths activities, despite the individual reactions within it, showed no significance. This could suggest that it is the group reaction to the unfamiliar mathematical knowledge that has not been known for the differing styles of questions across the four conditions and which has flattened the overall average scores. This could propose that the individual participants may well have consciously abandoned the mathematical tasks or reacted neutrally towards these sections which has been reflected in their EDA measurements, flattening the overall group levels. Comments from the participants themselves revealed that they had found the movement of the dog a distraction in the VR condition, particularly when 
concentrating during the maths activities. This could be due to the swapping of attention between the actual task to be completed and the natural tendency to want to view where the animal was. This may have created cognitive overload for some, but not all the participants.

\subsection{Limitations to the study}

\subsubsection{Size:}

The main limitation was the sample sizes. Seven children in one school, in one county of South West England, is not enough to generalise the efficacy of dogs in all schools for mental acuity when examining working memory and attention. The 24 adult students required for counterbalancing for order effects, rather than the full 84 as suggested by the prospective-power analysis (Faul et al., 2009) means that the statistical analysis was underpowered. A higher number of participants may have revealed that there are greater differences between subject areas or between virtual and live conditions with the dog. The reduction in participant numbers was due to the working conditions for the dogs involved. Had more dogs within a longer time frame been available, then the study numbers could have been increased. Other studies using therapy dogs with adults have also found this issue. For example, Gee et al., in 2014, required 53 students with two dogs working on a single occasion and again in 2015, with just 31 students and two dogs. In Trammell's smaller studies (2017) she used six dogs with 50 students, and five dogs with 56 students. Both Trammell and Gee et al., used incentives to gain participants. Despite the small numbers, in this thesis, the data do provide some valuable findings, as discussed. 


\subsubsection{Expectations from outside parties}

The study was difficult to set up as study was limited to the dogs and charities available at the time (2016.) Several charities were asked, initial meetings set up, interviews held, but the dogs became unavailable at the last minute. Only one newly formed volunteer-based charity was prepared to assist, who gave the contact for one of their local volunteer dog handler teams and were able to initiate introductions to the school. Many charities are not ready to receive, or have anyone in the role ready to assist, with researchers at any level examining the role of the dog in the classroom. One school dog charity refused all written or verbal contact with either the head office, or its dog handling teams, unless the researcher was from certain, 'approved,' in their opinion, universities.

\subsubsection{Collaboration}

Working in collaboration within a working school environment can also limit the scope of research. There are advantages and disadvantages for the often 'two way' process. For instance, the initial request for 60 , Year three children to be followed over an academic year was deemed too large a request, as was for time to work with a small group of the students and dog for mathematical activities in comparison to the reading. The school suggested the SEN group for 'Read to Dogs,' limited the project to 12 weeks (two school terms) and introduced the project to the parents. Despite sending out invitations to all the Year 3 SEN parents to allow permission for videoing, recording and extra working memory tests, only eight sets of parents replied with full permission. This was because the parents and the school were not happy for the children's blood pressure to be taken. Once this was realised, and with negotiation, a solution was found in using the handheld Pip. The before and after reading test was also chosen by the school as part of their overall 
data collection process, to fit in with their routines. Through the sharing of the immediate feedback of the working memory tests to the SENCO, at least one child was highlighted at an early age, for further diagnosis and intervention for specific literacy difficulties and the profiles of the other seven children were able to be monitored over the academic year. Ultimately the school-based research did take place and was completed on time, although slightly differently than was first planned. Collaboration requires flexibility, adaptability, and trust in the professional roles of others.

\subsubsection{Selection of participants}

The adult students were all selected through one university in the South West of England and despite advertising through four campuses, only one campus where both the education and psychology departments were situated replied. As was explained earlier in this chapter, the data sample appeared biased, due to the number of pet or animal owning, volunteer participants who responded.

\subsubsection{Methodology}

The methodology chosen involved first-hand gathering of data and the exploration of subsequent themes and issues. It was a mixed method, approach which gathered both the views and opinions of those taking part, while also collecting empirical data. This is a familiar approach, for an educational researcher. Skills, methods and techniques however were gathered from the areas of cognitive and developmental psychology and introduced into the overall research. Had a purely psychological approach been taken from the beginning, these studies would have been presented and interpreted differently. The overall methodology reflects this researchers background as a school-based teacher, but 
this thesis has explored how symbiotic the fields of psychology and education are. A future path may be for the researcher to re-train as a psychologist.

\subsection{Future direction}

The research into the skills for the identification of cognitive acuity with dogs in educational tasks has explored some issues and has raised further areas for research. The data showed that the presence of a 'live' dog is preferred by many over a virtual dog, for familiar school based tasks, such as multiple choice vocabulary questions, but dogs are also effective when in VR for achievement, despite the practicalities of trying to read text within that environment. This study widens research into the effects of virtual reality as a learning medium as the statistical evidence would imply that once skills are learned, the presence of the dog can help with the reinforcement through practice. This finding itself could create further areas for exploratory investigation as there appears to be no current research on animal assisted interventions using a virtual reality approach.

The evidence suggests that the facts and strategies for academic attainment are created internally for the learner, over which the dog has no influence. This finding was shown both in the children's and adults' attainment in the school-based tasks for reading, vocabulary and for mental maths. The dog's presence may motivate some, but as seen from both the adults and the children's studies, not all learners. Nor does the dog affect students on an equal basis. The numbers of the learners for which the presence of a dog may be beneficial appear to be smaller than most schools realise. The evidence from both child and adult studies, however, demonstrates that the presence of the dog can potentially affect both child and adult learners highlighting underlying cognitive skills and processes, rather than the maturity of learning strategies. These studies also identified 
issues associated with assessing the influence of dogs as simply reading speed fluency and electrodermal activity may be too crude to pick up subtle effects.

The working memory tests revealed that the dog may affect visuo-spatial processing and executive skills for some individuals, yet this still requires further investigation and development, particularly if the learner already has a lower working memory profile. A screener could be developed to measure any differences with and without the dog present using a mixture of the current sub tests from available commercial working memory tests. Linking these skills over time, to academic attainment would require regular testing with and without the dog available. Consequently, teachers would have to be introduced and trained for these screeners to be valid.

Working memory skills assist in cross-curricular subjects, including maths, but whether the dog, augmented these skills for maths was not established in this thesis due to too many different mathematical areas being included in the adults' samples, creating cognitive overload and task abandonment. Perhaps a single maths area should be considered in the future, or an adult based mathematical standardised test. Most maths tests are currently standardised within the school age range or stop at age 25 years.

Due to the lack of confidence and self-esteem shown by the adults, it may be prudent to use the fact that both the adults and children preferred having the dog present, and therefore create a maths to dogs programme which could be initiated in schools, simply to motivate both children and adults' mathematical confidence skills. That is, it is the effect of the dog may be to increase the motivation to engage with the subject, rather than directly influencing underlying cognitive processes. Again, as with the reading skills recorded in this thesis, the effect may be greater for some individuals than others. 
It was found that group average scores in literacy-based areas of reading and vocabulary showed different patterns for the child and adult participants, for example in the reading ages data gathered for the read to dogs' scheme. The group data tended to mask individual responses. This group 'result' can be interpreted differently, as was seen in the schools' responses to the Bark and Read data, in that when the data was in the dog's favour, this would be mediated by a 'confirmation' bias as to the efficacy of the dog assisting the learners. When examined in depth, reading for example, appeared to be too complicated with multi cognitive skills to establish where exactly the presence of the dog, made a difference. Caution then is required when promoting all animal assisted learning schemes and with those pertaining to endorse the skills of different learning groups, such as those with special needs or autism.

There was evidence from all three phases: the school survey, the children's school research and the adult study, to suggest that anthropomorphology with the dogs is beginning to dictate the roles, use and numbers of dogs in schools, rather than the data. In the studies described here, the presence of the dog only affected certain individuals, not mass groups, despite an increase of reading skills for some struggling readers. Bringing in a dog for 'the whole class' or just one group, such as all those with SEN may be too excessive, - perhaps the dog being brought in for one or two individuals, once or twice a week, may be all that is necessary. Perhaps by using the suggested working memory screener, these individuals could be selected and the intervention delivered by a particular teacher, or if a volunteer is used, with simple training, such as reading techniques, phonics or the school's system of 'pause prompt, praise,' or methods of teaching number bonds, thus equipping the volunteer with simple skills in line with other 
international charity counterparts. This approach could be considered a more personal intervention and may even promote higher levels of cognition and achievement for the individual in the long run. This focused 'intervention,' incorporating planned activities, a set time frame and a follow up assessment, allowing full interaction with the dog may help with literacy and mathematical skills, similar to a therapist approach for behaviour. General attainment and self-attainment would have to be measured on an individual basis. This would truly see the optimal use of Animal Assisted Intervention rather than the generally assumed interpretation that the presence of the animal is universally beneficial. Teachers can no longer expect 'en masse' interventions using the dog or by the dog simply being in the room, as being a valid justification for the dog's overall presence. The dog may well be regulating the emotions as has previously been argued by animal assisted research supported by the adult electrodermal activity data. The presence of the dog may also prime for further, but taught, educational cognition, but teachers will need to explain where and why the dog is necessary for the children in their particular class and place this in their planning (Silva et al., 2011, Beetz, 2013).

By focusing on the purpose for a dog within the class, whether for the socio-emotional or cognitive needs of the students, teachers and handlers may question the necessity for dogs to be in schools for long periods of time, which can include full, school days. This emphasis can guide the research into the educational benefits of having a dog in schools. The current guidelines all suggest part time roles of up to two to three hours maximum for a dog to visit, after which it should be offered complete rest in a safe space, implying that the dog, ideally should be resting at home (Kennel-Club, 2018, S.C.A.S., 2019). The role of the Animal Assisted Educator or teacher and their teaching contract now needs to 
reflect this so that there is enough time to teach, train and rest, placing both the emotional and physical wellbeing and welfare of both team members, handler and dog as a high priority for the school. This may also suggest that part time volunteers from visiting charities may better suit the needs and purposes of the role for the school. Focusing the use of animal assisted learning on those that can obtain the greater benefit is therefore beneficial both to the learners, and to the animals.

Ultimately, perhaps it is worrying to think that we teach our children In groups and classes hoping that the vast majority will learn and that the minority who have the difficulties, who are slightly behind, or require a different learning style, will just carry on along with the main group and will make the equivalent amount of progress. What is clear from the research in this thesis is that it is still very difficult to select individuals for whom working with an animal such as a dog would be an improvement. Whereas the wellbeing selfesteem and emotional sides of learning with an animal has been very highly successful, we are only beginning the journey into academic skill achievement. Hopefully this thesis will be a catalyst for further research. 


\section{References}

A.B.T.C. 2018. Animal Behaviour Training Council - Standards for Practitioners of Animal Training and Behaviour [Online]. Online: Animal Behaviour Training Council. Available: http://www.abtcouncil.org.uk/standards-for-practitioners.html [Accessed 31/08/20].

ACHESON, D. J., POSTLE, B. R. \& MACDONALD, M. C. 2010. The interaction of concreteness and phonological similarity in verbal working memory. Journal of experimental psychology. Learning, memory, and cognition, 36, 17-36.

ADAMS, J. W. 2007. Individual differences in mathematical ability: genetic, cognitive and behavioural factors. Journal of Research in Special Educational Needs, 7, 97-103.

AHN, S. J., JOHNSEN, K., MOORE, J., BROWN, S., BIERSMITH, M. \& BALL, C. 2016. Using Virtual Pets to Increase Fruit and Vegetable Consumption in Children: A Technology-Assisted Social Cognitive Theory Approach. Cyberpsychology, behavior and social networking, 19, 86-92.

AIRENTI, G. 2018. The Development of Anthropomorphism in Interaction: Intersubjectivity, Imagination, and Theory of Mind. Frontiers in psychology, 9, 2136-2136.

ALLEN, K. 2003. Are Pets a Healthy Pleasure? The Influence of Pets on Blood Pressure. Blackwell Publishers.

ALLOWAY, T. P. 2007. Working memory, reading, and mathematical skills in children with developmental coordination disorder. Journal of Experimental Child Psychology, 96, 2036.

ALLOWAY, T. P. \& GATHERCOLE, S. E. 2006. Working memory and neurodevelopmental disorders, Hove, East Sussex, England ; Psychology Press.

ALLOWAY, T. P., GATHERCOLE, S. E., KIRKWOOD, H. \& ELLIOTT, J. 2009. The Cognitive and Behavioral Characteristics of Children With Low Working Memory. Child Development, 80 , 606-621.

ALLOWAY, T. P., GATHERCOLE, S. E., WILLIS, C. \& ADAMS, A.-M. 2004. A structural analysis of working memory and related cognitive skills in young children. Journal of Experimental Child Psychology, 87, 85-106.

ALMEIDA, R. M. \& MARTINS DEGAGO, D. B. 2013. Effects of pleasant visual stimulation on attention, working memory, and anxiety in college students. Psychology \& Neuroscience, 6, 351-355.

AMIOT, C. E. \& BASTIAN, B. 2015. Toward a psychology of human-animal relations. Psychological Bulletin, 141, 6-47.

ANSARI, T. L. \& NAZANIN, D. 2010. Anxiety impairs inhibitory control but not volitional action control. Cognition \& Emotion, 24, 241-254.

ANSTICE, I. 2016. Leading the way? Reading dogs in public libraries. Public Libraries News. Online: http://www. publiclibrariesnews.com/aboutcontact/contact.

ANTZAKA, A., LALLIER, M., MEYER, S., DIARD, J., CARREIRAS, M. \& VALDOIS, S. 2017. Enhancing reading performance through action video games: the role of visual attention span. Scientific Reports, 7, 14563.

ASTLE, D. E., BATHELT, J. \& HOLMES, J. 2019. Remapping the cognitive and neural profiles of children who struggle at school. Developmental Science, 22, e12747.

AUTISM.ORG.UK. 2019. Diagnostic criteria - a guide for all audiences [Online]. online: The National Austistic Society. Available: https://www.autism.org.uk/advice-andguidance/topics/diagnosis/diagnostic-criteria/all-audiences [Accessed 03/06/19].

AYOTOLA, A. \& ADEDEJI, T. 2009. The relationship between mathematics self-efficacy and achievement in mathematics. Procedia - Social and Behavioral Sciences, 1, 953-957.

B.D.A. 2019. British Dyslexia Association Code of Ethics and Conduct for Accreditiation [Online]. online: British Dyslexia Association. Available: https://www.bdadyslexia.org.uk/terms/bda-code-of-ethics-v2-april-2019 [Accessed]. 
B.P.S. 2012. British Psychological Society Research Board: Guidelines for psychologists working with animals [Online]. Leicester: BPS. Available: https://www.bps.org.uk/sites/www.bps.org.uk/files/Policy/Policy\%20\%20Files/BPS\%20Guidelines\%20for\%20Psychologists\%20Working\%20with\%20Animals\% 20\%282012\%29.pdf [Accessed 26 Feb 2015].

B.P.S. 2018. British Psychological Association - Code of ethics and conduct [Online]. London: British Psychological Society. Available: https://www.bps.org.uk/news-and-policy/bps-codeethics-and-conduct [Accessed $01 \mathrm{Feb} 2019$ ].

BADDELEY, A. 2007. Working Memory, Thought and Action, Oxford, Oxford University Press.

BADDELEY, A., HITCH G. 1974. Working Memory, New York, Academic Press.

BADDELEY, A. D. 2002. Is working memory still working? European Psychologist, 7, 85-97.

BASHIRI, A., GHAZISAEEDI, M. \& SHAHMORADI, L. 2017. The opportunities of virtual reality in the rehabilitation of children with attention deficit hyperactivity disorder: a literature review. Korean Journal of Pediatrics, 60, 337.

BASSETTE, L. \& TABER-DOUGHTY, T. 2013. The Effects of a Dog Reading Visitation Program on Academic Engagement Behavior in Three Elementary Students with Emotional and Behavioral Disabilities: A Single Case Design. Child \& Youth Care Forum, 42, 239-256.

BECK, A. M. \& KATCHER, A. H. 2003. Future directions in human-animal bond research. The American Behavioral Scientist, 47, 79-93.

BECKER, J. L., ROGERS, E. C. \& BURROWS, B. 2017. Animal-assisted Social Skills Training for Children with Autism Spectrum Disorders. Anthrozoös, 30, 307-326.

BEDEWY, D. \& GABRIEL, A. 2015. Examining perceptions of academic stress and its sources among university students: The Perception of Academic Stress Scale. Health psychology open, 2, 2055102915596714.

BEETZ, A. 2013. Socio-emotional correlates of a schooldog-teacher-team in the classroom. Frontiers in psychology, 4, 886.

BEETZ, A. 2018. Animal Assisted Education Programmes and Research. Animal Assisted Education - Anthrozoology Module 3. Open Universiteit of the Nederlands / IAHAIO.

BEETZ, A., JULIUS, H., TURNER, D. \& KOTRSCHAL, K. 2012a. Effects of Social Support by a Dog on Stress Modulation in Male Children with Insecure Attachment. Frontiers in Psychology, 3.

BEETZ, A. \& MCCARDLE, P. 2017. Does Reading to a dog affect reading skills? How Animals Help Students to Learn. Abingdon, Oxon: Routledge.

BEETZ, A., UVNÄS-MOBERG, K., JULIUS, H. \& KOTRSCHAL, K. 2012b. Psychosocial and Psychophysiological Effects of Human-Animal Interactions: The Possible Role of Oxytocin. Frontiers in Psychology, 3.

BEKOFF, M. \& PIERCE, J. 2019. Unleashing your dog, Novato, California, New World Library.

BELL, A. 2013. Paws for a Study Break: Running an Animal Assisted Therapy Program at the Gerstein Science Information Centre. Partnership: The Canadian Journal of Library \& Information Practice \& Research, 8, 1-14.

BELL, J. 1992. Doing your research project - A guide for first-time researchers in education and social science, Milton Keynes, Open University Press.

BERA. 2011. British Educational Research Association - Ethical Guidelines for Educational Research [Online]. London: BERA. Available: https://www.bera.ac.uk/publication/ethicalguidelines-for-educational-research-2018-online\#community [Accessed 08 August 2016].

BERGEN, E., SNOWLING, M. J., ZEEUW, E. L., BEIJSTERVELDT, C. E. M., DOLAN, C. V. \& BOOMSMA, D. I. 2018. Why do children read more? The influence of reading ability on voluntary reading practices. Journal of Child Psychology and Psychiatry.

BESTER, G. \& BRAND, L. 2013. The effect of technology on learner attention and achievement in the classroom. South African Journal of Education [Online], 33.

BLACK, S. 2009. Sit, Stay, and Read. American School Board Journal, 196, 36-37. 
BLAIR, C. \& MCKINNON, R. D. 2016. Moderating effects of executive functions and the teacherchild relationship on the development of mathematics ability in kindergarten. Learning and Instruction, 41, 85-93.

BLANCHETTE, I. \& CAPAROS, S. 2016. Working memory function is linked to trauma exposure, independently of post-traumatic stress disorder symptoms. Cognitive Neuropsychiatry, 21, 494-509.

BLAZINA, C. B., G.; SHEN-MILLAR, D 2013. The Psychology of the Human - Animal Bond - A Resource for Clinicians and Researchers, New York, Springer.

BOULET-CRAIG, A., ROBAEY, P., LACOURSE, K., JERBI, K., OSWALD, V., KRAJINOVIC, M., LAVERDIÈRE, C., SINNETT, D., JOLICOEUR, P. \& LIPPÉ, S. 2017. Visual short term memory related brain activity predicts mathematical abilities. Neuropsychology, 31, 535-545.

BOWLBY, J. 1969. Attachment and Loss,, New York, Basic Books.

BRADSHAW, J. 2017. The Animals Among Us, St Ives, Clays Ltd (Penguin).

BRANDENBURG, J., FISCHBACH, A., SCHUCHARDT, K., GRUBE, D., HASSELHORN, M. \& BÜTTNER, G. 2018. Development of Working Memory from Grade 3 to 5: Differences between Children With and Without Mathematical Learning Difficulties AU - Klesczewski, Julia. International Journal of Disability, Development and Education, 65, 509-525.

BRAUN, C., STANGLER, T., NARVESON, J. \& PETTINGELL, S. 2009. Animal-assisted therapy as a pain relief intervention for children. Complementary Therapies in Clinical Practice, 15, 105-109.

BRELSFORD, V., MEINTS, K., GEE, N. \& PFEFFER, K. 2017. Animal-Assisted Interventions in the Classroom-A Systematic Review. International Journal of Environmental Research and Public Health, 14, 669.

BRENNING, K. M., SOENENS, B., BRAET, C. \& BOSMANS, G. 2012. Attachment and depressive symptoms in middle childhood and early adolescence: Testing the validity of the emotion regulation model of attachment. Personal Relationships, 19, 445-464.

BROCK, L. L., RIMM-KAUFMAN, S. E., NATHANSON, L. \& GRIMM, K. J. 2009. The contributions of 'hot' and 'cool' executive function to children's academic achievement, learning-related behaviors, and engagement in kindergarten. Early Childhood Research Quarterly, 24, 337349.

BROOKS, G. 2007. What works for pupils with literacy difficulties? the effectivness of intervention schemes. In: STRATEGIES, P. A. S. N. (ed.). Nottingham: Department for Children, Schools and Families.

BROOKS, G. 2016. What works for children and young people with literacy difficulties? - the effectiveness of intervention schemes. 5th ed.: The Dyslexia SpLD Trust.

BROWN, J., FISHCO, V. \& HANNA, G. 1993. Nelson-Denny Reading Test. Shoal Creek Boulevard, Austin, Texas: PRO-ED Inc.

BULL, R., ESPY, K. A. \& WIEBE, S. A. 2008. Short-Term Memory, Working Memory, and Executive Functioning in Preschoolers: Longitudinal Predictors of Mathematical Achievement at Age 7 Years. Developmental Neuropsychology, 33, 205-228.

BUSCH, C., TUCHA, L., TALAROVICOVA, A., FUERMAIER, A. B. M., LEWIS-EVANS, B. \& TUCHA, O. 2016. Animal-Assisted Interventions for Children with Attention Deficit/Hyperactivity Disorder: A Theoretical Review and Consideration of Future Research Directions. Psychological Reports, 118, 292-331.

BYSTRÖM, K. M., PERSSON, C. A. \& LUNDQVIST 2015. The Meaning of Companion Animals for Children and Adolescents with Autism: The Parents' Perspective. Anthrozoös, 28, 263-275.

CARAPETIAN, S., SIEDLARZ, M., JACKSON, S. \& PERLMUTER, L. C. 2008. Orthostatic blood pressure regulation predicts classroom effort in children. International Journal of Psychophysiology, 68, 70-74.

CARLISLE, G. 2015. The Social Skills and Attachment to Dogs of Children with Autism Spectrum Disorder. Journal of Autism \& Developmental Disorders, 45, 1137-1145. 
CHARRON, C., FISCHER, J.-P. \& MELJAC, C. 2008. Arithmetic after School: How Do Adults' Mental Arithmetic Abilities Evolve with Age? Research in the Schools, 15, 9-26.

CHEN, F. C., TSAI, C. L., BILTZ, G. R., STOFFREGEN, T. A. \& WADE, M. G. 2015. Variations in cognitive demand affect heart rate in typically developing children and children at risk for developmental coordination disorder. Research in developmental disabilities, 38, 362-71.

CHIARENZA, G. A., DI PIETRO, S. F. \& CASAROTTO, S. 2014. The psychophysiology of reading. International Journal of Psychophysiology, 94, 111-119.

CHILDS, S. 2018. Google - Canine Learning Initiative [Online]. https://www.thebearbonesofeducation.org/. Available: https://www.thebearbonesofeducation.org/ [Accessed 27/07/2019].

CHILDS, S. 2019. Google the Reading Dog, Great Britain, Lightning Source Ltd.

CHINN, S. 2004. The Trouble with Maths - A practical guide to helping learners with numeracy difficulties, Abingdon, Oxon., David Fulton Publishers (Routledge).

ÇIFTÇI, Ş. K. K. \& YıLDıZ, P. A. 2019. The Effect of Self-Confidence on Mathematics Achievement: The Meta-Analysis of Trends in International Mathematics and Science Study (TIMSS). International Journal of Instruction, 12, 683-694.

CLARK, S. D., SMIDT, J. M. \& BAUER, B. A. 2018. Welfare Considerations: Salivary Cortisol Concentrations on Frequency of Therapy Dog Visits in an Outpatient Hospital Setting: A Pilot Study. Journal of Veterinary Behavior.

CLAYTON, L. 2019. Teachers hit by allergy to therapy dog. Bailiwick Express.

COHEN, L., MANION, L. \& MORRISON, K. 2011. Research Methods in Education, London UK, Routledge.

COHEN, L., MANION, L. \& MORRISON, K. 2018. Research Methods in Education, Abingdon, Oxon, Routledge.

COLOMER, C., RE, A. M., MIRANDA, A. \& LUCANGELI, D. 2013. Numerical and Calculation Abilities in Children with ADHD. Learning Disabilities: A Contemporary Journal, 11, 1-15.

CONEJERO, A. \& RUEDA, M. R. 2017. Early Development of Executive Attention. Journal of Child and Adolescent Behavior, 05.

CONNELL, C. G., TEPPER, D. L., LANDRY, O. \& BENNETT, P. C. 2019. Dogs in Schools: The Impact of Specific Human-Dog Interactions on Reading Ability in Children Aged 6 to 8 Years. Anthrozoös, 32, 347-360.

COWAN, N. 2005. Working Memory Capacity - Essays in Cognitive Psychology, New York, USA, Psychology Press.

COWAN, N. 2010. Multiple Concurrent Thoughts: The Meaning and Developmental Neuropsychology of Working Memory. Developmental Neuropsychology, 35, 447-474.

COWAN, N. 2014. Working Memory Underpins Cognitive Development, Learning, and Education. Educational psychology review, 26, 197-223.

CRESSWELL, J. W. 2015. A concise introduction to Mixed Methods Research, Thousand Oaks California USA, Sage Publications Inc.

CROSSMAN, M. \& KAZDIN, A. 2016. Additional Evidence is Needed to Recommend Acquiring a Dog to Families of Children with Autism Spectrum Disorder: A Response to Wright and Colleagues...Wright, H. F., Hall, S., Hames, A., Hardiman, J., Mills, R., Mills, D. S., \& Team, P. (2015). Acquiring a pet dog significantly reduces stress of primary carers for children with autism spectrum disorder: A prospective case control study. Journal of Autism and Developmental Disorders. , $<$ Blank $>$ : Springer Science \& Business Media B.V.

DAVIS, T. N., SCALZO, R., BUTLER, E., STAUFFER, M., FARAH, Y. N., PEREZ, S., MAINOR, K., CLARK, C., MILLER, S., KOBYLECKY, A. \& COVIELLO, L. 2015. Animal Assisted Interventions for Children with Autism Spectrum Disorder: A Systematic Review. Education and Training in Autism and Developmental Disabilities, 50, 316-329.

DE KEERSMAECKER, J., DUNNING, D., PENNYCOOK, G., RAND, D. G., SANCHEZ, C., UNKELBACH, C. \& ROETS, A. 2019. Investigating the Robustness of the Illusory Truth Effect Across 
Individual Differences in Cognitive Ability, Need for Cognitive Closure, and Cognitive Style. Personality and Social Psychology Bulletin.

DE LISSNYDER, W., E. E. H., NAZANIN, D. \& RUDI, D. R. 2010. The association between depressive symptoms and executive control impairments in response to emotional and nonemotional information. Cognition \& Emotion, 24, 264-280.

DEBOER, C. 2019. Dyslexie-Font [Online]. Available: https://www.dyslexiefont.com/ [Accessed 04/08/2016].

DECHÊNE, A., STAHL, C., HANSEN, J. \& WÄNKE, M. 2010. The truth about the truth: a meta-analytic review of the truth effect. Personality and social psychology review : an official journal of the Society for Personality and Social Psychology, Inc, 14, 238-57.

DECI, E. L. \& RYAN, R. M. 2000. The "What" and "Why" of Goal Pursuits: Human Needs and the Self-Determination of Behavior. Psychological Inquiry, 11, 227-268.

DEHN, M. J. 2008. Working Memory and Academic Learning - Assessment and Intervention, Hoboken, New Jersey, Wiley.

DEHN, M. J. 2015. Essentials of working Memory Assessment and Intervention, Hoboken, New Jersey, Wiley.

DELTA-SOCIETY. 2019. Available: https://www.deltasociety.com.au/pages/volunteer-.html [Accessed 24/04/16].

DENNISON, P. 2015. You can train your dog - Mastering the art and science of modern dog training, Blairstown New Jersey, Shadow Publishing.

DERAKSHAN, N. \& EYSENCK, M. W. 2010. Emotional Sates, Attention and Working Memory, Hove and New York, Psychology Press.

DFE 2013. Teachers' Standards - Guidance for school leaders, school staff and governing bodies. In: EDUCATION, D. F. (ed.). London: Department for Education.

DFE 2014. The National Curriculum in England - Framework Document. In: EDUCATION, D. F. (ed.). London: Department for Education.

DFE/DOH 2015. Special Educational Needs and Disability Code of Practice: 0 to 25 years. London: Department for Education \& Department of Health.

DFES 2001. Special Educational Needs Code of Practice. In: SKILLS, D. F. E. A. (ed.). Annesley, Nottinghamshire: DFES Publications.

DHE, D. 2017. Transforming Children and Young People's Mental Health Provision: a Green Paper. In: DEPARTMENT OF HEALTH, D. F. E. (ed.). London: DHE, DfE.

DIMOLAREVA, M., GEE, N. R., PFEFFER, K., MARÉCHAL, L., PENNINGTON, K. \& MEINTS, K. 2018. Measuring Cortisol in the Classroom with School-Aged Children-A Systematic Review and Recommendations. International Journal of Environmental Research and Public Health, $15,1025$.

DODAJ, A., KRAJINA, M., SESAR, K. \& ŠIMIĆ, N. 2017. The Effects of Maltreatment in Childhood on Working Memory Capacity in Adulthood. Europe's journal of psychology, 13, 618-632.

DOGS-FOR-GOOD. Animal Assisted Intervention Insight Day. Animal Assisted Intervention Insight Day, 12/07/2019 2019 Banbury, Oxfordshire.

DOGS-HELPING-KIDS. 2017. School Dog Fact Sheet [Online]. Available: https://www.dogshelpingkids.co.uk/SCHOOL-DOG-FACTSHEET-APRIL2017.pdf [Accessed 29/07/19].

DOWKER, A. 2019. Individual differences in arithmetic - implications for psychology, neuroscience and education, Abingdon, Oxon, Routledge.

EEF 2017. Improving Literacy in Key Stage 2. Milbank, London: Education Endowment Foundation.

EEF 2018. Teaching and Learning Toolkit. In: TRUST, E. E. F. S. (ed.). https://educationendowmentfoundation.org.uk/: EEF.

EEF 2019. The Education Endowment Fund (EEF) Guide to the Pupil Premium. 
EL-SHEIKH, M., KEILEY, M. \& HINNANT, J. B. 2010. Developmental trajectories of skin conductance level in middle childhood: Sex, race, and externalizing behavior problems as predictors of growth. Biological Psychology, 83, 116-124.

ELLIOTT, J. G., GATHERCOLE, S. E., ALLOWAY, T. P., HOLMES, J. \& KIRKWOOD, H. 2010. An Evaluation of a Classroom-Based Intervention to Help Overcome Working Memory Difficulties and Improve Long-Term Academic Achievement. Journal of Cognitive Education and Psychology, 9, 227-250.

ENDERS-SLEGERS, M. J. 2018. Modules in Anthrozoology: Animal Assisted Education Module 3. [Online]. Open Universiteit Netherlands IAHAIO: www.ou.nl. Available: https://www.ou.nl/-/animal-assistededucation?inheritRedirect=true\&redirect=\%2Fweb\%2Fopenuniversiteit\%2Fzoekresultaten\%3Fq\%3DAnimal\%2BAssisted\%2BEducation [Accessed 21 November 2018 2018].

EWING, C., MACDONALD, P., TAYLOR, M. \& BOWERS, M. 2007. Equine-Facilitated Learning for Youths with Severe Emotional Disorders: A Quantitative and Qualitative Study. Child \& Youth Care Forum, 36, 59-72.

EYSENCK, M. W. \& CALVO, M. G. 1992. Anxiety and performance: The processing efficiency theory. Cognition and Emotion, 6.

FAUL, F., ERDFELDER, E., BUCHNER, A. \& LANG, A. G. 2009. Statistical power analyses using G* power 3.1: Tests for correlation and regression analyses. Behaviour Research Methods, $41,1149-1160$.

FINE, A. H., TEDESCHI, P. T. \& ELVOVE, E. 2015. Forward thinking: the evolving field of humananimal interactions. In: FINE, A. H. (ed.) Handbook on Animal Assisted Therapy Foundations and guidelines for animal assisted interventions. 4th ed. London: Elsevier.

FISHER, B. \& COZENS, M. 2014. The BaRK (Building Reading Confidence for Kids) canine assisted reading program: One child's experience. Literacy Learning: The Middle Years, 22, 70-80.

FISHER, S. \& MILLER, M. 2018. 100 Ways to Train the Perfect Dog, Cincinnati, OH, David and Charles F+W Publications Inc. Company.

FOWLER, H. W. \& FOWLER, F. G. 1989. The Concise Oxford Dictionary. In: SYKES, B. J. (ed.) The Concise Oxford Dictionary. 7th ed. Oxford UK: Oxford University Press.

FRANCISCO, A. A., GROEN, M. A., JESSE, A. \& MCQUEEN, J. M. 2017. Beyond the usual cognitive suspects: The importance of speechreading and audiovisual temporal sensitivity in reading ability. Learning and Individual Differences, 54, 60-72.

FREDERICKSON, N., FRITH, U. \& REASON, R. 1997. Phonological Assessment Battery (PhAB). London, UK.: G.L.Assessment Limited.

FRIESEN, L. 2010. Exploring Animal-Assisted Programs with Children in School and Therapeutic Contexts. Early Childhood Education Journal, 37, 261-267.

FRIESEN, L. \& DELISLE, E. 2012. Animal-Assisted Literacy: A Supportive Environment for Constrained and Unconstrained Learning. Childhood Education, 88, 102-107.

GABARD-DURNAM, L. J., O'MUIRCHEARTAIGH, J., DIRKS, H., DEAN, D. C., TOTTENHAM, N. \& DEONI, S. 2018. Human amygdala functional network development: A cross-sectional study from 3 months to 5 years of age. Developmental Cognitive Neuroscience, 34, 63-74.

GABRIELS, R. L., AGNEW, J. A., HOLT, K. D., SHOFFNER, A., ZHAOXING, P., RUZZANO, S., CLAYTON, G. H. \& MESIBOV, G. 2012. Pilot study measuring the effects of therapeutic horseback riding on school-age children and adolescents with autism spectrum disorders. Research in Autism Spectrum Disorders, 6, 578-588.

GANLEY, C. M. \& VASILYEVA, M. 2014. The role of anxiety and working memory in gender differences in mathematics. Journal of Educational Psychology, 106, 105-120.

GATHERCOLE, S. \& PICKERING, S. 2001. Research Section: Working memory deficits in children with special educational needs. British Journal of Special Education, 28, 89-97.

GATHERCOLE, S. E. 2008. Working memory in the classroom. Psychologist, 21, 382-385. 
GATHERCOLE, S. E. 2013. Working Memory Research and Practice. In: ASSOCIATION, B. D. (ed.) Working Memory and Dyslexia Conference. Holiday Inn, Regent's Park, London. UK.

GATHERCOLE, S. E. 2014. Unlocking Memory. In: ASSOCIATION, S. D. (ed.) Unlocking Memory. Blunsdon House Hotel, Swindon, Wilts. UK.

GATHERCOLE, S. E. \& ALLOWAY, T. 2008. Working Memory \& Learning - A Practical Guide for Teachers, London, Sage.

GATHERCOLE, S. E., ALLOWAY, T. P., KIRKWOOD, H. J., ELLIOTT, J. G., HOLMES, J. \& HILTON, K. A. 2008a. Attentional and executive function behaviours in children with poor working memory. Learning and Individual Differences, 18, 214-223.

GATHERCOLE, S. E., ALLOWAY, T. P., WILLIS, C. \& ADAMS, A.-M. 2006. Working memory in children with reading disabilities. Journal of Experimental Child Psychology, 93, 265-281.

GATHERCOLE, S. E., DURLING, E., EVANS, M., JEFFCOCK, S. \& STONE, S. 2008b. Working memory abilities and children's performance in laboratory analogues of classroom activities. Applied Cognitive Psychology, 22, 1019-1037.

GATHERCOLE, S. E., WOOLGAR, F., KIEVIT, R. A., ASTLE, D., MANLY, T. \& HOLMES, J. 2016. How Common are WM Deficits in Children with Difficulties in Reading and Mathematics? Journal of Applied Research in Memory and Cognition, 5, 384-394.

GEE, N., FINE, A. H., ESPOSITO, L. \& MCCUNE, S. 2017a. Creating an Atmosphere of Acceptance for $\mathrm{HAl}$ in Education - Future directions. How animals help students to learn - research and practice for educators and mental health professionals. New York: Routledge.

GEE, N., FINE, A. H. \& MCCARDLE, P. 2017b. How Animals Help Students Learn - Research and Practice for Educators and Mental-Health Professionals, New York, New York, Routledge.

GEE, N., FINE, A. H. \& SCHUCK, S. 2015a. Animals in Educational Settings: Research and Practice. In: FINE, A. H. (ed.) Handbook on Animal Assisted Therapy - Foundations and Guidelines for Animal Assisted Interventions. 4th Edition ed. London: Elsevier Academic Press.

GEE, N. R., BELCHER, J. M., GRABSKI, J. L., DEJESUS, M. \& RILEY, W. 2012a. The Presence of a Therapy Dog Results in Improved Object Recognition Performance in Preschool Children. Anthrozoös, 25, 289-300.

GEE, N. R., CHURCH, M. T. \& ALTOBELLI, C. L. 2010a. Preschoolers Make Fewer Errors on an Object Categorization Task in the Presence of a Dog. Anthrozoos, 23, 223-230.

GEE, N. R., CRIST, E. N. \& CARR, D. N. 2010b. Preschool Children Require Fewer Instructional Prompts to Perform a Memory Task in the Presence of a Dog. Anthrozoös, 23, 173-184.

GEE, N. R., FRIEDMANN, E., COGLITORE, V., FISK, A. \& STENDAHL, M. 2015b. Does Physical Contact with a Dog or Person Affect Performance of a Working Memory Task? Anthrozoös, 28, 483-500.

GEE, N. R., FRIEDMANN, E., STENDAHL, M., FISK, A. \& COGLITORE, V. 2014. Heart rate variability during a working memory task: Does touching a dog or person affect the response? Anthrozoös, 27, 513-528.

GEE, N. R., GOULD, J. K., SWANSON, C. C. \& WAGNER, A. K. 2012b. Preschoolers Categorize Animate Objects Better in the Presence of a Dog. Anthrozoös, 25, 187-198.

GEE, N. R., HARRIS, S. L. \& JOHNSON, K. L. 2007. The Role of Therapy Dogs in Speed and Accuracy to Complete Motor Skills Tasks for Preschool Children. Anthrozoös, 20, 375-386.

GEE, N. R., SHERLOCK, T. R., BENNETT, E. A. \& HARRIS, S. L. 2009. Preschoolers' Adherence to Instructions as a Function of Presence of a Dog and Motor Skills Task. Anthrozoös, 22, 267276.

GIBBS, S. \& BODMAN, S. 2014. Phonological Assessment Battery - Primary (PhAB 2). 2nd ed. London, U.K.: G.L. Assessment.

GILL, C. J. 1987. A New Social Perspective on Disability and Its Implications for Rehabilitation. Occupational Therapy In Health Care, 4, 49-55.

GLOUCESTERSHIRE, U. O. 2018. University of Gloucestershire Research Ethics: A handbook of principles and procedures [Online]. Cheltenham: University of Gloucestershire. Available: 
https://www.glos.ac.uk/docs/download/Research/handbook-of-principles-andprocedures.pdf [Accessed 01 March 2019].

GOSWAMI, U. 2004. Neuroscience, education and special education. British Journal of Special Education, 31, 175-183.

GOV.UK 2017a. Guidance Independent Schools and Colleges.

GOV.UK 2017b. Statistics for Special Needs in England - January 2017. In: NEEDS, S. E. (ed.). Kew, London: Department for Education.

GOV.UK 2019. Guidance: Pupil Premium 2018-2019: Conditions of Grant. In: AGENCY, E. S. A. F. (ed.). Gov.UK online: Gov.UK.

GRANDGEORGE, M., BOURREAU, Y., ALAVI, Z., LEMONNIER, E., TORDJMAN, S., DELEAU, M. \& HAUSBERGER, M. 2015. Interest towards human, animal and object in children with autism spectrum disorders: an ethological approach at home. European Child \& Adolescent Psychiatry, 24, 83-93.

GRANDGEORGE, M. \& HAUSBERGER, M. 2011. Human-animal relationships: from daily life to animal-assisted therapies. Annali dell'Istituto Superiore di Sanità, 47, 397-408.

GRANDGEORGE, M., LEMONNIER, E., TORDJMAN, S., DELEAU, M. \& HAUSBERGER, M. 2009. P02233 Autistic children and familiar animals: What types of interactions? A parent survey. European Psychiatry, 24, 5923.

GRANDIN, T. 2007. Autism from the Inside. Educational Leadership, 64, 29-32.

GRANDIN, T., JOHNSON, C. \& GALLO, A. 2009. Animals make us human : creating the best life for animals. Unabridged. ed. Prince Frederick, MD :: Recorded Books.

GROOME, D., BRACE, N., EDGAR, G., EDGAR, H., EYSENCK, M., MANLY, T., NESS, H., PIKE, G., SCOTT, S. \& STYLES, E. 2014. An Introduction to Cognitive Psychology - Processes and Disorders, Hove, East Sussex, UK., Psychology Press.

GRUSKA, A. 2010. Handbook of Individual Differences in Cognition, Memory and Executive Control. In: GRUSZKA. A. MATTHEWS, G. S., B. (ed.) 2010 ed. New York: Springer Science + Business Media.

HALL, S., S., WRIGHT, H., F. \& MILLS, D., S. 2017. Parent perceptions of the quality of life of pet dogs living with neuro-typically developing and neuro-atypically developing children: An exploratory study. PLOS ONE [Online], 12.

HALL, S. S., GEE, N. R. \& MILLS, D. S. 2016a. Children Reading to Dogs: A Systematic Review of the Literature. PLOS ONE, 11, 1.

HALL, S. S., WRIGHT, H. F., HAMES, A. \& MILLS, D. S. 2016b. The long-term benefits of dog ownership in families with children with autism. Journal of Veterinary Behavior: Clinical Applications and Research, 13, 46-54.

HALL, S. S., WRIGHT, H. F. \& MILLS, D. S. 2016c. What factors are associated with positive effects of dog ownership in families with children with autism spectrum disorder? The development of the Lincoln Autism Pet Dog Impact Scale. PLOS ONE, 11.

HART, C. 2005. Doing your Masters dissertation, London, Sage Publications Ltd.

HASHER, L., GOLDSTEIN, D. \& TOPPINO, T. 1977. Frequency and the conference of referential validity. Journal of Verbal Learning and Verbal Behavior, 16, 107-112.

HASSELHORN, M. \& GRUBE, D. 2003. The phonological similarity effect on memory span in children: Does it depend on age, speech rate, and articulatory suppression? International Journal of Behavioral Development, 27, 145.

HAWKINS, C. 2012. Animal-Assisted Therapy: Is there Room the Treatment Plan? Exceptional Parent, 42, 45-47.

HAWKINS, R. D. \& WILLIAMS, J. M. 2016. Children's Beliefs about Animal Minds (Child-BAM): Associations with Positive and Negative Child-Animal Interactions. Anthrozoös, 29, 503519.

HAYES, B. K., FRITZ, K. \& HEIT, E. 2013. The relationship between memory and inductive reasoning: Does it develop? Developmental Psychology, 49, 848-860. 
HEDIGER, K., GEE, N. \& GRIFFIN, J. A. 2017. Do animals in the classroom improve learning, attention or other aspects of cognition? How Animals Help Students Learn-Research and Practice for Educators and Mental-Health Professionals. New York, New York / Abingdon, Oxon: Routledge.

HEDIGER, K. \& TURNER, D. C. 2014. Can dogs increase children's attention and concentration performance? A randomised controlled trial. Human-animal Interaction Bulletin, Vol.2, 21-39.

HENRY, L. \& WINFIELD, J. 2010. Working memory and educational achievement in children with intellectual disabilities. Journal of Intellectual Disability Research, 54, 354-365.

HENRY, L. A., MESSER, D. J. \& NASH, G. 2015. Executive functioning and verbal fluency in children with language difficulties. Learning and Instruction, 39, 137-147.

HERGOVICH, A., MONSHI, B., SEMMLER, G. \& ZIEGLMAYER, V. 2002. The effects of the presence of a dog in the classroom. Anthrozoos: A Multidisciplinary Journal of The Interactions of People \& Animals, 15, 37-50.

HERZOG, H. 2010. Some we love, some we hate, some we eat - why it's hard to think straight about animals, New York, HarperCollins Publishers.

HERZOG, H. 2011. The Impact of Pets on Human Health and Psychological Well-Being. Current Directions in Psychological Science, 20, 236-239.

HINDES, M., O'HARA, K. \& PERLMUTER, L. C. 2009. Subsyndromal orthostatic blood pressure regulation correlates with motor skills in head start children. International Journal of Psychophysiology, 74, 101-105.

HOAGWOOD, K. E., ACRI, M., MORRISSEY, M. \& PETH-PIERCE, R. 2017. Animal-Assisted Therapies for Youth with or at risk for Mental Health Problems: A Systematic Review. Applied developmental science, 21, 1-13.

HOFFMANN, A. O. M., LEE, A. H., WERTENAUER, F., RICKEN, R., JANSEN, J. J., GALLINAT, J. \& LANG, U. E. 2009. Dog-assisted intervention significantly reduces anxiety in hospitalized patients with major depression. European Journal of Integrative Medicine, 1, 145-148.

HOLMES, J., ADAMS, J.W 2006. Working Memory and Children's Mathematical Skills: Implications for mathematical development and mathematics curricula. Educational Psychology, Vol.26, pp. 339-366.

HOLMES, J., DUNNING, D.L. 2017. A Psychological Perspective: Improving Working Memory to Enhance Maths Performance. In: ADAMS, J. B., P., MESSOUDI, A. (ed.) The Nature and Development of Mathematics Cross Disciplinary Perspectives on Cognition, Learning and Culture. Abingdon: Routledge.

HOLMES, J., GATHERCOLE, S. E., PLACE, M., DUNNING, D. L., HILTON, K. A. \& ELLIOTT, J. G. 2010. Working memory deficits can be overcome: Impacts of training and medication on working memory in children with ADHD. Applied Cognitive Psychology, 24, 827-836.

HOROWITZ-KRAUS, T., VANNEST, J. J., KADIS, D., CICCHINO, N., WANG, Y. Y. \& HOLLAND, S. K. 2014. Reading acceleration training changes brain circuitry in children with reading difficulties. Brain and Behavior, 4, 886-902.

HUANG, C. L., LUO, Y. F., YANG, S. C., LU, C. M. \& CHEN, A.-S. 2020. Influence of Students' Learning Style, Sense of Presence, and Cognitive Load on Learning Outcomes in an Immersive Virtual Reality Learning Environment. Journal of Educational Computing Research, 58, 596-615.

IAHAIO 2014 updated 2018. The IAHAIO Definitions for Animal Assisted Intervention and Guidelines for Wellness of Wellness of Animals Involved in AAI. IAHAIO.

INTERMOUNTAIN-THERAPY-ANIMALS. 2019. Available: https://www.goodtherapy.org/goodcause/intermountain-therapy-animals.html [Accessed 06/01/2019].

ISAZ. 2014. International Society of Anthrozoology [Online]. Online: International Society of Anthrozoology. Available: http://www.isaz.net/isaz/ [Accessed 2014].

JALONGO, M. 2006. On Behalf of Children. Early Childhood Education Journal. 
JALONGO, M. M. I. E. 2015. An Attachment Perspective on the Child-Dog Bond: Interdisciplinary and International Research Findings. Early Childhood Education Journal, 43, 395-405.

JALONGO, M. R. 2005. "What Are All These Dogs Doing at School?" Using Therapy Dogs to Promote Children's Reading Practice. Childhood Education, 81, 152.

JALONGO, M. R., ASTORINO, T. \& BOMBOY, N. 2004. Canine Visitors: The Influence of Therapy Dogs on Young Children's Learning and Well-Being in Classrooms and Hospitals. Early Childhood Education Journal, 32, 9-16.

JALONGO, M. R., E. LINDER, D., ROTZ, F. \& SCHULTZ, D. 2018. Future Directions in Canine-Assisted Activities, Education, and Therapy. Children, Dogs and Education : Caring for, Learning Alongside, and Gaining Support from Canine Companions. Cham : Springer International Publishing : Springer.

JAROSLAWSKA, A. J., GATHERCOLE, S. E., ALLEN, R. J. \& HOLMES, J. 2016a. Following instructions from working memory: Why does action at encoding and recall help? Memory \& Cognition, 44, 1183-1191.

JAROSLAWSKA, A. J., GATHERCOLE, S. E., LOGIE, M. R. \& HOLMES, J. 2016b. Following instructions in a virtual school: Does working memory play a role? Memory \& Cognition, 44, 580-589.

JEFFRIES, S. \& EVERATT, J. 2004. Working memory: Its role in dyslexia and other specific learning difficulties. Dyslexia (10769242), 10, 196-214.

JENKINS, C. D., LAUX, J. M., RITCHIE, M. H. \& TUCKER-GAIL, K. 2014. Animal-Assisted Therapy and Rogers' Core Components Among Middle School Students Receiving Counseling Services: A Descriptive Study. Journal of Creativity in Mental Health, 9, 174-187.

JOHNSEN, K., AHN, S. J., MOORE, J., BROWN, S., ROBERTSON, T. P., MARABLE, A. \& BASU, A. 2014. Mixed reality virtual pets to reduce childhood obesity. IEEE transactions on visualization and computer graphics, 20, 523-30.

JONES, A., \& KINDERSLEY, K. 2013. Dyslexia: Assessing and Reporting: the PATOSS Guide, London, Hodder.

KAHN, P. H. J. \& KELLERT, S., R. 2002. Children and Nature - Psychological, Sociocultural and Evolutionary Investigations, Cambridge, Massachusetts, Massachusetts Institute of Technology (MIT Press).

KAHNEMAN, D. 2011. Thinking Fast and Slow, London UK, Penguin Random House / Allen Lane.

KANE, M. J., POOLE, B. J., TUHOLSKI, S. W. \& ENGLE, R. W. 2006. Working memory capacity and the top-down control of visual search: Exploring the boundaries of 'executive attention'. Journal of Experimental Psychology: Learning, Memory, and Cognition, 32, 749-777.

KAUFFMAN, J. M. 2005. Characteristics of Emotional and Behavioural Disorders of Children and Youth, New Jersey, Pearson Prentice Hall.

KAUFMAN, C. 2010. Executive Function in the Classroom, Baltimore, Maryland USA, Brookes.

KEEFER, L. A., LANDAU, M. J. \& SULLIVAN, D. 2014. Non-human Support: Broadening the Scope of Attachment Theory. Social and Personality Psychology Compass, 8, 524-535.

KEINO, H., FUNAHASHI, A., MIWA, C., HOSOKAWA, M., HAYASHI, Y. \& KAWAKITA, K. 2010. Psychoeducational horseback riding to facilitate communication ability of children with pervasive developmental disorders. Journal of Equine Science, 20, 79-88.

KELLERT, S. R. \& WILSON, E. O. 1993. The Biophilia hypothesis, Washington, D.C. :, Island Press.

KENNEL-CLUB, T. 2015. UK's First School Resources To Tackle Dog Bite Incidents Involving Children [Online]. London: $\quad$ www.thekennekclub.org.uk. Available: https://www.thekennelclub.org.uk/press-releases/2015/june/uk-s-first-school-resourceto-tackle-dog-bite-incidents-involving-children-launched/ [Accessed 12/04/2020 2015].

KENNEL-CLUB, T. 2018. Bark and Read - Standards of Practice for Providers of Animal Assisted Intervention in Schools, London, The Kennel club charitable trust.

KENNEL-CLUB, T. 2020. Safe and Sound: Teaching kids how to behave around dogs [Online]. London: Kennel Club Limited. Available: 
https://www.thekennelclub.org.uk/training/safe-and-sound/safe-and-sound-sign-up/ [Accessed 2020].

KEREPESI, A., KUBINYI, E., JONSSON, G. K., MAGNUSSON, M. S. \& MIKLÓSI, Á. 2006. Behavioural comparison of human-animal (dog) and human-robot (AIBO) interactions. Behavioural Processes, 73, 92-99.

KIRÁLY, I., TAKÁCS, S., KALDY, Z. \& BLASER, E. 2017. Preschoolers have better long-term memory for rhyming text than adults. Developmental Science, 20.

KIRBY, A., PROF. 2020. DO-IT> People [Online]. online: @Do-IT Profiler Solutions Ltd. Available: https://doitprofiler.com/discover-do-it/about-us/ [Accessed 03/09/2020].

KIRNAN, J., SIMINERIO, S. \& WONG, Z. 2016. The Impact of a Therapy Dog Program on Children's Reading Skills and Attitudes toward Reading. Early Childhood Education Journal, 44, 637651.

KLOTZ, K. 2019. Reading Education Assistance Dogs [Online]. Intermountain Therapy Dogs. Available: http://www.readdogsmn.org/ [Accessed 20/08/2019].

KOGAN, L. R., GRANGER, B. P. \& FITCHETT, J. R. 1999. The human-animal team approach for children with emotional disorders: two case studies. Child \& Youth Care Forum, 28, 105121.

KOPONEN, T., SALMI, P., TORPPA, M., EKLUND, K., ARO, T., ARO, M., POIKKEUS, A.-M., LERKKANEN, M.-K. \& NURMI, J.-E. 2016. Counting and rapid naming predict the fluency of arithmetic and reading skills. Contemporary Educational Psychology, 44-45, 83-94.

KRŠKOVÁ, L., TALAROVIČOVÁ, A. B. \& OLEXOVÁ, L. 2010. Guinea pigs-The "Small Great" Therapist for Autistic Children, or: Do Guinea Pigs Have Positive Effects on Autistic Child Social Behavior? Society and Animals, 18, 139-151.

LANE, H. B. \& ZAVADA, S. D. W. 2013. When Reading Gets Ruff: Canine-Assisted Reading Programs. Reading Teacher, 67, 87-95.

LE ROUX, M., SWARTZ, L. \& SWART, E. 2014. The Effect of an Animal-Assisted Reading Program on the Reading Rate, Accuracy and Comprehension of Grade 3 Students: A Randomized Control Study. Child \& Youth Care Forum, 43, 655-673.

LEFEVRE, J.-A., BERRIGAN, L., VENDETTI, C., KAMAWAR, D., BISANZ, J., SKWARCHUK, S.-L. \& SMITH-CHANT, B. L. 2013. The role of executive attention in the acquisition of mathematical skills for children in Grades 2 through 4. Journal of Experimental Child Psychology, 114, 243-261.

LENIHAN, D., MCCOBB, E., DIURBA, A., LINDER, D. \& FREEMAN, L. 2016. Measuring the Effects of Reading Assistance Dogs on Reading Ability and Attitudes in Elementary Schoolchildren. Journal of Research in Childhood Education, 30, 252-259.

LEONARDI, R., J., BUCHANAN-SMITH, H., M., MCIVOR, G. \& VICK, S.-J. 2017. "You Think You're Helping Them, But They're Helping You Too": Experiences of Scottish Male Young Offenders Participating in a Dog Training Program. International Journal of Environmental Research and Public Health [Online], 14.

LILLEY, S. A., ANDRADE, J., TURPIN, G., SABIN-FARRELL, R. \& HOLMES, E. A. 2009. Visuospatial working memory interference with recollections of trauma. British Journal of Clinical Psychology, 48, 309-321.

LISSACK, K. 2018. Does labelling matter? Educational practicioners' experiences and perceptions of diagnostic labelling in education. British Psychological Society Review (South West of England Branch), 15-19.

LOBUE, V., BLOOM PICKARD, M., SHERMAN, K., AXFORD, C. \& DELOACHE, J. S. 2013. Young children's interest in live animals. BRITISH JOURNAL OF DEVELOPMENTAL PSYCHOLOGY, 31, 57-69.

LUDZIOM, Z. 2019. Dogs in Animal Assisted Therapy and Education Conference. Warsaw, Poland.

MARINE, G., SYLVIE, T., ALAIN, L., ERIC, L., MICHEL, D. \& MARTINE, H. 2012. Does Pet Arrival Trigger Prosocial Behaviors in Individuals with Autism? PLOS ONE [Online], 7. 
MARTIN, F. \& FARNUM, J. 2002. Animal-Assisted Therapy for Children with Pervasive Developmental Disorders. Western Journal of Nursing Research, 24, 657-670.

MASSENGILL SHAW, D. 2013. Man's Best Friend as a Reading Facilitator. Reading Teacher, 66, 365371.

MATTARELLA-MICKE, A., MATEO, J., KOZAK, M. N., FOSTER, K. \& BEILOCK, S. L. 2011. Choke or thrive? The relation between salivary cortisol and math performance depends on individual differences in working memory and math-anxiety. Emotion, 11, 1000-1005.

MATTHEWS, G. \& CAMPBELL, S. E. 2010. Dynamic relationships between stress states and working memory. Cognition and Emotion, 24, 357-373.

MCCARDLE, P., MCCUNE, S., GRIFFIN, J. A. \& MAHOLMES, V. 2011. How Animals Affect Us Examining the influence of Human-Animal Interaction on Child Development and Human Health, Washington DC, American Psychological Association.

MCCARTY, C. \& LALLAWAY, M. 2012. New Salford Sentence Reading Test. London, UK: Hodder Education

MCDANIEL PETERS, B. C. \& WOOD, W. 2017. Autism and Equine-Assisted Interventions: A Systematic Mapping Review. Journal of Autism and Developmental Disorders, 47, 32203242.

MCVAY, J. C. \& KANE, M. J. 2012. Why does working memory capacity predict variation in reading comprehension? On the influence of mind wandering and executive attention. Journal of Experimental Psychology: General, 141, 302-320.

MEINTS, K., BRESLFORD, V., GEE, N. \& FINE, A. H. 2017. Animals in Educational Settings - Safety for All. How Students Help Animals to Learn. New York: Routledge.

MELBY-LERVÅG, M. \& HULME, C. 2013. Is working memory training effective? A meta-analytic review. Developmental Psychology, 49, 270-291.

MELSON, G. F., KAHN, P. H., BECK, A., FRIEDMAN, B., ROBERTS, T., GARRETT, E. \& GILL, B. T. 2009. Children's behavior toward and understanding of robotic and living dogs. Journal of Applied Developmental Psychology, 30, 92-102.

MIYAKE, A., FRIEDMAN, N. P., EMERSON, M. J., WITZKI, A. H., HOWERTER, A. \& WAGER, T. D. 2000. The unity and diversity of executive functions and their contributions to complex "Frontal Lobe" tasks: a latent variable analysis. Cognitive psychology, 41, 49-100.

MOLL, K., SNOWLING, M. J., GÖBEL, S. M. \& HULME, C. 2015. Early language and executive skills predict variations in number and arithmetic skills in children at family-risk of dyslexia and typically developing controls. Learning and Instruction, 38, 53-62.

MUTER, V. \& SNOWLING, M. 1998. Concurrent and Longitudinal Predictors of Reading: The Role of Metalinguistic and Short-Term Memory Skills. International Reading Association.

NADAREVIC, L. \& AßFALG, A. 2017. Unveiling the truth: warnings reduce the repetition-based truth effect. Psychological Research : An International Journal of Perception, Attention, Memory, and Action, 81, 814-826.

NASEN 2014. The Pupil Premium - A quick guide to maximising the impact of additional funding for disadvantaged pupils. In: NEEDS), N. N. A. O. S. E. (ed.) www.nasen.org. Tamworth: NASEN.

NEELB. 2016. Schools health and safety risk assessments [Online]. Northern Ireland: North Eastern Education Library Board. Available: www.neelb.org.uk/schools/health-and-safety/riskassessments-for-schools/) [Accessed 2016].

NELSON, W., SCHULTZ, J. 2009. Managing Anger and Aggression in Students with Externalising Behaviour. In: MAYER, M., VAN ACKER, R., LOCHMAN, J., GRESHAM, F. (ed.) CognitiveBehavioural Interventions for Emotional and Behavioural Disorders. New York: Guilford Press.

NEZHAD, A. S. \& VAHEDI, M. 2011. The Role of Educational Psychology in Teacher Education Programs. Procedia - Social and Behavioral Sciences, 30, 327-330. 
O'HAIRE, M. 2013. Animal-Assisted Intervention for Autism Spectrum Disorder: A Systematic Literature Review. Journal of Autism \& Developmental Disorders, 43, 1606-1622.

O'HAIRE, M. E., MCKENZIE, S. J., BECK, A. M. \& SLAUGHTER, V. 2015. Animals may act as social buffers: Skin conductance arousal in children with autism spectrum disorder in a social context. Developmental Psychobiology, 57, 584-595.

OAKHILL, J., YUILL, N. \& GARNHAM, A. 2011. The differential relations between verbal, numerical and spatial working memory abilities and children's reading comprehension. International Electronic Journal of Elementary Education, 4, 83-106.

OCULUS. 2019. Oculus Quest (English) Health and Safety Warnings [Online]. Oculus.com. Available: https://www.oculus.com/legal/health-and-safety-warnings/?locale=en GB [Accessed 2019].

ODENDAAL, J. S. J. 2000. Animal-assisted therapy - magic or medicine? Journal of Psychosomatic Research, 49, 275-280.

OFSTED 2018. School Inspection Handbook: Handbook for inspecting schools in England under section 5 of the Education Act 2005. Manchester, UK.: Ofsted.

OFSTED 2019. The education inspection framework

Framework for inspections carried out, respectively, under section 5 of the Education Act 2005 (as amended), section 109 of the Education and Skills Act 2008, the Education and Inspections Act 2006 and the Childcare Act 2006. Manchester, UK.: Ofsted.

OWENS, M., STEVENSON, J., NORGATE, R. \& HADWIN, J. A. 2008. Processing efficiency theory in children: Working memory as a mediator between trait anxiety and academic performance. Anxiety, Stress \& Coping, 21.

P.A.T.O.S.S. 2016. Code of Ethics and Conduct [Online]. online: P.A.T.O.S.S. Available: https://www.patoss-dyslexia.org/write/MediaUploads/500 Code of Ethics.pdf [Accessed].

PACKIAM ALLOWAY, T. \& ALLOWAY, R. G. 2015. Understanding Working Memory, London, Sage.

PAKIAM-ALLOWAY, T. \& ALLOWAY, R. G. 2015. Understanding Working Memory, London, Sage Publications Inc.

PARRY, P. 2012. The Practical Illustrated Guide to Dog Training, Leicestershire, Anness Publishing Ltd.

PASSOLUNGHI, M. C. \& CORNOLDI, C. 2008. Working Memory Failures in Children with Arithmetical Difficulties. Child Neuropsychology, 14, 387-400.

PEARSON. 2020. Qualification Codes (UK version) [Online]. online: Pearson Assessments Education.

Available:

https://www.pearsonclinical.co.uk/information/qualificationcodes.aspx [Accessed 14 Sept 2016].

PEDROLI, E., PADULA, P., GUALA, A., MEARDI, M. T., RIVA, G. \& ALBANI, G. 2017. A Psychometric Tool for a Virtual Reality Rehabilitation Approach for Dyslexia. Computational and mathematical methods in medicine, 2017, 7048676.

PEMBROKE, J. 2019. Using Standardised Scores in Progress Matrices [Online]. Sigtonline. Available: https://www.sigplus.co.uk/2017/11/using-standardised-scores-in-progressmatrices/ [Accessed 03/09/2019].

PET-PARTNERS. 2019. Available: https://petpartners.org/ [Accessed 20/08/2019].

PETS-AS-THERAPY. 2016. https://petsastherapy.org/. [Accessed 2016 onwards].

PICKERING, S. J. \& GATHERCOLE, S. E. 2004. Distinctive Working Memory Profiles in Children with Special Educational Needs. Educational Psychology, 24, 393-408.

PILLOW-PRICE, K., YONTS, N. \& STINSON, L. 2014. Sit, Stay, Read: Improving Literacy Skills Using Dogs! Dimensions of Early Childhood, 42, 5-9.

POLHEBER, J. \& MATCHOCK, R. 2014. The presence of a dog attenuates cortisol and heart rate in the Trier Social Stress Test compared to human friends. Journal of Behavioral Medicine, $37,860-867$. 
POON, K. 2018. Hot and Cool Executive Functions in Adolescence: Development and Contributions to Important Developmental Outcomes. Frontiers in Psychology, 8.

POSNER, M. I. 2012. Attention in a social world, Oxford, Oxford Press.

POSNER, M. I. \& ROTHBART, M. K. 2007. Research on Attention Networks as a Model for the Integration of Psychological Science. Annual Review of Psychology, 58.

POSNER, M. I. \& ROTHBART, M. K. 2009. Toward a physical basis of attention and self regulation. Physics of life reviews, 6, 103-120.

POSNER, M. I. \& ROTHBART, M. K. 2017. Integrating brain, cognition and culture. Journal of Cultural Cognitive Science, 1, 3-15.

POSNER, M. I., ROTHBART, M. K., SHEESE, B. E. \& VOELKER, P. 2014. Developing Attention: Behavioral and Brain Mechanisms. Advances in Neuroscience, 2014, 1-9.

POSTLE 2006. Working Memory as an emergent property of the mind and brain. Neuroscience 139 (2006) 23-38.

POWELL, A. 2018. Coni the Dog Helps Ely St. Mary's Children with Reading (5th June 2018) [Online]. Spotted in Ely. Available: http://www.spottedinely.com/coni-dog-helps-ely-st-maryschildren-reading/ [Accessed 27 June 2018].

PROTHMANN, A., BIENERT, M. \& ETTRICH, C. 2006. Dogs in child psychotherapy: Effects on state of mind. Anthrozoös, 19, 265-277.

PROTHMANN, A., ETTRICH, C. \& PROTHMANN, S. 2009. Preference for, and Responsiveness to, People, Dogs and Objects in Children with Autism. Anthrozoös, 22, 161-171.

RABBITT, S. M., KAZDIN, A. E. \& HONG, J. E. 2014. Acceptability of Animal-Assisted Therapy: Attitudes toward AAT, Psychotherapy, and Medication for the Treatment of Child Disruptive Behavioral Problems. Anthrozoös, 27, 335-350.

REDEFER, L. A. \& GOODMAN, J. F. 1989. Brief report: Pet-facilitated therapy with autistic children. Journal of Autism and Developmental Disorders, 19, 461-467.

REILLY, K. M., ADESOPE, O. O. \& ERDMAN, P. 2020. The Effects of Dogs on Learning: A MetaAnalysis. Anthrozoös, 33, 339-360.

RENAISSANCE-LEARNING. 2019. Accelerated Reader [Online]. Available: http://www.renlearn.co.uk/accelerated-reader/ [Accessed 03/10/2017].

REYNOLDS, C. R. 1997. Forward and backward memory span should not be combined for clinical analysis. Archives of Clinical Neuropsychology, 12, 29-40.

REYNOLDS, C. R. \& VORESS, J. K. 2007. Test of Memory and Learning (TOMAL 2). 2nd ed. Texas, U.S.A.: PRO-ED Inc.

RICKER, T. J. \& COWAN, N. 2014. Differences between presentation methods in working memory procedures: a matter of working memory consolidation. Journal of experimental psychology. Learning, memory, and cognition, 40, 417-28.

ROBSON, C. 2002. Real World Research, Oxford, UK, Blackwell Publishing.

ROHANI, D. A. \& PUTHUSSERYPADY, S. 2015. BCI inside a virtual reality classroom: a potential training tool for attention. EPJ Nonlinear Biomedical Physics, 3, 1-14.

RUEDA, M. R., FAN, J., MCCANDLISS, B. D., HALPARIN, J. D., GRUBER, D. B., LERCARI, L. P. \& POSNER, M. I. 2004. Development of attentional networks in childhood. Neuropsychologia, 42, 1029-1040.

RUGAAS, T. 2006. On Talking Terms with Dogs: Calming Signals, Wentachee, WA, Dogwise Publishing.

RYAN, R. M. \& DECI, E. L. 2006. Self-Regulation and the Problem of Human Autonomy: Does Psychology Need Choice, Self-Determination, and Will? Journal of Personality, 74, 15571586.

S.C.A.S. 2019. Society for Companion Animal Study Animal Assisted Interventions: Code of Practice for the UK. https://www.scas.org.uk/.

SARKOVA, M., BACIKOVA-SLESKOVA, M., OROSOVA, O., MADARASOVA GECKOVA, A., KATRENIAKOVA, Z., KLEIN, D., HEUVEL, W. \& DIJK, J. P. 2013. Associations between 
assertiveness, psychological well-being, and self-esteem in adolescents. Journal of Applied Social Psychology, 43, 147-154.

SCALLION, S. 2010. Therapy Dogs Find a Role in School. Principal, 90, 48-49.

SCHOOLS-WEB-DIRECTORY. 2016. Schools-Web- Directory [Online]. Available: http://schoolswebdirectory.co.uk/index.php [Accessed 2016].

SCHRETZMAYER, L., KOTRSCHAL, K. \& BEETZ, A. 2017. Minor Immediate Effects of a Dog on Children's Reading Performance and Physiology. Frontiers in Veterinary Science, 4.

SCHUCK, S. E. B., EMMERSON, N. A., FINE, A. H. \& LAKES, K. D. 2015. Canine-Assisted Therapy for Children With ADHD: Preliminary Findings From The Positive Assertive Cooperative Kids Study. JOURNAL OF ATTENTION DISORDERS [Online]. Available: Item Resolution URL http://www.escholarship.org/uc/item/91z106c8.

SCHWEPPE, J. \& RUMMER, R. 2014. Attention, Working Memory, and Long-Term Memory in Multimedia Learning: An Integrated Perspective Based on Process Models of Working Memory. Educational Psychology Review, 26, 285-306.

SHANKWEILER, D., LIBERMAN, I. Y., MARK, L. S., FOWLER, C. A. \& FISCHER, F. W. 1979. The speech code and learning to read. Journal of Experimental Psychology: Human Learning and Memory, 5, 531-545.

SHEN, R. Z. Z., XIONG, P., CHOU, U. I. \& HALL, B. J. 2018. "We need them as much as they need us": A systematic review of the qualitative evidence for possible mechanisms of effectiveness of animal-assisted intervention (AAI). Complementary Therapies in Medicine, 41, 203-207.

SHIRAN, A. \& BREZNITZ, Z. 2011. The effect of cognitive training on recall range and speed of information processing in the working memory of dyslexic and skilled readers. Journal of Neurolinguistics, 24, 524-537.

SIGRID 2018. Animal Assisted Education: Teacher's perspectives of the benefits of and barriers to implementation - Questionnaire from Smith and Dale (2017) for use in Modules in Anthrozoology TM, Open Univeristy in the Netherlands / IAHAO. In: OOSTENDORP, J. (ed.).

SILVA, K., CORREIA, R., LIMA, M., MAGALHÃES, A. \& DE SOUSA, L. 2011. Can Dogs Prime Autistic Children for Therapy? Evidence from a Single Case Study. Journal of Alternative \& Complementary Medicine, 17, 655-659.

SMITH, B., P. \& DALE, A., A. 2016. Integrating animals in the classroom: The attitudes and experiences of Australian school teachers toward animal-assisted interventions for children with Autism Spectrum Disorder.

SMITH, K. A. 2010. Impact of Animal Assisted Therapy Reading Instruction on ReadingPerformance of Homeschooled Students.

SMITHSON, L. \& NICOLADIS, E. 2016. Visuospatial Working Memory Capacity Predicts Physiological Arousal in a Narrative Task. Applied Psychophysiology and Biofeedback : In association with the Association for Applied Psychophysiology and Biofeedback, 41, 203-214.

SNOWLING, M. J. 2001. From language to reading and dyslexia1. Dyslexia, 7, 37-46.

SNOWLING, M. J. 2013. Early identification and interventions for dyslexia: a contemporary view. Journal of research in special educational needs : JORSEN, 13, 7-14.

SNOWLING, M. J., STOTHARD, S. E., P., C., BOWYER-CRANE, C., HARRINGTON, A., TRUELOVE, E., NATION, K. \& HULME, C. 2009. The York Assessment of Reading Comprehension (Y.A.R.C.). London. UK: G.L. Assessment.

SOLOMON, O. 2010. What a Dog Can Do: Children with Autism and Therapy Dogs in Social Interaction. Ethos (00912131), 38, 143-166.

SOMERVILL, J. W., SWANSON, A. M., ROBERTSON, R. L., ARNETT, M. A. \& MACLIN, O. H. 2009. Handling a Dog by Children with Attention-Deficit/Hyperactivity Disorder: Calming or Exciting? North American Journal of Psychology, 11, 111-119.

ST CLAIR-THOMPSON, H., STEVENS, R., HUNT, A. \& BOLDER, E. 2010. Improving children's working memory and classroom performance. Educational Psychology, 30, 203-219. 
STANOVICH, K. E. 1994. Annotation: Does Dyslexia Exist? Journal of Child Psychology and Psychiatry, 35, 579-595.

STEVENSON, K., JARRED, S., HINCHCLIFFE, V. \& ROBERTS, K. 2015. Can a dog be used as a motivator to develop social interaction and engagement with teachers for students with autism? Support for Learning, 30, 341-363.

STOP-THE-77. 2019. Workshops and Videos for children and dogs [Online]. Available: https://www.thefamilydog.com/stop-the-77/ [Accessed 22/06/2016].

STROUD, C. 2012. Dogs in the classroom: reading with a fluffy friend. The Telegraph.

SUIED, C., DRETTAKIS, G., WARUSFEL, O. \& VIAUD-DELMON, I. 2013. Auditory-visual virtual reality as a diagnostic and therapeutic tool for cynophobia. Cyberpsychology, behavior and social networking, 16, 145-52.

SWANSON, H. L., KEHLER, P. \& JERMAN, O. 2010. Working Memory, Strategy Knowledge, and Strategy Instruction in Children With Reading Disabilities. Journal of Learning Disabilities, $43,24$.

SWIFT, C. H. 2009. Animal Assisted Therapy and the Reading Education Assistance Dogs[RTM] (R.E.A.D.) Program as Perceived by Volunteer R.E.A.D. Facilitators: A National Study. ProQuest LLC.

T.D.N. 2019. Therapy Dogs Nationwide [Online]. online: Therapy Dogs Nationwide. Available: http://www.tdn.org.uk/ [Accessed 2019].

TERRELL, C., PASSENGER, T. 2011. Understanding ADHD, Autism, Dyslexia \& Dyspraxia, Poole, Dorset, The British Medical Association.

THE-DOGS-TRUST. 2017. Fact Sheet - School Dogs [Online]. Available: https://www.dogstrust.org.uk/help-advice/factsheets-downloads/school\%20dogs.pdf [Accessed 27/07/2019].

THE-KENNEL-CLUB. 15/04/2019 2019. RE: Bark and Read Research. Type to DEPT., L. A. C.

THE-KEY. 2019. Available: https://schoolleaders.thekeysupport.com/about/ [Accessed 23/03/2016].

THE-SCHOOL-BUS. 2019. Available: https://www.theschoolbus.net/home [Accessed 27/03/2016].

THERAPY-DOGS-NATIONWIDE. 2020. Paws and Read Scheme Overview [Online]. Available: http://www.tdn.org.uk/test/wp-content/uploads/2019/02/PAWS-READ-IntroductionPack-1.pdf [Accessed 2020].

THOMPSON, P. A., HULME, C., NASH, H. M., GOOCH, D., HAYIOU-THOMAS, E. \& SNOWLING, M. J. 2015. Developmental dyslexia: predicting individual risk. Journal of Child Psychology \& Psychiatry, 56, 976-987.

TIMES-EDUCATIONAL-SUPPLEMENT. 2019. Available: https://www.tes.com/ [Accessed 17/09/16].

TRAMMELL, J. P. 2019. Therapy Dogs Improve Student Affect but Not Memory. Anthrozoös, 32, 691-699.

TSUN-JU, L. \& YU-JU, L. 2015. Language Learning in Virtual Reality Environments: Past, Present, and Future. Journal of Educational Technology \& Society, 18, 486-497.

VANFLEET, R. 2018a. International Institute of Animal Assisted Play Therapy ${ }^{\circledR}$ [Online]. online: International Institute of Animal Assisted Play Therapy ${ }^{\circledR}$. Available: http://risevanfleet.com/international/ [Accessed].

VANFLEET, R. 2018b. Relationship, Relationship, Relationship. In: VANFLEET, R. (ed.) Online Webinar. Division 17, Human Animal Interaction: Colorado State University.

VANFLEET, R. \& FAA-THOMPSON, T. 2017. Animal Assisted Play Therapy, Sarasota, Florida, USA, Professional Resouce Press.

VEENENDAAL, N. J., GROEN, M. A. \& VERHOEVEN, L. 2014. The role of speech prosody and text reading prosody in children's reading comprehension. British Journal of Educational Psychology, 84, 521-536. 
VISU-PETRA, L., CHEIE, L., BENGA, O. \& ALLOWAY, T. P. 2011. Effects of anxiety on memory storage and updating in young children. International Journal of Behavioral Development, 35, 38.

WARNOCK, H. M. 1978. Report of the Committee of Enquiry into the Education of Handicapped Children and Young People. In: PEOPLE, C. O. E. I. T. E. O. H. C. A. Y. (ed.). London: Her Majesty's Stationery Office.

WATERS, A. M. \& KERSHAW, R. 2015. Direction of attention bias to threat relates to differences in fear acquisition and extinction in anxious children. Behaviour Research and Therapy, 64, 56-65.

WATTS, K. \& EVERLY, J. S. 2009. Helping Children with Disabilities through Animal-Assisted Therapy. Exceptional Parent, 39, 34-35.

WESTGARTH, C., BROOKE, M. \& CHRISTLEY, R. M. 2018. How many people have been bitten by dogs? A cross-sectional survey of prevalence, incidence and factors associated with dog bites in a UK community. J Epidemiol Community Health, 72, 331-336.

WIEBE, S. A., SHEFFIELD, T., NELSON, J., MIZE CLARK, C. A. C., CHEVALIER, N. \& ESPY, K. A. 2011. The structure of executive function in 3-year-olds. Journal of Experimental Child Psychology, 108, 436-452.

WILKINSON, G. S. \& ROBERTSON, G. J. 2006. Wide Range Achievement Test (WRAT 4). 4th ed. Lutz, Florida, U.S.A.: PAR.

WILSON, A. J., ANDREWES, S. G., STRUTHERS, H., ROWE, V. M., BOGDANOVIC, R. \& WALDIE, K. E. 2015. Dyscalculia and dyslexia in adults: Cognitive bases of comorbidity. Learning and Individual Differences, 37, 118-132.

WILSON, B. J. D. D. R. 2006. A Computerized Task for Investigating the Relation Between Attentional and Emotional Processes in Children. Journal of Genetic Psychology, 167, 415431.

WINKLE, M., DICKSON, C., STEWART, L. A., CHANDLER, C., KERULO, G., SIMPSON, B., VANFLEET, R., JOHNSON, A., BRUNEAU, L. \& CALLAHAN, M. M. 2012. Animal-Assisted Intervention International

Recommended Competencies

for Animal Assisted Interactions.

WINKLE, M., ROGERS, J., KATALENIC, L., GORBING, P., HOPE GIBSONE, S., VAN COPPERNOLE, D., HEREMANS, J., MARISCAL, T., HULTMAN, S., HOOK, I., SIMPSON, B. \& DICKSON, C. 2019. Animal Assisted Intervention International Standards of Practice [Online]. Available: https://aai-int.org/wp-content/uploads/2019/02/AAll-Standards-of-Practice.pdf [Accessed].

WOHLFARTH, R., MUTSCHLER, B., BEETZ, A. \& SCHLEIDER, K. 2014. An investigation into the efficacy of therapy dogs on reading performance in 6-7 year old children. Human-animal Interaction Bulletin, 2, 60-73.

WOLFF, A., I. \& FRISHMAN, W., H. 2004. Animal-assisted therapy in cardiovascular disease. Seminars in Integrative Medicine, 2, 131-134.

WU, S. S., CHEN, L., BATTISTA, C., SMITH WATTS, A. K., WILLCUTT, E. G. \& MENON, V. 2017. Distinct influences of affective and cognitive factors on children's non-verbal and verbal mathematical abilities. Cognition, 166, 118-129.

YAIR, B.-H., AYA, K., DOMINIQUE, L. \& DAN, Z. 2010. When time slows down: The influence of threat on time perception in anxiety. Cognition \& Emotion, 24, 255-263. 
Appendices 


\section{Appendix A Qualification Codes from Pearson as of 05/09/2020}

Taken from : https://www.pearsonclinical.co.uk/information/qualificationcodes.aspx

\section{Qualifications}

Pearson is committed to maintaining professional standards in testing.

A central principle of professional test use is that individuals should use only those tests for which they have the appropriate training and expertise. Pearson supports this principle by stating qualifications for the use of particular tests and selling tests to individuals who provide credentials that meet those qualifications. The policies that Pearson uses to comply with professional testing practices are described below.

Additional policy information may be found on the qualifications policy page.

\section{Product qualification levels}

All Pearson Clinical Assessments products in the UK are assigned a qualifications level of either CLI, CL2, CL2R, or CL3 (with CLI being the highest qualification level).

In order to establish which tests are available to you, simply compare your training code against the product qualification code.

UNAS: Unassigned tests are available to all users, there are no special qualifications to purchase these products.

\section{Qualification Level CL3}

CL3 tests in general, are those which do not require an individual to have advanced training in assessment and interpretation. Qualified teachers would be given this code. A BSc in Psychology will only give access to $C L 3$ unless the individual has further training in test administration.

\section{Qualification Level CL2R}


CL2R tests require qualified teaching status and a further Post Graduate Qualification in SEN ie Post Graduate Diploma or Masters. This qualification would need to be in SEN, SpLD or a relevant field at Level 7.

For individuals in the Republic of Ireland only - CL2R tests require qualified teaching status + Certificate of Competence in Educational Testing (CCET - Test User) + evidence that you have signed up to the British Psychological Society's Register of Qualifications in Test Use (RQTU).

\section{Qualification Level CL2}

CL2 tests may be purchased by individuals who are certified by a professional organisation recognised by Pearson Assessment or have a graduate and/or post graduate qualification relevant to their profession. This qualification code would encompass all psychologists other than those mentioned for CLI, speech or occupational therapists, mental health professionals and health practitioners with appropriate Graduate and professional qualifications in their field of practice.

\section{Qualification Level CLI}

Tests with a CLI qualification require a high level of expertise in test interpretation, and can be purchased by individuals who:

- Are registered with the HCPC as a Practitioner Psychologist and/or is a Chartered Psychologist with the BPS.

- Are registered with the HCPC as a Practitioner Psychologist and also has the protected titles of Clinical Psychologist, Forensic Psychologist, Counselling Psychologist or Educational Psychologist.

NB Practitioner Psychologists registered with the HCPC, but who have no protected title or the protected titles of Health Psychologist or Sports and Exercise Psychologist, will be given a CL2 status. 


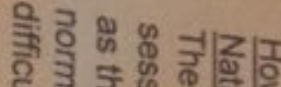

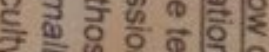
र

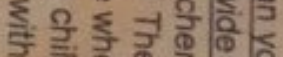
을

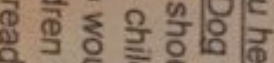

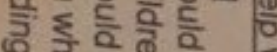
ㅇํㄱ 똟

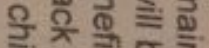
득 (1)

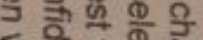
\용 옹 ₹응 。온 市需 $\because$ 을 ते 규 홍 ฏ

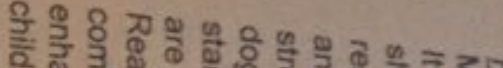

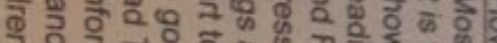
б \& ว

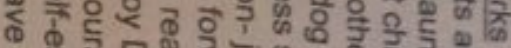

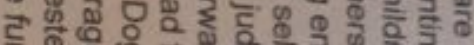
\$ क्ष

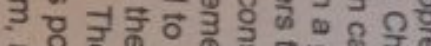

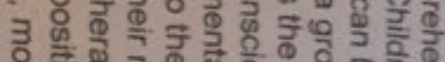

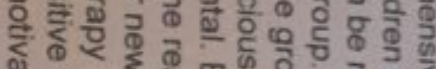

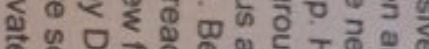

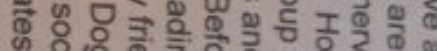

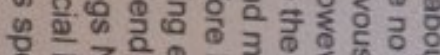

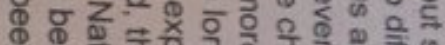

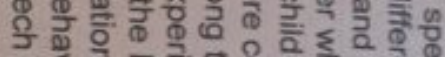
约

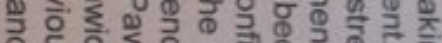

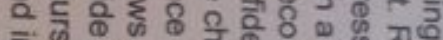
क्मे 믐

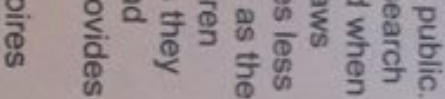

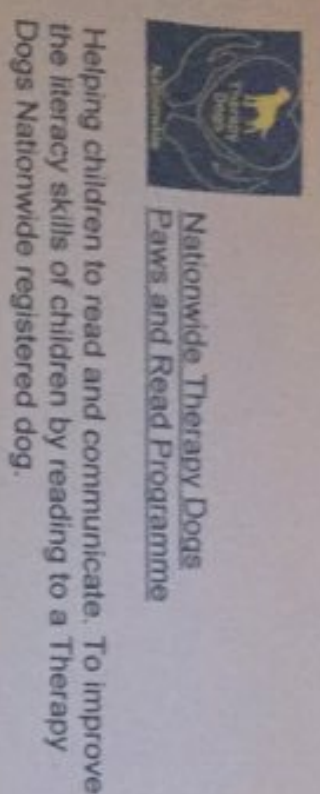

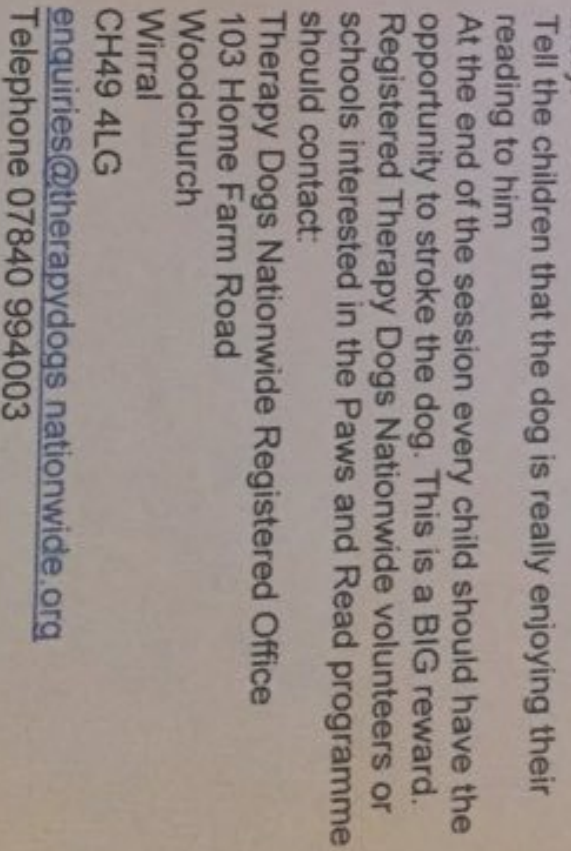
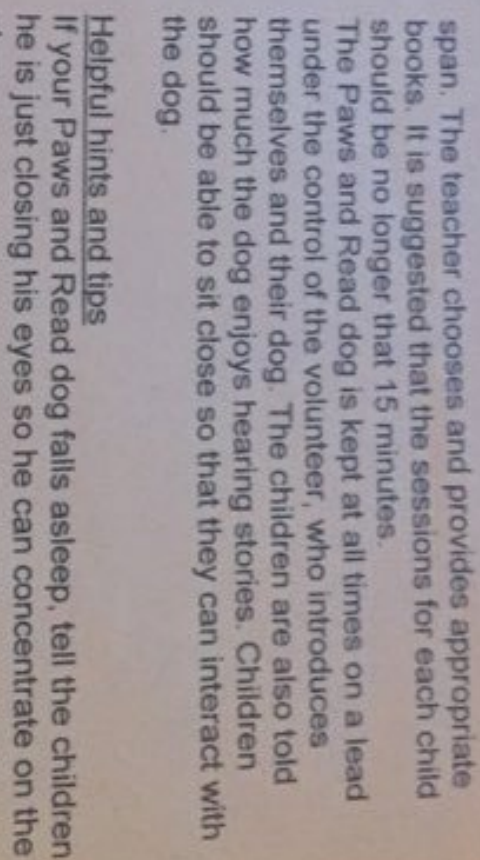


\section{Appendix C Children's Questionnaire}

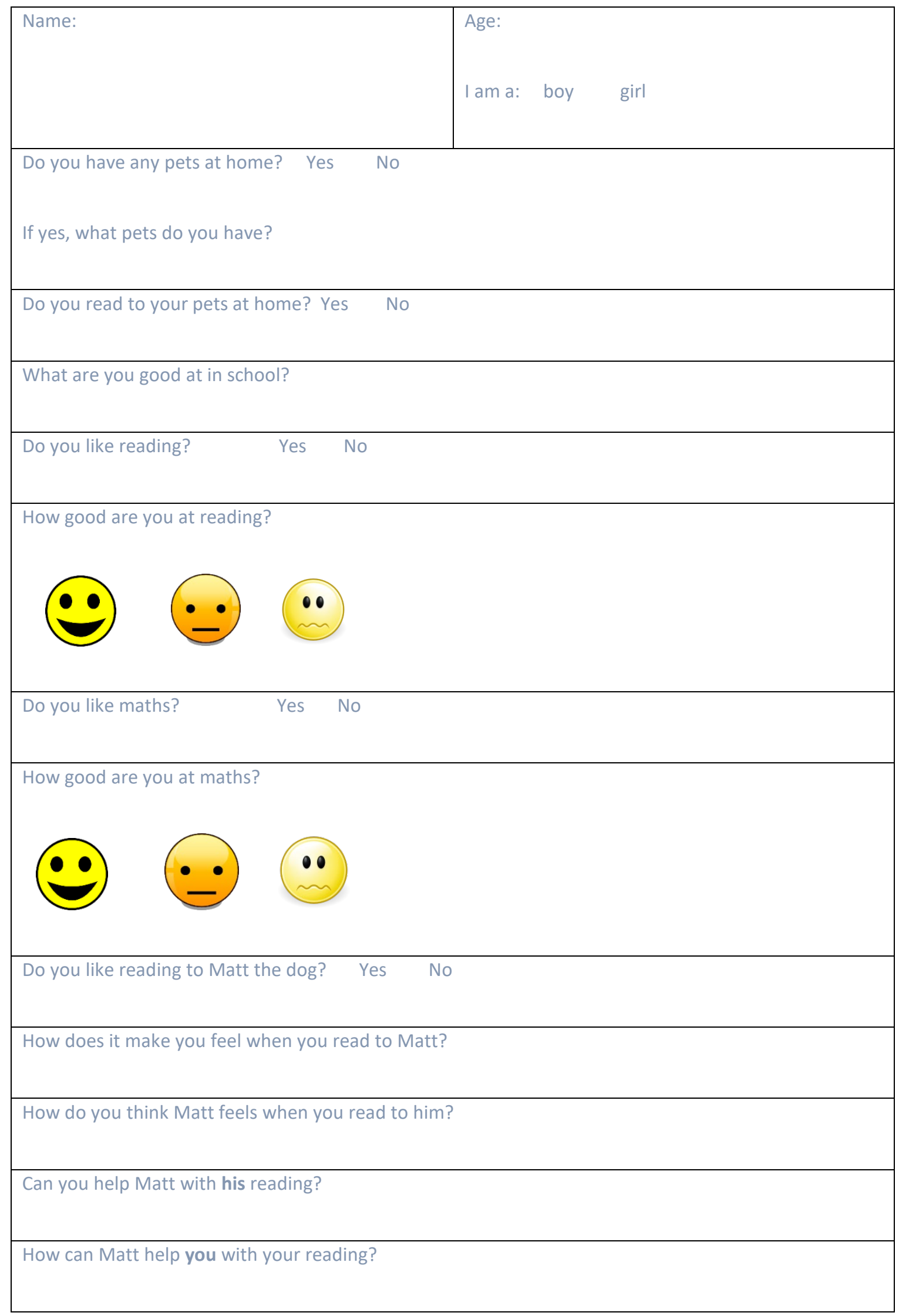

Thank you for filling in this questionnaire. 
Reading to Dogs Questionnaire - School Staff Version

- I have been observing and recording the 8 children who have been reading to Jerry and Matt lo dog (Paws and Read Scheme from Therapy Dogs Nationwide) and been measuring the levels of stress when reading, with and without Matt being present. Please would you help by giving your opinions as to the benefits (or not) of the scheme as you see it. Thank you.

1. What is your role in school?

Teacher

Teaching Assistant

Senior Management

Volunteer

2. Do you have any pets at home?

(1) Yes

No

3. Do you think that animals (such as dogs) have a role in education?

Yes

No

Not sure

4. How long have you been involved with classes who have read to a dog?

Never

This is my first year

$1-3$ years

3 Years and longer

5. What do you believe the Paws and Read (Jerry and Matt the dog) scheme is about? 
6. How do you select your readers for the Paws and Read Scheme?

I don't-someoritat sces

Iselect 11.

(s)

o you think that reading to a dog makes any difference for the individual child?

Yes

No

Don't know

8. Have you noticed any changes in the following for any child who has used the scheme?

Improved attitude to reading when back in class?

Increase in motivation to read when back in class?

Fluency and accuracy when reading back in class?

Overall confidence and self esteem when back in class?

The child has regressed in their reading

Other (please specify)

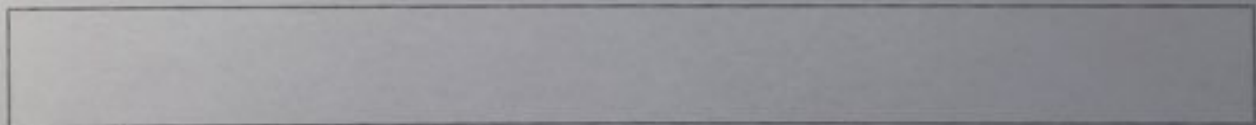

9. In your opinion, do the children who have read to the dog make

less progress than those who have not read to a dog?

about the same progress as those who have not read to a dog?

more progress than those who have not read to a dog?

10. When do you evaluate the paws and read scheme?

Weekly?

Termly?

Annually?

It's not up to mel 
11. Do you have any mecdotal evidence or stories of any particular success with the scheme? Please write below

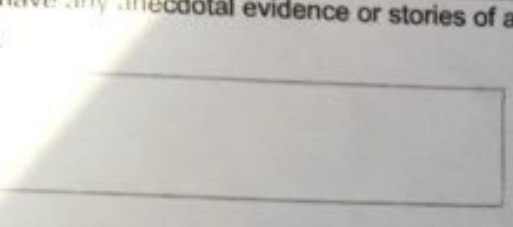

12. Would you continue the scheme for your class?

Yes

No

Maybe 
Appendix E Adult Questionnaire

Questionnaire for "Differences between the presence of "real" dogs or "virtual Reality" dogs for human academic learning skills

Section 1: Personal Data

What is your age? (Please tick a box): $18-25 \square$ 26-36 $\square$ 37-47 $\square \quad 48-58 \square 59$ or older $\square$

Are you: Male $\square$ Female $\square$

Which level of study are you? Under-grad $\square$ Post Grad $\square \quad$ Post Doctorate $\square$

Section 2: Previous experience with animals

Are you currently an animal / pet owner? Yes $\square$ No $\square$

Have you owned animals / family pets in the past? Yes $\square$ No

Did you have animals / family pets as a child? Yes $\square$ No

Section 3: Today's experiment

There were 4 conditions - Please rate the boxes from 1-4, (1 being the most and 4 being the least.)

In which condition did you feel the most relaxed?

\begin{tabular}{|l|l|}
\hline Condition & Your \\
\hline Vocab and Mental tasks in the presence of the researcher only \\
\hline Vocab and Mental Maths tasks in the presence of the researcher and the therapy dog & \\
\hline Vocab and Mental Maths tasks in VR with the researcher only & \\
\hline Vocab and Mental Maths tasks in VR with the researcher and the therapy dog & \\
\hline
\end{tabular}

In which condition did you feel you concentrated the best? 


\begin{tabular}{|l|l|}
\hline Condition & Your \\
\hline Vocab and Mental tasks in the presence of the researcher only \\
\hline Vocab and Mental Maths tasks in the presence of the researcher and the therapy dog & \\
\hline Vocab and Mental Maths tasks in VR with the researcher only & \\
\hline Vocab and Mental Maths tasks in VR with the researcher and the therapy dog & \\
\hline
\end{tabular}

Which tests did you prefer? Vocabulary $\square$ Mental Maths $\square$

Section 4: Any other information

Do you have any learning differences which you would like to share? (E.g. Dyslexia / Dyscalculia etc.) If so, what are they?

Please write in the box:

Please write in the box:

have any other comments / thoughts about today's experience that you wish to make?

Thank you very much for your time and support - it is very much appreciated,

Janet Oostendorp 


\title{
Appendix F Children's Poem
}

\section{Sitting by a Swamp}

By Kristyn Crow

\begin{abstract}
I was sitting by a swamp
Just humming a tune

With the fireflies dancing

'neath the fat gold moon
\end{abstract}

When off in the distance

Was a splashing sound

So I stood on my tippy toes

And looked around

I heard:

Splish splash

Rumba - Rumba

Bim bam slapping

BOOM!

Splish splash

Rumba - Rumba

Bim bam slapping

BOOM 
Appendix G Examples of Miscue analysis, Poem and Reading

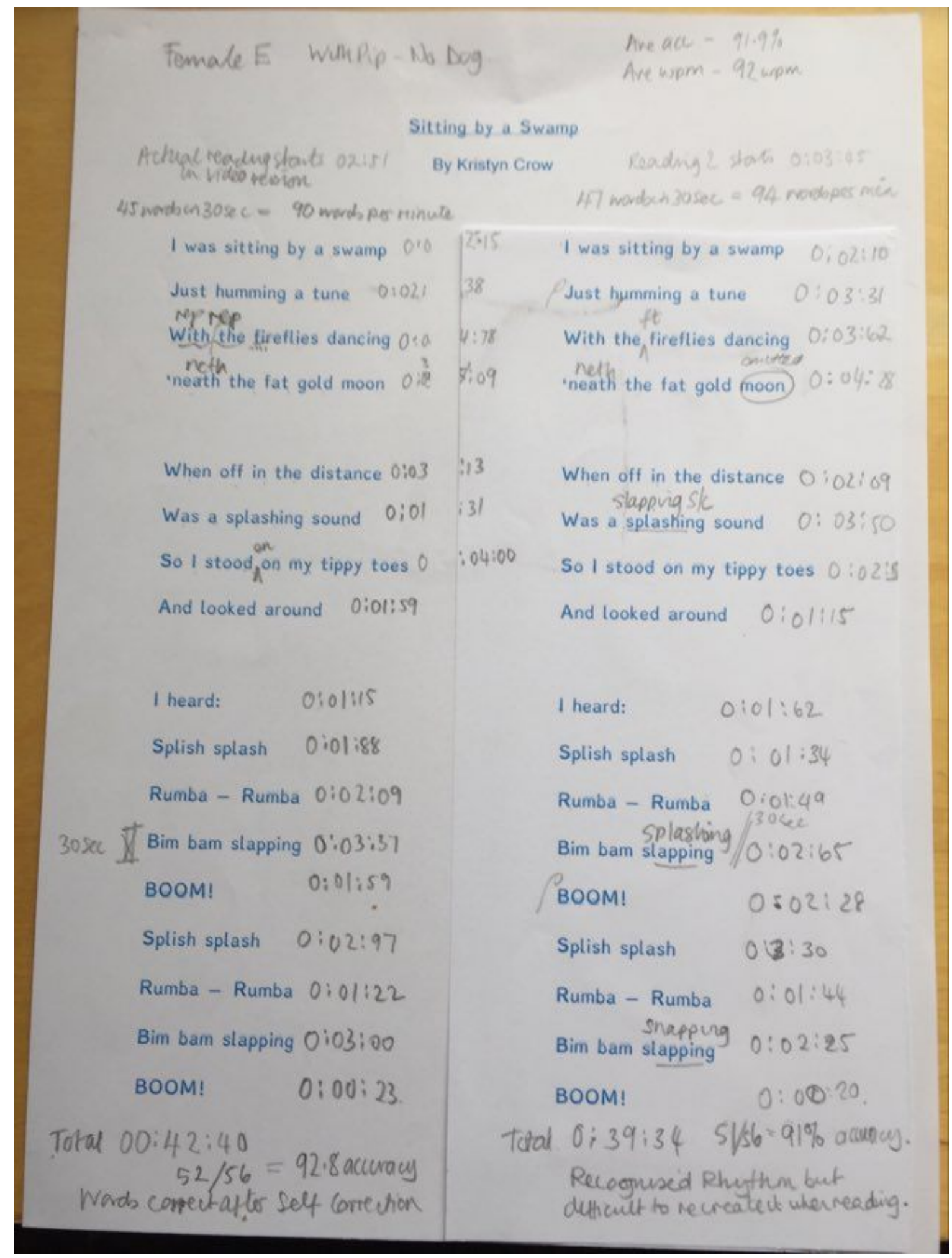




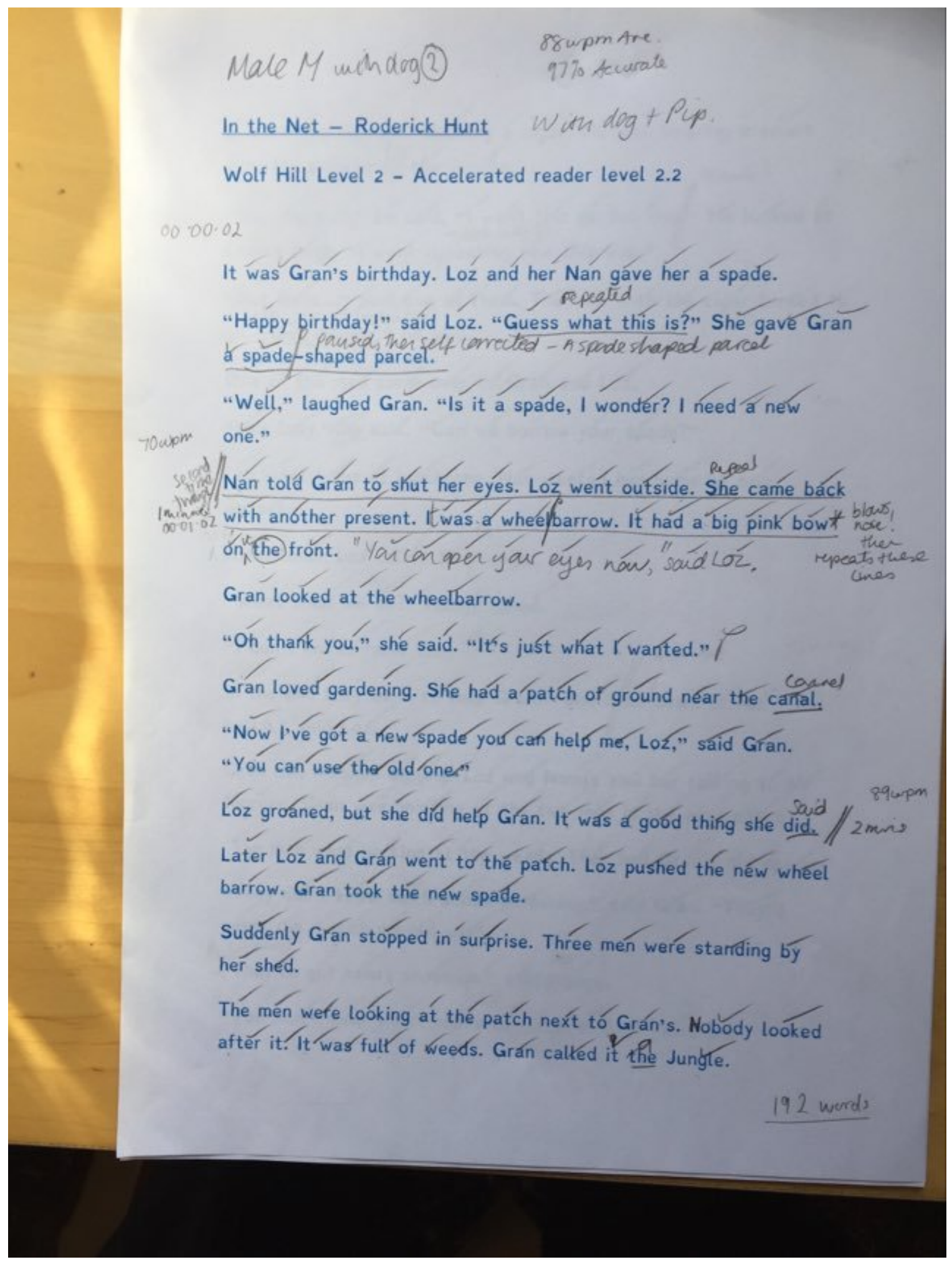




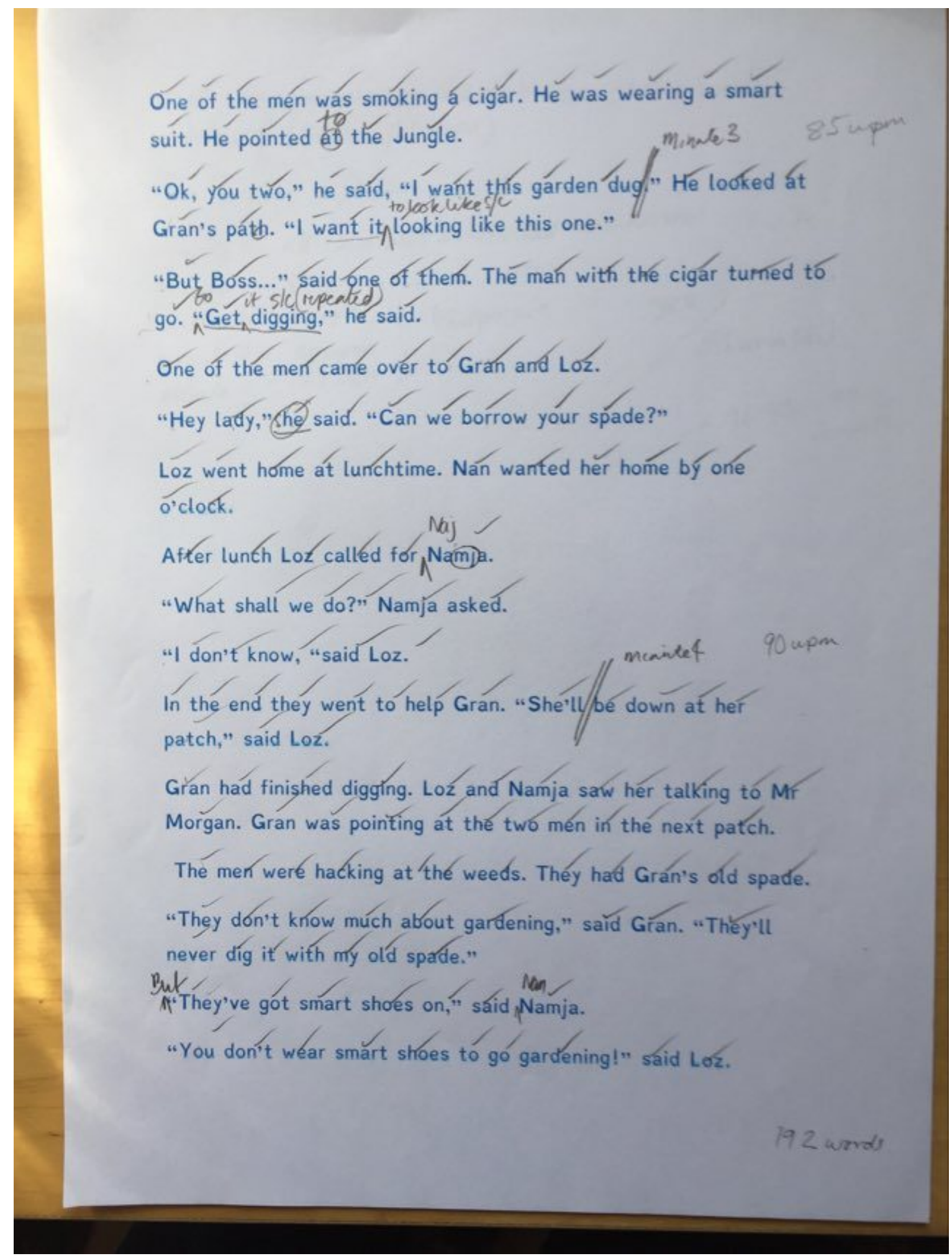


"They're up tó something," said Mr Morgan. "And it's not gardening!n (good eppesmoll)

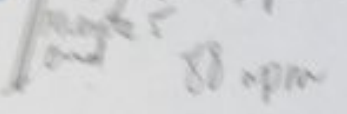

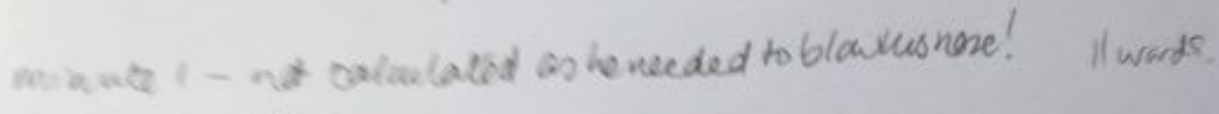

arencle 2 - 89apm

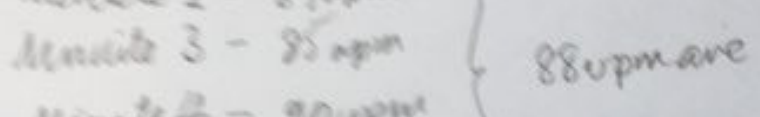

revetet- poupm

Alumale 5 - 88 morr.

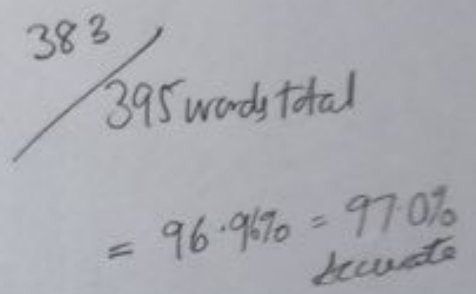


Appendix H Adult Mental Maths Questions

\begin{tabular}{|c|c|c|c|c|}
\hline Theme & Set 1 & Set 2 & Set 3 & Set 4 \\
\hline Range & $\begin{array}{c}\text { a) } \quad-6,0,1,1,1 \text {, } \\
\quad 1,2,2,2,3, \\
3,4,4 \\
\text { b) } 11,12,10, \\
9,9,10,26\end{array}$ & 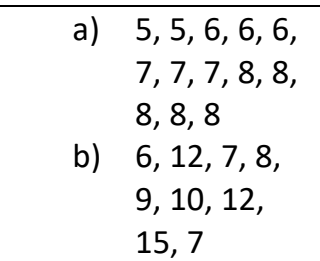 & 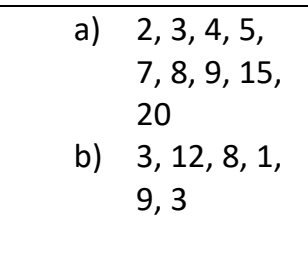 & $\begin{array}{l}\text { a) } 2,3,3,5,7 \\
\text { b) }-5,-4,-3,- \\
2,1,5\end{array}$ \\
\hline Mean & $\begin{array}{l}\text { a) } \quad 4,9,7,12 \\
\text { b) } \quad 3,2,2,3,0\end{array}$ & $\begin{array}{l}\text { a) } 8,11,8 \\
\text { b) } 1,9,8,6\end{array}$ & $\begin{array}{l}\text { a) 2, 2, 1, } \\
\text { 0,3 } \\
\text { b) } \quad 9,12,9\end{array}$ & $\begin{array}{l}\text { a) } 14,10,24, \\
12 \\
\text { b) } 2,8,9,5\end{array}$ \\
\hline Percentages & $\begin{array}{l}\text { a) } 10 \% \text { of } f 50 \\
\text { b) } 40 \% \text { of } 250 \\
\mathrm{~kg}\end{array}$ & $\begin{array}{l}\text { a) } 5 \% \text { of } 200 \mathrm{~g} \\
\text { b) } 20 \% \text { of } \\
3 \mathrm{~km}\end{array}$ & 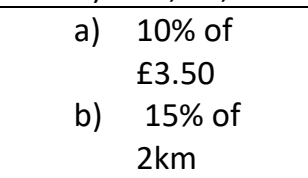 & $\begin{array}{l}\text { a) } 50 \% \text { of } f 64 \\
\text { b) } 60 \% \text { of } \\
750 \mathrm{~g}\end{array}$ \\
\hline Simplification & $\begin{array}{l}\text { a) } x^{2}-x+3 x^{2}-3 x \\
\text { b) }-3 m+2 m n \\
-4 m+5 m n\end{array}$ & $\begin{array}{l}\text { a) } 5 x+3 x-1 \\
\text { b) } 15-6 x-13- \\
7 x\end{array}$ & $\begin{array}{l}\text { a) } 8+3 y-y \\
\text { b) } 8+2 x+3+ \\
5 x\end{array}$ & $\begin{array}{l}\text { a) } a+b+a+b \\
\text { b) } 4 m^{2}+3 m- \\
2 m^{2}\end{array}$ \\
\hline $\begin{array}{l}\text { Ascending } \\
\text { Order }\end{array}$ & $\begin{array}{l}\text { a) } \quad-45,-27,- \\
33,38,14 \\
\text { b) } \quad-5,6,-7,8\end{array}$ & $\begin{array}{c}\text { a) }-2,0,-6,9 \\
\text { b) } 0.1,-2.3 \text {, } \\
0.5,-0.1\end{array}$ & $\begin{array}{l}\text { a) } 4,9,3,-3 \\
\text { b) }-15,-13,2 \text {, } \\
-17,8\end{array}$ & $\begin{array}{l}\text { a) }-2,5,-6,6 \\
\text { b) } 12,16,-5 \text {, } \\
-13,28\end{array}$ \\
\hline Decimals Add & $\begin{array}{l}\text { a) } 4.7+5.5 \\
\text { b) } 3.6+6.7\end{array}$ & $\begin{array}{l}\text { a) } 4.7+5.4 \\
\text { b) } 6.8+4.3\end{array}$ & $\begin{array}{l}\text { a) } 7.5+8.9 \\
\text { b) } 2.2+4.8\end{array}$ & $\begin{array}{l}\text { a) } \\
\text { b) } \\
2.55+4.22 \\
\text { b. } 3.12\end{array}$ \\
\hline $\begin{array}{l}\text { Decimals } \\
\text { Subtract }\end{array}$ & $\begin{array}{l}\text { a) } 5.17-4.09 \\
\text { b) } 15.46-8.32\end{array}$ & $\begin{array}{l}\text { a) } 3.1-1.7 \\
\text { b) } 7.3-6.6\end{array}$ & $\begin{array}{l}\text { a) } 2.16-1.42 \\
\text { b) } 1.51-0.46\end{array}$ & $\begin{array}{l}\text { a) } 6.6-4.1 \\
\text { b) } 7.5-5.4\end{array}$ \\
\hline Fractions & $\begin{array}{l}\text { a) } \frac{3}{8}=\frac{}{40} \\
\text { Calculate this addition } \\
\text { a) } \frac{1}{8}+\frac{1}{4}=\end{array}$ & $\begin{array}{l}\text { b) } \frac{7}{9}=\frac{7}{45} \text { this } \\
\text { Calculate } \\
\text { addition } \\
\text { a) } \frac{2}{5}+\frac{3}{10}=\end{array}$ & $\begin{array}{l}\text { a) } \frac{3}{7}=\frac{}{42} \\
\text { Calculate } \\
\text { this } \\
\text { addition } \frac{2}{3}+\frac{1}{6}=\end{array}$ & $\begin{array}{l}\text { a) } \frac{2}{3}=\frac{}{30} \text { this } \\
\text { Calculate } \\
\text { addition } \\
\text { a) } \frac{2}{5}+\frac{1}{10}=\end{array}$ \\
\hline $\begin{array}{l}\text { Negative } \\
\text { Numbers }\end{array}$ & $\begin{array}{l}\text { a) }+8--14 \\
\text { b) }+34+-23\end{array}$ & $\begin{array}{l}\text { a) }-9--7 \\
\text { b) }+3+-17\end{array}$ & $\begin{array}{l}\text { a) }+8--4 \\
\text { b) }+13+-1\end{array}$ & $\begin{array}{l}\text { a) }-19++4 \\
\text { b) }+34+-23\end{array}$ \\
\hline $\begin{array}{l}\text { BIDMAS } \\
\text { (order of } \\
\text { operations) }\end{array}$ & $\begin{array}{l}\text { a) }(4+6)^{2} \times 3= \\
\text { b) }(15-5)^{2}-55=\end{array}$ & 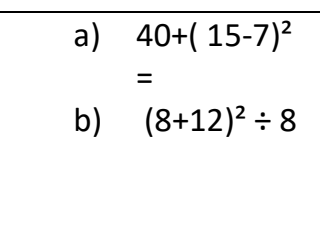 & $\begin{array}{l}\text { a) } 5(3 \times 7)^{2}= \\
\text { b) } 9(16-6)^{2}=\end{array}$ & $\begin{array}{l}\text { a) } 4(4+5)^{2}= \\
\text { b) }(10-8)^{2}-4 \\
=\end{array}$ \\
\hline
\end{tabular}


Appendix I Adult Nelson Denny Vocabulary Questions

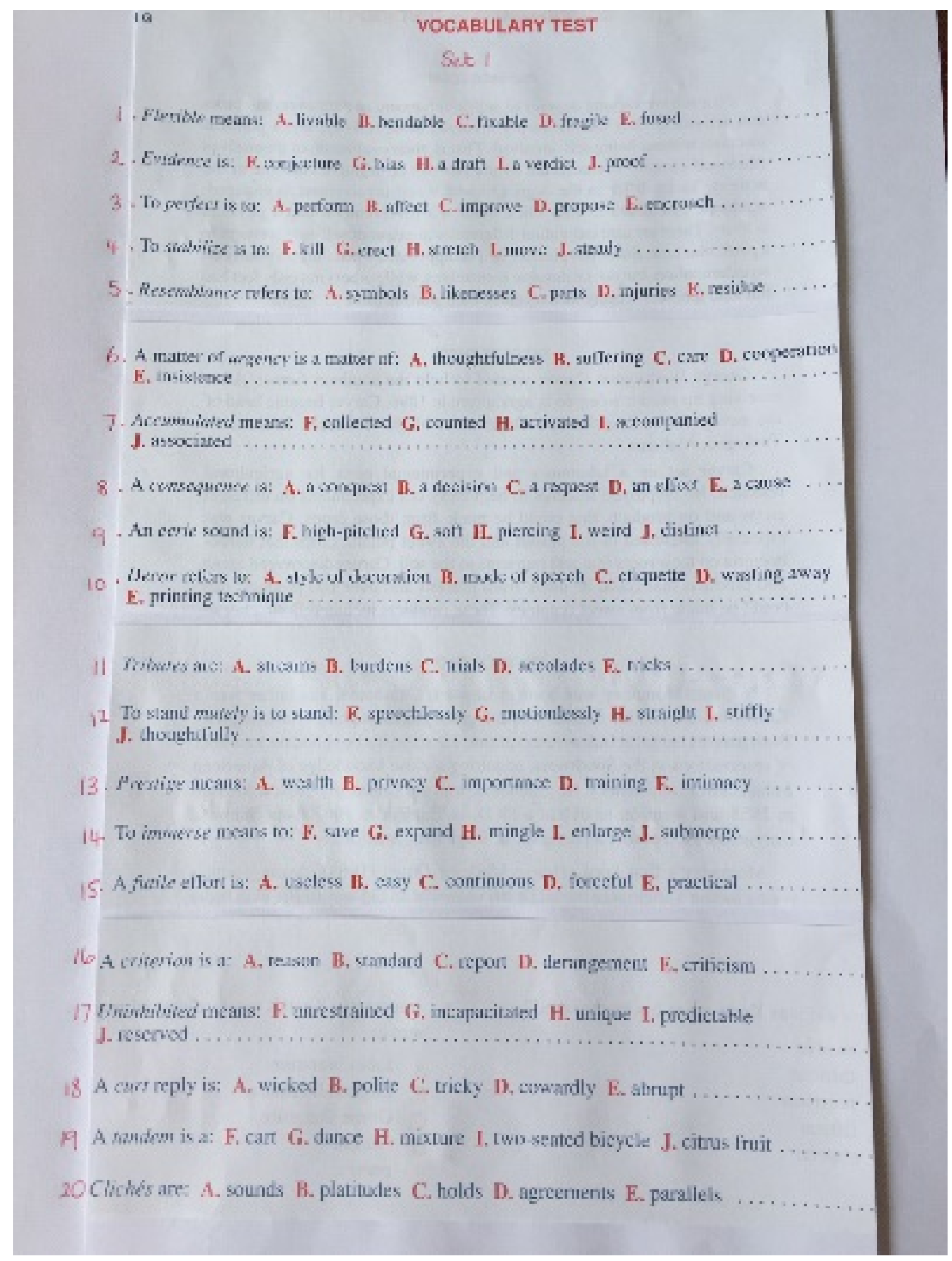




\section{Sict 2}

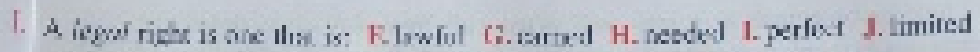

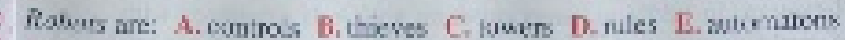

3 If it is irrified, it is: E. numed G. changed II. cha:ked L. aceed J. sonsikered

4.

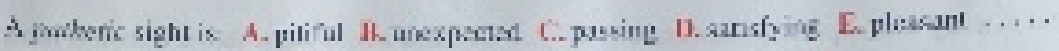

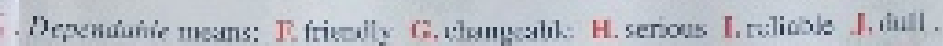

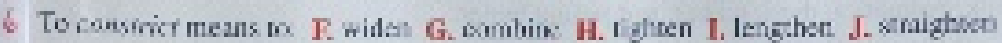

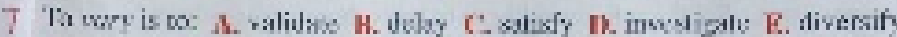

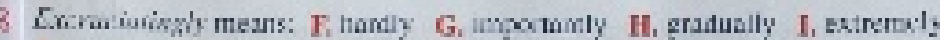

T. ste ilingly

9 Tacrics en: A. boces R. ideas $\mathrm{C}$, trooos $\mathrm{D}$, barles $\mathrm{k}$, machods

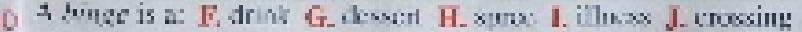

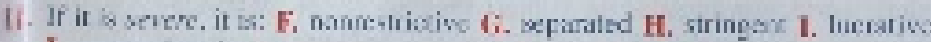

J. tumprehensive

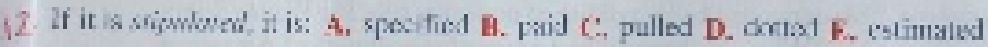

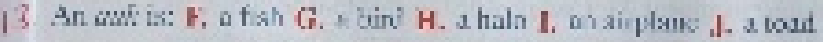

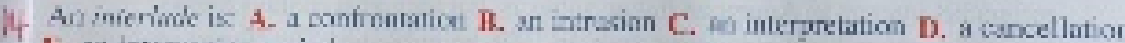
E. an interwening seried

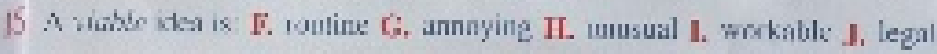

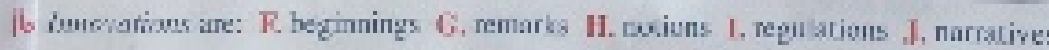

17 To speak ieunily is to spak: A. vigonosily B. sonly C.easily D. clearly E. haltiogely

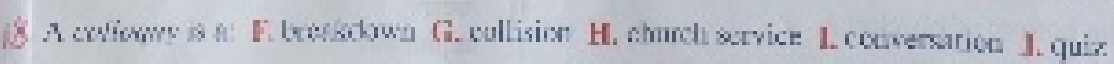

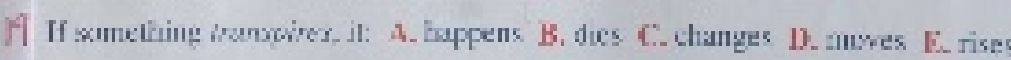

2C. One who is hespikate is: F, close-minata G, ill H, old I. chesriu] J. condial 


\section{Set 3}

- Canines are: A. binds B. dogs C. cats D. jungles E. canes

2. To retain means to: F. release (i. educate H. straightea I, forget J. reserve

3. To assist is to: A. ativise H. order C.aid D. join F. fail

4. If it is ssholvidied, it is: F, whole G.sepnented H. reduced I. enhanced I. superin

S. Agenda means: A. mil call If. agent C. program D. motion E.committee

b If it is probrofing, it is: A. hidden B. une sided C. even D, indented E. extending

1 . If yuu are gueried you are: F. imprisoned G. scarched H. releaced L questicned J. answeresd

8. A dweising is a: A, neighborbood B, fesilbence C. pit D, fountain E., tool

9. Humility means: F. modesty G. sadness H. hooesty I. quierness J. faimes

10. Mondatory means: A, unuecessary B. changed C. legal D. ahligatcory E. human

II Io be impelled is to he: $\mathbf{A}$. bindered $\mathbf{B}$, belped $\mathbf{C}$. improved $\mathbf{D}$. invited $\mathbf{C}$, driven

12. To be seicctive is to be: F, skilled $\mathbf{G}$. obvious H. discriminating $\mathbf{L}$. shacure J. lowable

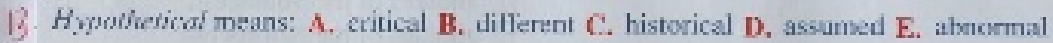

4 Ahurgtion means; P. regret $\mathbf{G}$, interest $\mathrm{H}$. haste I. relaxation J. pain

15 Resplenden means: A. responding B. darsling C. inhibiting D. respertable

E. resourceful

i6 A prognastic device: A. predicts B, treals C. prepures D, cures E. warn.

17 If something is nowghr, it is: F. round $G$, novel H. nothing L, original J. improper

18 Astithesis means: A. opposite B. old C.reasonable D, eager E. stubhom

19 An abomizable thing is: I, of the stumaxb f. pleasant H. loud 1. hateful J. of the mountains

20 A propersity is a: A. propeliant B, scheme C. tendency D, fear F. reaction 


\section{Set 4}

- Deferse is the act of: F. winning Ci, ruaning H-planning 1. tegressing .J resisting

2. Ar rondown means A, by chance B. frequently C. farorahly D. quicsily E. very quietiy

3. A figonous leacher is: F. absent G. rightenus H. Wirect I. strict J-helpless

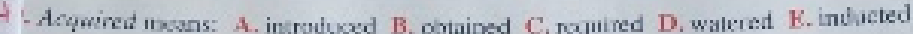

S. To burmow is tax F. blame G. loan II. tunnel I. bornow J. trap

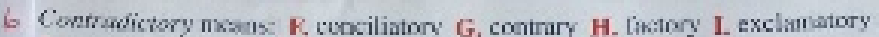

I. suference

Doficiencies are: A. assets $B$, inadequacies $C$, debss D. differences $\mathbf{B}$, defense:

8 Insomatia is: F, insight G. los of appetite H. titedness I. insbility lo sleap

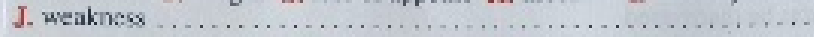

4 To devise is to: A. separate B, speak C, invent D, vivide E. double.

10. If it is rextured it is: F. teligious G, smowh H. politienl I. partemest J. educational

I1 A musarion is a: F, silenciog G. failure H. crippling I. notation .I, change

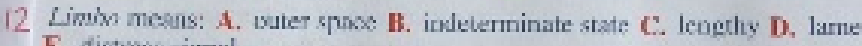
E. distress signal

13 Of terce' interest meaos: IC. broati G, timely II. Larrow I. favornble J, far-sighted

14. Ordivances are: A. unterstatemears B. repetitions C. military groups $\mathbf{D}$. movenents E. establisbet practices

15. A hatid reson is: F. vague G, thoughtless H. souod I. vain J. imaginative

16. To be asacte is to be: F, sample G, odd H. shrewd I. definite I, conservative

17. A cambonsex thing is: A. confortable B, valuable C. compact D, uld-fashioned E. unwoldy

18. An aibuistir act is: R, dranatic G, deliherate H. false I. unselfish ,I. useless

19. A sycoon is: A. a tropical stom B. a magnate C. a worker I), an animal E. a beginner 20. A fiasen is a: F. celehration G. big fure H. display I. complete failure J, riont . . 
Appendix J Example of Powerpoint questions used in VR and 'Live' Conditions

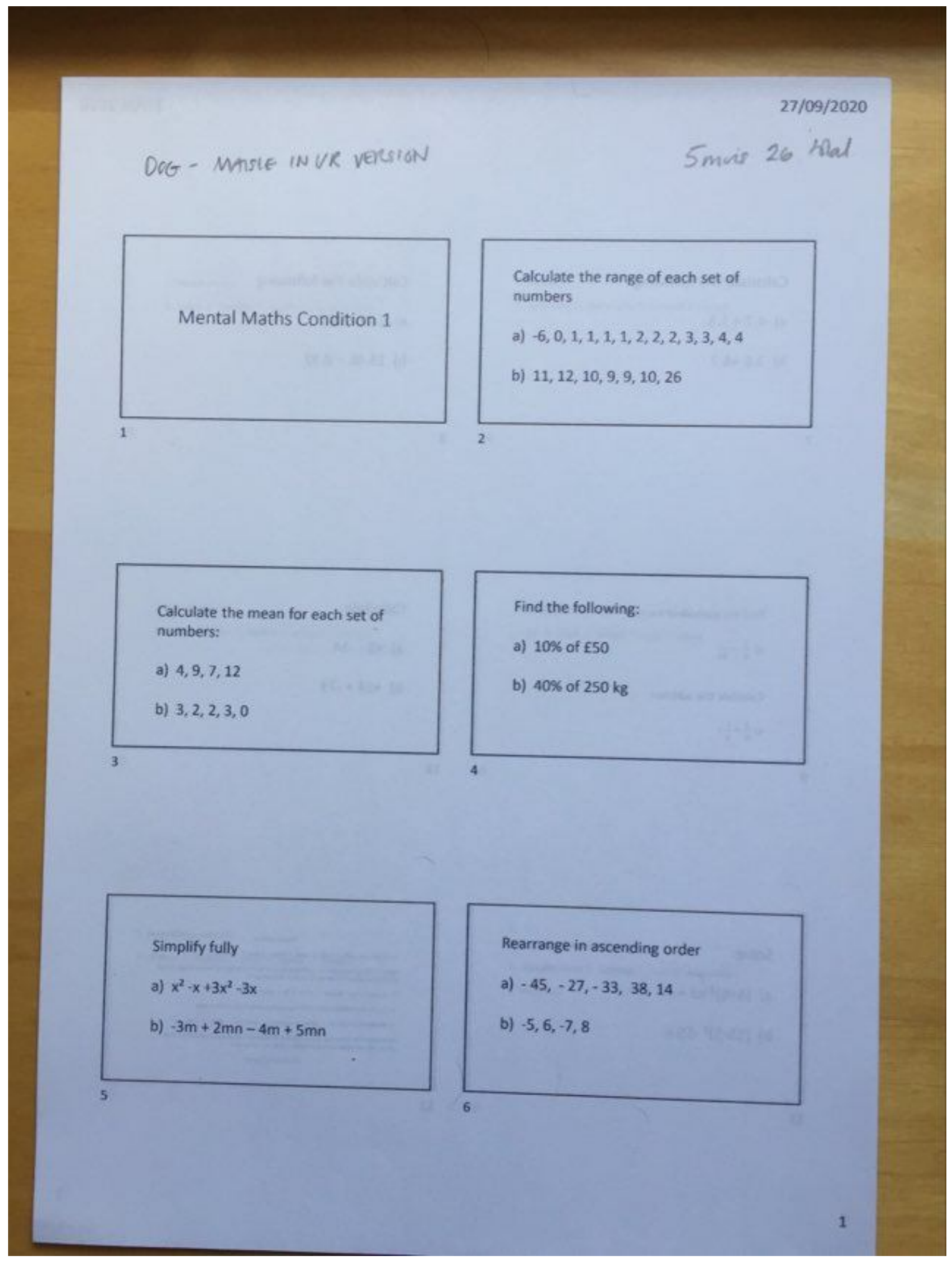



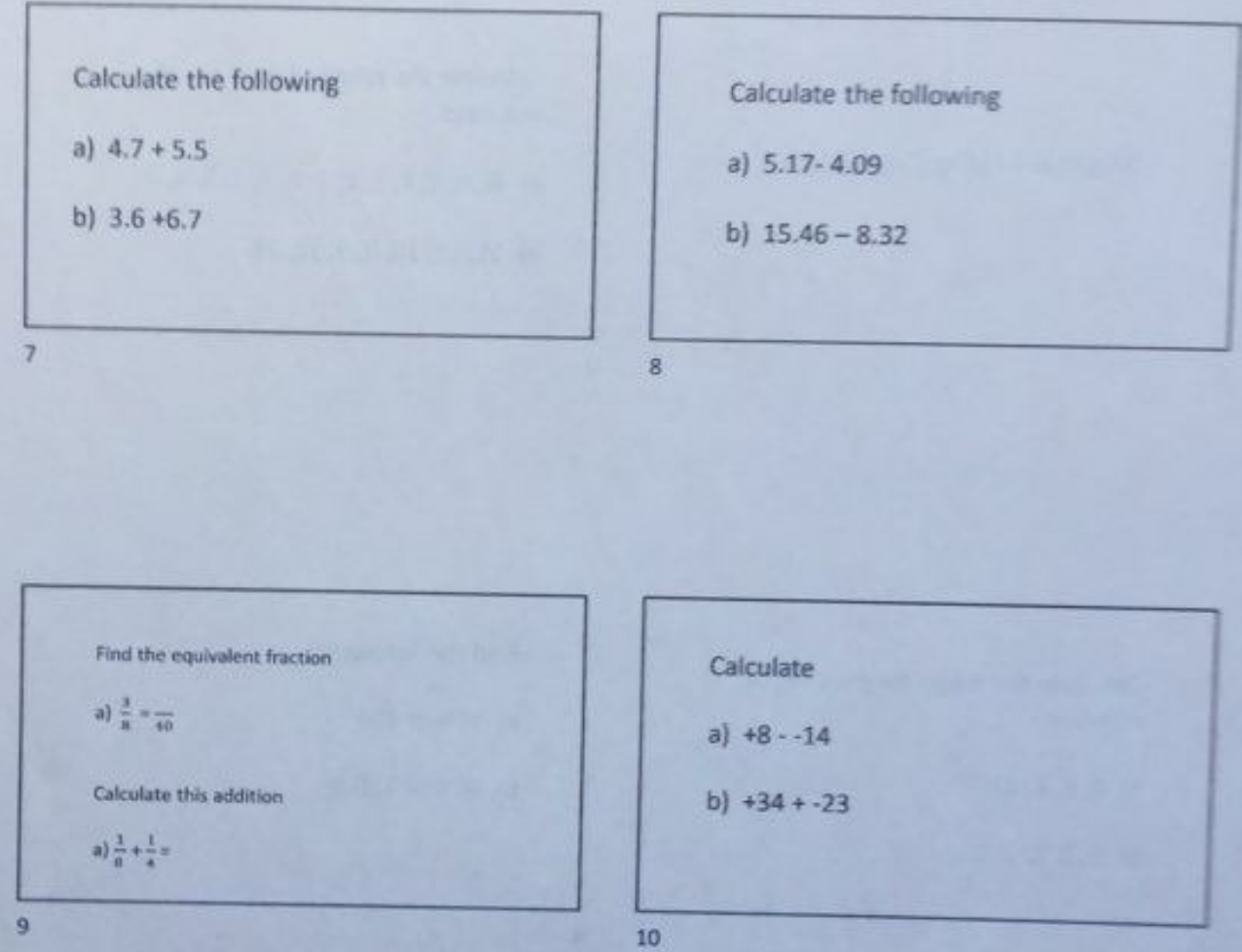

Solve:
a) $(4+6)^{2} \times 3=$
b) $(15-5)^{2}-55=$

11

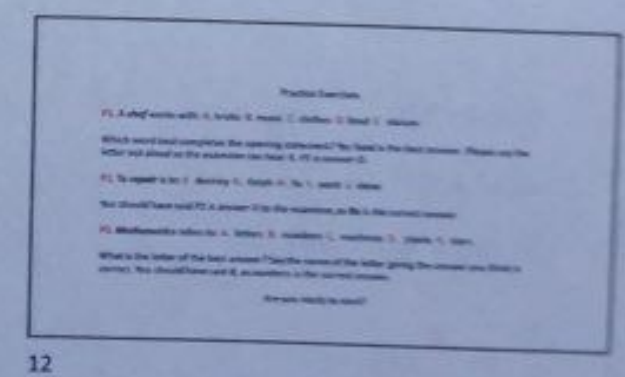




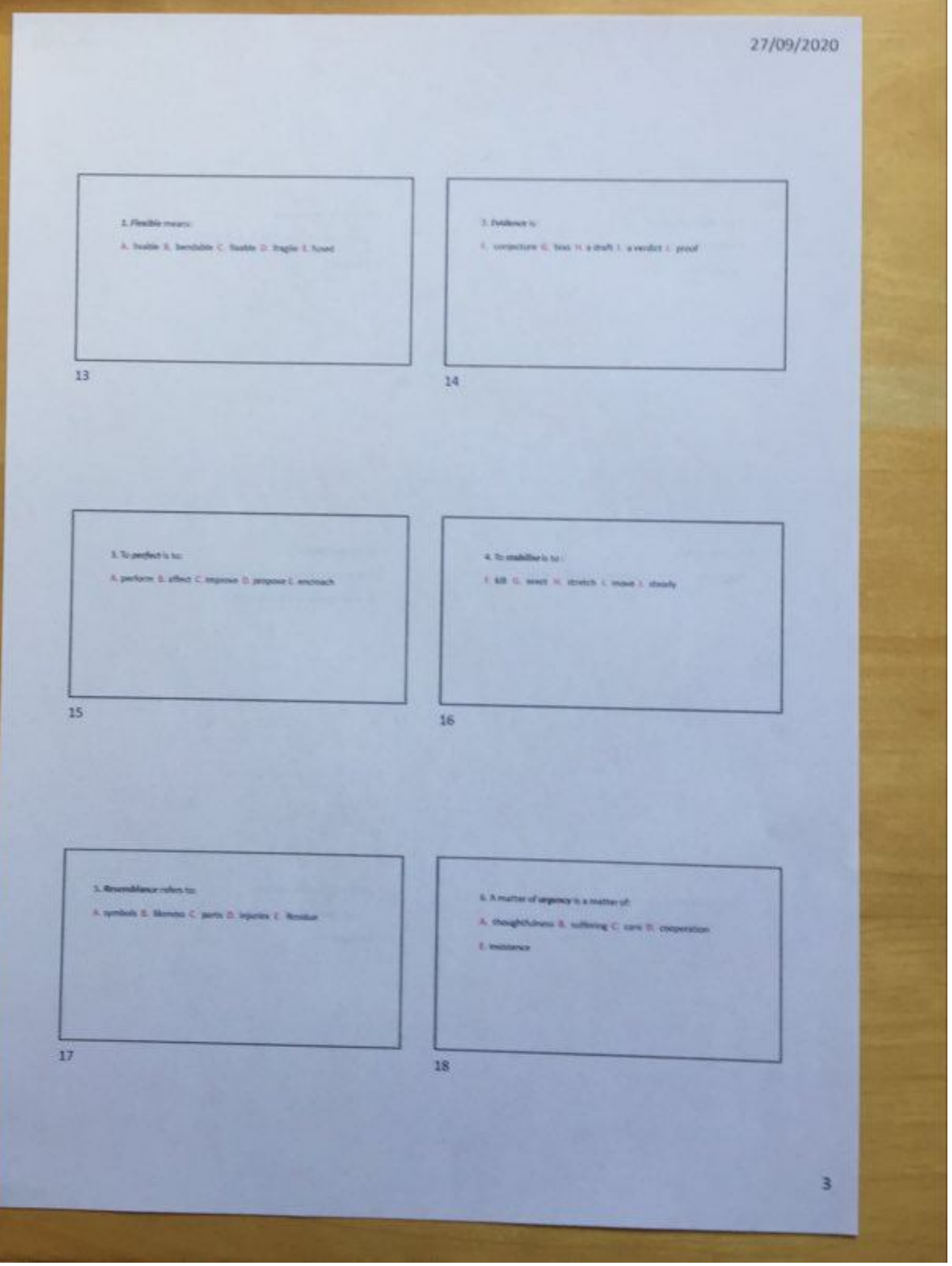




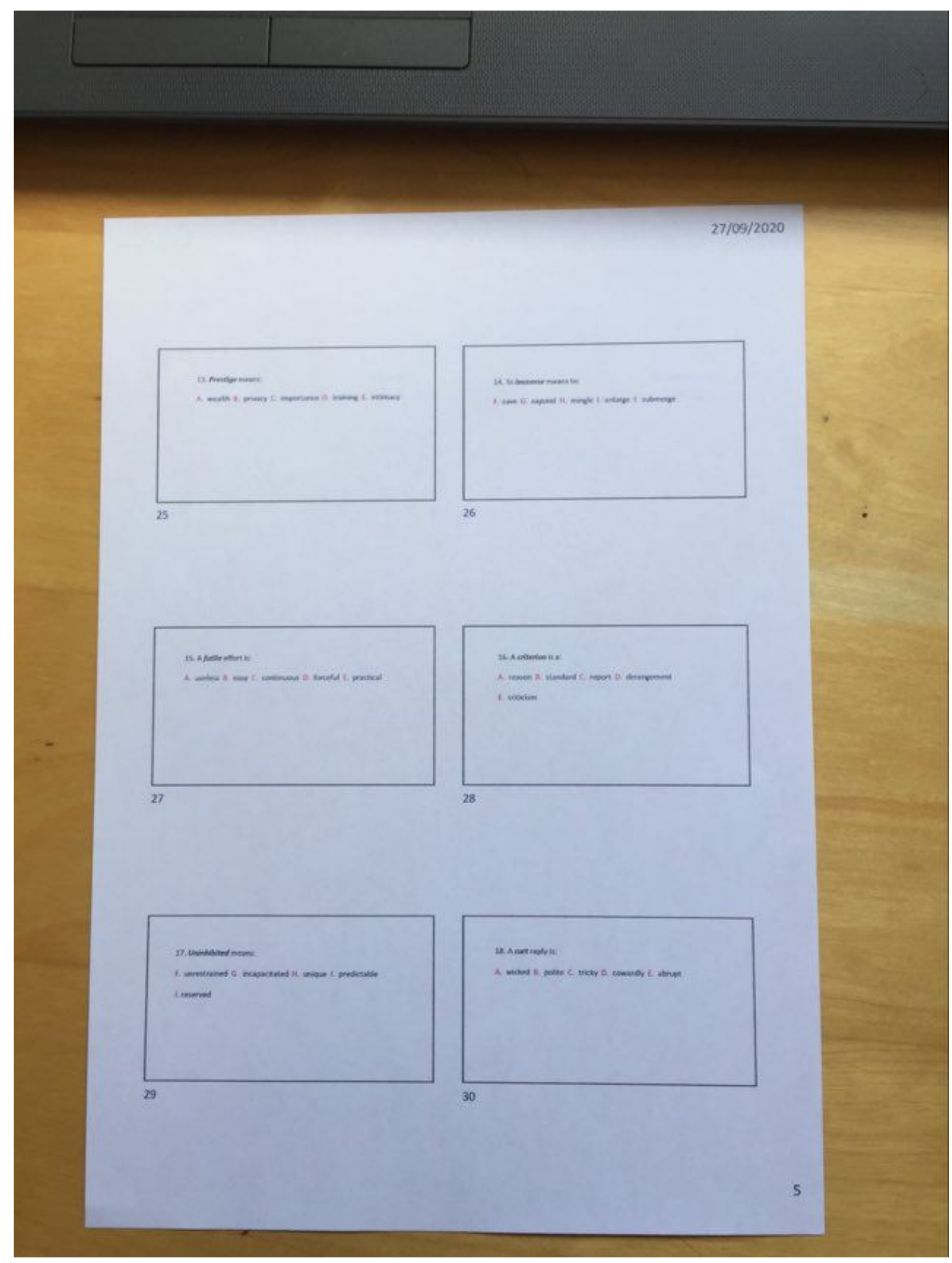




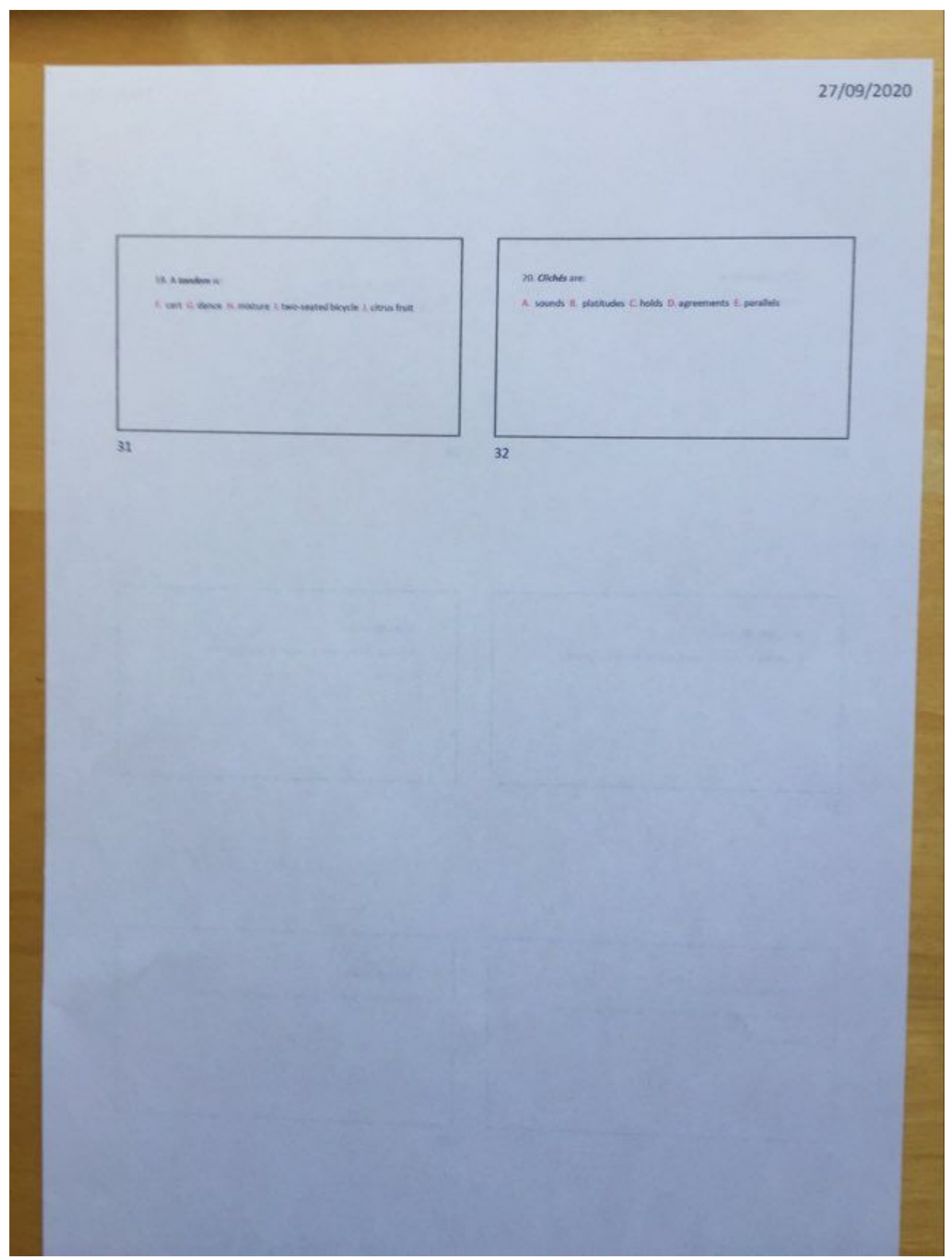


FORM RD1

RESEARCH ADMINISTRATION

APPLICATION TO REGISTER FOR A RESEARCH DEGREE

Application to register for (please delete as necessary):

Doctor of Philosophy (PhD)

This form must be typewritten, and applicants should read carefully the attached Notes of Guidance. The pagination must not be altered. Once signed, the completed form and any attachments should be forwarded to the appropriate Faculty Research Director/Research Degrees Tutor.

\section{SURNAME Oostendorp Godfrey TITLE Mrs FIRST NAME(S)Janet ADDRESS \\ 2 SOURCE OF FEES Self Funding \\ 3 QUALIFICATIONS (higher education only)}

\begin{tabular}{|l|l|l|l|l|l|}
\hline Institution & $\begin{array}{l}\text { Title of course } \\
\text { (e.g. BA Sociology) }\end{array}$ & Main subject(s) & $\begin{array}{l}\text { Classification } \\
\text { (e.g. 2i) }\end{array}$ & $\begin{array}{l}\text { Date of } \\
\text { Award }\end{array}$ & Awarding Body \\
\hline $\begin{array}{l}\text { University } \\
\text { Gloucestershire }\end{array}$ & $\begin{array}{l}\text { MEd. Supporting } \\
\text { Learning }\end{array}$ & $\begin{array}{l}\text { Working Memory } \\
\text { Identification of } \\
\text { Learning needs in } \\
\text { KS1 Classroom } \\
\text { (Diss) } \\
\text { Raising Student } \\
\text { Achievement } \\
\text { Specific Learning } \\
\text { Difficulties }\end{array}$ & 2012 & $\begin{array}{l}\text { University of } \\
\text { Gloucestershire }\end{array}$ \\
\hline Bath Spa University & $\begin{array}{l}\text { National Award } \\
\text { for SENCO }\end{array}$ & $\begin{array}{l}\text { Co-rodination of } \\
\text { Special Needs in } \\
\text { Education Setting }\end{array}$ & Pass & 2011 & Bath \\
University
\end{tabular}




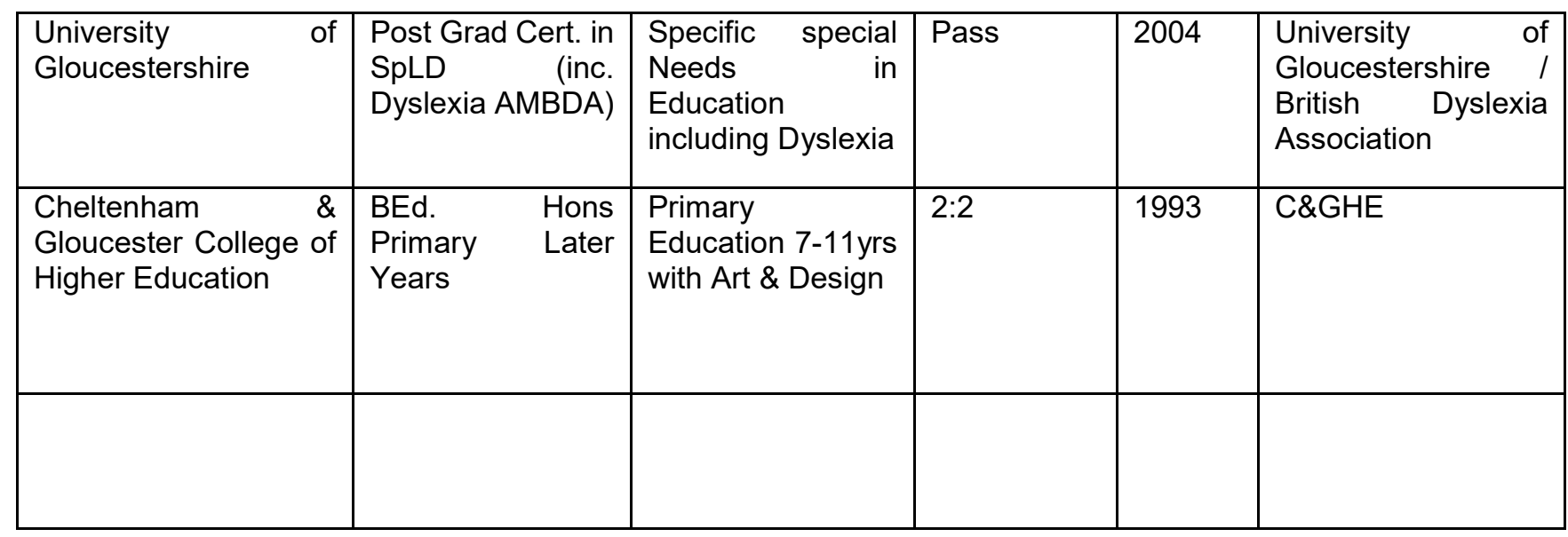

\section{PRESENT OCCUPATION AND PLACE OF WORK (if any)}

Part Time self-employed Private Tutor / Education Consultant - Catalyst Tuition / Aspire Tuition, Swindon 15), Swindon

Part Time specialist Teacher for Soundcheck Programme with Dyslexia Action (until Feb

Part Time Marker for Bath Spa University TT364 \& TT365 courses - SpLD dyslexia

5 PREVIOUS EMPLOYMENT, TRAINING OR EXPERIENCE (please give details relevant to this application, including brief details of any research or other relevant publications)

I have over twenty years' experience in primary teaching within specialist (special school) provision (Behavioural, Emotional and Social Difficulties) and mainstream primary teaching, including roles as a Special Needs Co-ordinator (SENCO) in two different mainstream primary schools. I have previously worked with the Specialist, Special Educational Needs (SSEN), Cognition and Learning Team as an advisory teacher for Wiltshire. Recently I have been a specialist teacher on a BDA and Government funded research project called "Soundcheck" which aimed for specific literacy and phonic based interventions to be given to Year 2 and 3 Primary pupils who had dyslexic or different learning profiles including memory difficulties. I also tutor, part time, as my own small business, with children with specific learning and literacy difficulties, at the weekends.I am also a member of several SEN professional associations: British Dyslexia Association, PATOSS, Dyslexia Action, National Autistic Society and am a specialist provision advisory panel member for NASEN.

I am interested in specific learning difficulties, special educational needs and cognition for learning. I achieved a first class Master's degree in 2012 and now wish to continue researching further into working memory, cognition and learning with primary aged children, in order to eventually disseminate any findings back into the world of teaching either through an education advisory service or from an academic basis. I attended University of Gloucestershire's PY210 Perception, Learning and Memory lectures to prepare myself for a more in-depth psychological view behind the theories of working memory and perception. I am very interested in the links between education and psychology of thinking, learning and teaching.I am also a dog owner who has seen her two young labradors work wonders with children with SEN in unofficial settings. I am very interested in the training of dogs as therapy dogs and as "school based dogs." 
I will require at least 2 primary schools (mainstream) and at least two special schools who work with the primary age range. These will be contacted through colleagues, SENCO networks and directly with the Head Teachers. Letters will also have to be sent to the Head of Governors seeking permission for such schools plus DBS checks required. There may well be a need for establishing a policy for the schools to work with animals directly.

I will also require the various services and advice of dog therapy charities / animal behaviour groups and in particular, therapy dog/s dogs who are already in school with their "handlers". I have a couple of potential leads - a special school and the "Dogs for Disabled" charity who were interested in my research area after a preliminary chat in August 2014.

Dogs for the Disabled have a lot of children with Autism and are looking themselves to develop the role of the dog in school. There is a possibility of some collaboration here.

I also have strong contacts at my local vet practice, including their animal behaviour team for advice, knowledge and support, should it be required.

7 FACILITIES (see Note 2. Please give details of special facilities available for the research, e.g. laboratory, database, specialist equipment etc)

I will also have access to the necessary helpful staff, labs and ICT technicians within the Psychology and CRACKLE unit at the University.

8 TRAINING IN RESEARCH METHODS (please specify which courses will be/have been taken, or indicate if exemption has been approved by the Faculty Research Director and the grounds for this)

MR401: Philosophy and Approaches to Research

MR402: Methodologies and Methods

$\mathrm{N}$

Other (please state):

Exemption: Masters in Education EDM 444 University of Gloucestershire

9 REGISTRATION (see Note 3):

Date of first enrolment:

(This will be used to calculate your maximum period of registration)

1 October 2013

Mode of study (full time or part time):

Part Time

Hours per week on average allowed for the programme:

20 Hours 
Expected duration of programme (in years):

5-7 years

RESEARCH ETHICS (see Note 4):

1. I have read and understood the University of Gloucestershire's Research Ethics: $A$ Handbook of Principles and Procedures

Signed: ...

2. My research will be conducted under the guidelines of (please tick):

$x$ The University of Gloucestershire's Handbook of Research Ethics

$\square$ The University of Gloucestershire's standard protocols in the exercise physiology laboratory

$\square$ The NHS Research Governance Framework

$\square$ The British Sociological Association

X The British Psychological Society Code of Conduct

x The British Educational Research Association

$\square$ The Market Research Society

$\square$ The Oral History Association

$\square$ Other (please state and attach copy)

3. Does this proposal contain elements that make reference to RESC mandatory?

$Y$

(Please see Research Ethics: A Handbook of Principles and Procedures Part A, section 6 , and Guidelines for Working with Children and Young People: http://insight.glos.ac.uk/researchmainpage/researchoffice/Pages/ResearchEthics.asp $\mathrm{x})$

4. Any specific issues concerning the ethics of this research that require particular comment are detailed in section 14 on page ..... [please enter page number]

Can the use of the "school therapy dog" increase attention and improve working memory functioning and attainment in children with learning difficulties?

RESEARCH QUESTIONS (see Note 5)

1. What do children regarded as having attention and memory difficulties, pay attention to when being taught in class? 
2. What are the learning implications for a child's differing attentional focus in taught lessons?

3. What are the emotional consequences for those with attention and memory difficulties being unable to focus the memory appropriately in terms of measured stress (blood pressure / heart rate) levels?

4. Would the use of a "therapy" animal ("school dog") lower measured stress for learning with those who have memory and attention problems?

5. Can interaction with a school dog during lessons, help to improve attention and memory performance of children with attention and memory difficulties?

1. Use first hand research and evidence to design and carry out an Action Research Enquiry into the effects of classroom dogs on attention, memory and stress in children with learning difficulties

2. Using this approach, to firstly determine the nature of attentional focus and associated stress levels for young children with learning difficulties in the classroom setting

3. Using this approach, to then determine the emotional and learning (attention and memory) consequences for children with learning difficulties of having an animal (dog) "working" within the classroom.

14 PROPOSED PLAN OF WORK (see Note 7; maximum 1500 words, not including bibliography and temporal plan; minimum 10pt typeface)

Aims:

- to investigate whether a "therapy" dog improves attention span and memory for children with learning difficulties

- to triangulate and consolidate links between educational pedagogical SEN research, with a psychological, cognitive based stance and animal-therapy psychology.

To the knowledge of the researcher, there have been no similar studies linking dogs to actual children's attention and learning within the UK.

\section{Contextualisation:}

The emotional benefits of having therapy dogs in classrooms for children with special educational needs has been studied, (Friesen, 2010, Haight \& Drew, 2012, Hergovich et al, 2002, Jalongo et al, 2004, Scallion, 2010) but there is still little quantitative evidence of how this assists with academic learning. Stress can be a major inhibitor to the function of working memory (Dehn, 2008,Cowan, 2005, and Henry, 2012) in children with learning difficulties.

So far, research has focused on the emotional impact on self-esteem, confidence and achievement, building on the work of Fisher \& Couzens (2014),Swift (2010) and Black (2009). Traditionally, blood pressure, usually diastolic, has been measured to show that the dogs can reduce the effects of stress on a person or child (Jalaongo et al, 2004, Allen, 2003, Somervill et al, 2008 and Bassette, 2013). It is thus proposed that this research, by using a dog in the classroom, may also find quantitative evidence for improving attention and memory. 
Working memory and attention are both core cognitive abilities for learning. Working memory refers to the temporary manipulation of information in the brain, while attention is the ability to select information (the mental "spotlight") (Purves et al, 2008). The two concepts and abilities are interrelated in that attention can direct the information that is held in working memory. Difficulties in working memory may arise in the first instance from problems with attention. The focus for this thesis will thus be, whether improvements can be achieved in both these key abilities for children, judged to have learning difficulties and so determine if improving attention can also improve children's working memory (and so their overall learning).

Memory and attention issues affect children with ADHD (Westerberg et al, 2004, Klingberg 2002, Dobler et al, 2005), Autism, Asperger's Syndrome (Cui et al, 2010), Dyslexia (Jefferies \& Everatt, 2004, Elliott \& Gibbs, 2008), Dyspraxia (DCD) (Alloway, 2006, Alloway \& Archibald, 2008), Speech and Language, reading difficulties (Swanson et al, 2010, Oakhill et al 2011. Aaron et al 2008), Dyscalculia, (Auerbach et al, 2008), Downs Syndrome, and other overall moderate and severe learning difficulties.

Class teachers are expected to know the impact of such issues and implement accurate, personal, pedagogical styles to cater for such pupils (SEND Code of Practice, 2014). Currently interventions for working memory in schools consist of meta-cognitive based programmes (mainly literacy) or expensive, computer based packages which are open to criticism (Lervag \& Hulme, 2013, Gathercole et al, 2012, Holmes \& Gathercole, 2013). Schools are accountable for measuring the impact of their interventions. The possibility of using a dog to reduce stress and enhance attention and memory of a child (and hopefully other associated aspects of academic achievement) is warranted for further research.

\section{Why work in mainstream and special schools?}

Cognitive psychology (eg., Cowan, 2010, 2005; Henry, 2012; Postle, 2006; Posner, 2012; Posner et al 2013; Ricker \& Cowan, 2014), shows that executive brain functions are implicated in working memory but also that memories are multi-sensual, emotional and environment dependent. The main implication is that any memory difficulties for children may reflect not only high-order brain functions but also emotions. Any attempt to remediate memory issues may need to address both intellectual and emotional dimensions of learning. Introducing a classroom animal may meet this requirement. If a dog was present, would attention and memory improve due to the impact on both cognitive and emotional functions for the child?

The focus sample of children would be those who either have a diagnosis or have recognised strong characteristics of autism / $A D(H) D$ (Attention Deficit (Hyperactivity) Disorder). Ideally they would be of primary school age (5-11 years), however initial investigations of local schools indicate secondary ages (11 - 19 years) may also be involved. There may be varying degrees of difficulty for any such sample of children. Children with Attention Deficit Disorder or with Autism have been shown to have differing alert states for attention and memory (Posner, 2012) and such factors will be accounted for. Allowances would also be made for differing school cultures, sizes and teaching strategies in regard to the issues of interest. Ideally comparisons between two mainstream and two special schools should yield some interesting data (see below).

\section{Research methods and strategy}


This multi- design strategy will be within the "critical realist" stance (Robson, 2002 p163) which allows for both qualitative and quantitative design, so that one construct can support the other. It will follow the action research model in that a hypothesis is created to improve the practice around them, following a clear cycle of stages (McNiff \& Whitehead, 2006,p9). The teacher-researcher role also collaborates with partners including teachers, SENCOs, pupils, parents and dog handlers, their ideas and opinions. The approach will thus be to observe children's attentional behaviour in class as well with more standardised assessments of attention and working memory abilities.

The quantitative aspect will involve comparing a baseline, pre-dog, assessment with a postintervention assessment to determine any intervention effects. The tools for these assessments will be recognised standardised tests including the ANT-C (Attentional Network Test for Children, Rueda et al, 2004). If permitted, children's heart rate and blood pressure will also be checked with a blood-pressure unit to reflect background stress levels.

Additionally, an instruction-based activity both with and without the dog present will be observed to obtain more natural indication of the children's attentional behaviour with timed observation by the researcher, with tick charts of key behaviours, digital recordings, narrative journal notes and measures of the child's "situation awareness" (Edgar \& Edgar, 2007).

The intervention will involve two parts: firstly, time with a dog in particular lessons over a week, as suggested above, and secondly, over 6 months following a group through a literacy or numeracy intervention, appropriate to the school. This is to gather data over a longer period of time and to try to establish or eliminate any "novelty" factors of having an animal in the classroom. With each, a control group, who does not interact with the dog, will also be required.

The dog trained for such therapy work will attend classes and either "settle" within the environment while being petted or actively take part in the lesson following commands from the handler. A fully trained handler will be in control of the dog and will oversee the animal's welfare.

\section{Sample Size}

Mainstream class sizes average about 30 children, while specialist provision classes average between 6 and 12 pupils. Ideally, two mainstream and two special school classes with approximately 80 children will be involved in the project.

\section{Ethical Considerations}

There are particular Ethical issues when working with young children and animals. Scallion (2010) noted that some children suffer from Cynophobia while others may be unintentionally provocative towards dogs with risk of dog bites (Jalongo, 2006). Different religions and customs may also wish to avoid contact with a dog. These issues will be addressed. The children's safety will be paramount and only an already trained therapy dog already familiar with the school, the surroundings and young children will be employed. The dogs will hopefully be accessed via charities who train such dogs (eg., Dogs for the Disabled, Pets as Therapy and Dogs helping Kids). 
The Ethical considerations will follow the "Guidelines for Involving Children and Young People in Research" from the appendix of the "Handbook of Principles and Procedures" of the University Research Degrees Committee, and the British Psychological Society Ethical Principles for conducting research with human participants, and for Psychologists working with animals, ensuring that:

- The rights of children and young persons are protected to ensure freedom from harm, physically, mentally, emotionally ethically and to ensure that they are not exploited in any way

- Schools and charities are consulted and permissions granted before any research takes place

- The children are asked for their consent to participate

- Their Parent or Carer's consent is given if it does not override the participant's wishes

- All children remain anonymous and any data destroyed after 7 years after the project has finished

- Children have the right to withdraw if they so wish, without any repercussions

- Any disclosures under the Child Protection Act are taken straight to the appropriate child protection officer in that establishment

- All participating adults are enhanced DBS cleared

- Any situations rising from the assessments are shared with appropriate teaching colleagues (it will be at the schools discretion whether to adjust children's teaching plans and to inform the parents).

- The dog is monitored at all times, receives adequate rest periods, exercise and cognitive stimulation, has a clean healthy environment and is not subjected to any pain or deliberate harm.

- The dog has been assessed as suitable for therapy, with a naturally calm, placid and sociable nature and some previous experience in therapeutic situations.

- The dog has the right to be withdrawn from any situation

- All contact between the dog and children is closely monitored for safety

\section{TEMPORAL PLAN FOR PROJECT}

The plan is to work in seven phases (see detailed timeline below). 
Phase 1 - To review literature, discuss the issues and approaches further with supervisors, possible charities, staff, schools and dog handlers

Phase 2 - Design the activities intervention with the dog handlers and teachers

Phase 3 - Do the pre-assessments of the children's attention and memory abilities using the standard tests (and if permitted, stress levels via heart rate and blood pressure) and do a preintervention observation of the children in the classroom.

Phase 4 - Introduce the dog as detailed above

Phase 5 - Repeat the standard assessments and the classroom observation

Phase 6 - Perform the analyses and discuss the results with the teachers and dog handling staff / charities

Phase $7-$ Write the thesis

\begin{tabular}{|c|c|c|}
\hline Phase 1 & $\begin{array}{l}\text { Review Literature, discuss } \\
\text { issues and gain permissions } \\
\text { of schools, possible } \\
\text { charities and handlers }\end{array}$ & April 2015 - December 2015 \\
\hline Phase 2 & $\begin{array}{l}\text { Design the interventions } \\
\text { with dog handlers and } \\
\text { teachers }\end{array}$ & January 2016 - July 2016 \\
\hline Phase 3 & $\begin{array}{l}\text { Pre-assessments and pre- } \\
\text { observations }\end{array}$ & $\begin{array}{l}\text { September } 2016 \text { - Dec } 2016 \\
\text { (Dependent on number of } \\
\text { schools) }\end{array}$ \\
\hline Phase 4 & $\begin{array}{l}\text { Introduce the dog and carry } \\
\text { out interventions }\end{array}$ & $\begin{array}{l}\text { Dec } 2016 \text { - April / May } 2017 \\
\text { (Dependent on number of } \\
\text { schools) }\end{array}$ \\
\hline Phase 5 & $\begin{array}{lr}\text { Repeat } & \text { standard } \\
\text { assessments } & \text { and } \\
\text { observations } & \end{array}$ & $\begin{array}{l}\text { Dec } 2016 \text { - July } 2017 \text { (both } \\
\text { instant and long term } \\
\text { interventions) }\end{array}$ \\
\hline Phase 6 & $\begin{array}{l}\text { Perform the Analyses and } \\
\text { discuss with teachers }\end{array}$ & July 2017 - October 2017 \\
\hline Phase 7 & Write the Thesis & $\begin{array}{l}\text { October } 2017 \text { - October } \\
\text { 2018(?) }\end{array}$ \\
\hline
\end{tabular}

\section{Bibliography}

Aaron, PG., Joshi, RM., Gooden, R., Bentum, KE. (2008) Diagnosis and treatment of reading disabilities based on the component model of reading Journal of Learning Disabilities Vol 41. No. 1 Jan/Feb 2008 pp 67-84

Allen, K. (2003) Are Pets a Healthy Pleasure? The Influence of Pets on Blood Pressure Current Directions in Psychological Science, Vol 12 No. 6 
Alloway T. (2006) Working Memory, reading and mathematical skills in children with developmental coordination disorder Journal of Experimental Child Psychology 96 (2007) 20-36

Alloway, T., Archibald, L. (2008) Working memory and learning in children with Developmental Coordintaion Disorder and Specific Language Impairment Journal of Learning Disabilities Vol 14 No. 3 May/June 2008 251-262

Auerbach, J., Gross - Tsur, V., Manor, O., Shalev, R. (2008) Emotional and Behavioural characteristics over a six year period in youths with persistant and nonpersistant Dyscalculia Journal of Learning Disabilities Vol 41. No. 3 May/June pp 263-273

Bassette, L., Taber-Doughty, T. (2013) The Effects of a Dog Reading Program on Academic Engagement Behaviour in Three Elementary Students with Emotional and Behavioural Disabilities: A Single Case Design Child Youth Care Forum (2013) 42:239-256

Black, S., (2009) Sit, Stay and Read American School Board Journal, December 2009

Cowan, N., (2010) Multiple concurrent thoughts: the meaning and developmental neuropsychology of Working Memory Developmental Neuropsychology, 35:5 447-474

Cowan, N. (2005) Working Memory Capacity New York: Psychology Press

Cui, J., Gao, D., Chen, Y., Wang, Y. (2010) Working memory in early school age children with asperger's syndrome Journal of Autism Development Disorder (2010) 40:958-967

Dehn, M. (2008) Working Memory and Academic Learning - Assessment and Intervention New Jersey: Wiley

Department for Education / Department for Health (June 2014) Special Educational Needs and disability code of practice: $0-25$ years Available at https://www.gov.uk/government/publications/send-code-of-practice-0-to-25

Dobler VB, Anker S, Gilmore J, Robertson IH, Atkinson J, Manly T (2005) Asymmetric deterioration of spatial awareness with diminishing levels of alertness in normal children and children with ADHD Journal of Child Psychology and Psychiatry 46, 1230-1248

Edgar, G., \& Edgar, H. (2007). Using Signal Detection Theory to Measure Situation Awareness: The

Technique, The Tool (QUASATM), the TEST, the Way Forward. In M. Cook, J. Noyes \& Y. Masakowski (Eds.), Decision making in complex environments (pp. 373-385). Aldershot, UK: Ashgate.

Elliott J., Gibbs S., Does Dyslexia Exist? Journal of Philosophy of Education Vol.42 No.3-4 pp 475 $-491$

Fisher, B., Couzens, M., (2014) The BaRk (Building Reading Confidence for Kids) canine assisted reading program: One child's experience Literacy Learning: The Middle Years Vol 22 No. 1

Friesen, L. (2010) Exploring animal assisted programs with children in school and therapeutic contexts Early Childhood Education Journal 37:261-267

Gathercole S.E, Dunning, D., Holmes, J. Cogmed Training: Let's be realistic about intervention research Journal of Applied Research in Memory and Cognition 1 (2012) 210-203

Holmes, J. Gathercole S.E., (2013) Taking working memory training from the laboratory into schools Educational Psychology: an International Journal of Experimental Educational Psychology available at DOI:10.1080/01443410.2013.797338

Height, D, Drew, U (2012) Will the use of therapy dogs provide greater motivation for multiply disabled children? Dissertation Abstracts International: Section B: The Sciences and Engineering, Vol $72(8-B)$ 
Henry, L. (2012) The Development of Working Memory in Children London: Sage

Hergovich, A., Manshi, B., Semmler, G., Zeiglmayer, V.(2002) The effects of the presence of a dog in the classroom Anthrozoos, 15 (1)

Jalongo, M. (2006) On behalf of children Early Childhood Education Journal Vol 33. No. 5

Jalongo, M., Astorino, T., Bomboy, N. (2004) Canine Visitors: the influence of therapy dogs on young children's learning and well-being in classrooms and hospitals Early Childhood Education Journal, Vol 32, No. 1 August 2004

Jefferies, S. Everatt, J. (2004) Working memory: Its role in dyslexia and other specific difficulties Dyslexia 10: 196-214

Klingberg, T., Forssberg, H., Westerberg, H. (2002) Training of Working Memory in children with $A D H D$ Journal of Clinical and Experimental Neuropsychology 2002 Vol. 24 No. 6

Lervag, M., Hulme, C., (2013) Is working memory training effective? A meta-analytic review Developmental Psychology 2013 Vol 49 No.2 270-291

McNiff, J., Whitehead, J., (2006) All you need to know about action research London: Sage

Oakhill, J., Yuill, N., Garnham, A. (2011) The differential relations between verbal, numerical and spatial working memory abilities and children's reading comprehension International Electronic journal of Elementary Education 20114 (1) 83-106

Posner, M. (2012) Attention in a social world Oxford: Oxford University Press

Posner, M., Rothbart, M., Tang, Y.,(2013) Developing self-regulation in early childhood Trends Neuroscience Education 20132 (3-4) 107-110

Postle (2006) Working Memory as an emergent property of the mind and brain Neuroscience 139 (2006) 23-38

Purves, D., Augustine, G., Fitspatrick, D., Hall, W., LaMantia, A-S., McNamara, J., White,L. (2007) Neuroscience $4^{\text {th }}$ Edition New York: Sinauer Associates Inc.

Ricker, T., Cowan N., (2014) Differences between presentation methods in working memory procedures: A matter of working memory consolidation Journal of Experimental Psychology, Learning, Memory and Cognition Mar 2014, Vol 40, Issue 2 p 417-428

Robson, C., (2002) Real World Research (second edition) Oxford: Blackwell Publishing

Scallion, S., (2010) Therapy dogs find a role in school Principal, September / October 2010

Somervill, J., Swanson, A., Robertson, R., Arnett, M., MacLin, O. (2009) Handling a dog by children with Attention Deficit/Hyperactivity Disorder: Calming or exciting? North American Journal of Psychology, 2009 Vol 11, No.1 111-120

Somervill, J., Kruglikova, Y., Robertson, R., Hanson, L., MacLin, O., (2008) Physiological Responses by College Students to a Dog and a Cat: Implications for Pet Therapy North American Journal of Psychology, 2008 Vol 10, No. 3 519-528

Swanson, H.L., Kehler, P. Jerman, O., (2010) Working Memory, strategy knowledge and strategy instructions in children with reading disabilities Journal of Learning disabilities 43 (1) 24-47

Swift, C, (2010) AAT and Reading Education Assistance Dog (READ) program as perceived by volunteer READ facilitators: a National Study Dissertation Abstracts International Section A, Vol 71 (3-A) 2010 pp 822

University of Gloucestershire (2008) Research Ethics: A handbook of principles and procedures: Appendix 3, Guidelines for involving children and young people in research, Appendix 5, British 
Psychological Society's Code of Conduct available at http://insight.glos.ac.uk/researchdevelopment/pages/researchethics.aspx

Westerberg, H., Hirvikoski, T., Fossberg, H., Klingberg, T. (2004) Brief Report: Visuo-spatial Working Memory Span: a sensitive measure of cognitive deficits in children with ADHD Child Neuropsychology, Vol 10, No.3 pp155 - 161

\section{Possible Assessments}

Martin, N. ( 2006) Test of Visual Perceptual Skills available at http://www.annarbor.co.uk/index.php?main page=index\&cPath=250 43587

Martin, N., Brownell, R., ( 2005) Test of Auditory Perceptual Skills available at http://www.annarbor.co.uk/index.php?main page=product info\&products id $=552$

Pakiam Alloway, T., (2007) The Automated Working Memory Assessment available at http://www.pearsonclinical.co.uk/Psychology/ChildCognitionNeuropsychologyandLangua ge/ChildMemory/AutomatedWorkingMemoryAssessment(AWMA)/AutomatedWorkingMe moryAssessment(AWMA).aspx

Reynolds, C., Biglar, E. (2007) Test for Memory and Learning available at http://www.annarbor.co.uk/index.php?main page=index\&cPath=253 425437

Rueda, M. R., Fan, J., McCandlis, B. D., Halparin, J. D., Gruber, D. B., Pappert Lercari, L., \& Posner, M.I (2004) Attentional Network Test for Children (2004) available at http://www.millisecond.com/download/library/ANT/

\section{Charity contacts}

Dogs Helping kids, available at http://www.puppygurus.co.uk/dhk/index.php

Dogs for the Disabled, available at http://www.dogsforthedisabled.org/

Pets as Therapy, available at https://www.petsastherapy.org/

15 FIRST SUPERVISOR (See Note 7; include name, qualifications, post held, School. A brief CV of no more than 2 pages should be attached for any proposed supervisor not previously approved by the URDC.)

Dr. Graham Edgar, Reader in Psychology, Natural \& Social Sciences

Experience of supervision of research degree candidates:

Currently supervising* $\quad 3 \mathrm{PhD} \ldots$... EdD .... DBA .....MPhil ......MRes 2 MA/MSc by Research

Previous successful completions $9 \mathrm{PhD} \ldots . \mathrm{EdD} \quad$.......DBA .... MPhil ......MRes ....MA/MSc by Research

\section{${ }^{*}$ total supervisions should include the current candidate}

Please outline research supervisor training that has been undertaken (including the date of any courses attended - see Note 8): 
SECOND SUPERVISOR(S) (See Note 7; include name, qualifications, post held, department \& institution. A brief $\mathrm{CV}$ of no more than 2 pages should be attached for any proposed supervisor not previously approved by the URDC. Please provide a brief rationale for the appointment of an external supervisor.)

Professor Di Catherwood, Professor of Psychology, Natural \& Social Sciences

Experience of supervision of research degree candidates:

Currently supervising* $3 \mathrm{PhD} \quad \ldots . \mathrm{EdD} \quad \ldots . . \mathrm{DBA} \ldots . . \mathrm{MPhil} \quad \ldots . . . \mathrm{MRes} 2 \mathrm{MA} / \mathrm{MSc}$ by Research

Previous successful completions $11 . \mathrm{PhD} \ldots$. EdD ......DBA .... MPhil .......MRes 2 MA/MSc by Research

* total supervisions should include the current candidate

Please outline research supervisor training that has been undertaken (including the date of any courses attended - see Note 8): VARIOUS COURSES eg.,: SUPERVISOR FORUM 10/7/13

Dr. Kerry Hughes, Senior Tutor and Lecturer in Psychological Sciences

Experience of supervision of research degree candidates:

Currently supervising* PhD $\quad \ldots . . \mathrm{EdD}$ .. DBA .....MPhil .....MRes ...MA/MSc by

Research

Previous successful completions PhD $\quad \ldots . . \mathrm{EdD}$ DBA MPhil ...MRes MA/MSc by

Research

\section{* total supervisions should include the current candidate}

Please outline research supervisor training that has been undertaken (including the date of any courses attended - see Note 8):

16 ADVISOR(S) (name, qualifications, post held and place of work)

\section{STATEMENT BY THE APPLICANT}

I wish to apply for registration for the degree of on the basis of the proposals given in this application. I confirm that the particulars given are correct and I understand that, except with specific permission, I must 
prepare and defend my thesis in English. I agree to abide by the regulations, and the Research Ethics Principles and Procedures of the University.

Signed .... $\quad$.... Date ............03/04/15

We support this application and believe that the candidate has the potential to complete successfully the programme of work proposed. We recommend that the applicant be registered for the above research degree.

Signed

Date 30 April, 2015

First Supervisor

Signed ....

Date 30 April, 2015

Second Supervisor

Signed

Date 12 May 2015

Second Supervisor(s)

\section{RECOMMENDATION BY THE FACULTY RESEARCH DIRECTOR/FACULTY RESEARCH DEGREES TUTOR/DIRECTOR OF STUDIES (RESEARCH DEGREES)}

I support this application for registration and confirm that :

a) the resources are available for the proposed programme of research; 
b) adequate and appropriate arrangements for supervision have been made and any internal supervisor who has not supervised to successful completion has followed or will follow the University's Research Supervisors' Support Programme (see Note 8);

c) all parties are aware of and have agreed to operate by the regulations and the Research Ethics Principles and Procedures of the University. 
RD1 FORM COMMENT PROFORMA POST-FRDC

\begin{tabular}{|l|l|}
\hline Student Name & Janet Oostendorp-Godfrey \\
\hline
\end{tabular}

Please submit this with the corrections made noting your actions in the space provided below, and e-mail to Helen Wright and Mark De Ste Croix.

\begin{tabular}{|c|c|c|}
\hline $\begin{array}{l}\text { Category of } \\
\text { feedback }\end{array}$ & Comments & Action \\
\hline $\begin{array}{l}\text { Substantive } \\
\text { issues }\end{array}$ & $\begin{array}{l}\text { The RQs are well articulated, } \\
\text { although question } 3 \text { is perhaps } \\
\text { problematic as there could be a } \\
\text { further explanation as to why } \\
\text { emotion is related to blood } \\
\text { pressure. The hypothesis } \\
\text { appears to be that stress can be } \\
\text { determined from blood } \\
\text { pressure, but not entirely clear } \\
\text { what is the causal factor. The } \\
\text { order of questions could run } \\
\text { from } 2,1,3,4,5 \text { moving from the } \\
\text { general to the more specific. As } \\
\text { the overarching question is that } \\
\text { stated is } 2 \text {, and the specific } \\
\text { focus of the study is question } 1 . \\
\text { The committee felt that the } \\
\text { biggest issue with the study at } \\
\text { present is that there is not } \\
\text { sufficient consideration of how } \\
\text { the various streams of data will } \\
\text { be analysed together. There } \\
\text { would appear to be physical } \\
\text { measurements (blood } \\
\text { pressure), educational data and } \\
\text { observational data. This will be } \\
\text { then acted on in an Action } \\
\text { Research approach, which } \\
\text { implies on-going analysis and } \\
\text { that such an analysis will be } \\
\text { available early in the project. } \\
\text { Such an analytical framework is } \\
\text { not a trivial task and the results } \\
\text { of the project could hinge on it, } \\
\text { so some suggestions of how this } \\
\text { will be integrated, weightings } \\
\text { and priorities would be helpful. }\end{array}$ & \\
\hline $\begin{array}{l}\text { Correction to } \\
\text { technical } \\
\text { presentation of } \\
\text { form }\end{array}$ & & \\
\hline
\end{tabular}




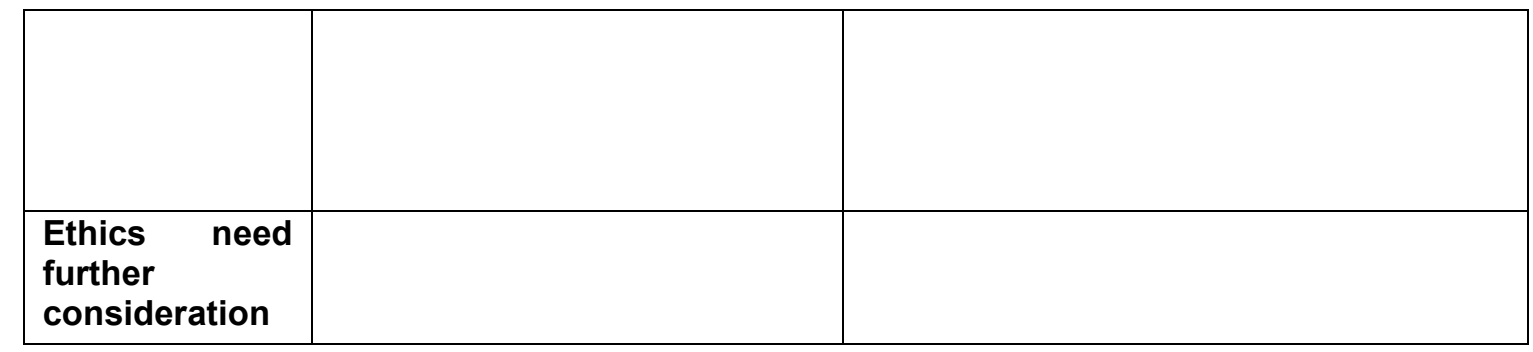

Decision by Committee: Approved Chairs Action

FRD Further comments: Committee felt that this was a well developed RD1 that will significantly contribute new knowledge to the field of study.

Date: $22 / 06 / 2015$

From: XXXXX

Sent: 23

June

2015

To: $X X X X X X X$

Subject: RD1 Feedback

Dear Janet

Your RD1 was considered at the recent Faculty Research Degrees Committee and I am pleased to tell you that it was approved on chair's action subject to some minor changes, which are listed on the action sheet attached. Please arrange to discuss the changes with your supervisors as soon as possible.

Please forward your amended RD1 and the completed action sheet electronically to myself and XXXX (with electronic signatures) to sign off.

Regards

XXXX

Administrator (Assignment Room)

Academic Registry

University of Gloucestershire

Room LC024/JP012, Oxstalls Campus

Oxstalls Lane, Gloucester, GL2 9HW

Tel: 01242715131 


\section{Appendix L Permissions from Charity}

Swindon

Wilts

Therapy Dogs Nationwide

$31 / 05 / 16$

Dear Ms. Chairperson,

Please may I have the charity's permission to go into the schools in the South West (namely Gloucestershire and Wiltshire) and work with the Therapy Dogs Nationwide handlers and their dogs. I am trying to investigate the use of "therapy dogs" in raising the attention and hopefully, the working memory capacity of children in schools. Although I have been offered other noncharity dogs to use, I am very aware that safety is paramount and so I only want to use dogs which have been "assessed" as being suitable, both for the benefits of the dog and of the children. This is also one of the conditions of the PhD permission.

As reading is often seen as using high quantities of working, short and long term memory, it would be interesting to see how the schools and children perceive the success rates of the dogs, plus how, if possible, dogs could also contribute to attention and working memory for maths and other subject areas. This would require before and after data, plus school interventions for literacy and numeracy, so this will mean that I will need to develop longer term relationships with the school, dogs and handlers, both in mainstream and special schools.

Ultimately I would like to see more dogs in schools, as, in just using my two in an unofficial capacity to see how this project could work, I have already seen positive changes in anxiety levels and attainment in the pupils I tutor. I would also be interested in how I could have my two dogs officially assessed for possible future work in schools with Therapy Dogs Nationwide.

Please find enclosed copies of the following documents:

- My PhD proposal Form

- Permission from the FRDC

- Letter from my University supervisors

- My current CV

Many thanks for your help and support,

Yours sincerely

Janet Oostendorp 


\title{
Answer from Chairperson
}

\author{
$03 / 05 / 2016$
}

Hi (Chairperson's name) Thank you for the add. I wonder if you can help me? I've got nowhere with PAT and trying to find a local co-ordinator for my area! Do you have dogs in schools in the Wiltshire Swindon area at all? I am trying to do a PhD about school dogs and increasing attention for memory and would like to see dogs in schools to check feasibility and suitability for my study. Although PAT are talking slowly to me - I've had to give my whole Phd outline and CV just to ask for their local co-ordinator's name which I still haven't received. Dogs for Good are considering letting me work with one of their schools, but I would like a wider scope of other charities in the UK. Also, and on another note, how can I get my dogs assessed? I can't use them in my research of course but after I finally finish my studies I would like to take them into school / Uni - thanks

\section{$17 / 5 / 2016$}

it is wonderful of our volunteers to want to help you.... but will are talking about this this evening....can you wait to contact volunteers after our meeting. Chairperson

$30 / 05 / 2016$

$\mathrm{Hi}$ - is there an official address for Therapy dogs nationwide? I have a letter from my PhD supervisors and was wondering where to send it. Would I need to address it to you? thank you

Yes please send it to me : - (address given)

$10 / 06 / 2016$

Hi - I hope all is going well and that you are happy for me to go into schools before the summer holidays start. 
Hello Janet....recovering, been a bit ill....sorry I didn't get back to you. You must have you dogs assessed by us and be a registered volunteer.... have no objection to you school work as long as the dogs pass our test and the correct paper work has been completed. Love Chairperson 
Inclusion co-ordinator /SENCO

$14 / 06 / 2016$

Dear MrsXXXXX,

We are two of the university supervisors for the doctoral research project of Janet Oostendorp Godfrey. We are contacting you to show our full support and complete endorsement for her project on the value of school therapy dogs as support for the learning of children with specific learning difficulties. This is a project that is likely to provide results of great value to those with an interest in the education of children with special needs and to organizations that offer school dog services for this purpose.

Janet is uniquely qualified to conduct such a study. She has over 20 years of experience as a Special Needs Teacher, Co-ordinator and tutor, is a member of several SEN professional associations and has already achieved an excellent Masters degree in this field of Special Needs. Without any doubt she would perform her studies in a professional and sensitive manner that respected the children and the teaching and dog-handling staff and she would work collaboratively and openly with the dog therapy organizations and schools.

The children's records would of course be kept confidentially according to the regulations and ethical requirements of the British Psychological Society. The general results of the project would be made available to the organizations and schools involved. The project has the full ethical approval of the university and the supervisory team.

We do hope that you are able to offer Janet support in this highly worthwhile project. If you have any queries, Janet would be very happy to answer them or please contact either of us.

Yours sincerely,

\section{Dr. Graham Edgar and Professor Dianne Catherwood}

School of Natural and Social Sciences, University of Gloucestershire, Francis Close Hall, University of Gloucestershire, Cheltenham, GL50 4AZ 
Hello Janet

Here's the info as promised.

Our point of contact is, who is the Inclusion Coordinator (SENCO in old money!). Her email is xxxx If you email xxx perhaps you could copy me on it?

The school address is: $\mathrm{xxxxxxx}$

Matt and I go into school every Monday from 1400 to 1500 . For the rest of this term that means we should be in school on Mondays from 13 June to 18 July inclusive (though there may be the odd exam/sports day/end of term jolly which gets in the way).

Since Matt and I started in 2012 the method of selecting the children the school asks us to see has varied, although it has essentially been Y3 throughout. We started off with a small group of children who needed support with their reading. Recently school has asked us to see all those Y3 children who want to read to Matt, regardless of reading ability; having worked through the year group we then give extra turns to those who need more support with reading. Personally I think it's better to concentrate on the children who need support, but it's up to the school how they use the resource.

Some parents refuse permission because their child is not keen on dogs. Others give permission BECAUSE their child is not keen on dogs. Obviously we treat these with caution, but so far they have just been a bit wary and have soon warmed up when they see that Matt is not fierce! We haven't had a child who is really phobic - obviously we'd hope that their parents wouldn't put them forward.

If you arrange with $\mathrm{xxx}$ to observe a session I'm sure we could then stay on past the hour for a chat if you like. Alternatively, we could go into school at say 1330 for a chat beforehand.

Looking forward to meeting and working with you

$\operatorname{XXX}$ 


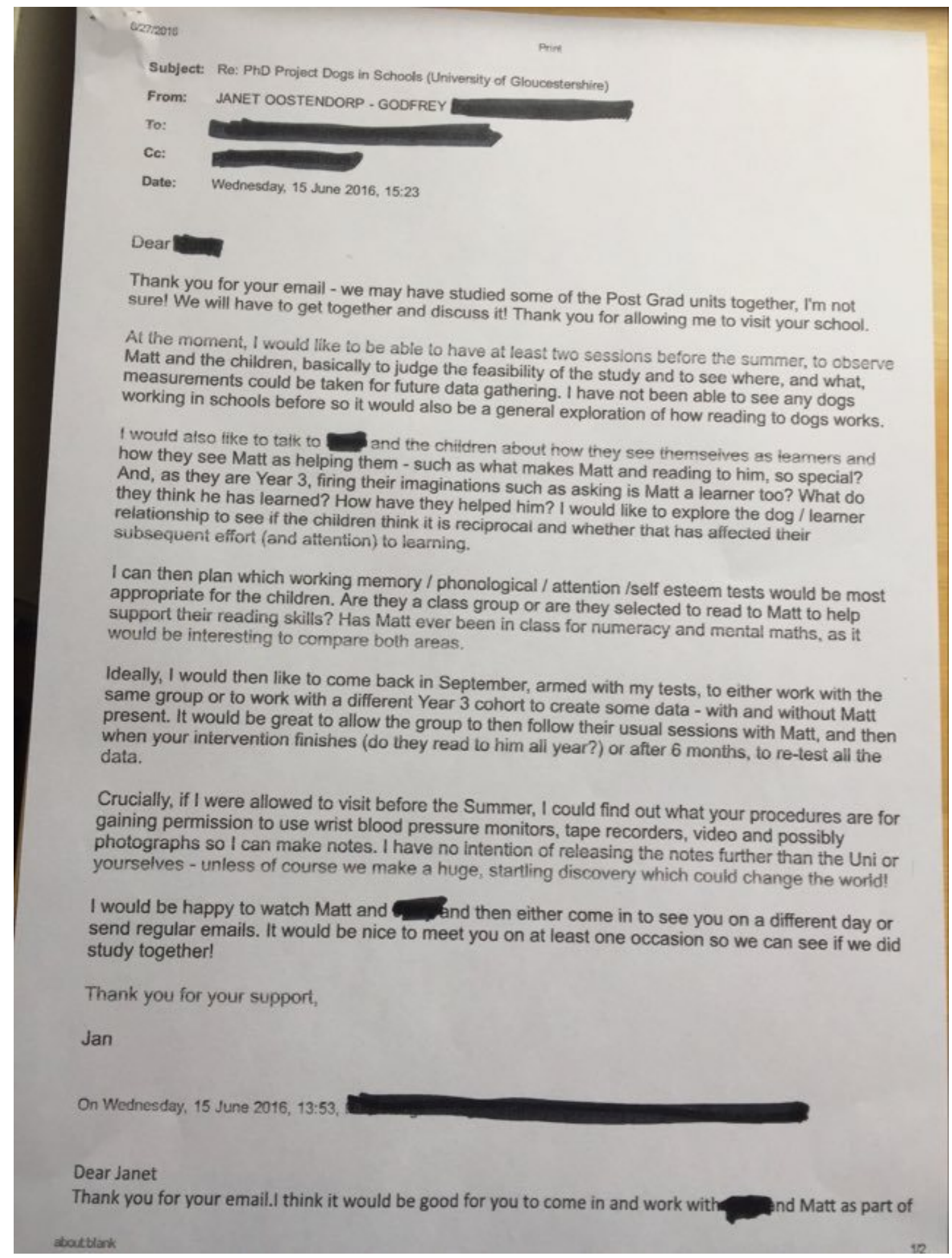


your PHD project. What would your involvement consist of? Will it be now or starting in September?The contact Jerry via email and currently Matt visits on a Monday and I do not work on a Monday-l tend to you coming in to see me on a different day or possibly hapervise the session. We could get round this by inclusion Co-ordinator

Looking at your CV did we do our Inclusive education Post grad together?

From: JANET OOSTENDORP - GODFREY

Sent: 14 June 2016 08:34:58

To:

Cc: 1

Subject: PhD Project Dogs in Schools (University of Gloucestershire)

Dear

I have been given your school's name as you have a regular visiting dog from Therapy Dogs Nationwide with his handler / owner

Would it be possible to come and observe Jerry and Matt working with your children as part of my project? I am trying to look at working memory and how a dog can affect stress levels to hopefully inprove attention of primary aged children in literacy and maths. Would your school be interested

I am a qualified, specialist SEN teacher (also dyslexia specialist AMBDA and past SENCO) who has worked in both special and mainstream schools and I already have a clear DBS which is updated annually. I am studying at the University of Gloucestershire and although I am originally from the Education department I am studying with the Psychology school at Francis Close Hall. also have sought and gained permission from from Therapy Dogs international.

I have attached my RD1 form (my study outline) a letter from my supervisors and my CV.

Thank you,

Janet Oostendorp

June 2016 - Proposed Plan to be discussed with SENCO / Inclusion Officer 
(June 2016 Plan for discussion Original RD1 and CV already sent to school)

Situation

- $\mathrm{XXXXX}$ is a mainstream school in XXXXX with currently 240 children on roll.

- I would be collecting data from a group of Year 3 pupils - usually there are 2 classes per year - approx. 60 children. Both classes take it in turns to weekly visit XXX and Matt, his dog (Border Terrier.)

- XXX and Matt come in once a week to hear readers, "Read to Dogs Scheme"

- Each reading session lasts one hour -2-3pm on Monday afternoons in the music room.

- Each child reads for 5 mins at a time which means that approximately 6 children can be heard in each reading session. - In theory it would take 5 weeks for each class to all be heard, creating 10 weeks for all Year 3 in total. Do not want to rush the reading!

- Bearing in mind that school holidays (ie half terms and Christmas) are also counted, in order for the children to have read at least twice, if not 3 times to Matt, I will probably be involved until Easter Holidays (April) Due to any delays, I may have to be involved all year!

- To get the before and after 6 months data, I will also need to be able to work with the children on a 1:1 basis and may require a couple of weeks to get through all the children

- It would be easier to try to get the non-dog data than the with dog data.

- Tests will need to be quick to administer due to time pressures from class timetable

\begin{tabular}{|l|l|l|}
\hline Activities & Notes... & Data \\
\hline $\begin{array}{l}\text { Wrist worn blood pressure } \\
\text { monitors when with or } \\
\text { without dog }\end{array}$ & $\begin{array}{l}\text { Borrow from Uni - will } \\
\text { probably only need 5-6 at any } \\
\text { one time. }\end{array}$ & $\begin{array}{l}\text { Need to teach children to } \\
\text { "read" their data to "help" - } \\
\text { will BP change with and } \\
\text { without dog? }\end{array}$ \\
\hline $\begin{array}{l}2 \times \text { Working Memory Auditory } \\
\text { Tests with / without dog } \\
\text { present before and after 6 } \\
\text { months }\end{array}$ & $\begin{array}{l}\text { Test of Auditory Processing } \\
\text { Skills (TAPS -3) Selected tests } \\
\text { Test of Memory and Learning } \\
\text { (TOMAL-2) Selected tests }\end{array}$ & $\begin{array}{l}\text { (Wots of data } \\
\text { (Will need a gap timewise } \\
\text { between dog present and } \\
\text { not) }\end{array}$ \\
\hline $\begin{array}{l}2 \times \text { Working Memory Visual } \\
\text { Tests with / without dog } \\
\text { present before and after 6 } \\
\text { months }\end{array}$ & $\begin{array}{l}\text { Test of Visual Perceptual Skills } \\
\text { (TVPS-3) Selected tests } \\
\text { Test of Memory and Learning } \\
\text { (TOMAL-2) Selected tests }\end{array}$ & $\begin{array}{l}\text { 4lots of data } \\
\text { (Will need a gap timewise } \\
\text { between dog present and } \\
\text { not) }\end{array}$ \\
\hline
\end{tabular}




\begin{tabular}{|c|c|c|}
\hline $\begin{array}{l}\text { Reading and Comprehension } \\
\text { results before and after } 6 \\
\text { months }\end{array}$ & $\begin{array}{l}\text { Use School's own - York } \\
\text { Assessment of Reading } \\
\text { Comprehension (YARC?) }\end{array}$ & $\begin{array}{l}2 \text { lots of data (possibly with } \\
\text { some internal diagnosis) }\end{array}$ \\
\hline $\begin{array}{l}\text { Self Esteem questionnaires } \\
\text { before and after } 6 \text { months }\end{array}$ & $\begin{array}{l}\text { Need to develop using "child } \\
\text { friendly" language - Likert } \\
\text { scales with pictures }\end{array}$ & $\begin{array}{l}2 \text { lots of data - may also have } \\
\text { to ask questions during } \\
\text { sessions as children are young } \\
\text { and perhaps cannot } \\
\text { remember things through to } \\
6 \text { months }\end{array}$ \\
\hline "Matt's" Open Journal & $\begin{array}{l}\text { For the children to record any } \\
\text { feelings or comments about } \\
\text { their reading to Matt each } \\
\text { week (voluntary) or for Matt } \\
\text { to comment back! }\end{array}$ & $\begin{array}{l}\text { Data from the children - can } \\
\text { be transcribed by adults } \\
\text { (Or comments from "Matt!") }\end{array}$ \\
\hline Questionnaires to teachers & & $\begin{array}{l}\text { Find out teacher's opinions of } \\
\text { the read to dog's scheme }\end{array}$ \\
\hline Questionnaires to Parents? & & $\begin{array}{l}\text { Have parents any feelings } \\
\text { towards their child's memory } \\
\text { for reading? }\end{array}$ \\
\hline $\begin{array}{l}\text { Questionnaire to handlers / } \\
\text { volunteers } \\
\text { (Could also try internet } \\
\text { polling) }\end{array}$ & & $\begin{array}{l}\text { Questionnaire for other } \\
\text { handlers / volunteers } \\
\text { including XXX as to their } \\
\text { opinions of the effectiveness } \\
\text { of the scheme - what stories } \\
\text { / accounts have they had? }\end{array}$ \\
\hline $\begin{array}{l}2 \times \text { Maths Mental activities - } \\
\text { with without dog present } \\
\text { before and after } 6 \text { months }\end{array}$ & $\begin{array}{l}\text { Use times tables from Year } 3 \\
\text { Maths curriculum or mentally } \\
\text { subtract and add number to } \\
\text { larger numbers or count } \\
\text { forwards / backwards in } 3 \mathrm{~s} \\
\text { etc. }\end{array}$ & 4 lots of data \\
\hline Video of sessions with the dog & $\begin{array}{l}\text { Could be videoed weekly - } \\
\text { looking for body language / } \\
\text { fluency when reading OR } \\
\text { could be done once a month }\end{array}$ & $\begin{array}{l}\text { Visual data of body language } \\
\text { - stress / relax both for dog } \\
\text { and child }\end{array}$ \\
\hline "Matt-Cam" of sessions & Use Go-pro Camera on dog & $\begin{array}{l}\text { Could be recorded weekly or } \\
\text { monthly - looking for body } \\
\text { language - investigate what } \\
\text { Matt "sees" from the humans }\end{array}$ \\
\hline $\begin{array}{l}\text { Voice recordings of reading } \\
\text { sessions - with and without } \\
\text { dog present }\end{array}$ & $\begin{array}{l}\text { Looking for speed of } \\
\text { processing, fluency, tone and } \\
\text { expression - does this change } \\
\text { when child reads to dog? }\end{array}$ & $\begin{array}{l}\text { Data from reading to an adult } \\
\text { and compare to reading with } \\
\text { the dog }\end{array}$ \\
\hline
\end{tabular}




\begin{tabular}{|c|c|c|}
\hline $\begin{array}{l}\text { Stop the } 77 \text { workshop } \\
\text { (General safety workshops for } \\
\text { dogs and children outside } \\
\text { school situations) }\end{array}$ & Record children's opinions & $\begin{array}{l}\text { General purpose information } \\
\text { - more to help reduce any } \\
\text { risks of any dog / child attacks } \\
\text { in society }- \text { teaching } \\
\text { awareness of dog body } \\
\text { language etc. }\end{array}$ \\
\hline
\end{tabular}

What needs to happen next:

- Permission from the school and parents for children to see Matt for reading

- Permission from the school and parents (also governors?) for the study to take place

- Permission from the parents and school to record the children both on video and audibly

- Permission from the parents for the children to wear the BP wrist monitors

- Reassurance to the parents that any notes will be kept confidential and for research purposes only (any significant profiles will be identified to the SENCO)

- Request to XXX to see if he could possibly be free for extra time to gather the pre and post dog data with Matt. 


\section{Appendix N Letter to parents}

September 2016

Dear Parents,

My name is Janet and I am a PhD student at the University of Gloucestershire. I am investigating the underlying skills of memory and its use for learning in children.

Therapy Dogs Nationwide and the school have allowed me to come into work with XXX and his therapy dog Matt, to look at the differences Matt can make to learning.

Previous research has shown that positive impact contact with pets can boost children's confidence and self-esteem. Dogs give unconditional acceptance as they are non-judgemental. They can also provide confidence to the children by giving the child time to work out and correct themselves. They can reduce stress and make amazing listeners!

There is also some research that children who read to these dogs show an increase in reading levels, word recognition, a higher desire to read and write, and an increase in intra and interpersonal skills among the children they mix with.

My project involves videoing, voice-recording and data logging what is happening while the children are working with Matt and comparing it to when they are not. I will also be working on some short, simple memory activities with your children to see if having Matt present can make a difference. I will also be using questionnaires for teachers, parents and the children themselves to gather everyone's thoughts about any benefits of the project. I should only be in the school for a few weeks until Christmas!

Any records would of course be kept confidentially according to the regulations and ethical requirements of the British Psychological Society. The general results of the project will be made available to Therapy Dogs Nationwide and the school. The project has the full ethical approval of the university and the supervisory team, who can be contacted below.

I hope you will allow your child to take part in this project.

Thank you for your help,

Yours sincerely,

Janet Oostendorp Godfrey

Dr. Graham Edgar and Professor Dianne Catherwood 


\section{Please ensure you have read the University of Gloucestershire Research Ethics Handbook of Principles and Procedures before completing this form}

Please make sure you have completed the Ethics Self-Assessment Checklist prior to filling out this form. This will give you an indication on the types of research designs that require scrutiny from either the School Research Ethics Panel (SREP) or the Research Ethics Committee (REC). On occasion, some research proposals will also need the scrutiny of an external research ethics committee. Please ensure you have discussed this with your module tutor/supervisor/coresearchers.

\section{Contact Details of Lead Researcher}

\begin{tabular}{|l|l|}
\hline Name: & Janet Oostendorp-Godfrey \\
\hline Student or staff number: & $0111997 / 3$ \\
\hline $\begin{array}{l}\text { Please state in which capacity } \\
\text { this application is being made } \\
\text { (as this affects the gatekeeper } \\
\text { process) }\end{array}$ & Postgraduate Researcher \\
\hline $\begin{array}{l}\text { University e-mail address: } \\
\text { s0111997@connect.glos.ac.uk }\end{array}$ \\
\hline
\end{tabular}

\section{Contact Details of research supervisor(s) OR module tutor OR co-researcher(s)}

\begin{tabular}{|l|l|l|l|}
\hline Name: & Graham Edgar & Name: & Alex Masardo \\
\hline $\begin{array}{l}\text { University e-mail } \\
\text { Address: }\end{array}$ & gedgar@glos.ac.uk & $\begin{array}{l}\text { University e-mail } \\
\text { Address: }\end{array}$ & amasardo@glos.ac.uk \\
\hline
\end{tabular}

\section{About the Project}

\begin{tabular}{|l|l|l|l|}
\hline Project Title: & \multicolumn{3}{|l|}{$\begin{array}{l}\text { Can the cognitive benefits of animal assisted therapy increase attention and } \\
\text { working memory skills for young people in educational settings? }\end{array}$} \\
\hline Start Date: & 2015 & Completion Date: & 2020 \\
\hline
\end{tabular}

\section{Project Research Questions (include as many as applicable)}

1. Can the presence of a therapy dog lower stress while performing cognitive tasks? 


\begin{tabular}{|l|l|}
\hline 2. & Can the presence of a therapy dog improve performance on cognitive tasks? \\
\hline 3. & $\begin{array}{l}\text { If there is any effect of a therapy dog, can this effect be replicated in a virtual reality } \\
\text { setting? }\end{array}$ \\
\hline 4. & \\
\hline
\end{tabular}

\section{Brief description of the project (using no more than $\mathbf{5 0 0}$ words):}

Include answers to the following questions (unless non-applicable):

- Why is the project being carried out?

- How has it been designed to answer the research questions?

- Who are your participants?

- How will they be recruited?

- How will data be collected?

- Where will data collection take place?

- How will data be analysed?

- Has the project got any external funding?

- Provide a sample size calculation (for quantitative data)

The project is being carried out to see whether the presence of a therapy dog can lower stress and/or improve performance when adults are engaged in cognitive tasks (mathematical and verbal comprehension). The comprehension task is a widely used reading test - the Nelson-Denny reading test (https://en.wikipedia.org/wiki/NelsonDenny Reading Test). The mathematic task is taken from Key Stage 4 Maths paper questions at Foundation Level.

The project will use an opportunity sample of adults (over 18) recruited by adverts around the University of Gloucestershire and word-of-mouth. The project will use an experimental paradigm to answer the research conditions. Each participant will take part in four experimental conditions.

- Completing arithmetic and verbal comprehension tasks in the presence of the researcher.

- Completing arithmetic and verbal comprehension tasks in the presence of the researcher and a therapy dog.

- Completing arithmetic and verbal comprehension tasks in virtual reality. The VR environment will be a filmed environment including the researcher.

- Completing arithmetic and verbal comprehension tasks in virtual reality. The VR environment will be a filmed environment including the researcher and a therapy dog. 
Performance on the cognitive tasks will be assessed and stress levels will be assessed using a widely used 'PIP' - a device held between thumb and forefinger (see below and https://thepip.com/en-gb/how-it-works/) that measures electrodermal activity (EDA). The PIP is widely used as an aid to relaxation and is marketed as such.

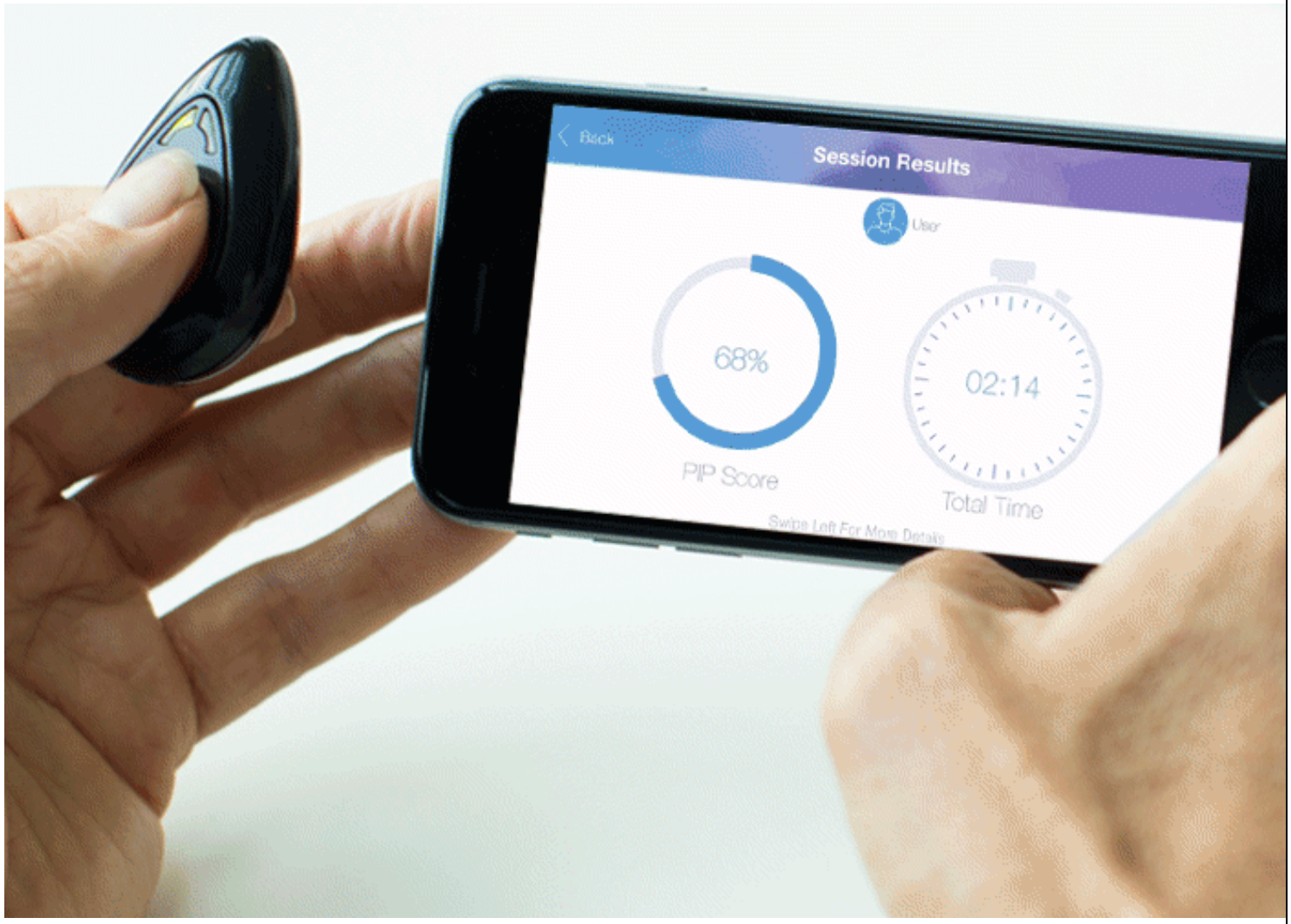

The EDA data will be analysed using in-house analysis software to provide a running average of EDA. Performance on the tasks will be analysed using a one-way ANOVA. Prospective power analysis (Cohen, 1995) suggests a total of 84 participants would be needed for a medium effect size. There is, however, a constraint on the number of times the trials can be run within a reasonable time period without stressing the therapy dogs. It is therefore proposed to recruit a minimum of 24 participants as that will allow full counterbalancing of the four conditions.

There is no external funding for this project. 
Does this proposal contain any REC/SREP mandatory criteria or elements from the Ethics SelfAssessment Checklist?

No

If yes, please indicate which

1) research which involves biomedical or clinical intervention (with the exception of those approved under standard protocols, e.g. those contained in the UoG laboratory handbook)

2) deceptive research

3) covert research or where the data are not recorded in a manner that protects the anonymity of subjects or participants

4) where the research topic is one dealing with sensitive aspects of the subject's or participant's behaviour, or where proposals for research involve vulnerable populations

5) research where participants are under 18

6) research involving work outside the UK

7) research involving assent-based participation

8) research requiring external institutional approval (e.g. NHS, looked after children, young offenders, military personnel)

9) research which involves staff using students as research participants 
If you answer yes to any of the following questions, please give further details as required.

\begin{tabular}{|c|c|c|c|}
\hline 1) & $\begin{array}{l}\text { Have actions been taken to ensure } \\
\text { compliance with the Data Protection } \\
\text { Legislation? }\end{array}$ & Actions taken: & $\begin{array}{l}\text { Compliance with university } \\
\text { data protection guidelines. }\end{array}$ \\
\hline 2) & $\begin{array}{l}\text { Is partnership /collaboration with } \\
\text { another institution involved? }\end{array}$ & $\begin{array}{l}\text { Name of } \\
\text { institution: }\end{array}$ & No \\
\hline 3) & $\begin{array}{l}\text { Has another Ethics Committee } \\
\text { approved the project? }\end{array}$ & $\begin{array}{ll}\text { Ethics } & \\
\text { Committee } & \& \\
\text { date } & \text { of } \\
\text { approval: } & \end{array}$ & No \\
\hline 4) & $\begin{array}{l}\text { Have any training needs been } \\
\text { identified as necessary for the } \\
\text { researcher(s) to complete prior to } \\
\text { undertaking the research? }\end{array}$ & Brief details: & No \\
\hline 5) & $\begin{array}{l}\text { Have relevant professional guidelines } \\
\text { been consulted? }\end{array}$ & $\begin{array}{l}\text { Source of } \\
\text { guidelines: }\end{array}$ & Yes, BPS \\
\hline 6) & $\begin{array}{l}\text { Has another form of 'risk assessment' } \\
\text { been undertaken (in addition to this } \\
\text { form)? }\end{array}$ & $\begin{array}{l}\text { Brief details } \\
\text { (and, if } \\
\text { appropriate, } \\
\text { please append } \\
\text { documents): }\end{array}$ & No \\
\hline
\end{tabular}

\section{Clinical trials insurance: Does the research involve:}

If you answer yes to any of the following questions, please give further details as required.

Important: If your research involves any of the following categories, you must refer your application to the University insurance office in order that the relevant liability insurance can be arranged. Under no circumstances can your project commence until you receive confirmation that liability insurance is in place. Contact details for the insurance office: insurance@glos.ac.uk. 


\begin{tabular}{|c|c|c|}
\hline & $\begin{array}{l}\text { investigating or participating in methods } \\
\text { of contraception? }\end{array}$ & Brief details: \\
\hline b) & $\begin{array}{l}\text { assisting with or altering the process of } \\
\text { conception? }\end{array}$ & Brief details: \\
\hline c) & the use of drugs? & Brief details: \\
\hline d) & the use of surgery (other than biopsy)? & Brief details: \\
\hline e) & genetic engineering? & Brief details: \\
\hline f) & subjects under 5 years of age? & Brief details: \\
\hline g) & subjects known to be pregnant? & Brief details: \\
\hline h) & $\begin{array}{l}\text { pharmaceutical product/appliance } \\
\text { designed or manufactured by the } \\
\text { institution? }\end{array}$ & Brief details: \\
\hline i) & work outside of the United Kingdom? & Brief details: \\
\hline
\end{tabular}

\section{Voluntary Informed Consent}

\begin{tabular}{|c|c|c|}
\hline a) & $\begin{array}{l}\text { Please indicate what form of consent will } \\
\text { be used in this investigation }\end{array}$ & Written \\
\hline
\end{tabular}




b) How and by whom will the voluntary informed consent from participants be
undertaken?
Please indicate in particular if participants/respondents/subjects are children or young
people, or are members of other 'vulnerable populations'.
The study will provide participants with an information sheet and consent form (see Appendices)
stating what they will do in the study, and their ethical rights as a participant (see Appendices).
The researcher will be on hand to answer any questions the participants may have, and to make
sure they have fully understood the information provided to them. Participants will be informed
that they can ask questions at any point of the study, and that they can stop their participation
at any point without having to provide a reason. No members of vulnerable populations will be
used in the study.

\begin{tabular}{|c|c|c|c|}
\hline \multicolumn{4}{|l|}{ Risk of Harm } \\
\hline \multicolumn{4}{|c|}{ Please indicate any possible risks to the researchers, participants, other persons } \\
\hline Tick all that apply & RESEARCHERS & PARTICIPANTS & $\begin{array}{c}\text { OTHER } \\
\text { PERSONS }\end{array}$ \\
\hline \multicolumn{4}{|l|}{ ENVIRONMENTAL } \\
\hline Undue environmental damage & $\square$ & $\square$ & $\square$ \\
\hline \multicolumn{4}{|l|}{ LEGAL } \\
\hline $\begin{array}{l}\text { Contravention of legislation on any of: gender, race, } \\
\text { human rights, data protection, obscenity, } \\
\text { environment }\end{array}$ & $\square$ & $\square$ & $\square$ \\
\hline Defamation & $\square$ & $\square$ & $\square$ \\
\hline \multicolumn{4}{|l|}{ PHYSICAL } \\
\hline Bodily Contact & $\square$ & $\square$ & $\square$ \\
\hline Lone working & $\square$ & $\square$ & $\square$ \\
\hline Physical danger/violence (or threat of either) & $\square$ & 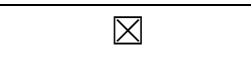 & $\square$ \\
\hline Research outside of the UK & $\square$ & $\square$ & $\square$ \\
\hline
\end{tabular}




\begin{tabular}{|c|c|c|c|}
\hline \multicolumn{4}{|l|}{ PHYSIOLOGICAL } \\
\hline Ingestion of foods, fluids or drugs & $\square$ & $\square$ & $\square$ \\
\hline Undue physical stress or exertion & $\square$ & $\square$ & $\square$ \\
\hline $\begin{array}{l}\text { Sampling of human tissue, body fluids including } \\
\text { venepuncture }\end{array}$ & $\square$ & $\square$ & $\square$ \\
\hline \multicolumn{4}{|l|}{ PSYCHOLOGICAL } \\
\hline $\begin{array}{l}\text { Psychological intrusion from questionnaires, } \\
\text { interview schedules, observation techniques }\end{array}$ & $\square$ & $\square$ & $\square$ \\
\hline \multicolumn{4}{|l|}{ SOCIAL, CULTURAL \& PROFESSIONAL } \\
\hline Contravention of social/cultural boundaries & $\square$ & $\square$ & $\square$ \\
\hline Nudity, loss of dignity & $\square$ & $\square$ & $\square$ \\
\hline $\begin{array}{l}\text { Compromising professional boundaries with } \\
\text { participants, students, colleagues }\end{array}$ & $\square$ & $\square$ & $\square$ \\
\hline \multicolumn{4}{|l|}{ REPUTATIONAL } \\
\hline $\begin{array}{l}\text { Research that unduly and adversely affects the } \\
\text { reputation of the institutions involved }\end{array}$ & $\square$ & $\square$ & $\square$ \\
\hline \multicolumn{4}{|l|}{ Other risks identified, but not listed above: } \\
\hline \multicolumn{4}{|c|}{$\begin{array}{l}\text { If you have ticked any of the previous remarks, please describe the actions that will be taken to minimise the } \\
\text { risk. }\end{array}$} \\
\hline \multicolumn{4}{|c|}{$\begin{array}{l}\text { The participant will be in the presence of a therapy dog for one of the four conditions. The participant will be } \\
\text { given the option to withdraw from the study without ever meeting the dog should they wish. Two dogs will } \\
\text { be used, both certified and insured therapy dogs (See Appendix A). Both dogs are trained as therapy dogs and } \\
\text { are very used to interacting with people. }\end{array}$} \\
\hline \multicolumn{4}{|c|}{$\begin{array}{l}\text { The dogs are both over } 9 \text { months old, and have been with their owners for at least } 6 \text { months. They are } \\
\text { groomed, clean, vaccinated, ticked and flea'd, wormed and in good health. They have been assessed by the } \\
\text { charity for their calm and friendly temperament with gentle behaviour. Only those who have passed this test } \\
\text { become registered therapy dogs. }\end{array}$} \\
\hline \multicolumn{4}{|c|}{$\begin{array}{l}\text { Jan is very experienced with her dogs and able to chat about practically anything to make people feel } \\
\text { comfortable and less stressed. Jan and her husband (who may assist with dog handling are Mental Health First } \\
\text { Aiders. }\end{array}$} \\
\hline
\end{tabular}


The particular dogs that will be used in this study, Maisie and Isla (both Labradors) have regularly worked in schools, hospices, the Uni of Glos and with Swindon Mind / Youth Mind for the past 2 years.

The dogs have regularly been into $\mathrm{FCH}$, due to visiting the Psychology Department and taking part in Mental Health and Stress Weeks with the students over the past 3 years. Both dogs have also been at the Park for a virtual reality conference where Jan was presenting a poster in 2017, and for Freshers Week (Oct 2018) and have also visited Oxtalls for a Post Grad Conference in 2016 and for Exam Stress research (May 2018).

Possible Risks

Dogs can bite (Not in reality as they are both very experienced, assessed dogs - low risk).

Dogs could move suddenly and scare someone (medium to low risk).

Dogs can lick - we try very hard to discourage this as a charity, but people still think dogs want to give "kisses." Dogs also lick to "sense" the area around them - we suggest washing and hygiene are a priority, should this happen (usually hands) Medium to low risk .

Allergies to dogs - depends on the person - some have taken precautions such as inhalers in the past, others have chosen to avoid the area with the dogs (possible high risk)

Phobias - the dogs are used to people who are nervous around them, including children. There is no pressure on anyone to interact with the dogs if they don't want to.

Cultural or Religious Reasons - again no pressure to have to visit the dogs by anyone.

The information sheet (Appendix B) will make it clear that dogs will be involved in this study and that participants are free to withdraw if concerned about any of the above.

Current TDN Insurance document are in Appendix A. 


\section{Anonymity / Confidentiality}

Please indicate measures that will be taken to protect and maintain the anonymity and/or confidentiality of participants.

All participants will be assigned a unique participant number, and it will be this number that is directly linked to their data. Therefore, no participant will be individually identifiable from their data as no names or personal information will be linked directly to the data. No information that will allow the participant to be uniquely identified will be stored with the data.

All information gathered will be stored securely, with only the research team having access to this. Personal information will be destroyed in line with BPS and data protection guidelines when the project is complete.

Data from each participant will be combined with that of other participants, and it is this data, rather than individual data, that will be analysed.

How are you anonymising your data?

No information that will allow the participant to be uniquely identified will be stored with the data. All participants will be identified by a letter/number code only. 


\begin{tabular}{|l|l|}
\hline $\begin{array}{l}\text { What secure storage do } \\
\text { you propose to hold your } \\
\text { data in? }\end{array}$ & Password-protected computer. \\
\hline What secure premises will & \\
the data be stored in? (e.g., & Locked premises. \\
locked lab or office) & \\
\hline What are you going to do & All raw data will be destroyed when no longer required by the project (e.g. \\
with your data once the & to support publications). \\
\hline project is complete? & \\
\hline How are you ensuring & All computer-based data will be completely anonymous (i.e., no individual will be \\
\hline compliance with relevant & identifiable from it), and will be stored securely in accordance with the data \\
data protection legislation? & protection act. \\
\hline (Please attach the Privacy notice / PIS as an appendix) \\
\hline
\end{tabular}

\begin{tabular}{|c|l|l|}
\hline \multirow{2}{*}{ Signature of researcher(s) } & Name & Date \\
\cline { 2 - 4 } & & \\
\cline { 2 - 4 } & & \\
\hline Signature of supervisor(s) & & \\
\hline (if appropriate) & & $3 / 3 / 2019$ \\
\hline
\end{tabular}




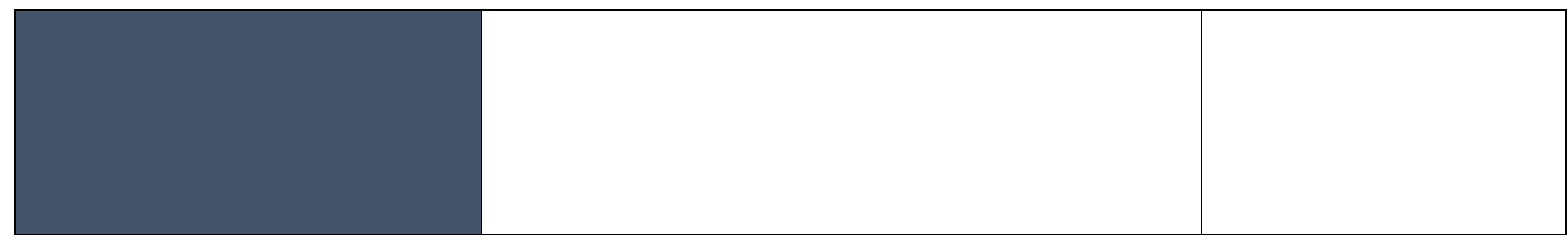

NB: forms not fully completed and signed by researcher/s and supervisor/s (where appropriate) will be returned without consideration for approval.

\section{Supporting documentation.}

Please ensure that when preparing applications to SREP/REC using the Research Ethics Proforma that the following are included on the form or within the project description attached to the form, where these are of relevance:

a) sample information sheet to be given to participants and sample letters about voluntary informed consent and withdrawal (beware of any possible problems of coercion), written in good clear English. University addresses should be used; (depending on timing of the REC/SREP consideration this may be inappropriate)

b) statement about any conflict of interest;

c) statement about DBS check (Criminal Records Bureau) https://www.gov.uk/government/organisations/disclosure-and-barring-service ;

d) copies of questionnaires and/or interview schedules and questions, even if in early draft form.

REC's/SREP's decision-making will be more straightforward if you have covered the following issues in your project description. If you need to, attach further information.

(i) the nature of the observation of human participants;

(ii) the outline planning and procedures for focus group research or one-to-one interviews to include establishing ground rules affecting revelation of any personal details to the group;

(iii) that issues of power relations are taken into consideration;

(iv) that issues of any guilty knowledge likely to arise from the research are thought through;

(v) the details and number of participants (age, gender, whether a vulnerable group noting that this often depends on the specific research project, size of group);

(vi) whether this is a double-blind study;

(vii) the justification of use of photography or video and that permission concerning these are planned for; 
(viii) that issues of physical and/or psychological and personal danger affecting either the participants or the researcher have been considered fully;

(ix) whether interviews are to be recorded electronically or manually.

(x) if your research involves any of the subject matters described in the Clinical trials section, you must refer your application to the insurance office in order that the relevant liability insurance can be put in place. Under no circumstances can your project commence until you receive confirmation that liability insurance has been agreed. Contact details for the insurance office: insurance@glos.ac.uk

Research form Appendix A

Details of therapy dog certification, insurance, etc. 

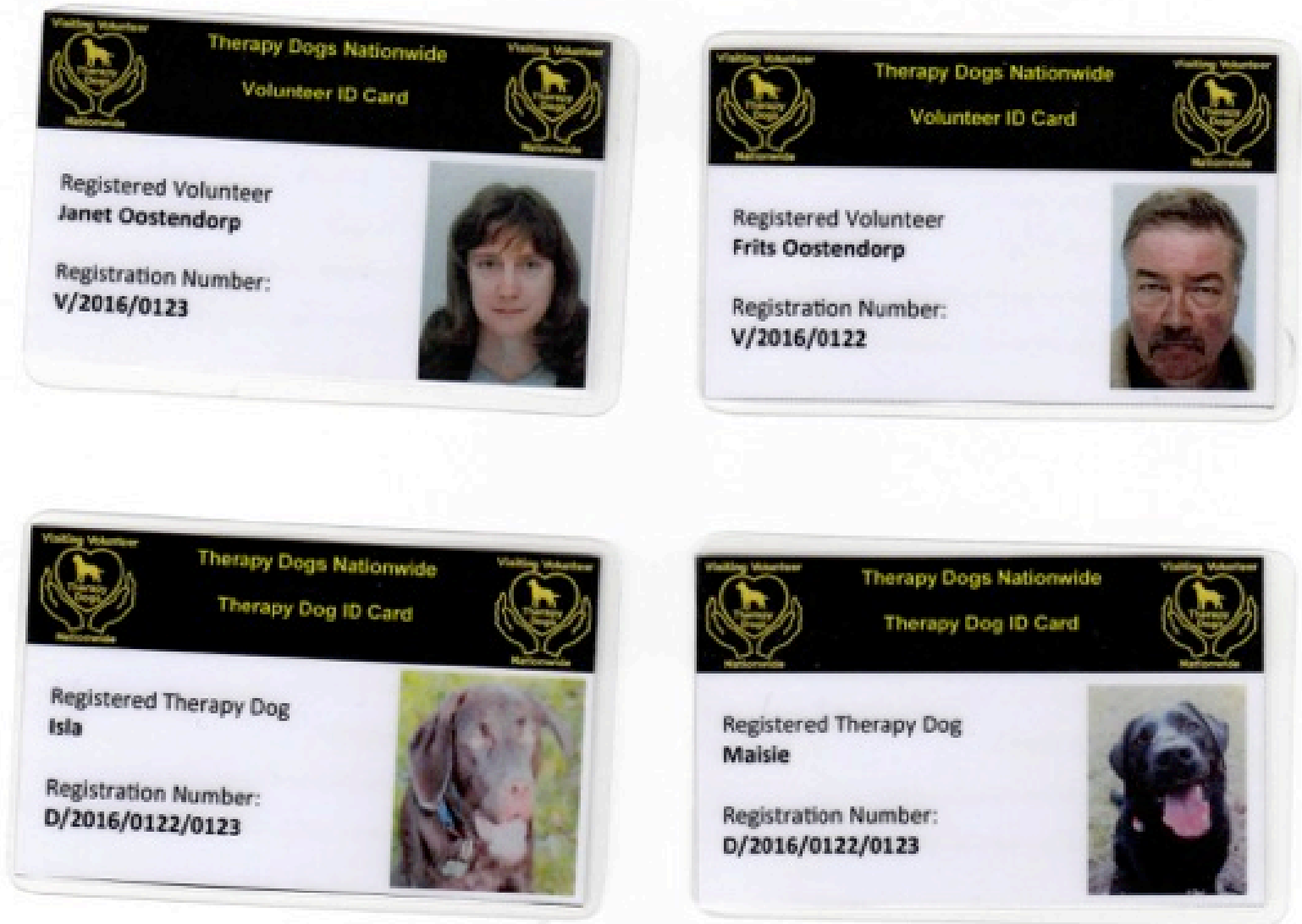


\section{Speclallst Insurance for Charities and the Voluntary Sector \\ Policy Schedule}

Thank you for choosing Arthur. $\downarrow$ Gallagher Insurance Brokars Ltd Charties and Voluntary Sector Ineuramox, uecierwitten by Royal 8 Sun Alliance Insurence pic (RSA).

You should read thia Schedule in conjunction with your Policy Wording

These details are a rocord of the irformation provided to RSA. It is also eseential that you reed all of the clauses applying to your Policy as these contain important information that may altect your Pollicy cover.

Your Delalis:

Policyholder:

Therapy Dogs Natlonwide

Pollcyholder's Addres: $\quad 103$ Hcme Farm Foed, Wirtal, Nerseyslde

CH4S 7LG

Business Deecriptlon: Voluntary organisation taking dogs into cave and supportad living environments offering an animal therapy service

Your Insurance Broker / Intemedlary's Detalls:

Insurance Broker / Intermedkary Name:

Addrese:
Arthur J. Gallagher Insurance Brokers Ltd

Magden Park, Groen Meadow, Llentrisant CF72 8XL

Your Policy Datea:

Perlod of insurance:

From:

01 March 2018

To:

Renerral Date:
28 February 2019

01 March 2019

Your Premium Information:

Renewal Pramium:

Insurance Premium Tac:

Total Amount Due:

A full copy of your Pollcy Wording is zwailable on request. Please contect your Intemedlarylngurance Broker at the addrens ghown if you wish to request a copy. 


\section{To Whom It May Concern}

Client: Therapy Dogs Nationwide

Date: $21^{\text {st }}$ February 2017

I can confirm, as Insurance Brokers for Therapy Dogs Nationwide the following covers are in force:

Public/Products Lability (Policy No: RKL23407/03/20)

Insurer:- $\quad$ INK Underwriting Agencies Ltd

Period of cover:- 12 months from $1^{\text {st }}$ March 2017

Limit of Indemnity:- $\quad £ 5$ million

Employers' Liability (Policy No: RKL23407/03/20)

Insurer:- $\quad$ INK Underwriting Agencies Ltd

Period of cover:- $\quad 12$ months from $1^{\text {st }}$ March 2017

Limit of Indemnity:- $\quad$ £10 million

Yours Faithfully

Emily Clarke

Commercial Admin Clerk 


\section{THERAPY DOGS NATIONWIDE}

103 Home Farm Road, Woodchurch

Wirral, CH49 7LG Tekphone: 07840994003

Email: enquiries@therapydogsnationwide.org

Website: www.therapydogsnationwide.org

VOLUNTEER AND VOLUNTARY AREA TEAM IEADER (ATL) DETAILS SHEET

Please use this sheet in your department to record the contact details of your visiting Therapy Dogs

nationwide volunteer and Therapy dog.

Please do let our volunteer know in advance if you do not wish them to visit at the arranged time.

Many of our volunteers may travel some distance with their animal, often on public transport and at their own expense, in order to visit your establishment.

VOLUNTEER NAME AND ID NUMBER

Janet and Frits Oostendorp V/2016/0122 and V/2016/0123

NAME OF THERAPY DOG

Maisie (Black Labrador) and Isla (Chocolate Labrador)

THE TDN VOLUNTEER USUALLY VISITS HERE ON:

When required!

VOLUNTEER CONTACT TELEPHONE NUMBER/S

01793823266 (Swindon)

NAME OF LOCAL VOLUNTARY AREA TEAM LEADER (ATL)

Leisl de Lafontaine, 07725 040117, leisl@btinternet.com

VOLUNTARY AREA TEAM LEADER (AT'S) TELEPHONE NUMBER/S

Therapy Dogs Nationwide: 103 Home Farm Road, Woodchurch, Wirral, CH49 7 LG Trustees: Eikeen Slattery (Chair), Eileen Hodge, Anne Jones, Mary Orver MBE

Patron: Tony Hodge Trustees: Eileen Slattery (Chair), Eileen Hodge, Anne
Consultant: Maureen Hennis

Charity No. 1167622 


\section{Research Ethics Form Appendix B}

\section{Participant information sheet.}

\section{Participant Information sheet}

\section{Are there differences between the presence of "real" dogs or "Virtual Reality" dogs for human academic learning skills?}

The use of dogs within school-based settings is increasing. Animal Assisted Education (AAE) or Animal Assisted Intervention (AAI) has shown that there have been benefits for individuals for socio-emotional learning when dogs are present. This investigation is to investigate whether "virtual reality" dogs can also affect the executive memory skills associated with vocabulary learning and mathematics.

If the investigation has positive results then not only will it strengthen the case for the inclusion of suitably trained and prepared animals within the school curriculum, but could also lead to further research for other, alternative approaches for students who cannot be present in schools, for whatever reason e.g. school phobia, mental health issues, or Education Other Than At School (EOTAS).

The research will consist of two short videos presented within a virtual reality (VR) system and two short sessions without VR. You will therefore be asked to take part in four short sessions as follows (not necessarily in this order):

Session 1. Completing arithmetic and verbal comprehension tasks in the presence of the researcher.

Session 2. Completing arithmetic and verbal comprehension tasks in the presence of the researcher and a therapy dog.

Session 3. Completing arithmetic and verbal comprehension tasks in virtual reality. The $V R$ environment will be a filmed environment including the researcher. 
Session 4. Completing arithmetic and verbal comprehension tasks in virtual reality. The $V R$ environment will be a filmed environment including the researcher and a therapy dog.

The VR system that we will use is a standard, commercially-available, system (Oculus Rift). A researcher will be present at all times while you are using the system, and you may stop using the system at any point if you wish.

You will also be asked to hold a "PiP" to measure skin conductance (based on sweat levels) throughout each session. The PiP is a commercially available system marketed as an aid to stress-reduction. It is a small device that you will be asked to hold between thumb and forefinger in each session. The PiP will be shown to you and its used explained. You can let go of the PiP at any time if you wish.

Each activity is less than 4 minutes in length and will involve multiple choice vocabulary definitions and a selection of mental mathematical questions. Afterwards there will be a short semi-structured interview asking for your personal preferences of the different conditions. It is therefore likely to take up to 30 minutes in total. To enable the scoring of performance on the tasks only, a video will be recording throughout. Once the data have been analysed the video will be destroyed. Note that the study is only concerned with individual differences in performance across the four conditions - not in comparing between individuals.

Your data will be anonymised and your data will only be identified by a number. You will be provided with this number that you can use to withdraw your data from the study if you should so wish.

Data will be stored on a password protected computer and only the researcher and the research supervisory team will have access. No information identifying you as an individual will be contained within the student's doctoral dissertation, but the general findings may be published by both the student and the University of Gloucestershire in the future.

\section{Risks}

There are some risks to using Virtual Reality headsets. Those who are susceptible or diagnosed with epilepsy, anxiety, PTSD, have eye conditions involving eye strain, or suffer from nausea. If you have any of these conditions, or if you believe there is any reason that you may suffer adverse affects from immersion in VR, you should not take part. You do not have to give a reason. It is entirely up to you whether you wish to take part.

There are also risks when working with live, sentient animals such as dogs. If you are allergic to either dander or dogs, or Cynophobic (phobic towards dogs) you may also not 
wish to participate in this study. There is also a risk of being licked, with possible subsequent cross contamination. The advice would be to wash your hands and any affected area after working with the dogs and hand cleanser will be available. The handler will try to intervene to reduce any unwanted behaviours and it is advisable to try to retain a calm approach around animals, as dogs can easily read and respond to human body language and energy levels very quickly. Dogs can bite (Not in reality as they are both very experienced, assessed dogs - low risk) and can move suddenly with the possibility of scaring someone (medium to low risk).

If you have any concerns about any of the above, then please do not take part in this study. You do not have to give any reason.

\section{Dog welfare}

The dogs are experienced and qualified, registered "therapy" dogs under the "Therapy Dogs Nationwide" charity and are insured as such. They have regularly visited the University of Gloucestershire's campuses and the CEAL building in the past.

The dogs will only work for up to two hours maximum, outside of any breaks in any given day and will be given "rest days" between visits as per charity rules.

\section{Consent}

If you wish to take part in the study, please complete the consent form.

Thank you.

Janet Oostendorp Godfrey s0111997@connect.glos.ac.uk oostendorp@btinternet.com

Supervisors: Graham Edgar (gedgar@glos.ac.uk), Alex Masardo (amasardo@glos.ac.uk). 


\section{Research Ethics Form Appendix C}

\section{Consent forms.}

UNIVERSITY OF GLOUCESTERSHIRE

SCHOOL OF NATURAL AND SOCIAL SCIENCES

\section{CONSENT FORM - RESEARCHER COPY}

\section{Name of Principal Investigator}

Janet Oostendorp-Godfrey

Title of Research

Are there differences between the presence of "real" dogs or "Virtual Reality" dogs for facilitating human cognitive skills?

Please initial the boxes to indicate acceptance of each point.

I understand that my participation in this project will involve attempting arithmetic and verbal comprehension tasks with and without a therapy dog present. The tasks will also be performed either in virtual reality (VR) or in the, 'real world.':

I understand that my participation in this study is entirely voluntary and that I can withdraw at any time for any reason without negative consequences by telling the researcher that I wish to stop. I also understand that I can withdraw my data from the study up to $31^{\text {st }}$ June 2019 by contacting the researcher by e-mail and quoting my participant number (the details of which will be noted on the debrief form). I understand that I will not be asked to provide a reason for this withdrawal:

I understand that I am free to ask questions at any time throughout the study and can email the researcher if I have any questions after my participation: 
I understand that the information provided by me will be held confidentially and securely, and that any material with my name on will be held separately from my data. My data will be identifiable only by a participant number, and not by my name. When the study is written up, my data will be anonymously integrated with the other participants such that I will not be personally identifiable from it. I understand that the information I have provided will be processed and retained in accordance with the Data Protection Legislation. No personal information provided may be retained indefinitely. Finally, I also understand that at the end of the study I will be provided with additional information and will be fully debriefed as to the aims of the study.

I understand that all sessions will be videoed and that the videos will be destroyed as soon as the data obtained has been scored.

I understand that if I feel any negative effects after the study, I can contact my $\underline{\text { GP }}$, the student Helpzone (if I am a student at UoG; 01242 714444) or the Samaritans (116 123, jo@samaritans.org).

I understand that the Principal Investigator of this work will have attempted, as far as possible, to avoid any risks, and that safety and health risks will have been separately assessed by appropriate authorities.

Under these circumstances, I agree to participate in the research.

Name:

Signature of participant:

Date:

Signature of researcher: 
SCHOOL OF NATURAL AND SOCIAL SCIENCES

\section{CONSENT FORM - PARTICIPANT COPY}

\section{Name of Principal Investigator}

Janet Oostendorp-Godfrey

\section{Title of Research}

Are there differences between the presence of "real" dogs or "Virtual Reality" dogs for facilitating human cognitive skills?

Please initial the boxes to indicate acceptance of each point.

I understand that my participation in this project will involve attempting arithmetic and verbal comprehension tasks with and without a therapy dog present. The tasks will also be performed either in virtual reality (VR) or in the, 'real world.':

I understand that my participation in this study is entirely voluntary and that I can withdraw at any time for any reason without negative consequences by telling the researcher that I wish to stop. I also understand that I can withdraw my data from the study up to $31^{\text {st }}$ June 2019 by contacting the researcher by e-mail and quoting my participant number (the details of which will be noted on the debrief form). I understand that I will not be asked to provide a reason for this withdrawal:

I understand that I am free to ask questions at any time throughout the study and can email the researcher if I have any questions after my participation: 
I understand that the information provided by me will be held confidentially and securely, and that any material with my name on will be held separately from my data. My data will be identifiable only by a participant number, and not by my name. When the study is written up, my data will be anonymously integrated with the other participants such that I will not be personally identifiable from it. I understand that the information I have provided will be processed and retained in accordance with the Data Protection Legislation. No personal information provided may be retained indefinitely. Finally, I also understand that at the end of the study I will be provided with additional information and will be fully debriefed as to the aims of the study.

I understand that all sessions will be videoed and that the videos will be destroyed as soon as the data obtained has been scored.

I understand that if I feel any negative effects after the study, I can contact my $\underline{\mathrm{GP}}$, the student Helpzone (if I am a student at UoG; 01242 714444) or the Samaritans (116 123, jo@samaritans.org).

I understand that the Principal Investigator of this work will have attempted, as far as possible, to avoid any risks, and that safety and health risks will have been separately assessed by appropriate authorities.

Under these circumstances, I agree to participate in the research.

Name:

Signature of participant:

Date:

Signature of researcher: 
Research Ethics Form Appendix D

\section{Debrief.}

UNIVERSITY OF GLOUCESTERSHIRE

SCHOOL OF NATURAL AND SOCIAL SCIENCES

\section{DEBRIEF FORM}

Title of Research

Are there differences between the presence of "real" dogs or "Virtual Reality" dogs for facilitating human cognitive skills?

\section{Debrief}

Thank you for giving your time to participate in this research.

As mentioned in the briefing, Animal Assisted Education (AAE) or Animal Assisted Intervention (AAI) has shown that there have been benefits for individuals for socio-emotional learning when dogs are present. This investigation was to investigate whether "virtual reality" dogs can also affect the executive memory skills associated with vocabulary learning and mathematics.

You were asked to perform mathematical and verbal comprehension tasks either in VR or in the, 'real world' and with, or without, a dog present. We are looking to see whether the presence of a dog can:

- Improve performance on cognitive tasks (as assessed by the tasks you were asked to do).

- Decrease stress (as assessed by the PiP you were asked to hold. 
We are also interested to see whether a 'virtual dog' has the same effect as a real one. This is something that is useful to know as a virtual dog is cheaper and more accessible than a real one - and so could allow any benefits to be spread more widely.

You are reminded that your data will be kept confidentially and anonymously, and that you will not be personally identifiable from your data. You are also reminded that your data will be kept securely, with access only given to the research team. This will not be kept indefinitely, but will be destroyed in accordance with the ethical guidelines provided by the British Psychological Society (BPS) and data protection guidelines.

You are also reminded that you are free to withdraw from the research at any stage without penalty, and you can ask for your data to be destroyed up until 31 $31^{\text {st }}$ December 2018 if you wish (please note that no reason will be required for your withdrawal). If you wish to withdraw your data, please contact Janet Oostendorp-Godfrey (oostendorp@xxxxxxxx.com) and quote the number below.

If you have any questions about the research, or are dissatisfied with the way the research is conducted, please contact Graham Edgar in the first instance (gedgar@glos.ac.uk). You can also contact the chair of the ethics committee that approved this study (Rachel Sumner rsumner@glos.ac.uk).

Thank you again for your participation in this research. Your help is much appreciated.

If you feel any negative effects after the study, please contact your GP, the student Helpzone at the University of Gloucestershire if you are a student there (01242 714444) or the Samaritans (116 123, jo@samaritans.org). 
Thank you for submitting an ethics application to the NSS School Research Ethics Panel (SREP).

Before the panel can grant ethical approval please address the following comments and revisions. Please use the following table to describe the changes you have made and if necessary show where you have amended your original ethics application (see example)

Once you have amended your ethics application, please return this form with a copy of ethics application to nssethics@glos.ac.uk

\begin{tabular}{ll|ll}
\hline Name: & Janet Oostendorp-Godfrey & Name: & Prof Graham Edgar \\
University e-mail & $\underline{\text { s0111997@connect.glos.ac.uk }}$ & University e-mail & gedgar@glos.ac.uk \\
Address: & & Address: &
\end{tabular}

Date of original $05 / 03 / 2019$

submission

Date of revision Click here to enter a date.

\section{Title of study}

Title of applicant's research

Can the cognitive benefits of animal assisted therapy

increase attention and working memory skills for young

people in educational settings? 
EXAMPLE:

Please show how you will recruit your participants

The primary concern here is that the ethical and safety issues are mostly focussed on the human participants. There needs to be a little more focus on the welfare of the dogs involved. I actually have no qualms whatsoever about the project BUT the issues need to be identified on this form so that there is evidence that the dogs' welfare is a priority. Given they are certified and insured therapy dogs this isn't going to be a problem at all but I would suggest identifying:

- That the dogs can be withdrawn from the study at any time under the direction of the handler

- That a quiet space be identified for the dogs and handlers to "retreat" to if necessary

- That fresh water be provided

- That regular breaks be given under the guidance of the handler

- That at all times the welfare of the dogs be given due consideration
I will recruit participants by emails sent out to the team coaches. I have attached the email I will be using to my original application (see appendix F). Changes to my participant recruitment can be found on page 5 . I have highlighted changes in red text.

I am glad you have mentioned this!

Absolutely they will have their "5 Freedoms!" (I wouldn't bring them in otherwise)

Yes the dogs will have access to water, their beds and a quiet place to settle when they are not "working." They have at all times the choice of whether to work or not. They will let the "handlers" know as the handlers know their body language behaviour very well. They will also have access to their treats and toys too.

There will be breaks between each student and we are only planning for the dogs to be "at work" for up to two hours in student sessions only, but this will not be in "solid time" as there will be gaps 


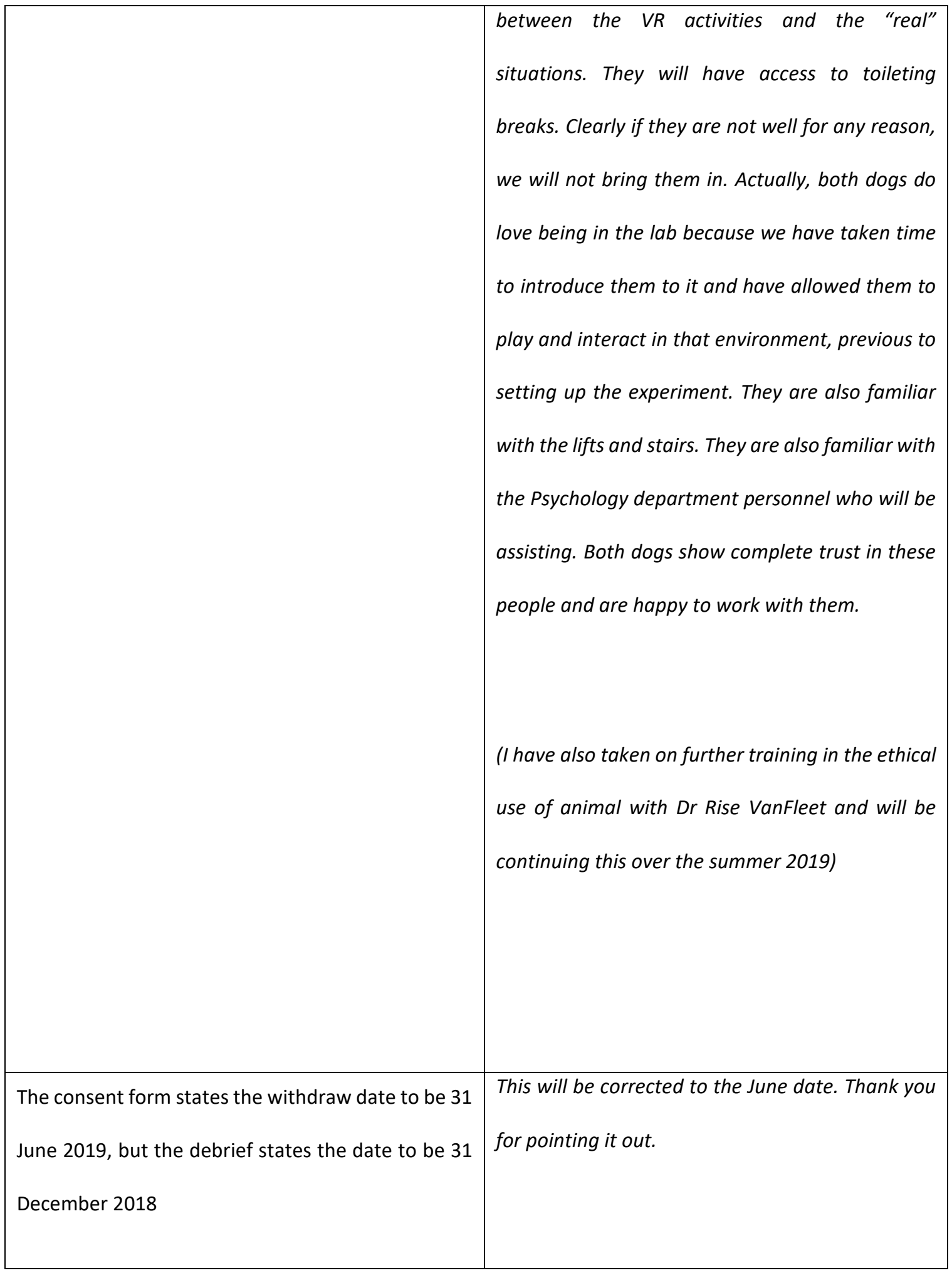

Tel 08448010001 www.glos.ac.uk Registered office: The Park, Cheltenham, GL50 2RH University of Gloucestershire The Park Cheltenham GL50 2RH The University of Gloucestershire is a company limited by guarantee registered in England and Wales. Registered number: 06023243 . Registered office: The Park, Cheltenham, GL50 2RH. 


\section{Permission Granted}

20тн March 2019

Dear Janet,

Thank you for your application to the School of Natural \& Social Sciences - School Research Ethics Panel (NSS-SREP).

Following institutional ethical review, I am pleased to confirm ethical clearance.

Please keep a record of this letter as a Can the cognitive benefits of animal assisted confirmation of ethical approval for your therapy increase attention and working study (detailed below), reviewed by the memory skills for young people in School Research Ethics Panel of the School educational settings?

of Natural \& Social Sciences, University of

Gloucestershire, on 5th March 2019.

Project Title:

Start Date:

20th March 2019

Projected Completion Date:

1st January 2020

NSS-REP Clearance code:

NSS_OOSTENDORPGODFREY_190320 


\section{Appendix P Risk Assessment}

\section{Therapy Dogs Nationwide - Risk Assessment adapted from "Bark and Read" Risk Assessment}

for schools.

\section{Risk Assessment for Phase 2}

\begin{tabular}{|c|c|c|c|}
\hline $\begin{array}{l}\text { Identifying the } \\
\text { Risk }\end{array}$ & $\begin{array}{l}\text { Risk } \\
\text { Rating }\end{array}$ & Control Measures & $\begin{array}{l}\text { Person } \\
\text { Responsible }\end{array}$ \\
\hline $\begin{array}{l}\text { Hygiene } \\
\text { Concerns }\end{array}$ & Low & $\begin{array}{l}\text { Only registered Therapy Dogs Nationwide dogs } \\
\text { to be involved as they are immunised and } \\
\text { vaccinated, and up to date with flea and } \\
\text { worming treatments in accordance to Therapy } \\
\text { Dogs Nationwide policy. These particular two } \\
\text { dogs are kibble fed. Dogs will be free from } \\
\text { disease or injury and in good health. } \\
\text { Adult to use hand gel after touching dog. } \\
\text { Encouraged to wash hands in nearest toilets. } \\
\text { Volunteer to ensure all dog excrement is taken } \\
\text { off premises to be disposed of in an appropriate } \\
\text { manner, i.e. dog poo bin }\end{array}$ & $\begin{array}{l}\text { TDN } \\
\text { volunteer / } \\
\text { handler }\end{array}$ \\
\hline Allergies to dogs & low & $\begin{array}{l}\text { All participants to notify TDN volunteer handler } \\
\text { before meeting dog. } \\
\text { Sign to say dogs are in building at entrance. Dog } \\
\text { "spaces" have access to windows for ventilation. } \\
\text { Dogs regularly groomed before arrival and use of } \\
\text { wipes during visit } \\
24 \text { hour "gaps" between visits to allow for } \\
\text { cleaning }\end{array}$ & $\begin{array}{l}\text { TDN } \\
\text { Volunteer / } \\
\text { Handler }\end{array}$ \\
\hline $\begin{array}{l}\text { Participant } \\
\text { safety }\end{array}$ & medium & $\begin{array}{l}\text { TDN dogs will be appropriately socialised and } \\
\text { orientated within the university environment } \\
\text { Dogs will remain on lead and under full control } \\
\text { of handler at all times } \\
\text { Dogs will be the sole focus of the handlers } \\
\text { throughout - including during rest periods } \\
\text { The dogs will be removed by the handlers from } \\
\text { any situation they deem to be detrimental to the } \\
\text { dog, or the dog has shown that they wish to be } \\
\text { removed, for the sake of the dogs' welfare. An }\end{array}$ & $\begin{array}{l}\text { TDN } \\
\text { Volunteer / } \\
\text { Handler }\end{array}$ \\
\hline
\end{tabular}




\begin{tabular}{|l|l|l|l|}
\hline $\begin{array}{l}\text { Inexperienced / } \\
\text { phobias } \\
\text { cultural or } \\
\text { religious reasons }\end{array}$ & low & $\begin{array}{l}\text { alternative known "safe space" is available to } \\
\text { the dogs within the car. }\end{array}$ & $\begin{array}{l}\text { Dogs will remain on lead and under full control } \\
\text { of handler at all times } \\
\text { Handlers are willing to walk the dogs in the } \\
\text { opposite direction if wished and may choose to } \\
\text { do so depending on the level of behaviour } \\
\text { towards the dog, however the dogs do have a lot } \\
\text { of experience with nervous people and will look } \\
\text { to the handlers for guidance. Dogs are } \\
\text { temperament assessed to remain calm as much } \\
\text { as possible. Handlers can show how to be } \\
\text { introduced to a dog properly if wanted by } \\
\text { participant. }\end{array}$ \\
Holunteer \\
\hline $\begin{array}{l}\text { Dog bites } \\
\text { scratches }\end{array}$
\end{tabular}

103 Home Farm Road, Woodchurch, Wirral, CH49 4LG enquiries@therapydognationwide.org 07840994003

Registered Charity 1167622 


\section{Appendix Q Locations of school dogs in special school sample 2016}

Local Authority
BANES
Bexley
Birmingham
Brent
Brighton \& Hove
Bromley
Cambridgeshire
Cumbria
Doncaster
Dorset
Essex
Glos
Hackney
Haringey

\begin{tabular}{l} 
Name if \\
known \\
Milo \\
Tai \\
? \\
Poppy \\
Elvis \\
Dudley \\
? \\
Henry / Archie \\
Herbert \\
? \\
Finn \\
? \\
\hline Gracie \\
\hline
\end{tabular}

Handler
PAT dog
PAT
Students x3
D/K
PAT dog
D/K
Staff
PAT 2
D/K
PAT
Head \& Counsellor
Staff x5
Speech \& Lang
PAT dog

\begin{tabular}{|c|c|}
\hline In school & In school \\
\hline 2016 & 2018 \\
\hline 1 & 1 \\
\hline 1 & 0 \\
\hline 3 & 0 \\
\hline 1 & 1 \\
\hline 1 & 0 \\
\hline 1 & 1 \\
\hline 1 & 1 \\
\hline 2 & 0 \\
\hline 1 & 1 \\
\hline 1 & 0 \\
\hline 1 & 1 \\
\hline 5 & 0 \\
\hline 1 & 0 \\
\hline 1 & 0 \\
\hline
\end{tabular}




\begin{tabular}{|c|c|}
\hline Herefordshire & Casper (at least) \\
\hline Kent & ??? \\
\hline Lancashire & Rufus / Archie \\
\hline Lewisham & Jasmine \\
\hline Liverpool & Henry \\
\hline Luton & William \\
\hline Northumberland & $?$ \\
\hline Oxon & $?$ \\
\hline Poole & Moses / PAT dog \\
\hline Rotherham & Henry \\
\hline Somerset & Molly / Lucy \\
\hline Swindon & Ruby /Mack \\
\hline Tyne \& Wear & Oscar \\
\hline West Buckinghamshire & $?$ \\
\hline Wirral & Dylan \\
\hline Barnet & $?$ \\
\hline Barnet & Dexter \\
\hline Barnet & $?$ \\
\hline Blackpool & Doodles \\
\hline
\end{tabular}

Students $x 2$

Students x3

Teacher / TA

PAT dog

Teacher

PAT dog

$D / K$

PAT dog

puppy walker/ staff

Head Teacher

D/K

Student / Head

PAT

PAT $\operatorname{dog} x 2$

PAT dog

Patient / PAT

PAT dog

PAT

Assistant Head
0

2

0

1

0

1

1

1

1

0

1

1

0

1 
Brent

Brighton \& Hove

Brighton \& Hove

Bristol

Buckinghamshire

Bury

Cheshire

Cleveland

Dudley

East Sussex

Essex

Exeter

Glos

Gloucestershire

Hampshire

Hampshire

Hampshire

Hampshire

Hampshire
Millie

Einstein

Patience

Angie

Fozzy / Finn

Polly

Bella

$?$

$?$

Billy

?

$?$

Mollie

Murphy

Nimmo*

Perdy

Nimmo*

\section{Staff $\times 4$}

Student

Staff

\section{PAT dog}

Staff Handler

Staff

Occ. Ther/ Outdoor Ins.

PAT dog

$D / K$

teacher

$\mathrm{D} / \mathrm{K}$

Support Services

PAT dog

PAT dog

PAT dog

PAT dog

Staff

PAT dog

staff
0

0

1

1

1

0

0

0

0

0

0 


\begin{tabular}{|c|c|c|c|}
\hline Haringey & Piglet & $\mathrm{D} / \mathrm{K}$ & 1 \\
\hline Hartlepool & Archie & PAT dog & 1 \\
\hline Hertfordshire & Gibson & Staff & 1 \\
\hline Kent & ? & PAT dog & 1 \\
\hline Lancashire & Ellis & Deputy Head & 1 \\
\hline Lancashire & Albert & Staff & 1 \\
\hline Lancashire & Ellie / Matilda & Teacher $x 2$ & 2 \\
\hline Lancashire & Mervyn & PAT & 1 \\
\hline Leicestershire & $?$ & $\mathrm{D} / \mathrm{K}$ & 1 \\
\hline Lincolnshire & Willow & $\mathrm{D} / \mathrm{K}$ & 1 \\
\hline Luton & Poppy & PAT dog & 1 \\
\hline Manchester & Dora & Head Teacher & 1 \\
\hline Manchester & Buddy & PAT & 1 \\
\hline Manchester & Josh & Dir. of Ed. & 1 \\
\hline Milton Keynes & $?$ & PAT dog & 1 \\
\hline North Lincolnshire & $?$ & TDN Dog & 1 \\
\hline Northamptonshire & Blake & $\mathrm{D} / \mathrm{K}$ & 1 \\
\hline Northamptonshire & Danny & PAT dog & 1 \\
\hline Oldham & Flynn & Head Teacher & 1 \\
\hline
\end{tabular}




\begin{tabular}{|c|c|c|c|c|}
\hline Oldham & Benji & $\mathrm{D} / \mathrm{K}$ & 1 & 1 \\
\hline Oxon & Betsy & Teaching Assistant & 1 & 1 \\
\hline Oxon & ? & Staff $\times 2$ & 2 & 2 \\
\hline Plymouth & Lulu & safeguarding officer & 1 & 0 \\
\hline S.Glos & Travis $+1 /$ Jess & PAT $\operatorname{dog} x 2$ & 2 & 2 \\
\hline Shropshire & ? & $\mathrm{D} / \mathrm{K}$ & 1 & 1 \\
\hline Solihull & Oakleigh* & PAT dog & 1 & 1 \\
\hline Solihull & Oakleigh* & PAT dog & 1 & 1 \\
\hline Solihull & Jasper/Milo/Fozzy & Deputy Head x2 & 2 & 2 \\
\hline South Leeds & Bailey & $\mathrm{D} / \mathrm{K}$ & 1 & 0 \\
\hline Southampton & (was 2PAT now Fudge) & Staff $\times 2$ & 2 & 1 \\
\hline Staffs & Eric & Deputy Head & 1 & 1 \\
\hline Surrey & ? & Staff 3 PAT2 & 5 & 6 \\
\hline Surrey & Tarka & PAT dog & 1 & 0 \\
\hline Sussex & Nobby & $\mathrm{D} / \mathrm{K}$ & 1 & 0 \\
\hline Sussex & Marley & $\mathrm{D} / \mathrm{K}$ & 1 & 0 \\
\hline Swindon & Maisie & Teacher & 1 & 1 \\
\hline Swindon & Buddy & Teaching Assistant & 1 & 0 \\
\hline Wakefield & ? & PAT dog & 1 & 1 \\
\hline
\end{tabular}




\begin{tabular}{|c|c|c|c|c|}
\hline Wandsworth & Faith & PAT & 1 & 0 \\
\hline Warwickshire & Archie & PAT dog & 1 & 0 \\
\hline Warwickshire & Cracker & Teacher & 1 & 1 \\
\hline Warwickshire & Pringle & PAT dog & 1 & 1 \\
\hline Warwickshire & George & Staff & 1 & 1 \\
\hline Warwickshire & Bertie & Therapist & 1 & 1 \\
\hline Warwickshire & ? & PAT dogs 3 & 3 & 0 \\
\hline Weymouth & Ollie & Gracie & 1 & 0 \\
\hline Wigan & Tilly & $\mathrm{D} / \mathrm{K}$ & 1 & 1 \\
\hline Wilts & Freddie & Teaching Assistant & 1 & 0 \\
\hline Wilts & Ollie & Staff Handler & 1 & 0 \\
\hline Wokingham & Bonzo & PAT dog & 1 & 1 \\
\hline Worcestershire & Ralph & $\mathrm{D} / \mathrm{K}$ & 1 & 0 \\
\hline & & Total Dogs & 135 & 73 \\
\hline
\end{tabular}

Key: PAT - Pets as Therapy, D/K - Handler of dog unknown *Dog works in more than one school 
Appendix R Owner /Trainer of dogs

\begin{tabular}{|c|c|c|c|c|c|c|}
\hline School & owned by & Name & 2016 & FT or PT? & Training? & Policy? \\
\hline BANES & PAT dog & Milo & 1 & $\mathrm{P} / \mathrm{T}$ & РAT & No \\
\hline Barnet & Patients' / PAT & ? & 2 & $\mathrm{P} / \mathrm{T}$ both & Ind/PAT & No \\
\hline Barnet & PAT dog & Dexter & 1 & $\mathrm{P} / \mathrm{T}$ & РАT & No \\
\hline Barnet & PAT & ? & 1 & $\mathrm{P} / \mathrm{T}$ & PAT & \\
\hline Bexley & PAT & Tai & 1 & $\mathrm{P} / \mathrm{T}$ & PAT & No \\
\hline Birmingham & students & ? & 3 & $\mathrm{~F} / \mathrm{T}$ & Guide dogs & Yes \\
\hline Blackpool & Assistant Head & Doodles & 1 & $\mathrm{~F} / \mathrm{T}$ & Independent & No \\
\hline Brent & ? & Poppy & 1 & $F / T$ & Autism assist ASD & \\
\hline Brighton\& Hove & Student & Zeb / Cola & 2 & F/T both & Hearing Dog & No \\
\hline Brighton \& Hove & Staff & Millie & 1 & $\mathrm{~F} / \mathrm{T}$ & Independent KC? & No \\
\hline Brighton \& Hove & PAT dog & Elvis & 1 & $\mathrm{P} / \mathrm{T}$ & РАT & No \\
\hline Bristol & PAT dog & Einstein & 1 & $\mathrm{P} / \mathrm{T}$ & PAT & No \\
\hline Bromley & ? & Dudley & 1 & $\mathrm{~F} / \mathrm{T}$ & $\mathrm{D} / \mathrm{K}$ & Yes \\
\hline Buckinghamshire & Staff Handler & Patience & 1 & $\mathrm{~F} / \mathrm{T}$ & Dogs for Good & No \\
\hline Bury & Staff & Angie & 1 & $\mathrm{~F} / \mathrm{T}$ & Buddy Dogs & No \\
\hline
\end{tabular}




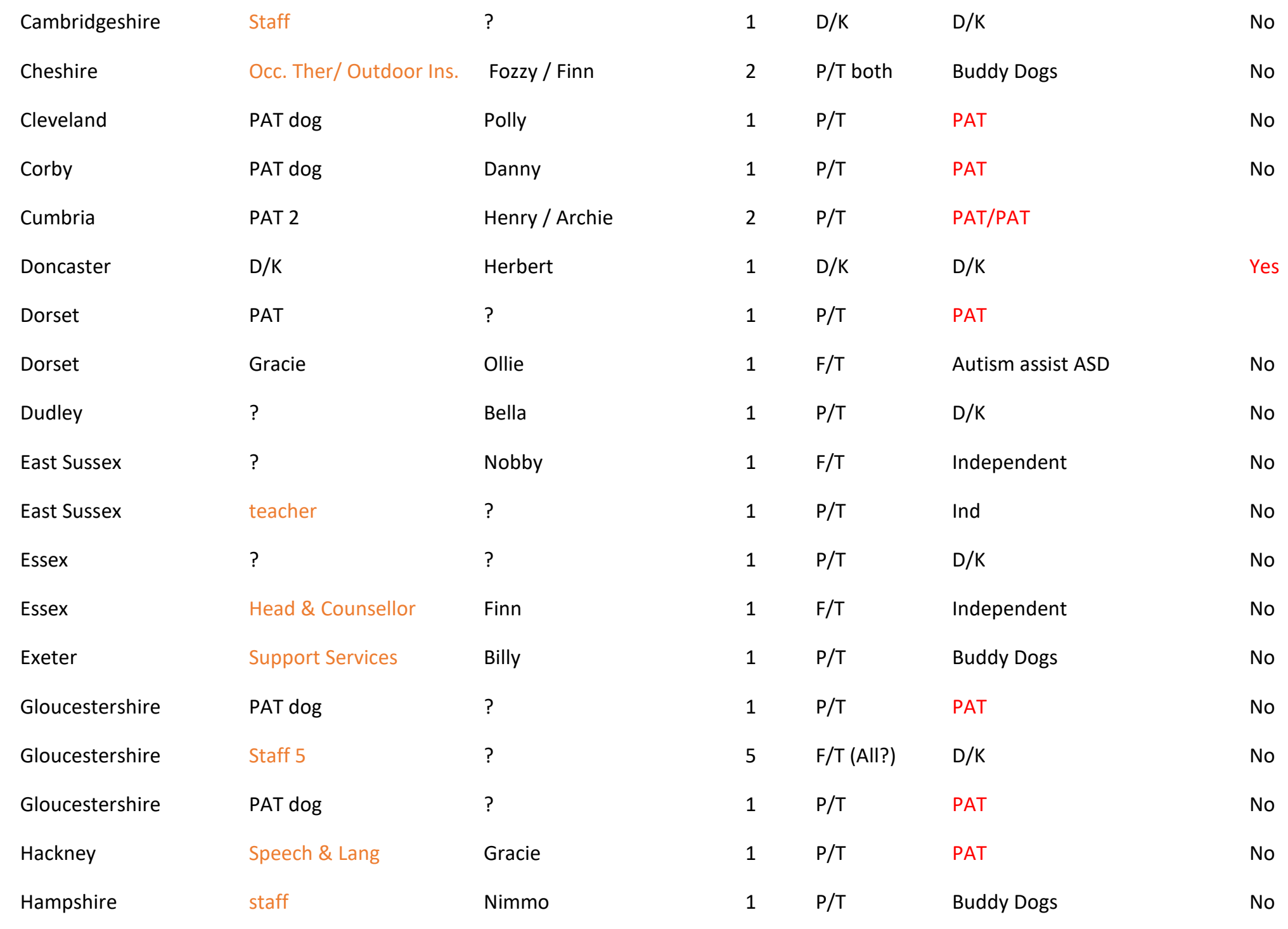




\begin{tabular}{|c|c|c|c|c|c|c|}
\hline Hampshire & PAT dog & Mollie & 1 & $\mathrm{P} / \mathrm{T}$ & PAT & No \\
\hline Hampshire & PAT dog & Murphy & 1 & $\mathrm{P} / \mathrm{T}$ & PAT & No \\
\hline Hampshire & Staff & Nimmo & 1 & $\mathrm{~F} / \mathrm{T}$ & Buddy Dogs & No \\
\hline Hampshire & PAT dog & Perdy & 1 & $\mathrm{P} / \mathrm{T}$ & PAT & No \\
\hline Haringey & $?$ & Piglet & 1 & $\mathrm{P} / \mathrm{T}$ & $\mathrm{D} / \mathrm{K}$ & No \\
\hline Hartlepool & PAT dog & Archie & 1 & $\mathrm{P} / \mathrm{T}$ & PAT & No \\
\hline Herefordshire & Students & Casper (at least) & 2 & $\mathrm{~F} / \mathrm{T}$ & Guide Dogs & Yes \\
\hline Hertfordshire & Staff & Gibson & 1 & $\mathrm{~F} / \mathrm{T}$ & Buddy Dogs & No \\
\hline Kent & PAT dog & $?$ & 1 & $\mathrm{P} / \mathrm{T}$ & PAT & no \\
\hline Kent & PAT dog & ? & 1 & $\mathrm{P} / \mathrm{T}$ & PAT & No \\
\hline Kent & Students & ??? & 3 & $\mathrm{~F} / \mathrm{T}$ & Guide dogs & Yes \\
\hline Kettering & $?$ & Blake & 1 & $\mathrm{~F} / \mathrm{T}$ & Buddy Dogs & No \\
\hline Lancashire & РAT & Mervyn & 1 & $\mathrm{P} / \mathrm{T}$ & PAT & No \\
\hline Lancashire & Teacher / TA & Rufus / Archie & 2 & $\mathrm{P} / \mathrm{T}$ both & $\mathrm{D} / \mathrm{K}$ & no \\
\hline Lancashire & Deputy Head & Ellis & 1 & $\mathrm{~F} / \mathrm{T}$ & Independent & No \\
\hline Lancashire & Staff & Albert & 1 & $\mathrm{~F} / \mathrm{T}$ & Buddy Dogs & No \\
\hline Leicestershire & $?$ & ? & 1 & $\mathrm{D} / \mathrm{K}$ & Guide Dogs & No \\
\hline Lewisham & PAT dog & Jasmine & 1 & $\mathrm{P} / \mathrm{T}$ & PAT & No \\
\hline Lincolnshire & $?$ & Willow & 1 & $\mathrm{P} / \mathrm{T}$ & $\mathrm{D} / \mathrm{K}$ & 0 \\
\hline
\end{tabular}




\begin{tabular}{|c|c|c|c|c|c|c|}
\hline Liverpool & Teacher & Henry & 1 & $\mathrm{~F} / \mathrm{T}$ & Independent & No \\
\hline Luton & PAT dog & William & 1 & $\mathrm{P} / \mathrm{T}$ & PAT & No \\
\hline Luton & PAT dog & Poppy & 1 & $\mathrm{P} / \mathrm{T}$ & PAT & No \\
\hline Manchester & Head Teacher & Dora & 1 & $\mathrm{~F} / \mathrm{T}$ & $\mathrm{D} / \mathrm{K}$ & No \\
\hline Manchester & Dir. of Ed. & Josh & 1 & $\mathrm{~F} / \mathrm{T}$ & Buddy Dogs & No \\
\hline Milton Keynes & PAT dog & ? & 1 & $\mathrm{P} / \mathrm{T}$ & PAT & No \\
\hline North Lincolnshire & TDN Dog & ? & 1 & $\mathrm{P} / \mathrm{T}$ & TDN & No \\
\hline Northumberland & $\mathrm{D} / \mathrm{K}$ & ? & 1 & $\mathrm{D} / \mathrm{K}$ & $\mathrm{D} / \mathrm{K}$ & No \\
\hline Oldham & $\mathrm{D} / \mathrm{K}$ & Benji & 1 & $\mathrm{~F} / \mathrm{T}$ & $\mathrm{D} / \mathrm{K}$ & No \\
\hline Oldham & Head Teacher & Flynn & 1 & $\mathrm{P} / \mathrm{T}$ & $\mathrm{D} / \mathrm{K}$ & No \\
\hline Oxfordshire & Teaching Assistant & Betsy & 1 & $\mathrm{~F} / \mathrm{T}$ & Dogs Helping Kids & No \\
\hline Oxfordshire & PAT dog & ? & 1 & $\mathrm{P} / \mathrm{T}$ & PAT & No \\
\hline Oxfordshire & Staff 2 & ? & 2 & F/T both & Ind / ind & Yes \\
\hline Poole & pup walker/ staff & Moses / PAT dog & 2 & $\mathrm{P} / \mathrm{T}$ both & Canine Partners/PAT & No \\
\hline Plymouth & safeguarding officer & Lulu & 1 & $\mathrm{~F} / \mathrm{T}$ & Dogs Helping Kids & No \\
\hline Shropshire & $\mathrm{D} / \mathrm{K}$ & ? & 1 & $\mathrm{P} / \mathrm{T}$ & $\mathrm{D} / \mathrm{K}$ & No \\
\hline Solihull & PAT dog & Oakleigh & 1 & $\mathrm{P} / \mathrm{T}$ & PAT & No \\
\hline Solihull & PAT dog & Oakleigh & 1 & $\mathrm{P} / \mathrm{T}$ & PAT & No \\
\hline Solihull & Deputy Head & Jasper / Milo / (Fozzy) & 2 & F/T both & PAT/PAT & No \\
\hline
\end{tabular}




\begin{tabular}{|c|c|c|c|c|c|c|}
\hline Somerset & $\mathrm{D} / \mathrm{K}$ & Molly / Lucy & 2 & $\mathrm{~F} / \mathrm{T}$ & $\mathrm{D} / \mathrm{K}$ & No \\
\hline South Gloucestershire & PAT dog2 & Travis +1 / Jess & 2 & $\mathrm{P} / \mathrm{T}$ both & PAT/PAT & No \\
\hline Southampton & Staff 2 & (was 2PAT now Fudge) & 2 & $\mathrm{~F} / \mathrm{T}$ & Independent / PAT & No \\
\hline South Leeds & $\mathrm{D} / \mathrm{K}$ & Bailey & 1 & $\mathrm{P} / \mathrm{T}$ & $\mathrm{D} / \mathrm{K}$ & No \\
\hline Southwark & Staff 4 & Lexi, Lily, Missy, Ted & 4 & $\mathrm{~F} / \mathrm{T}$ all? & РАТ/РАТ/РАТ/РАТ & Yes \\
\hline Staffordshire & Deputy Head & Eric & 1 & $\mathrm{~F} / \mathrm{T}$ & Dogs Helping Kids & No \\
\hline Staffordshire & Teacher & Cracker & 1 & $\mathrm{~F} / \mathrm{T}$ & Buddy Dogs & No \\
\hline Surrey & Staff 3? PAT2 & ? & 5 & $\mathrm{P} / \mathrm{T}$ All? & Independent / PAT & Yes \\
\hline Surrey & PAT dog & Tarka & 1 & $P / T$ & PAT & No \\
\hline Sussex & $\mathrm{D} / \mathrm{K}$ & Marley & 1 & $\mathrm{~F} / \mathrm{T}$ & Independent & No \\
\hline Swindon & Teacher & Maisie & 1 & $P / T$ & PAT & No \\
\hline Swindon & Teaching Assistant & Buddy & 1 & $P / T$ & Independent & No \\
\hline Swindon & Student / Head & Ruby /Mack & 2 & $\mathrm{~F} / \mathrm{T}$ (both) & ASD / Ind & No \\
\hline Trafford & PAT & Buddy & 1 & $P / T$ & PAT & no \\
\hline Tyne \& Wear & PAT & Oscar & 1 & $P / T$ & PAT & No \\
\hline Wandsworth & PAT & Faith & 1 & $P / T$ & PAT & No \\
\hline Warwickshire & PAT dog & Pringle & 1 & $\mathrm{P} / \mathrm{T}$ & PAT & No \\
\hline Warwickshire & Staff & George & 1 & $\mathrm{~F} / \mathrm{T}$ & Buddy Dogs & No \\
\hline Warwickshire & Therapist & Bertie & 1 & $\mathrm{P} / \mathrm{T}$ & Dogs for Good & No \\
\hline
\end{tabular}




\begin{tabular}{|c|c|c|c|c|c|c|}
\hline Warwickshire & PAT dog & Archie & 1 & $\mathrm{P} / \mathrm{T}$ & РAT & No \\
\hline Warwickshire & PAT dogs 3 & ? & 3 & $\mathrm{P} / \mathrm{T}$ all? & РAT / РAT / РAT & No \\
\hline West Buckinghamshire & PAT $\operatorname{dog} 2$ & ? & 2 & $\mathrm{P} / \mathrm{T}$ both & PAT/ PAT & No \\
\hline Wigan & $\mathrm{D} / \mathrm{K}$ & Tilly & 1 & $\mathrm{~F} / \mathrm{T}$ & $\mathrm{D} / \mathrm{K}$ & No \\
\hline Wigan & Teacher 2 & Ellie / Matilda & 2 & $\mathrm{P} / \mathrm{T}$ & $\mathrm{D} / \mathrm{K}$ & No \\
\hline Wiltshire & Teaching Assistant & Freddie & 1 & $\mathrm{~F} / \mathrm{T}$ & $\mathrm{D} / \mathrm{K}$ & No \\
\hline Wiltshire & Staff Handler & Ollie & 1 & $\mathrm{~F} / \mathrm{T}$ & Dogs for Good & No \\
\hline Wirral & PAT dog & Dylan & 1 & $\mathrm{P} / \mathrm{T}$ & PAT & No \\
\hline Wokingham & PAT dog & Bonzo & 1 & $\mathrm{P} / \mathrm{T}$ & PAT & No \\
\hline Worcestershire & $D / K$ & Ralph & 1 & $\mathrm{P} / \mathrm{T}$ & $\mathrm{D} / \mathrm{K}$ & No \\
\hline
\end{tabular}

Key:

D/K- Don't Know - information not given PAT - Pets as Therapy Charity

TDN - Therapy Dogs Nationwide Charity

Ind - Independently trained - training received but source not known 


\section{Appendix S School Policies}

\section{School polvey(1)}

\section{introduction}

The school therapy dog will give educational and emotional benefits to the children at some of the benefits include the potential to calm children, lower anxiety, increase confidence and teaches chlldren to nurture.

The aim of this policy and attached risk assessment demonstrates how the school has thought carefully about having a school therapy dog. The policy will outline the expectations on staff and pupils to keep the children and therapy dog safe.

\section{Principles}

- The school therapy dog will be owned by a nominated member of staff.

- Ongoing costs will be covered by the owner and the school budget will not be effected.

- The dog will be a Labradoodie chosen for their good temperament and non-moulting coat.

- A risk assessment will be completed and reviewed annually.

- Staff and Parents have been made aware of the school therapy dog.

- New parents and staff will be made aware of the school therapy dog.

- The dog wili be crate trained and will travel around school on the lead.

- The dog will have a safe place to stay.

- If the dog is unwell, it should not come into school.

- The dog will be vaccinated and regularly wormed and flead.

- The dog will be trained and work towards a Pets As Therapy certification.

- Dogs express their feelings through their body language. Growling or baring of teeth indicate that the dog is feeling angry or threatened. Flattened ears, tail lowered or between their legs, hiding behind their owner, whining or growling are signs that the dog is frightened or nervous. If the dog is displaying any of these warning signs he should be immediately removed from that particular situation or environment, to it's safe place.

\section{Staff}

- Must always be present with the dog whilst near children.

- Should prevent children from crowding around the dog.

- Who have a known allergy must not go near the dog.

- Should never let a child go near or disturb a sleeping dog

- Must not let children play roughly with the dog.

- Must make sure children are never left alone with the dog.

- Should make sure children do not feed or eat close to the dog.

- Must make sure children always wash their hands after petting the dog 
- Beardies are a medium sized dog, who grow a long coat of hair if the owner wishes them to do so. Their coats change colour for the first few years of their lives.

- They are very enthusiastic dogs who are eager to please and learn.

- Herbert is a member of the Kennel Club.

- Herbert loves to go for walks, and to chase balls.

- Herbert is a very playful, friendly and loving dog. He is still learning as he is so young, and the pupils will be taught to approach and work with Herby appropriately.

- Numerous research studies have supported the benefits dogs may have on children.

- Dogs can enhance children's psychological development, improve social skills, and increase self-esteem among other benefits.

- Dogs can also teach responsibility, compassion, and respect for other living things.

- Dogs in the classroom can be used to calm fears, relieve anxiety, and teach skills.

Physical - interaction with a furry friend reduces blood pressure, provides tactile stimulation, assists with pain management, gives motivation to move, walk and stimulates the senses

Social - a visit with a dog provides a positive mutual topic for discussion, promotes greater self-esteem and well-being, and focused interaction with others

Cognitive - companionship with a dog stimulates memory, problem solving and game playing

Emotional - an adorable four-legged visitor improves self-esteem, acceptance from others, and lifts mood often provoking laughter

Environmental - a dog in a facility decreases the feeling of a sterile environment, lifts mood and this continues after visit

It is inevitable that questions and concerns will be raised when introducing a dog at

The RSPCA estimate that there are approximately 8.5 million dogs kept as pets in the UK. Schools are beginning to recognise how dogs can be an asset to our children's lives, however, it can still be seen as quite 'radical' introducing a dog into a school.

- Allergic reactions - Parents have been asked to state whether their child suffers from allergic reactions, and if they want their child to have access to the dog. School staff will keep a keen eye on pupils, to see if potential allergies are occurring. Herbert is showered and groomed at least once a 


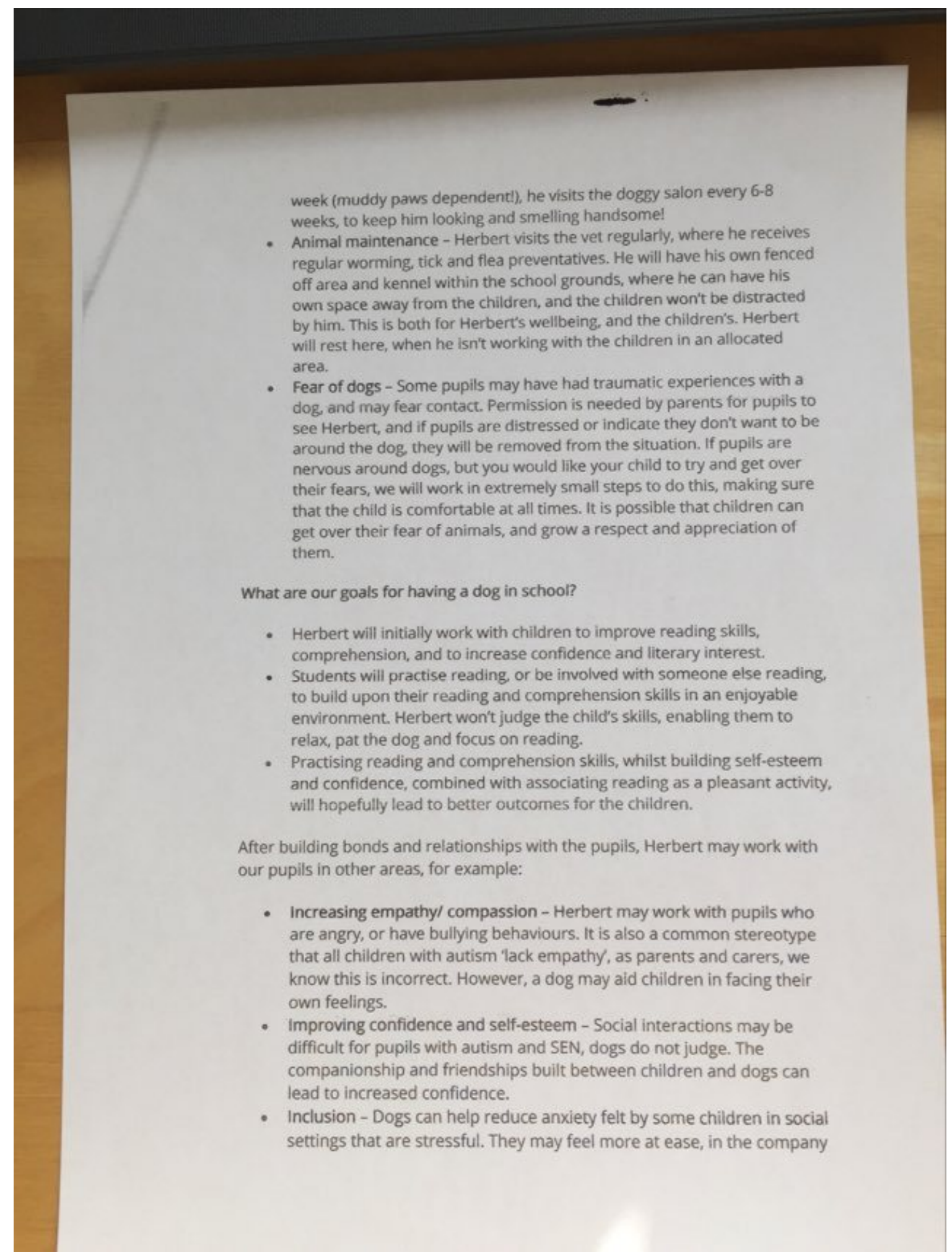




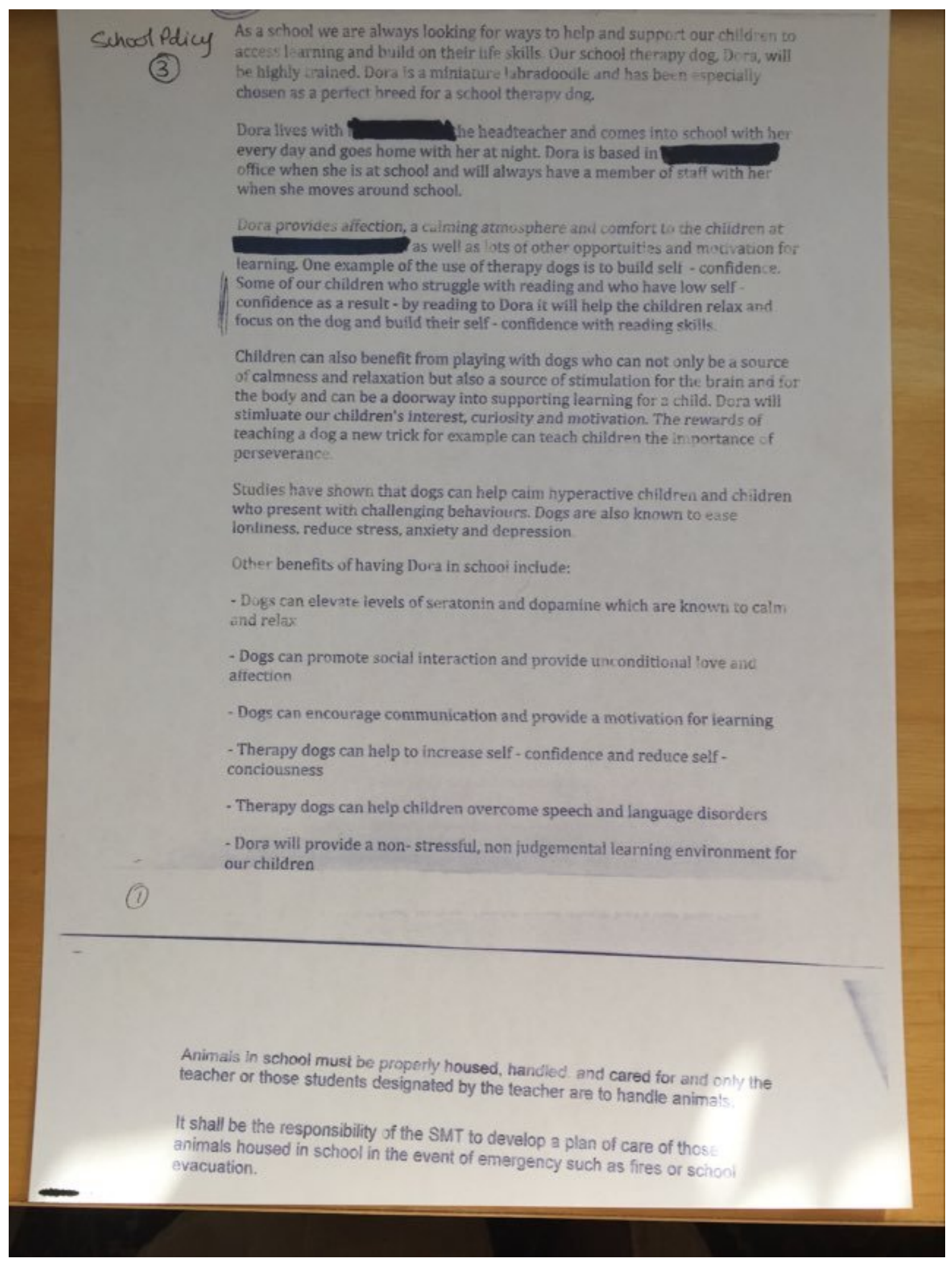




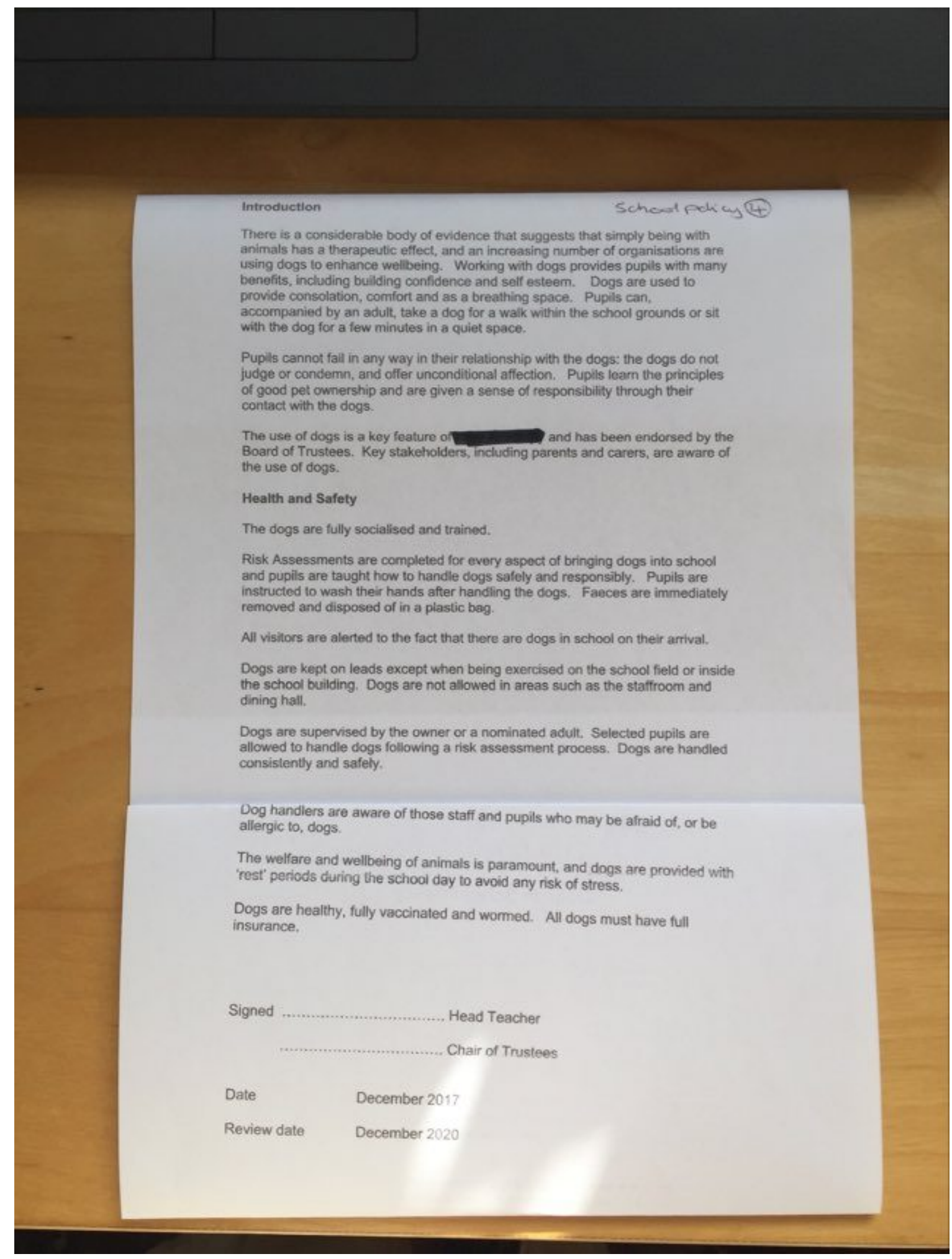


Shoot pdicy/website

(5).

Prior to purchasing the puppy, the Deputy Headteacher has visited a number of breeders for different breeds of dogs and taken advice from a variety of sources before making the final decision. The top priority for any dog has been to ensure that the temperament of the parents was suitable for interaction with children. The breeder that has been selected is a registered and accredited breeder with the Kennel Club and has vast experience of breeding and training therapy dogs that enhance the lives of Autistic children.

To find out more about the chosen breeder please

visit wuw.steadypaws.co.uk. Here you will find information and videos about the work of Andrew Preston. The chosen breed is an English springer spaniel bred from a working line chosen for their very mild temperament and trainability.

It is accepted that interacting with animals is not appropriate for all children but that for some it has the potential to provide many positive benefits. Any parent who does not wish their child to interact with the puppy is invited to write to inform $\mathrm{Mr}$ Lamb of their wishes. This risk assessment will be reviewed regularly and the impact of a school dog will be evaluated by the school senior management team.

Chidren can benefit educationally and emotionally, increase their understanding of responsibility and develop empathy and nurturing skills through contact with a dog. In addition to these benefits, children take great enjoyment from interaction with a dog. By having a dog in school we want to encourage those children specifically who are vulnerable, or those who are less confident with learning to have a friendly audience and to look forward to a challenge. Having a dog in school can encourage reluctant children to come to school. The vast majority of dogs are gentle and loving, offering children opportunities to improve social development skills, unconditional acceptance and the chance to do something really well. For some children, a dog will be a special friend, helping them to build self-esteern, relax and have fun. For others, time spent with a dog will be a reward for excellent effort with a difficult challenge.

Over the past two years we have had weekly visits from a PAT dog (Pets As Therapy) that children have groomed and read storles to. Children who might be embarrassed to read aloud to the class or even adults are likely to be less scared to read to a dog. It might be less stressful for a child to read aloud to a dog than to a teacher or a peer. After all, a dog won't judge or correct you. Dogs are used to encourage struggling readers to practise reading aloud. With the presence of a "calm and well-trained dog" children find social support and peer interaction. Dogs prove to be incredibly calm and happy to have a children read to them or join a group of children in the library whilst they are having a book reading session. Dogs give unconditional acceptance, as they are non-judgmental, which is especially crucial to struggling emerging readers. The dogs also provide confidence to children as they do not make fun of them when they read, but above all they make amazing listeners, providing the children with a sense of comfort and love.

Research has proved that students who read to dogs show an increase in reading levels, word recognition, a higher desire to read and write, and an increase in intra and interpersonal skills among the children they mix with. 
There are many benefits to having a therapy dog in a school like Highturlong. For example, studies have shown that confidence and self esteem are improved in the presence of a school dog. Children who do not have animals at home can

experience collective ownership of an animal and

him

Wathin school Doodies has a large job specification. These are the activities he will Wechin school Docdies has a large job spectication. These are the activities he therapy dog

He wil listen to children read without interrupting which helps to produce contident readers:

readers.

He will give cudides to any children who need it and also be used as a reward for children who have worked exceptionally hard

Doodles will encourage improved speech and language and be used to encourage

descriptive writing.

Doodles will calm those who are frightened of dogs while also teaching respect for animals. He is already being very successful in encouraging our children and young people to take exercise during break times and have fun outside in the fresh air.

Docdles has a put a smile on the faces of the staff and brought an extra feel good factor to the school. 


\section{Animal Assisted Interventions}

The human - animal relationship is significant in supporting emotional well-being. it is also important supporting physical health.

Interactions with animals changes physical and mental states. At Newbridge the students interact with 'Tilly' the dog and many take part in equine assisted learning at a local equine centre.

\section{Canine}

Tilly is an eight year old Golden Retriever who has been working with students at our school since she was very young. Qualified and accredited to undertake the role, Tilly is an important member of the school community. Students 'read' to her, groom her, teach her and sometimes just "be with her'.

Much research has been undertaken into the benefits of animal assisted activities/therapies, in particular:

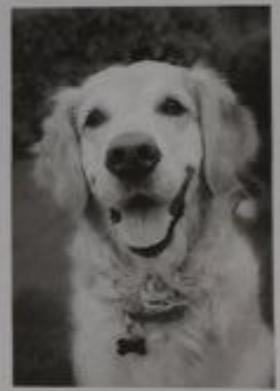

- Positive influence on self - esteem

- Improves self-confidence and self-concept

- Improves attention span

- Improves problem solving skills

- Develops time keeping

- Develops and expands communication

- Encourages team work

- Promotes self-discipline and a sense of responsibility

- Improves learning using the animal (dog or horse) as inspiration and a motivating tool

Equine

The centre is run by Patrick Gracy, a highly competent and recognised member of 'intelligent horsemanship' whose founder is the world renowned and famous 


\section{Appendix T Dog Cam observation Samples}

\section{Matt Cam}

Child 1: $\mathrm{H}$ had forgotten book and the child is upset

Matt on (collar and lead) straight up to the child side on show sniffing child on the beanbag. Child reciprocates putting hand on Matt's head to stroke. Scratch ears and neck. Matt moves head left and right. Sniffs the child's legs and bottom half. Stands in front of child. Child is still scratching ears. Full eye contact on Matt. Matt goes in for a full body sniff-turns to his left, side on to the human. Full body shake with the child's hand on the back of his collar. Turns back to child sniffing shoes and socks. Child still giving ear scratches. Matt moves his head left and right, now child's hand over the back of his neck and is scratching his side upper leg. Researcher comes over, Matt's attention is towards the researcher who is trying to ignore the dog. He is looking at the researcher's hands who is holding the PIP ready for the child. The dog is very much following the researcher's hands as she goes and gives the child the PIP and he sniffs the researcher's hand and looks up at her hands as she withdraws them away from the child. Matt wants to go to the researcher who is still ignoring the dog but is restrained by his collar. He starts to move with the child as the child leaves the room but still restrained by the collar and lead. As the researcher speaks, he turns full-bodied towards her, follows her movement around the room. Is he still watches the researcher's hands.

H now with book

Matt is standing watching researcher handing the PIP to the child who is on a cushion ready to read with the book. He follows the researcher's body as she moves around the room. It centres back on the child when the book is opened. He still follows the researcher's movements away. He is told to lie down with a hand movement from his handler. He sits with his back against the wall and turned his head towards the handler watching the handler. The child starts to read, as the 
child does so, he turns his head and leans towards the child. He looks forward while reading. Matt looks around the room while the child reads. When the child pauses Matt automatically looks to the handler.

He settles into a lying down facing forwards and adjusts himself. When the handler moves to help the child, he is very aware of the movements and he has a clear path of escape and movement but the collar and lead were prevented from leaving.

He settles into a light doze and wiggles and moves his head. He is lying on a front pose but is very attentive to the handler's knees. When the handler adjusted his seating or a stranger walks and quietly he monitors them but still remains settled next to the child's cushion. When the stranger goes and heads for the door Matt is restrained and pulled back by the collar and lead put back into position by the handler. The handler has put his hand on the quality pool. Matt moves back to the child, whines, is watches the handler's signal to lie down which is a click of the fingers and does as he is asked. He gets up to follow the researcher who is going to shut the door and sees a warning finger remains where he is. He sits back down whines five times. He is asked to be quiet and to sit down properly by the handler. Matt's attention and body then turned to the child. Couple more whines and he curls up to settle and then to get comfy with the camera on his back. He starts gentle panting. Then quietly sniffs the floor and put his head on the floor.

The child is asked to stop reading by the handler. Matt's head goes up and he watches the handler's hands. He turns his head between the child and the handler, sits up and avidly watches the handler. Both the handler and the child stroke the back of his neck. He looks at the handler first and then the child, back to the handler and his head definitely goes up at the word "biscuit." He still watching the handler and the child is still stroking the back of his head and ears. The bag rattles, Matt is then up and standing by the handler then he "sits." Matt looks up at the handler's face. He moves back in front of the handler. He looks alternatively at the face of the handler and the child and sits beautifully opposite them. He is watching the "down" signal that he receives and 
then gently takes the biscuit from the child. He then sniffs the floor for crumbs while the child scratches his neck. He lifts his head up from sniffing and checks both the handler and child again to make sure there are no extra crumbs. Matt then moved towards the door and sniffs the shoes. He then watches with full attention researcher as she quietly switches the camera off.

Child 2: $\mathrm{G}$ with book - she settles to read

3:43 Matt settles on his side and gets a quick tummy rub from $G$

7:02 the sudden noise in the room and his head is up while he is still in the down position and he is looking to the source of the noise, he gets up whines goes towards the table and is not really looking at the handler. He first sits and then stands and gets back in ear scratch from G. He looks up at the handler giving a down signal. He is then verbally told to do down. The handler touches the dog's nose to make the dog to sit child's arm is still cuddling and scratching the dog as she reads. Matt settles into a down watching the handler. He has a full head on the floor and he sighs and relaxes. He gets a good from his handler.

7:41 a secondary good from the handler signalling that the child can finish reading has Matt on all four paws standing looking to the handler for food. The child calls him and gives him a cuddle from behind, both sides rubbed but all attention is still on the handler. Matt's eyes follow the handler's hands to the biscuits. The handler is taking too long so Matt walks around under the table and stands next to the hands. He then walks backwards with his eyes intently on the owner's face. He does a beautiful sit and he gets in ear scratch from the child. The biscuit is at the tip of his nose he hopped gently up in the air to eat it while his head was being patted. Straightaway his head is down sniffing for crumbs. He then wanders around the beanbag and sniffs where the child was sitting. He sniffs the mats on the floor, he sniffs the beanbag and sniffs the floor again. He is relaxed in body posture. He then ducks under the handler's legs towards the sand the researcher's voice but is restrained by the lead. The child with two hands and full face on now gives an ear 
tickles. He sniffs into the crouching child before giving a full body shake. He wonders back and forth shaking, sniffing loudly and pushing through the space, even though he is restrained by the lead. He watches the face of the child as she gets up. He moves his head left and right as she plays on the ukulele. He wanders about as much as he can avoiding her legs as he is still restrained by the lead. He faces the door but at the mention of his name, he turns around. Matt appears to like the sounds of the tin whistles - he slowly looks at the child as she goes through the music trolley and all the instruments. As the researcher gets up suddenly he pulls around to her. The child then rubs the dog after cleaning her hands. Matt shakes his head.

10:14 There is lots of whining as the child and researcher leave and his gaze follows them looking at the door.

Child three: E- meet and greet

first 56 seconds.

There's lots of enthusiastic sniffing of the torso and legs of the child as they sit down on the beanbag. Matt is a slightly restrained by the lead, but gets ear scratches in return. He is told (and actually physically moved) to turn around and to settle. But he wants more sniffs of the child. He stepped forward to sniff and also checks at the cushion. He then sneezes and then does a full body shake, followed by a second full body shake. He looks around the room and then at the feet of the researcher. He moves his head between the conversation of the child with the researcher as each person speaks. He whines three times and then tries to move back into the child's body and legs. He lies down facing the child. He gets a stroke on the head, sniffs the child's knee and then turns anticlockwise back to the handler. The handler then uses the lead to getting back "into position." He is told to lie down with the click of the fingers. He sits and watches the researcher help the child with the PiP. He is encouraged to be down, facing the trolley. Gradually his body relaxes and his head goes down onto the floor. 
Child 3 Reading

While she is reading Matt settles really well is quietly snoozing, judging by his breathing.

05:25 "very good" from the handler denoting that the child can stop reading. Matt's head is up and alert, while he remains in the down position. He watches the hands. The handler crosses over the dog's lead to reach the book from the child, and gets an immediate sit from Matt, who is following the hand. Matt whines. Matt gives the handler a full focus "sit" and whines and squeaks at the handler. He gets in ear scratch from the handler and a pat on the head from the child. Matt's full focus is still on the handler. The child strokes him, he gives to whines, and then he is asked if he would like biscuit. This gives a long whine, a full attention "sit" and full concentration on the handler. Matt goes around to the front of the handler when he reaches for biscuit. Matt's eyes are on the table and the handler, ignoring the child completely. He goes back to a sitting position beside the handler and whines. He follows the biscuit with his nose as it is handed to the child. The child holds the biscuit a little above Matt's nose so he has to stretch up to take it. He munches it while facing the handler and gets an ear scratch from the handler himself. Matt takes ages to munch the biscuit as he is really enjoying it. He gives a quick look at the researcher as she goes to the child. His eyes then turn back to the handler, follows the handler's hand while the camera is switched off.

Questions Posed by MattCam...

- Is there a point where the dog gets bored?

- Is there a point with a reading takes over, rather than having the dog present?

- What is the optimal time for the dog/child interaction?

Is the attention span affected by the task and the previous learning?

When reading to the dog, do the reading skills takeover and the dog become secondary? 
Does this depend on how much previous experience the child has had reading and also how much experience the child has had with the dog?

Dog

What is it's about the dog's physical presence? Is it the shape, is it the smell or the feel of the warmth of having a living thing, or is it's the dog's reaction to you? How does the dog learn to ignore/inhibit being distracted?

Burnham et al. 2014, "attentional capture was longer when visual and spatial working memory influenced selective attention, particularly when visual or spatial working memory was loaded specific components of working memory influenced the visual selective attention." 
Appendix U Female A - Session 1 Reading Observations

\begin{tabular}{|c|c|c|c|}
\hline Time & Observed Behaviour - Dog & Time & Observed behaviour - Reader \\
\hline & & 000000 & Sitting on bean bag, book open ready to start. Dog to her right \\
\hline & & 000002 & Wriggles in seat position, scratches forehead \\
\hline \multirow[t]{2}{*}{000005} & Lies prone, belly facing towards reader - at a distance away & & \\
\hline & & 000006 & $\begin{array}{l}\text { Looks at dog, reaches to pet with RH. Smiles. Full eye contact } \\
\text { with dog }\end{array}$ \\
\hline \multirow[t]{2}{*}{000007} & Receives stroke from reader & & \\
\hline & & 000008 & $\begin{array}{l}\text { Gently tickles dog's tummy, moves to get better contact on } \\
\text { bean bag. Eye contact on dog. }\end{array}$ \\
\hline 000011 & Is physically moved closer by handler - same position & 000011 & Can't get good contact so dog is moved by handler \\
\hline
\end{tabular}




\begin{tabular}{|c|c|c|c|}
\hline & & 000015 & Strokes dog's neck, eyes on dog, relaxed body open posture \\
\hline \multirow[t]{3}{*}{000016} & Receives neck stroke from reader & & \\
\hline & & 000018 & Opens book again, leans forward tickles dog's ear \\
\hline & & 000020 & Tickle dog's tummy looks up at researcher's comment - smiles \\
\hline \multirow[t]{2}{*}{000021} & Lies on side "hooks" paw over reader's arm & & \\
\hline & & 000025 & Gently tickles dog's shoulder \\
\hline \multirow[t]{3}{*}{000030} & Dog Cam switched on - no reaction & & \\
\hline & & 000033 & Wipes nose with flat of hand, looks at book \\
\hline & & 000036 & Wriggles on cushion, scratched forehead with $\mathrm{RH}$ \\
\hline 000039 & Pushes left front paw into reader's bean bag & & \\
\hline
\end{tabular}




\begin{tabular}{|c|c|c|c|}
\hline & & 000040 & $\begin{array}{l}\text { Starts to read very quietly (almost inaudible on tape) still } \\
\text { scratching forehead, slow hesitant reading }\end{array}$ \\
\hline & & 000043 & Wiggles feet, hands back on book \\
\hline & & 000045 & Pushes hair out of her eyes R.H -still focused on book \\
\hline & & 000052 & Pushes back hair twice \\
\hline & & 000059 & $\begin{array}{l}\text { Now continually flicking hair our of eyes (hair is too short to be } \\
\text { in them) shift weight on cushion }\end{array}$ \\
\hline & & 000107 & Puts finger on word in book \\
\hline & & 000109 & Back to flicking hair, wiggles feet \\
\hline & & 000123 & Has touched hair 4 times. Then both hands on book \\
\hline & & 000126 & $\begin{array}{l}\text { Stuck on a word - wipes nose with palm of RH. Handler helps } \\
\text { her }\end{array}$ \\
\hline
\end{tabular}




\begin{tabular}{|c|c|c|c|}
\hline & & 000130 & Hand on chest then back to nose while hearing explanation \\
\hline & & 000137 & $\begin{array}{l}\text { Pulls on bottom lip until sure of word 'ok' then both hands on } \\
\text { book }\end{array}$ \\
\hline & & 000142 & Turns page, pushes hair as she does so, back to 2 hands on book \\
\hline & & 000149 & Feet wiggling, still reading pushed hair with $\mathrm{RH}$ \\
\hline & & 000157 & Reads slowly from book but gently flexes pages as she does so \\
\hline & & 000202 & $\begin{array}{l}\text { Uses RH finger to keep place, body turns slightly away from dog } \\
\text { and handler }\end{array}$ \\
\hline & & 000210 & Stuck on word, brushes hair RH, handler helps. Looks at book \\
\hline & & 000216 & Looks at book, says word correctly, gets praise, scratches hair \\
\hline & & 000218 & $\begin{array}{l}\text { Head turns away from handler, looks at book, finger under } \\
\text { words }\end{array}$ \\
\hline
\end{tabular}




\begin{tabular}{|c|c|c|c|}
\hline & & 000221 & Back to hair pushing, voice a little louder \\
\hline \multirow[t]{2}{*}{000224} & Gently pushes paw in and out of bean bag & & \\
\hline & & 000237 & Constant hair pushing, feet wriggling \\
\hline \multirow[t]{7}{*}{000252} & Wriggles into comfy position on left side breathing slows & 000252 & Shows no attention to the dog or even has noticed his wiggling \\
\hline & & 000257 & $\begin{array}{l}\text { Uses finger under word and has good expression, "Oh no!" said } \\
\text { Biff }\end{array}$ \\
\hline & & 000305 & Points out each word then turns the page \\
\hline & & 000312 & Back to pushing hair \\
\hline & & 000318 & Hands back on book, wiggles feet \\
\hline & & 000322 & RH pushed hair back into pony tail carries on reading \\
\hline & & 000330 & Hair twiddling in pony tail while still reading \\
\hline
\end{tabular}




\begin{tabular}{|c|c|c|c|}
\hline & & 000337 & Hand back down across body while she is still reading \\
\hline & & 000344 & $\begin{array}{l}\text { Scratches other arm whole body shifts slightly sideways away } \\
\text { from handler and dog }\end{array}$ \\
\hline & & 000346 & Pushes back hair again fiddles with $\mathrm{RH}$ ear \\
\hline & & 000349 & Hand back in lap. Then back on book \\
\hline & & 000354 & Points under next words with $\mathrm{RH}$ \\
\hline & & 000358 & Wriggles with book, voice a little louder again \\
\hline & & 000406 & Finger pointing to words as she reads Staccato reading \\
\hline & & 000430 & Stuck. Scratches chin. Handler to help. He points to first section \\
\hline & & 000453 & Works with handler to deconstruct "windy day" then repeats it \\
\hline & & 000458 & Finger back on book points to every word. Turns page \\
\hline
\end{tabular}




\begin{tabular}{|c|c|c|c|}
\hline & & 000501 & Hands back in hair, scratching pony tail \\
\hline & & 000508 & Wiggles feet. Rocks forward gently while reading \\
\hline & & 000515 & Twiddles hair then arm across body scrunching jumper \\
\hline & & 000529 & Finger back under words again \\
\hline & & 000550 & Using both hands to underline words on each page, turns page \\
\hline & & 000552 & $\begin{array}{l}\text { Wiggles feet and body - momentary pause while works out next } \\
\text { word }\end{array}$ \\
\hline & & 000550 & Using both hands on book wriggles on cushion \\
\hline & & 000607 & $\begin{array}{l}\text { Pauses. Handler corrects missing word, wriggles index finger } \\
\text { while being corrected }\end{array}$ \\
\hline & & 000614 & Pauses reading, uses index finger to find word, but still stuck \\
\hline & & 000618 & Handler assists again, help her to split word \\
\hline
\end{tabular}




\begin{tabular}{|c|c|c|c|}
\hline 000627 & Wriggles onto front tummy as hearing handler's voice "good" & 000627 & "Good" from handler praising reader \\
\hline & & 000630 & Shows no notice of dog moving and re-settling \\
\hline \multirow[t]{8}{*}{000631} & Looks at reader, stays on belly, looks forward, head on paws & & \\
\hline & & 000635 & Right arm on left wrist still concentrating on book \\
\hline & & 000641 & Wiggles cushion taps fingers on arm while reading \\
\hline & & 000650 & Pauses, then attempts word, putting finger underneath it \\
\hline & & 000652 & $\begin{array}{l}\text { Attempts word again, head beginning to rock, tension now } \\
\text { shown in shoulders }\end{array}$ \\
\hline & & 000657 & Full concentration on page but.. \\
\hline & & 000702 & Long pause, wipes nose with $\mathrm{LH}$, right finger under word \\
\hline & & 000706 & Wipes hand down shirt still looking at word \\
\hline
\end{tabular}




\begin{tabular}{|l|l|l|l|}
\hline & & 000708 & Handler assists, slowly sound out the phonemes together \\
\hline & & 000714 & She bends it and get it - approval from hander, "that's it!" \\
\hline 000723 & Hears handler's voice "good place to stop" is up on all fours, & 000718 & Lots of confidence and speed through next 3 words and finishes \\
\hline & whines and turns to face handler & sentence & \\
\hline 000727 & Looks around room, back to handler, does a "sit" - direct eye & 000727 & Watches dog but remains seated on cushion. Rh up to scratch \\
& contact with handler & ear. & movement towards it. \\
\hline
\end{tabular}


Appendix V Observations between dog and adult participants

1.Interacts when not concentrating. More interaction during vocab, body language closed during maths. Was allergic to dogs!

2.Not a lot of contact with dog, more concentrating on task

3. Pets dog to start but then concentrates. Eye contact then back to task. Says he's relaxed and "dogs having an effect

4.Lots of interaction, stroking dog on the floor giving belly rubs etc. "Dog definitely helps"

5.Dog trying to attract her but is ignored during tasks - lots of petting when over. Subject mouths out her thinking when working

6.Not over familiar with dogs. Dog showed signs of not wanting to be with subject. Tentative strokes only

7.Dog eventually settles. Lots of strokes during vocab which increases to full belly rub. Not in maths where no contact.

8.No interaction with dog. Could do good baseline reading at 11:00. Closed body language throughout. ADHD said later versions meant she was better prepared

9. Lots of interaction, stroking dog. Dog settles for vocab for belly rubs. Lots of eye contact when not looking at the screen

10. Lots of stroking to ears, dog relaxed, lots of eye contact, dog settles on floor

lots of stroking to start but sits back when concentrating, not focused on dog at all.

11. Hardly any interaction. Said hard to read in VR and couldn't see dogs in VR as was concentrating so hard!

12. lots of interaction and stroking ears. Dog wandered off 3 times but returned to participant, settled. Less interaction when doing maths sections

13. lots of slow stroking action, conversations with the dog, very happy dog, belly rubs, dog trying to attract attention, mutual appreciation

14. lots of interaction, gets on floor with dog, dog enjoying interaction got a little over excited but calmed, lots of belly rubs offered "I felt so calm"

15. lots of interest in dog ear tickles chest rubs, dog had difficulty initially settling, lots of smiles, got the dog to settle belly rubs concentrated on question then back to dog, interaction throughout

16. Not over interested in dog more concentration on the exercises. Dog then sat behind chair as no more interaction until the end.

17. lots of interaction ear scratching, chest rubs, eye contact conversations "really cute". Dog wandered off but settled back. Concentrated on question then stroked dog, lots of fuss at end. "Really enjoyable" 
18. lots of petting talking to dog, chest rubs scratching ears, eye contact, during tasks occasional contact while thinking. Uses hands a lot while thinking and talks through process out loud.

19. Lots of eye contact neck rubs and interaction, loose waggy tail around dog shows happy behaviour dog sits at feet lots of long body strokes and tickles full belly rubs. Dog gets up twice to go to handler, back to subject gives kisses! Back into belly rubs - eye contact and stroking in between questions. Dog happy to be with subject.

20. Lots of eye contact, smiles and stroking. Dog immediately happy with subject, straight into full belly rub and relaxed in position throughout, pawing subject if stroking stopped. Subject lots of eye contact and smiles between the questions - clearly liking the experience

21. Lots of eye contact and smiles at dog, lots of ear tickles and body strokes, dog eventually allows full belly rubs, settles at subject's feet. In between questions lots of eye contact and gentle strokes to dog - even after finishing. Dog relaxed in subject's presence

22. Lots of initial ear stroking, dog rooted to spot but allows it. Lots of eye contact. Dog moves forwards to initiate more contact, but participant moves back. Tense body language from subject, very focused on task, dog gives no belly rubs. Eventually invites belly rubs but subject still focused on task, dog eventually gives up and sits waiting to leave

23. Dog sat at feet lots of ear stroked and eye contact, reassuring pats from subject. Dog then moves to handler and vocalises. Both have relaxed body postures, lots of ear tickles, dog then sits behind chair in direction of door (has signalled to handler that dog needs the loo!)

24. Did not seem to want to engage with dog. Dog waited patiently at side of subject, then laid down and waited until end. Closed body language from client - minimal interaction 


\section{Appendix W Tests of parametric assumptions}

Tests of assumption of normal distribution by inspection of frequency distribution. At least some data violated the assumption of normality in a way that would be difficult to correct by transformation (e.g. bimodal distributions).

Test of assumption of sphericity using Mauchly's Test. Assumption violated for at least some data.

No data sets satisfied both assumptions.

Frequency distributions - Vocab scores

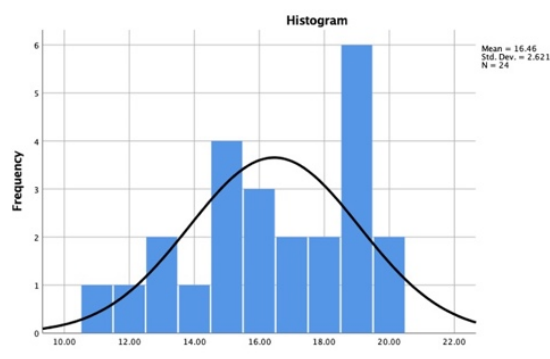

Vocab scores with dog - not VR

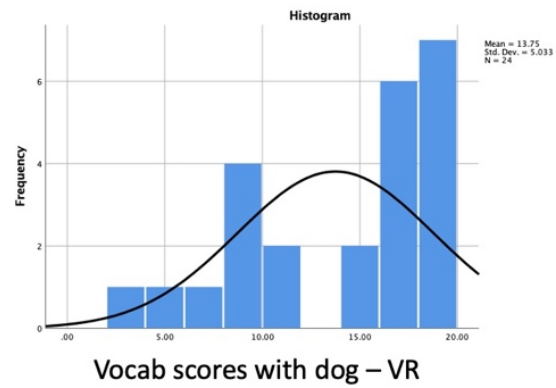

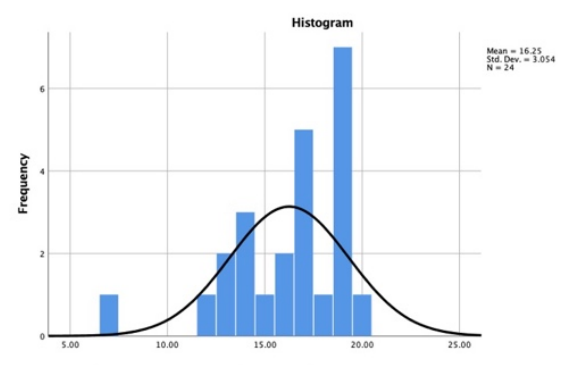

Vocab scores with no dog - not VR

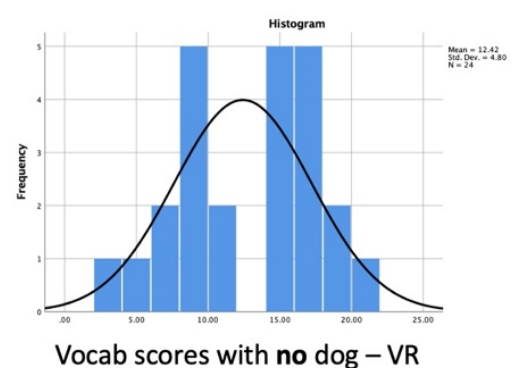




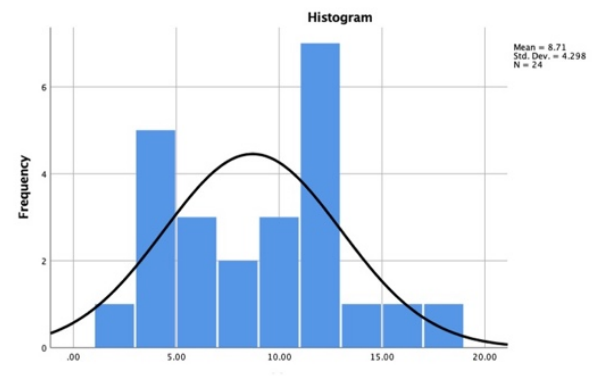

Maths scores with dog - not VR

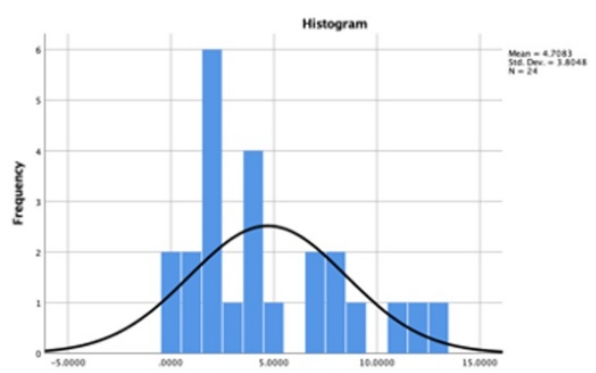

Maths scores with dog - VR
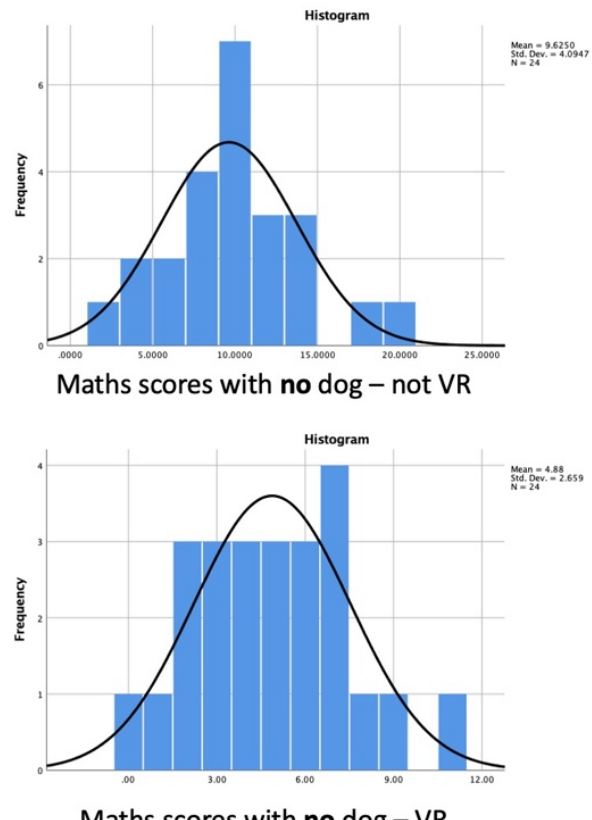

Maths scores with no dog - VR

Frequency distributions - EDA (Pip) scores - Vocab.

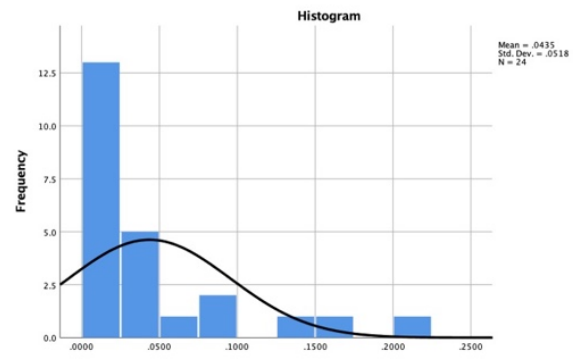

Vocab EDA (Pip) scores with dog - not VR

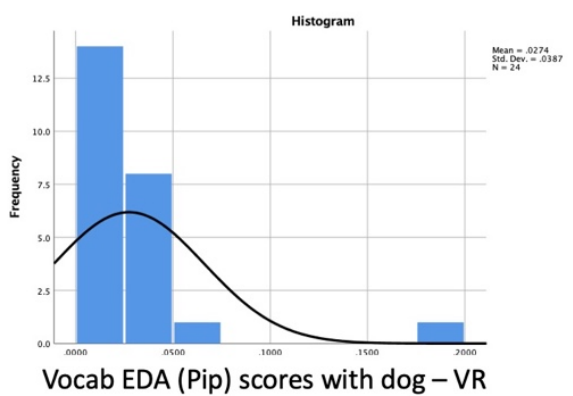

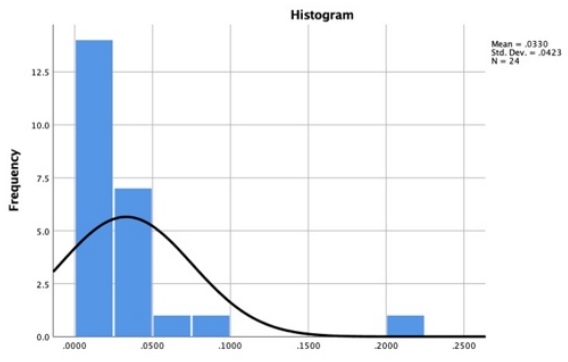

Vocab EDA (Pip)scores with no dog - not VR

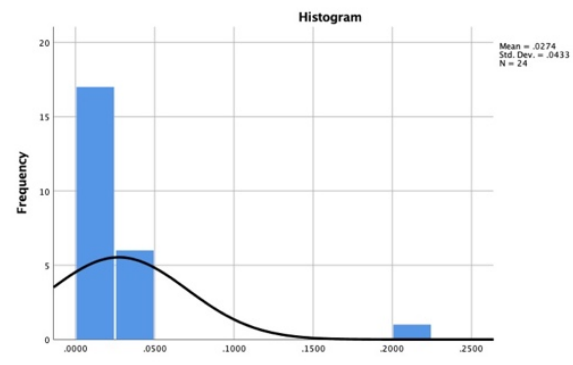

Vocab EDA (Pip) scores with no dog - VR 
Frequency distributions - EDA (Pip) scores - Maths.

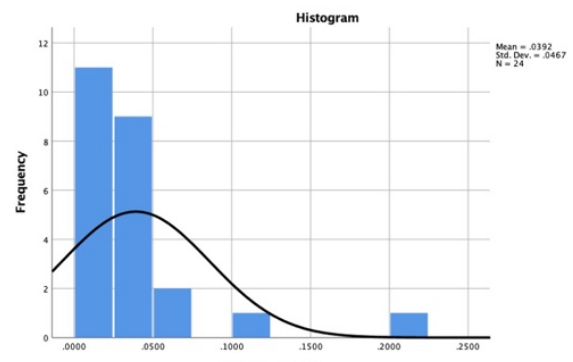

Maths EDA (Pip) scores with dog - not VR

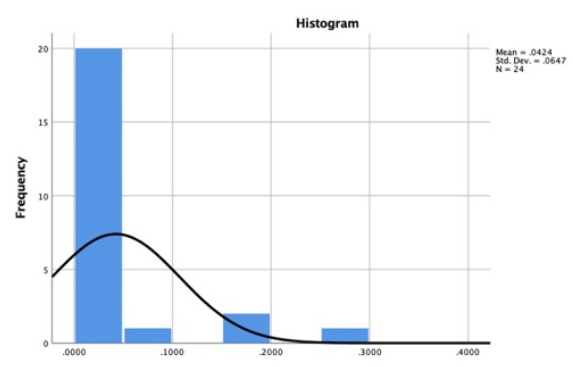

Maths EDA (Pip) scores with dog - VR

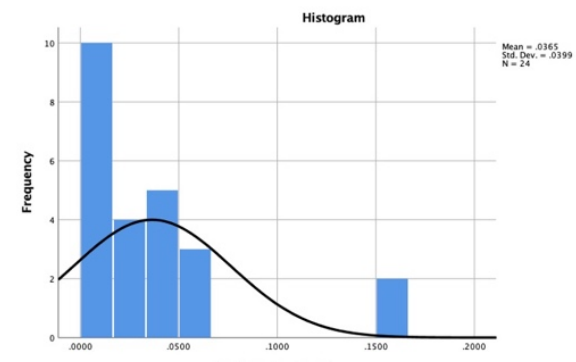

Maths EDA (Pip)scores with no dog - not VR

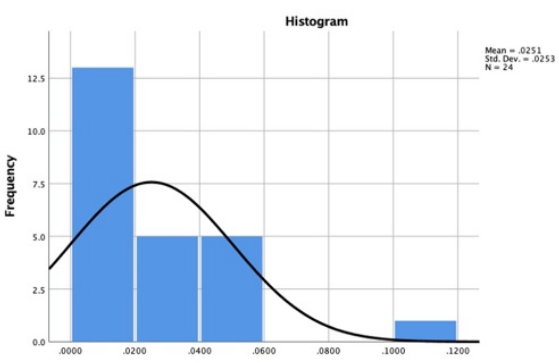

Maths EDA (Pip) scores with no dog - VR 
Appendix $\mathbf{W}$ Tests of the assumption of sphericity

\section{Mauchly's Test of Sphericity}

Measure: MEASURE_1

\begin{tabular}{|c|c|c|c|c|c|c|c|}
\hline \multirow[b]{2}{*}{ Within Subjects Effect } & \multirow[b]{2}{*}{ Mauchly's W } & \multirow[b]{2}{*}{$\begin{array}{l}\text { Approx. Chi- } \\
\text { Square }\end{array}$} & \multirow[b]{2}{*}{ df } & \multirow[b]{2}{*}{ Sig. } & \multicolumn{3}{|c|}{ Epsilon ${ }^{b}$} \\
\hline & & & & & $\begin{array}{c}\text { Greenhouse- } \\
\text { Geisser }\end{array}$ & Huynh-Feldt & Lower-bound \\
\hline Vocab & .309 & 25.478 & 5 & .000 & .577 & .619 & .333 \\
\hline
\end{tabular}

Tests the null hypothesis that the error covariance matrix of the orthonormalized transformed dependent variables is proportional to an identity matrix.

a. Design: Intercept

Within Subjects Design: Vocab

Vocabulary scores 
b. May be used to adjust the degrees of freedom for the averaged tests of significance. Corrected tests are displayed in the Tests of Within-Subjects Effects table.

Maths scores

\section{Mauchly's Test of Sphericity ${ }^{a}$}

Measure: MEASURE_1

Within Subjects Effect Mauchly's W

df

Sig.

Epsilon $^{b}$ 


\begin{tabular}{|c|c|c|c|c|c|c|c|}
\hline & & $\begin{array}{l}\text { Approx. Chi- } \\
\text { Square }\end{array}$ & & & $\begin{array}{c}\text { Greenhouse- } \\
\text { Geisser }\end{array}$ & Huynh-Feldt & Lower-bound \\
\hline Maths & .813 & 4.497 & 5 & .481 & .884 & 1.000 & .333 \\
\hline
\end{tabular}

Tests the null hypothesis that the error covariance matrix of the orthonormalized transformed dependent variables is proportional to an identity matrix.

a. Design: Intercept

Within Subjects Design: Maths

b. May be used to adjust the degrees of freedom for the averaged tests of significance. Corrected tests are displayed in the Tests of Within-Subjects Effects table.

EDA (Pip) Maths scores

\section{Mauchly's Test of Sphericity ${ }^{a}$}


Measure: MEASURE_1

\begin{tabular}{|c|c|c|c|c|c|c|c|}
\hline & & & & & \multicolumn{3}{|c|}{ Epsilon $^{b}$} \\
\hline Within Subjects Effect & Mauchly's W & $\begin{array}{l}\text { Approx. Chi- } \\
\text { Square }\end{array}$ & $d f$ & Sig. & $\begin{array}{c}\text { Greenhouse- } \\
\text { Geisser }\end{array}$ & Huynh-Feldt & Lower-bound \\
\hline EDA_Maths & .627 & 10.136 & 5 & .072 & .767 & .857 & .333 \\
\hline
\end{tabular}

Tests the null hypothesis that the error covariance matrix of the orthonormalized transformed dependent variables is proportional to an identity matrix.

a. Design: Intercept

Within Subjects Design: EDA_Maths

b. May be used to adjust the degrees of freedom for the averaged tests of significance. Corrected tests are displayed in the Tests of Within-Subjects Effects table. 


\section{EDA (Pip) Vocab scores}

\section{Mauchly's Test of Sphericity}

Measure: MEASURE_1

\begin{tabular}{lr|r|r|r|r|r|} 
& & & & \\
Within Subjects Effect & Mauchly's W & $\begin{array}{c}\text { Approx. Chi- } \\
\text { Square }\end{array}$ & df & Sig. & Greenhouse- \\
Geisser & Huynh-Feldt & Lower-bound \\
\hline EDA_Vocab & .234 & 31.580 & 5 & .000 & .650 & .710
\end{tabular}

Tests the null hypothesis that the error covariance matrix of the orthonormalized transformed dependent variables is proportional to an identity matrix.

a. Design: Intercept

Within Subjects Design: EDA_Vocab

b. May be used to adjust the degrees of freedom for the averaged tests of significance. Corrected tests are displayed in the Tests of Within-Subjects Effects table. 


\section{SPSS output}

Vocabulary scores - Friedman test.

\section{Descriptive Statistics}

\begin{tabular}{lr|r|r|r|r} 
& N & Mean & Std. Deviation & Minimum & Maximum \\
\hline VRdogV & 24 & 13.7500 & 5.03250 & 3.00 & 19.00 \\
\hline VRnodogV & 24 & 12.4167 & 4.79961 & 3.00 & 20.00 \\
\hline RealdogV & 24 & 16.4583 & 2.62064 & 11.00 & 20.00 \\
\hline RealnodogV & 24 & 16.2500 & 3.05386 & 7.00 & 20.00 \\
\hline
\end{tabular}

\section{Ranks}

Mean Rank

\begin{tabular}{lc}
\hline VRdogV & 2.25 \\
\hline VRnodogV & 1.65 \\
\hline RealdogV & 3.06 \\
\hline
\end{tabular}




\section{Test Statistics ${ }^{a}$}

\begin{tabular}{lr} 
N & 24 \\
\hline Chi-Square & 22.123 \\
\hline df & 3 \\
\hline Asymp. Sig. & .000 \\
\hline
\end{tabular}

a. Friedman Test 
SPSS output

Wilcoxon signed ranks test

Vocab scores

VR dog v no dog

\section{Descriptive Statistics}

\begin{tabular}{lr|r|r|r|r} 
& N & Mean & \multicolumn{1}{c}{$\begin{array}{c}\text { Std. } \\
\text { Deviation }\end{array}$} & Minimum & Maximum \\
\hline VRdogV & 24 & 13.7500 & 5.03250 & 3.00 & 19.00 \\
\hline VRnodogV & 24 & 12.4167 & 4.79961 & 3.00 & 20.00 \\
\hline
\end{tabular}

\section{Ranks}

\begin{tabular}{llr|r|r} 
& & & Mean Rank & Sum of Ranks \\
\hline VRnodogV - VRdogV & & & & \\
\hline & Negative Ranks & $13^{\mathrm{a}}$ & 9.54 & 124.00 \\
\cline { 2 - 5 } & Positive Ranks & $4^{\mathrm{b}}$ & 7.25 & 29.00 \\
\hline Ties & $7^{\mathrm{c}}$ & & \\
\hline Total & & & \\
\hline
\end{tabular}
a. VRnodog $V<$ VRdogV
b. VRnodogV > VRdogV
c. $V R$ nodogV $=$ VRdogV 


\section{Test Statistics ${ }^{\mathrm{a}}$}

\begin{tabular}{lr} 
& $\begin{array}{c}\text { VRnodogV - } \\
\text { VRdogV }\end{array}$ \\
\hline$Z$ & $-2.259^{\mathrm{b}}$ \\
\hline Asymp. Sig. (2-tailed) & .024 \\
\hline
\end{tabular}

a. Wilcoxon Signed Ranks Test

b. Based on positive ranks. 
SPSS output

Wilcoxon signed ranks test

Vocab scores

Non-VR dog v no dog

Descriptive Statistics

\begin{tabular}{lr|r|r|r|r} 
& N & Mean & $\begin{array}{c}\text { Std. } \\
\text { Deviation }\end{array}$ & Minimum & Maximum \\
\hline RealdogV & 24 & 16.4583 & 2.62064 & 11.00 & 20.00 \\
\hline RealnodogV & 24 & 16.2500 & 3.05386 & 7.00 & 20.00 \\
\hline
\end{tabular}

Ranks

\begin{tabular}{ll|r|r|r} 
& N & Mean Rank & Sum of Ranks \\
\hline RealnodogV - RealdogV Negative Ranks & $9^{\text {a }}$ & 10.17 & 91.50 \\
\cline { 2 - 5 } & Positive Ranks & $9^{\text {b }}$ & 8.83 & 79.50 \\
\hline Ties & $6^{c}$ & & \\
\hline Total & & & \\
\hline
\end{tabular}



a. Realnodog $\mathrm{V}<$ RealdogV
b. Realnodog $V>$ RealdogV
c. Realnodog $\mathrm{V}=$ Realdog $\mathrm{V}$

\section{Test Statistics $^{\mathrm{a}}$}

\begin{tabular}{lr} 
& $\begin{array}{r}\text { RealnodogV - } \\
\text { RealdogV }\end{array}$ \\
\hline Z & $-.265^{\mathrm{b}}$ \\
\hline Asymp. Sig. (2-tailed) & .791 \\
\hline a. Wilcoxon Signed Ranks Test \\
b. Based on positive ranks.
\end{tabular}


SPSS output

Maths scores - Friedman test.

\section{Descriptive Statistics}

\begin{tabular}{lr|r|r|r|r} 
& N & \multicolumn{1}{|c|}{ Mean } & \multicolumn{1}{c}{$\begin{array}{c}\text { Std. } \\
\text { Deviation }\end{array}$} & Minimum & Maximum \\
\hline VRdogM & 24 & 4.708333 & 3.8047929 & .0000 & 13.0000 \\
\hline VRnodogM & 24 & 4.8750 & 2.65907 & .00 & 11.00 \\
\hline RealdogM & 24 & 8.7083 & 4.29842 & 2.00 & 17.00 \\
\hline RealnodogM & 24 & 9.625000 & 4.0946678 & 2.0000 & 19.0000 \\
\hline
\end{tabular}

\section{Ranks}

Mean Rank

\begin{tabular}{lc}
\hline VRdogM & 1.52 \\
\hline VRnodogM & 1.81 \\
\hline RealdogM & 3.15 \\
\hline RealnodogM & 3.52 \\
\hline
\end{tabular}




\section{Test Statistics ${ }^{\mathrm{a}}$}

\begin{tabular}{l|r}
\hline N & 24 \\
\hline Chi-Square & 43.060 \\
\hline df & 3 \\
\hline Asymp. Sig. & .000 \\
\hline
\end{tabular}

a. Friedman Test 
Wilcoxon signed ranks test

Maths scores

VR dog v no dog

Descriptive Statistics

\begin{tabular}{lr|r|r|r|r} 
& \multirow{2}{*}{$\mathrm{N}$} & \multicolumn{1}{c|}{ Mean } & $\begin{array}{c}\text { Std. } \\
\text { Deviation }\end{array}$ & Minimum & Maximum \\
\hline VRdogM & 24 & 4.708333 & 3.8047929 & .0000 & 13.0000 \\
\hline VRnodogM & 24 & 4.8750 & 2.65907 & .00 & 11.00 \\
\hline
\end{tabular}

\begin{tabular}{|c|c|c|c|c|}
\hline \multicolumn{5}{|c|}{ Ranks } \\
\hline & & $\mathrm{N}$ & Mean Rank & Sum of Ranks \\
\hline \multirow[t]{4}{*}{ VRnodogM - VRdogM } & Negative Ranks & $9^{a}$ & 14.83 & 133.50 \\
\hline & Positive Ranks & $14^{b}$ & 10.18 & 142.50 \\
\hline & Ties & $1^{\mathrm{c}}$ & & \\
\hline & Total & 24 & & \\
\hline
\end{tabular}

a. VRnodog $\mathrm{M}<$ VRdog $\mathrm{M}$

b. VRnodogM > VRdogM

c. VRnodogM = VRdogM 


\section{Test Statistics $^{\mathrm{a}}$}

\begin{tabular}{lr} 
& $\begin{array}{c}\text { VRnodogM - } \\
\text { VRdogM }\end{array}$ \\
\hline Z & $-.138^{\mathrm{b}}$ \\
\hline Asymp. Sig. (2-tailed) & .890 \\
\hline
\end{tabular}
a. Wilcoxon Signed Ranks Test
b. Based on negative ranks. 
Wilcoxon signed ranks test

Maths scores

Non-VR dog v no dog

Descriptive Statistics

\begin{tabular}{lr|r|r|r|r} 
& $\mathrm{N}$ & Mean & $\begin{array}{c}\text { Std. } \\
\text { Deviation }\end{array}$ & $\begin{array}{c}\text { Minimu } \\
\mathrm{m}\end{array}$ & $\begin{array}{c}\text { Maximu } \\
\mathrm{m}\end{array}$ \\
\hline RealdogM & 24 & 8.7083 & 4.29842 & 2.00 & 17.00 \\
\hline $\begin{array}{l}\text { Realnodog } \\
\mathrm{M}\end{array}$ & 24 & 9.625000 & 4.0946678 & 2.0000 & 19.0000 \\
\hline
\end{tabular}

Ranks

\begin{tabular}{llr|r|r} 
& $\mathrm{N}$ & $\begin{array}{c}\text { Mean } \\
\text { Rank }\end{array}$ & \multicolumn{1}{c}{$\begin{array}{c}\text { Sum of } \\
\text { Ranks }\end{array}$} \\
\hline $\begin{array}{l}\text { RealnodogM } \\
\text { RealdogM }\end{array}$ & $\begin{array}{l}\text { Negative } \\
\text { Ranks }\end{array}$ & $8^{\mathrm{a}}$ & 8.81 & 70.50 \\
\cline { 2 - 4 } & $\begin{array}{l}\text { Positive } \\
\text { Ranks }\end{array}$ & $13^{\mathrm{b}}$ & 12.35 & 160.50 \\
\hline Ties & & & \\
\hline Total & $3^{\mathrm{c}}$ & & \\
\hline
\end{tabular}

a. Realnodog $\mathrm{M}<$ Realdog $\mathrm{M}$ 
b. RealnodogM > RealdogM

C. Realnodog $M=$ Realdog $M$

\section{Test Statistics ${ }^{a}$}

\begin{tabular}{|c|c|}
\hline & $\begin{array}{l}\text { Realnodog } \\
\qquad \text { M - } \\
\text { RealdogM }\end{array}$ \\
\hline Z & $-1.576^{b}$ \\
\hline $\begin{array}{l}\text { Asymp. Sig. } \quad \text { (2- } \\
\text { tailed) }\end{array}$ & .115 \\
\hline
\end{tabular}
a. Wilcoxon Signed Ranks Test
b. Based on negative ranks. 
Friedman test

Entire session EDA (PiP) scores

\section{Descriptive Statistics}

\begin{tabular}{lr|r|r|r|r} 
& $\mathrm{N}$ & Mean & $\begin{array}{c}\text { Std. } \\
\text { Deviation }\end{array}$ & $\begin{array}{c}\text { Minimu } \\
\mathrm{m}\end{array}$ & $\begin{array}{c}\text { Maximu } \\
\mathrm{m}\end{array}$ \\
\hline VRdogPip & 24 & .037119 & .0489658 & .0024 & .2381 \\
\hline VRnodogPip & 24 & .031659 & .0355876 & .0007 & .1354 \\
\hline RealdogPip & 24 & .048193 & .0586022 & .0035 & .2203 \\
\hline $\begin{array}{l}\text { RealnodogPi } \\
\mathrm{p}\end{array}$ & 24 & .031189 & .0389668 & .0021 & .1872 \\
\hline
\end{tabular}

Ranks

\begin{tabular}{lr} 
& $\begin{array}{l}\text { Mean } \\
\text { Rank }\end{array}$ \\
\hline VRdogPip & 2.42 \\
\hline VRnodogPip & 2.17 \\
\hline RealdogPip & 3.13 \\
\hline $\begin{array}{l}\text { RealnodogPi } \\
\text { p }\end{array}$ & 2.29 \\
\hline
\end{tabular}




\section{Test Statistics ${ }^{a}$}

\begin{tabular}{l|r}
\hline $\mathrm{N}$ & 24 \\
\hline Chi-Square & 7.950 \\
\hline df & 3 \\
\hline Asymp. & .047 \\
Sig. & \\
\hline
\end{tabular}

a. Friedman Test 
Wilcoxon signed ranks test

Entire session EDA (PiP) scores

VR dog v no dog

\section{Descriptive Statistics}

\begin{tabular}{|c|c|c|c|c|c|}
\hline & $\mathrm{N}$ & Mean & $\begin{array}{c}\text { Std. } \\
\text { Deviation }\end{array}$ & $\begin{array}{l}\text { Minimu } \\
\mathrm{m}\end{array}$ & $\begin{array}{l}\text { Maximu } \\
\mathrm{m}\end{array}$ \\
\hline VRdogPip & 24 & .037119 & .0489658 & .0024 & .2381 \\
\hline $\begin{array}{l}\text { VRnodogPi } \\
\mathrm{p}\end{array}$ & 24 & .031659 & .0355876 & .0007 & .1354 \\
\hline
\end{tabular}

Ranks

\begin{tabular}{llr|r|r} 
& & N & $\begin{array}{c}\text { Mean } \\
\text { Rank }\end{array}$ & $\begin{array}{c}\text { Sum of } \\
\text { Ranks }\end{array}$ \\
\hline $\begin{array}{l}\text { VRnodogPip } \\
\text { VRdogPip }\end{array}$ & $\begin{array}{l}\text { Negative } \\
\text { Ranks }\end{array}$ & $15^{\mathrm{a}}$ & 12.07 & 181.00 \\
\cline { 2 - 4 } & $\begin{array}{l}\text { Positive } \\
\text { Ranks }\end{array}$ & $9^{\mathrm{b}}$ & 13.22 & 119.00 \\
\hline Ties & & & \\
\hline Total & $0^{\mathrm{c}}$ & & \\
\hline
\end{tabular}
a. VRnodogPip < VRdogPip
b. VRnodogPip > VRdogPip
c. VRnodogPip = VRdogPip 


\section{Test Statistics ${ }^{a}$}

\begin{tabular}{lrr} 
& & $\begin{array}{r}\text { VRnodogPip } \\
\text { - VRdogPip }\end{array}$ \\
\hline$Z$ & & $-.886^{\mathrm{b}}$ \\
\hline $\begin{array}{l}\text { Asymp. Sig. } \\
\text { tailed) }\end{array}$ & & \\
\hline
\end{tabular}
a. Wilcoxon Signed Ranks Test
b. Based on positive ranks. 
Wilcoxon signed ranks test

Entire session EDA (PiP) scores

Non-VR dog v no dog

Descriptive Statistics

\begin{tabular}{l|c|c|c|r|r} 
& $\mathrm{N}$ & Mean & $\begin{array}{c}\text { Std. } \\
\text { Deviation }\end{array}$ & $\begin{array}{c}\text { Minimu } \\
\mathrm{m}\end{array}$ & $\begin{array}{c}\text { Maximu } \\
\mathrm{m}\end{array}$ \\
\hline RealdogPip & 24 & .048193 & .0586022 & .0035 & .2203 \\
\hline $\begin{array}{l}\text { RealnodogPi } \\
\mathrm{p}\end{array}$ & 24 & .031189 & .0389668 & .0021 & .1872 \\
\hline
\end{tabular}

Ranks

\begin{tabular}{llr|r|r} 
& $\mathrm{N}$ & $\begin{array}{c}\text { Mean } \\
\text { Rank }\end{array}$ & $\begin{array}{c}\text { Sum of } \\
\text { Ranks }\end{array}$ \\
\hline $\begin{array}{l}\text { RealnodogPip } \\
\text { RealdogPip }\end{array}$ & $\begin{array}{l}\text { Negative } \\
\text { Ranks }\end{array}$ & $18^{\mathrm{a}}$ & 13.94 & 251.00 \\
\cline { 2 - 3 } & $\begin{array}{l}\text { Positive } \\
\text { Ranks }\end{array}$ & $6^{\mathrm{b}}$ & 8.17 & 49.00 \\
\hline Ties & & & \\
\hline Total & $0^{\mathrm{c}}$ & & \\
\hline
\end{tabular}

a. RealnodogPip < RealdogPip 

b. RealnodogPip > RealdogPip
c. RealnodogPip = RealdogPip

\section{Test Statistics ${ }^{a}$}

\begin{tabular}{lr} 
& $\begin{array}{c}\text { RealnodogP } \\
\text { ip - } \\
\text { RealdogPip }\end{array}$ \\
\hline$Z$ & $-2.886^{\mathrm{b}}$ \\
\hline $\begin{array}{l}\text { Asymp. Sig. (2- } \\
\text { tailed) }\end{array}$ & .004 \\
\hline $\begin{array}{l}\text { a. Wilcoxon Signed Ranks Test } \\
\text { b. Based on positive ranks. }\end{array}$
\end{tabular}


SPSS output

Vocabulary EDA (Pip) - Friedman test.

\section{Descriptive Statistics}

\begin{tabular}{l|r|r|r|r|r} 
& & & $\begin{array}{c}\text { Std. } \\
\text { Deviation }\end{array}$ & $\begin{array}{c}\text { Minimu } \\
\mathrm{m}\end{array}$ & $\begin{array}{c}\text { Maximu } \\
\mathrm{m}\end{array}$ \\
\hline VocabVRdogPip & 24 & .027408 & .0386792 & .0025 & .1968 \\
\hline $\begin{array}{l}\text { VocabVRnodogPi } \\
\mathrm{p}\end{array}$ & 24 & .027353 & .0432529 & .0011 & .2183 \\
\hline $\begin{array}{l}\text { VocabRealdogPip } \\
\text { VocabRealnodog }\end{array}$ & 24 & .043543 & .0518131 & .0021 & .2065 \\
\hline \begin{tabular}{l} 
Pip \\
\hline
\end{tabular} & 24 & .032975 & .0423211 & .0029 & .2076 \\
\hline
\end{tabular}

\section{Ranks}

Mean

Rank

\begin{tabular}{ll}
\hline VocabVRdogPip & 2.33 \\
\hline $\begin{array}{l}\text { VocabVRnodogPi } \\
p\end{array}$ & 2.06 \\
\hline VocabRealdogPip & 3.08
\end{tabular}


VocabRealnodog $\quad 2.52$

Pip

\section{Test Statistics ${ }^{a}$}

\begin{tabular}{lr} 
N & 24 \\
\hline Chi-Square & 8.096 \\
\hline df & 3 \\
\hline $\begin{array}{l}\text { Asymp. } \\
\text { Sig. }\end{array}$ & .044 \\
\hline
\end{tabular}

a. Friedman Test 
Wilcoxon signed ranks test

Vocabulary EDA (PiP) scores

VR dog v no dog

\section{Descriptive Statistics}

\begin{tabular}{|c|c|c|c|c|c|}
\hline & $\mathrm{N}$ & Mean & $\begin{array}{c}\text { Std. } \\
\text { Deviation }\end{array}$ & $\begin{array}{l}\text { Minimu } \\
\mathrm{m}\end{array}$ & $\begin{array}{l}\text { Maximu } \\
\mathrm{m}\end{array}$ \\
\hline VocabVRdogPip & 24 & .027408 & .0386792 & .0025 & .1968 \\
\hline $\begin{array}{l}\text { VocabVRnodog } \\
\text { Pip }\end{array}$ & 24 & .027353 & .0432529 & .0011 & .2183 \\
\hline
\end{tabular}

Ranks

\begin{tabular}{llr|r|r} 
& $\mathrm{N}$ & $\begin{array}{c}\text { Mean } \\
\text { Rank }\end{array}$ & $\begin{array}{c}\text { Sum of } \\
\text { Ranks }\end{array}$ \\
\hline $\begin{array}{l}\text { VocabVRnodogPip } \\
\text { VocabVRdogPip }\end{array}$ & $\begin{array}{l}\text { Negative } \\
\text { Ranks }\end{array}$ & $14^{\mathrm{a}}$ & 11.86 & 166.00 \\
\cline { 2 - 4 } & $\begin{array}{l}\text { Positive } \\
\text { Ranks }\end{array}$ & $10^{\mathrm{b}}$ & 13.40 & 134.00 \\
& Ties & $0^{\mathrm{c}}$ & & \\
\hline & & & \\
\hline Total & 24 & & \\
\hline
\end{tabular}



a. VocabVRnodogPip < VocabVRdogPip
b. VocabVRnodogPip > VocabVRdogPip
c. VocabVRnodogPip = VocabVRdogPip

\section{Test Statistics ${ }^{a}$}

\begin{tabular}{|c|c|}
\hline & $\begin{array}{c}\text { VocabVRno } \\
\text { dogPip - } \\
\text { VocabVRdo } \\
\text { gPip }\end{array}$ \\
\hline Z & $-.457^{b}$ \\
\hline $\begin{array}{l}\text { Asymp. Sig. (2- } \\
\text { tailed) }\end{array}$ & .648 \\
\hline
\end{tabular}

a. Wilcoxon Signed Ranks Test

b. Based on positive ranks. 
Wilcoxon signed ranks test

Vocabulary EDA (PiP) scores

Non-VR dog v no dog

\section{Descriptive Statistics}

\begin{tabular}{l|c|c|c|c|c} 
& $\mathrm{N}$ & Mean & $\begin{array}{c}\text { Std. } \\
\text { Deviation }\end{array}$ & $\begin{array}{c}\text { Minimu } \\
\mathrm{m}\end{array}$ & $\begin{array}{c}\text { Maximu } \\
\mathrm{m}\end{array}$ \\
\hline VocabRealdogPip & 24 & .043543 & .0518131 & .0021 & .2065 \\
\hline $\begin{array}{l}\text { VocabRealnodog } \\
\text { Pip }\end{array}$ & 24 & .032975 & .0423211 & .0029 & .2076 \\
\hline
\end{tabular}

\section{Ranks}

\begin{tabular}{llr|r|r} 
& $\mathrm{N}$ & $\begin{array}{c}\text { Mean } \\
\text { Rank }\end{array}$ & $\begin{array}{c}\text { Sum of } \\
\text { Ranks }\end{array}$ \\
\hline $\begin{array}{l}\text { VocabRealnodogPip } \\
\text { VocabRealdogPip }\end{array}$ & \begin{tabular}{ll|l} 
Negative \\
Ranks
\end{tabular} & $16^{\mathrm{a}}$ & 12.81 & 205.00 \\
\cline { 2 - 4 } & $\begin{array}{l}\text { Positive } \\
\text { Ranks }\end{array}$ & $8^{\mathrm{b}}$ & 11.88 & 95.00 \\
\hline Ties & $0^{\mathrm{c}}$ & & \\
\hline Total & & & \\
\hline
\end{tabular}



a. VocabRealnodogPip < VocabRealdogPip
b. VocabRealnodogPip > VocabRealdogPip
c. VocabRealnodogPip $=$ VocabRealdogPip

\section{Test Statistics ${ }^{a}$}

\begin{tabular}{lrr} 
& $\begin{array}{c}\text { VocabRealn } \\
\text { odogPip - } \\
\text { VocabReald } \\
\text { ogPip }\end{array}$ \\
\hline$Z$ & & $-1.571^{\mathrm{b}}$ \\
\hline $\begin{array}{l}\text { Asymp. } \\
\text { tailed })\end{array}$ & & \\
\hline
\end{tabular}
a. Wilcoxon Signed Ranks Test
b. Based on positive ranks. 


\section{Descriptive Statistics}

\begin{tabular}{l|r|r|r|r|r} 
& & & \multicolumn{1}{c}{$\begin{array}{c}\text { Std. } \\
\text { Deviation }\end{array}$} & $\begin{array}{c}\text { Minimu } \\
\mathrm{m}\end{array}$ & $\begin{array}{c}\text { Maximu } \\
\mathrm{m}\end{array}$ \\
\hline MathsVRdogPip & 24 & .042433 & .0646778 & .0016 & .2804 \\
\hline $\begin{array}{l}\text { MathsVRnodogPi } \\
\mathrm{p}\end{array}$ & 24 & .025080 & .0253016 & .0003 & .1057 \\
\hline $\begin{array}{l}\text { MathsRealdogPip } \\
\text { MathsRealnodog }\end{array}$ & 24 & .039182 & .0466699 & .0043 & .2246 \\
\hline \begin{tabular}{l} 
Pip \\
\hline
\end{tabular} & 24 & .036466 & .0399354 & .0009 & .1548 \\
\hline
\end{tabular}

\section{Ranks}

\begin{tabular}{lr} 
& $\begin{array}{c}\text { Mean } \\
\text { Rank }\end{array}$ \\
\hline MathsVRdogPip & 2.46 \\
\hline $\begin{array}{l}\text { MathsVRnodogPi } \\
\text { p }\end{array}$ & 2.02 \\
\hline $\begin{array}{l}\text { MathsRealdogPip } \\
\text { MathsRealnodog }\end{array}$ & 3.00 \\
\hline \begin{tabular}{l} 
Pip \\
\hline
\end{tabular}
\end{tabular}




\section{Test Statistics ${ }^{a}$}

\begin{tabular}{l|r}
\hline $\mathrm{N}$ & 24 \\
\hline Chi-Square & 6.967 \\
\hline df & 3 \\
\hline Asymp. & .073 \\
Sig. & \\
\hline
\end{tabular}

a. Friedman Test 
Wilcoxon signed ranks test

Maths EDA (PiP) scores

VR dog v no dog

\section{Descriptive Statistics}

\begin{tabular}{lr|r|r|r|r} 
& & & $\begin{array}{c}\text { Std. } \\
\text { Deviation }\end{array}$ & $\begin{array}{c}\text { Minimu } \\
\mathrm{m}\end{array}$ & $\begin{array}{c}\text { Maximu } \\
\mathrm{m}\end{array}$ \\
\hline MathsVRdogPip & 24 & .042433 & .0646778 & .0016 & .2804 \\
\hline $\begin{array}{l}\text { MathsVRnodog } \\
\text { Pip }\end{array}$ & 24 & .025080 & .0253016 & .0003 & .1057 \\
\hline
\end{tabular}

\section{Ranks}

\begin{tabular}{|c|c|c|c|c|}
\hline & & $\mathrm{N}$ & $\begin{array}{l}\text { Mean } \\
\text { Rank }\end{array}$ & $\begin{array}{l}\text { Sum of } \\
\text { Ranks }\end{array}$ \\
\hline \multirow[t]{4}{*}{$\begin{array}{l}\text { MathsVRnodogPip } \\
\text { MathsVRdogPip }\end{array}$} & $\begin{array}{l}\text { - Negative } \\
\text { Ranks }\end{array}$ & $14^{a}$ & 13.71 & 192.00 \\
\hline & $\begin{array}{l}\text { Positive } \\
\text { Ranks }\end{array}$ & $10^{b}$ & 10.80 & 108.00 \\
\hline & Ties & $0^{c}$ & & \\
\hline & Total & 24 & & \\
\hline
\end{tabular}



a. MathsVRnodogPip < MathsVRdogPip
b. MathsVRnodogPip > MathsVRdogPip
c. MathsVRnodogPip = MathsVRdogPip

\section{Test Statistics ${ }^{a}$}

\begin{tabular}{|c|c|}
\hline & $\begin{array}{c}\text { MathsVRno } \\
\text { dogPip - } \\
\text { MathsVRdo } \\
\text { gPip }\end{array}$ \\
\hline Z & $-1.200^{b}$ \\
\hline $\begin{array}{l}\text { Asymp. Sig. (2- } \\
\text { tailed) }\end{array}$ & .230 \\
\hline
\end{tabular}
a. Wilcoxon Signed Ranks Test
b. Based on positive ranks. 
Wilcoxon signed ranks test

Maths EDA (PiP) scores

Non-VR dog v no dog

\section{Descriptive Statistics}

\begin{tabular}{lr|r|r|r|r} 
& & & \multicolumn{1}{|c|}{$\begin{array}{c}\text { Std. } \\
\text { Deviation }\end{array}$} & $\begin{array}{c}\text { Minimu } \\
\mathrm{m}\end{array}$ & $\begin{array}{c}\text { Maximu } \\
\mathrm{m}\end{array}$ \\
\hline MathsRealdogPip & 24 & .039182 & .0466699 & .0043 & .2246 \\
\hline $\begin{array}{l}\text { MathsRealnodog } \\
\text { Pip }\end{array}$ & 24 & .036466 & .0399354 & .0009 & .1548 \\
\hline
\end{tabular}

Ranks

\begin{tabular}{|c|c|c|c|c|}
\hline & & $\mathrm{N}$ & $\begin{array}{l}\text { Mean } \\
\text { Rank }\end{array}$ & $\begin{array}{l}\text { Sum of } \\
\text { Ranks }\end{array}$ \\
\hline \multirow[t]{3}{*}{$\begin{array}{l}\text { MathsRealnodogPip - } \\
\text { MathsRealdogPip }\end{array}$} & $\begin{array}{l}\text { Negative } \\
\text { Ranks }\end{array}$ & $15^{\mathrm{a}}$ & 11.27 & 169.00 \\
\hline & $\begin{array}{l}\text { Positive } \\
\text { Ranks }\end{array}$ & $9^{b}$ & 14.56 & 131.00 \\
\hline & Ties & $0^{c}$ & & \\
\hline
\end{tabular}


a. MathsRealnodogPip < MathsRealdogPip

b. MathsRealnodogPip > MathsRealdogPip

c. MathsRealnodogPip $=$ MathsRealdogPip

\section{Test Statistics ${ }^{a}$}

\begin{tabular}{lrr} 
& $\begin{array}{c}\text { MathsRealn } \\
\text { odogPip - } \\
\text { MathsReald } \\
\text { ogPip }\end{array}$ \\
\hline$Z$ & & $-.543^{\text {b }}$ \\
\hline $\begin{array}{l}\text { Asymp. Sig. } \\
\text { tailed) }\end{array}$ & (2- & .587 \\
\hline
\end{tabular}
a. Wilcoxon Signed Ranks Test
b. Based on positive ranks. 